xin.

Straits Settlements and Federated

Malay States Agri-Horticultural

Show, 1904 - 1910 


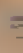

$-1$ $+4$

STRAITS SETTLEMENTS

$A(1)$

FEDERATED MALAY STATES.

\section{THE SECOND JOINT-ANNUAL}

\section{Agri-Morticulural Show. 1905.}

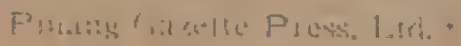





\section{PENANG \\ Agri-Horticultural Show, 1905.}

\section{Programme for Division D.}

Classes $1,2,3,6,9,14$ will be judged on Wednesday morning. Judging commencing at 7 o'clock.

Classes 4, 5, will be judged on Wednesday afternoon. Judging commencing at 5 o'clock.

Classes $7,8,12,13,15$ will be judged on Thursday morning. Judging commencing at 7 o'clock.

Classes 10,11, will be judged on Thursday afternoon. Judging commencing at 4:45.

All exhibits must be on the Show Ground each day not later than 6-30 a.m.

G. H. STITT, Hon. Secretary. 

Class.

1. Polo Pony, registered $\mathbf{1 4 . 1}$ or under: the bom fide proporty of $n$ member of my recormised Polo Club in the Colony or the Federation Malia States To be shown moler saddle, and tested with stick :m-1 ball if required.

1st. Prize. A cup presented by Butterworth Po'o Club. 2ud. Prize. Valuo $\$ 10$

Exhibitor's Nos.

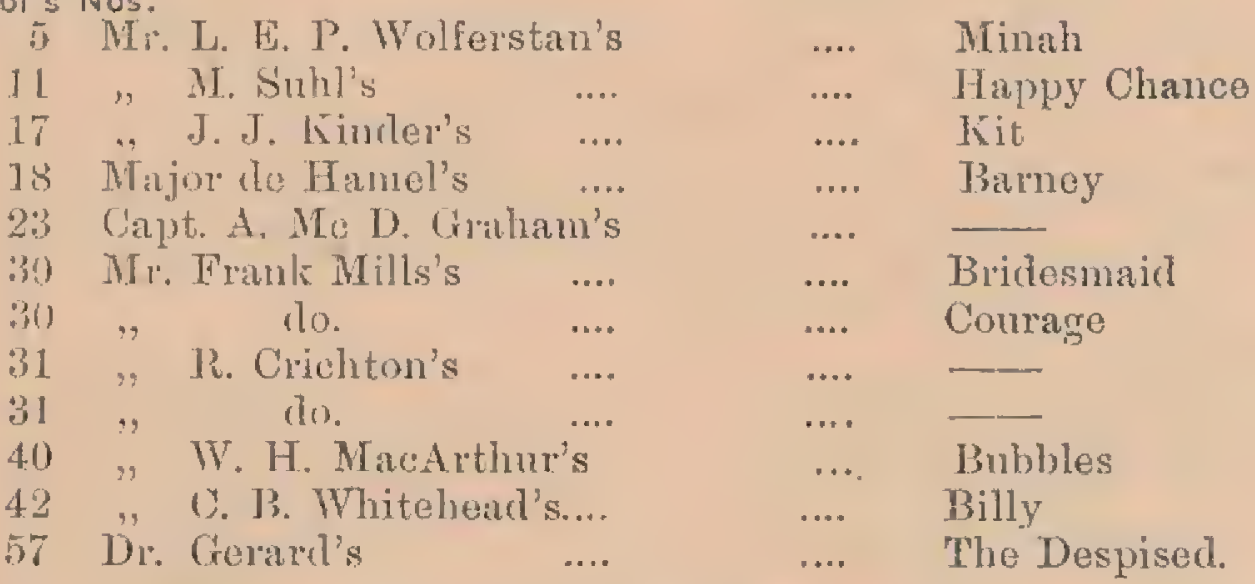

2. Gentleman's Hack, 14.2 and under: to be shown under saddle. 1st Prize. A cup, presented liy O. V. Thomas, Esq. 2ud Prize. Valne $\$ 10$.

\section{Exhibitor's Nos.}

2 Capt. Macelntyre's

1. Mr.M. Suhl's

17 Mr. F. J. Kinder's

18 Cipt. Molynenx's

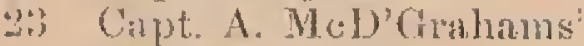

:30 Mr. Fomk Mills?

2 Capt. Maclutyre's

3 Hon. ol. Brombend Mathews'

$40 \mathrm{Mr}$. IV. H. Macistliurs'

42 Mr. C. B. Whiteheal's

34 Mr. C. H. Lees..

47 Rajah Mudah, Kedtah

$49 \mathrm{Mr}, \mathrm{M}$. R. Thornton's

50 Dr. Travers'

i) Miss Anterson's

it Dr. Geratris

$\begin{array}{lll} & & \\ \ldots & \ldots & \text { Sadi } \\ \ldots & \ldots & \text { Happy Chance } \\ \ldots & \ldots & \text { Kit } \\ \ldots & \ldots & \text { The Friar } \\ \ldots & \ldots & \text { Counge } \\ \ldots & \ldots & \\ \ldots & \ldots & \text { Comedian } \\ \ldots & \ldots & \text { Bubbles } \\ \ldots & \ldots & \text { Billy } \\ \ldots & \ldots & \text { Boxer } \\ \ldots & \ldots & \text { Rex } \\ \ldots & \ldots & \text { Georgina } \\ \ldots & \ldots & \text { Bushrat } \\ \ldots & \ldots & \text { The Despised }\end{array}$


3. Gentloman's Hack urer 14.2 to bo show'u moler satdle.

P'rize. A cmp' presenterl hy J. E. Berkhnijsen, Escl.

Exhititor's Nos.

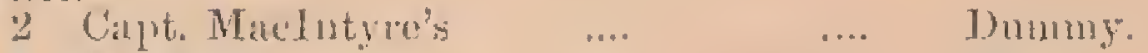

4 Mr. C. Sugrlen's $\quad$... $\quad$... Masetom.

1: Mrs. G. H. Stitt's N... ... Patrick.

29) Mr. H. H. Hudsom's ...

2 Capt. Marelutyre's $\quad$... $\quad$...

37 Mr. H. Learsm's

4. Mr. 1:. 1. Tanw's

$48 \mathrm{Mr} .0$. Sieleken's

48 Do.

13 Mr. (t, H. Stitt's

5.) M.. J. Pickunpack's

....

... The Bam.

... Manfrest.

… Evoning state.

.... sineerly.

4. Lady's Hack orer 1.23, to be shown muler satile mut richen by a laty.

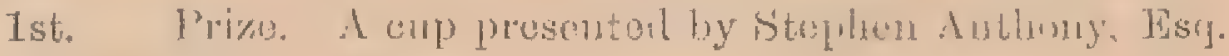

mint. Jine, Value $\$ 10$.

\section{Exhlbitor's Nos.}

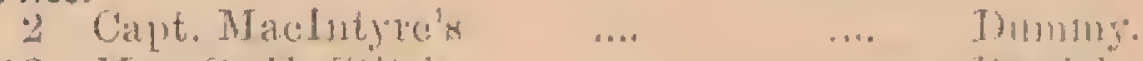

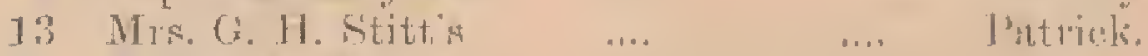

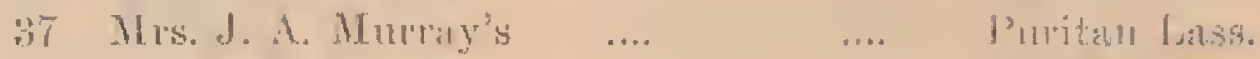

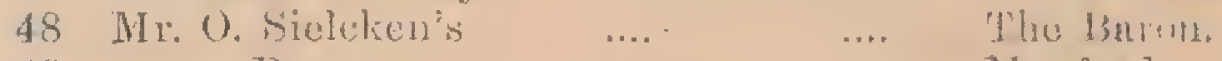

48 Do.

Nanforer.

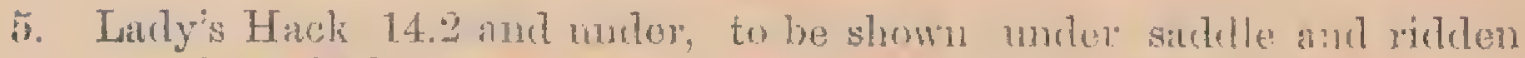
by a Likly.

1st. Irize. A Gohl Bracelet presenterl by (4. H. Lues, Eur. 2nd. T'rize. $\$ 10$.

\section{Exhibitor's Nos.}

2 Capt. Macintyre's

5 Mr. L. E. P. Wolferstan's ....

11, M. Snlli's ....

17, F. . Kinter's

30 , Frank Mills

Capt. MacIntye's

33 Hon. J. Bromiheal Matthew

39 Mr. H. Tearson's

42 : C. B. Whitelead's

49 " M. R. Thiorntou's

51. Miss. Anderson's

$53 \mathrm{Mr} . \mathrm{R} . \mathrm{C}$. Gnimnesss'
Simli

Mimaly

Haplyy Chance

iit

Bridesmair

Gomerlim

Peory

Billy

Georgina

Iarborough 
6. Best Ghary and Pony, 14.2 mal under, to te shown in eart or carriagu.

1st. Prize. $\$ 20$.

211i. Prize, $\$ 10$,

Exhibitor's Nos.

2!) Mr. H. H. Hutam ....

(60) "Chre Saing ....

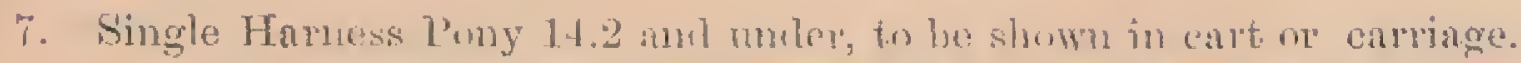

1st. Pri\%e. A enp presenterl by Messia. McAlister \& Co.

2nrl. Prize. Value $\$ 10$.

\section{Exhibibtter's Nos.}

\begin{tabular}{|c|c|c|c|}
\hline$!$ & ins. Huoley's & & The Boy \\
\hline $7 \overline{7}$ & Mr. In A. Kincler"s & $\ldots$ & hit \\
\hline 24 & Mr. W. Willes Douglas' & $\ldots$ & \\
\hline 31 & Ni: R. Crichtom's... & $\ldots$ & \\
\hline 31 & $\mathrm{D}_{0} \quad \ldots$ & $\ldots$ & \\
\hline 2 & Crupt. Wactotyre's. & .. & Sali \\
\hline 83 & Hon. J. Bromhead Watthows & .. & cmmertian \\
\hline 40 & Mr. W. H. MacArthms & $\ldots$ & Bubbles \\
\hline 42 & Mr. C. 3. Whitelead's & .... & l'illy \\
\hline 44 & Mr. C A. Jaw's ... & .... & \\
\hline 41 & Hons, E. W. Birch... & $\cdots$ & 1. \\
\hline 46 & Mr. J. Huesses .... & ... & Jintily \\
\hline 50 & Dr. Thurers $\quad \ldots$ & … & Bushrat \\
\hline $6(i$ & Mr. Kaw foo Kent & $\cdots \cdot$ & 5 \\
\hline $5 ! ?$ & Mr. Jannes Starlo is & $\cdots$ & Mnly \\
\hline $18>+8$ & $\begin{array}{l}\text { Mr. Chee simgs } \\
\text { C'apt. Molyeux's }\end{array}$ & 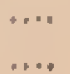 & $\begin{array}{l}\text { Chop Dohlat } \\
\text { Ariminat }\end{array}$ \\
\hline & & & \\
\hline
\end{tabular}

8. Single Haness Horse over 14.2 to be shown in cart or carriage. Ist Irize. $\$ 20$.

2nd Prize. \$10.

Exhibitors' Nos.
$10 \mathrm{Mr}$. H. IWools'...
13 Mrs. G. H. Stitts
18 Capt. Molynenx's
10) Mr. (i. Mughlistons'
25 Mr. olas. Fintr's
44 Mr. U. L. I. Itw'
47 Mr. M. R. Thorntons?

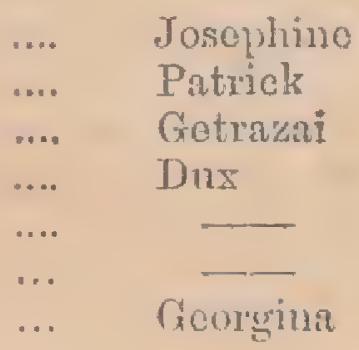


9. Registered Gharry Pony of any description : the bout fide property of acensed Gharry owner (Australian horse banred.)

1st Prize. $\$ 20$.

znd Prize. \$10. 
10. Jumping Pony 14.2 and under, to be riddenover at least four jumps. Ist. Pri\%e A cup presented ly Capt. Macintyre.

2ud. Trize. Value $\$ 10$.

xhibitors' Nos.

1 Majol de Hamel's

2 (ant. Maciotyre's

$11 \mathrm{Mr} . \mathrm{M} . \mathrm{M} \| \mathrm{l} ! \mathrm{s}$

18 Capt. Mulynenx's

24 Wr: Wr. Wille's Donglis

30 . Frank Mills: ...

$\$ 1 \quad$. R. Grichtom's ...

31 : 310.

30 Mr. H. Pentson

$\therefore 4$ " do.

42 (C. I) Whitehearls

$4 ! 1$ "M. R. Thum"utur's ....

52 . 1). Kimus

... Haply Chaner

... Comage

11. Jumping Horse owe 14.2, to be ridten over at least fonr jumps.

Is.. Prize. A cup presenter lyy Chung Thye Phin, Esc.

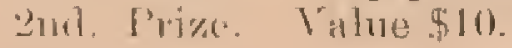

Exhibitors" Nos.

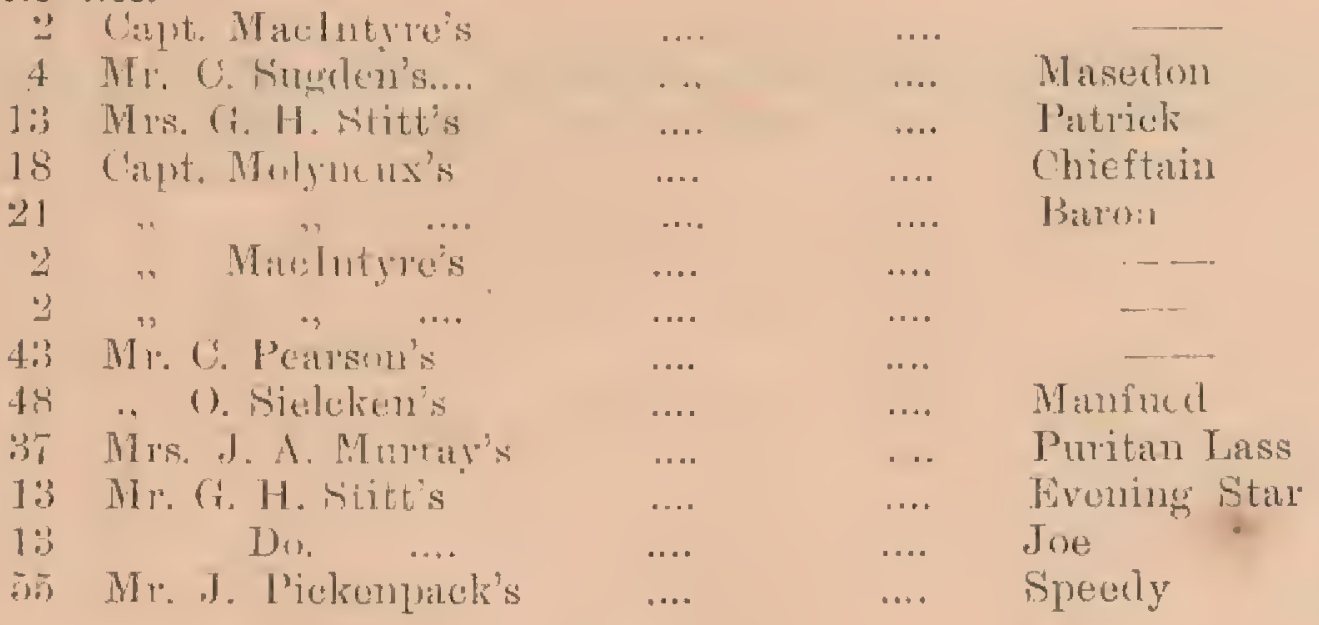

12. Single 'lnnout. Ist. Prize. A cup presented by 'l. Scott Gardner, Esc., (I poh cup.) zud. Trize. Value $\$ 10$.

Exhibitors' Nos.

10. H. Wool.

21. Mrr. W. Willes Donglas.

33. Hon. J Inrombearl Matthews

34. Mr. C. H. Lees

40. Mr. W. H. MacArthor
Exhibitutors Nos.

45. Hon. E. W, Birch

46. Mr. J. Huesse

37. Mr. J. A Muray

55. Mr. J. Pickenpark

59. Mr. Jas Stark 
6

13. Double Turnout : of any description, pairs, tandems, ete.

1st. Prize. A cup presented by Cheah Tek Thye, Esq.

2nd. Prize. Value $\$ 10$.

50. Mr. Ohnng Thye Phin

60. , Cheah Tat Toi

14. Gharry Turnout: the fom fule property of a licensed gharry owner.

(Australiau horses barred) 1st. Prize. Value $\$ 20$. 
15. Shandrydan lurrout, the bome file property of a licensed shandry. dan owner.

1st. Prize \$20. (local pouy.)

16. The best all round Horse 14.2 or over in the Show on the halter.

1st. Prize. A cup presented ly The Members of the Civil Service. 
17. The best and round Pony 14.2 and under to bo showin or: the halter 1st. Prize. A cup presented by C. Cummess, Es j.

18. The hest Strats Settlements or Native States breul horse on mare, :uyy are, over 1.2 liands.

Exhibitors' Nos. 1st. Prize. A cup presenterl hy Thon. John 'I'urmer.

!.. Mr. W. Hosley

16. Mr. Gen Stothard

20. Dr. 'T'. C. Muglistum

26. Mr. Chung Nh Yong

$2(i . \quad$ Dis

:36. Mr. H. W. H.tuman's

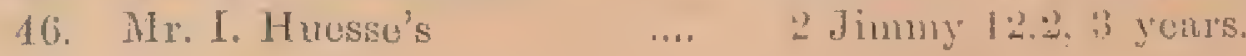

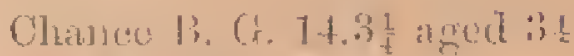
nusuths.

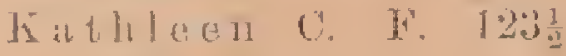
Yonting 
Cin? 1, 1, Ni:

\section{JUDGES.}

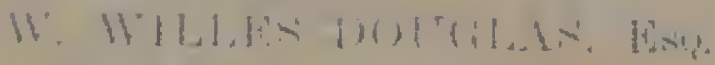

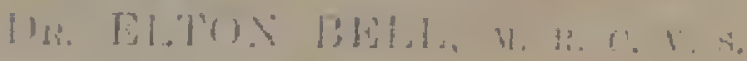

\section{SUB-COMMITTEE.}

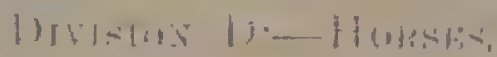

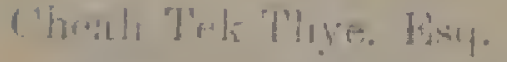

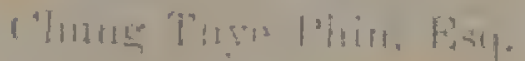

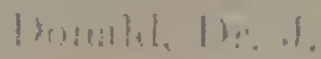

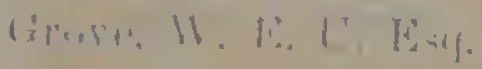

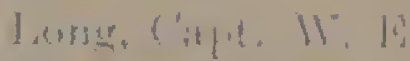

Inedrthe. W. H. Fis.

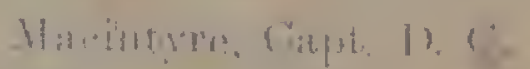

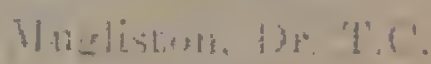

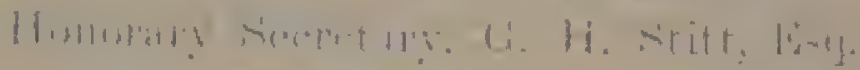




\section{Straits Settlements}

AND

Fiederated Malay States.

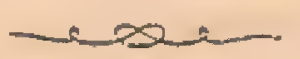

TILE FIRST JOLNT ANNUAL

\section{AGRI-HORTICULTURAL SHOW}

WILL BE HELD ON 'I'HE HACE COURSE

$\mathrm{A}^{\prime} \mathrm{T}$

KUALA LUMPUR, SELANGOR,

uN

Friday, Saturday and sumday, sth, 6th and " th August, 1904.

\section{PRIZE LIST.}

The Show will be open on Friday, th August, from 11 a.m. until 6 p.m.; and on Saturday and Sunday, bth and thl August, from 8 a.m. until 6 p.m.

Adnession: T'wexty Chints.

Orluning Ceremony (1st Day, 11 a.m.-2 p.m.) ... \$2 


\section{PATRONS.}

H.E. THE GOVERNOR AND HIGH COMMISSIONER

(Sir John Anderson, K.c.M.Æ.)

H.H. THE SUL'IAN OF PERAK

(Sin Idris Mersid-el-Aazam Shah, G.c.M.g.)

H.H. 'I'HE SULTAN OF SELANGOR

(Suleman bin Almemeum Raja Musa).

H.H. THE YANG DI PERIUAN BESAR, NEGRI SEMBILAN ('T'unte Mohamad, c.m.g.)

H.H. THE SULIAN OF PAHANG

(Sir Ahmad Mafimam Shah Ahuad, K.c.m.g.)

THE RESIDENT-GENERAL, FEDERATED MALAY STATES

(W. Hood Treacher, Ese., c.m.g.)

\section{PRESIDENT.}

THE BRITISH RESIDENT, SELANGOR

(b. G. CAMPBELL, EsQ, acting).

VICE-PRESIDENTS.

'THE HON. THE RESIDEN'I' COUNCILLOR, PENANG

(J. K. Birch, Ese., acting).

I'HE HON. 'THE RESIDEN'l COUNCIILOK, MALACCA

(R. N. Brand, Esq., acting).

THE BRITISH RESIDENT, PERAK

(H. Conway Betafietd, Ese, acting).

THE BRITISH RESTDIN'T, NEGRI SEMBHAN

(F. J. WELD, Lse, acting).

THE BRITISH RESLUENI, PAHANG

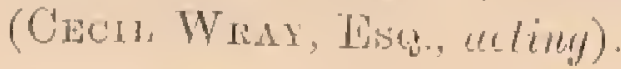

THE RAJ HUDA. SELANGOR

(Raja taut bint Sultan Mohamed). 


\section{STANDING COMNITTEEE.}

\section{SINGAPORE.}

Assistant Supementendent, Bonatic Gardens, Singapore. The Director on Botanic Galidens, S.S. (Hon. Secretary).

\section{PENANG AND PROVINCE WELIGSLEY.}

'The Seniok District Ofhicer, P. W

The Collecrior of Tand Revenue.

'The Supetemtendent, Botanic Gardens and Furests ( $H$ om. Secretary).

$$
\text { MALACCA. }
$$

The Collegerolk on Land Revenus.

The Hon, 'the Resident Councilaol: (Hon. Secretury).

\section{PHRAK}

I'He DHector of Museuns, F.M.S.

Tue Superintindent, Government Plantrations.

'The Distuetot Officer, Ĺuala Kangean (Hon. Secretury).

\section{SELANGOR.}

'The Collector uh Land Revenue, Kuata Lumpul.

The Champlan, United Pran'sejus' Association.

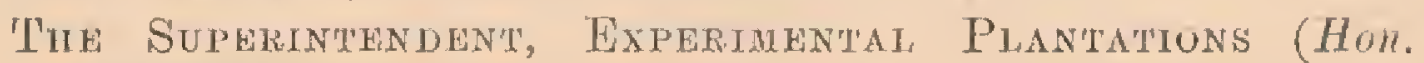
Secretury).

\section{NEGRI SEMBILAN.}

The Districh Officer, Thmpin.

The Cojinector on Iand Revenum, Seremban.

'J'he Chairman, Negri Semibilan Plantelis' a disociation.

W. H. Mackiay, Hon. Secretary.

\section{PAHANG}

'I'he Shenion Magristrate.

'The Distriet Ofreferi, haub.

The Distreter Orficer, Plikan. 


\section{GENERAL COMMITTEE.}

Arden, Stanley Bailey, W. W.

Brown, Is. C. Browell, H. F.

Baxendiale, C. E.

Burn-Murdoch, A. M.

Byrne, H. E.

Carey, E. V.

Chan sow Lin

Clayton, T. W.

Cumming, $G$.

Daniels, Dr. C. W.

Dewir, A. R. J.

Doraisani Pillai

Dykes, F. J. B.

Ford, 'T. A.

Graham, Capt. A. MeD.

Hewgill, C, W.

Horley, Rer. W. E.

Inche Tamby Abdullath

Jinkson, I. C., K.c.

Kemny, WV. Eyre

Lee Kong Lam

Toke Chow 'Thye

Meikle, W.

Meikle, R. S.

Mills, Frmil

Nicholas, W

Parinlanim Pillai, K. T.
Partinson, P. W.

Parry, M. S.

Prior, E. B.

Raja Haji Bôt

Raja Hassan

Ritja Mahmud

Rendle, H. C.

Robertson, J. Argyll

Robinson, H. C.

Russell, J.

San Ah Wing

Severn, Claud

Skimner, E. B.

Suith, Sydney R.

Spooner, J. C.

Swettenham, J. P.

Swindell, Rev. F. G.

Talbot, Capt. H. I.

Tickell, G. T.

Tranchell, E. C. J.

Travers, Dr. E. A. O.

'Tuck, Dr. G. L.

Veming, A. R.

Von Donop, L. B.

Watson, R. G.

Wee Hap Lang

Wilkinson, R. J.

Yeomans, S. C.

Zacharias, H. C. E.

The District Officer, Ulu Selangor' (C. D. Bowen)
Do.
Klang (H. B. S. Cox)
Do.
Ulu Lamerat (O. F. Stonor)
Do.
Kualia Liangat (A. S. Jelf)
Do.
Kuala Selangor' (E. F. Towulev)

\section{GENERAL PURPOSES COMMTTIEE.}

Bivon. I. C.

Byrne, H. E.

Carey, E. F

Claytun, 'I' W.

Cumming, $\mathrm{G}$.

Diniels, Dr. C. W.

Dykes, F. J. B.

Inche Tauby Abdullah

Kenny, W. Eyre

Iolke Chow 'Thye
Meikle, W.

Robinson, H. C.

Rusissell, J.

Sall Ah Wing

Smith, Sydney R.

Spooner, J. C.

Tickell, G. T'.

Tranchell, E. C. J.

Tuck, Dr. G. L.

Zachirias, H. C. E.

The District Officer, Ulu Selanger" (C. D. Bowen)

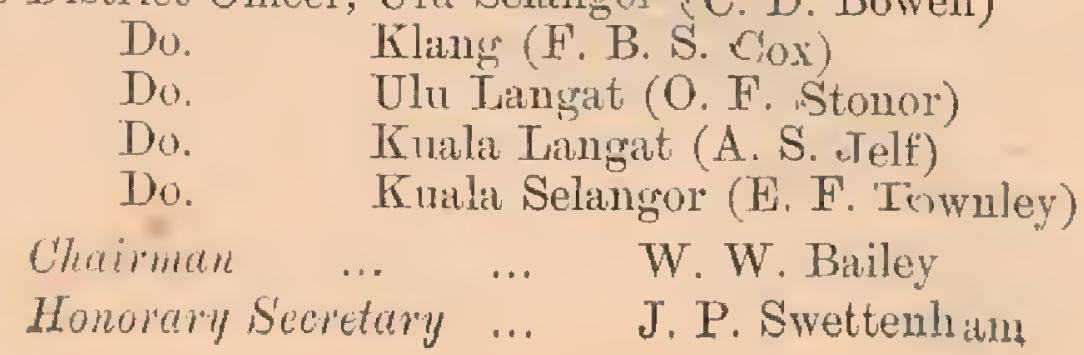




\section{SUB-COMMTTTEES.}

Drvision A.

AGRICULITURAT PRODUCE.

Bailey, W. W.

Baxendale, C. E.

Browell, H. F.
Curey, E. V.

Parkinson, P. W.

Parry, M. S.
Honorary Secretary
T. C. Brown

Divisron B.

FLOWFRS, FRUITS AND VEGETABLES.

Horley, Rev. W. F.

Tee Kong Lam

Meikle, W.

Parimanam Pillai, K. T.

Prior, E. B.
Rendle, H. C.

Veming, A. R.

Von Doinop, T. B.

Wee Hilp Jang

Lee Kong Lam

Homorary Secretary ... H. E. Ḅ̧rne

Division C.

STOCK AND DAIRY PRODUCE.

Brewster, E. J.

Carey, E. V:

Daniels, Dr. C. W.

Doraisami Pillai

Dylies, F. J. B.

Ford, 'T. A.

Meikle, R. S.

Skinmer, F, 1?.

Honorny Serretary ... T. W. Chaton

Diviston D.

HORSES AND DOGS.

Bailey, W. W.

Belfield, $\mathrm{H}$. Conway

Burn-Murdoch, A. M.

Dennys, F. O. B.

Dewar, A. R. J.

Ford, T. A.

Graham, Capt. A. MeD.
Kenny, W. Erre

Mills, Frank

Smith, Sydney R.

Swettenhiam, J. P.

Talbot, Cupt. H. T.

'l'ravers, 1)!. F, A. O.

$\begin{array}{lll}\text { Chaiman } \quad \ldots & \ldots & \text { G. Cumming } \\ \text { Honmary Secretary } & \ldots & \text { J. O. Spooner }\end{array}$ 
Diviston E.

\section{NA'TTVE TNDUSTRTES AND MANUTACTURES}

\section{IMPTIEMENTS AND MTSCWLILANEOUS.}

Tranchell, E. J. C.

Zacharias, H. C. E.

Wilkinson, R. J.

The District Officer, Ulu Selingor (C. D. Bowen)

Do, Klang (F.B. S. Cox)

Do. Ulu Litngat (O. F. Stonor)

Do. Kuala Langat (A. S. Jelf)

Do. Kuala Selangor' (E. F. 'Lownley)

The Penghulu, Knala Knbu (Dato Penglima Kiri)

Do. Klang (Rilja Hatssan)

Do. Kajang (Rajua Ibrahim)

Do. Bandar (Haji Ilorahim)

Do. Kuala Selangor (Raja Saipolib)

Hommary Secretary $\quad$... H. C. Robinson

H. C. F. TACHARIAS,

Honoway Treastror.

STANLEY ARIDEN,

Honmary General Secretary. 


\section{CONTENTS.}

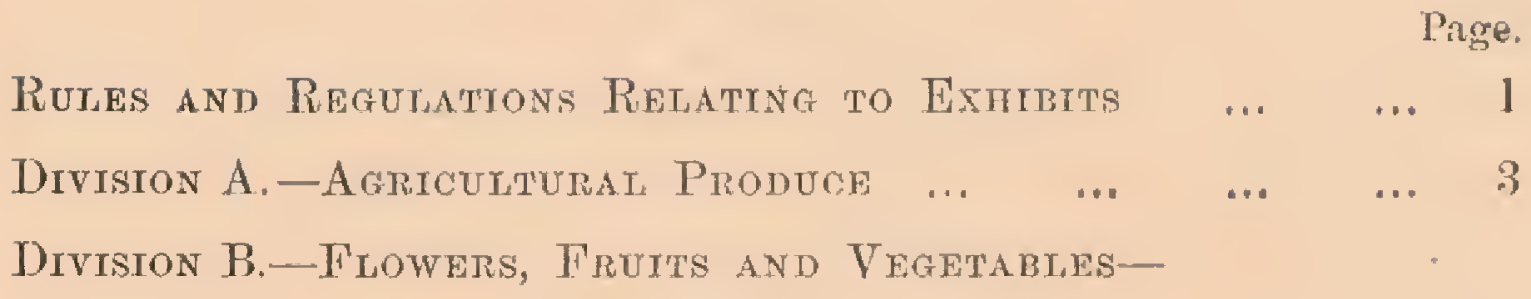

Section 1-Flowers:

Ornamental Foliage Plants, in pots 5

Plants in Flower , 5

Plants whether in Flower or not... 6

Cut Flowers and Table Decorations 6

$\begin{array}{llllllll} & 2 \text { - } & 2 \text {-Fruits ... } & \ldots & \ldots & \ldots & \ldots & 7\end{array}$

$\begin{array}{lllllll}\text {, } 3 \text {-Vegetables } & \ldots & \ldots & \ldots & \ldots & 8\end{array}$

Diviston C.-Stock and Dairy Pronuce-

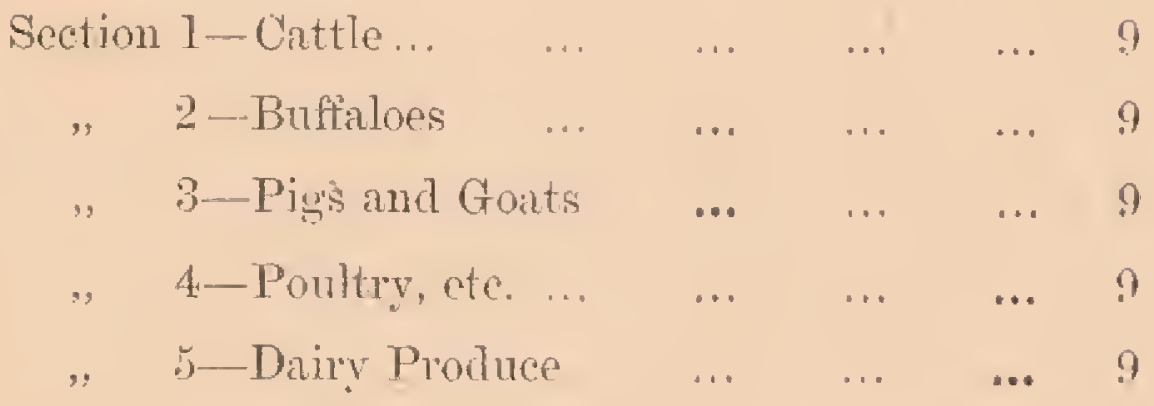

Drvision D.- Horeses and Doas--

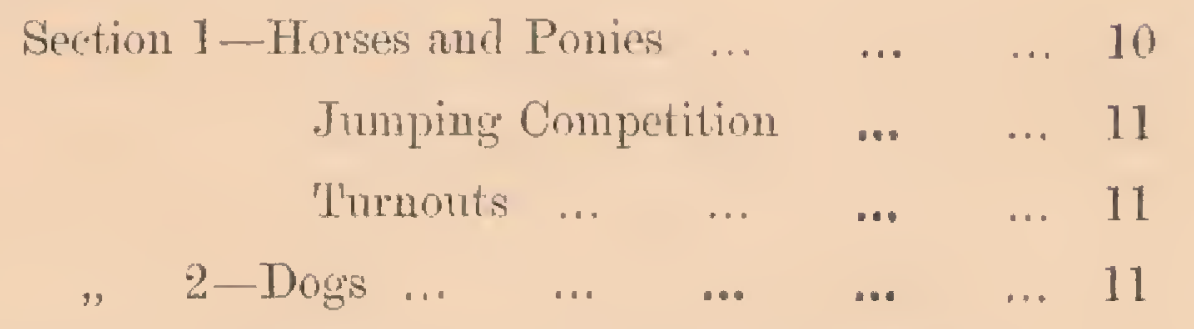

Drviston F.-Native Tndustries and Manufactures:

Tmptements and Miscemitaneoug ... $\quad \ldots \quad 12$ 



\section{RULES AND REGULATIONS RELATING TO EXHIBITS.}

1. All exhibits must be bona fide the property of the exhibitor.

2. Competition for prizes shall be open only to exhibitors resident in the Colony of the Straits Settlements or the States of the Malay Peninsula.

3. Exhibits for competition in division $E$, "Native Industries, etc.," shall be confined to articles manufactured in the Colony of the Straits Settlements or the States of the Malay Peninsula.

4. The Committee will appoint Judges, from whose decision there shall be no appeal.

5. The Judges will have authority to withhold a prize when they are of opinion that there is not sufficient merit to justify an award, and also to award special prizes for anything not contained in the schedule.

6. No exhibitor shall be awarded two prizes in any one class, or more than five prizes in the same division.

7. The arrangement of the exhibits shall be subject to the directions of the Committee.

8. The Committee reserves to itself the right to refuse any entries that they may think fit to reject, and to cancel any class at any time up to seren days previouk to the first day of the Show.

9. Exhibitors desiring free transport must give notice to the District Officer or the Secretary, who will, if they approve of their exhibits, provide them with numbered tickets and a free pass on the railway for themselves and their exhibits.

10. Exhibits will be received between noon and 6 p.m. on the day previous to the show, and between 6 a.m. and 8 a.m. on the opening day. No exhibit will be received after 8 a.m. 
11. Entries for Horses and Dogs close on 25th July. Entries for Horses under Classes 1, 2, 3, 5, 6, 7, 9 and 10 must be accompanied by a certificate of height from any Sporting, Turt or Polo Club in the Colony or F.M.S., $n$ from a qualified Veterinary Surgeon.

12. Exhibitors in Divisions $\mathrm{C}$ and $\mathrm{D}$ must provide for the care and maintenance of their own exhibits.

1:3. No exhibit (except live animals) may be removed from the ground before the close of the Show. Exhibitors will be provided with a card which must be produced at the close of the Show before they can be allowed to remove their exhibits.

14. The General Secretary is empowered to exclude, ol' remove, any animal which in his opinion is not in a fit state for exhibition.

1.). Exhibitors in Divisions C and D will only be permitted to remove their animals from the Show Ground on obtaining an order of removal from a nember of the ('ommittee.

16. Al? reasonable care will be taken to provide for the safety and careful preservation of exhibits of every kind sent to the Sliow; but it must be clearly understood that the Committee does not hold itself responsible for the loss of, or the damage to, any exhibit either in transit, or during exhibition. Provision will be made for keeping valuables moder lock and key at night.

17. Space will be provided for tradesmen and others wishing to exhibit articles for sale but not for competition. All purchases will be subject to the above rules and regulations, and the sale price must be clearly specified.

18. Govermment exhibits shall not be eligible for prizes in any class.

Winner's of prizes of $\$ 10$ and upwards--Silver medal or value, optional. 
Division $A$.

\section{AGRICULTURAL PRODUCE.}

\begin{tabular}{|c|c|c|c|c|c|}
\hline \multirow{2}{*}{ 量 } & \multirow{2}{*}{ Nature of Exhibit. } & \multirow{2}{*}{$\begin{array}{l}\text { Number or } \\
\text { quantity to be } \\
\text { exhibited. }\end{array}$} & \multicolumn{3}{|c|}{ Prizes. } \\
\hline & & & 1st. & $2 n d$ & $3 \mathrm{r} d$. \\
\hline & & & $\$$ & s & $\$$ \\
\hline 1 & Arrowroot, fresh, best sample & 25 roots & 5 & 2 & I \\
\hline 2 & " prepared ", & 1 catty & 5 & 2 & 1 \\
\hline 3 & Bumboos, best collection & & 5 & 2 & $\mathrm{I}$ \\
\hline 4 & Betel-nuts, fresh ... & 50 & 3 & 2 & 1 \\
\hline 5 & ". dried and split & 1 catty & 5 & 3 & I \\
\hline 6 & Cloves, best sample & $2 \quad$, & 10 & 5 & 2 \\
\hline 7 & Coconuts, unhusked, best sample & 10 nuts & 7 & 3 & $I$ \\
\hline 9 & 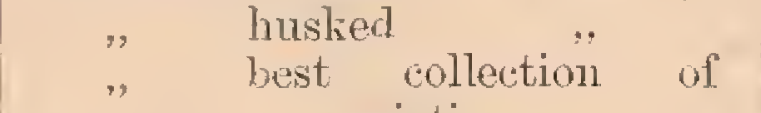 & $10 \quad$, & 7 & 3 & 1. \\
\hline 10 & $\begin{array}{lll}\text { varieties } & \ldots & \ldots \\
\text { Copra, best sample } & \ldots & \ldots\end{array}$ & 3 of each & 10 & 5 & 2 \\
\hline 11 & Coconut Palm, best collection of & 5 cutties & 5 & 3 & 1 \\
\hline 12 & $\begin{array}{l}\text { products } \\
\text { Cotton "Kapok" } \text { "best sample } \quad \text {... }\end{array}$ & 1 catty & $\begin{array}{r}10 \\
5\end{array}$ & $\begin{array}{l}5 \\
3\end{array}$ & $\frac{2}{1}$ \\
\hline 1. & $\begin{array}{c}\text { ", any other variety, best } \\
\text { sample }\end{array}$ & & 10 & 5 & 2 \\
\hline 14 & Cocoa, fresh pods, best sample ... & 5 puds & 5 & 3 & 1 \\
\hline 15 & Coffee, Liberian & 2 cat & 10 & 5 & 2 \\
\hline 16 & ,, any other variety... & 1 catty & 5 & 3 & 1 \\
\hline 17 & Fibres, best collection ... & $\begin{array}{l}1 \text { bundle } \\
\text { of each }\end{array}$ & 15 & 7 & 2 \\
\hline 18 & Fodder Grass, best collection & & 5 & 3 & 1 \\
\hline 19 & Gambier & $\frac{1}{3}$ catty & 5 & 3 & 1 \\
\hline 20 & Getahs, best collection of local ... & $\frac{1}{2}$, of ea. & 7 & 3 & 1 \\
\hline 21 & Gums and Damars ," & $\frac{1}{2},, \quad$, & 7 & 3 & 1 \\
\hline 22 & Ginger, best sample & 20 roots & 5 & 3 & 1 \\
\hline 23 & Incligo, prepared, best sample ... & & 5 & 3 & 1 \\
\hline 24 & Mace, dried & 1 catty & 5 & 3 & 1 \\
\hline 2 & Maize & 12 heads & 5 & 3 & 1 \\
\hline 26 & Medicinal Plants, best collection & & 10 & 5 & 2 \\
\hline 27 & Nutmegs, fresh, best sample & 50 & 5 & 3 & 1 \\
\hline & dried & 50 & 10 & 5 & 2 \\
\hline 29 & Oil, citronella & 1 pint & 10 & 5 & 2 \\
\hline 30 & ", coconut , ".. & $1 "$ & 10 & 5 & 2 \\
\hline 0 & $\begin{array}{c}\text { " teel seed (Minyak Iengah) } \\
\text { best sample }\end{array}$ & & 5. & & \\
\hline & , cistor & $1 "$, & 7 & 5 & $\begin{array}{l}1 \\
2\end{array}$ \\
\hline & in of essential ... & & 10 & 5 & 2 \\
\hline 0 & Oil-cake, best sample & 5 catties & 5 & 3 & 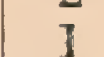 \\
\hline 35 & Pepper, white, best sample & 1 catty & 10 & 5 & 2 \\
\hline & black & 1, & 10 & 5 & 2 \\
\hline 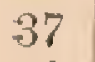 & $"$ Trang & $1 \quad$, & 10 & 5 & 2 \\
\hline 3 & Patchouli, best sample & 1. & 5 & 3 & 1 \\
\hline
\end{tabular}


Division A.-(cont.)

AGPICULTURAT PRODUCE-(cont.)

\begin{tabular}{|c|c|c|c|c|c|c|}
\hline \multirow{2}{*}{ 昜 } & & \multirow{2}{*}{$\begin{array}{l}\text { Number or } \\
\text { quantity to be } \\
\text { exlibited. }\end{array}$} & \multicolumn{3}{|c|}{ Prizes. } \\
\hline & \multicolumn{2}{|l|}{ Nature of Exhibit. } & & 1st. & 2nd. & $3 \mathrm{rd}$ \\
\hline & \multirow{3}{*}{\multicolumn{2}{|c|}{ Padi, any variety $\ldots$ best collection of varieties }} & & $\$$ & $\$$ & 8 \\
\hline 39 & & & $\frac{1}{2}$ gantang & 10 & 5 & 2 \\
\hline 40 & & & & & 10 & 2 \\
\hline 41 & \multicolumn{2}{|l|}{$\begin{array}{l}\text { Pulut, best sample } \\
\text { „, best collection of varieties }\end{array}$} & $\frac{1}{2}$ gantang & $\begin{array}{l}5 \\
7\end{array}$ & 3 & 1 \\
\hline 43 & \multirow{2}{*}{\multicolumn{2}{|c|}{$\begin{array}{l}\text { Rotans, best collection ... } \\
\text { Rubber (rambong) best sample }\end{array}$}} & & 5 & 3 & 1 \\
\hline 44 & \multirow{2}{*}{\multicolumn{2}{|c|}{ Kubver (rambong) best simple... }} & 20 biscuits & + & 10 & 0 \\
\hline & & & $\begin{array}{l}\text { exce } \\
5 \text { cat }\end{array}$ & & & \\
\hline 45 & \multicolumn{2}{|l|}{$\begin{array}{c}\text { " } \quad \text { "Para" best sample } \ldots \\
\text {, free from chemicals }\end{array}$} & di & $t$ & 10 & 0 \\
\hline 4.7 & \multirow{2}{*}{\multicolumn{2}{|c|}{ Rum shrub $\quad ", \quad \ldots$}} & $\begin{array}{l}\text { optiont } \\
1 \text { pint }\end{array}$ & 10 & 0 & 0 \\
\hline 48 & & & & 10 & 0 & 0 \\
\hline 49 & \multicolumn{2}{|l|}{ Sago "prepared" " $\quad$ ".. } & $1 \mathrm{gt}$ & 10 & 5 & 2 \\
\hline 50 & \multirow{2}{*}{\multicolumn{2}{|c|}{ 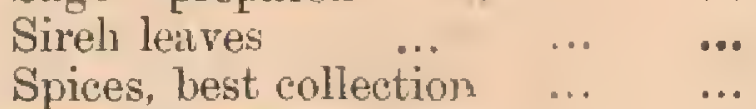 }} & 100 leav & 5 & 3 & 1 \\
\hline 51 & & & ... & 10 & 3 & 2 \\
\hline 52 & \multirow{2}{*}{\multicolumn{2}{|c|}{ Sugar Canes best ". collection of }} & $10 \mathrm{ca}$ & 10 & 0 & 0 \\
\hline & & & & 10 & 0 & 0 \\
\hline 54 & \multicolumn{2}{|l|}{ Sugar, cocomut, best sample ... } & 2 cattic & 5 & 3 & 1 \\
\hline & \multicolumn{2}{|l|}{ " nipah , , $\quad$... } & & 5 & 3 & 1 \\
\hline 56 & \multirow{2}{*}{\multicolumn{2}{|c|}{ 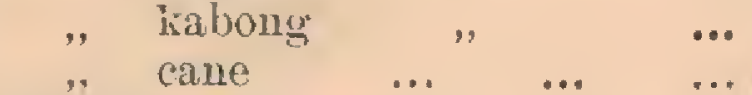 }} & & & & 1 \\
\hline & & & & 10 & 0 & 0 \\
\hline$\theta$ & \multicolumn{2}{|l|}{ "Epioca roots best collection of } & 3 roots & 5 & 3 & 1 \\
\hline 59 & \multirow{2}{*}{\multicolumn{2}{|c|}{$\begin{array}{c}", \quad \text { best collection } \\
\text { varieties } \\
\text { ". pearl, best sample }\end{array}$}} & 1 of & 10 & 5 & 3 \\
\hline 60 & & & 1 catty & 5 & 3 & 1 \\
\hline & \multirow{3}{*}{\multicolumn{2}{|c|}{ 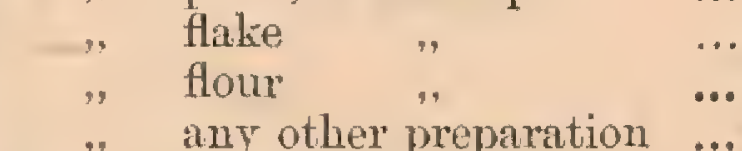 }} & & 5 & 3 & 1 \\
\hline & & & & 5 & 3 & 1 \\
\hline & & & & 5 & 3 & 1 \\
\hline 64 & \multicolumn{2}{|l|}{$\begin{array}{l}\text { any other preparation } \\
\text { Tea, best sample ... }\end{array}$} & & 10 & 5 & 2 \\
\hline 6 & \multicolumn{2}{|l|}{$\begin{array}{lllll}\text { Toddy } & , & \ldots & \ldots & \ldots\end{array}$} & 1 pint & 3 & 2 & 0 \\
\hline 6 & \multirow{2}{*}{\multicolumn{2}{|c|}{$\begin{array}{l}\text { Turmeric }, \\
\text { Tubah (Akar) best sample }\end{array}$}} & 25 roots & 5 & 3 & 1 \\
\hline or & & & 1 catty & 0 & 2 & 0 \\
\hline 68 & \multicolumn{2}{|l|}{ Walking Sticks, best collection ... } & ... & 10 & 5 & 2 \\
\hline
\end{tabular}

* Cup presented by Towkay Loke Yew.

† " " the Negri Sembilan Plinters' Association.

¥ ,,$\quad$ the Selangor Planters' Association.

$\$$ Specin prize, value $\$ 25$, presented by M. S. Parry, Esq. 


\section{Diviston B.}

\section{FLOWERS, FRUITS AND VEGETABLES.}

SEction 1.-FLOWERS.

\begin{tabular}{|c|c|c|c|c|c|c|}
\hline \multirow{2}{*}{ 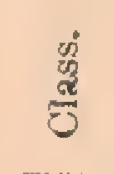 } & \multirow{2}{*}{\multicolumn{2}{|c|}{ Nature of Exhibit, }} & \multirow{2}{*}{\multicolumn{2}{|c|}{$\begin{array}{l}\text { Number or } \\
\text { quantity to be } \\
\text { exhibited. }\end{array}$}} & \multicolumn{2}{|c|}{ Prizes. } \\
\hline & & & & & 1st. & 2nd. \\
\hline \multirow{5}{*}{$\begin{array}{l}1 \\
2 \\
3 \\
4\end{array}$} & \multicolumn{2}{|c|}{ Onamental Foliage Plants, in pots. } & \multirow{2}{*}{\multicolumn{2}{|c|}{6 plants }} & 8 & 8 \\
\hline & \multicolumn{2}{|c|}{ Aroids other than Caladiums ... $\quad$... } & & & 3 & 0 \\
\hline & ," best specimen & $\ldots \quad \ldots$ & & & 2 & 0 \\
\hline & Caladinms $\quad \ldots \quad \ldots$ & $\cdots$ & 3 & $"$ & 3 & 0 \\
\hline & (1) & $\cdots$ & & $"$ & 2 & 0 \\
\hline 5 & $\ldots \quad \ldots$ & $\ldots$ & 3 & , & 2 & 0 \\
\hline 6 & Crotons & $\ldots$ & 6 & , & 3 & 2 \\
\hline 7 & " best specimen ... & $\ldots$ & 1 & ", & 2 & 0 \\
\hline 8 & Dracœnas & $\ldots$ & 3 & , & 3 & 0 \\
\hline 9 & ", best specimen & $\ldots$ & 1 &, & 2 & 0 \\
\hline 10 & Ferns (any variety), distinct & $\ldots$ & 6 & $"$ & 5 & 2 \\
\hline 11 & " (Adiantums) ., & $\ldots$ & 3 & , & 5 & 0 \\
\hline 12 &, & $\ldots$ & 3 & ," & 3 & 0 \\
\hline 13 & " (any variety), best spr & ecimen ... & 1 & ", & 2 & 0 \\
\hline 14 & Marantas and Calathens & $\ldots$ & 3 & , & 3 & 0 \\
\hline 15 & , , best specimen & $\ldots$ & 1 & $"$ & 2 & 0 \\
\hline 16 & Palms, distinct linds & ... & 3 & ,. & 10 & 5 \\
\hline 17 & best specimen & $\ldots$ & 1 & ", & 3 & 0 \\
\hline 18 & Panax and Aralia & $\ldots$ & 3 & , & 2 & 0 \\
\hline 19 & Selaginella, distinct kinds & $\ldots$ & 3 &, & 3 & 0 \\
\hline 20 & best specimen & & 1 & ," & 2 & 0 \\
\hline 21 & Any Ornamental Foliage & Plants not & & & & \\
\hline 22 & $\begin{array}{l}\text { included in the above } \\
\text { Ditto }\end{array}$ & $\cdots$ & 3 & ", & 3 & 0 \\
\hline 23 & $\begin{array}{l}\text { Ditito best specimen } \\
\text { Foliage Plant of auy kind, }\end{array}$ & best speci- & 1 & $"$ & 2 & 0 \\
\hline & $\ldots \quad \ldots$ & $\ldots$ & 1 & , & 5 & 0 \\
\hline & Ornamental Plants in Flow & er, in pots. & & & & \\
\hline 24 & Achimenes & $\ldots$ & 3 & $"$ & 3 & 0 \\
\hline 25 & Amaryllis and Lilies ... & $\ldots$ & 3 & ". & 3 & 0 \\
\hline 26 & Asters $\quad \ldots \quad \ldots$ & $\cdots$ & 3 & ", & 2 & 0 \\
\hline 27 & Balsams ... & $\ldots$ & 3 &, & 2 & 0 \\
\hline 28 & Camnas ... & $\ldots$ & 6 & $"$ & 5 & 2 \\
\hline 29 & 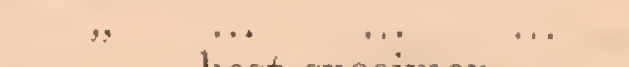 & $\cdots$ & 3 & ", & 3 & 0 \\
\hline 30 & best specimen ... & $\ldots$ & 1 & ,. & 2 & 0 \\
\hline 31 & Cocksconbs $\quad \ldots$ & $\ldots$ & 3 &, & 2 & 0 \\
\hline 32 & Chrysanthemums & .. & 3 & ", & 3 & 0 \\
\hline 38 & Dahlias ... & $\ldots$ & 3 & , & 2 & 0 \\
\hline 34 & Dianthus (Indian Pinls) & $\ldots$ & 3 & 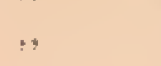 & 2 & 0 \\
\hline 35 & Fncharis ... $\quad \ldots \quad \quad \ldots$ & $\ldots$ & 3 & $"$ & 3 & 0 \\
\hline 36 & Gloxinias & $\ldots$ & 3 & ", & 3 & 0 \\
\hline 37 & Orehids ... & $\cdots$ & 3 & $\because$ & 10 & 3 \\
\hline
\end{tabular}


Diviston B.-( $\mathrm{cminl}$.)

SRCTTON 1.-TTOWERS- $(\mathrm{cml}$.)

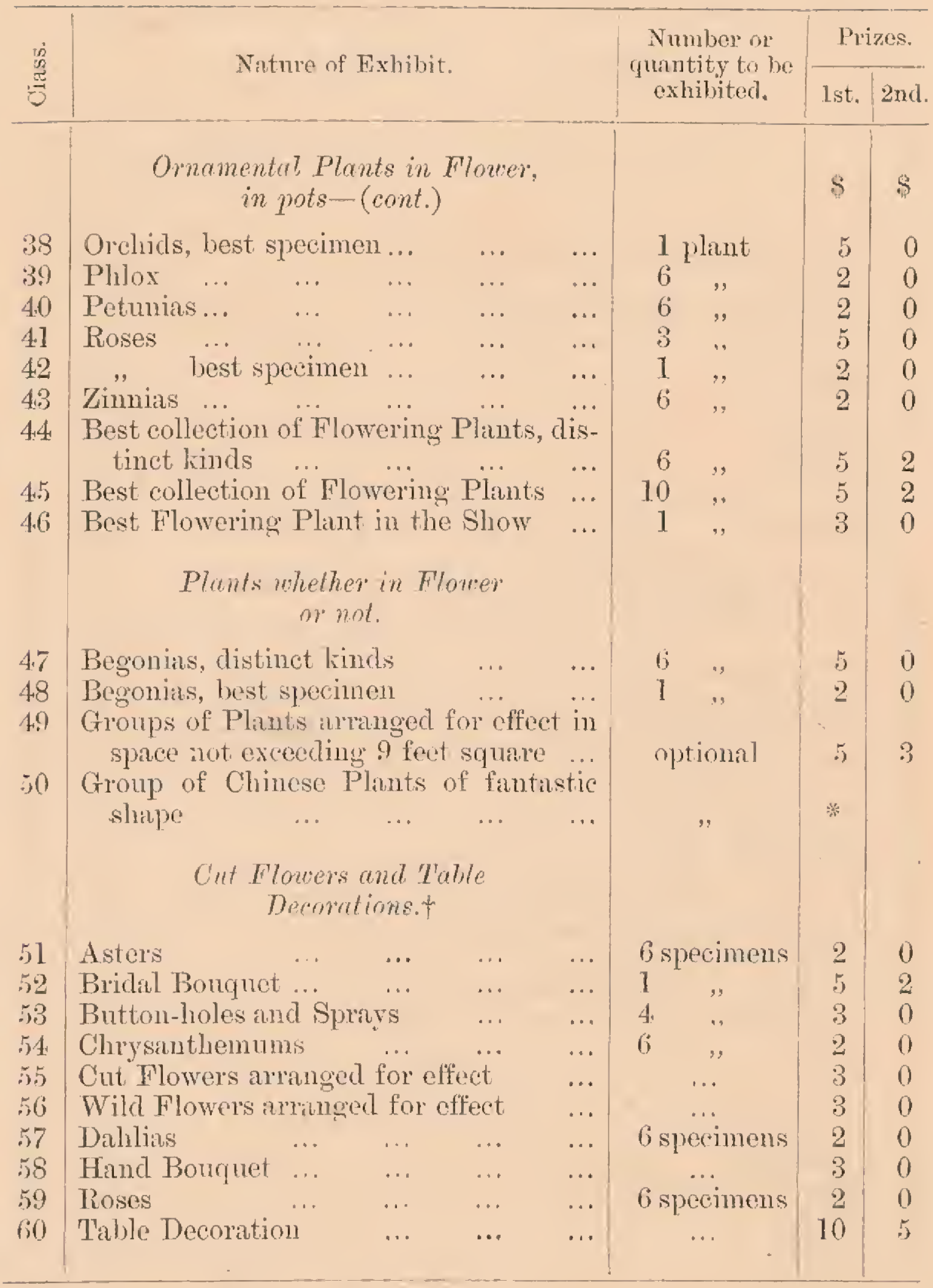

* Cup presented by Towkay Loke Yew.

+ Competition in Classes 51-60 will be repeated on the second day of the Show. Exhibits must be staged before 8 a.m.

Exhibitors must provide their own stmnds for cut flowers.

'The Committee will provide tubles of a uniform sime $(7 \mathrm{ft} . \times 4 \mathrm{ft}$.) for Competitors in Class 60. 
DTVTROA B.--(nnt.)

SECTION 2.-TPUTTS.

\begin{tabular}{|c|c|c|c|c|}
\hline \multirow[b]{2}{*}{ 造 } & \multirow[b]{2}{*}{ Nature of Exhibit. } & \multirow{2}{*}{$\begin{array}{l}\text { Number or } \\
\text { quantity to be } \\
\text { exhilited. }\end{array}$} & \multicolumn{2}{|c|}{ Prizes, } \\
\hline & & & 1st. & 2nd. \\
\hline & & & $s$ & $\$$ \\
\hline 1 & Binanas, "Pisangs," best collection ... & 6 of ea. kind & 10 & 5 \\
\hline & " $\quad$ best bunch, any & 1 bunch & 3 & 2 \\
\hline 3 & $\begin{array}{llll}\text { Champedak } & \ldots & \ldots & \ldots\end{array}$ & 3 fruits & 2 & 1 \\
\hline 4 & Chiku ... & & 3 & 2 \\
\hline 5 & Custard Apple ... & 10 & 2 & 1 \\
\hline 6 & $\begin{array}{c}\text { Cultivated Fruits, best collection, any } \\
\text { number of kinds } \ldots\end{array}$ & $\begin{array}{l}\text { not less than } \\
3 \text { of ea. kind }\end{array}$ & * & \\
\hline$i$ & $\begin{array}{cccc}\text { Cultivated Fruits, best collection, } & 6 \\
\text { distinct kinds } & \ldots & \ldots & \ldots\end{array}$ & & 5 & 3 \\
\hline 8 & 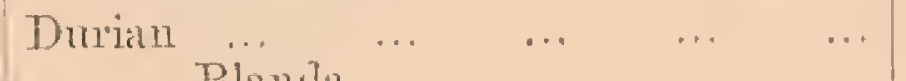 & 3 fruits & 5 & 2 \\
\hline 9 & , Blanda ... & & 2 & 0 \\
\hline 10 & Duliu $\quad \ldots \quad \ldots$ & 20 & 3 & \\
\hline 11 & Binjai ... & 6 & 2 & 1 \\
\hline 12 & Jack Pruit & 1 & 2 & 0 \\
\hline 1: & Jambi $\quad \ldots$ & 20 & 2 & \\
\hline 14: & Langsat ... & 3 bunches & 2 & 1 \\
\hline 15 & Limes $\quad \ldots$ & 12 fruits & 5 & 3 \\
\hline 16 & Mingoes.. & 6, & 3 & 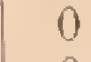 \\
\hline 17 & Machang & $6 \quad$, & 2 & 0 \\
\hline 18 & Mangosteens ... & $12 \quad$. & 5 & 3 \\
\hline 19 & Mata Kuching .. & 20, & 2 & 0 \\
\hline 20 & Melon, any kind & 1 & 3 & 0 \\
\hline 21 & Oranges $\ldots \quad \ldots, \quad \ldots$ & $6 \quad$. & 3 & 2 \\
\hline 22 & Pineapple (Mauritins) ... & $B \quad$. & 3 & 2 \\
\hline 23 & ". (any other variety) ... & , " & 3 & 2 \\
\hline 24 & Pomeloes & ," & 2 & 1 \\
\hline 25 & Pulissan ... & 20 & 2 & 1 \\
\hline 26 & Rambai ... & 3 bunches & 2 & 1 \\
\hline 27 & Rambutan , $\cdots, \quad \ldots \quad \ldots$ & 20 fruits & 5 & 2 \\
\hline 28 & Wild Erible Fruits, best collection ... & optional & 5 & 3 \\
\hline 2 & $\begin{array}{l}\text { Any kind of Fruit not included in the } \\
\text { ahove } \quad \ldots \quad \text {... }\end{array}$ & & 3 & 2 \\
\hline & Preserved Fruits and Vegetables. & & & \\
\hline 30 & $\begin{array}{c}\text { Preserved Fruits, best sample, any } \\
\text { method } \quad \ldots \text {... }\end{array}$ & optional & 5 & 0 \\
\hline 31 & Chutney, hest simple ... & " & 5 & 0 \\
\hline 32 & Pickles & ", & 5 & 0 \\
\hline
\end{tabular}

* Special prize, value $\$ 25$, presented by Stanley drden, Esq. 
Division B.-(cont.)

SECTION 3.-VEGETABLES.

\begin{tabular}{|c|c|c|c|c|}
\hline \multirow{2}{*}{ 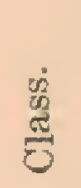 } & \multirow{2}{*}{ Nature of Exhilsit. } & \multirow{2}{*}{$\begin{array}{l}\text { Number or } \\
\text { quantity to be } \\
\text { exhilbited. }\end{array}$} & \multicolumn{2}{|c|}{ Prizes. } \\
\hline & & & 1st. & 2nd. \\
\hline & & & 8 & $\$$ \\
\hline 1 & Artichokes & 12 tubers & 2 & 0 \\
\hline 2 & Beetroot... & 6 specimens & 2 & 0 \\
\hline 3 & Brinjals & & 2 & 0 \\
\hline 4 & Beans, best collection ... & $\begin{array}{l}1 \text { bundle of } \\
\text { each }\end{array}$ & 3 & 2 \\
\hline 5 & Cabbages & 3 specimens & 2 & 0 \\
\hline 6 & Chilies, best collection ... & 30 of each & 3 & 2 \\
\hline 7 & Cucumbers $\quad \ldots \quad \ldots$ & 6 specimens & 2 & 0 \\
\hline 8 & $\begin{array}{c}\text { Herbs used in curries and sambals, } \\
\text { best collection } \quad \ldots\end{array}$ & $\begin{array}{l}1 \text { bundle of } \\
\text { each }\end{array}$ & 2 & 0 \\
\hline 9 & Lettuces & 4 specimens & 2 & 0 \\
\hline 10 & $\begin{array}{c}\text { Onions, Shallots and Garlic, best } \\
\text { collection } \quad . . \\
\text {... }\end{array}$ & 1 bundle & 2 & 0 \\
\hline 11 & $\begin{array}{lllll}\text { Pumpkins } & \ldots & \ldots & \ldots & \ldots\end{array}$ & ens & 2 & 0 \\
\hline 12 & Radishes & 10 specimens & 2 & 0 \\
\hline 13 & 'Tomatoes, best dish ... & 6 specimens & 5 & 3 \\
\hline 14 & Vegetables, best collection ... & $\begin{array}{l}\text { at least } 3 \\
\text { specimens } \\
\text { of each }\end{array}$ & * & 5 \\
\hline 15 & $\begin{array}{l}\text { Vegetables and Herbs for maling a } \\
\text { Silad, best collection }\end{array}$ & & 5 & 3 \\
\hline 16 & $\begin{array}{l}\text { Water-melons, Gourds, Squashes, } \\
\text { Tuffas, etc., best collection }\end{array}$ & 1 of each & 5 & 3 \\
\hline 17 & $\begin{array}{ccc}\text { Yams, Kladi and } & \text { Sweet } & \text { Potatoes, } \\
\text { best collection } & \ldots & \ldots\end{array}$ & 60 & 5 & 3 \\
\hline 18 & Any Vegetable not in the above & optional & 3 & 2 \\
\hline
\end{tabular}

\section{Spectat Crass.-PLAN' DISEASES.}

The best specimen of parasitic disease-animal or regetable-affecting plants of economic value.

Prize value $\$ 25$, presented by Dr. C. W. Daniels.

[Government servants other than Europeans will be cligible for this prize.]

* Special prize, value $\$ 25$, presented by H. N. Ridley, Esq. 


\section{Division C.}

\section{S'LOCK AND DAIRY PRODUCE.}

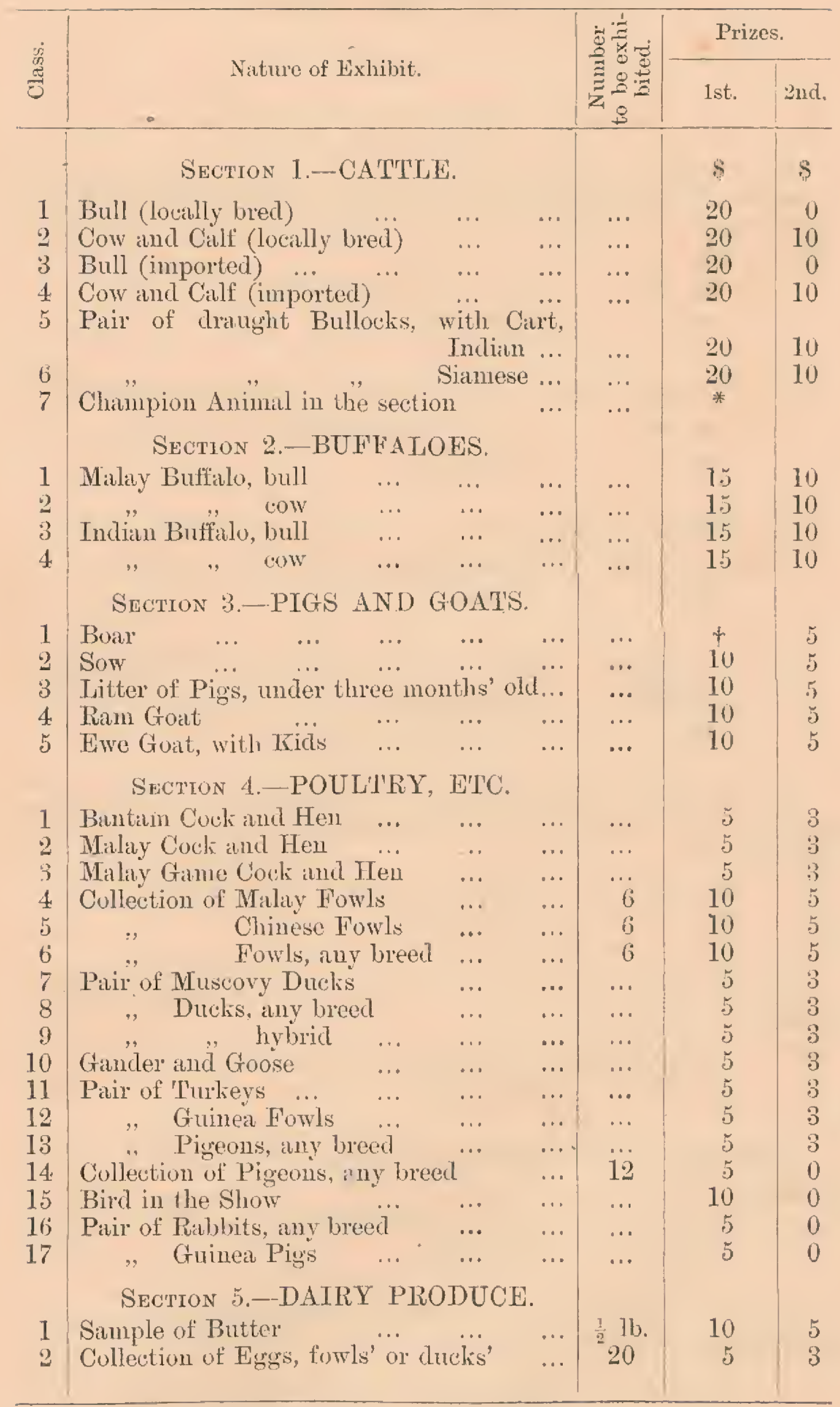

* Cup presented by F. J. B. Dykes, Nsq.

$t, \quad$ I'uwkay Loke Yew. 


\section{DIVISLOX D.}

\section{HORSES AND DOGS.}

\section{SEUTION 1.-HORSES AND PONIES.}

1. Polo Pony, resistered 14.1 or unter: the bmin fide property of an nember of any recognised Polo Club in the Colony or the Federated Malay States. To be shown under siddle, and tested with stick and ball if required.

$$
\begin{array}{lll}
\text { Ist prize } & \ldots & \text { A cup, presented by F. J. Weld, Esq. } \\
\text { 2nd }, & \ldots & \text { Value } \$ 10
\end{array}
$$

9. Gentleman's Hack, 14.2 and under : to be shown under sild lle.

1st prize ... A cup, presented by Cecil Wray, Est.

2nd,$\quad \ldots \quad$ Value $\$ 10$

3. Gentleman's Hack, over 14.2: to be shown under suddle.

1st prize ... A cup, presented by R. N. Blimd, Esip.

end,$\quad \ldots \quad$ Value $\$ 10$

4. Latdy's Hatck: to be shown under saddle and ridden by at Lady,

Ist prize ... A cup, presented by Fred. Belfield, Esu.

2ud , ... Value $\$ 10$

5. Ruw Pony. 14.1 and undere; suitable for Polo but that hilis not yet plaved: to be shown under saddle. The property of a dealer. and fur sale.

1st prize ... A cup, presented by J. K. Birch, Esq.

2nd , ... Vilue $\$ 10$

6. Single Harness Pony, 14.2 and under: to be shown in cart or cólriblige.

1st prize ... A cup, presented by H. Vaue, Esc.

and,$\quad \ldots$ Value $\$ 10$

7. Single Harness Horse, over 14.2: to be shown in cart or calriage.

1st prize ... A cup, presented by Towking Wee Hap Lang 2nd , $\quad \ldots$ Value $\$ 10$

8. Ghary Pony of any description: the bon fate property of a liconsed ghinry owner. (Austrulian liorses barred.)

1st prize ... $\$ 50$, R. Dordisimy Pilliti

zad,$\quad \ldots \quad 10$ 
Division D.- (comt. $)$

SECTION 1.-HORSES AND PONIES-(conl.)

Jumping Compelition.

9. Jumping Pony, 14.2 and under: to be ridden over at leust four fiences.

]st prize ... A cup, presented by Towkay Chin Sow Lin end , ... Value $\$ 10$

10. Jumping Horse, over 14.2: to be ridden over at letst four fences.

1st prize ... A cup, presented by 'Towkity Low Bown Kin end,$\quad \ldots \quad$ Value $\$ 10$

11. Single Turnout.

\section{Turnouts.}

1st prize ... A cup, presented by J. C Parqual, Lisy. end , $\quad \ldots$ Value $\$ 10$

12. Double 'l'umout of any description, pair's, tandems, etc.

1st prize ... A cup, presented by L'owkay Loke Yew and , ... Value $\$ 10$

13. Gharry l'mmout : the bomit fide property of a licensod ghame owner. (Austrulian horses barred.)

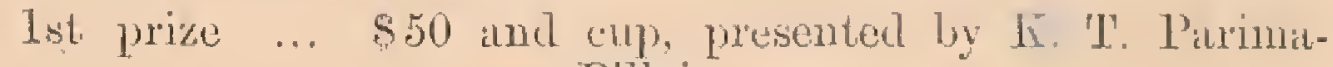 nam Pillai
ind , $\quad$.. Value $\$ 10$
Championship Cless.

14. 'the best Hurse in the Show: to be shuwn on the balter.

Prize ... A cup, presented by D. G. Camploell, Esid.

\section{SECTION 2.-DOGS.}

1. Smooth-haired Fox Terrier, Dog $\quad \ldots \quad$... Prize value $\$ 10$

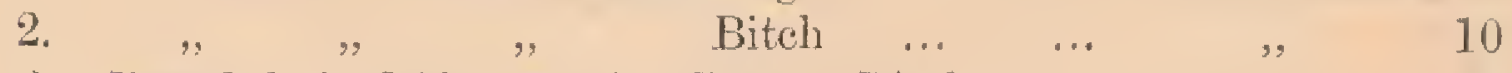

3. Rough-haired Fox Termier, Dog or Bitch ... $\quad$ " 10

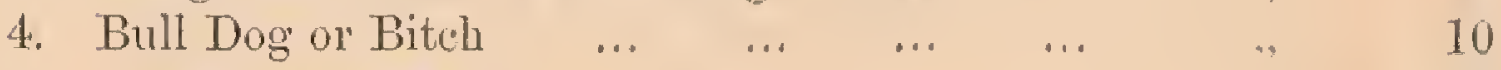

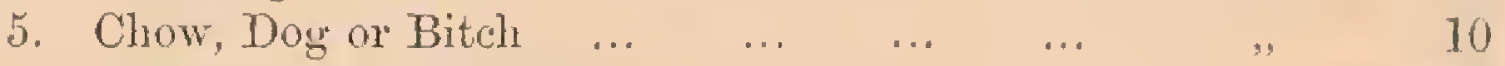

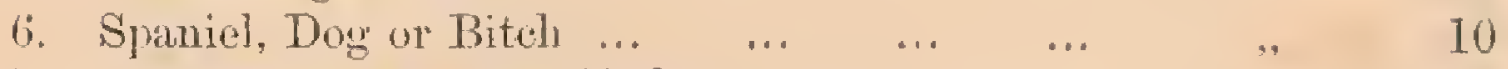

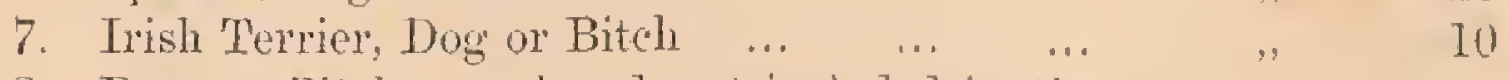

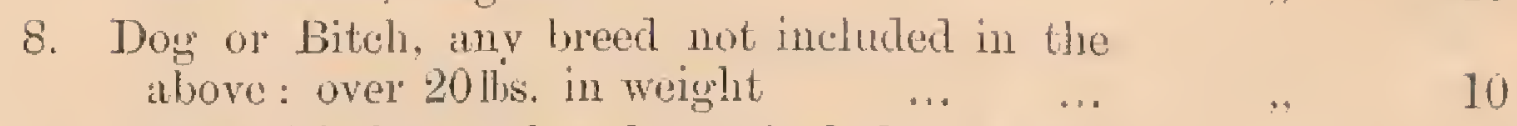

9. Dow or Bitch, my breed not included in the
above: under 20 ins. in weisht ... ...

\section{Chempionship Cless.}

10. 'I'le best Dogr or' Bitch in the Show.

Prize ... A cup, presented by W. Hood 'Trencher. Esi., с.м.6. 
Division E.

NATIVE INDUSTRIES AND MANUEACTURES: AGRICULIURAL IMPLEMENI'S AND MISCELLANEOUS.

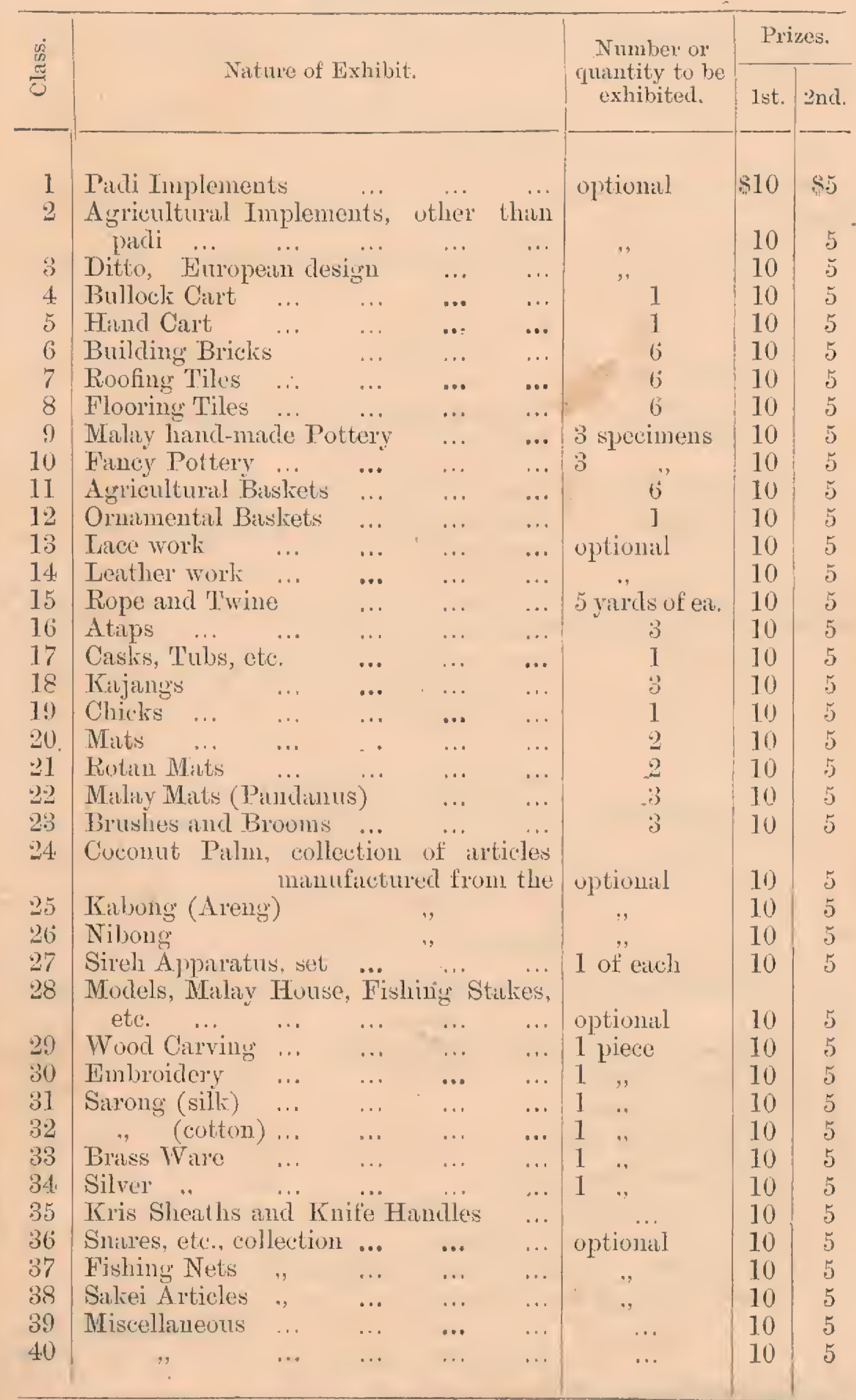

A cup, presented by 'Lowkay Loke Yew, will be awarded for the best specimen of native uratsmanship exhibited in this division. 
ISt. Afri Hortical Show 1904 P. J-O 
In one particular instance where the plants were badly affected, I attributed the attack to the fact that the young seedlings had suffered from an insufficiency of water at the roots. The nurseries had been made under, the shade of large trees, and the seedlings had suffered owing to the fact that during a comparatively dry spell of weather, the soil, being full of the roots of the older trees, had become too dry'to support a large number of seedlings growing close together in nursery beds. Moreover the beneficial effect of heavy showers in cleansing the foliage, such ats we are accustomed to in a dry season, is lost to the plants when growing under shade.

This disease is chiefly limited to plants growing under unfavourable conditions, and is not likely to affect trees when once established. I am of opinion that it may be entirely avoided by making the nursery beds in a damp locality in such a position that the seedlings will derive the full benefit of a free circulation of air and be subjected to the full effect of heavy showers from time to time. The soil should be free and well drained, but capable of retaining plenty of moisture even in comparatively dry weather : if necessary, artificial irrigation must be resorted to, but care must be exercised that this is done thoroughly as opposed to frequently, an operation which would be worse than useless in the case of small seedlings exposed to the full blaze of a tropical sun.

\section{STANLEY ARDEN, \\ Superintendent Experimental Plantations.}

Experimental Plantation,

BATU TIGA, SELANGOR,

4th May, Igo5.

\section{THE AGRICULTURAL SHOW AT KWALA LUIMPUR, 1904.}

The Report on this Show, held in Kiwala Lumpur, 5th, 6th and 7 th August, has just been published. As it is rather toolong to publish completely in the "Bulletin" we extract from it such facts as are interesting specially to the general public. It was anreed on all hands that the Show was a success, and the President, Mr. D. G. CampBELL, attributes this to the members of the various Committees and District Officers who laboured so energetically for this end. "It cannot," he says, "be said that "the Show was held as the outcome of any special interest on the "part of the general public or even of the planting community, "on the contrary it was held at the instigation of the Govern"ment." And it must be admitted it is true that a certain 


\section{$(231)$}

number of those who might have been expected to have been willing to assist for the benefit of the community appeared to misunderstand the rationale of such exhibitions, which are not for the benelit of the Government but strictly for the benefit of each and every resident in the Peninsula. As to the utility of these exhibitions Mr. CAMpBELL writes as follows:-

The Utility of A gricultural Shores. - The utility of such shows has often been questioned, but no one who saw the large and varied exhibits in the "Native Industry" section, and the ready sale which these exhibits met with, can deny that a great stimulus was thereby afforded to native industries; and, so long as the natives can rely upon being able to dispose of their productions at these annual shows, there is good reason to believe that some of the Malay arts and handicrafts, which, is the Hon. Secretary for that division remarks in his report, are rapidly becoming obsolete, may, at least to some extent, be revived.

Educational Value of Shows.-The practical demonstration on the preparation of rubber given by Mr. Burgess, Government Analyst, Singapore, was of the greatest value, and my Committee is deeply indebted to him, as also to Mr. G. DEARIE Russelt, Manager, Federated Engineering Company, who constructed a rubber-washing machine on the principles recommended by Weber-for an excedingly interesting and instructive demonstration; and one which will probably have a very important bearing upon the preparation of rubber in the States.

The educational value of these shows is a point which should always be kept to the fore, and every effort should be made to bring the natives within their educative influence. It is sugrgested that, in addition to such demonstration as the one referred to above, prizes should be offered for agricultural implements as the result of competitive trials, not merely as exhibits in the ordinary way.

\section{REPORT ON DIVISION" "A."-IGRICULTURAl PRODUCE.}

Mr. L. C. Brow hat the chare of this department, which was really very well represented. He reports:-

As regards Division " A." I feel every reason to be proud of the exhibits, not only from the most satisfactory-but that the exhibits themselves would. as a whole, do credit to any show as the products of tropicil arriculture.

The exhibits received came from the following districts:Perak.-Upper and Lower Perak, Krian, New Territory, Matang, Larut and Kinala Kangsar.

Tampin.

Negri Sembilan.-Seremban, Jelebu, the Coast and Kuala Langa. 
Pihang.-Only a small exhibit of padi. tributed.

Pening, Province Wellesley and Malacca, also con-

A few remarks about some of the exhibits deserving, I consider, special mention. may not be out of place, the more so as it will serve for reference and comparison with any future show.

Coconuts, of which there was a magnificent collection, both specimen and variety, may be said to have taken first honoms, in so much that it was in this product for a very tine collection of varieties that Mr. E. B. PrIor, Golden Hope Estate, was awarded the cup kindly presented by H. E. the High Commissioner for the best collection of agricultural products. The same estate received a first prize for an excellent sample of copra, while the Selangor Oil Mills were equally successful for a very fine sample of poonac minufactured in their factory. The priducts derivable from the coconut tree and its fruit were very much in evidence, and with respect to this, one exhibitor had baskets, brushes, ladles of all sorts, sticks, and some articles of such intrinsic value that he refused to part with them at any price. Excellent samples of sugar, coir fibre and twine wore also on view, and it is to be hoped that at some future show. when the industry expands in all its branches, we maly see rope. soap and cocont butter exhibited from some factory situated in the Federated Malay States or the Colony.

Rubber.-I was certainly disappointed that there were not more exhibits in this class; on the other hand, most of the samples were of excellent quality. Mr. A. B. LAKF took both the cups presented for Para: Kent Estate the one given by the Selangor Planters" Assuciation; and Uganda Estate Mr. PAIRI"s prize; Mr. F. A. STEPHEN's exhibit being "higlly commended," as also that of Mr. W'. MV. PArkinson. Mr. IV. IV. BAIIEI succeded in carrying of the cup presented by the Negri Sembilan Planters' Association for the best simple of rambong.

Padi.-The virions linds, both as regards specimens and collections, were uxceptionally good, but great difficulty was found in obtaining competent Judges for awarding the prizes, as the Judges appointed for this division expressed themselves unwilling to undertake the work, believing that they could not give fair justice in the selection, and it would be better if, in future, some special arrangement wis made about this. Mr. BeLreled, the Acting Resident of Perak, was good enough to present a prize for the best sample of pald, and this was awarded to Penghulu Kotu Lama Kiri, Kuala Kangsar.

The Judges commented most favourably on the exhibit that took first in Gambier, and they were also very much taken by the best sample in white pepper, grown on Padang Rengas Estate. Kuala Kangsar. An interesting feature of the show was an excellent display of varions fibres by Messrs. Hogan \& Co., which 


\section{$(233)$}

attracted much attention and easily sined the first prize. lixhibits of Liberian coffee, cotton, and aproca (pearl, flake and Honir) were all very gook.

Special thanlis are due to the Hon. J. Allinson, Messis. RIDLE and Fox, who lindly offichated as Julges, and $I$ an particularly indebted to Mr. Fox for the valuable assistance he wave me in arranging and stasing the cxhibits, as also to Mr. Aliten and Mr. J. P. Swettenhas for the help wiven me from time to time in the necessary preparations for the exhibition.

L. (. 13ROWN,

Hon. Secreary, Dirision "A."

REPORT ON DNISION "B."-FLMWRRS, FRUTS

(N) TEGETABLI:S.

Dealing with the three sections separately and commencing with that for llowers, plants, etc, the exhihits staged were poor, and had it not been for the enterprise of mily alront half-i-dozen whibitors this class would have been a hopeless failure.

As it was, the value offered in first prizes amounted to $\$ 230$. while only w60 were awarded by the Judges; and as regards second prizes, awards of the value of $\$ 5$ mly were made out of a provision of $\$ 35$.

The collections sent hy Lady Thlachrir, Mk. I). G.

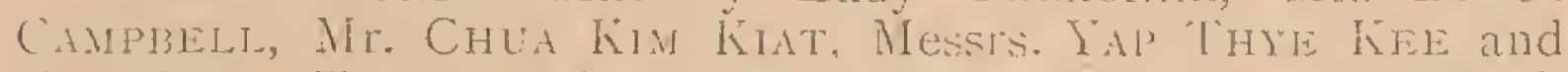
TH SEOW TExG and by Mis. AH YEok, dirided practicilly all the prizes between them. Mr. D. G. C.Mprust. winning six first prizos, two second prizes, and one commenderl exhibit, while Mr. ChUA KIM KIAT won six first prizes.

Lally TREACHER Won two first prizes for her collections of ferns, and Mr. LokF YEw's cup for the best wronp of Chinese plants arranged in fontastic shapes was awirded to Messrs. YAl

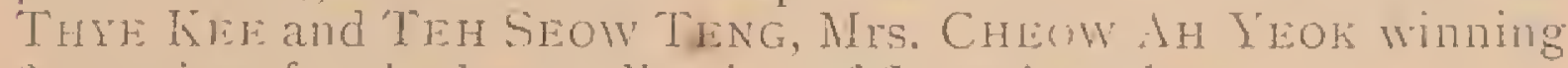
first prizes for the best collection of flowering plants.

The only exhibit worthy of mention among the class fur cut flower was the bowl of magniticent ruses shown by Mr. Elhkaums, of Negri Sembilan.

The class for table decoration was well filled, and Miss INDERSON, who very kindly consented to awart the prizes, had some difficulty in discriminating among the six pretty tables of the ladies who competed.

The sroup of ferns, etc., staged by the Govermment Gardens. Selangor, were exceedingly effective and as a general exhibit of flowers, fruits and vegetibles. 100 yreat praise cannot be awarded to the Government Gardens. I'erak, for their rery representative collection staged by Mr. CAMpblat, the Governmont Superintendent. 
In the section for fruit, prizes to the value of \$ $\$ 06$ (first) and $\$ 42$ (second) were offered for competition, the actual awards made by the Judges, however, only amounted to $\$ 67$ (first), $\$ 30$ (second), and $\$ 6$ (special).

Mr. STANLEY ARDEN had very kindly offered a special prize to the value of $\$ 25$ in this section for the best collection of cultivated fruit, but the Judges considered that there was not any collection of sufficient merit to warrant its being awaded.

The Land Office, Penang, won in this section five first prizes and five second, while next to theirs the best exhibits were those from the district of Jugra, which carried off three first and two second.

Mrs. Moorhouse won first prize in classes $3 \mathrm{I}$ and 32 for chutney and pickles, respectively; and Mrs. RevNe was awarded first prize in class 30 , for preserved fruits.

Perhaps the most interesting class in the fruit section was No. 29 that for any variety of frut not included in the official prize list. The first prize was awaded to Mr. W. J. CoATs for his Brazilian pears, and a special prize to Mr. R. D. ToldemachE, for some exceptionally fine lemons.

The limes exhibited by Batu Caves and Kamuning Estates, and also the oranges grown by Mr. CHUA Kim KeAT, of Singapore, are well worthy of mention as showing what can be produced in the Straits.

The fruits exhibited purely by Malays were very poor, both in quality and quantity. The poomess in quality is due principally to the reason that the fruit season was only just bexinning. but a great deal of it is attributable, as is also the paucity of exhibits. to the fact that only in the one district of Jurra did the District Officer seem to take any real interest in working up his Malays to exhibit, and having done so, in seeing that their collections were properly classified for staging.

This applies also to the section for vegetables, and until more interest is taken by the officers who are in touch with the Malays in their own homes, it will be impossible to ever get together a representatire collection of what the Malays can do in the cultivation of the ordinary fruits or vegetables, for which there is the greatest demand.

As an illustration, and taking the section for fruits, prizes were offered in thirty-two separate classes, in twenty-one of which second prizes were also offered. Ont of these only six firsts and six seconds were awarded to Malays of the F. M. S., and of that number again, three firsts and two seconds went to the district of Jusra alone.

To proceed to the section for vegetables. There wure eighteen different classes in which first prizes to the value of $\$ 49$, and seconds to the value of $\$ 23$, were offered, the value of the prizes actually awarded being $\$ 34$ in firsts, and $\$ 18$ in seconds. 
Mr. H. N. Ridlir's special prize in this section was won by Mr. Foo Wha Cineng, of Kualat Lumpur, for his collection of vegetables, the other principal exhibitors being the Land Office, Penang, with three firsts and one second, and the Jusra District with one first and two seconds.

The most representative classes were perhaps those for brinjals, pumpkins, watermelons and kladi, and it is perhaps worthy of mention that in the classes for such ordinary vegetables as beetroot, cabbages, onions and artichokes, no exhibits were entered for competition.

Speaking renerally; I do not consider that this division of the show was a very representative one. The plants and flowers are not of so much importance, perlaps, as the fruits and vegetables, and it is a matter to be regretted that the classes for them were not better filled.

\section{H. E. BYRNE, Hon. Secretary, Division "B."}

REPORT ON DIVISION "C."-STOCK AND DAIRT PIROTUCE.

Sections I and 2 were cancelled a week before the first day of the Show, owing to the existence of rinderpest in Kuala Iumpur. Section I would have had some interesting exhibits, as several Europeans in Selangor and Perak had intended to exhibit.

Section 3 only provided one exhibit in class $I$. In classes 2 and 3 there vere no exhibits. In the classes for guats, classes $f$ and 5, there were four and six exhibits respectively. All the exhibits in these sections, except one from Klang, were from Kunla Lumpur and district, and none of them call for special motice. One prize was awarded in class $\mathrm{r}$, and two in each of classes + and 5 .

Two extra prizes were given for sheep.

The Chinamen were backward in bring their pigs, owing to trouble of transporting them, keeping them under restraint, and feeding them on the show ground. The prizes would probably have to be increased in ralue to induce people to show their pigs, and I doubt whether, even if pigs were exhibited in large numbers, it would prove a great advantage or help on the purposes of the Show.

In section 4, poultry, etc, there was a large number of exhibits. Far ahead of the rest were Mr. FARRER BAYNES' light Brahma fowls. There were many kinds and varieties of fow's exhibited by natives.

In section 5 there were two exhibits of butter, both from Europeans, and several exhibits of eggs.

There were also miscellaneous exhibits which it was found impossible to class muder any of the scheduled headings, such as cats, plandoks, birds of various coluurs and sizes, and a crocodile. These exhibits excite considerable interest among the spectators: 


\title{
$(236)$
}

but it should be decided, I think, to keep within the classes scheduled and not to accept miscellaneous exhibits, and clear instructions should be issued to local secretaries on the subject.

Many of the native exhibitors appeared to imagine that deformity in an animal is a special merit, and the mere fact of an exhibit being unique $i_{1}$ its gait or the malformation of its limbs entitles it to a prize. There was also an impression that the Judges were to make a daily circuit of the show. awarding fresh prizes on each occasion.

Thanks are due to Messrs. Drkes, R. MerkLE, E. B. SRINAER and E. F. HownLE for the work they did in amanging the exhibits, and to the two last-named and Mrs. VENNING and Mrs. EpHraums for judging the exhibits. In conclusion, I would recommend that on future occasions prize winners should receive their money prizes on the last day of the Show, as far as possible. This would save a considerable amount of trouble to secretaries I think, and would he rery popular among the native prize winners.

\author{
T. M. CLAYTON. \\ Hon. Scretmy', Dinison "C."
}

REPORT ON DINSION "E."-NATHL INIDSTRIES ANH

\section{MANUFACTURTES.}

The section of the Agri-Horticultural Show devoted to Native Industries and Manufactures was certainly by far the largest and probably attracted the grentest attention of any on the ground. its success being almost entirely due to the efforts made by the various District Officers in the Colony and the Federated Malay States to induce the natives to cxhibil-by no means an easy matter. The total number of individual exhibits could not have been less than eight or ten thousand, thongh, of conrse, this inchudes series of twenty or thirty objects exhibited inder one heal.

As might be expected, the bulk of the prizes were carried off br exhibitors from Perak and Negri Sembilan, white a few, chiefly connected with agriculture, were won by Malacca. Selaneror exhibits formed a very small proportion of the whole, and Pahang, owing to distance and difficulties of transport, was but poorly represented; nearly erery specimen sent by this State, however, was ararded a prize. Special mention should be made of the magnificent exhibit of stamped cloth, lain telcpol, and silver, from the Kuala Langat district; of the carefully executed series of model fishing boats and nets from linantan : of the miscellaneous exhibits of the Kuala Kangsa Art School and the Perak Vernacular Schools; and of the kris sheaths and handles from Upper Perak. 


\section{$(237)$}

The Show was instrumental in bringing to light numerous implements, etc., now quite obsolete in the Federated Malay States, such as the gobi api or fire syringe; but the general impression given was that Malay arts and handicrafts are rapidly becoming obsolete (in the WVestern States, at any rate) with but little prospect of revival. To take only two instances, the examples of recent silver-work and wood-carving entered were both poor in execution and tasteless and debased in design. showing marked Chinese and Indian influence. Terrible ornaments made of Berlin wool of the most riolent analine shades, where the most popular exhibit in the show among the natives, and were eagerly competed for by Chinese, natives of India, and Malays alike.

\section{H. C. ROBINSON, \\ Ilon. Secretnry", Division "E."}

Balance Sheet of Agri-Horticutidull Show, Kuala

LUMPUR, IgO5.

As per 2 th February, ino5.

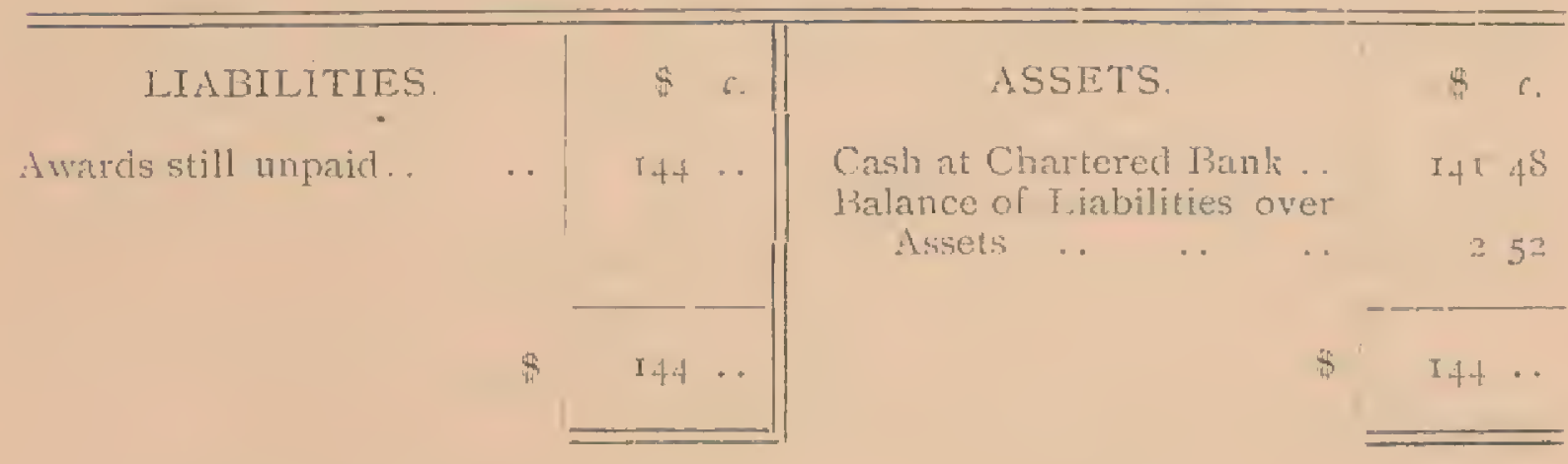

$1 \%$.

Profits and Loss Account.

$\mathrm{C} r$

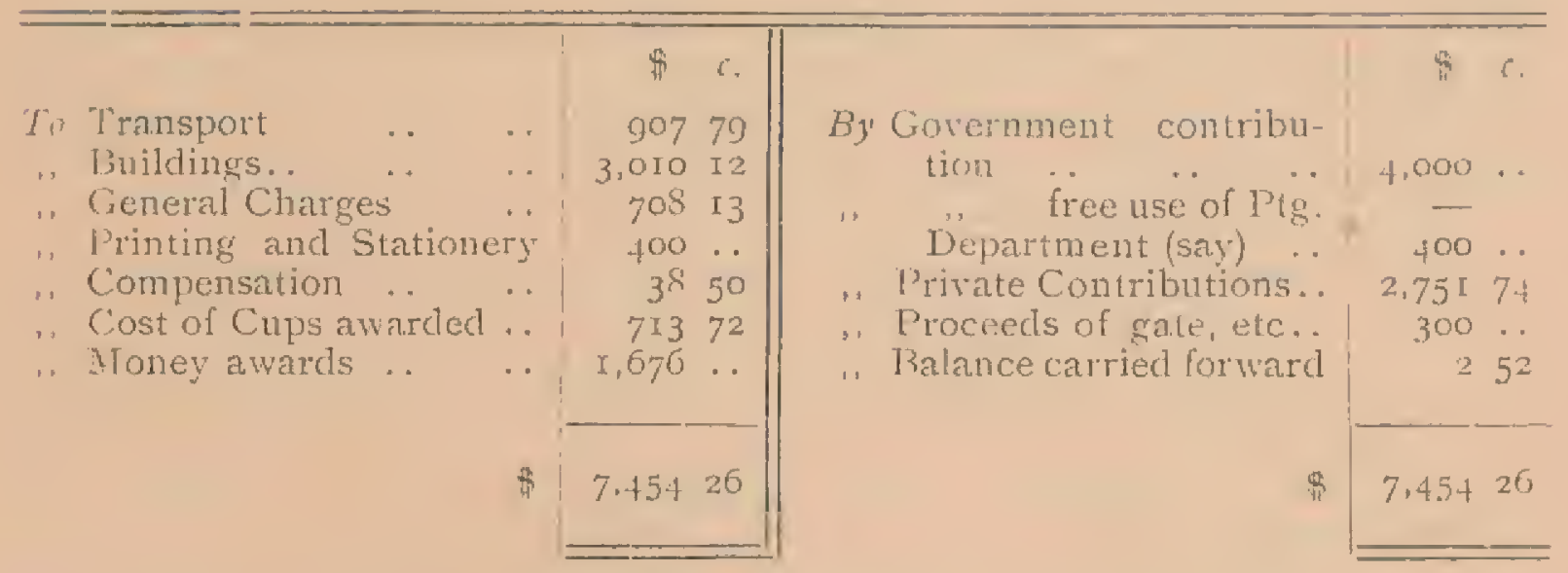

The amount of awards unpaid is based on statements made to me by the General Secretary. No charge was made for printing and stationcry, the F.M.S. Government Press having 


\section{$(238)$}

been placed at the Committee's disposal free of charge. No acconnt has been taken sub "Liabilities" of diplomas that at one time it was resolved to issue to prize winners.

LiUMA LUMPUR, 24 th February, I 905 .
H. ( . E. ZACHARIAS, Hon. Treasurer.

Audited and found correct with rouchers and receipts produced.

H. VANE,

$7^{\text {th }}$. March, I905.

Auditor.

\section{AGRICULTURAL SHOW FOR 1905.}

This year's Agri-Horticultural Show is to be held at Penany on the gth, roth and IIth of August next. The Prize List has just been published, and copies can be had of the Superintendent of Gardens at Penang, or the Director of Gardens at Sirrapore, on application. Exhibitors wishing free transport on the railway must sive notice to a District Officer or General Secretary, who will, if they approve of their cxhibits, provide them with numberen tickets and a free third-class pass on the railway for themselves and their exhibits. A reduction of 25 per cent. off the usual freight will be made by the Steamship Company on all bonat fide exhibits, other reasonable expenses connected with transport of exhbits will be considered by the Committce.

Entries in all divisions must be made in writing and reach the Secretary at least seven days before the opening day. In the case of horses and dogs three weeks.

The price of admission to the exhibition is two dollars for the opening ceremony the first day, and 20 cents every succeeding day.

Among the additional exhibits for which prizes are offered are Para rubber-seed oil and clore oil, the best collection of varieties of padi srown in any one Mukim and accompanied by an affidavit from the Penghulu that the whole collection was grown in his Mukim during the previous padi season. Para and Rambong rubber not less than 60 lbs. packed as sent from the estate.

Cups are offered as follows:-

For the best exhibit in the Agricultural Produce section

by H. E. THE Governor.

For best sample of rice prepared by machinery by Kis KI:K CHUAN.

Best collection of varieties of rice by Hon. A.

HUTTENBACH.

Rambong, best sample, by Negri Sembilan Planters AsSOCIATION.

Para and Rambong packed for shipment by W. IV. BAIIEY, EsQ. 
Para Rubber free from chemicals, SELANGor PLANTIRS Assuctation.

Sugar, best refined white, by LIM Eow HoNG.

Cannas, 6, presented by Dr. S. Kat.

Table decoration, T. GawTHorke, Eso.

Best collections of fruits, Hon. J. K. BrRCH.

Draught Bulls, pair, A. O. MERICAN.

Bull or Bullock, Peninsula, PFang Cattle Butcheks.

Buffalo, Sre AH THOON.

Champion animal in Cattle Section. Dr. Locke.

Boar, HOKIEN PORK BuTCHERS.

Sow, Pig Market Dealers.

Sow and Litter, AH KOON.

Six pigs, El,ToN Belz. Eso.

Best Pig over 300 catties, CANTONEs: PORK BUtCHJ.R.

Champion Pig, TEAM KEY.

Six Shcep, Indan MUtTon TRaming Co.

Sheep (pair), The Piginaleks.

Pail of Turkeys, H. STARR. Eso.

Best specimen of Native Work, GaN NGOH BEE.

Rubber Machinery, MeMBERS of ENGINEERS' INSTIT LTL.

Also a number of Cups for Horses and Dogs.

Penang has always shown well in cattle and ponltry, and we may hope for a rood display this time. The fruit crop, at least down South, promises very ill this season. but the Northern part of the Peninsula may be more productive. The rematiable success last year in the Malay arts section, and the subsequent sale of almost everything the natives brought at very sood figures, onght to bring as good an exhibition at least. Flowers and ornamental plants too should be much better than at last year's show, where they were remarkably poorly stiged, and we shall hope to see a really good exhibition of rubbers, not perhaps better in quality, for that shown last year at Kwala Lumpur was about as good as it could be, but in sreater grantity, and more representative of this great industry.

\section{SIR HUGH LOW.}

It is with much regiet that we have to chronicle the denth of one who did so much for agriculture and botany as Sir HugH Low, who died on April i 8 th, Igo5, very nearly eighty-one years old.

He was born on May Ioth, I 824 , and ahout I 8 to obtained an appointment in the Hon. East India Company and travelled out to the East with Mr. (Sir) JoHx BRoOkE, which ended in his quickly resigning his Indian appointment and joining the Rajal as secretary. He remained there about thrce years and then 
retmined to England where he published a well-known work in "Sarawal, its Inhabitants and Productions." In I8+\$ Mr. BROOKE became Actine Governor of Labuan, and HUGH Low accompanied him and became Colonial Truasurer of the Island, whence he risited Lawas and Brunei and made the first ascent of Mt. Kinabalu in I85 $\mathrm{T}$, where he collected the grand species of pitcher plants, Nepouthes Rajah, Lowii, Edrurdsianus, and iillusa.

He ascended the sane monntain again in 1858 , and again added much to the kuowledge of its flora. During his star in Borneo inficed he discosered a great number of plants now well known in our Gardens, besides the pitcher plants, among other Cypripedium Lowii, Fanda Luacii, Coelogyne fandurnta, the fine Rhododendrons of Borneo R. Brovermm, R. Loximmm, and others. So well was he know 1 by the natives to be an enthusiastic cullector of plints, that the prety follage Orchids Anoetoctilus and Hacmarta an knomm to this day by the Malays as loun Lo, or Low"s lear.

In 1877 he hecime Resident of Purak after the murder of Mn. BrkCH and held the appointment till he retired in ISSo. He Was created C.M.G. in 1879 . K.C.M.G. in I883, and C.C.M.G. in $\operatorname{sis} 9$.

During his residence in Perak le maintained his interest in botany and especially in agriculture. Starting with the Finalia Kinnsar Garden where he planted most of the best fruits of the country and introduced the fine naturilized Bornean lemon which is now fairly common in the State. Liman Bali, from the Island of binli, undonbtedly the bert pumoloe, llas also introduced by him and is cultivated by many Malays up and down the river. Of useful timbers. Mahogany, Cedrela toona, and Tuak were planted and mone of economics that were then proculable but what received attention. He was the first to plant Hered braziliensis in the Native States from seeds and plants. supplied by the Butanic Grulens, Simgapore. and planted at Cinila Kangsar, and from there planted in many parts of the State. The oldest trees on Fimming Estate and the large tree w two at Lady WEID S bungaluw as well as those at S"tiawan were all supplied from the Rinula Kingsar trees. Some stock tress of ficus dastion and Manihot Glasionit were also cultivated in this girden.

Cinchona and coffee Were tried at Waterloo and also at the Hermitage, and at the later warden many excollent English vegetables were successfully wromen.

The Cicely garden half way between Lady IVILD'S bungalow and the Hermitage was planted with tea ind liberian coffec and the Gapi's Garden surrounding. Lady WELn's bunaralow planted with pepper, liberian coffee and fruits. 


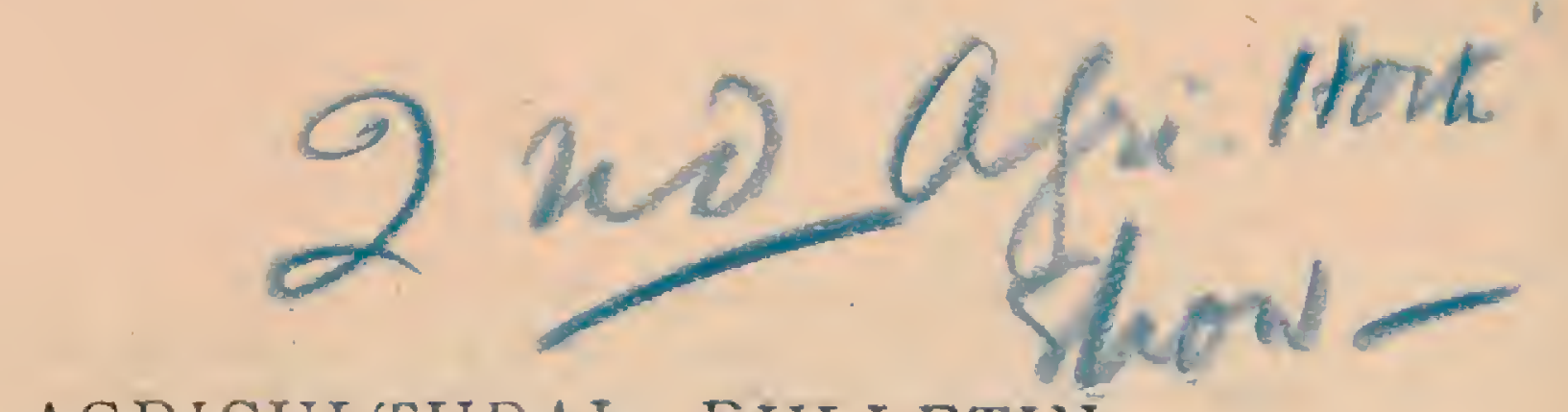

AGRICUL'TURAL BULLETIN

OF THE

STRAITS

AND

FEDERATED MALAY STATES.

No. 9.] SEPTEMBER, 1905. [VoL. IV.

\section{THE AGRICULTURAL EXHIBITION HELD IN PENANG.}

The second Agricultural Exhibition for the whole of the Malay Peninsula which was held at Penang this year was undoubtedly the finest display of the produce of the country both in agricultural products and in artistic work ever exhibited to the public in the Peninsula. Some Ceylon visitors were heard to ask why cannot we have Shows like this in Ceylon? Nothing approaching such an exhibition having ever been seen in the neighbouring Colony. The display indeed reflected the greatest credit on the Secretary, Committees and the numerous exhibitors and others who contributed to the success of the Show. The weather was perfect, hardly any rain falling during the three days of the Show, and the crowds of Europeans and Natives who came to see the exhibits testified to the interest taken in the produce of the country. Indeed, Penang was so crowded with visitors that many found some difficulty in finding places to stay in the town.

It was noticed that the Native exhibitors were this year more apt at the work of exhibiting their produce than on previous occasions, and seemed to understand the methods and regulations adopted for the arrangements of the different classes better than last year, thus telieving the Secretaries and others of a good deal of work.

In the Art Section which was very large, the Natives had no cause for regret that they had brought their works to Penang, for those who did not get prizes were gratified by the way in which the public bought their exhibits, and more would doubtless have been sold had not the unsold stock been repacked and carried off by the exhibitors on the last day with surprising rapidity. It would, perhaps, be worth considering if on future occasions some arrangement could be come to to have a sale-day after the Show.

The exhibition was opened at eleven o'clock on the first day by His Excellency Sir JOHN ANDERSON who had previously made a 
tour of the buildings. After a speech by Mr. J. K. BIRCH, His Excellency spoke for a considerable time on the subject of the exhibition and on the progress of agriculture; his remarks being very much to the point and very well received, after which he declared the Show open. The exbibition remained open till Friday evening when the Cups which had been presented by various donors were distributed to the winners by Mr. BIRCH.

\section{Agricultural Produce.}

The section for agricultural produce was the biggest and perhaps the most interesting part of the Show and both in quantity and quality exceeded all previous exhibitions. Taking the classes as they were arranged in the schedule, arrowroot was well shown, Mr. W. W. NORRIS of Singapore taking first prize and diploma for roots as he did last year and a second prize for prepared arrowroot. The second prize for roots fell to Caledonia Estate, the third to Penghulu Mat ChiE. Prepared arrowroot was represented by a number of good samples, the first prize being allotted to $\mathrm{Mr}$. CHOw AfI NEAN, his sample being a little finer than that of Mr. NoRRIS. In this class, a sample of the roots of Canna edulis, Tous les mois, a form of arrowroot seldom cultivated, was shewn.

Betel-nuts both fresh in the husk and dried and split were extensively exhibited, over fifty samples of the latter class being entered. The prize for fresh Betel-nuts fell to Mr. C. J. WiLliams with a very fine bunch. Dried nuts were more difficult to judge owing to the great number shown and the excellence of the samples. The comparative values of this product are estimated not only by the size of the nut and its fully ripe condition but also by its fullness and absence of a crack or split in the centre.

There were but few entries for articles made from the Coco-nut palm, and those not very first class collections but many of such articles were to be found in the Art Section of the Show. Coco-nut oil was clearly a popular exhibit, over eighty bottles of good, bad and indifferent oil being staged.

Toddy was represented by five or six exhibits, that of Caledonia Estate being considered by the expert employed to judge, a Tamil man, as very superior.

The great Show thus of products of the Coco-nut palm illustrated the great importance of this plant to the Malay Peninsula and the excellence of the produce in all its forms.

Dragon's blood, the red resin from the shells of the fruit of Doemonorops was represented by several high class specimens. ABDUL WAHAB taking the first prize with a good bright coloured sample of powder, and block with a bunch of the fruit.

Of Indigo there were several samples chiefly liquid and paste, Caledonia Estate showed a bottle of Indigo powder, dry, rather pale in colour, but considered worthy of special commendation by the judges on account of the difficulty and importance of preparing it in this form.

Fibres were poorly shewn, compared with the fine lot shewn last 
year by Mr. SCHIRMER, who did not exhibit on this occasion. Chiefly noticeable was some excellent rope of Sanseviera fibre prepared by Mr. PRIOR. There is a future for fibre in the Malay Peninsula and we shall hope to see it better shewn at future Shows.

Some samples of Raphia bast prepared in the Botanic Gardens, Singapore, from the leaves of the Raphia ruffia palm were shewn in this section.

Cocoa pods were only represented by two lots, neither ripe and of very inferior quality and no prize was awarded.

Cloves had thirteen exhibits and were fairly good. The sample that obtained the first prize being excellent, but we remember to have seen a better Show of this spice in former exhibits in Penang.

Nutmegs were very good and a considerable number both in the husk and in the shell were shewn, and Mace was also well represented.

There were several entries for collections of spices used in curries, very nicely got up and arranged.

Ginger was not as finely shewn as we have seen, though the sample which obtained the first prize shewn by Mr. CHIN KIN GOoN was by no means a bad one. The other samples were small and rather poor. The same might be said of Turmeric, the first sample, however, shewn by Mr. KANG THEAN SUNG, was exceptionally good. Perhaps the dry season at the beginning of the year may have been the cause of the poorness of the exhibits in these classes.

Pepper, both white and black, was represented by excellent examples from Gapis and Kamuning Estates and also from Sepang. In this class some excellent pepper was staged which was disqualified by the judges as it was discovered that the exhibitor was a dealer in Penang who had imported his samples from Achin.

There were one or two other cases in agricultural produce in which the exhibitor had shewn products purchased by him in the market, and unfortunately, by some oversight, the regulations did not prevent an exhibitor purchasing samples in the market and showing them as his own so long as they were grown in the Peninsula. Such a thing is hardly likely to occur except when the exhibitions are held in the large towns, Singapore and Penang, but the rules in this respect will require alteration.

Of Copra, over a hundred samples were shown, chiefly sundried, but Kiln-dried Copra was not wanting; many of the specimens were very fine.

The classes for Coco-nuts husked and unhusked, and collections of different kinds were, as at last year's Show, represented by a vast array, occupying one side of the building allotted to agricultural produce, and gave no small work to the judges to decide on which were the best. A Ceylon visitor was heard to remark that if he had 
known that the Malay Peninsula could produce such nuts he would have started planting here years ago. Mr. PRIOR as usual carried off the best prizes in this section both for collection and sample. In the collections of Coco-nuts some very odd varieties appeared, such as the horned Coco-nut with its short horn-like additional carpels, and some very narrow and small fruits, and many more useful varieties.

Coco-nut Sugar was well shewn, the samples being numerous and good, and giving some trouble to the judges to decide on their respective merits. There were five or six exhibits of Nipah Sugar, and plenty of the sugar from the Kabong palm Arenga, most of which was very good and some very excellent. Tea was not unrepresented; Durian Tunggal estate taking first prize.

Gambir was fairly shewn, Penghulu KASAN's Cube, and Nail gambir being a good sample.

Coffee shewed by the scantiness of the exhibits how much this product has fallen before the advance of rubber, still what was shewn was of very good quality. Mr. BAILEY carried off a prize for his hybrid Stenophylla-liberica, and was seconded by Mr. PRIOR, and the latter won on Liberian coffee both new and old.

Of Essential Oils there were a number of interesting kinds shewn. Mr. Hardouin staged a very pretty series of Patchouli, Nutmeg, Clove, Lemon-grass and Citronella. Mr. MACHADo shewed also several good and unusual oils including Calamus Oil, (Acorus Calamus) and Lemon Oil.

Citronella Oil was shewn also separately but not in large series.

Castor Oil had eight exhibits and was good. Sesamum Oil was also shewn, and one sample of Rubber Seed Oil very clear and bright was exhibited by Mr. COATES. Para rubber seed is too much in demand this year for planting to produce a large display of its value as an oil.

Sago Flour and Pearl Sago were not as well shewn as might have been expected, the samples being few and poor.

Tapioca roots were fairly good and some were large, and the display on the whole was about as good as usual.

Tapioca Flour and Pearl, and Flake Tapioca were well shewn and of good quality and colour.

Sugar Canes were not very extensively shewn, but it is unnecessary to say that those coming from Caledonia Estate were very fine. The variety of kinds shewn by other exhibitors was not large, but both crushing and eating canes were represented. A branched cane, not by the way a great rarity, attracted some attention. In refined Sugar, Caledonia Estate was the only exhibitor, for a very nice looking sample refined in Hongkong was promptly disqualified by the judges. Rum and Rum shrub also were only represented by samples from the great Sugar Estate, whence also came a sample bag of Molascuit, a new food for horses mostly composed of sugar, and said to be very popular with these animals. 
Brown Sugar was well shewn, LEONG LAK HING of Bagan Serai getting a prize for this.

The Shows of Jungle Produce as represented by Guttas and Rubbers, and also Damars, were poor, and call for no special remarks. Rattans were well represented, a considerable number of collections being shewn. Many of those exhibited were, however, hardly trade rattans, still as collections they were good and interesting. Bamboos were well shown, ordinary kinds as well as the curious spotted bamboo, twisted ones, and somie large specimens of the big Dendrocalamus giganteus.

Native herbs used in medicine were very much more extensively shewn than at last year's Show where there were but few entries. One competitor took a prize with a hundred and four different herbs with a catalogue of their native names and uses.

Mr. MACHADO brought from Kamuning estate some valuable drugs he had grown and prepared including Ipecacuanha, Jeringu (Acorus calamus) Brucea sumatrana. Hydrocotyle asiatica, dried leaves, and Papain for the latter he received a special prize.

Fodder grasses were represented by several collections, but none were particularly well selected. The exhibitors seemed to think that any grasses or sedges could be classed as fodder grasses, and some of the collections contained coarse sedges quite unsuited for fodder. Of Tuba roots a considerable number of samples were shewn, chiefly differing in thickness of root and neatness of preparation.

Patchouli was well shewn, twenty-two samples being staged, $\mathrm{Mr}$. MACHADO again this year carried off first prize with fine and well dried leaves. Of Sirih leaves there were twenty-six specimens, both of the Chinese and Malay strains, nearly all were very fine large-leaved samples. The Malay who was asked to judge this difficult class, gave his verdict for the red veined form.

Indian corn was good and abundant, some fine colès being shown. There was little variety in the kinds staged, only one sample of the red corn being shown.

Cotton was chiefly and largely represented by the short stapled Javanese cotton, but Mr. LoGAN won on a fine sample of Egyptian prepared with great care, and grown in the North of Province Wellesley, Mr. MACHADo also showed Egyptian.

Kapok was very abundant, and many of the samples very good. Para rubber was naturally one of the most interesting and attractive exhibits and the collection was for the most part superb. $\mathrm{Mr}$. BAILEY's crepe and fancy rubber were highly admired. Mr. PRIOR's crepe, and the samples shown by Mr. CoATES, Mr. MACHADO and Sandy Croft estate were all of the finest qualities. The old fashioned biscuits were represented but the crepe and flat oblong sheets were more in favour, and the round biscuits seem to be almost a thing of the past. Scrap was well prepared by $\mathrm{Mr}$. BAILEY and Mr. MEIKLE, and was really very good. Rambong was not wanting, Mr. PRIOR'S crepe strips being the best sample 
of this rubber we have seen, Mr. BAILEy shewed excellent samples. One untoward thing occurred in the judging of the rubber classes, Mr. LAKE and PAGETS' cup for any rubber of other class than what took first prize in Para rubber, was awarded to a good sample of sheet rubber, entered in the name of a Chinaman, who it proved later was merely a buyer and not a cultivator. In cases like this the prize should really belong to the grower and preparer of the rubber and not the mere agent, who has no claim to it.

A prize offered for improved rubber machinery was awarded. to an improved roller for making sheet rubber. It is needless to say that both in quantity and quality the exhibition of rubber was an immense improvement on the display of last year's Show, and was an exhibition of which the planters might well be proud.

\section{Flowers and Plants.}

The exhibition of horticulture was by no means what it has been at previous Shows, and was little if at ail better than that of last year's Show at Kwala Lumpur, a very large number of the classes in the schedule were not at all represented. The flowers were scanty and on the whole poor, the foliage plants not up to standard, why this should be one cannot say, but one cannot help feeling that we have not now-a-days the horticulturists of some years back. In a country where so many fine and beautiful plants can be easily grown, it is regrettable to see so poor a Show staged.

A fine Grammatophyllum speciosum with eight flower spikes shewn by Mr. A. OECHALE was very attractive. The Cantonese Club showed a fine series of cock's combs and of asters, but beside this there was practically nothing in the way of flowering plants to look at. The Botanic Gardens of Penang shewed some fine Cattleyas and other orchids, and the beautiful white, red and pink Tobaccos, Nicotiana Sanderiana and affnis and a number of pinks which brightened up the exhibition considerably of foliage-plants. Mr. JOHN BROWN shewed a good group of aroids and Mr. MACHADO brought from Kamuning a very fine pot of white Caladium; Mrs. LoGAN took a prize with a large Asparagus plant. Palms were somewhat better shewn, Kow Jo Tok took the first prize for six palms, well grown but common kinds, Mr. MACHADO showed some of the rarer nature palms from the Kamuning woods, including both forms of new Pinanga acaulis and Iguanuara Wallichii, and took the first prize for specimen palm with a well grown Licuala grandis.

Mr. TAN TIN BEE shewed a nice little lot of palms also including the gem of the whole of the foliage plants, a perfect specimen of the beautiful and rare Licuala orbicularis from Borneo.

Two lots of the Chinese grotesque plants were shown, both good of their kind.

Mrs. J. BROWN shewed a fairly good group of various plants.

A few medium Crotons, a poor lot of Begonias, some mediocre ferns and Selaginiellas made up the rest of this part of the exhibition. The cut and arranged flowers were scanty, the roses were fairly 
good, those of Kamuning being very good for low country cultivation. There were several entries for table decoration, Mrs. Fox securing the first prize for a pretty arrangement of roses, a table decorated with pink and white Honolulu creeper was the next best arrangement.

\section{VEGETABLES.}

In the classes for vegetables there were some fa rly good exhibits but the attraction in this part of the Show was the superb series of vegetables from the Perak Hills Gardens shown by Mr. CAMPBELL. Here on a circular table were shown peas, carrots of two varieties, very large, excellent cabbages, potatoes of unusual size for even our hill stations, excellent beet root, tomatos, leeks, radishes, vegetable marrows, two varieties, turnips, kohlrabi, celery (exceptionally large) artichokes, chocho, parsley, mint and several other vegetables. Undoubtedly the finest exhibition of European vegetables ever seen in the Peninsula.

With these were staged some good Cocoa-pods, and some thin sheets of Ceara rubber (very clean and good) Castilloa, Rambong, and Para rubber. The whole display reflected the greatest credit on Mr. CAMPBELl. Of the vegetables shown for competition the first prize for collection of vegetables was won by a very poor lot, Cucumbers were fairly good, and so were beans of different kinds, the rest of the vegetables were very ordinary.

\section{FRUIT.}

The fruit classes were better on the whole and contained some very good exhibits, but it was difficult to see on what grounds the judges awarded their decisions, as in the cases of clurians, pineapples, papayas and pumeloes, the fruits were not even opened, so that flavour and condition seemed to be eliminated in deciding the comparative value of the fruits. In the case of any fruit not specially classed a prize was given to some fruits supposed to be lemons, and by some thought to be oranges, but which were really one of the citrons of inferior quality. The most noticcable fruits in this class were some good avocado pears shown by $\mathrm{Mr}$. COATES, and some good pomegranates shewn by Mr. KHEW KHOOI of Balik Pulau, but neither of these exhibits obtained any mark of commendation.

But few Rambutans were shown, although the crop seemed to be very fine in Penang this year, but the samples were fairly good, and the Pulasans better, Tampunet, (Artocarpus rigida) a fruit less well known to the Eurpoean than it should be was well shown. A good number of pines were shown, chiefly Mauritius pines, of which the sample which received the first prize was hardly in condition. There were two samples of black West Indian, one of which large but overripe obtained a first prize, the second in better condition, were smaller. A second prize was won by some field pines, (of the Red Ripley style) large but of no particular merit. A special prize was awarded to a tall-stemmed pine with a small and almost rotten fruit. Perhaps the most interesting pine shewn was the Pernambuco (Abacaxi) of which three fruits hardly ripe, how- 
ever, were shown by Mr. CoATES. This white fleshed pine is perhaps the finest eating pine in the world.

Of other fruits especially noticeable were some very fine Binjai of great size, which well deserved the prize they obtained. The Guavas were also good and there were ten exhibits of Dukus.

Bachangs, Champedaks, Watermelons (as far as one could judge from unopened fruits) were all good, and the Chikus (Sapodillas) were of good size but not ripe. The Pumeloes were good in point of size. The Durians it was impossible to form an opinion on as none were opened. Oranges were only represented by two exhibits, one sent by Mr. KIM KEAT of Singapore was deservedly given a first prize. Mangosteens considering the poor season were well up to standard. Of Papayas hardly any but the very large green kind were shown. These though highly suitable for extracting papain, are usually very inferior as fruit to the smaller orange variety, which was only represented by one or two fruits. Bananas were plentiful but most were overripe, some of the bunches however were in good condition and of good strains. The prize for the collection of fruit was won by Mr. LoGAN with a good and well arranged set in excellent condition. On the whole, considering that the fruit season has been a bad one this year in most places, the display of fruit was good, and very superior so the show of vegetables.

\section{ART.}

The Art Section of the Show was even better than that of last year and was indeed overcrowded. Among the exhibits which had a botanical interest were some hats of the style of Panama hats made by the Sakais under the direction of Mr. CERRUTI. These hats which were soft and flexible and of good form and texture were made of strips of Banana fibre. Mr. CERRUTI at first attempted to cultivate the Panama hat-plant (Carludovica palmata) for this purpose and plants were sent from the Singapore Botanic Gardens, but this plant is of slow grouth, at least in this country, and so he taught the Sakais to use the cultivated Banana instead. Only some half dozen hats were exhibited, but they were so highly appreciated by the public that any number could probably be disposed of. Mr. CERRUTI may be congratulated on his inducting the Sakais into manufactures of useful articles for which there is some demand.

\section{STOCK.}

The exhibition of stock was by no means as good as might have been expected, or indeed as was shewn at the Show held at Penang some years ago.

Cattle were hardly shown at all. Mr. Douglas won a prize however with a small but nice looking Australian bull, as champion animal in the Cattle section. Local bred and Indian Cattle were poorly represented. Buffalos were represented by a couple of bulls of no great merit.

Pigs were better shown, there were some very good sows on view. The champion animal being an enormous and very sulky brute, 
weighing 480 catties $\left(5 \frac{1}{2} \mathrm{cwt}\right)$. There were a good number of litters of pigs also. Goats were few and not very good. Sheep had all classes filled, the first prize Ewe sheep being exceptionally fine, the other exhibits were fair.

\section{Poultry.}

The exhibition of poultry was very good, and far in advance of last year's Show. Mr. H. WOOL won the prize for the champion with a grand pair of fowl. Two pens of Buff Cochins were highly admired and there was a good show of Bantams, Malay fowl, Silkies, and other breeds. Guinea fowl were not as good as last year, only one entry of a pair of good white birds being made. Muscovy ducks and Manila ducks were well shown, and the Geese were fine and in good condition.

The Pigeons, Antwerps and Fantails were very good, but there aught to have been more entries.

Of Cage-birds a number of different kinds were shown, Nicobar Pigeons, Pergam (Carpophaga), Hornbill, Parakeets, Crested Partridges, Avadabats and other birds.

Rabbits, at one time a feature of Penang Shows, were not exhibited. Two lots of Guinea Pigs however created much interest among the Natives.

\section{PRODUCE.}

The Butter which was rather extensively shown was very good, and there were many entries for Eggs, both of fowls and ducks, and some of the samples were exceptionally good.

\section{The Governor's Cup.}

For the best general exhibit in Agricultural produce was carried off by Caledonia Estate, Kamuning coming second. This cup was judged for by a system of marks for.first, second and third prizes, and for exhibits which did not take prizes but were worthy of being shown, Mr. MACHADO of Kamuning Estate won a cup given for the best general exhibit of the Show, shewing Para Rubber, Coconuts, Ginger, Oils, Papain and other drugs, Pepper black and white, Patchouli, Arrowroot, 'Betelnuts, Kapok, Coffee, Fibre, Citronella Oil, Coconut Oil, Sirih, Tapioca roots, Tea, Tuba, Turmeric, Chilis, Limes, Roses, Palms, Fowls; two breeds, Eggs, Caladiums, not a bad' exhibition for a comparatively small estate. Caledonia estate showed Betelnuts, Arrowroöt, Indigo, Rum, Rum shrub, Sugarcane, Sugar, Patchouli, Cotton, Kapok, Coffee, Oil, Coconuts, Tapioca, Toddy and Rubber. Mr. PRIOR though showing less in variety did very well with his fine lot of produce, taking three prizes for Coconuts, one for Copra, two for Coffee, two for Rubber and a highly commended Fibre, which was about all he showed.

NeXT YeAR'S. ExHIBITION.

At the suggestion of His Excellency the Governor the exhibition in 1906 will be held at Singapore about the end of July or beginning of August, and we shall hope to have as successful an exhibition as the last. 


\section{PRIZES AWARDED AT THE PENANG AGRICULTURAL SHOW 1905.}

\section{DIVISION $A$.}

\section{Agricultural Prodnce.}

Class.

Ist. 2nd. 3Td Extra.

I. Arrow-root, fresh, best sample.

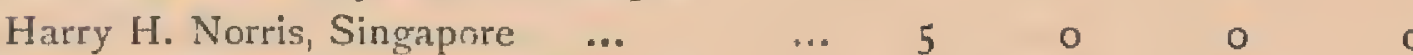

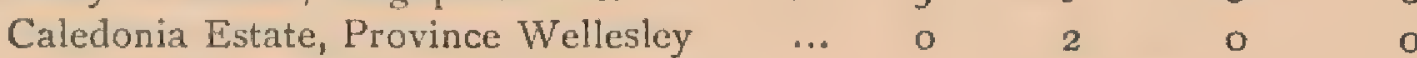

Penghulu Mohamed Sahid, Nibong Tebal o $\quad 0 \quad$ I

2.

Arroze-root, prepared, best sample.

Chow Ah Nyen, Penang

Harry H. Norris, Singapore ... ...

Sahid bin Lebai Mohamed Salleh, Nibong Tebal o

Bamboos, best collection.

Penghulu of Pulau Tiga, Lower Perak

Penghulu Pulau Kemeri, Kuala Kangsa

Sahat bin Rahman, Malacca

$\begin{array}{ccccc}\ldots & 5 & 0 & 0 & 0 \\ \ldots & 0 & 2 & 0 & 0 \\ \ldots & 0 & 0 & \text { I } & 0\end{array}$

Betel-nuts, fresh.

C. J. Williams, Bukit Mertajam

Penghulu Mamud, Negri Sembilan

Gan Pow, Butterworth

...

5.

Betel-nuts, dried and split.

Mohamed Hassin, Bukit Mertajam

Kung Thean Sung, Penang -...

Penghulu Mamud, Malacca ...

Cloves, best sample.

Law Chit Mun, Penang

S. M. Peralta, Malacca

Cheah Soon Soon, Penang

$\ldots \quad \ldots \quad$ 10

... $3 \quad 0$

... 0

...

$\begin{array}{lllll}\cdots & 5 & 0 & 0 & 0\end{array}$

.. $00-3$

.

Coconuts, unhusked, be'st sample.

Penghulu Mat Hassan, Bukit Mertajam $\quad \ldots \quad 7 \quad 7 \quad 0 \quad 000$

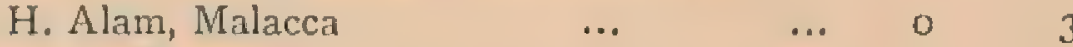

Bertam Estate

8.

Coconuts, husked, best sample.

Pachee, Province Wellesley

Penghulu Lehir, Telok Anson

Penghulu Mat Assan, Bukit Mertajan

Penghulu Yusop, Malacca (Extra Prize)

...

$\$$

o

o

o

o

$$
\text { o }
$$$$
\text { o }
$$

o

9. Coconuts, best collection of varietics.
E. B. Prior, Selangor, Medal
Abu Kassim, Kriars
Syed Hamad, Penang
$\begin{array}{ccc}\ldots & \ldots & 10 \\ \cdots & \cdots & 0 \\ \cdots & \cdots & 0 \\ \text { mextra } & \text { Prize) } & 0\end{array}$

$\begin{array}{lllll}\cdots & 7 & 0 & 0 & 0 \\ \cdots & 0 & 3 & 0 & 0 \\ \cdots & 0 & 0 & 1 & 0 \\ & 0 & 0 & 0 & 3\end{array}$

10. Copra, best sample sun dried,

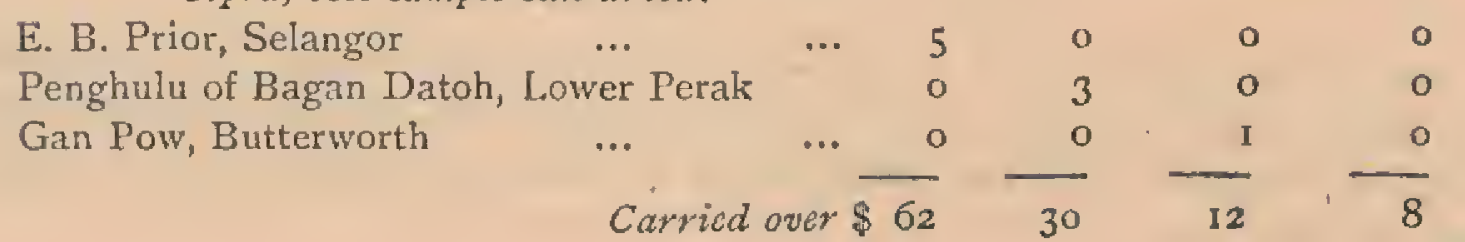




\section{Division A.-Continued.}

Class.

\begin{tabular}{|c|c|c|c|}
\hline 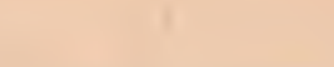 & $\$$ & $\$$ & $\$$ \\
\hline Brought forward & 62 & 30 & 12 \\
\hline
\end{tabular}

I I. Copra, best sample kiln dried.

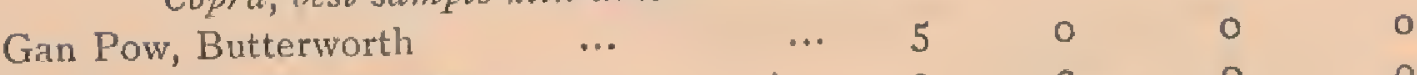

Koe Tiang Hock, Penang ... ‘... o 3030

12. Coconut palm, best collection of products.

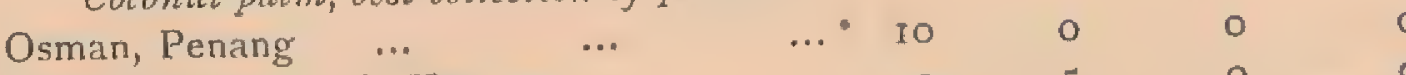

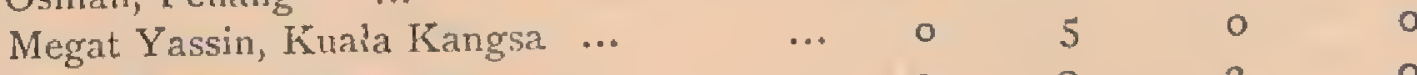

Ngah Ma Aruf, Kuala Kangsa

Cotton, "Kabu Kabu" best sample.

Haji Mohamed Sahaat, Penang $\quad$... 5 - 5 o

Syed Ahmad, Penang $\quad$... $\quad \ldots .003$

Mohamed Hassin, Bukit Mertajam

14. Cotton "Kapas" any other variety best sample.

D. Logan, Penang, Medal …

A. D. Machado ...

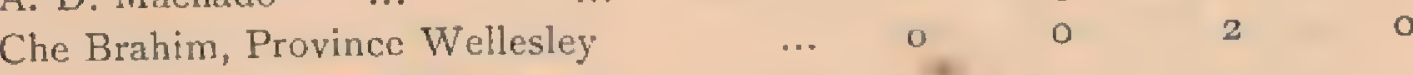

Coffee, Liberian.

E. B. Prior, (Selangor) Medal ... $\quad$... 10 10 0 o

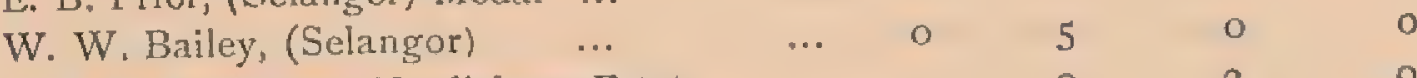

C. \& R. S. Meikle, Wardieburn Estate $\quad . .00020$

I 7.

Coffee, any other varicty.

W. W. Bailey, (Selangor)

E. B. Prior, Selangor

Dragon's Blood, best sample.

Abdul Wahab, Krian

Penghulu Hiassin, Krian

P. Cornelius, Penang

$$
\begin{array}{lll}
\ldots & \ldots & 5 \\
\ldots & \ldots & 0
\end{array}
$$

Fibres, best collection.

19.

Syed Ahmed, Penang

Mydin, Penang ...

Shaik Hossain, Penang

20. Fodder, Grass best collection.

Alli, Penáng

Mohamed Arasat, Penang

Chek Lah, Penang

Gambier, best sample.

$2 I$

Penghulu Kassan, Ayer Molek, Malacca ...

Penghulu Haji Jawal, Ayer Molek, Malacca

Penghulu Haji Mamud, Ayer Molek, Malacca

22. Getalus, best collection of local.

Penghulu Grik, Perak ...
23. Gums and Dammars, best collection of local.

Haji Saman bin Itam, Malacca

Penghulu of Pulau Tiga, Lower Perak ...

Penghulu Grik, Perak

Ginger, best sample.

Chin Kim Yoon, Penang

$$
\text { Carried over } \$ 156 \quad \frac{5}{73} \quad \frac{0}{25} \frac{0}{8}
$$




$\begin{array}{ccccc} & \text { Ist. } & \text { 2nd, } & \text { 3rd. } & \text { Extra. } \\ \text { Brought forward } & \$ & \$ & \$ & \$ \\ 15 & 73 & 25 & 8\end{array}$

Ginger, best sample-continued.

Kim Kwee, Penang... $\quad \ldots \quad$...

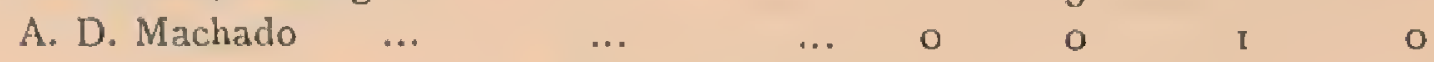

25. Indigo, prepared, hest sample.

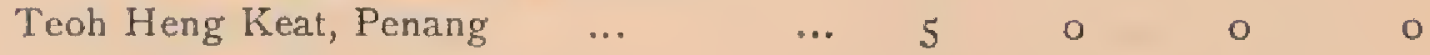

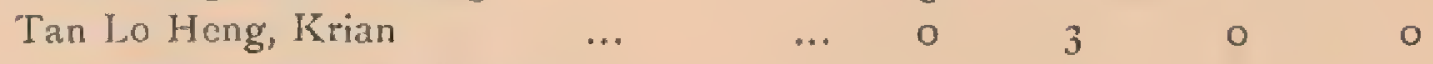

C. J. Williams, Bukit Mertajam $\quad \ldots .000000$

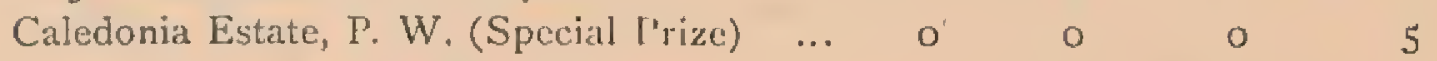

27. illace dried, red.

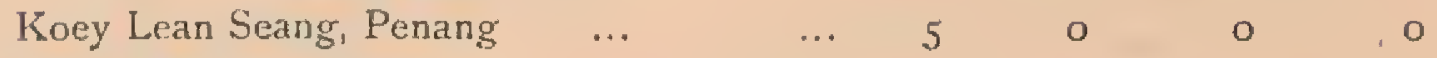

$\begin{array}{lllllll}\text { Chang Kim On, Penang } & \ldots & \ldots & 0 & 3 & 0 & 0\end{array}$

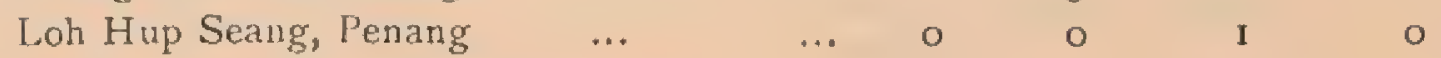

28. Maize.

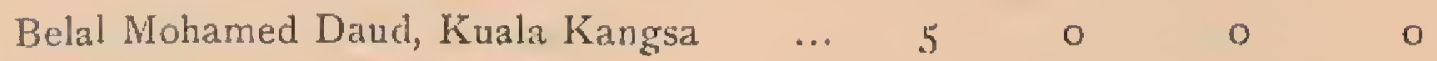

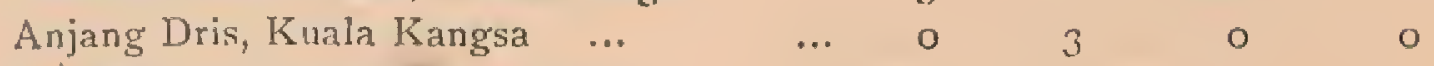

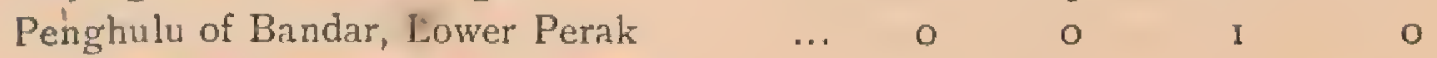

29. Medicinal Plants, best collection.

Penghulu of Durian Sebatang, Lower Perak Io 0 o

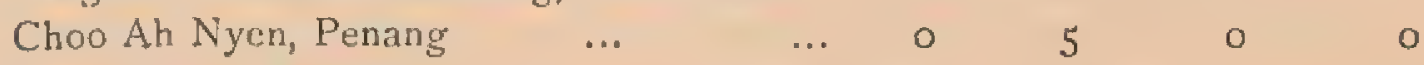

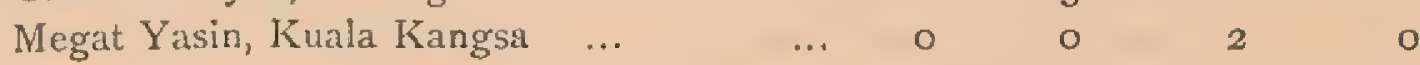

30. Nutmegs, fresh, best sample.

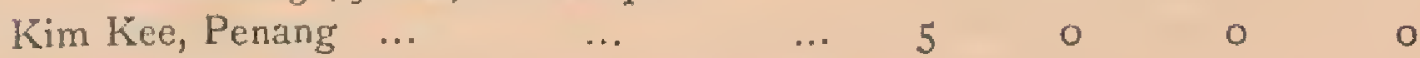

C. \& R. S. Meikle, Wardiebum Estate, Selangor $0 \quad 3 \quad 00$

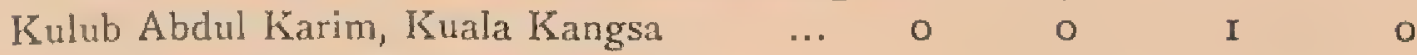

31. Nutmegs, dried, best sample.

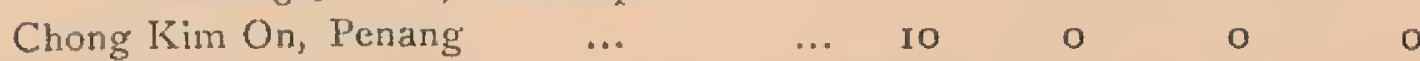

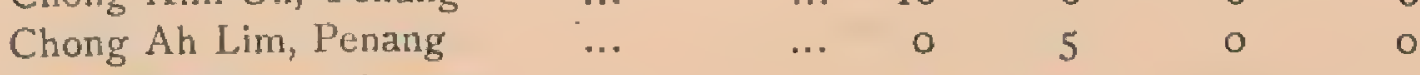

$\begin{array}{lllllll}\text { Teoh Tiang Siew, Penang } & \ldots & \ldots & 0 & 0 & 2 & 0\end{array}$

32. Oil, citronella, best sample.

Mohamed Zain, Penang $\quad \ldots \quad$... 10 ro 0 o

$\begin{array}{llllllll}\text { E. E. Chasseriau } & \ldots & \ldots & \ldots & 0 & 5 & 0 & 0\end{array}$

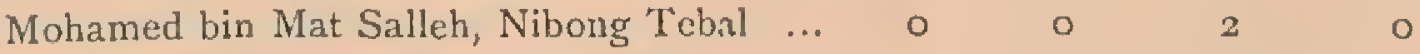

33. Oil, Coconut, best sample.

Lim Soo Lim, Penang $\quad \ldots \quad$...

William Kellie Smith, Batu Gajah $\quad \ldots .0000$

$\begin{array}{lllllll}\text { G. B. Baptist, Penang } \quad \ldots & \ldots & 0 & 0 & 2 & 0\end{array}$

34. Oil, tee! seed (Minyak lengah) best sample.
William Kellie Smith, Batu Gajah

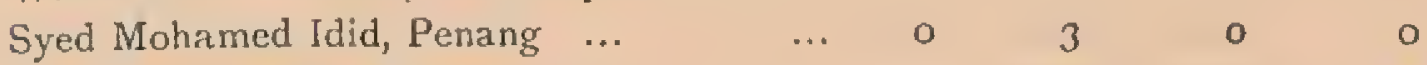

$\begin{array}{lllllll}\text { Chain bin Che Mat, Penang } & \ldots & \ldots & 0 & 0 & \text { I } & \text { o }\end{array}$

35. Oil, Castor, best sample.

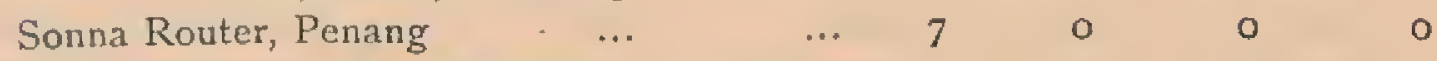

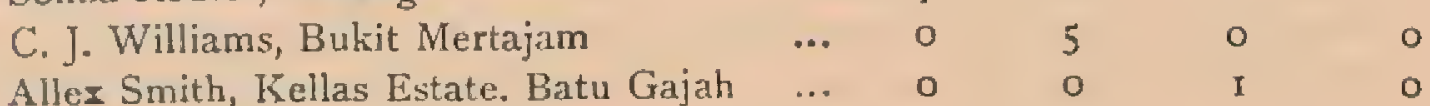

36. Oil, Para Rubber, best sample.

W. J. Coates, Negri Sembilan.

Carried over $\frac{5}{\$ 233} \quad \frac{0}{116} \quad \frac{0}{40} \quad-\frac{0}{13}$ 


\section{Division $A$.-Continued.}

$3^{8}$.

$\begin{array}{ccccc} & \text { rst. } & \text { 2nd. } & 3 \text { rd. } & \text { Extra. } \\ \text { Brought forzuard } & 233 & \$ & \$ & \$ \\ \text { II } 6 & 40 & \text { I3 }\end{array}$

Oil cake, best sample.

D. Mohamed Noor, Penang

Oils, best collection of essential.

A. D. Machado ... $\quad . . . \quad \ldots$, o

William Kellie Smith, Kellas Estate, Batu Gajah o Pepper, white, best sample.

40.

E. R. Salisbury, Padang Rengas

Lee Seng Nam, Selangor

A. D. Machado

Pepper, black, best sample.

E. R. Salisbury, Padang Rengas

A. D. Machado

Loh Kong Yew, Negri Sembilan

42 .

Pepper, Trang, best sample.

Ban Tek Hong, Penang

Patchouli, best sample.

A. D. Machado ... ...

- Mad Tahir, Kuala Kangsa ...

IYaji Gabor, Kuala Kangsa ...

44 .

Padi, any variety.

Penghulu Che Puteh, Krian ...

Penghulu Mohamed, Krian ...

Haji Othman, Butterworth ...

$\begin{array}{lllll}. . & 10 & 0 & 0 & 0\end{array}$

$\begin{array}{lllll}\text {.. } & 0 & 5 & 0 & 0\end{array}$

$\therefore \quad$ o

$\begin{array}{llll}. & 10 & 0 & 0\end{array}$

$\begin{array}{lllll}. . & 0 & 5 & 0 & 0\end{array}$

.. 0 o 2

Padi, best collection of varieties.

Penghulu Haji Mohamed Ahib, Krian $\quad . .550$ 50 0 o

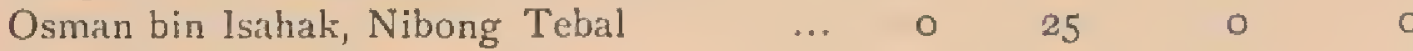

Haji Mamudin, Perak

Pulot, best sample.
Penghulu Cheh Puteh, Krian...

$4^{6}$.

Kung Thean Sung ... …

47. Pulot best collection of varieties.

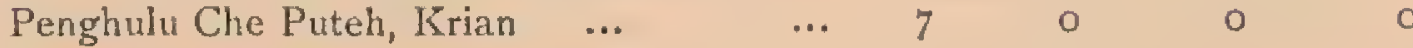

Osman bin Isahak, Nibong Tebal $\quad$... 003000

Mukim ljok, Perak

48. Rice, prepared by machinery, best sample.

Daub, Malacca … ... ...

49. Rice, prepared by pounding, best sample.

Haji Abas, Bukit Mertajam ... $\quad$...

P'enghulu Hasim, Krian

50. hice, hest collection of varieties.

Haji Mamudin, Perak

Muntri Tengah, Malacca Rotans, best collection.
Mat Asat ...
Haji Mohamed Sahat, Penang
Penghulu Grik, Perak


Barried over

... 
Division A.-Continued.

$\begin{array}{ccccc} & \text { 1st. } & \text { 2nd. } & 3 \text { rd. } & \text { Extra. } \\ \text { Brought forwart } & \$ & \$ & \$ & \$ \\ & 370 & 18 S & 68 & 13\end{array}$

53. Rubber, (rambong) best sample:

W. W. Bailey, Selangor

54. Rubber, (Para \& Rambong) general exhibit which must be packed in boxes as sent from the Estate, and guaranteed that such exhibits have received no special treatment.

C. E. Symonds, Yam Seng Estate

55. Rubber, (Para) free from chemicals.

W. J. Coates, Negri Sembilan ...

59. Sago flour, best sample.

Noodin bin Kali, Malacca ...

Set Kee Ann, Malacca _..

60.

Penghulu Mat Sahat, Malacca ...

Sireh leaves.

Penghulu Mamud, Malacca

Kulop Sotan, Kuala Kangsa ...

Mohamed Salleh, Penang

$6 x$.

Spices, best collection.

D. Mohamed Noor, Penang ...

Sh. Mohamed Zinalabdin, Penang

Mohamed Ariffin, Penang

$\cdots \quad \ldots \quad 5$

10

o

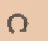

62.

Sugar Canes.

Leong Lok Hing, Penang

Sugar Canes, best collection of varietics.

Caledonia Estate, P. W., Medal

64.

Sugar, Coconut, best sample.

Haji Hamid, Selangor

Penghulu Mohamed

Ismail, Butterworth

$\begin{array}{lll}\ldots & \cdots & 5 \\ \cdots & \cdots & 0\end{array}$

... $10 \quad 0$

... 0 o 3

.. 0 o

o

o$$
0
$$$$
\text { o }
$$

65. Sugar, Nipah, best sample.

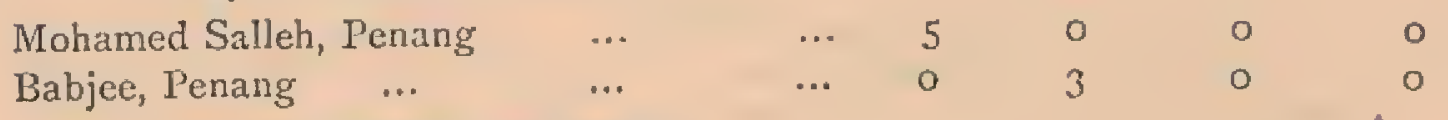

66. Sugar, Kabong, best sample.

$\begin{array}{llllllll}\text { No Name } & \ldots & \ldots & \ldots & 5 & 0 & 0 & 0\end{array}$

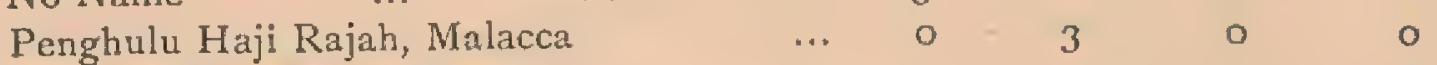

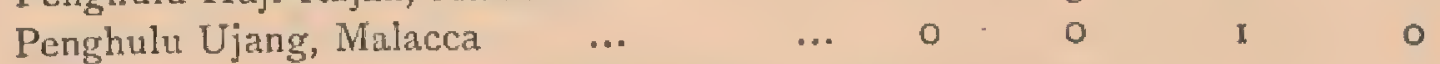

67. Sugar (cane) brown, best sample

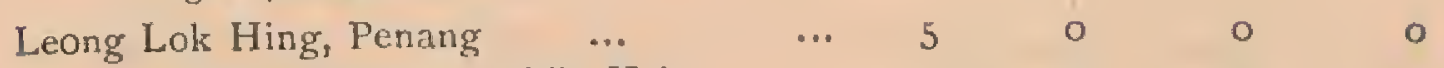

Penghulu Haji Mohamed Akib, Krian ...

Ong Yu Tong Siang, Ek Estate, Krian … $\quad 0 \quad 00 \quad 1 \quad$ o

68. Sugar (cane) refined white, best sample.

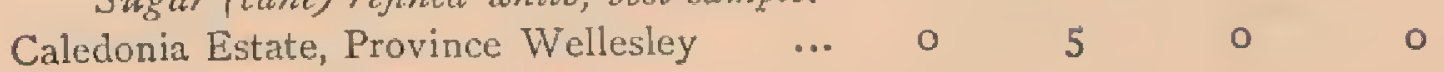

69. Tapioict, roots.

Geo. Stothard, Malakoff Estate, P. W. ... 5 , o $\quad$ o

Jatin bin Mat Akib, Nibong Tcbal $\quad \ldots .0000$

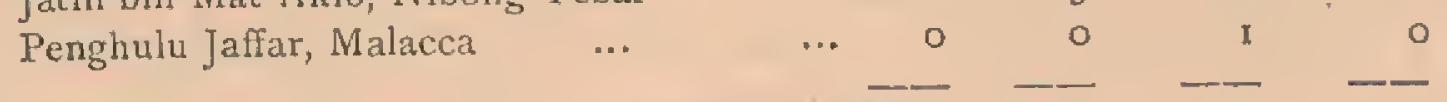

Carried over $\begin{array}{llll}\$ 440 & \overline{249} & 77 & -\end{array}$ 
Drvision A.-Continued.

$\begin{array}{ccccc} & \text { Ist. } & \text { 2nd. } & \text { 3rd. } & \text { Extra } \\ \text { Brought forward } & \$ 40 & 249 & 77 & \text { 13 }\end{array}$

7o. Tapioca roots, best collection of varicties.

Penghulu Mohamed Sitid, Nibong Tebal ... 10 o 0

Alma Estate

Tapioca, pearl, best sample.

71 .

Chan Say Peng, Malacca

Penghulu Alom, Malacca

Khon Soo Tat, Penang

Kung Cheng, Malacca (Special Prize)

72.

Tapioca, flake, best sample.

Geo. Stothard, Malakoff Estate, P. W.

Chan Koon Cheng, Malacca

Diamond Jubilee Estate

$\begin{array}{lllll}\ldots & 5 & 0 & 0 & 0 \\ \ldots & 0 & 3 & 0 & 0 \\ \ldots & 0 & 0 & 1 & 0\end{array}$

73.

Tapioca, flour, best sample.

Chan Koon Cheng, Malacca ...

Caledonia Estate, Province Wellesley $\quad \ldots \quad 5$

Sahid bin Lebai Mohamed Saleh, Nibong Tebal o

74. Tapioca, any other preparation. .

Geo. Stothard, Malakoff Estate, F. W. ... 5 o $\quad$ o

C. Tuah Choo, Bukit Mertajam

‥ 03

75.

Tea, best sample.

S. M. Peralta, Malacca

Penghulu Tongsah, Malacca .

76.

Toddy, best sample.

Pariathumbee Pillai, Penang ...

Narayanasany Pillai, Penang (Special Prize)

... $10 \quad 0$

o

0

$$
\text { o }
$$

77. Tuba (akar) best sample.

H. H. Cornelius, Penang

Ma' akir, Negri Sembilan

$\begin{array}{llll}\ldots & \ldots & 3 & 0 \\ \ldots & \ldots & 0 & 2\end{array}$

Termeric, best sample.

78.

Kung Thean Sung, Penang

Lean Loi Fat, Penang $\quad$...

Wok, Negri Sembilan

$\cdots$

... 5

...

$\cdots$

red.

79. Walking sticks, best collection unprepared,

Hassan, Pulau Jerejak

Daub, Malacca ...

Penghulu Sahid, Malacca

...

a. 10

… $\quad \therefore \quad 0$

8o. C. \& R. S. Meikle, Selangor (2nd prize) ... o

W. W. Bailey, (ist prize) $\quad$... $\quad$... I 5

8I. C. \& R. S. Meikle, Selangor (2nd prize) ... o Io

\begin{tabular}{|c|c|c|c|c|}
\hline ze) ... & 0 & 10 & 0 & 0 \\
\hline Total ... & 5 I3 & 305 & 83 & 16 \\
\hline
\end{tabular}




\section{Flowers, Fruit and Vegetables.}

SECTION I.-FLOWERS.

\section{Ornamental Foliage Plants in Pots.}

Class. Ist. 2nd. 3rd. Extra.

I.

Aroids other than Caladiums.

John A. Brown, Penang Aroids best specinten.

2.

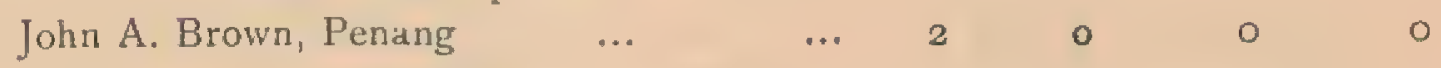

3.

Caladiums.

Lim Kean Thuan, Penang $\quad \ldots \quad$... 3

$4 \cdot$

Caladirms.

6.

$\begin{array}{llllllll}\text { Mr. Schule } & \ldots & \ldots & \ldots & 3 & 0 & 0 & 0\end{array}$

10. Ferns (any variety) distinct.

Mr. Schule

A. Stephen Anthony, Penang ...

$\begin{array}{lllll}\ldots & 5 & 0 & 0 & 0\end{array}$

I6.

Palms, distinct kinds.

Khau Joo Tok, Penang _..

John A. Brown, Penang $\quad$...
Palms, best specimen.

17.

A. D. Machado ‥

I9. Selaginella, distinct kinds.

Mr. Schule

23. Foliage Plant of any kind best specimen.

Mr. D. Lngan, Penang

Ornamental Plants in flowers in Pots.

26. Asters.

3 I.

Contonese Club, Penang

Cockscombs.

Lim Ah Chum, Province Wellesley

38.

Orchid, best specimen.

Arthur Oechsle, Penang

$4 \mathrm{I}$.

Coses.

... Io $\quad$ Io 00

... 0 o 5 5 0

44. Best collection of fincuering plants distinct
kinds.

Cantonese Club, Penang

45. Best collection of flowering plants lopen to Subscribers for flower seed's only.)

L. M. Murray, Penang (Cup) ...

46.

Cantonese Club, Penang

Plants whether in Flower or not.

49. Gronps of plants arranged for effect in space not exceeding ofeet square.

Mirs. J. A. Brown, Penang

$$
\begin{array}{llrrr}
\cdots & 5 & 5 & 0 & \circ \\
\text { Carried over, } & 68 & 7 & 0 & 0
\end{array}
$$


Division $B$.-Continued.

Class.

$\begin{array}{ccccc} & \text { 1st. } & 2 n d . & 3^{\text {rd. }} & \text { Extra. } \\ \text { Brought forward, } & \$ & \$ & \$ & \$ \\ \text { B } & 7 & 0 & 0\end{array}$

5o. Group of Chinese Plants of fantastic shape.

Cantonese Club, Penang

Cut Flowers and Table Decorations.

5 s.

Asters.

Khoo Moh Kaw, Penang ...

52.

Bridal Bouquet.

Miss V. Brown, Penang … …

53. Bution-holes and Sprays.

Miss Constance Law, Penang $\begin{array}{lllllll}. . . & \ldots & 3 & 0 & 0 & 0\end{array}$

55. Cut Flozvers arranged fur elfet.

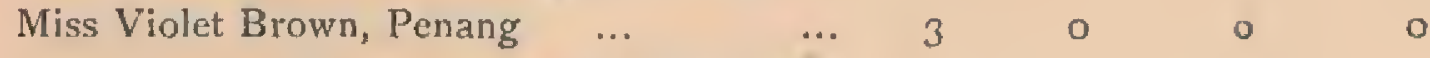

56. Wild Flowers arranged for efect.

K. Bromhead Matthews, Penang

57. Dahlias:

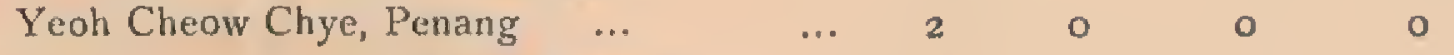

$5^{8}$

A. D. Machado

Hand bouquet.

59.

60.

Table Decoration.
Teon Cheow Chye, Penang

Mrs. Fox (Cup)

\section{SECTION II.-FruITS.}

61. Bananas, "Pisang," best collection.

Takaya B. H. Mohamed, Penang $\quad \ldots \quad 004500$

62. Bananus, "Pisang" best bunch any kind.

Serban, Negri Sembilan $\quad \ldots \quad$... 300000

63.

David Brown, (extra prize) $\quad \ldots \quad$... 3

Champedak.

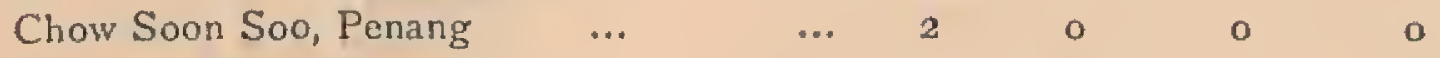

$\begin{array}{lllllll}\text { Penghulu, Mat Said } & \ldots & \ldots & 0 & \text { I } & 0 & 0\end{array}$

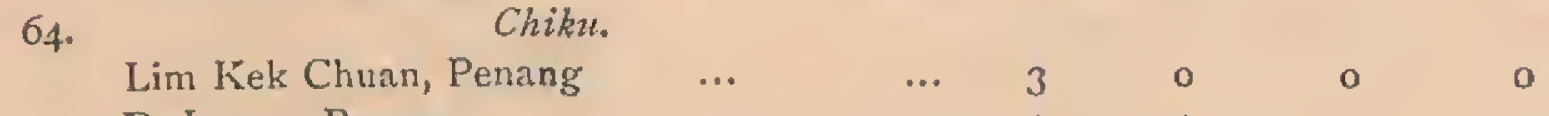

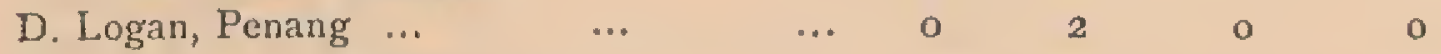

65. Custard Apple.

Ahmad bin Mat Tahir, Province Wellesley $2 \quad 0^{\circ} \quad 0^{0} 0$

66 Cultivated fruits, best collection any number.

D. Logan, Penang (Cup) $\quad \ldots \quad$ …

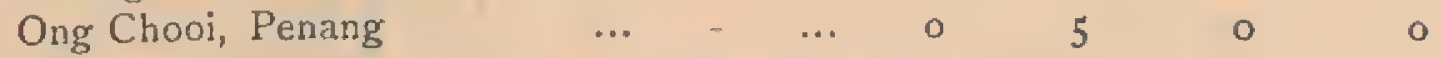

67. Cultivated fruits, best collection 6 distinct kinds.
Mohamed Zain, Penang $\quad$...
M

$68 . \quad$ Durian.

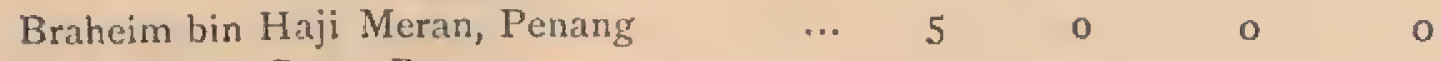

69. Durian Blanda.

Chin Kim Toon, Penang

$\begin{array}{lllll}\cdots & 2 & 0 & 0 & 0 \\ \text { Carried over, } \$ 116 & -\end{array}$ 
Drvision $B_{0}-$ Continued.

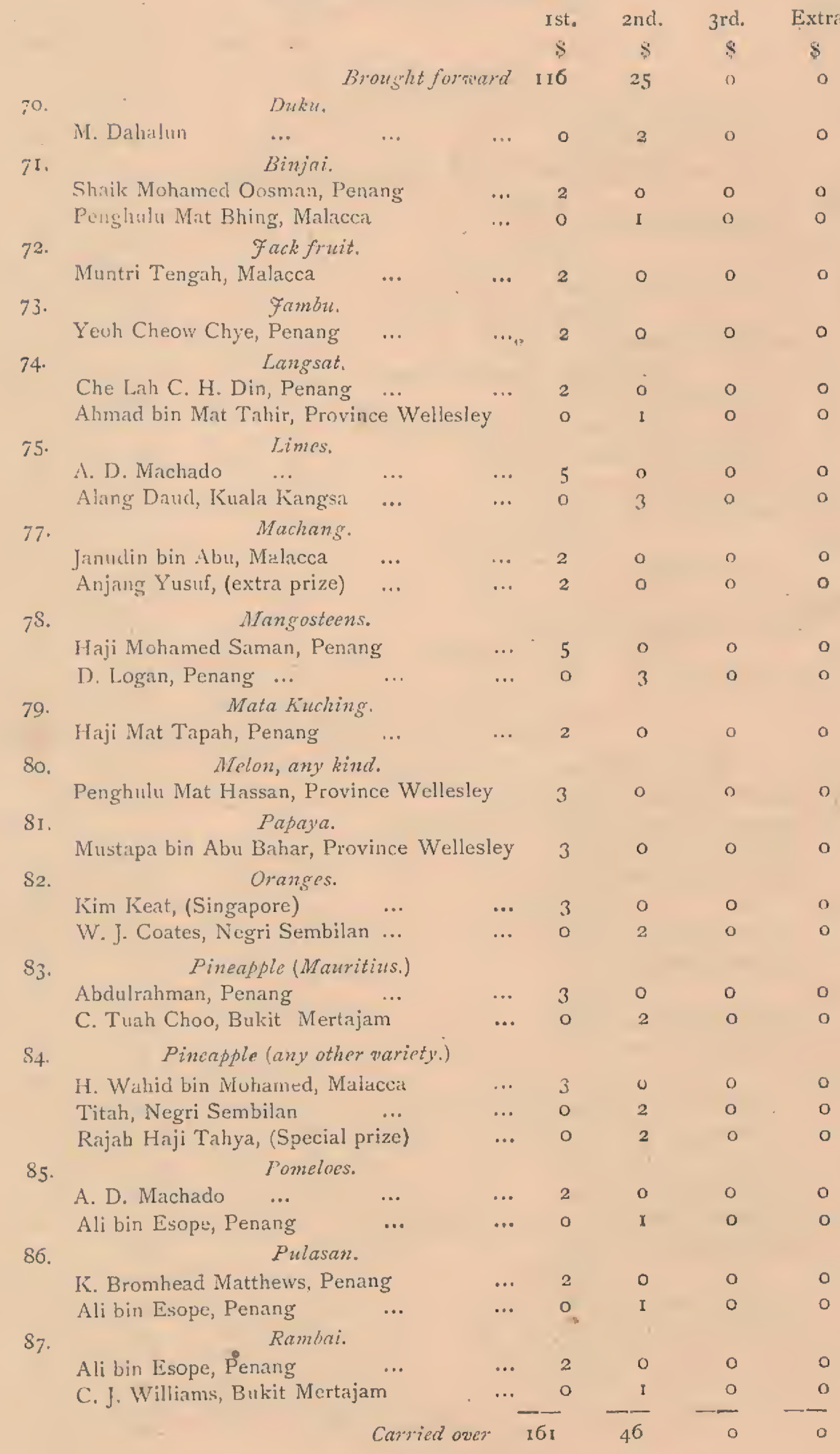


Division B.-Continued.

Class,

88.

$\begin{array}{crrr}1 \text { st. } & \text { 2nd. } & \text { 3rd. } & \text { Extra. } \\ \$ & \$ & \$ & \$ \\ 16 \mathrm{x} & 46 & 0 & 0\end{array}$
Kiambirtan.

Lim Kek Chuan, Penang

A. O, Merican, Penang

Wild edible fruits, best collection.

89.

Penghulu Mat Sahat, Malacca ... ... ... 5 o

$\begin{array}{llllllllllll}\text { Yab, Penang } & \ldots & \ldots & \ldots & 0 & 3\end{array}$

90. Any kind of fruit not included in the above.

R. Douglas Tollemache, Selangor

Penghulu Telok Bahru

‥ $3 \quad 0$

Preserved Fruits and Vegetables.

91. Preserved fruits, best sample any method.

Mohamed Kassim, Penang ... ... 10

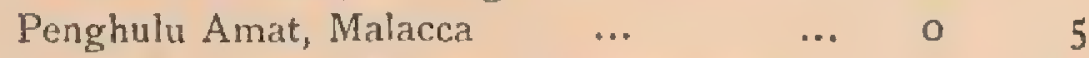

Penghulu Mamud, Malacca (Special prize) o

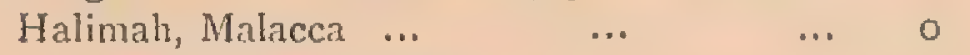

93.

Pickles.

M. Pasqual, Penang:

Fellies.

94.

Awang, Penang ...
Benny Fruits.

97.

Nai Kong, Penang

98.

John Lamb

\section{Brinjals.}

99.

Beans, best collection.

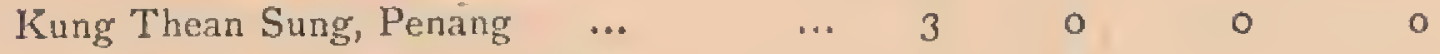

Mohamed Hashime, Penang ... $\quad \ldots \quad 0 \quad 2 \quad 0 \quad 0 \quad 0$

Ioo. Cabbages.

Chong Ah Yong, Penang

IOI. Chillies, best collection.

A. D. Machado … …

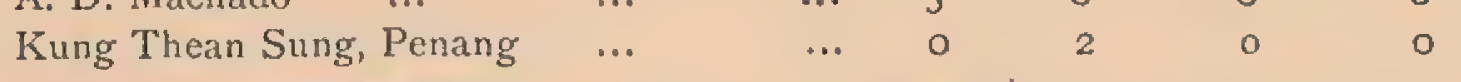

102. Cucumbers.

Penghulu Mat Hassan, Bukit Mertajam ... 2 200

103. Herbs used in curries and sambals, best collection.

Shaik Hossain, Penang … ... 2

I04. Ladies Fingers.

Kung Thean Sung, Penang

105.

Lettuces.

Chong $\Lambda$ h Yong, Penang

Io6. Onions, shallots and garlic, best collection.

Kung Thean Sung, Penang

107.

Mat Taib, Krian

Pumpkins.

108. Radishes.

Takaya bin Haji Mohamed, Penang

I10. Vegetables, best collection.

G. B. Cerruti, Perak

$\begin{array}{llrrrr}\cdots & \cdots & 5 & 0 & 0 & 0 \\ \text { Carried nver } & 2 I 7 & -64 & 6\end{array}$ 


\section{Division B.-Concluded.}

Class.

$\begin{array}{rrccc} & \text { st. } & \text { 2nd. } & 3 \text { rd. } & \text { Extra. } \\ \text { Brought forward } & \$ & \$ & \$ & \$ \\ \text { II } & 64 & 6 & 0\end{array}$

I13. Yams, Kladi and Sreet potatoes, best collection.

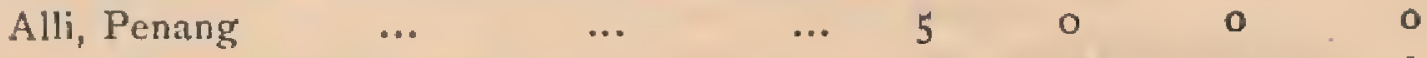

Kung Thean Sung, Penang . ... ..... 0 o 3 o

114. Any Vepet.thle not in the above.

Alli, Penang

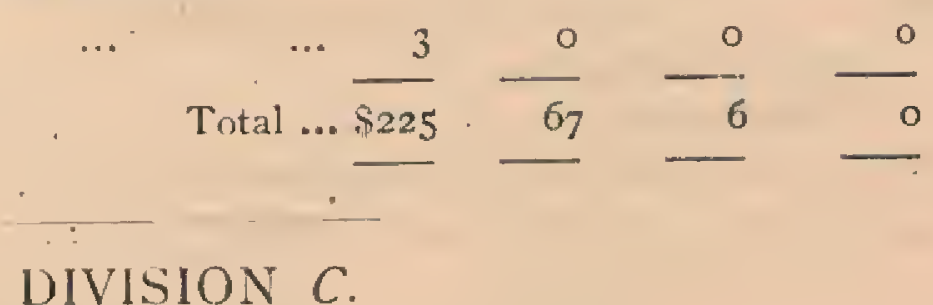

Stcck and Dairy Produce.

Class.

1st. 2nd. 3rd. Extra.

\& $\$ \$ \$$

I.

Bull, locally hred.

Mahomed Ariff, Penang

... $\quad \ldots \quad 20$

Puteh, Penang, (Extra).

4.

Bull or Bullo-k imported, Peninsular.

D. Douglas, Province Wellesley

6.

Cow and Calf imported, Indian.

D. Logan, Penang ...

.... $\quad \cdots \ldots$ 20. 0

Bull or Bullock, Peninsular, best.

8.

Puteh, Penang

9.

Buffalo Bull.

16.

Lye Thoon

Best pên of 6 pigs

Sung Hup, Penang

Sung Leong, Penang, (Extra prize)

Best pig over 300 catties.

17.

Soo Swee

Goat, Ram.

19.

Plot bin Noh, Nibong Tebal

Kamaludin bin Haji Bahudin, Penang

20.

Goas, Ewe with kids.

Plot bin Noh, Nibong Tebal

21.

Sheep, Ram.

M. Gregory, Penang

Sheep, Eve.

W. Yang Beng, Penang

Bantam Cock and Hen.

Mrs. D. Logan, Penang

Khoo Moh Kow, Penang

Malay Cock and Hen.

Law Chit Man, Penang

Penghulu Lenggong, U. Bernum

$$
\begin{array}{lrrrrrr}
\ldots & \ldots & 5 & 0 & 0 & 0 \\
\text { Carried over, } & \$ 125 & & 0 & 3 & 0 & 0 \\
\hline 1 & & 0 &
\end{array}
$$




\section{Division C.-Contimued.}

$\begin{array}{rrccc} & \text { ist. } & \text { 2nd. } & 3 \text { rd. } & \text { Extra. } \\ \text { Brought forward, } & \$ & \$ & \$ & \$ \\ \text { I } 25 & 4 \mathrm{I} & 0 & 20\end{array}$

27. Malay Game Cock and Hen.

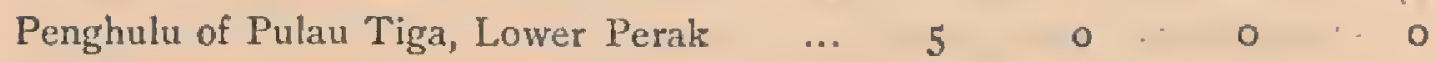

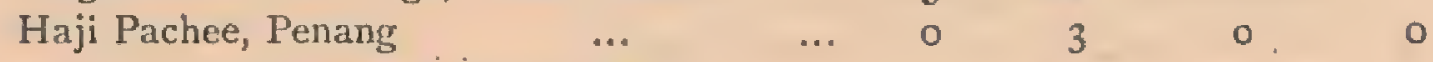

28.

Collection of Malay Fowls.

W. Willis Douglas

Kung Thean Sung, Penang (Extra prize) $\cdots$

30.

Collection of Fonvls, any breed.

L. E. P. Wolferstan

Braheim, Botanic Gardens, Penang (Extra prize) o

31. Pair of Muscory Ducks, Duck and Drake.

K. Bromhead Matthews $\quad \ldots \quad$... 10

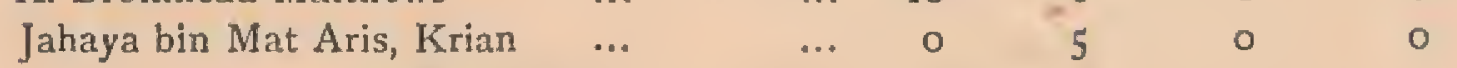

32. Pair of Manila Ducks.

Syed Sallim, Penang

Mustapa bin Abu Baker

Moonah, Penang (Extra prize) ...

... 5

... 0

$\begin{array}{lll}0 & 0 & 0\end{array}$

53. Pair of Ducks, any breed, Duck and Drake.

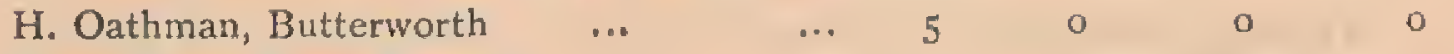

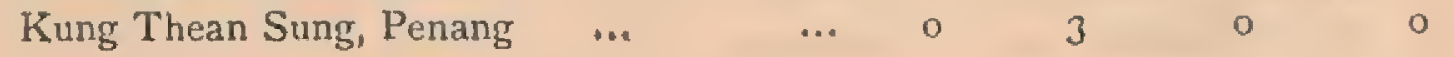

35.

Gander and Goose.

Mrs. D. Logan, Penang

K, Bromhead Matthews

$\begin{array}{llllll}\cdots & \cdots & 5 & 0 & 0 & 0\end{array}$

37. Pair of Guinea Fowls, Cock and Hon.

$\begin{array}{llllllll}\text { Mrs. D. Logan } & \ldots & \ldots & \ldots & 5 & 0 . & 0 & 0\end{array}$

38. Pair of Pigeons, any brect.

Mrs. D. Logan

Extra prize $\$ 3$.

Penghulu Haji Salleh, P. W. (Extra prize) o 003

40.

H. Wool

Best Bird in the Show.

42 .

Pair of Guinea Pigs.

Sample of Butter.

H. H. Cornelins, Penang

Geo. Stothard, Malakoff Estate

Extra prize.

44. Collection of Eggs, Fowls' or Ducks'.

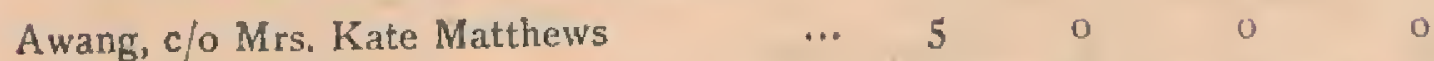

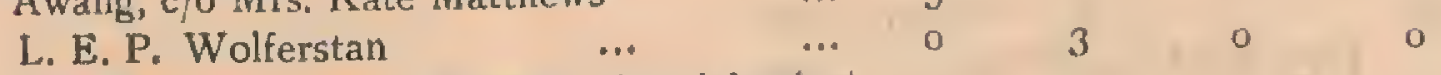

Haji Mohamed Sahat, Penang, (Special prize) o 0 o 3

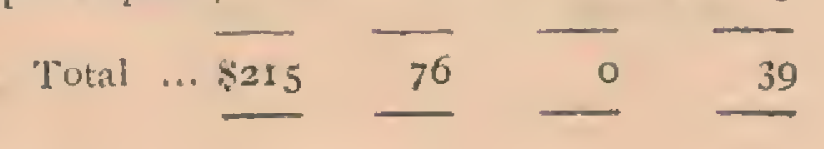


DIVISION $D$.

\section{Horses and Dogs.}

Class.

Ist. 2nd. $\quad 3^{\text {rd. }}$

I. Polo Pony, registzred 14.I or untder.

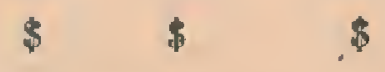

Capt. A. Mc. D. Grahams "Bessie" (Cup)

Mrs. M. Suhls "Happy Chance" (Medal)

10

o

2. Gentleman's Hack, rf.2 and under.

C. R. Molyneux's "The Friar" (Cup)

W. H. MacArthur "Bubbles" (Medal)

G. H. Stitts "Patrick" (Cup)

3.

Gentlemant's Hack, over 14.2.

O. Sielckens "Mantred" (No second prize)

4. Lady's Hack over 14.2 , to be shown under saddle and ridden by a Lady.

Mrs. Jamieson's "Nickolas" (Cup)

Mrs, J. A. Murray's "Puritan Lass'

5. Lady's Hack I4,2 and under, to be shozun under saddle and radden by a Lady.

Mr. M. Thornton's "Georgina " Bracelet

Mr. MacArthur's "Bubbles". (Medal)

6. Best Gharry and I'ony, 14.2"and under, to be shown in cart or carriage.

Mr. Lim Chee Siang, Medal and \$ro

Mr. Hudson's

$\therefore$

$\cdots \quad 10$

o

Single Harness Pony I 4.2 and under, to be shown in cart or carriage.

Khaw Joo Keat's (Cup)

W. W. Douglas

8. Single Harness Horse over 14.2 and under, to be Mr. H. Wool shown in cart or carriage.

Mr. G. H. Stitt "Patrick" $\quad$.. $\quad$... $\quad$...

9. Registered Gharry Pony of any description: the bona fide property of a licensed Gharry owner (Australian horse barred.)

No entry

10. Fumping Pony 14.2 and under, to be ridden over at least four jumps.

Capt. MacIntyre, "Sadi" (Cup)

Mr. Suhls "Happy chance" ..

I1: Fumping Horse over I4.2, to be ridden over at least four jumps.

C. R. Molyneux "Chieftain" (Cup)

G. H. Stitts "Patrick" …

12.

Single Trirnout.

G. B. Whitehead "Billy" (Cup)

H. Wool

Carried over, $\frac{\cdots}{\$ 20} \frac{10}{130}-\frac{0}{0}$ 


\section{Drvision D.-Continued.}

Class.

$\begin{array}{cccc} & \text { 1st. } & \text { 2nd. } & .3 \mathrm{rd} . \\ \text { Brought forward, } & \$ & \$ & \$ \\ & 20 & 130 & 0\end{array}$

13. Double Turnout, of any description, pairs, tandems, etc.

Cheang Thye Pin, (Cup)

Chea Tat Toi

14. Gharry Tumout, the hona fide property of a licensed

Shandrydan. Turnout, the bona fide property of a ghary ormer.

licensed Shandrydan ovner.

No entry.

16. The best all round Horse 14.2 or over in the Show: to be shoron out the halter.

G. H. Stitt "Watrick" (Cup)

17. The best all round Pony 14.2 and under to be shown on the halter.

G. B. Whitehead "Billy" (Cup)

18. The best Straits Settlements or Native States bred horse or mave, any age over 12 hands.

"Geo. Scothard, (Cup)

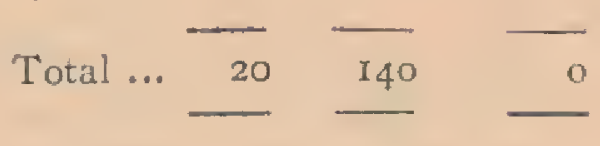

\section{DIVISION $E$.}

\section{Native Industries and Manufactuies.}

Class.

I.

Ornamental Baskets.

Mahani bin Mamud, Malacca

Penghulu of Bandar, Lower Perak

Ist. 2nd. 3 rd.

Lace work (bikii)

Penghulu Baba Pringgit, Malacia

Mohamed Kassin

3.

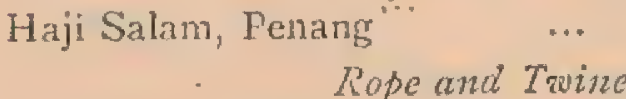

4.

J. Abrams, Penang

Rope and Truine

Penghulu of Pulau Tiga, Lower l'erak

5.

Tali ljok.

Noodin bin Katli, Malace:

Penghulu of Bachang, Malacca

6.

Attaps.

Penghulu of Kota Stia, Lower l'erak

Penghulu of T. Baharu, Lower Perak

Kajangs.

7

l'enghulu of Pulau "liga, Lower Perak

Penghulu of T. Baharu, Lower Perak

Carried over

$\begin{array}{ccccc}\ldots & \ldots & 10 & 0 & 0 \\ \ldots & \ldots & 0 & 5 & 0 \\ \ldots & \ldots & 10 & 0 & 0 \\ \ldots & \ldots & 0 & 5 & 0 \\ \ldots & \ldots & 10 & 0 & 0 \\ \ldots & \ldots & 10 & 0 & 0 \\ \ldots & \ldots & 0 & 5 & 1 \\ \ldots & \ldots & 10 & 0 & 0 \\ \ldots & \ldots & 0 & 5 & 0 \\ \ldots & \ldots & 10 & 0 & 0 \\ \ldots & \ldots & 0 & 5 & 0 \\ \ldots & \ldots & 10 & 0 & 0 \\ \text { Caried oven } & & \$ 70 & 30 & 0\end{array}$




\section{Drvision E-Continued.}

Class.

$\begin{array}{cccc} & \text { rst. } & \text { 2nd. } & 3 \text { rd. } \\ \text { Brought forward, } & \$ & \$ & \$ \\ & & 30 & 0\end{array}$

8.

Chicks.

D Mohamed Noor, Penang

Arifin, Penang

9.

L. Daud, Krian

Mats.

ro.

Penghulu Jaffar, Malacca

Mats Rotan.

Asin, Krian

Malay Mats (Padanus.)

Haji Mohamed Sahaat, Penang

Haji Mohamed Rasaid, Penang

12.

Hats.

Haji Salam, Penang

Penghulu of Bagan Dato, Lower Perak ...

Shaik Hossain, Penang

Brushes and Brooms.

D. Mohamed Noor, Penang

14.

Coco-Nut Palms, Collection of articles manufactured from.

Mohamed Akib, Butterworth

Muntri Tengah, Malacca

15. Kabong (arenga.)

Kulop Mohamed Ali, Kuala Kangsa

I6.

No Prize

Nibong.

17.

Sireh apparatus set.

Mohamed Hussein, Province Wellesley ...

Haji Mohamed Salleh, Negri Sembilan ...

Said (Special prize)

18.

Model Malay House.

Kwala Muda, c/o A. D. Neubronner

Kulop Lotan, Kuala Kangsa

19.

Penghulu Yusof, Malacca

Model Fishing stakes.

Lebei Sidik, Butterworth

20.

Model Snares and Traps.

Ahmad bin Mohamed Taib, Butterworth

D. Mohamed Noor, Penang

Wood carving I piece.

Penghulu H. Mohamed Akib, Krian

Penghulu Abdul Majid, Selangor

L. E. P. Wolferstan (Special prize)

... $\quad \ldots$ 10

... 0

$\circ$

5

... IO

$\circ$

22.

Embroidery I piece.

Malay Art School, Kuala Kangsa

Penghulu Dol C. Bidin, Malacca

23. Sarong Silk.

Mohaned Yatim, Kuala Kangsit

Shaik Mohamed, Penang

$\begin{array}{lrrrr}\ldots & \ldots & 10 & 5 & 0 \\ \ldots & \ldots & 0 & 5 & 0 \\ \ldots & \ldots & 0 & 5 & 0 \\ \ldots & \ldots & 10 & 0 & 0 \\ \ldots & \cdots & 0 & 5 & 0 \\ \ldots & \ldots & 10 & 0 & 0 \\ \ldots & \ldots & 0 & 5 & 0 \\ \text { Carricd over, } & \$ 220 & 110 & 0\end{array}$




\section{DIVISION E.-Continued.}

$\begin{array}{cccc} & \text { 1st. } & \text { 2nd. } & 3 \text { rd. } \\ \text { Brought forcuard, } & \$ & \$ & \$ \\ 220 & \text { IIO } & 0\end{array}$

24. Sarong cotton.

Mad Saman, Kuala Kangsa ...

Moh Arshat, Butterworth ... 25.

Narayanasamy Pillai, Penang

$\begin{array}{lllll}\ldots & \ldots & \text { I0 } & 0 & 0\end{array}$

Haji Mohamed Sahid, Penang 26. Silver rvare.

Chas. G. Garrard, Malacca ...

To Gampar Maharaja Zacharia, Negri Sembilan

27. Tin ware.

Lam Chay, Negri Sembilan ...

Kuan Fun, Negri Sembilan ...

28.

Krisses sheaths and knife handles.

To Gampar Maharaja, Negri Sembilan ...

Koo Aim K. Kudam, Penang Fishing Nets.

Haji Mustapa, Penang ... 30.

Bakar Bin Omar, Butterworth
Sakei articles.

G. B. Cerruti, Perak, Medal and Diploma

Pan Dewa Sakti, Batang Padang, Perak

$\begin{array}{rrrrr}\ldots & \ldots & 10 & 0 & 0 \\ . . & \ldots & 0 & 5 & 0\end{array}$

31. Collection of photographs illustrating native life

No Prize or industrics.

32. Miscellaneous.

H. Mohamed, Penang

Kuala Muda, c/o A. D. Neubrọner

$$
\begin{array}{rrrrr} 
& \ldots & 10 & 0 & 0 \\
\text { Total } & \ldots & \frac{1}{300} & \frac{5}{150} & -0
\end{array}
$$

DIVISION $E$.

SECTION I .

Native Industries and Manufactures confined to Competitors from Schools of the Colony and Malay Peninsula.

Class.

- Ist. and. 3rd.

$\$$ \&

I.

Larut

Krian

2.

Lower Perak

Upper Perak

$3 \cdot$

Krian

Malay hand-made Pottery.

Kuala Kangsa

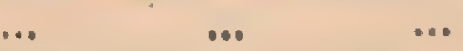

$\cdots$

...

Ornamental Baskets.

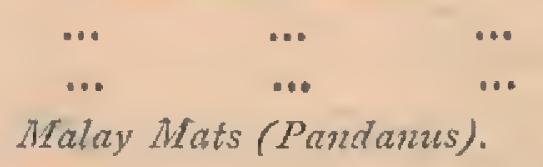




\section{Division E-Section I.-Continued.}

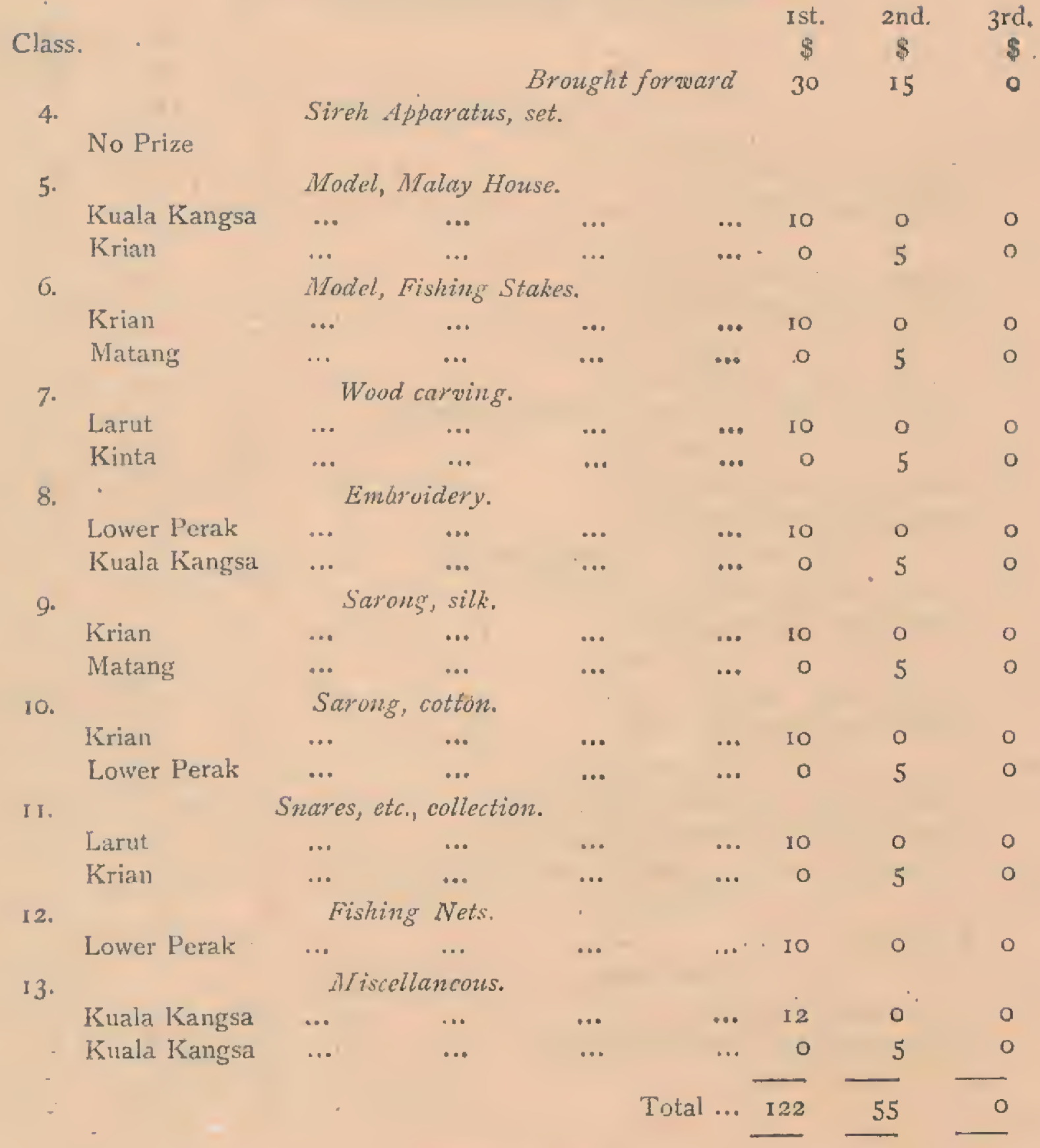

\section{DIVISION $F$.}

\section{Agricultural Implements and Miscellaneous.}

Class.

I. Padi Implements (Collection).

Ist. 2 ind.

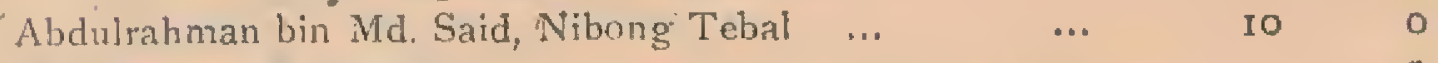

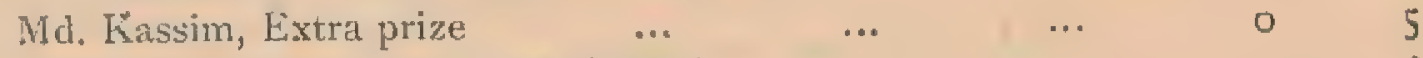

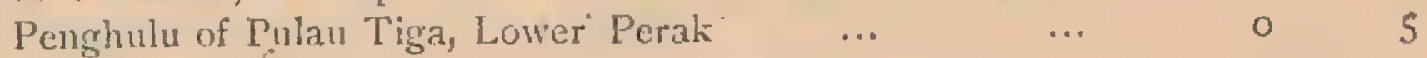

2. Agricultural Implements, other than for Padi or" Rubber. locally made.

Khamis, Butterworth ...

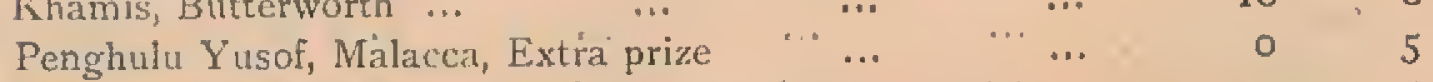

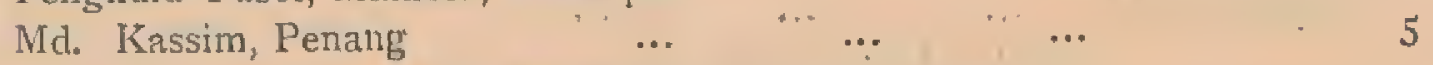

3. Agricultural Implenonts, European made.

McAlister \& Co., Pénang … $\quad \ldots \quad \ldots \quad$... 


\section{Division F.-Continued.}

Class.

\begin{tabular}{|c|c|}
\hline & Ist. \\
\hline Brought formard & 30 \\
\hline$\therefore$ & IO \\
\hline
\end{tabular}

5

McAlister \& Co., Penang

Bullock-Cart.

\section{No Prize}

Hand Cart.

6.

Buildiug Bricks.

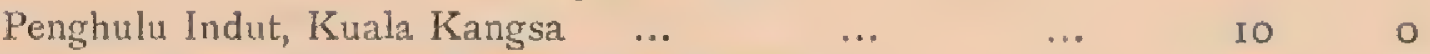

Penghulu Indut, Kuala Kangsa, Extra prize ... $\quad$.. $\quad$ o 5

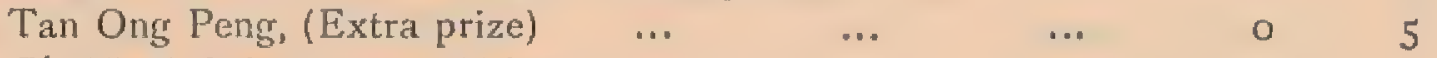

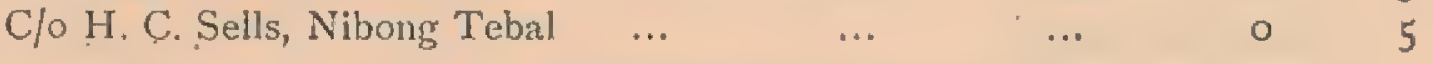

7.

Roofing Tiles.

Penghulu Indut, Kuala Kangsa

Tan Choon Lean, Butterworth

Penghulu Shaik Yabya, Extra

Flooring Tiles.

Tan Ong Peng, Kuala Kangsa, Medal ...

Caledonia Estate, Province Wellesley, ...

9.

Malay hand-nidide Pottery.

Abdul Wahid, Krian 0.

Penghulu of Pulau Tiga, Lower Perak ...

Faney Pottery.

Malay Art School, Kuala Kangsa

Penghulu of Pulau Tiga, Lower Perak ...

II.

Syed Ali, Penang

Agricultural Baskets.

12.

Mukin Ijok, Perak

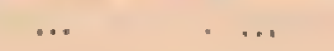

Casks, Tubs, etc.

Penghulu Yusof, Malacca

13. Carriages four zohcels, Phaton or Waggonette.

C. Chin Seng, Penang, Medal

$\cdots$ 


\section{Drvision $F$-Continued.}

22. Shandridan (two wheel Gharry.)

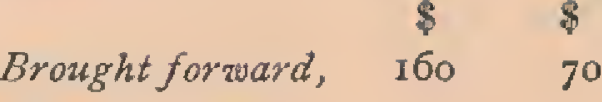

C. Chin Seng, Penang, Medal Mat, Penang

23.

Shandridan, Improved Construction.

No Prizes

24.

Best Exhibit of mining implements.

No Prizes

25.

Miscellaneous.

Hassim bin Kadir

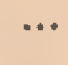

$\cdots$

Penghulu Haji Mohamed, Extra
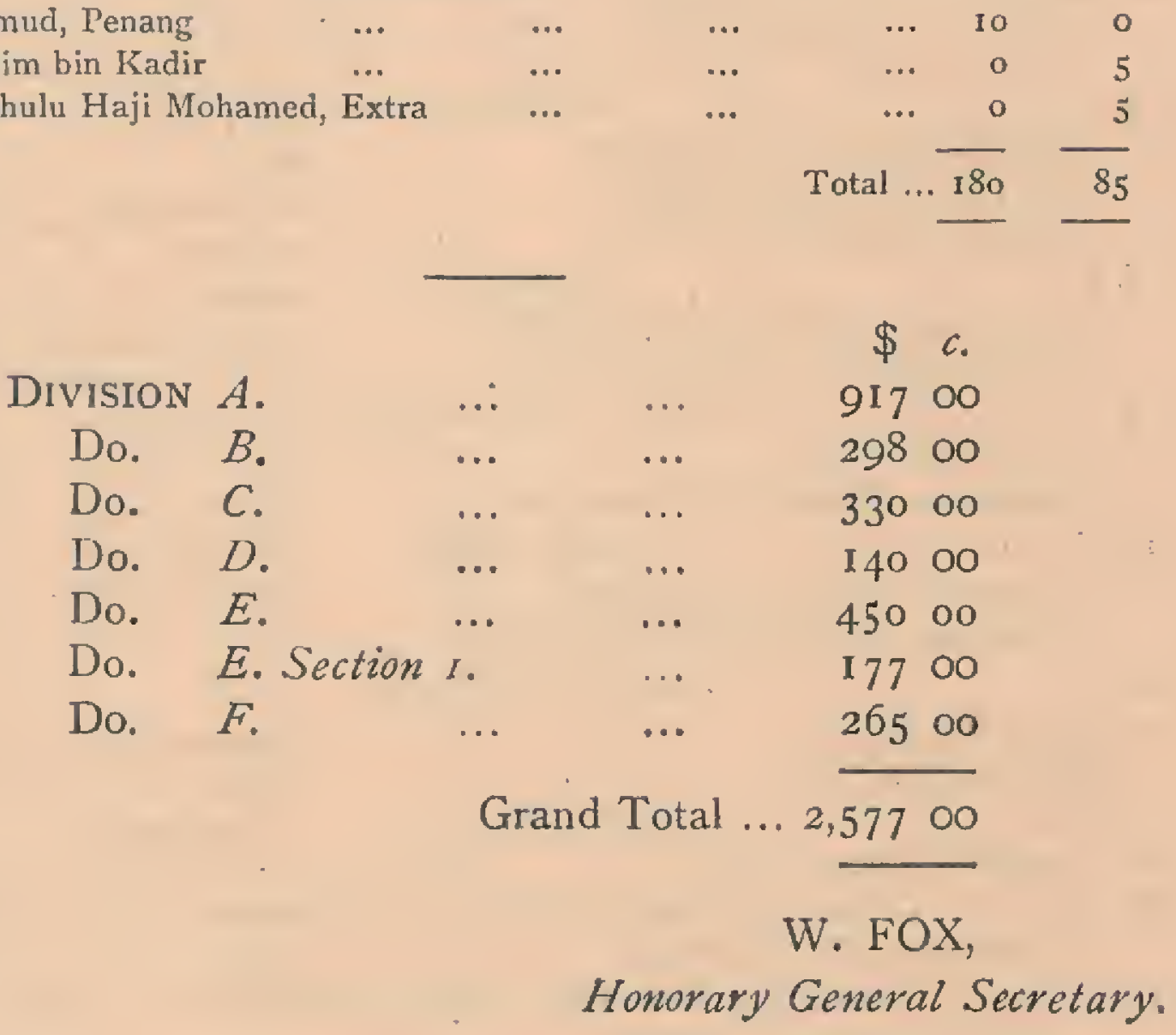


\section{AGRICULTURAL SHOW, PENANG, 1905.}

\section{DIPLOMAS WERE AWARDED}

TO THE FOLLOWING:

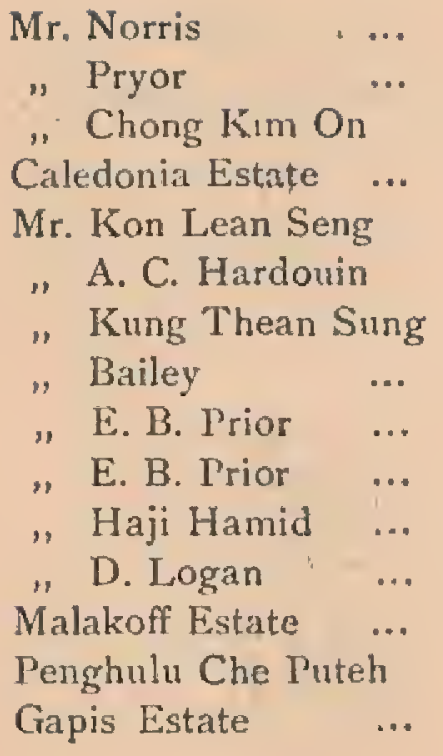

\section{Division $A$.}

\begin{tabular}{|c|c|c|}
\hline$\ldots$ & For & Arrowroot. \\
\hline$\ldots$ & " & Copra Sun-dried. \\
\hline .. & "1 & Nutmegs. \\
\hline & "' & Dry Indigo. \\
\hline & " & Mace. \\
\hline & ", & Essential Oil. \\
\hline & $"$ & Turmeric. \\
\hline & " & Rubber class (54) \\
\hline & " & Rubber (Rambong) \\
\hline & $"$ & Coconuts, best Coll \\
\hline & ", & Sugar (Coconut) \\
\hline & " & Cotton "Kapas" \\
\hline & $"$ & Tapioca, flake. \\
\hline & $"$ & Padi, any variety. \\
\hline & $n$ & Pepper, white. \\
\hline
\end{tabular}

DIVISION $B$.

$\begin{array}{ccl}\ldots & \text { For } & \text { Preserved fruits. } \\ \ldots & , & \text { Palms. } \\ \ldots & n & \text { Avocadn pears. } \\ \ldots & \text { ". } & \text { Watermelons. } \\ \ldots & \text { " } & \text { Lemons. } \\ \ldots & \text { " } & \text { Binjai. } \\ \ldots & \text { ". } & \text { Collection of Fruits. }\end{array}$

Division $E$.

Mr. L. E. P. Wolferstan , Chas. Garrard ... Mahanib Mamat Penghulu Baba, Pringgit Noodin bin Kali ...

Asiah ... ...

Mohamed Akib ...

Penghulu Dol

Rajah Bila

Moh. Yatim

Narayanasamy Pillai

To Gampar Maharaja Zacharia Lam Chay

Kwan Fun

Mustapa

Haji Mustapa

Mr. G. B. Cerruti

Major De Hamel

\begin{tabular}{|c|c|}
\hline For & Wood Carving. \\
\hline " & Silver Ware. \\
\hline$"$ & Ornamental Baskets. \\
\hline$n$ & Lace work. \\
\hline$"$ & Tali Ijok. \\
\hline " & Hats. \\
\hline$"$ & Coconut Palms, Coll : \\
\hline$"$ & Embroidery. \\
\hline , & Sarongs, Silk. \\
\hline$"$ & Brassware. \\
\hline$"$ & Silverware. \\
\hline$"$ & Tinware. \\
\hline$"$ & Do. \\
\hline " & Kriss handles. \\
\hline " & Fishing Nets. \\
\hline$"$ & Sakai Articles. \\
\hline " & Miscellaneous exhibits \\
\hline
\end{tabular}

Arrowroot.

Nutmegs.

Dry Indigo.

(54)

Rubber (Rambong)

(Coll:

Cotton "Kapas"

Padi, any variety.

Pepper, white.
Turmeric. 
362

Division E., Section I, Schools only.

\begin{tabular}{|c|c|c|c|c|}
\hline $\begin{array}{l}\text { S. Krian School } \\
\text { L. Perak School }\end{array}$ & $\begin{array}{l}\cdots \\
\cdots\end{array}$ & $\begin{array}{l}\cdots \\
\cdots\end{array}$ & $\begin{array}{c}\text { For } \\
" \prime\end{array}$ & $\begin{array}{l}\text { Ornamental Baskets. } \\
\text { Embroidery. }\end{array}$ \\
\hline \multicolumn{5}{|c|}{ DIVISION $F$. } \\
\hline W. R. Swan & $\cdots$ & $\cdots$ & For & $\begin{array}{l}\text { Machinety in connection with } \\
\text { Tapping and preparing rub- } \\
\text { ber. }\end{array}$ \\
\hline Caledonia Estate & $\cdots$ & $\cdots$ & $"$ & Roofing Tiles. \\
\hline & & & & $\begin{array}{c}\text { W. FOX, } \\
\text { orary General Secretary. }\end{array}$ \\
\hline
\end{tabular}


PEVISED LIST.

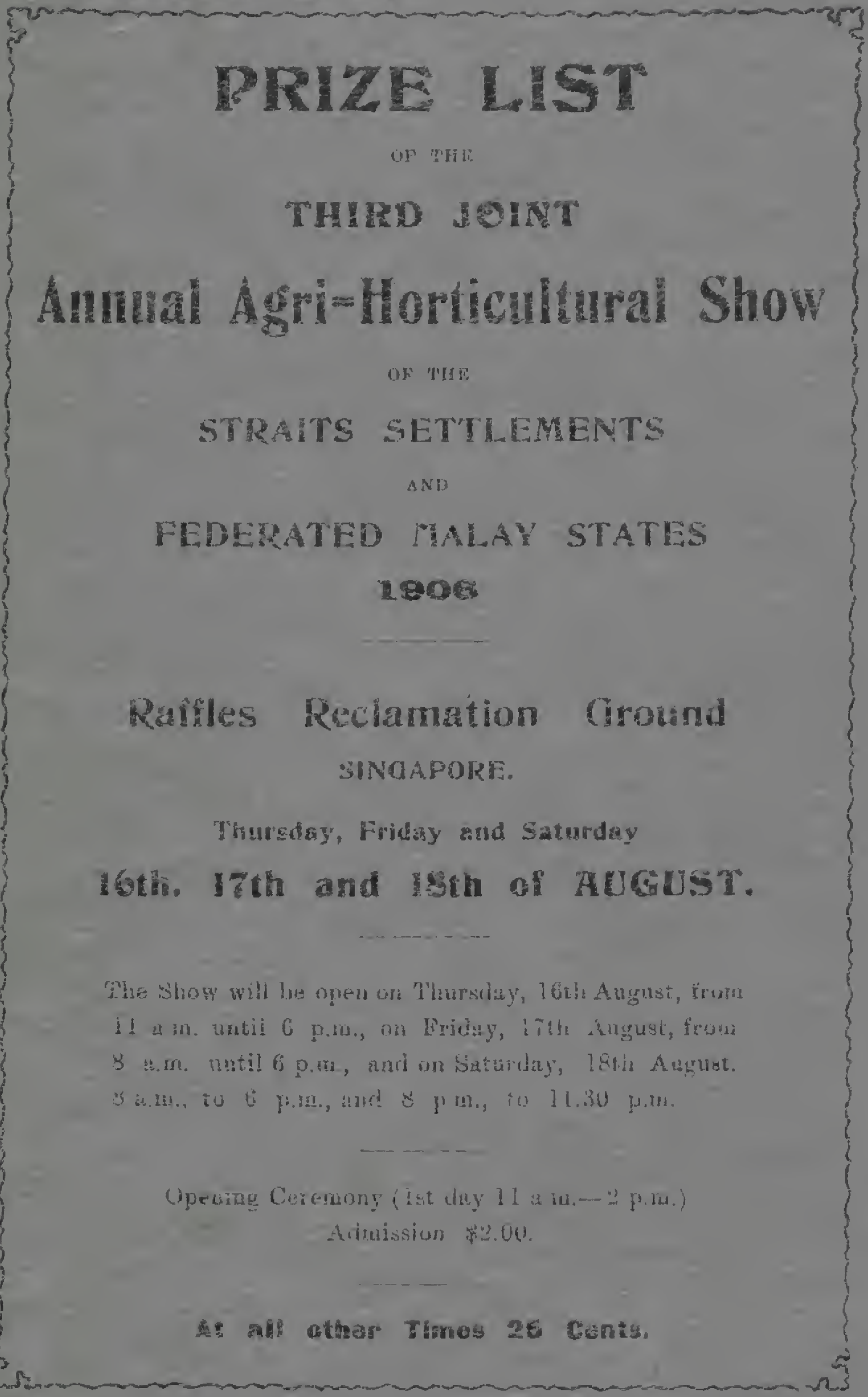




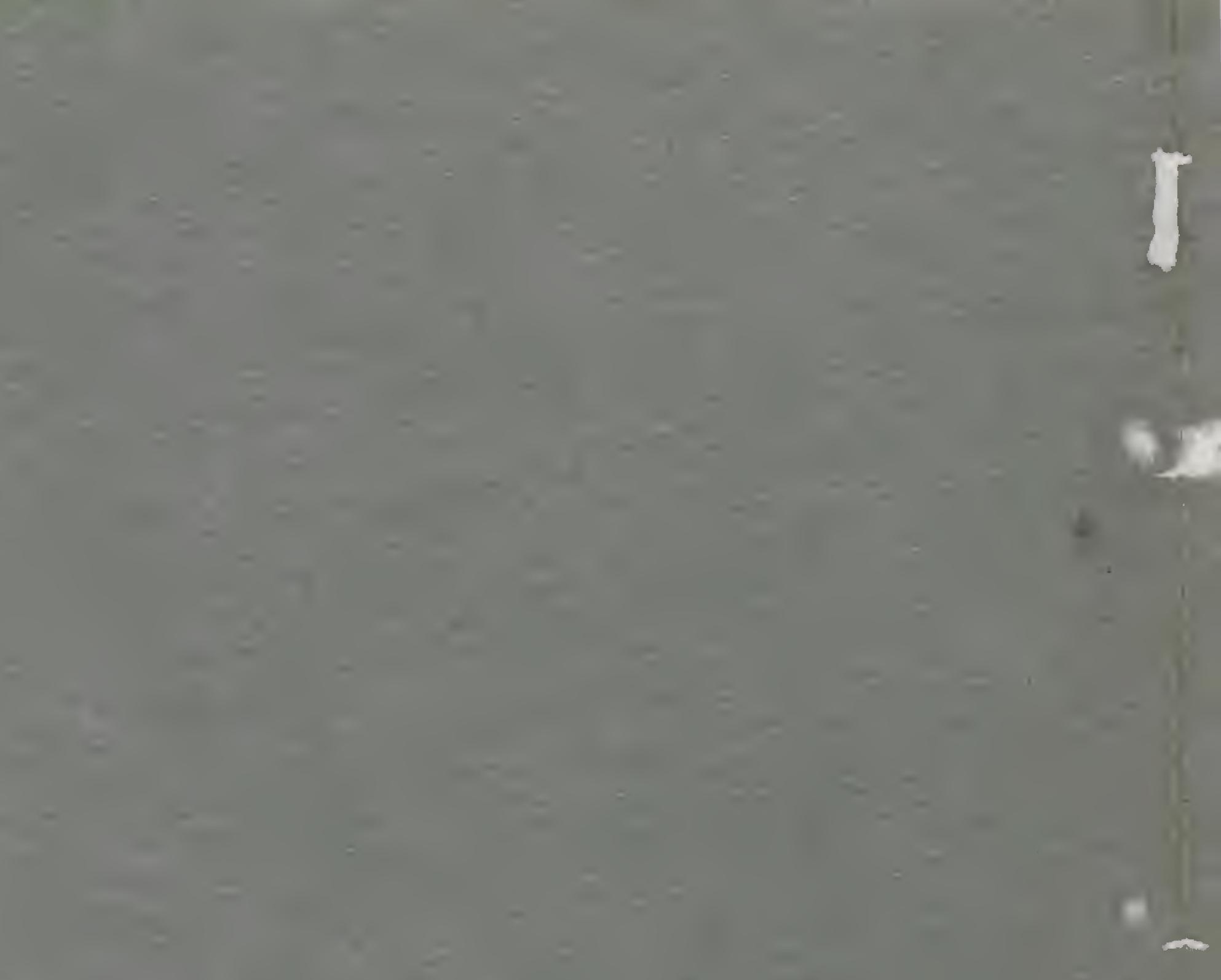




\section{REVISED LIST.}

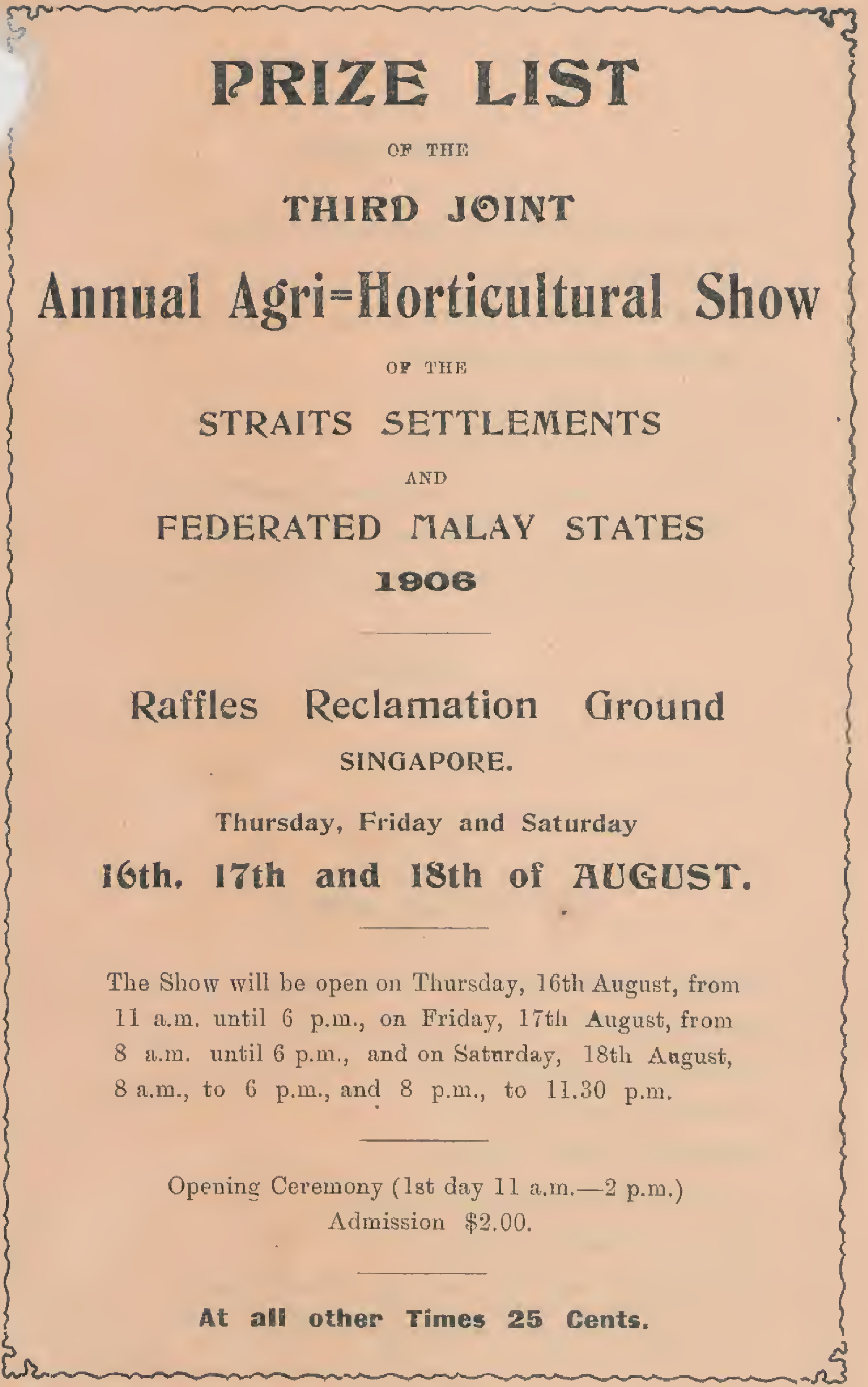




\section{PATRONS.}

] H. E. The Governor and High Commissioner.

2 H. H. The Snttan of Perak.

$\therefore$ H. H. The Sultan of Selangor.

4 H. H. The Sultan of Pahang.

5 H. H. The Sultan of Johore.

G H. H. The Sultan of Kedah.

7 H. H. The Yang di Pertuan Besar, Negri Sembilan.

8 The Resident General Federated Malay States.

(H. E. The High Commissioner of Western Siamese States.

PRESIDENT.

\section{- VICE-PRESIDENTS.}

The Hon. The Resident Councillos, Penang.

the Hon. The Resident Councillor, Malacea.

The Hon. John Anderson M.L.C.

The British Resident, Perak.

The British Resident, Selangor.

The British Resident, Negri Sembilan.

The British Resident, Pahang.

The President, Municipal Commissioners, Singapore.

The President, Chamber of Commerce, Singapore. 


\section{STANDING COMMITTEE.}

\section{SINGAPORE.}

The Director of Botanic Gardens, S. S. (Hon. Secretary).

Assistant Superintendent, Botanic Gardens, Singapore.

The Collector of Land Revenue.

PENANG AND PROVINCE WELLESLEY.

The Senior District Officer, P. W.

The Collector of Land Revenue.

The Superintendent, Botanic Gardens and Forests (Hon. Secretary).

MALACCA.

The Hon. 'The Resident Councillor (Hon. Secretary).

The Collector of Land Revenue.

PERAK.

'The Director of Museums, P. M. S.

The Superintendent, Government Plantations (Hou. Secretary).

The District Oflicer, Kuala Kangsar.

'the Director of Agriculture.

STLANGOR.

The Collector of Land Revenue. Knala Lumpur.

'The Chairman, United Planters' Association.

The Superintendent, Experimental Plantations (Hon. Secretary),

\section{NEGRI SEMBILAN.}

The District Officer, 'Tampin.

The Collector of Land Revenue, Seremban.

The Chairman Negri Sembilan Planters' Association.

J. W. Simmons, Eson: (Hon. Secretary).

The Senior Magistrate.

PABANG.

'I'he District Officer, Raub.

'l'he District Officer, Pekan (Hon. Secretary).

\section{GENERAL CONMITTEE.}

Iton. E. L. Brockman

A. W. Bailey

J. B. Young

Noel T'rotter

Alex. Gunn

W. D. Barnes

J. W. Van de Stadt

C. Sugden

Alex, Gentle

A. Knight
'J.. S. Baker

Rev. Father Gex

Dr. Lim Boon Keng

Dr. Murray Robertson

D. 'J.' Boyd

C. S. Crane

A. D. Allan

J. Denniston

W. G. St. Clair

W. S. Coutts 
GENERAL COMMITTEE.

R. A. J. Bidwell

Tan Khean Hock

Yeow Ngan Pan

Rev. Dr. West

E. J. Khory

W. S. Wigley

P. S. Falshaw

S. I. Symonds

Dr. Gibbs

D. K. Sommerville

W. J. Nutt

Seab Liang Seah

R. J. Wilkinson

Dr. D. J. Galloway

Col. Pennefather

Hon. A. 'T. Bryant

John Somerville

Choa Kim Keat

\#on. J. O. Anthonis\%

A. Emslie Benzie

H. Payne Gallwey

J. Polglase

G. A. Hall

F. Hilton

E. M. Janion

G. Muhlenbein

R. Little

G. Gansloser

J. A. Drysdale

F. H. Carr

H. P. Bagley

A. Morrison

G. P. Nicholson

K. A. Stevens

F. Bramall

P. Cunliffe
R. 'T'. Gibson Fleming-

IIon. 'Tan Jiak Kim

ज. Kiliani

F. F. H. Edlin

Hon. J. Anderson

R. Derry

W. Nanson

Cunte de Bondy

R. Scott

Dr. bane

H. Spakler

A. H. Lemon

D. W. Lovell

W. Diepenheim

Hon. J. R. Inmes

Hon. W. J. Napier.

W. R. Hawtrey

R. W. Braddell

H. Marriott

Rev. H. O. Izard

Syed Mohamed Alsagoff

Rajan Hitam Nongchio

Col. Hon. Alex. Murray

E. Scott Russell

St. V. B. Down

W. C. Michell

W. Dunman

E. J. Khory

Dr. Glennie

A. J. Macdonald

F. U. Wreford

W. Mackean

II. Becker

R. Allen

Dr. Fllis

R. W. Egerton Eastwick

\section{GENERAL PURPOSES COMIMTTEE.}

Hon. John Anderson (Chairman).

Col. Hon. Alex, Murray.

Dr. D. J. Galloway.

Col. Pennefather.

IV. O. Hichell.

H Payne Gallwey
Hon. 'Tan Jiak Kim.

Seah Liang Seah.

Choa Kim Kert.

Syed Mohamed Alsagoff

J. Polglase.

Hon. A. 'I'. Bryant. 


\section{GENERAL PURPOSES COIIIIITTEE.}

st. V. B. Down.

W. Nanson.

W. D. Barnes.

W. G. St. Clair.
John Somerville

Robert Derry.

H. Becker.

Rajah Hitam Nongchie.

\section{SUB-COMMITTEES.}

Division A.-Agricultural Produce.

John Somerville.

W. Diepenheim (IIon. Secretary).

D. W. Lovell.

R. Little.
R. Scott.

IIon. J. R. Innes.

W. Dunman.

\section{Division B.-Flowers, Fruits and Vegetables.}

St. V. B. Down.

W. Nanson.

Hon. W. J. Napier.

Choa Kim Keat.
E. J. Khory.

IIon. J. O. Anthonisz.

Rev. Father Gex.

Dr. Eillis (Hon. Sec.)

Division C.-Stock and Dairy Produce.

Hon: A. I'. Bryant.

f)r. Glennie.

P. S. Falshaw (Hon. Secretary).
S. L. Symonds.

C. S. Urane.

W. R. J. Hawtrey.

\section{Division D.-Horses and Dogs.}

C. Sugden.

H. Payne Gallwey.

J. B. Young.

F. Hilton.
Col. Pennefather.

P. S. Falshaw.

Rowland Allen.

L. E. Gaunt (Hon. Secretary).

Division E.-Native Industries and Manufactures.

Alex. Gentle.

A. H. Lemon.

W. C. Michell,

E. F. H. Edlin.

A. W. Bailey.
Dr. Dane.

H. Marriott.

G. A. Hall (Hon, Secretary).

Rev. H. O. Izard.

W. S. Coutts.

Division F.-Agricultural Implements and Miscellaneous.

A. Emslie Benzie.

W. H. Nutt.

A. J. Macdonald.

A. D. Allan.
K. A. Stevens.

E. Scott Russell.

F. C. Wreford (Hon. Sec.)

\author{
W. MACBEAN, \\ Honorary iTreasurer. \\ H. N. RIDLEY, \\ Hon. General Secretary.
}




\section{RULES AND REGULATIONS RELATING}

\section{TO EXHIBITS.}

1. All exhibits must be the bona fule property of the exhibitor.

2. Competition for prizes shall be open only to exhibitors resident in the Colony of the Straits Settlements or the States of the Malay Peninsula.

3. Competition in Divisions A. B. and E. shall be confined to exhibits grown or manufactured in the Colony of the Straits Settlements, or the States of the Malay Peninsula.

4. The Committee will appoint judges, from whose decision there shall be no appeal.

5. The judges withold a prize when they are of opinion that there is not sufficient merit to justify an award, and may award special prizes for any exhibit not contained in the schedule.

6. No Exbibitor shall be awarded two prizes in any one class.

7. 'The arrangement of the exhibits shall be subject to the directions of the Committee.

8. 'The Committee reserves to itself the right to refuse any entries that it may think fit to reject, and to cancel any class at any time before the 9th August 1906.

9. Exhibitors desiring free transport by Railway, must give notice to a District Ofticer, or the General Secretary, who if the exhibits are approved, will provide them with numbered tickets and a free 3rd class pass on the Railway for themselves and their exhibits. A reduction of 25 per cent off the usual freight will be made hy the Straits Steam Sbip Co. on all bon $\hat{a}$ ficle exhibits. Other reasonable expenses in connection with transport of exhibits will be considered by the committee.

10. All entries must be in writing; and must reach the General Secretary at least 7 days, in the case of Horses and Dogs 3 weeks. before the first day of show.

No exhibit in Divisions A. C. E. and N. will be received later than 6 p. m. on the 15th August 1906.

Exhibits in Divisions B. and D. will be received up to $5 \mathrm{a} . \mathrm{m}$. on the 16 th dugust 1906.

11. Entries fo: Horses under Classes 252, to 260 must be accompanied br a certificate of height fro:n any sporting, Turf or Polo Club in the Gulony or F. M. S. or from a qualified Veterimary Surgeon.

12. Exhibitors in Division (. and D. must provide for the care and maintenance of their own exbibits.

13. No exhibit (except live animals) may be removed from the ground before the close of the Show. Exhititors will be provided with a card which must be produced at the close of the Show before they can be allowed to remove their exhibits.

14. The Committee is empowered to exclude, or remove, any animal which in its opinion is not in a fit state for exhibition.

15. Exhibitors in Division C. and D. will only be permitted to remove their animals from the Show Ground on obtaining an order of removal from the General Secretary or the Hon. Secretaries of these Divisions. 
16. All reasonable care will be taken to provide for the safety and careful preservation of exhibits of every kind sent to the Show but it must be clearly understood that the Committee does not hold itself responsible for the loss of, or the damage to, any exhibit either in transit or during exhibition. Provision will be made for keeping small articles of value under lock and key at night.

17. Space will be provided for tradesmen and others wishing to exhibit articles for sale but not for competition. The sale price must be clearly specilied.

18. Government exhihts shall not be eligible for prizes in any class.

19. Wimners of prizes of $\$ 10$ and upwards, my have a silver medal if they prefer it to money.

\section{SCHEDULE.}

DIVISION A.

AGRIOULIURAL PRODUOH.

Nature of Lxhibit.

1. Arrow-root, fresh, best sample ...

2. Arrow root, prepared. best sample

3. Barrboos, best collection

1. Betel-nuts, fresh, best sample ...

J. Betel-nuts, dried and split, best sample

6. Clovn, best simple

7. Cocontits, unhusked best sample

8. Coconuts, husked best sample

9. Coconuts, best collection of varieties

10. Copra, sun dried best sample

11. Copra, klin dried best sample

12. Cotton, "Kapok" best sample ...

13. Cotton, "Kapok," any variety, best sample

14. Cocoi, ir'sh pods, best sample ...

15. Colfee, Liberian

16. Colfee, any other variety

17. Dragon's Blood, best sample

18. Fibres, best collection

19. Gambier, best sample

20. Getahs, best collection of local ...

21. Gums and Danmars best collection of lecal

22. Ginger, best sample ...

23 . Indigo, prepared, best sampla $\ldots$

24. Mace, dried yellow, best sample...

25. Nace dried, red, best sample
Number or quantity to be exhibited.

... 25 roots

... 1 catty

... optional

... 50

... 1 catty

... $\frac{1}{2}$ :

... 10 muts

... 10

... 3 of each

... 5 catties

... 5,

... 1 catty

... 1 ,

... 5 pods

... 2 catties

... I catty

... $\frac{1}{2}$,

buudle of each is

I catty

... I catty

$\frac{1}{2}$ carty of each 10

... $\frac{1}{2}, ", 7$

... 20 roots

... 1 catty

... 1 ,

... 1 ,
Prizes. 1st 2nd ôrd $\$ \$ \$$ $\begin{array}{lll}5 & 2 & 1\end{array}$ $5 \quad 21$ $\begin{array}{lll}5 & 2 & 1\end{array}$ $\begin{array}{lll}3 & 2 & 1\end{array}$

521

$\begin{array}{lll}10 & 5 & 2\end{array}$

$\begin{array}{lll}7 & 3 & 1\end{array}$

$\begin{array}{lll}7 & 3 & 1\end{array}$

$10 \quad 5 \quad 2$

$\begin{array}{lll}5 & 3 & 1\end{array}$

$\begin{array}{lll}5 & 3 & 1\end{array}$

$\begin{array}{lll}5 & 3 & 1\end{array}$

$\begin{array}{lll}10 & 5 & 2\end{array}$

$\begin{array}{lll}5 & 3 & 1\end{array}$

$\begin{array}{lll}10 & 5 & 2\end{array}$

$\begin{array}{lll}5 & 3 & 1\end{array}$

$\begin{array}{lll}5 & 3 & 1\end{array}$

$\begin{array}{lll}5 & 7 & 2\end{array}$

$\begin{array}{lll}5 & 3 & 1\end{array}$

$\begin{array}{lll}0 & 5 & 2\end{array}$

7 : 1

$\begin{array}{lll}5 & 3 & 1\end{array}$

531

531

531 
DIVISION $A$.

Nature of Exhibit.

26. Maize, best sample

27. Medicinal Plants, best collection

28. Nutmegs. fresh, best simple .

29. Nutmegs, dried, best sample ...

30. Oil, citronelia, best sumple

31. Lemongrass, best sample

32. Oil, Uoconut, best sample

33. Oil, teel seed (Minyak lengah) best sample

34. Uil, Castor, best sample

35. Oil, Para liubber, best sample ...

36. Oil, clove, best sample

37. Oil cake best simple

38. Oils, best collection of essential...

39. Pepper, white, best sample ...

40. Pepper, black, best sample ...

41. Patchouli, best sample

42. Padi, any variety

4:3. * Padi, best collection of varieties

44. Pulot, best sample

45. Pulot, best collection of varieties

46. Rice, prepared by machinery, best sample ...

47. Rice, prepared by pounding, best sample ...

48. Rice, best collection of varieties

49. Rice, boiled, best sample

o. Rotans, best collection

51. Rubber (Rambong)

52. Rubber, (Rambong)

53. do do

53.(a) Rubber (Para)

כ4. do do

55. do do

56. do do

57. do do

58. do do

59. do do
N'umber or

Prizes. quantity to 1st 2nd 3rd be exhibited.

... 12 heads $5 \quad 31$

... optional

... 50

... 50

... 1 pint

... 1 pint

... 1,

... 1 ,

... 1,

... 1 ,

... 1 ,

... 5 catties

... $\frac{1}{4}$ pint of each 10

$\therefore 1$ catty

... l ,

... I ,

... $\frac{1}{2}$ gantang

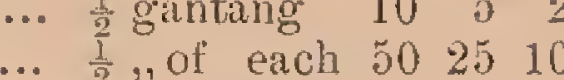

... $\frac{1}{2}$ gantang 5

$\frac{1}{2}$ gantang of each $7 \quad 3 \quad 1$

1 gantang $10 \quad 5 \quad 3$

.. $\frac{1}{2}$ gantang $10 \quad 53$

... $\frac{\sqrt{2}}{2}$ do of each $10 \quad 5 \quad 3$

$\begin{array}{lllrrr}\ldots & \ldots & \frac{1}{2} \text { grantang } & 10 & 5 & 3 \\ \ldots & \ldots & \text { optional } & 5 & 3 & 1\end{array}$

$\begin{array}{lllrll}\ldots & \ldots & \frac{1}{2} \text { grantang } & 10 & 5 & 3 \\ \ldots & \ldots & \text { optional } & 5 & 3 & 1\end{array}$

... Sheets ... 10. Cup presented by the

Negri Sembilan planter's association.

... biscuits $10 \quad 100$

... ball 10 catties 100

... sheets 10. Cup presented

by Hon. W. J. Napier.

... biscuits 10. Cup presented by Hon. Tan Jiak Kim.

... crepe 10 catties 100

... lace 10,100

... worm 10,100

... 100

... scrap 10 catties 100

60. Rubber (Para): General exhibit, to be packed in boxes as sent

from estate, weight not less than 50 lbs. Cup presented

by Mr. H. N. Ridley.

61. Rum, best sample ...

$\begin{array}{lll}\cdots & \cdots & 1 \text { pint } \\ \cdots & \cdots & 1 \text { pint }\end{array}$

1000

62. Rum Shrub, best sample

$10 \quad 0 \quad 0$

* Grown in any one Mukim, and accompanied by an'affidavit furnished by the Penghulu to the effect that the whole was grown in his own Mukim during the previous Padi season. 
Number or

Prizes.

Nature of Exhibit.

quantity to

1 st 2nd $3 \mathrm{rd}$

63. Sago: pearl, best sample

be exhibited.

64. Sago, flour, best sample

6 . Sireh leaves best sample

66. Spices, best collection

... 1 gantang

$\$ \$ \$$

67. Sugar cane best sample

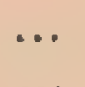

68. Sugar canes, best collection of varieties

69. Sugar, Coconut, best sample ...

70. Sugar, Nipah, best sample ...

71. Sugar, Kabong, best sample ...

72. Sugar (cane) brown, best sample

.. 1 ,

... 100 leaves

$\begin{array}{lll}10 & 5 & 2\end{array}$

.. optional

$10 \quad 5 \quad 2$

.. 10 canes

$\begin{array}{lll}5 & 3 & 1\end{array}$

$10 \quad 3 \quad 2$

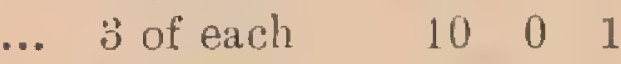

... 2 catities $\quad 5 \quad 501$

$\begin{array}{llllll}\ldots & 2 & 1 & 5 & 3 & 1\end{array}$

73. Sugar (cane) refined, white, best sample $\quad \begin{array}{lll} & 2\end{array}$

74. Honey in the comb best sample

75. Tapioca, roots, best sample

... 3 roots

76. Tapioca, roots, best collection of varieties

77. 'Tapioca, pearl, best sample ...

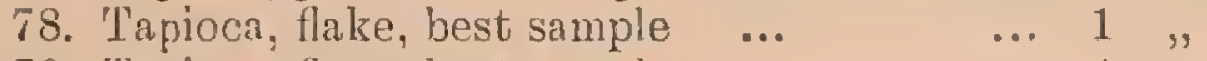

79. 'Tapioca, flour, best sample $\quad . \quad$... 1 ",

80. Tapioca, any other preparation, best sample... 1 "

81. Tea, best sample ...

82. Toddy, best sample

83. 'T'uba (akar) best sample

84. Turmeric, best sample

... 1.

5301

531

$\begin{array}{lll}5 & 3 & 2\end{array}$

$5 \quad 2 \quad 0$

$53 \quad 1$

$10 \quad 5 \quad 3$

$\begin{array}{lll}5 & 3 & 1\end{array}$

$\begin{array}{lll}5 & 3 & 1\end{array}$

$\begin{array}{lll}\text { a } & 3 & 1\end{array}$

53.1

$\begin{array}{lll}10 & 5 & 2\end{array}$

325

... $\quad$... 25 roots $\quad 5030$

85. Walking sticks, best collection unprepared $\begin{array}{llll}1 & \text { of each } & 10 & 5\end{array}$

Best exhibit in this section. Gup presented by H. E, Sir John Anderson.

\section{DIVISION B. \\ FLOWERS, FRUITS AND VEGETABLES. \\ SEOTION I.-FLUWEHS.}

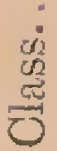

Nature of Exhibit

Ornamental Foliage Plants in pots.

86. droids other than Caladiums

87. . best specimen ...

58. Caladiums...

89. do

90. Coleus

91. Specimen Coleus

92. Crotons

93. " best specimen ...

94, Dracienas ...

95. do best specimen

36. Ferns (any variety), distino

37. do (adiantums) do
Number ol Prizes. quantity to 1st 2 nd be exhibited.

\begin{tabular}{cccccc}
\multicolumn{1}{c}{} & \multicolumn{3}{c}{$\$$} & & $\$$ \\
$\ldots$ & 6 & plants & $\ldots$ & 5 & \\
$\ldots$ & 1 &, & $\ldots$ & 4 & 2 \\
$\ldots$ & 6 &, & $\ldots$ & 5 & 5 \\
$\ldots$ & 1 &, & $\ldots$ & 4 & 0 \\
$\ldots$ & 6 &, & $\ldots$ & 4 & 2 \\
$\ldots$ & & & $\ldots$ & 3 & 0 \\
$\ldots$ & 6 &., & $\ldots$ & 6 & 4 \\
$\ldots$ & 1 &, & $\ldots$ & 5 & 0 \\
$\ldots$ & 3 &, & $\ldots$ & 5 & 3 \\
$\ldots$ & 1 &, & $\ldots$ & 3 & 0 \\
$\ldots$ & 12 &, & $\ldots$ & 10 & 6 \\
$\ldots$ & 6 &, & $\ldots$ & 8 & 4
\end{tabular}


Division B.

Nature of Exhibit.

98. Ferns (adiantums) distinct 99. do. (any variety), best specimen 100. Marantas and Calatheas

101. do. best specimen

102. PaIms, distinct kinds

103. Palms, best specimen

104. Panax and Aralia ...

105. Selaginella, distinct kinds

106. Selayinella, best specimen

107. Any ornamental Foliage Plants not included

Number or quantity to $\begin{array}{cccc}\text { be eshibited. } & & \$ & \$ \\ \ldots & 3 & \ldots & 3\end{array}$

Prizes.

1st 2nd

$\begin{array}{llllll}\ldots & 1 & 1 & \ldots & 4 & 0\end{array}$

... $3,1, \ldots 8 \quad 4$

... 1 ,

... 40

$\ldots 10$. 5

... 5

... 4.2

... 84

.. 40

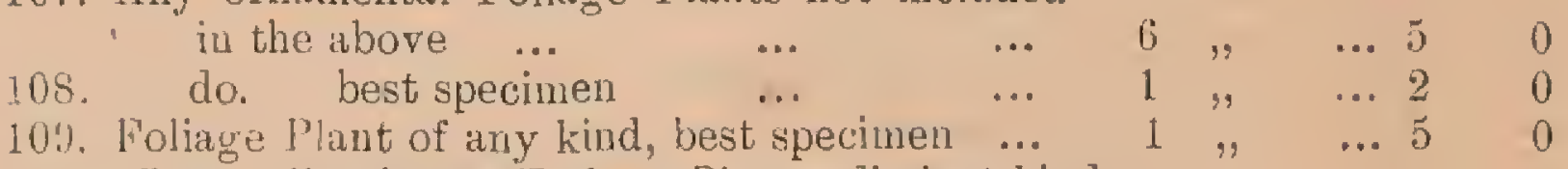
$10 \%$, . Best collection of Foliage Plants, distinct kinds.

Cup presented by Hon. 'l'an Jiak Kim.

Ornamental Plants in Flower, in pots.

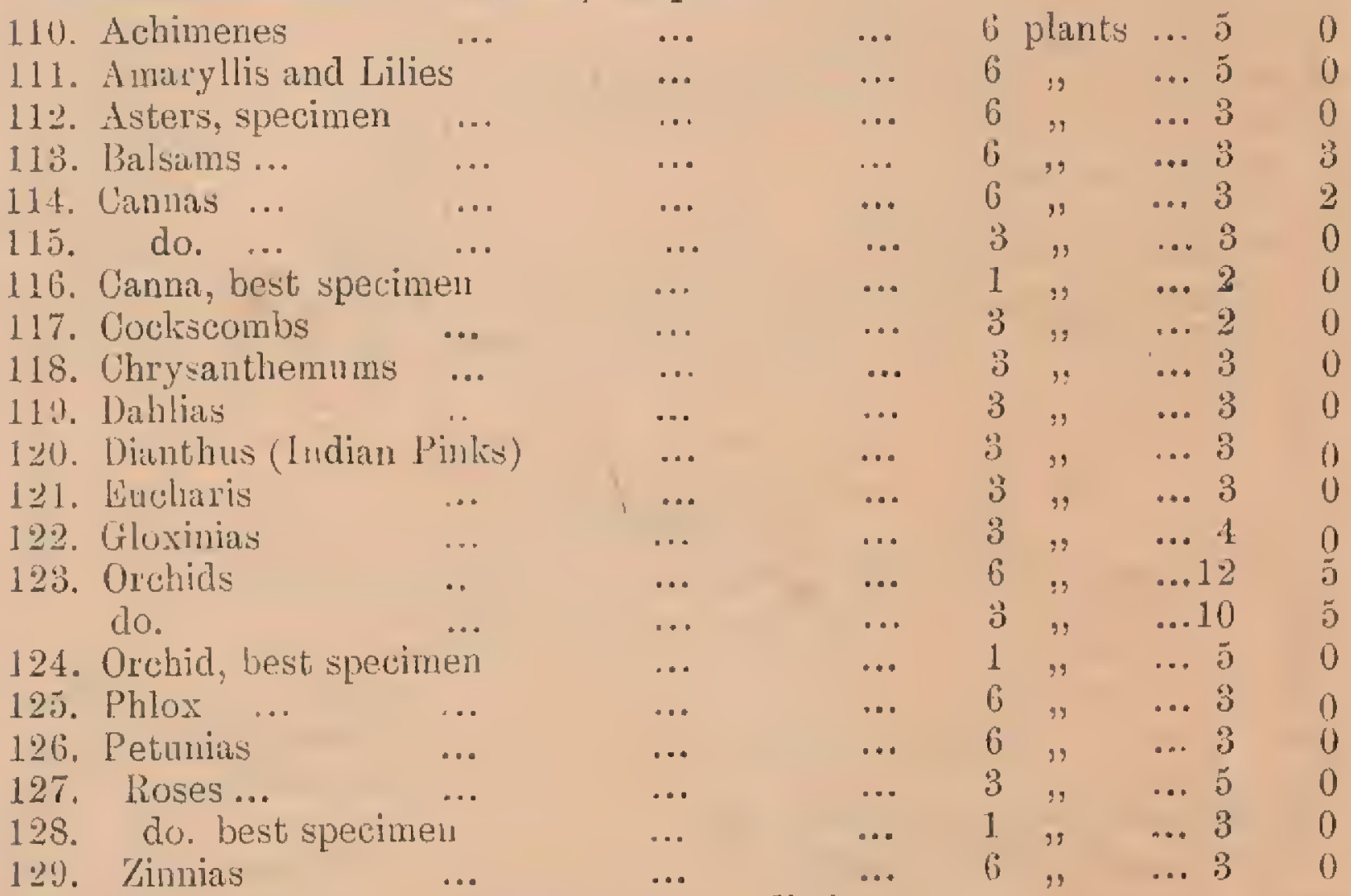

130. Best collection of flowering plants distinet. kinds. Cup presented by Mr. St. V. B. Down. $\quad \ldots$...

131. Best plant in Flower in Show not included in above. Plants whether in Flower or not.

132. Begonlias, distinct kinds

133. Begonia, best specimen

134. Groups of plants arranged for effect in srace

6 kinds not exceeding 9 feet square

... 1 plant ... 5

$\begin{array}{llllll}\ldots & 6 & & & & \end{array}$

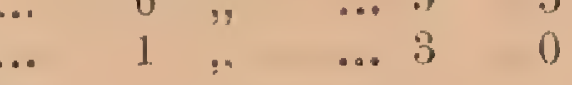

ce

optional $\ldots 885$ 


\begin{tabular}{|c|c|c|}
\hline Division B. & Nature of Exhibit. & $\begin{array}{l}\text { Number or } \\
\text { quantity to } \\
\text { be exhibited. }\end{array}$ \\
\hline
\end{tabular}

Cut Flowers and Table Decorations.

135. Group of Chinese Plants of fantastic shape... optional $\ldots 50$

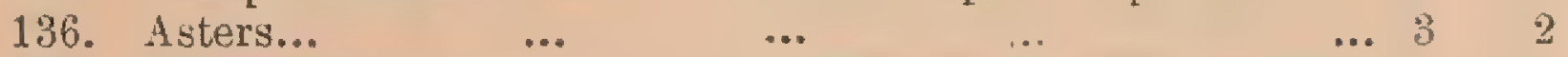

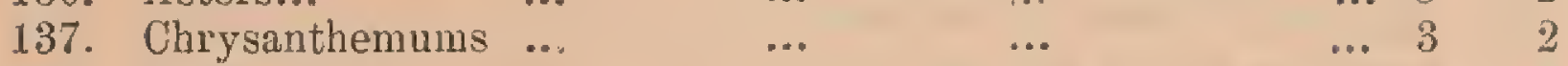

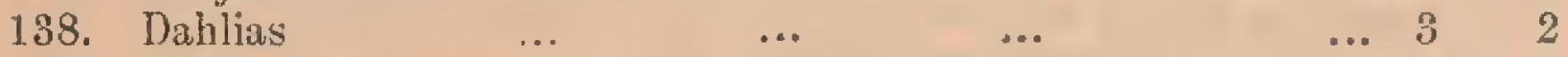

139. $\begin{array}{lllllll}\text { Roses ... } & \ldots & \ldots & \ldots & \ldots & 3 & 2\end{array}$

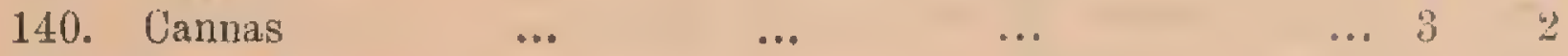

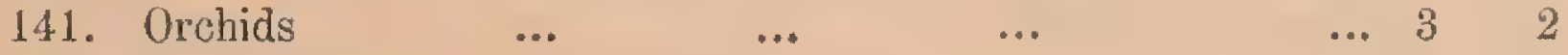

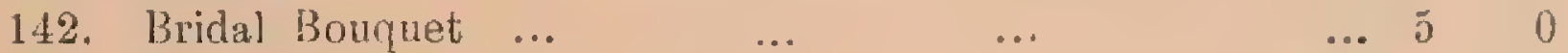

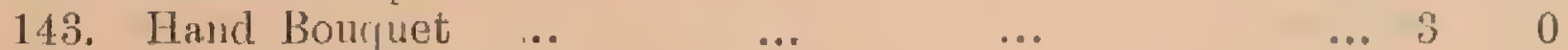

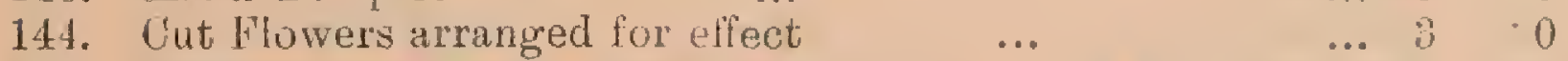

145. Collection of cut flowers not less than ... 12 kinds ... 50

146. Table Decaration. Gup presented by Mr. Choa Kim Keat

147. Wild Flowers arranged for effect $\quad \ldots \quad$... 330

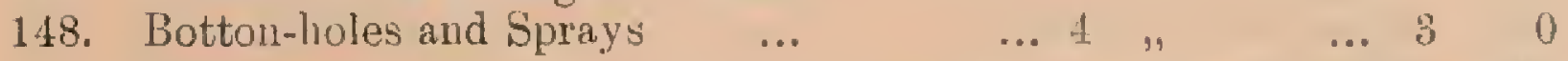

Exhibitors in classes 136-145 must provide their own stands for cut flowers and must stage their exhibits before $8 \mathrm{am.m}$. The Committee will provide tables of a uniform size $\left(7^{\prime}+3 \frac{1}{2}\right.$ for competitors in class 1.46 .

SEGTIUN 2. FRUITS.

14\%. Banauas, "Pisangs," best collection _.. 6 of ench kind $10 \quad 5$

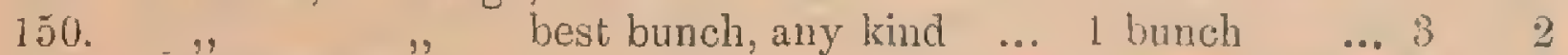

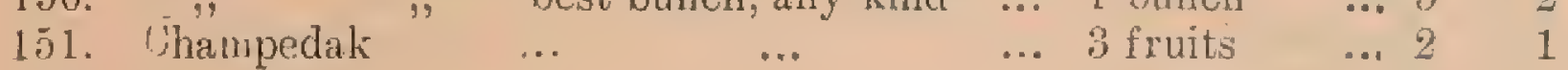

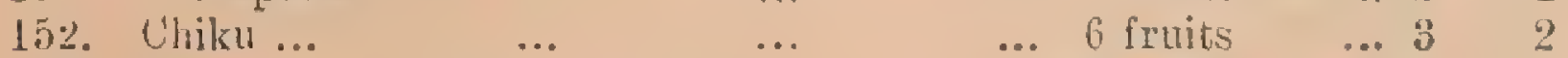

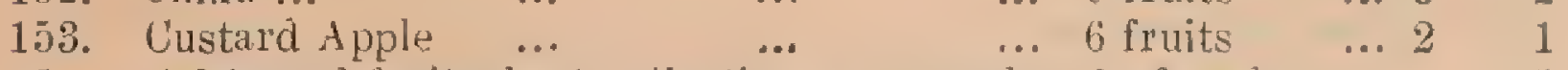

154. Cultivated fruits, best collection any number 3 of each ' 15\%. Durian kind. Cup presented by Mr. H. Marriott

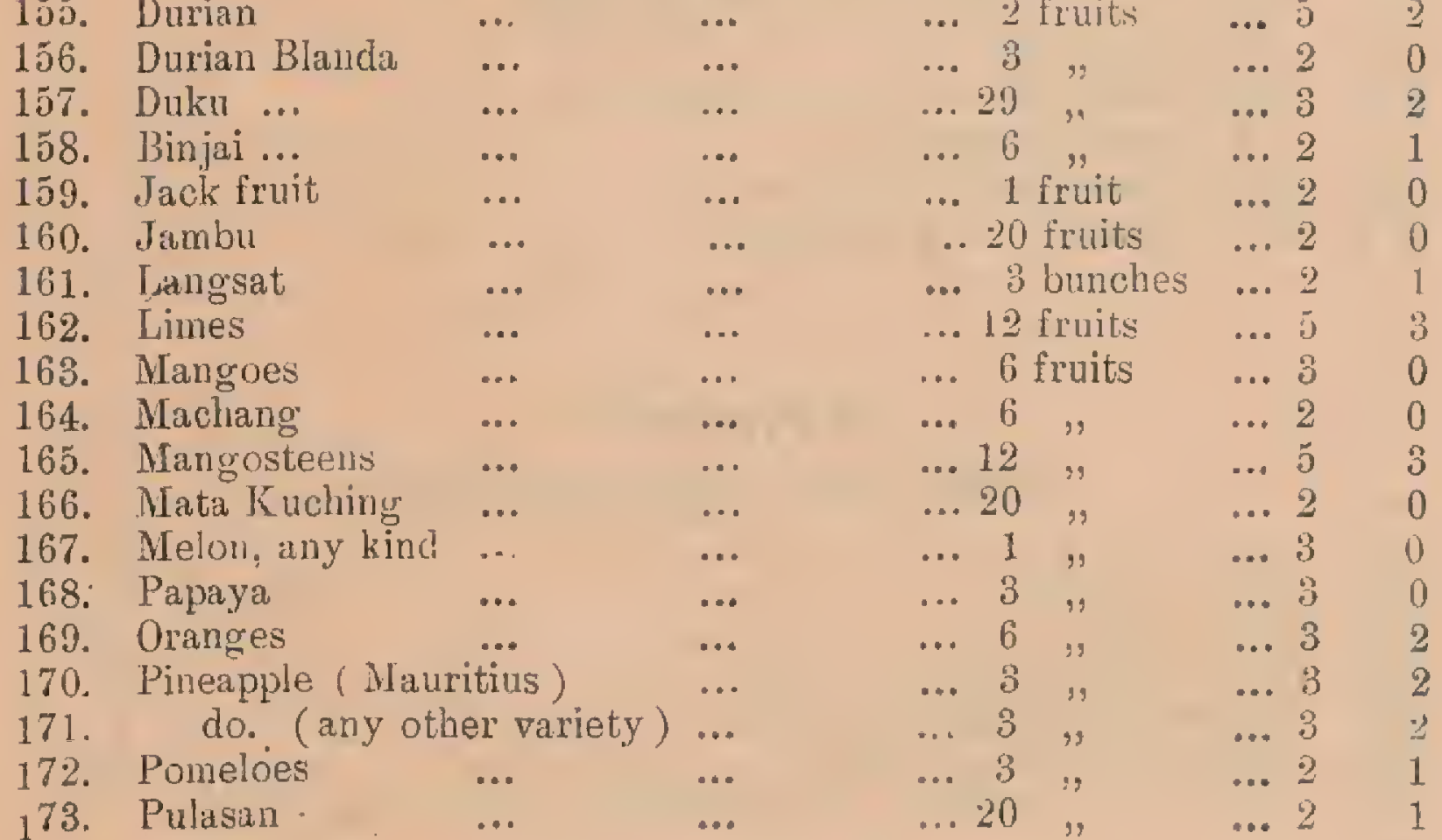


DIVISION $B$.

Nature of Exhibit

Number or

Prizes. quantity to

1st 2 nd

174. Rambai

175. Rambutan

be exhibited.

$\$ \quad \$$

176. Wild edible fruits, best collection

... 3 bunches ... 21

...20 fruits $\ldots 22$ 1

... optional ... 5 5 3

177 Any kind of fruit not included in the above... , $\quad \ldots .32$

Preserved Fruits and Vegetables. All exhibits in the following classes must be growin or frepured by the exhibitors.

178. Preserved Fruits, best sample, any method ... optional ... $10 \quad 5 \quad 3$

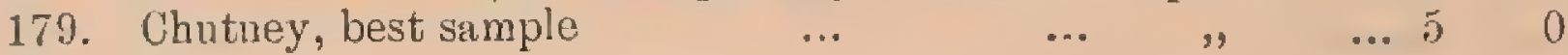

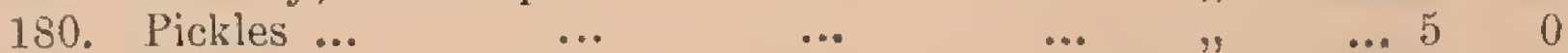

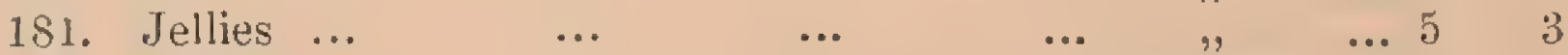

182. Artichokes $\quad \ldots . \quad \ldots \quad \ldots 12$ tubers $\ldots 2$... 20

183. Beetroot $\quad \ldots \quad$... $\quad \ldots 6$ specimens $2 \quad 0$

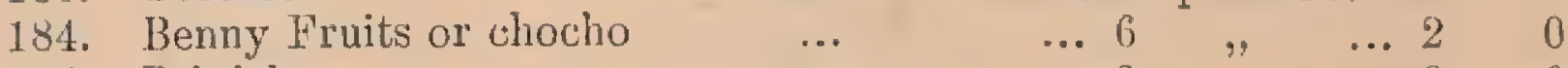

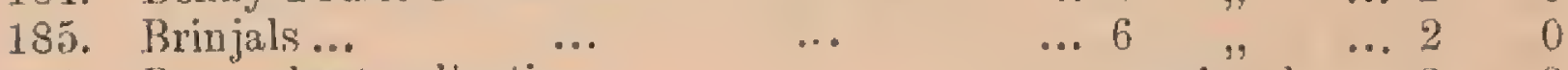

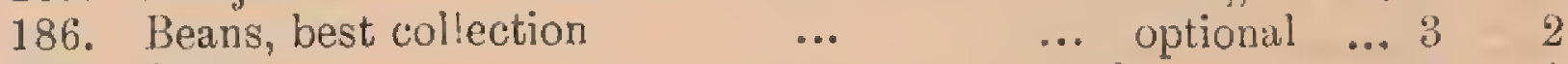

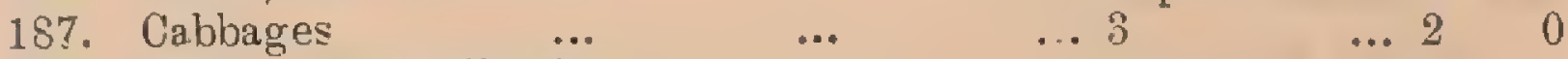

188. Chillies, best collections $\quad \ldots \quad \ldots 30$ of each $\ldots 3 \quad$...

189. Cucumbers $\quad . .6 \quad \ldots 6$ specimens... 2 ... 0

190. Herbs used in curries \& sambals, best collection 1 bule. of each $2 \quad 0$

191. Ladies Fingers $\quad . .6 \quad \ldots 6$ specimens... 2 ...

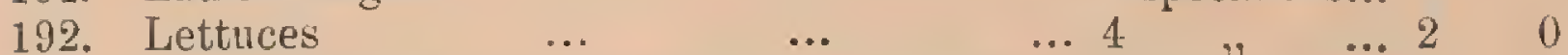

193. Onions, Shallots and Garlic, best collection ... 1 bdle. of each 20

194. Pumpkins $\quad$... $\quad$.. $\quad \ldots 3$ specimens... $2 \quad 0$

195. Radishes $\quad \ldots \quad \ldots .10$ specimens... " 20

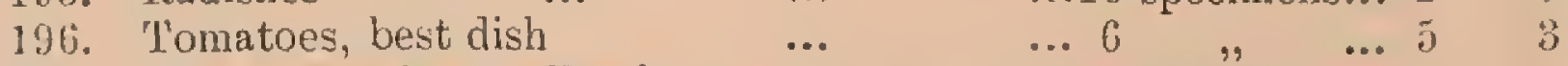

197. Vegetables, best collection ... ...at lestst 3 specimens of each. Cup presented by Hon. 'Tan Jiak Kim... 3

198. Vegretables and Herbs for making a Salad,

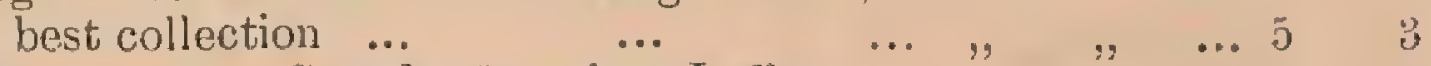

199. Water-melons, Gourds, Squashes, Luffas, \&c., best collection $\quad \ldots \quad \ldots 1$ of each $\ldots 55$

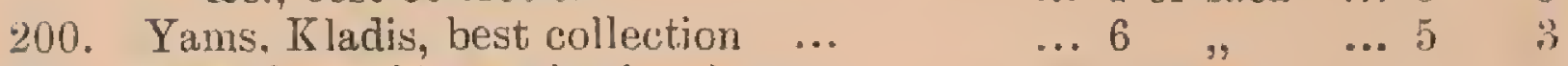

201. Any Vegetable not in the above $\quad$... optional .. 3 z

\section{DIVISION C. \\ STOCK AND DAIRY PRODUCE.}

202. Bull, locally bred

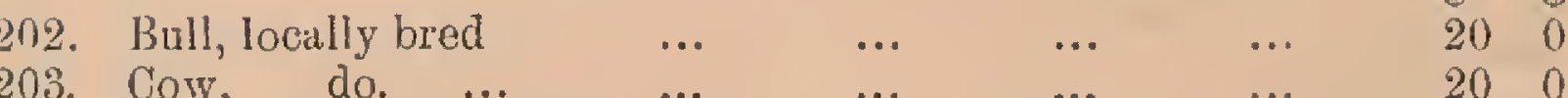

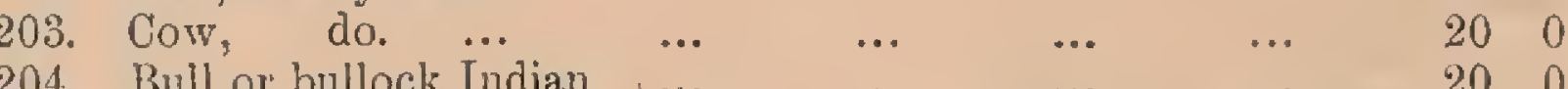

$\begin{array}{llllll} & & & & & \\ & \ldots & \ldots & \ldots & 20 & 0\end{array}$

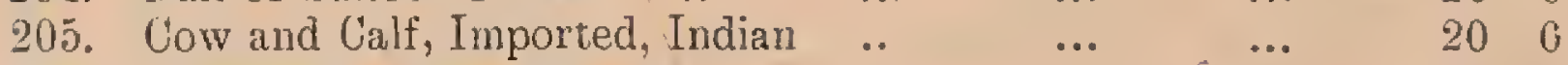

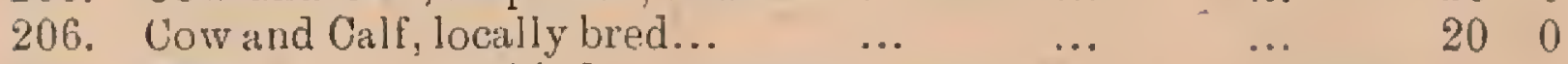

207. Milch Gow of any kind. Cup presented by Hon. A. T. Bryant. 
DIVISTON $\mathrm{C}$.

Nature of exhibit.

208. Pair of draught Oxen, Siamese breed

209. do. do. do. Indian do.

210. Pair of draught Buffalos ...

211. Buffalo, Bull, Indian ..

21\% do. Cow, do.

213. Buffalo, Bull, locally bred.

214. do. Uow, do.

215. Champion animal in classes $202,204,208,209$.

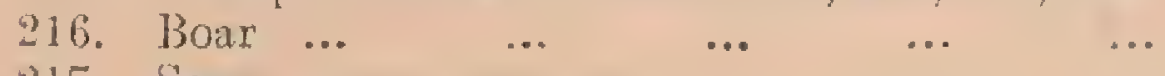

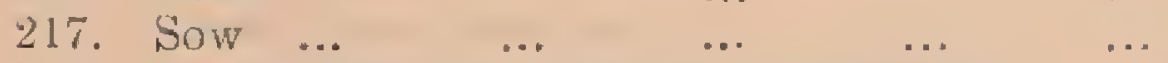

218. Sow and litter under three months old ...

219. Pen of 6 pigs. ... $\quad . . \quad \ldots \quad \ldots$

220. Pig over 300 catties $\quad \ldots \quad \ldots$

221. Champion Pig in the Show.

222. Goat, Ram

223. Goat, kwe with lids $\cdots$

224. Best Milk goat ...

225. Sheep, Ram ...

2.25. Shep, Ra

227. Sheep, hest pen of 6 . Indian breed

228. do. do. do. Javanese do.

229. Bantam Cock and Hen ...

230. Malay Cock and Hen ...

231. Malay Game Cock and Hen

232. Collection of Malay Fowls

233. Collection of Chinese Fowls

234. Collection of Fowls, any breed

235. Cock and hen, any breed, not included in above

236. Nuscory Duck and Drake

237. Manila Duck and Drake ...

238. Duck and Drake of any breed

239. Duck and Drake hybrid ",

240. Gander and Goose

241. Pair of 'l'urkeys, Cock and Hen

242. Pair of Guinea Fowls, Cock and Hen

243. Pair of Pigeons, any breed Cock and Hen

244. Collection of Pigeous, any breed ...

245 . Best Cage Bird

245. Best Uage Bird... ... ...

246. Best Collection of Cage Birds ... ...

247. Pair of Rabbits, any breed, Buck and Doe .
Number or Prizes.

quantity to 1st 2nd

be exhibited. \$\$

100

$10 \quad 0$

$10 \quad 0$

1510

150

Cup

1510

Cup

$\cdots \quad 10 \quad 0$

$\begin{array}{lll}\cdots & 10 & 0\end{array}$

$\begin{array}{lll}\ldots & 10 & 0\end{array}$

$\begin{array}{lll}\ldots & 15 & 0\end{array}$

150

$1 \%$

105

$10 \quad 5$

100

105

$10 \quad 5$

$10 \quad 0$

100

5 3

53

5) 3

105

105

$10 \quad 5$

50

105

5 3

53

53

5 3

5 3

53

53

50

50

100

50

50

53

250. Collection of Eggs, fowls' ducks' or turkeys', optional

251. Best Turnout Bullock-cart and Bullocks, any kind. A cup presented by Dr. J. A. R. Glennie. 


\section{DIVISION D.}

\section{HORSES AND DOGS.}

252. Polo Pony, registered 14.1 or under; bona file property of a member of any recognised Polo Club in the Colony or F. M. S. 'lo be shown under saddle.

1st. Prize. $\Lambda$ cup presented by Mr. M. Payne Gallwey. 2nd. Prize. A cup.

253. Gentleman's Hack, 14.2 and under. 'lo be shown under saddle.

1st. Prize. A cup presented by Mr. C. Sugden.

2nd. Prize. A cup.

254. Gentleman's Ilack, 14.2 and over. 'T'o be shown under saddle.

1st. Prize. A cup presented by Mr. F. F. H. Fdlin.

2nd. Prize. A'cup.

255. Single Harness Pony 14.2 and under. 'To be shown in cart or carriage.

1st. Prize. A cup prespnted by Dr. Galloway.

2nd. Prize. A cup.

256. Single Harness IIorse 14.2 and over. To be shown in cart or carriage.

1st. Prize. A cup presented by the Bankers.

2ud. Prize. A cup presented by the Bankers.

257. Lady's Hack. Any size. To be shown under siddle and ridden by a lady.

1st. Prize. A cup presented by Mr. J. B. Yomng.

2nd. Prize. A cup.

25s. Jumping Pony. 14.2 and under. To be ridden over at least four jumps.

1st. Prize. A cup presented by Mr. W. I). Barmes.

2nd. Prize. A cup.

259. Tumping Horse 14.2 and over. 'To be ridden ovar at least four jumps.

1st. Prize. A cup presented by The Brokers.

2nd. Prize. A cup.

260. Double Turnout of any description, pars, tandems etc.

1st. Prize, A cup presented by Mr. Toel 'Trotter.

2nd. Prize, A cup.

261. Gharry 'Lurnont: the bone fide property of a licensed Gharry owner (Australian Horses barred).

1st Prize $\$ 20$.

262. Best Horse or Pony in the Show, to be shown on a halter. Wimners in Classes 252 to 260 only eligible.

1st Prize. A cup presented by 'l'he Merchants.

lintries Close Saturday July 28.

Entry forms may be obtained from Mr. T. H. Gaunt.

\section{DOGS.}

263. Smooth haired Fox Terrier Dog

264. Smooth-haired Fox Terrier Bitch

265. Rough-haired Fox Terrier Dug ...

266. Rough-haired Fox Terrier Bitch
Ist. 2nd.

\$ $\$$

$10 \quad 5$

$10 \quad 5$

105

$10 \quad 5$ 


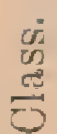

DIVISION D.

Nature of Exhibit.

Number or Prizes. quantity to 1 st. 2nd be exhibited. $s \$$

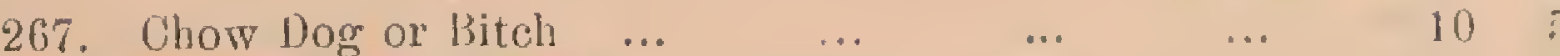

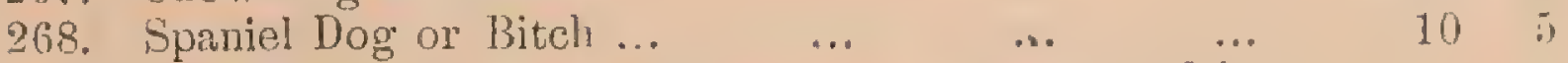

269. Irish 'Terrier 1 ogg. 1st Prize. A cup presented by Mr. F. Hilton

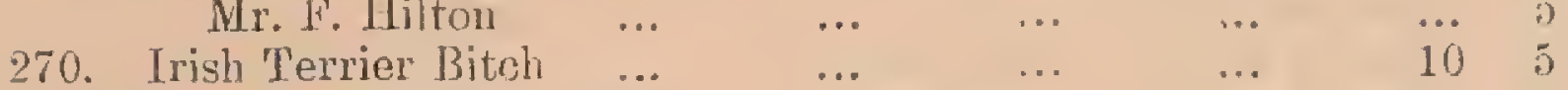

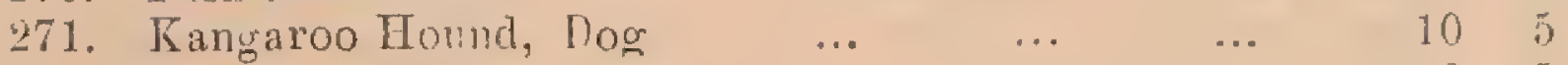

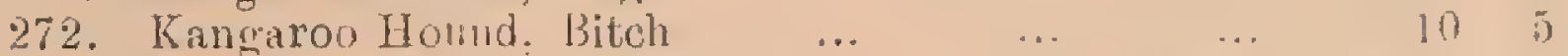

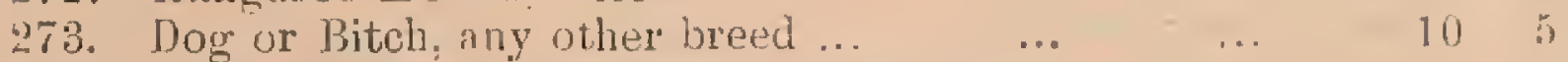

274. Champion Dog in the show - 1 Borneo cup.

\section{DIVISION E.}

NATIVE INDUSTRIES AND MANUFACTURES.

CLASS I.

275. Ornamental Baskets

270. Lace work (biku)

277. Leather work

278. Rope and T'wine ...

279. Tali Ijok

280. Attaps

281. Kajangs

282. Chicks

283. Mats

284. Mats, Rotan

285. Malay Mats

286. Hats

287. Brushes and Brooms

288. Coconut Palms. Collection of articles manufactured from

289. Kabong (arenga)

290. Nibong

291. Sireh apparatus set

292. Model Malay House

293. Model Fishing stakes

294. Model Snares and Traps

295. Wood carving, 1 piece

296. Embroidery, 1 piece

297. Sarong (silk)

298. Sarong (cotton) ...

299. Brass ware

300. Silver ware

301. 'T'in ware

302. Krisses sheaths and knife handles

303. Fishing Nets

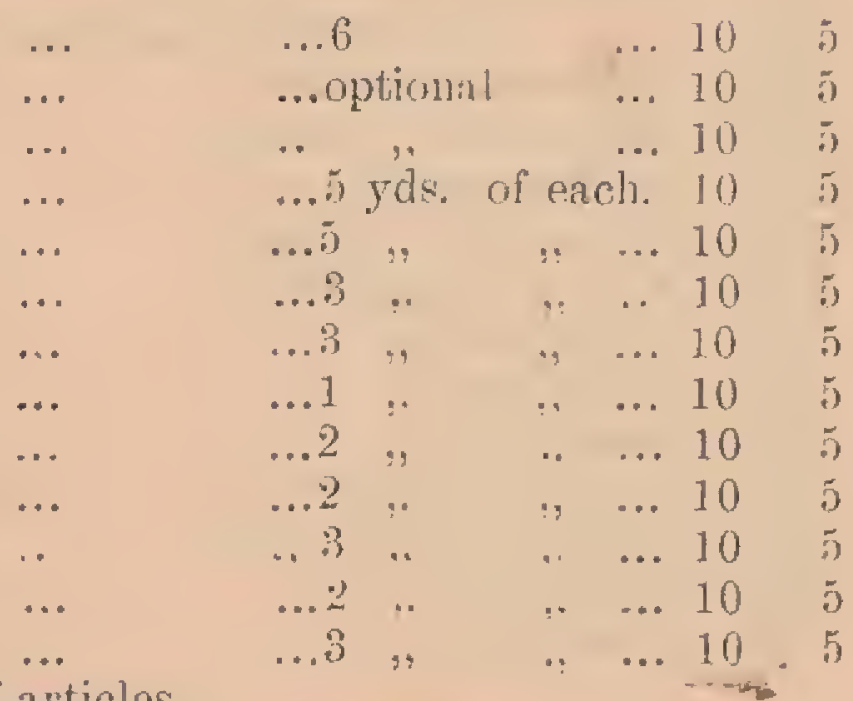

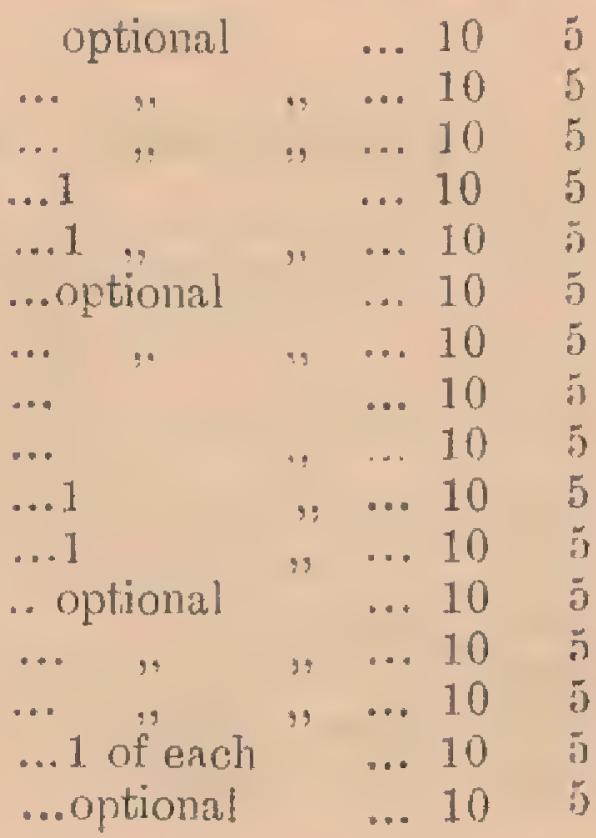


Division E.

Nature of Exhibit.
Number or Prizes. quantity to 1st. 2nd. be exhibited. \$\$ \$ $\$$

304. Sakei articles $\quad \ldots \quad$... $\quad$...

305. Collection of photographs illustrating native life or industries $10 \quad 5$

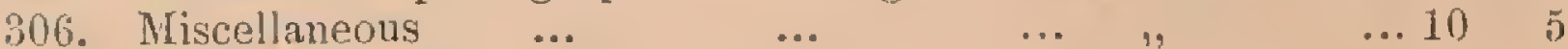

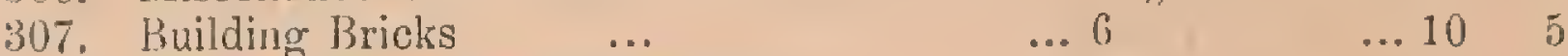

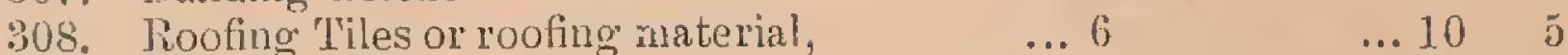

309. Flooring 'liles do.

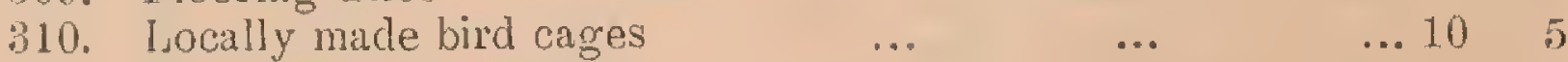

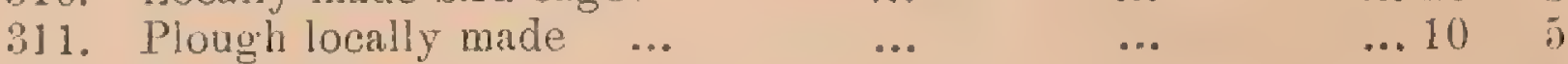

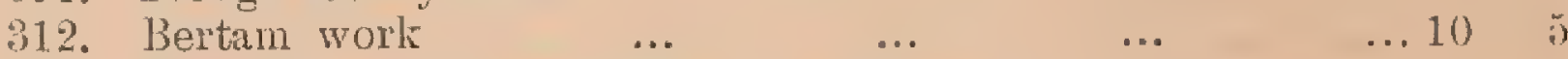

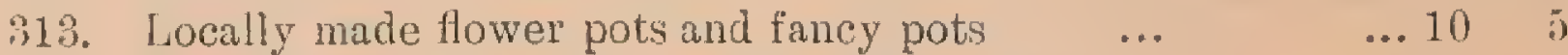

314, Locally made Chatties $\quad \ldots$ (earthenware) ... $\quad \ldots 10 \quad 5$

\section{CLASS II.}

Native Industries and Manufactures confined to Competitors from Schools of the Colony and Malay Peninsula.

315. Nalay hand-made Pottery

316. " land-made fancy Pottery

317. Ornamertal Baskets

318. Malay Mats (Pandanus)

319. Sireh Apparatus, set

$3: 0$. Model, Malay House

321. Mode!, Fishing Stakes

322. Wood carving ...

32.5. limbroidery

324. Sarong (silk)

325. Sarong (cotton) ...

326. Snares etc., collection

327. Fishing Nets

328. Miscellaneous

$\cdots$

... 3 specimens ...10 5

$\begin{array}{llll}. .3 & \ldots 10 & 5\end{array}$

$\ldots 1$ of each ...10 5

... 3 specimens ... $10 \quad 5$

... 1 of each ...10 5

$\begin{array}{lll}\ldots 1 & \ldots 10 & \end{array}$

... optional ... 10

. .1 piece $\quad . .1010$

$\ldots 1, \quad \ldots 10 \quad 5$

$\ldots 1, \quad \ldots 10 \quad 5$

$\begin{array}{llll}\ldots 1 & 110 & \ldots & \end{array}$

... optional ... 10 5

... $\quad \ldots 10 \quad 5$

$\begin{array}{llll}\ldots & \ldots 10 & & \end{array}$

\section{DIVISION F.}

AGRICULTURAL IMPLEMENTS AND MISCELLANEOUS.

329. Padi Implements. (Collection) $\quad$... optional $\quad \ldots 10 \quad$ j

330. Agricu!tural implements, other than for padi

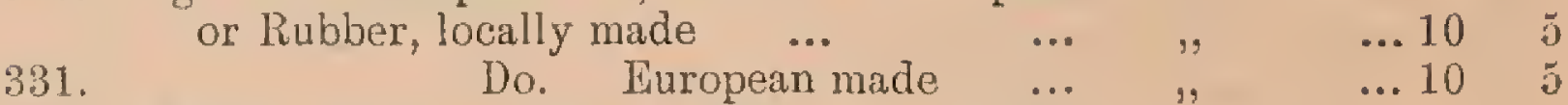

332. Machinery in connection with preparing Rubber. To be exhibited at work $\quad$...,$\quad$ Cup

333. Best collection of tools for tapping rubber ... n $\quad . .10$

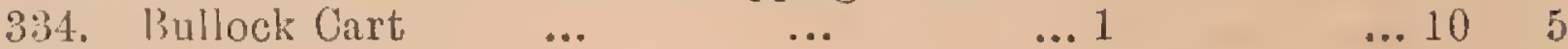

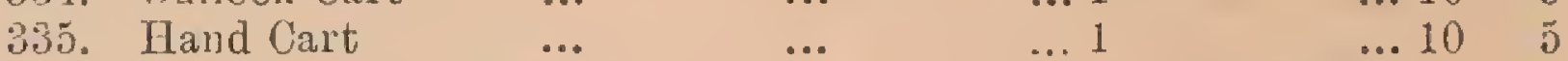

336. Agricultural Baskets $\quad$... $\quad \ldots 2$, of each kind $10 \quad 5$

337. Cusks, 'lubs, etc. ... $\quad \ldots \quad$... 3 specimens $\ldots 10 \quad$

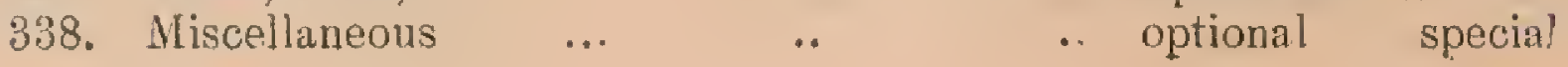




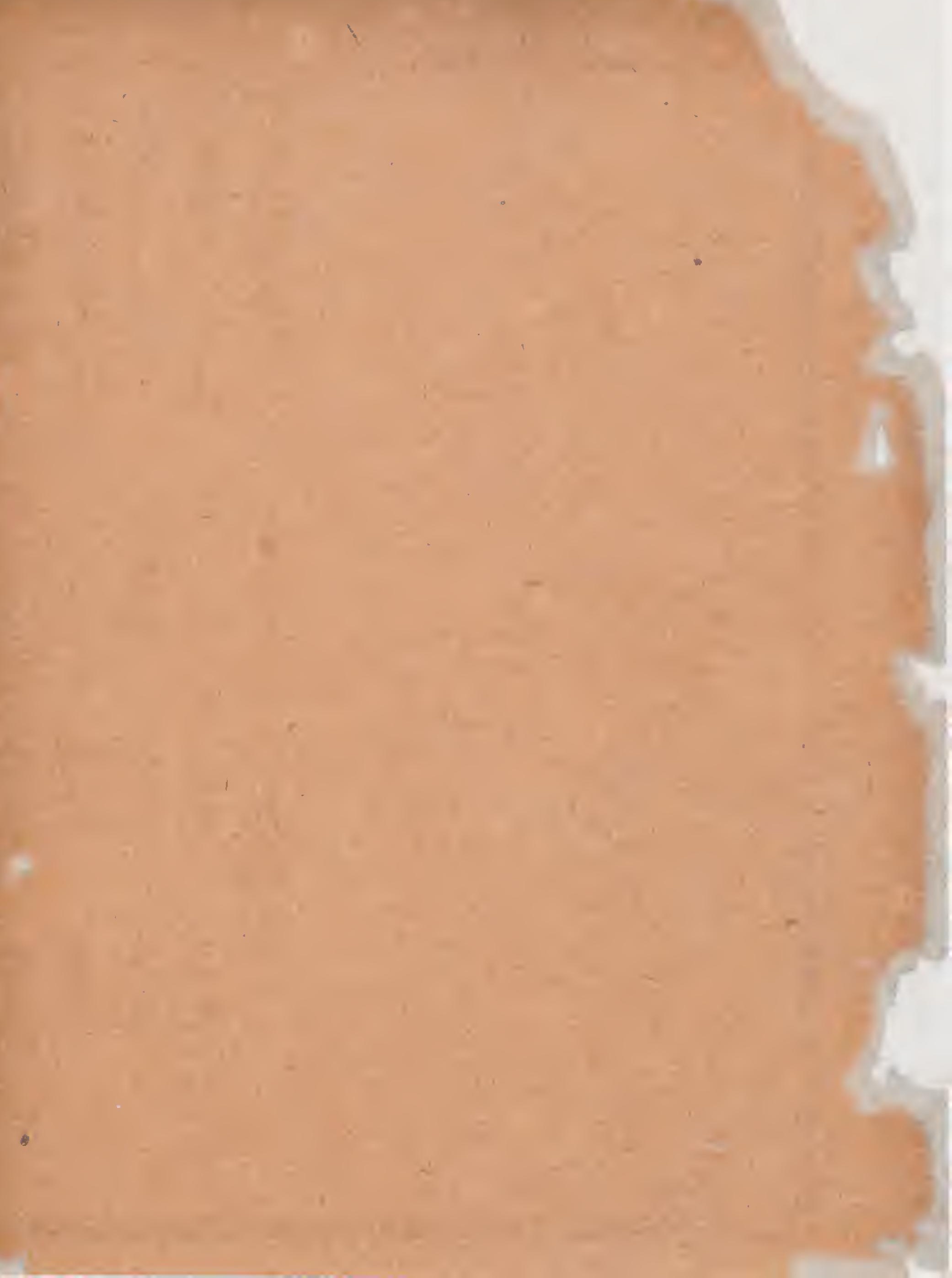




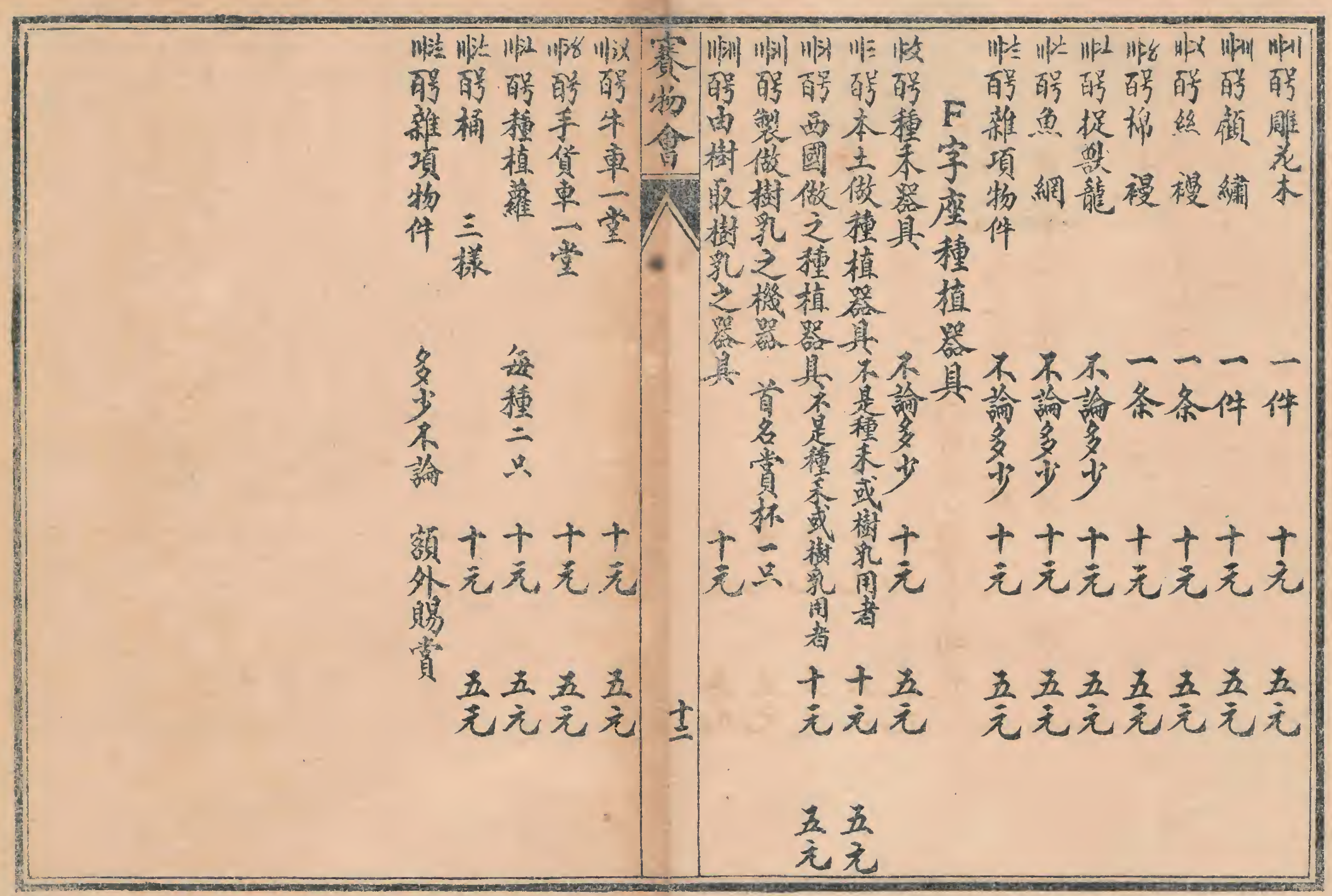




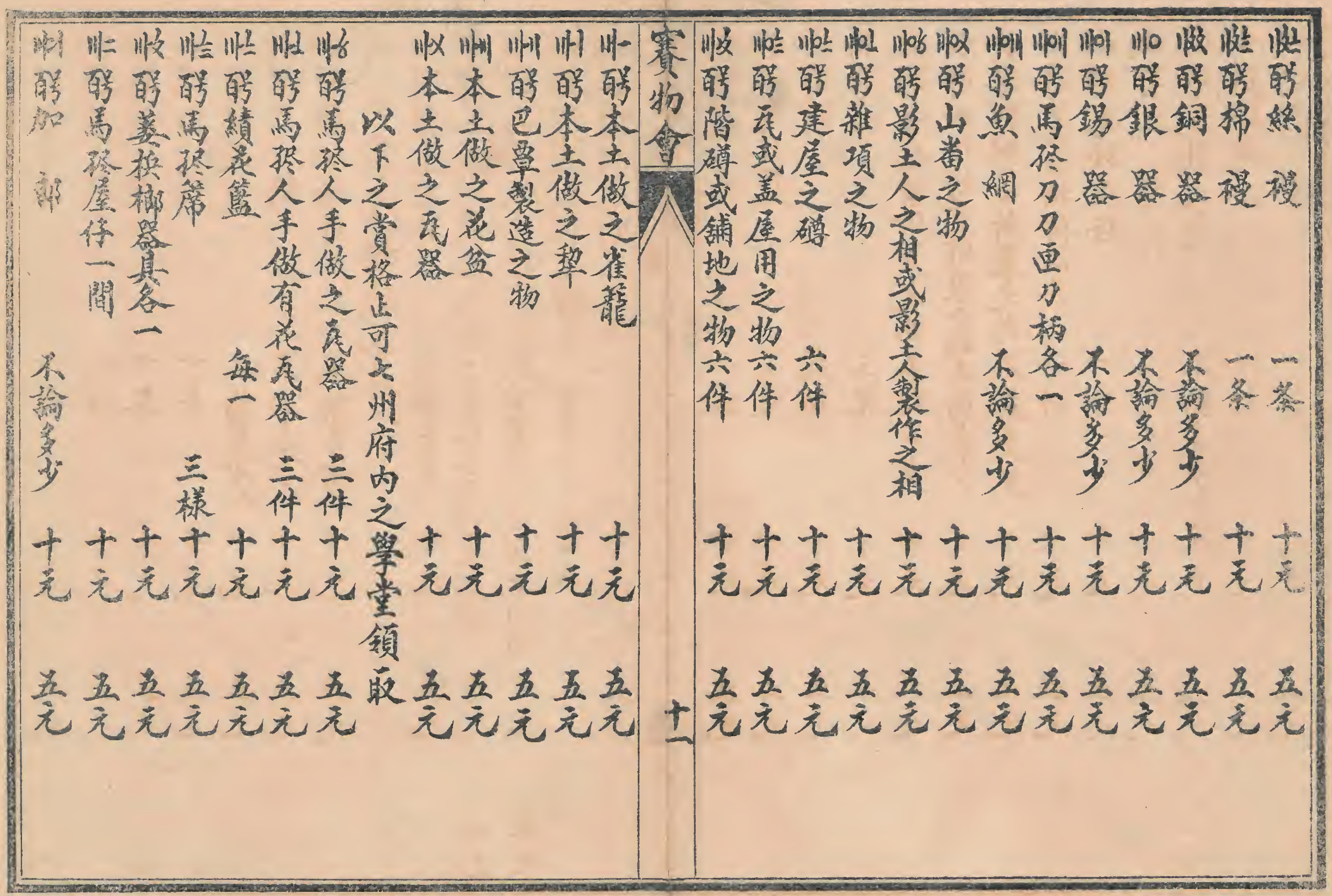




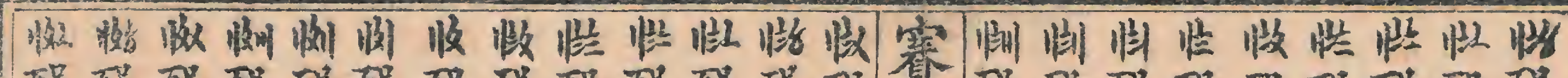

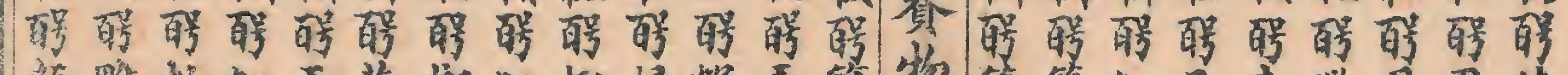

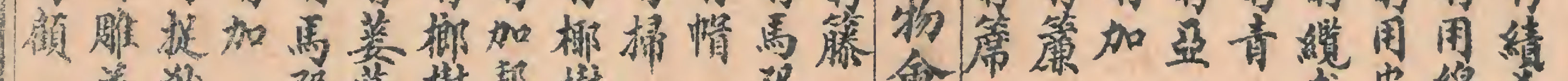

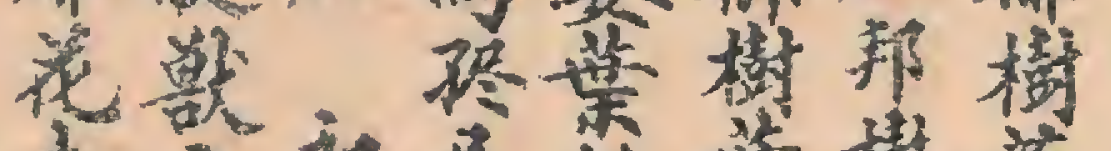

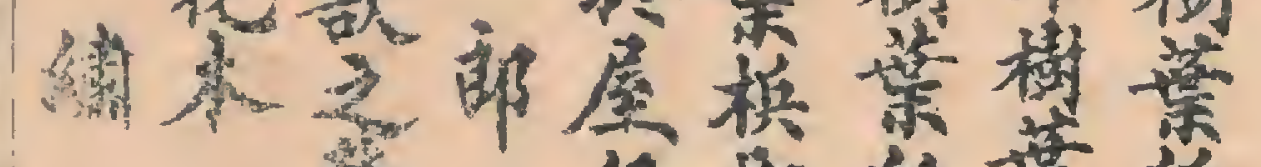

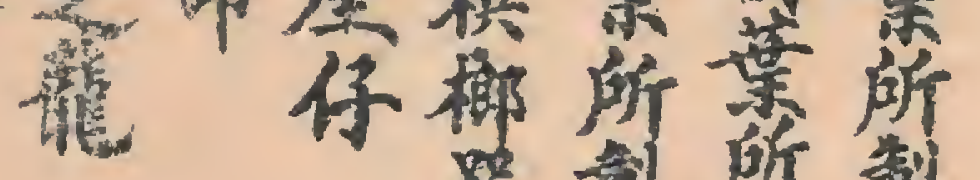

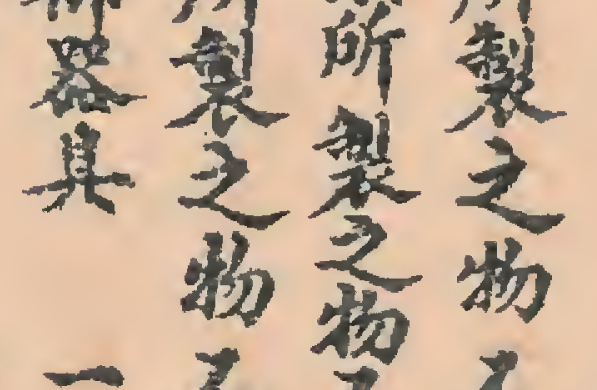

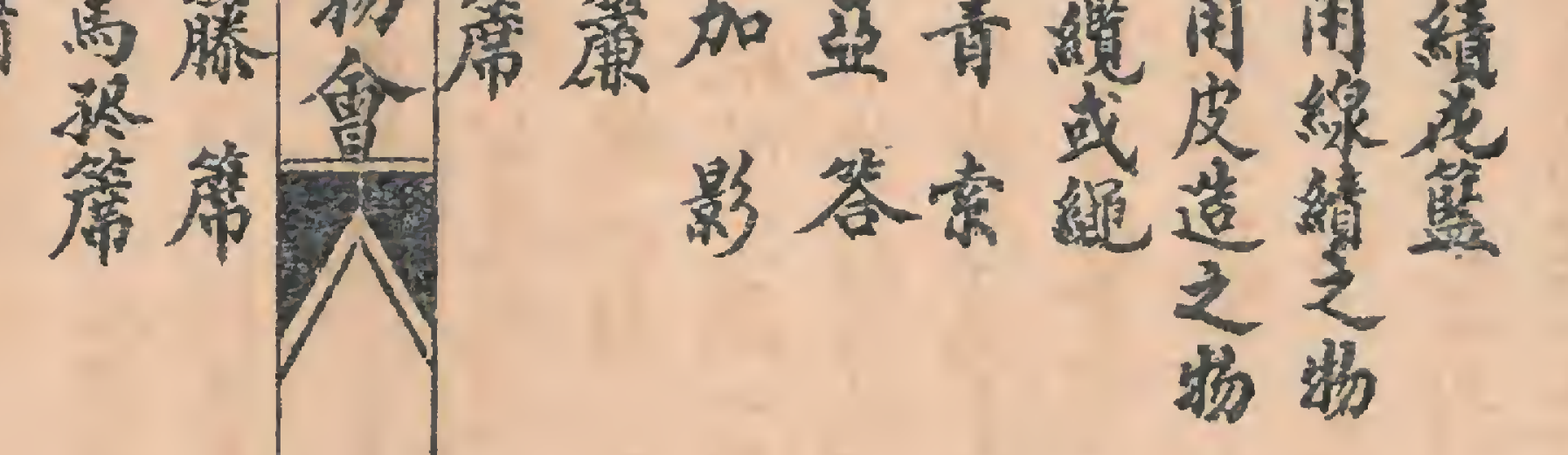

E $\rightarrow$ B

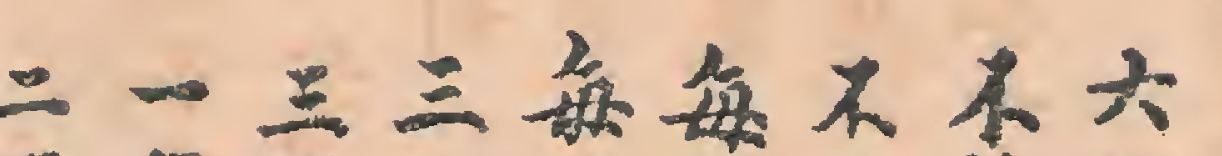

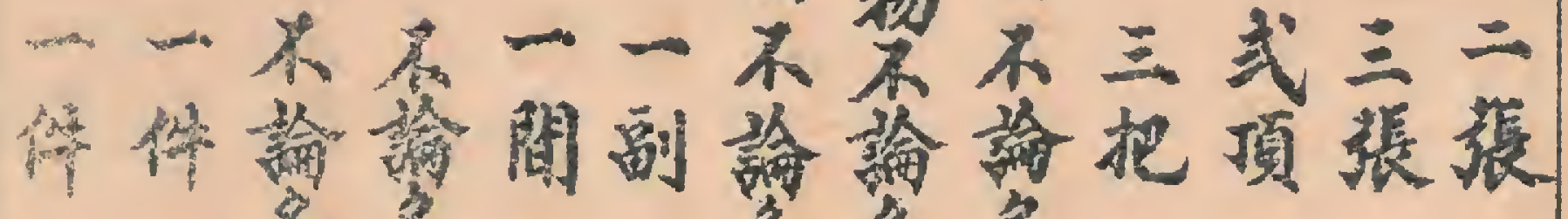

$$
\text { 多严等少少 }
$$

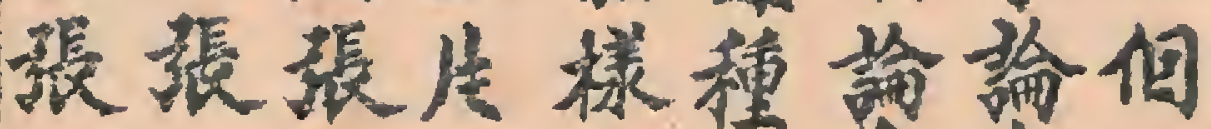

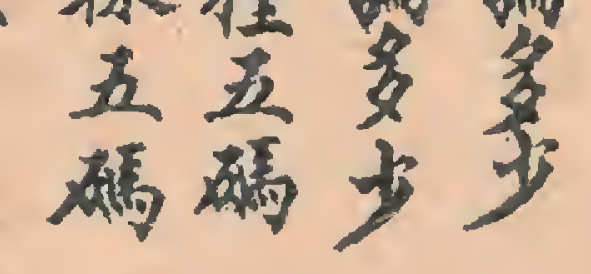

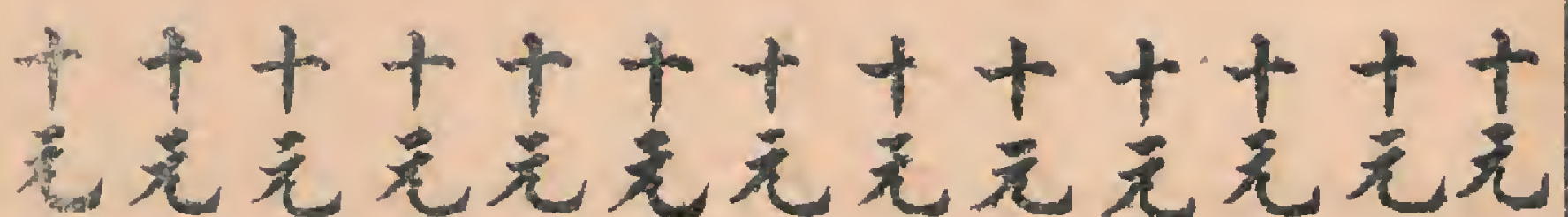

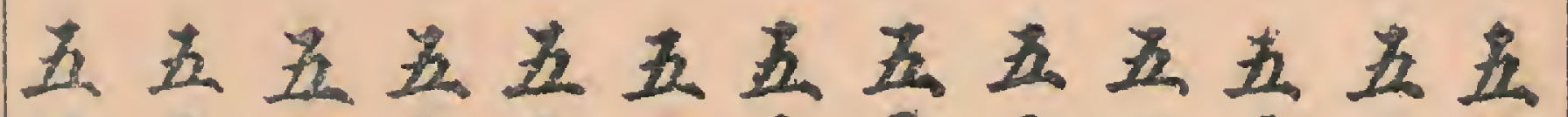

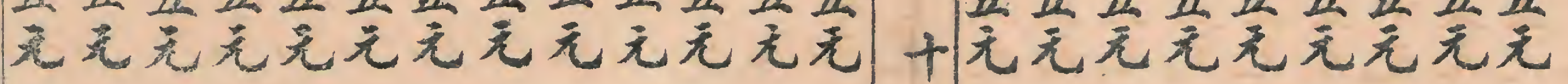

等无変

座者问 去吾盟

座并起

慗务告

涪有紧 银的 各地业 㰩十俱 九是 品 偻举 各、電 $\$$ 及 然 商基 都 然 透最 


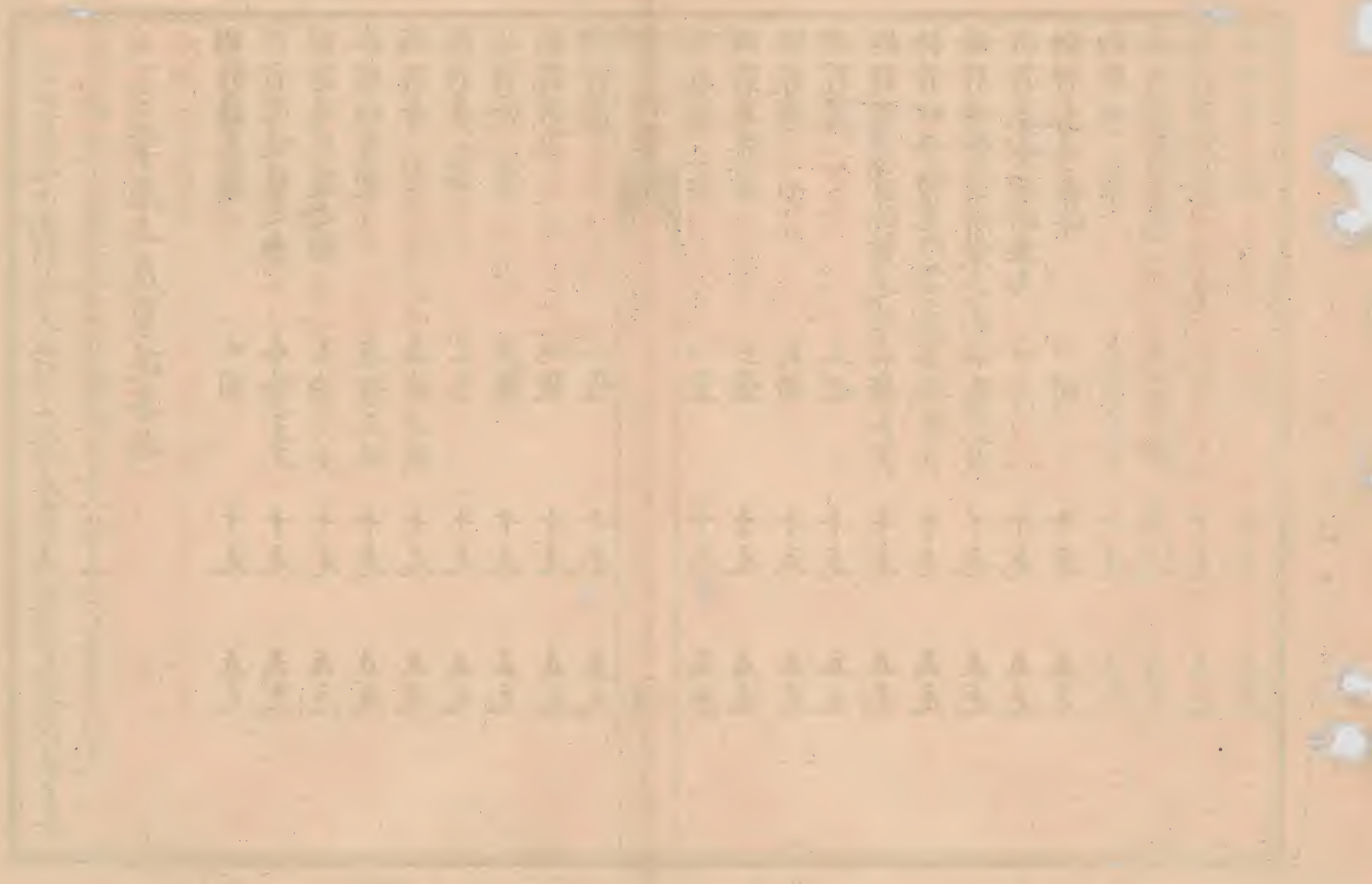




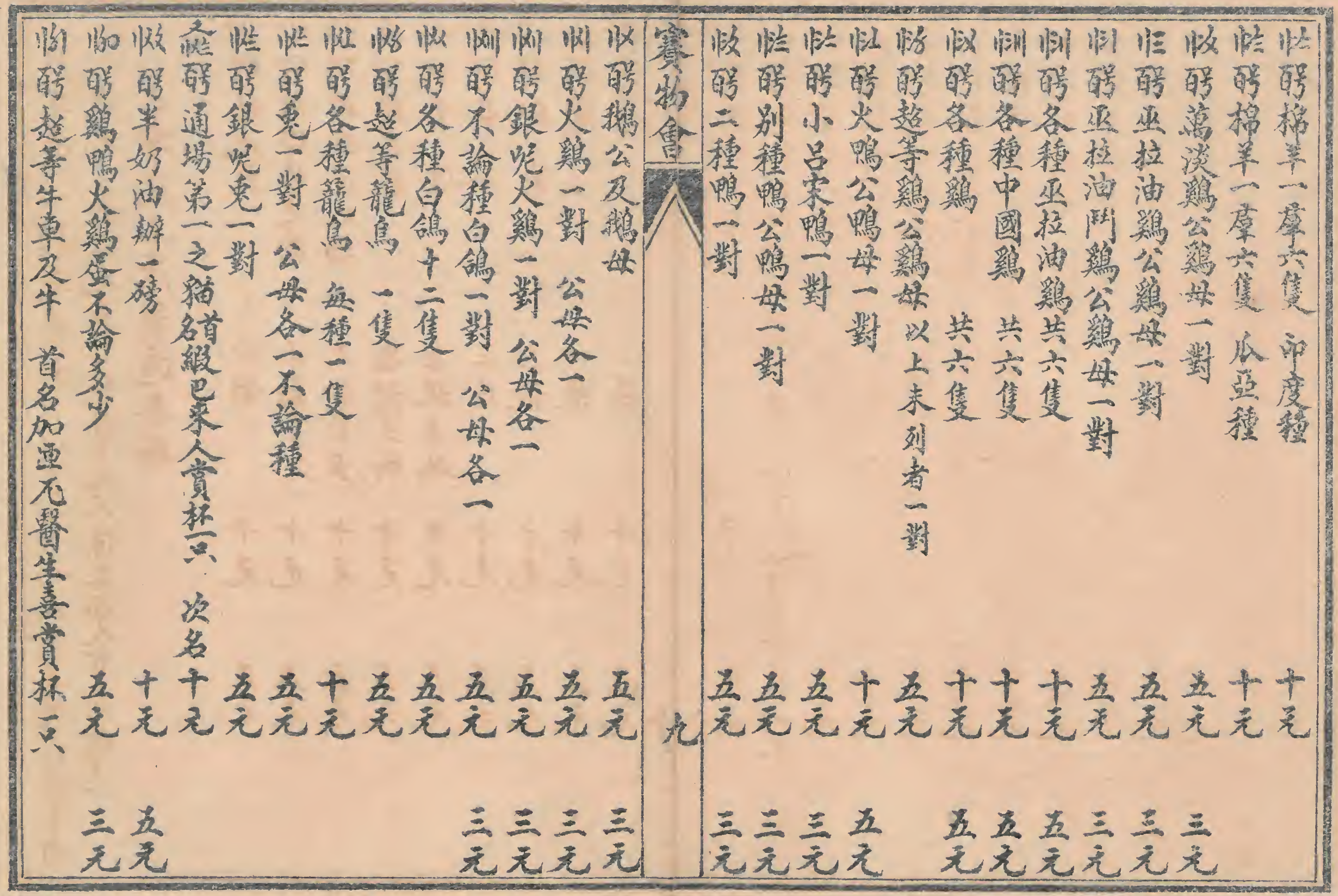





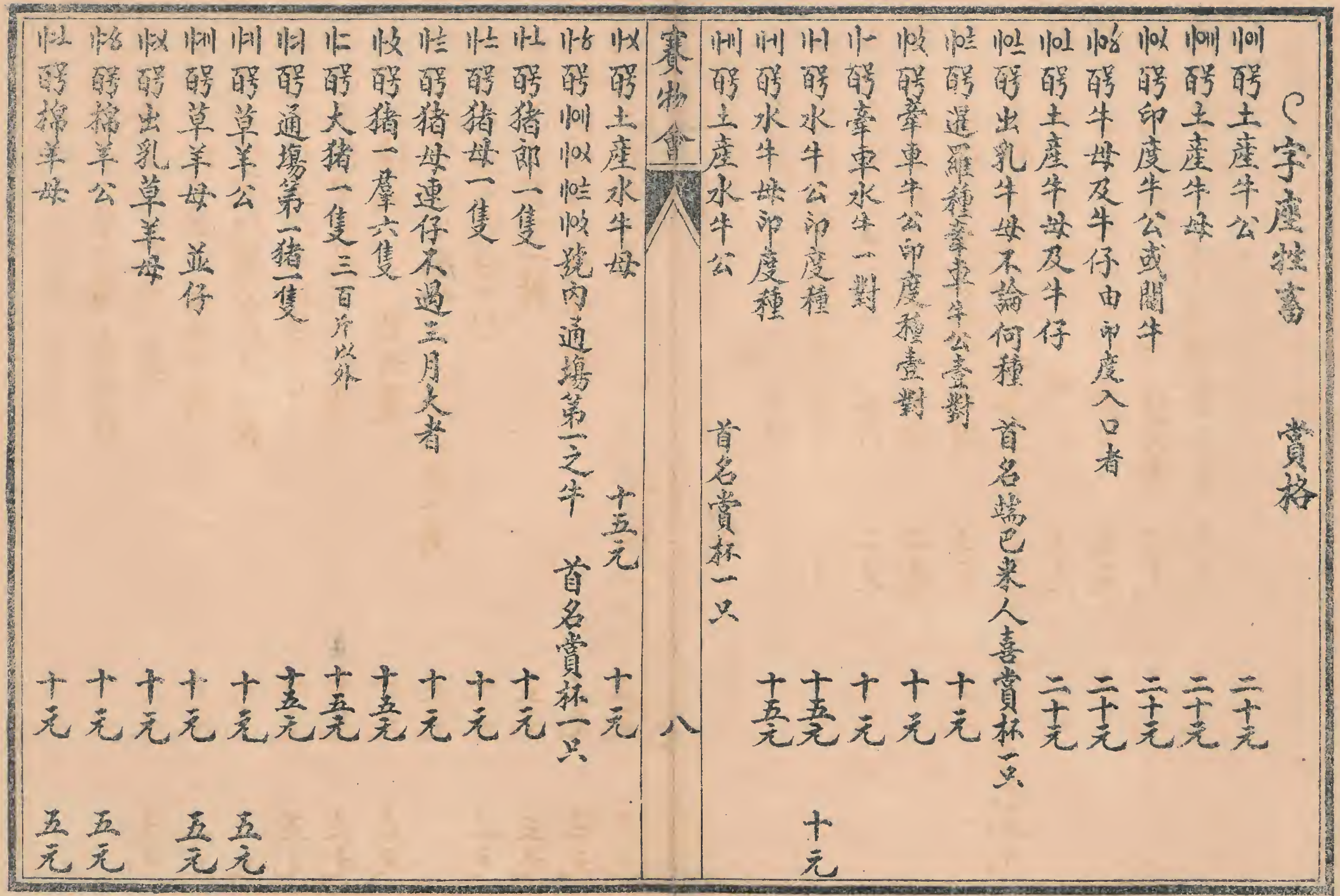





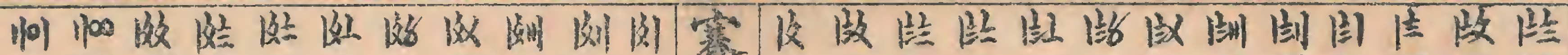

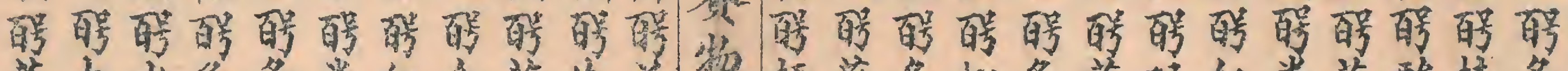

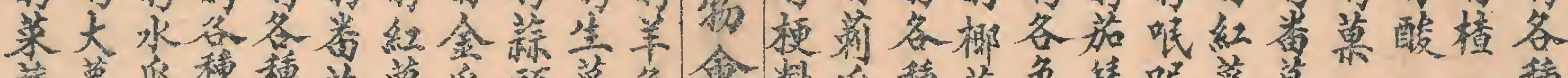

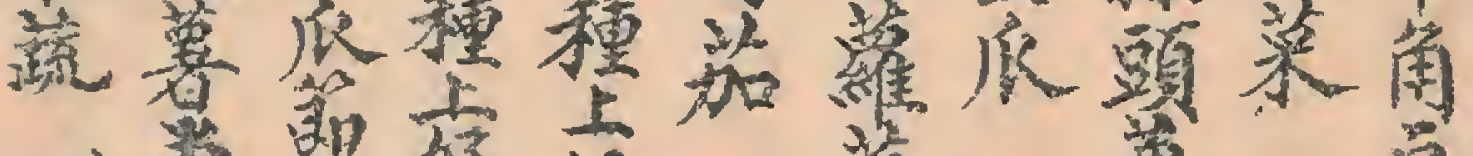

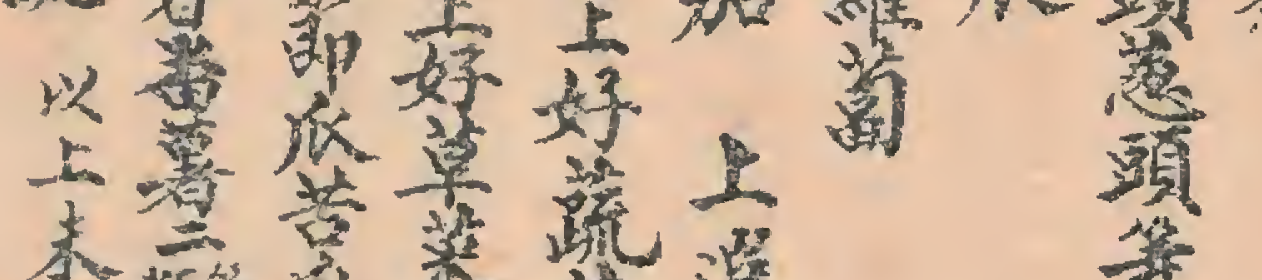

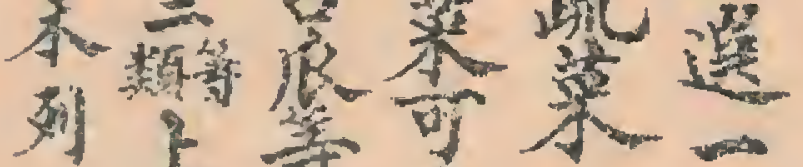

明血等等

者遙色瀁食 盤

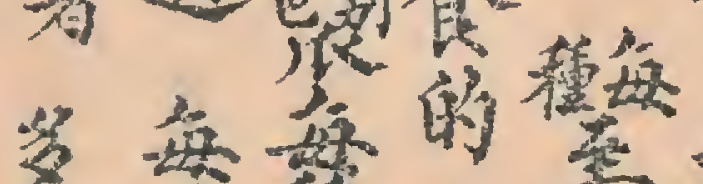

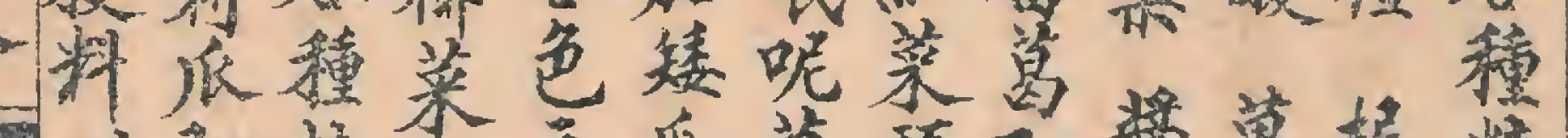

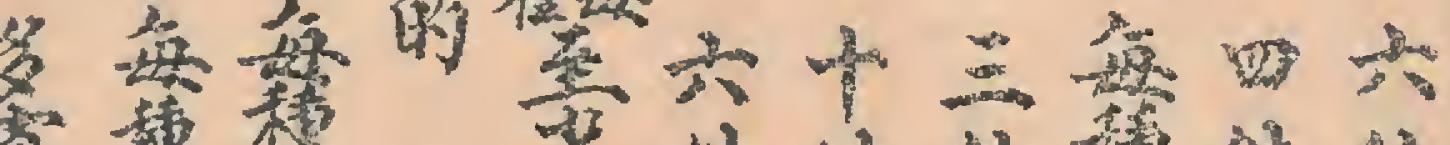

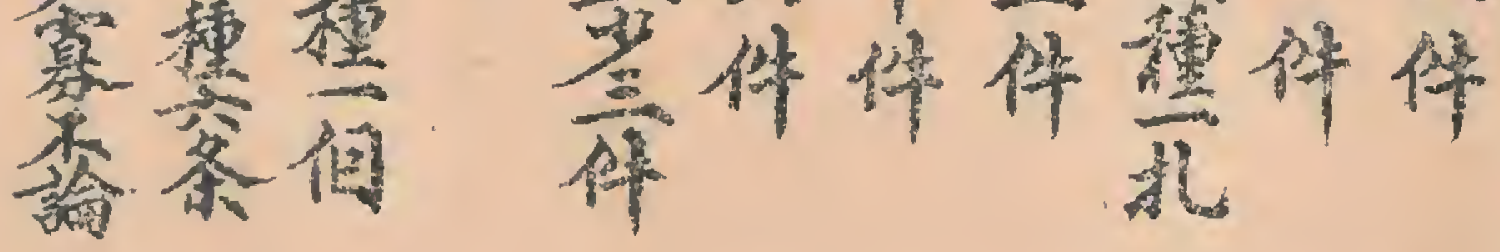

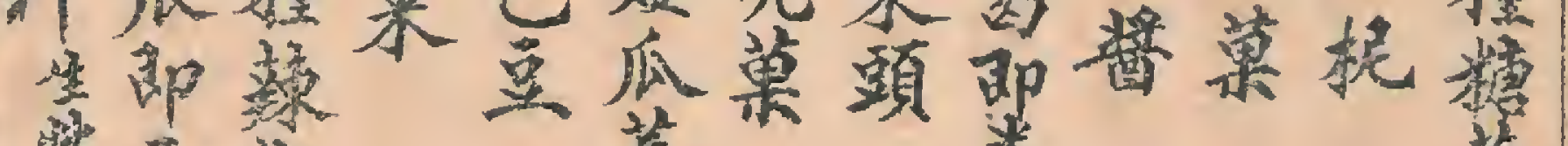

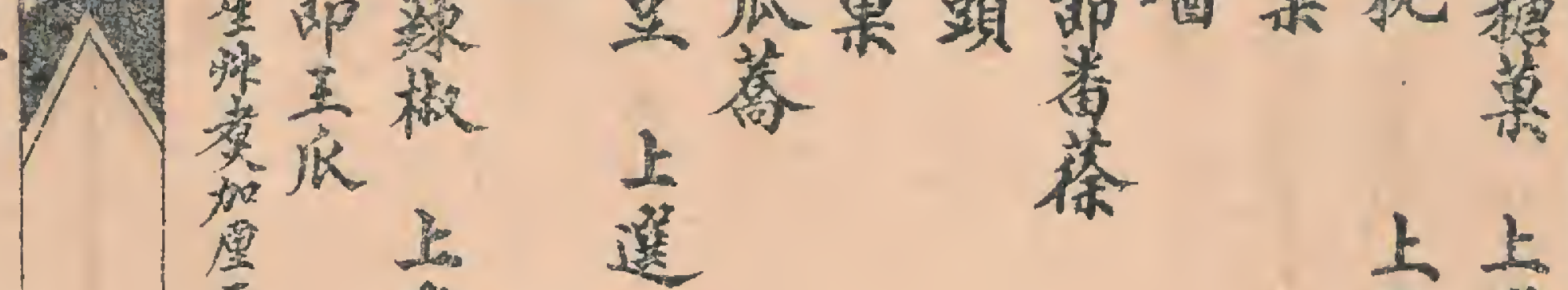
㱉

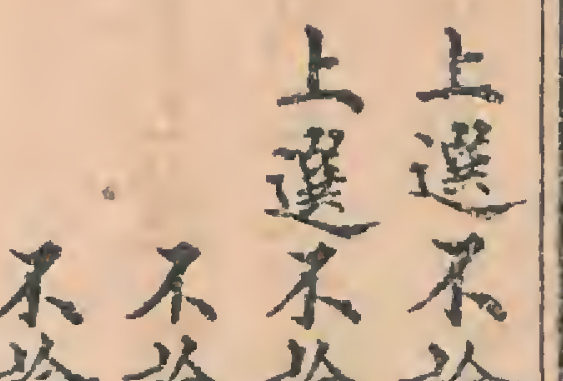

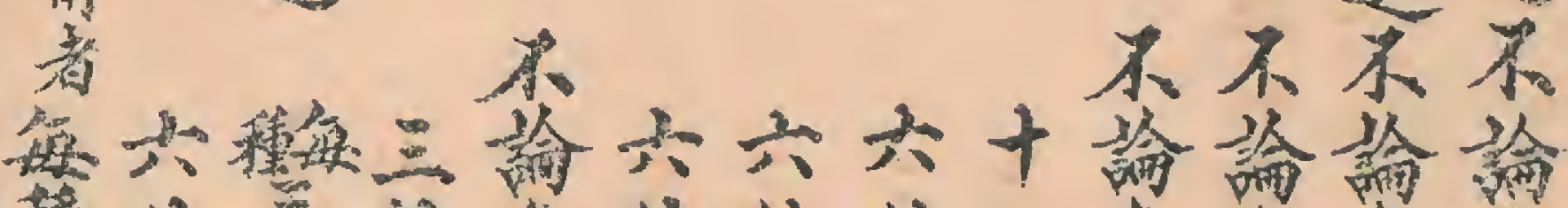

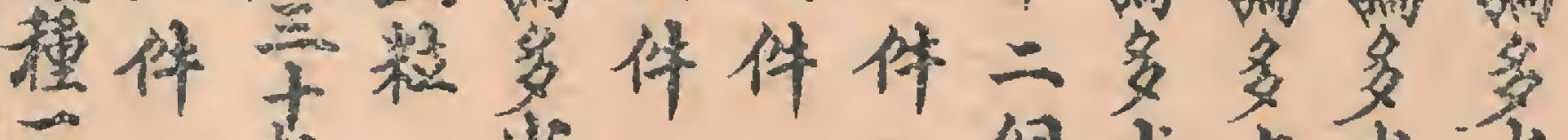

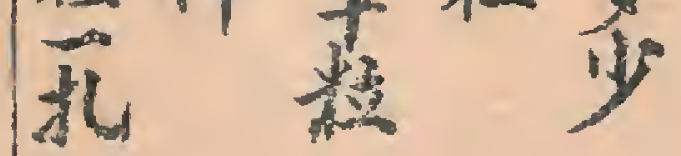
㑑少少少少

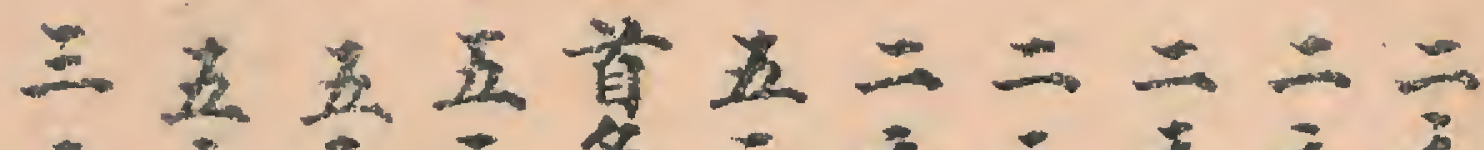
无无无藮无无无无无

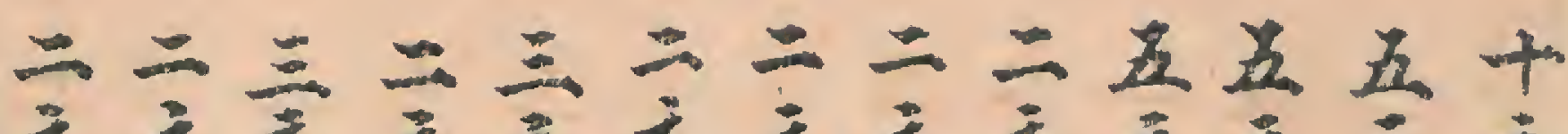

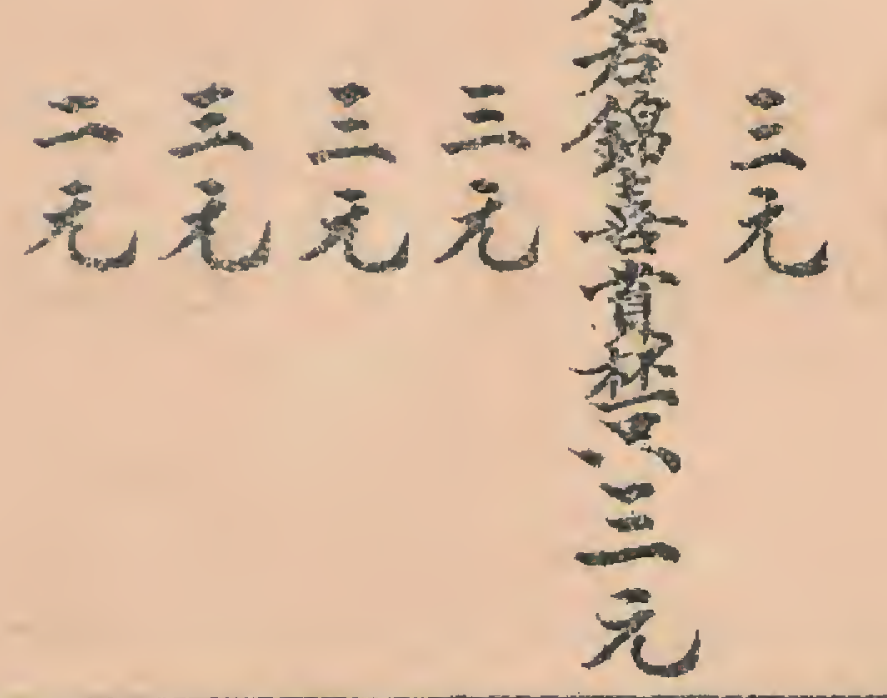

竞
宾 


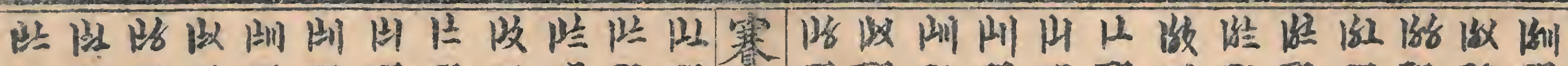

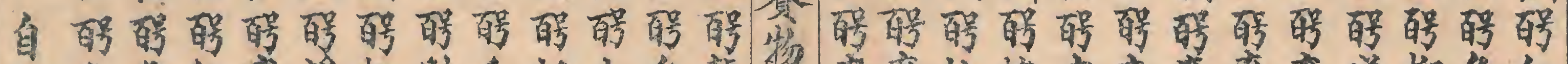

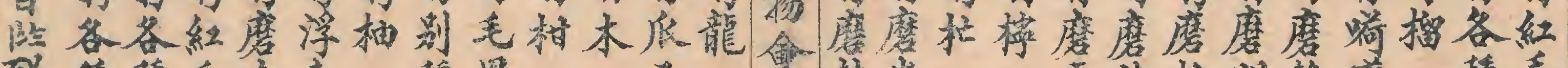

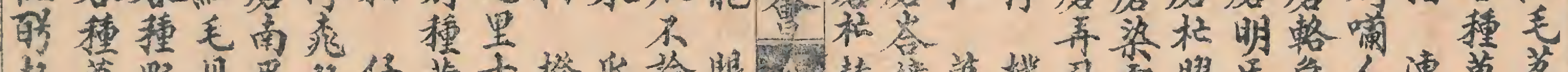

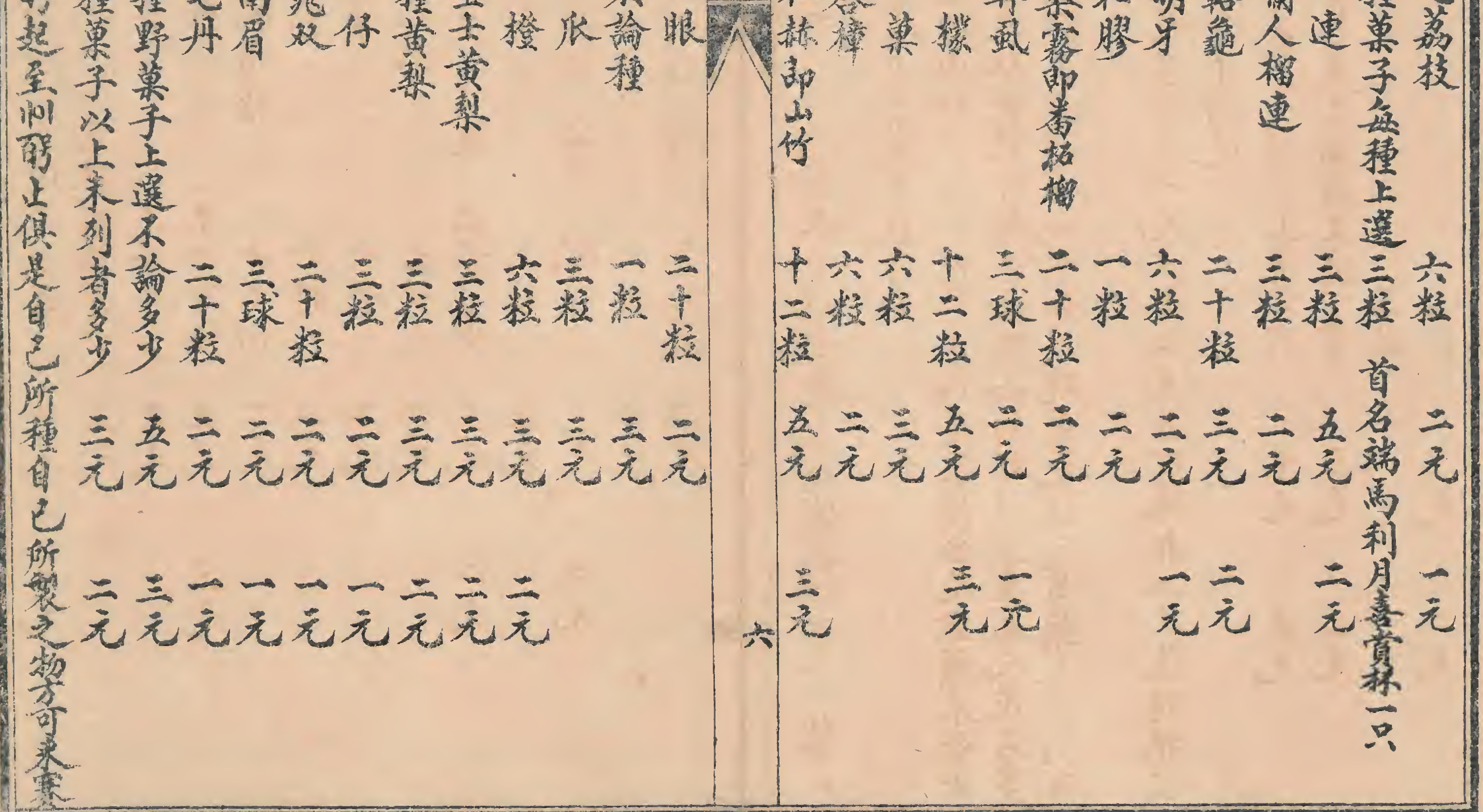




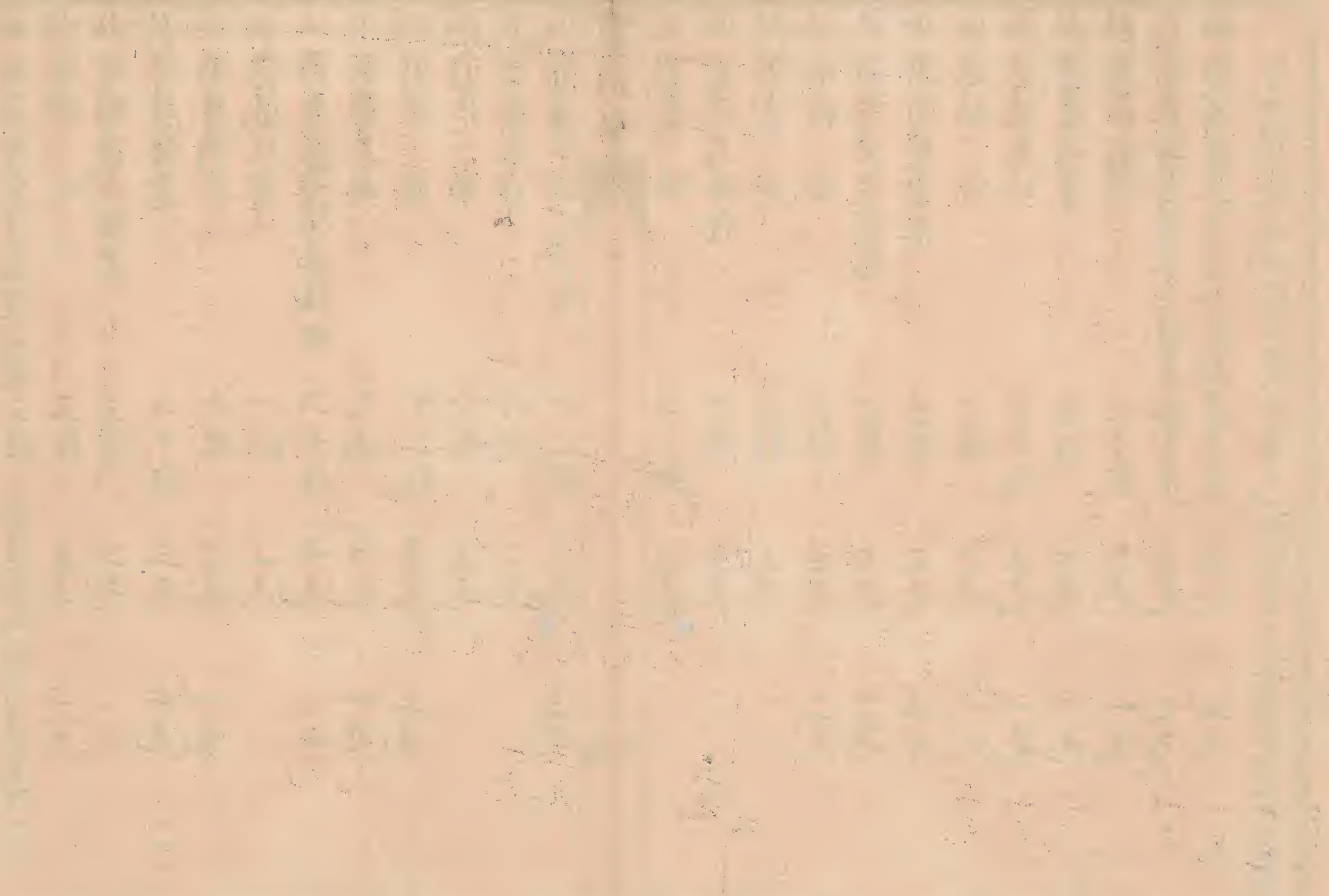

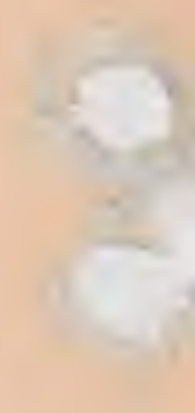




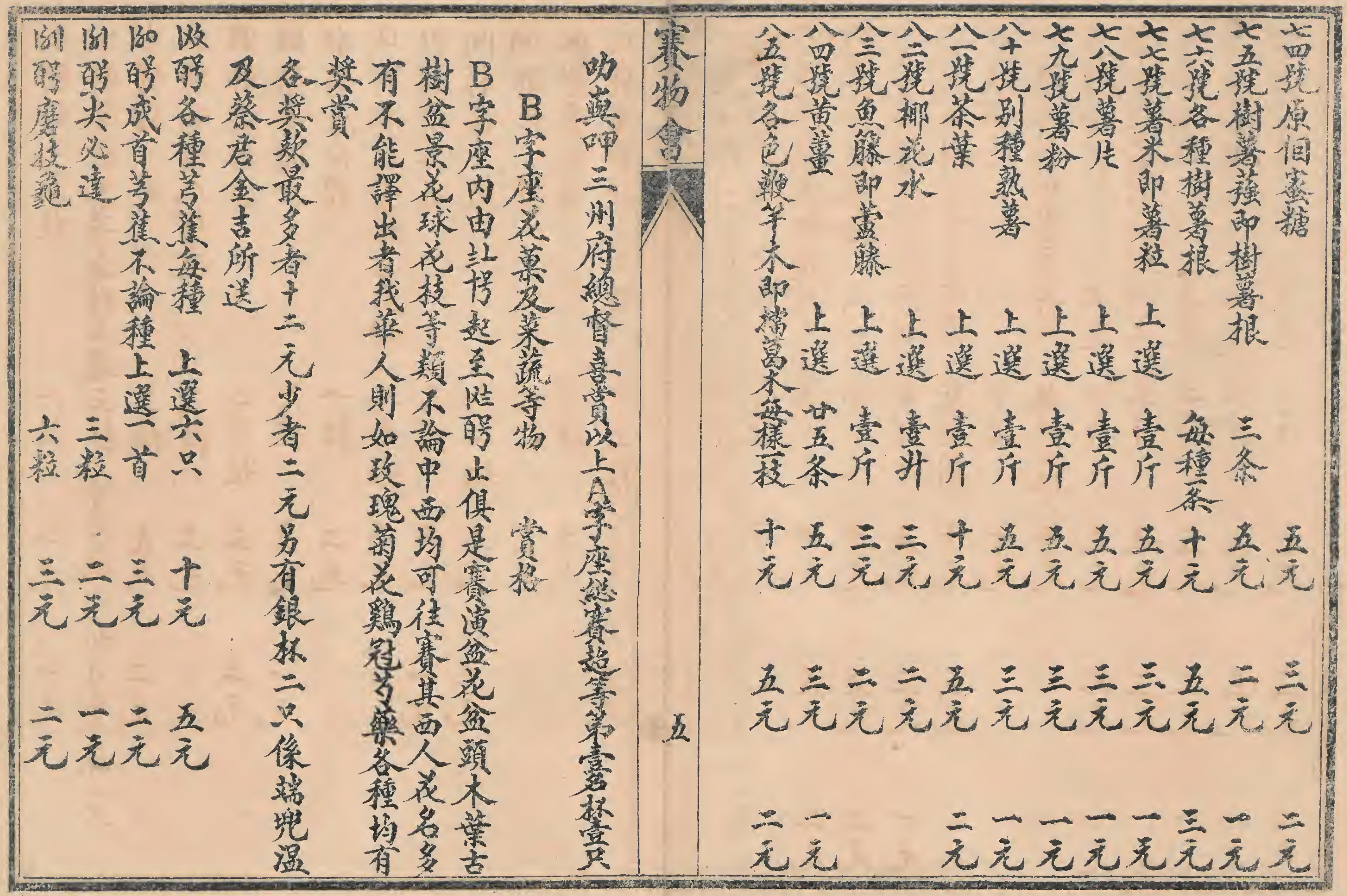


3 


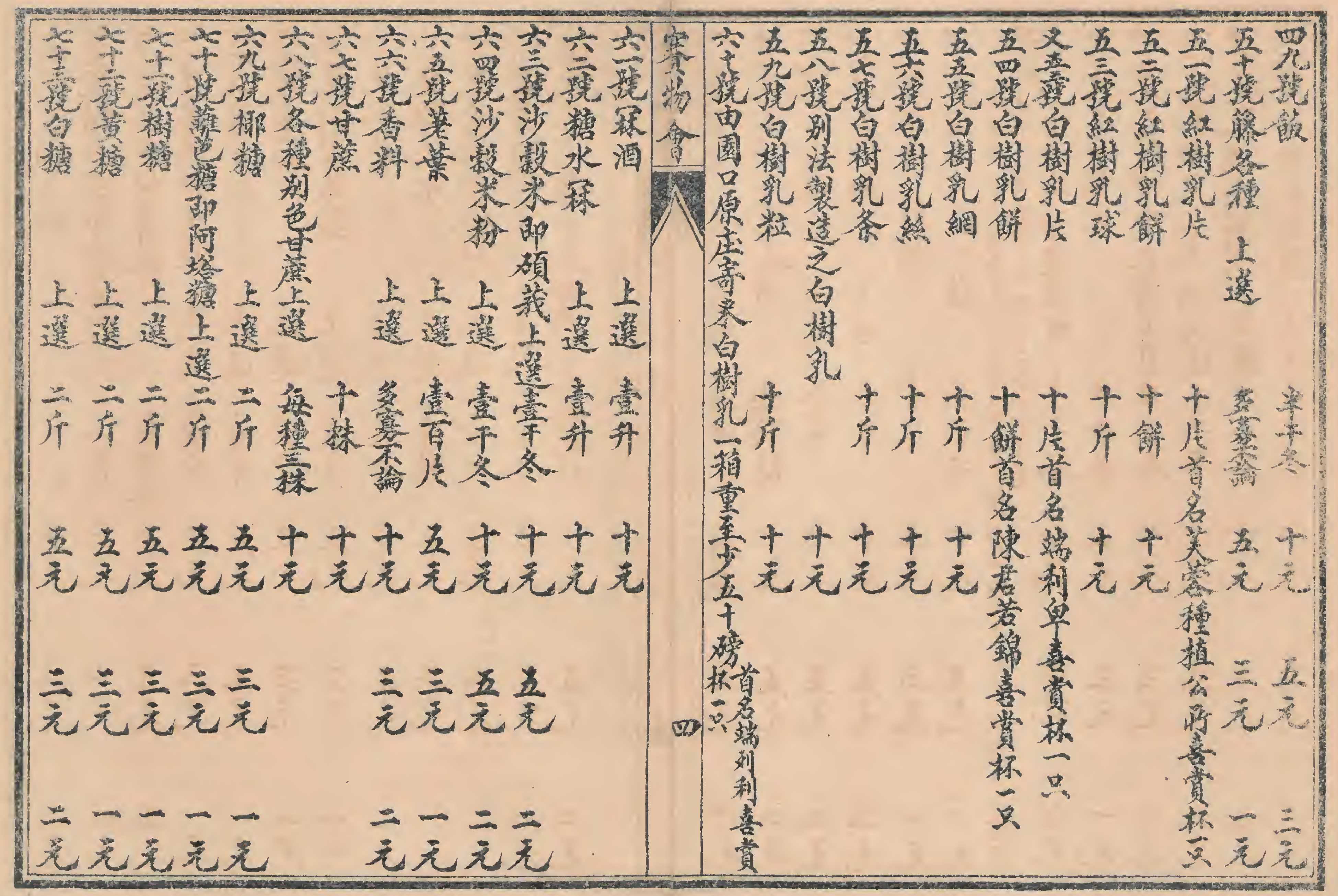




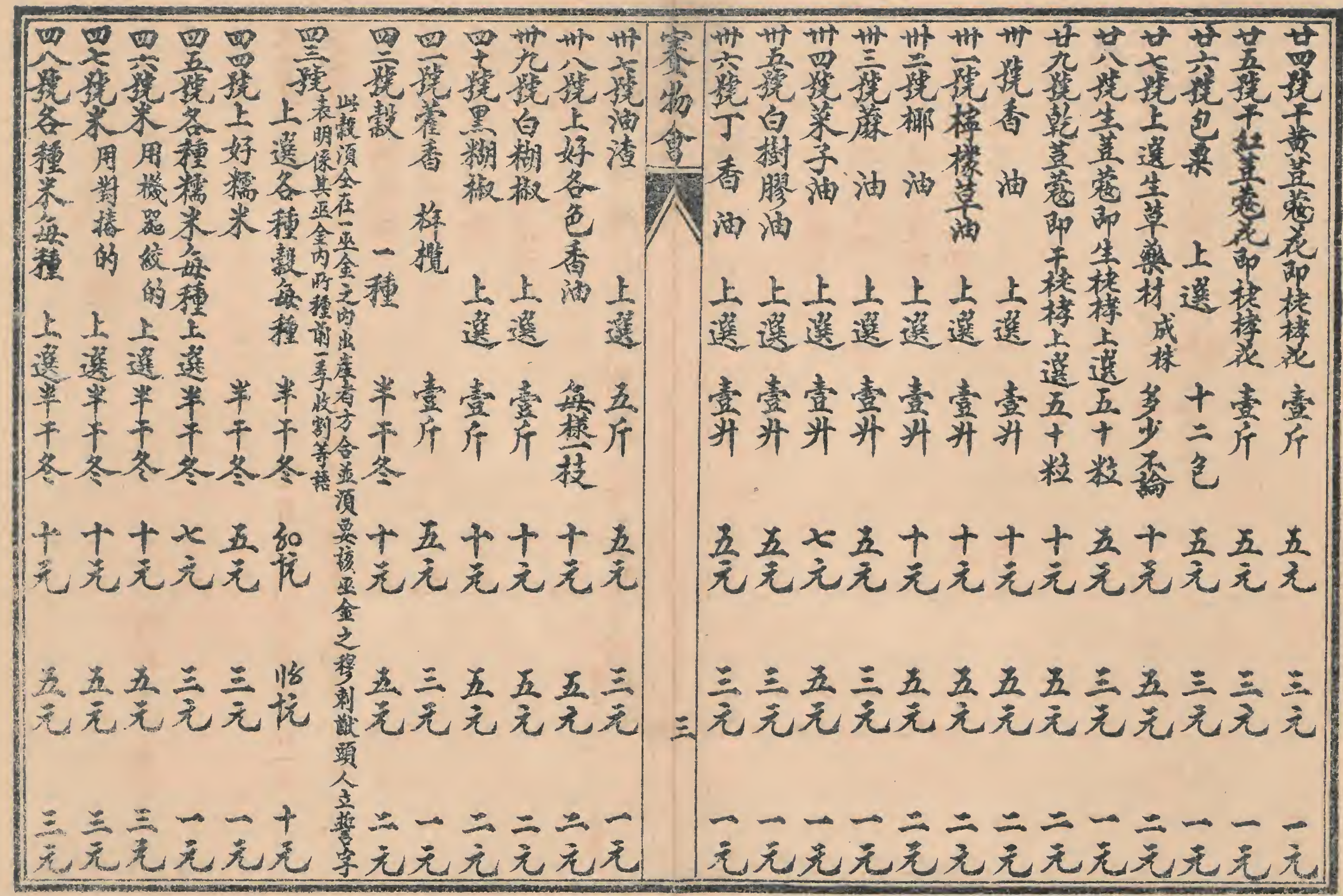


28 


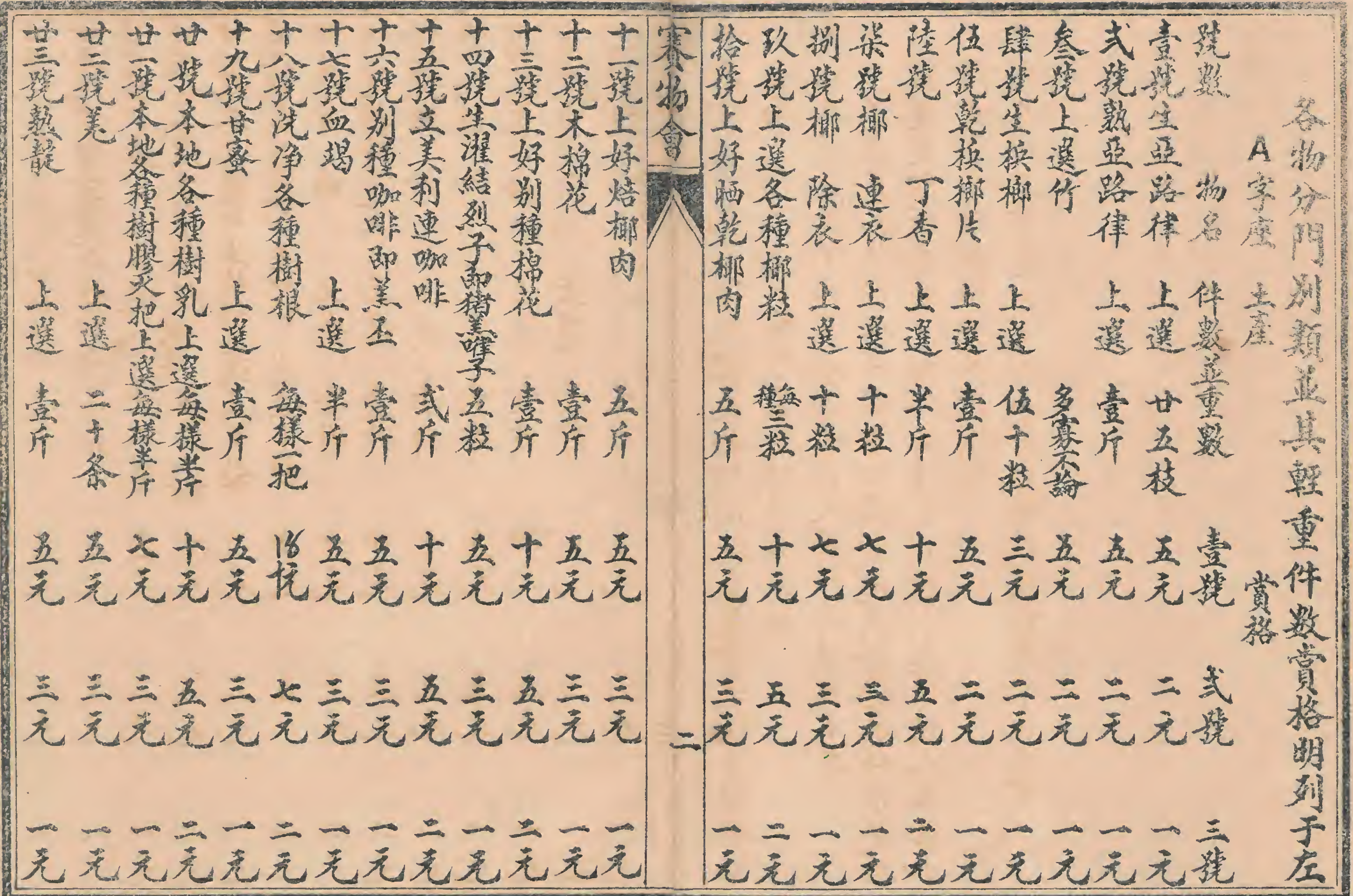




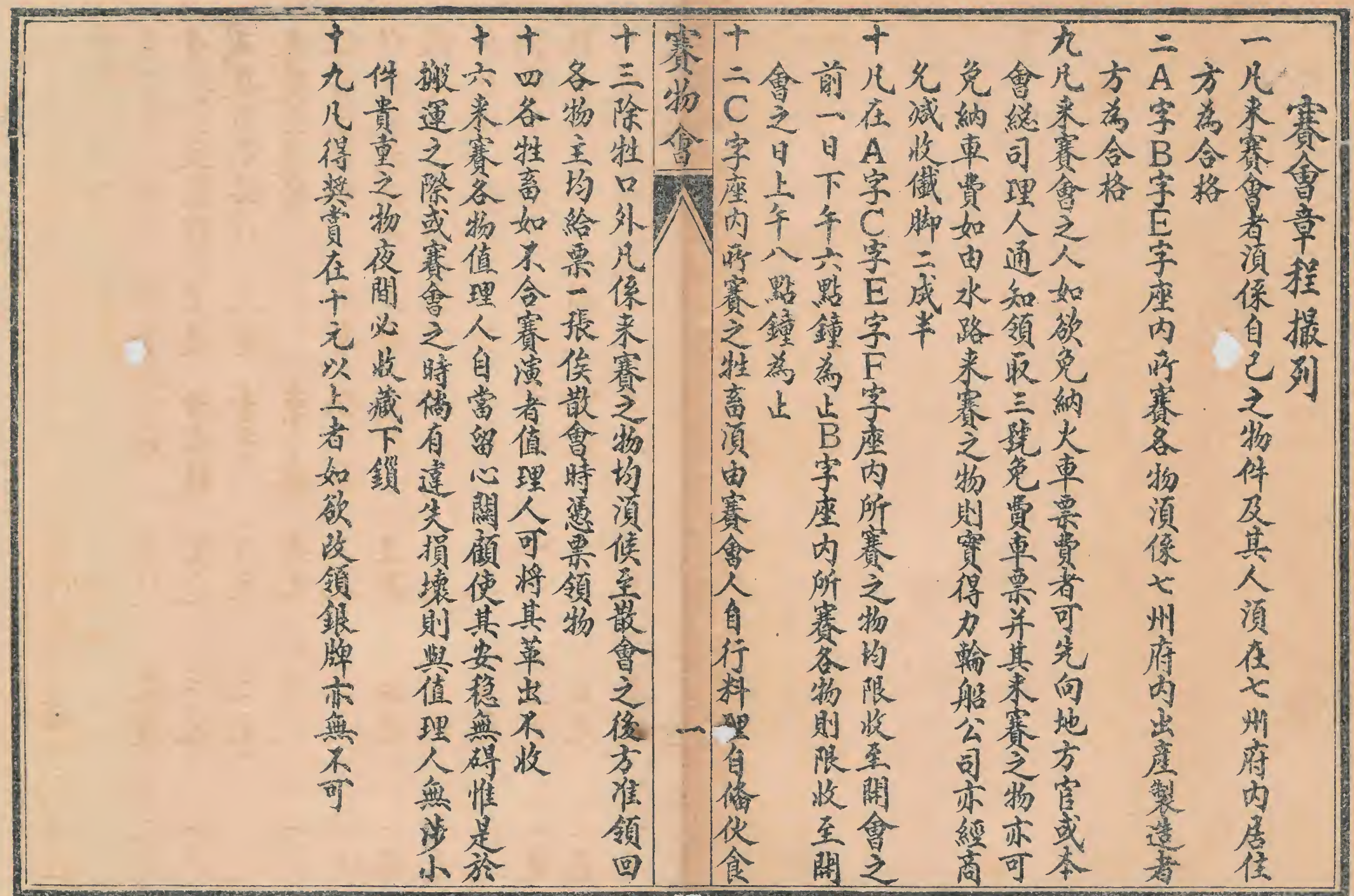




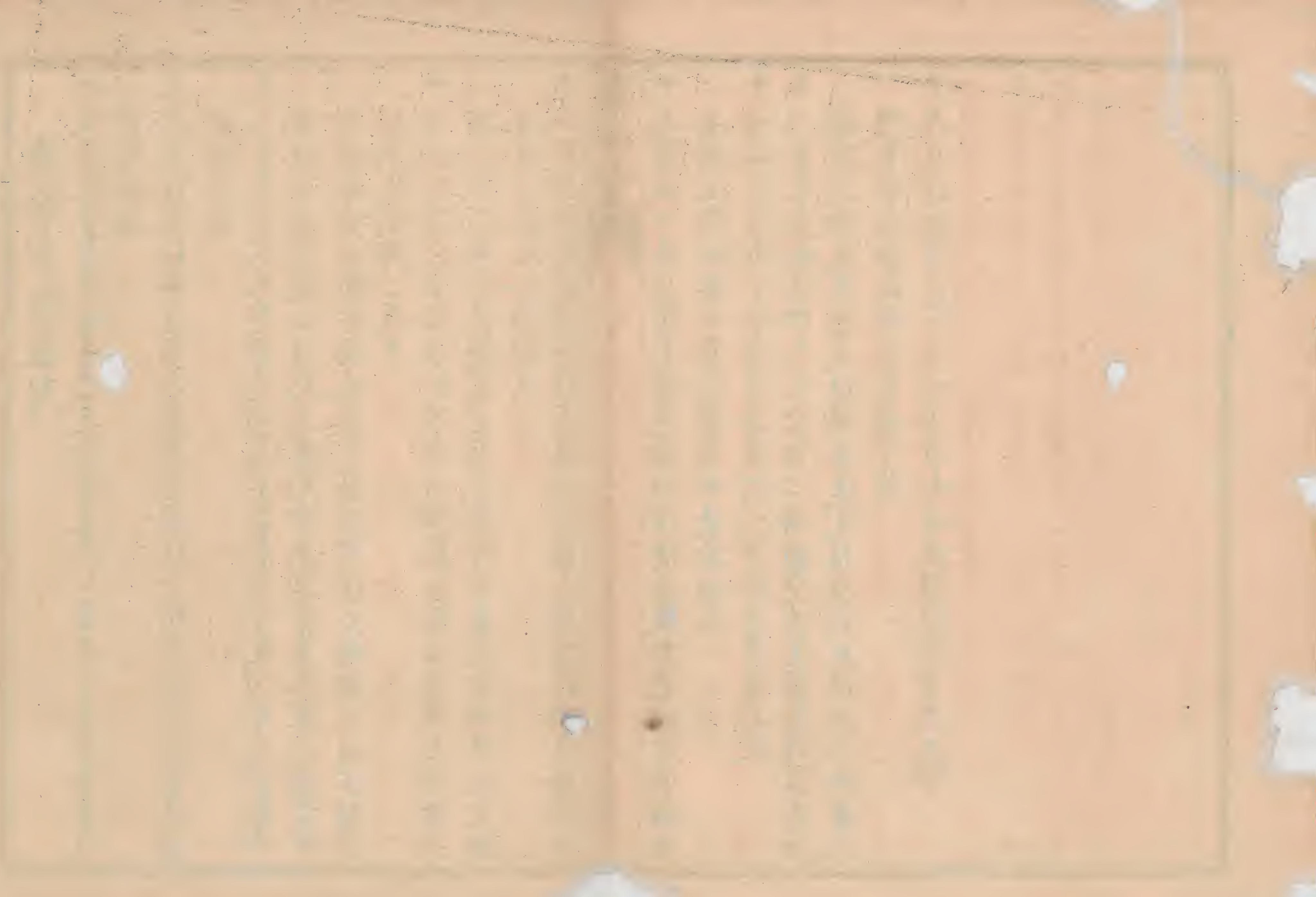




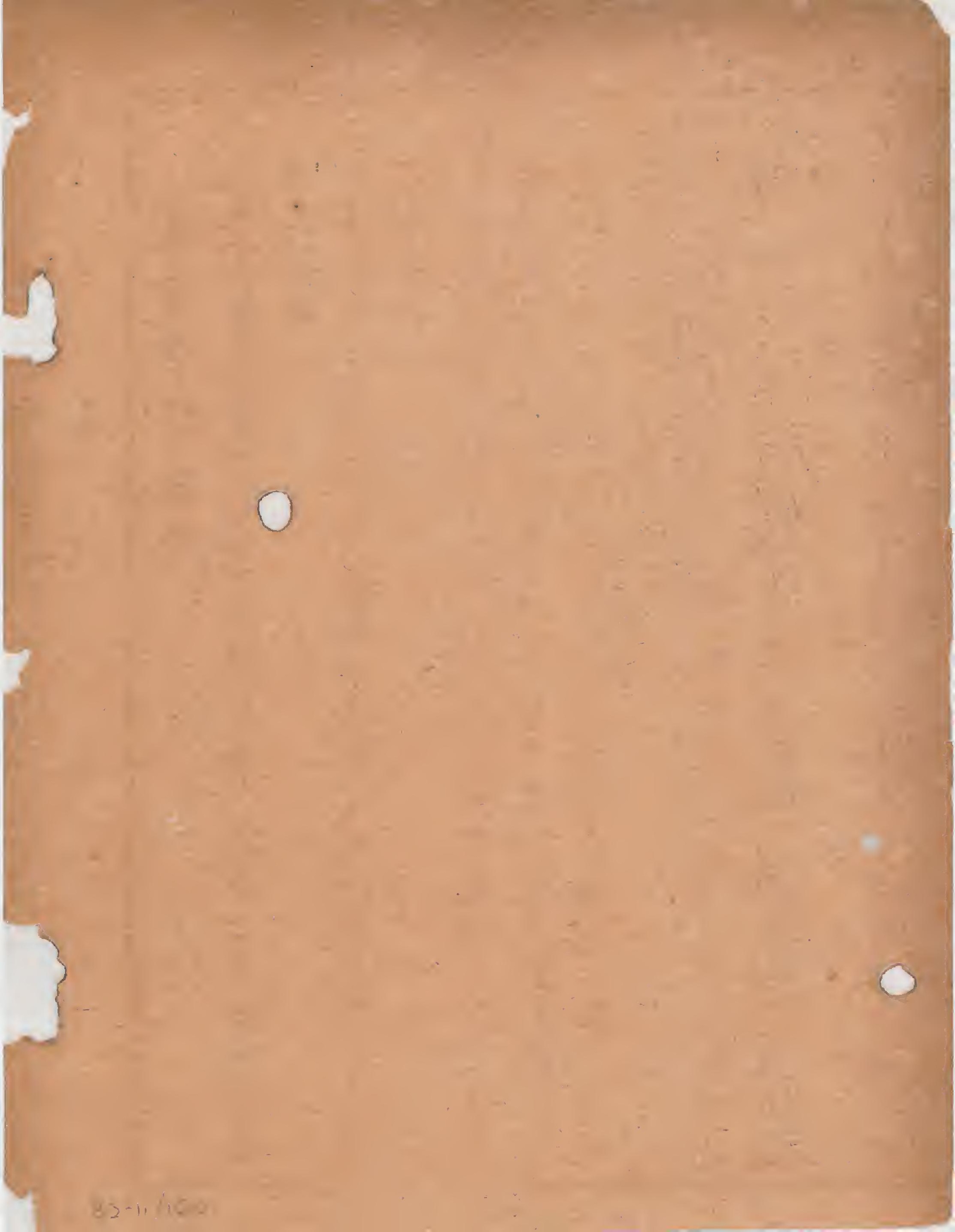




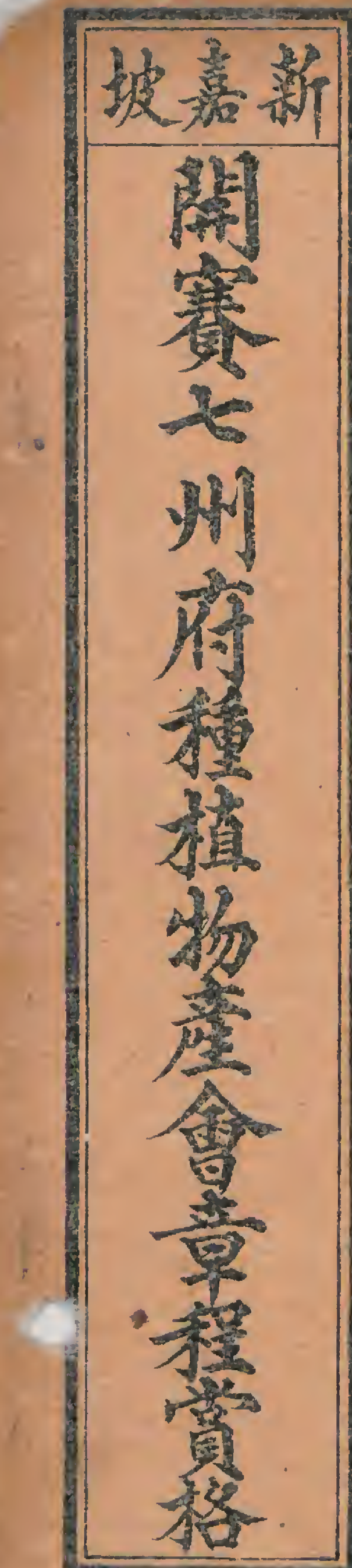




\section{வெகுய की पित}

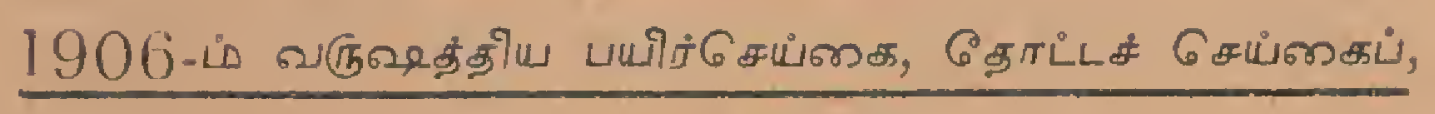

பบாருட் कாட்கी.

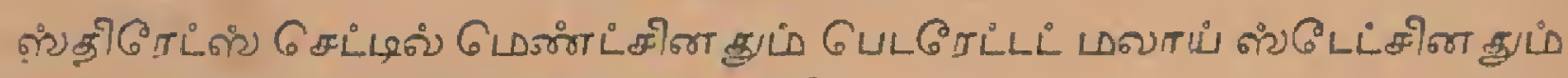

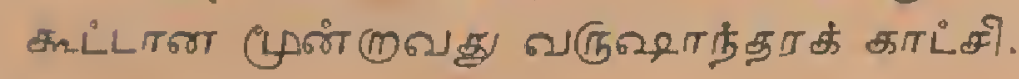

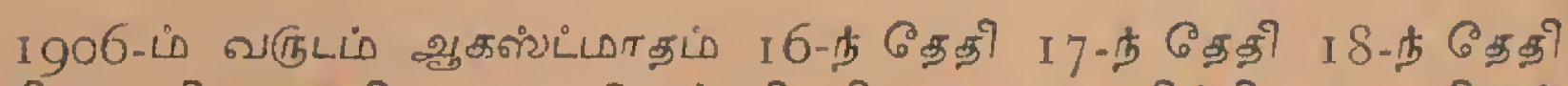

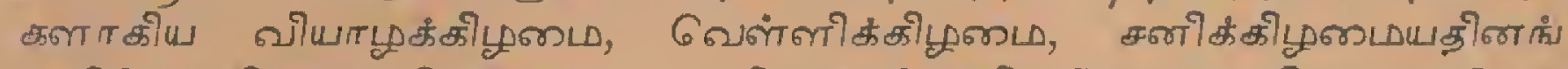

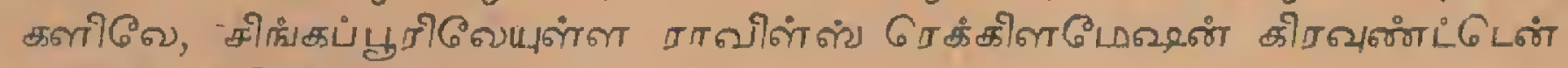
ஜுட் இடத்திலே நடத்தப்படும்.

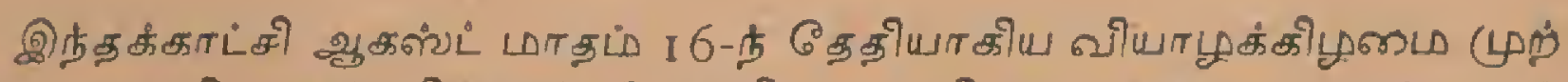

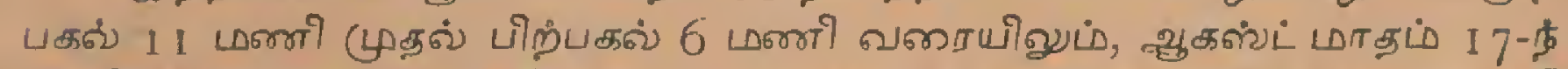

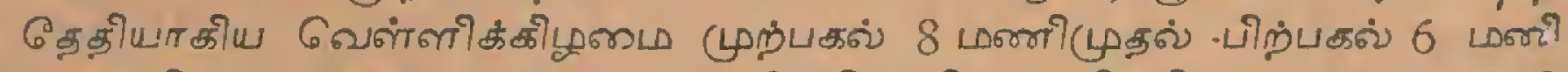

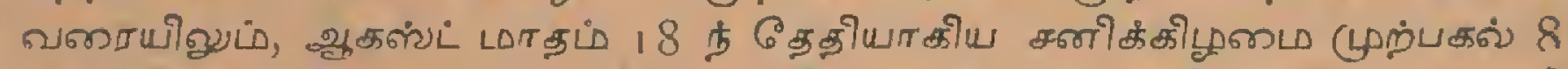

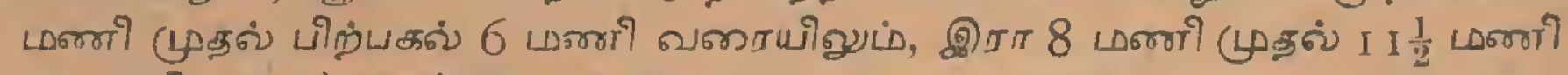

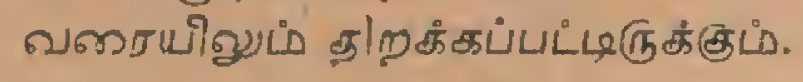

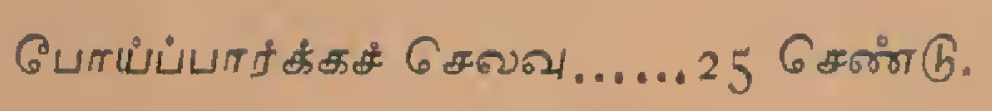

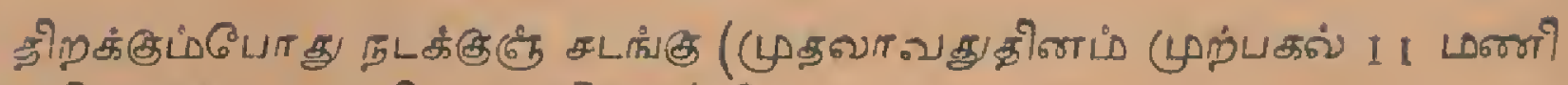

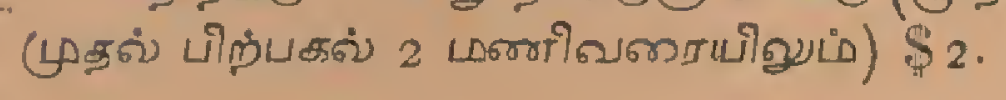

எย์. எช์. โிட்வி,

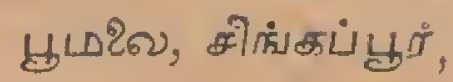

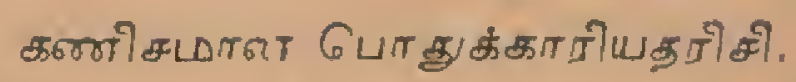




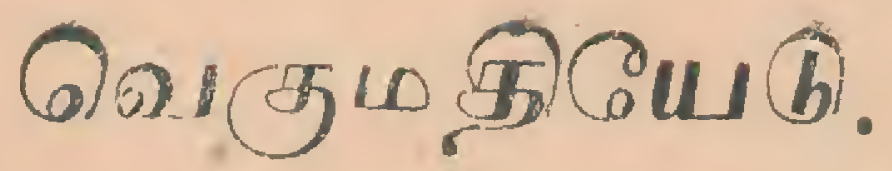

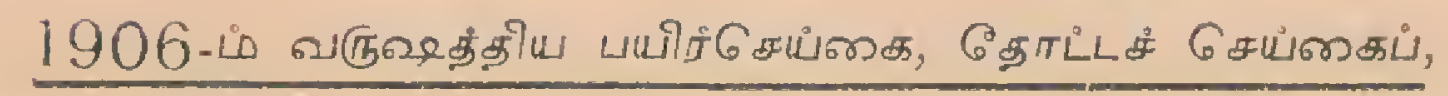

போரூட காடீசி.

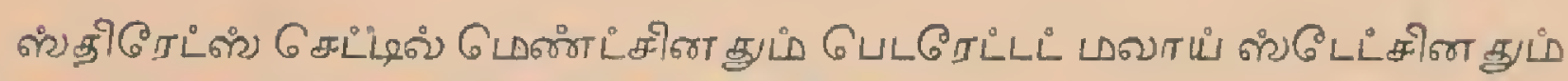

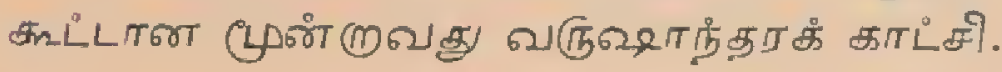

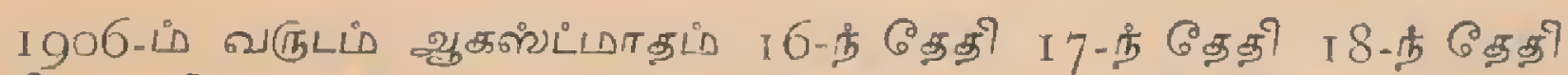

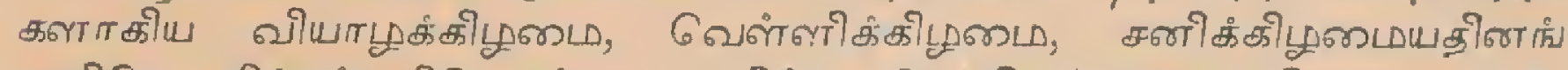

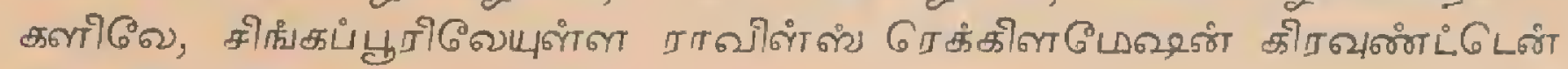
ชூம் இடத்திலே நடதீதப்படும்.

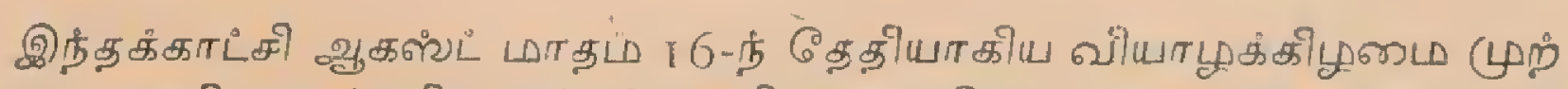

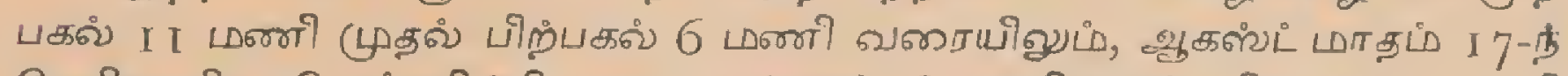

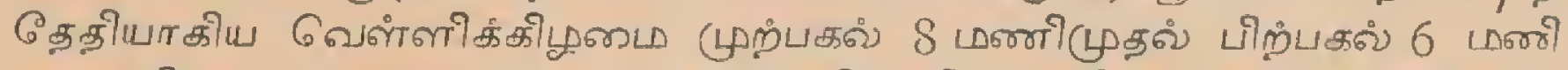

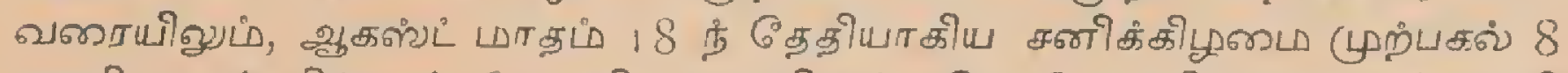

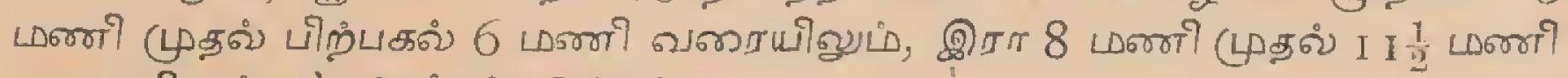

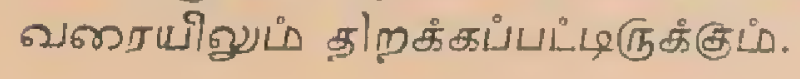

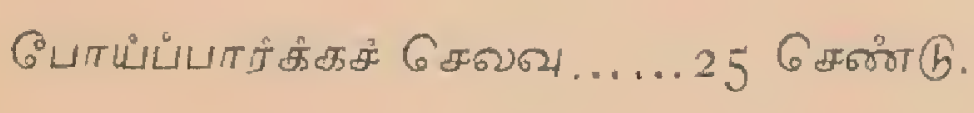

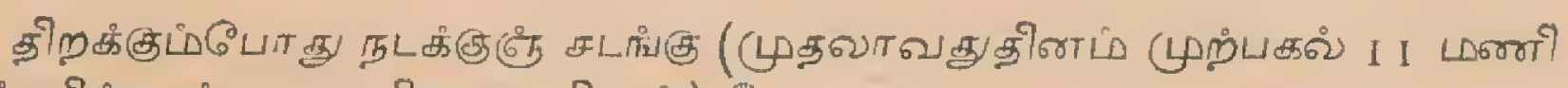
(ுுதவ் பிต்பகலீ 2 மணிவ

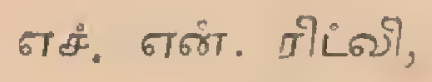 \\ பூம2ல, சிก็கப் பூரீ,

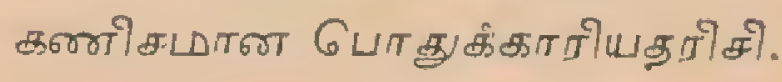



சிங்கப்பூரிலே நடடதீதப்படுங் காடீயிகீகான ஒழுங்குகள்.

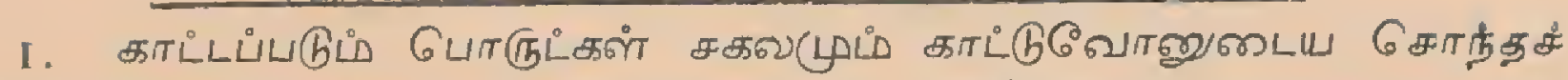

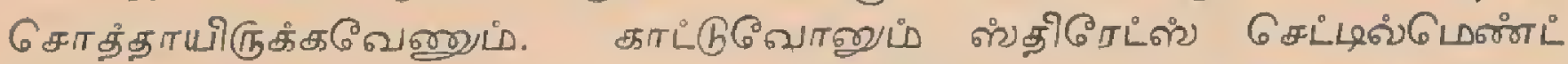

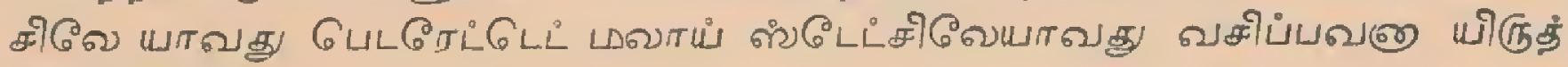
தவेவேண்டும்.

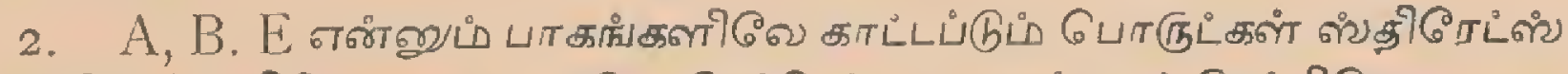

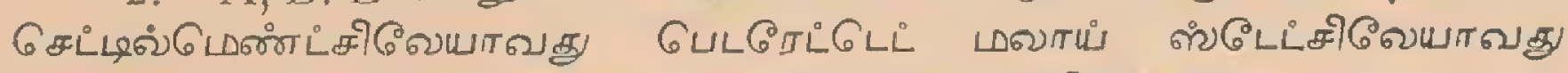

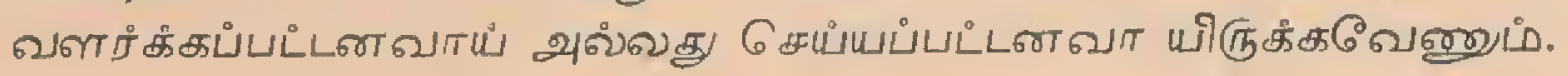

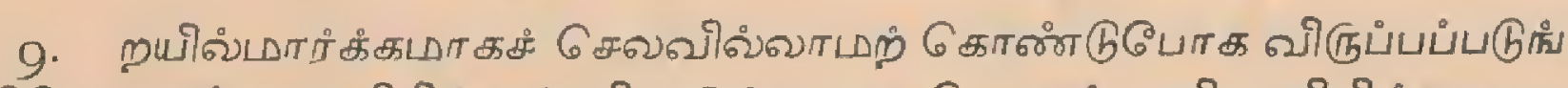

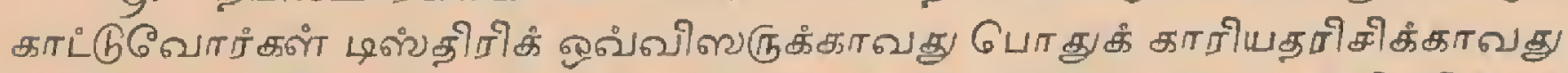

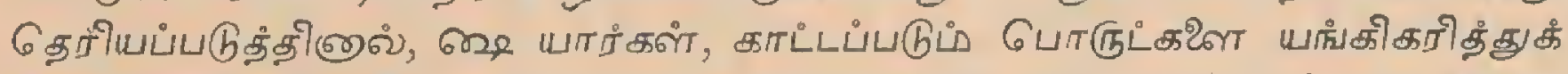

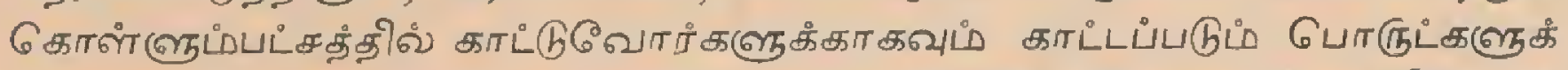

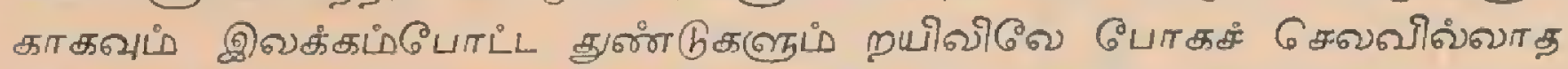

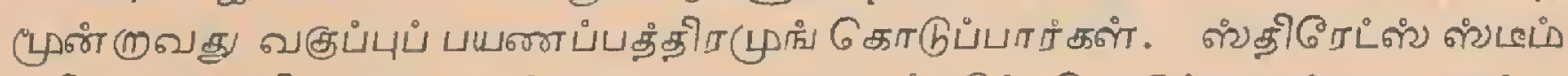

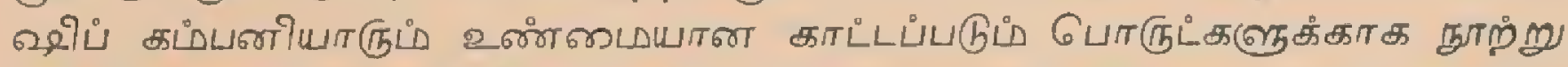

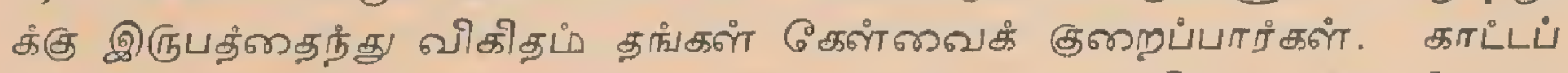

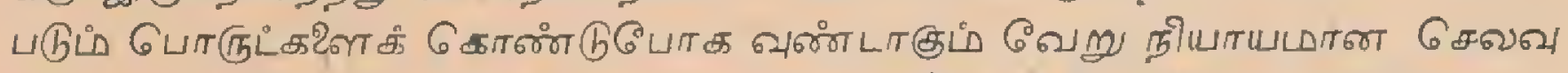

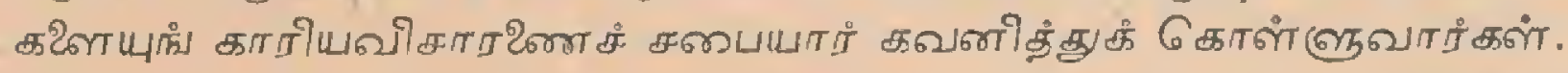

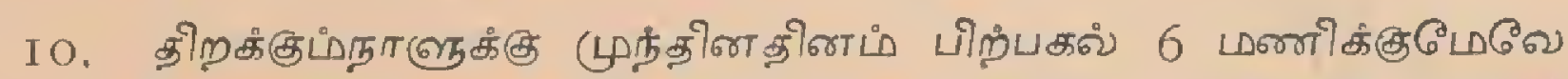

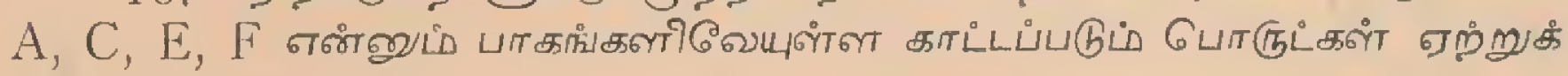
கோளำกப்பเレாடீடா.

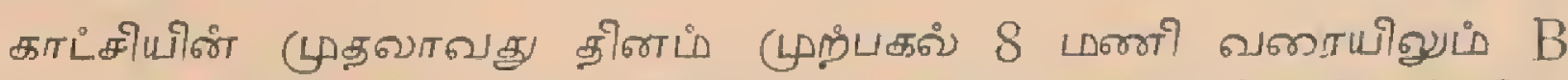

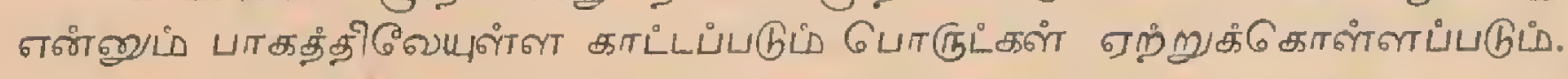

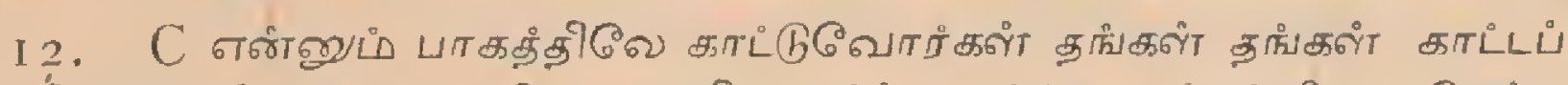

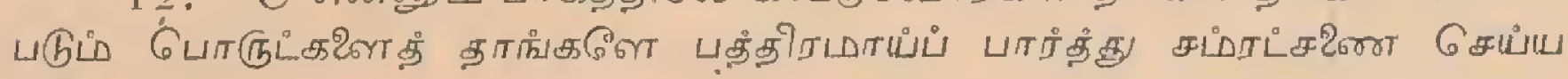
வேண்டும்.

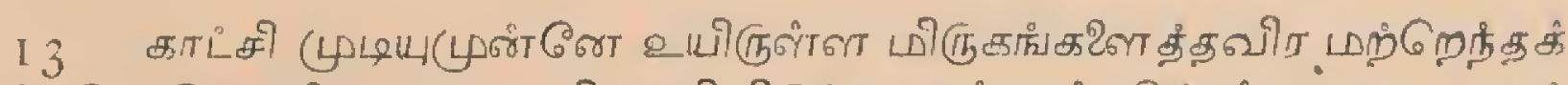

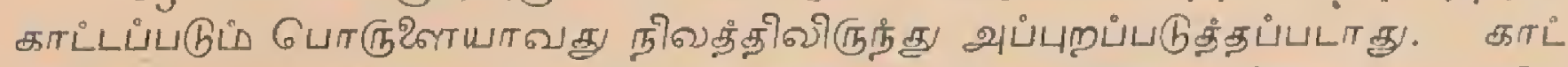

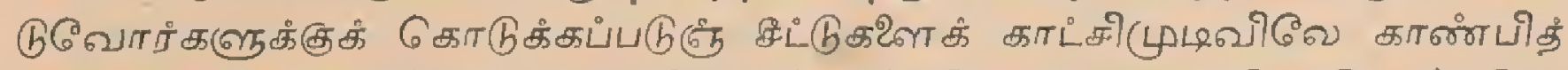
தாற்தான் காடீடப்படுட் டொருட்க2ள யப்புறப்படுத்த உத்தரவு 6 காடுக்கபபடுட்.

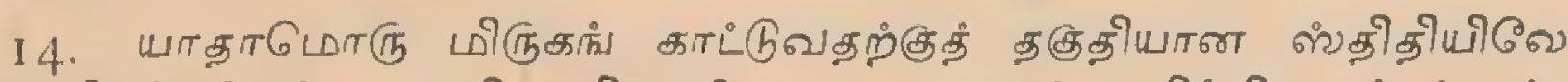

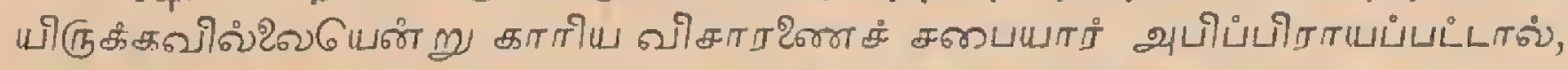

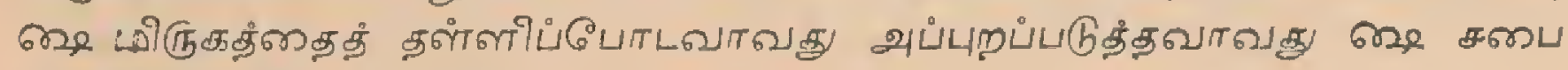
யாருக்கு श्थதிकார முண்டு.

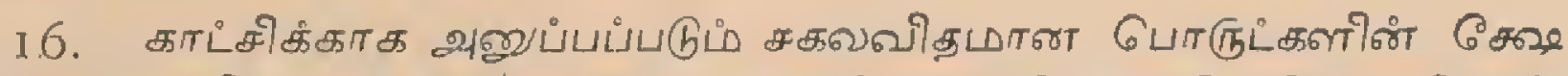

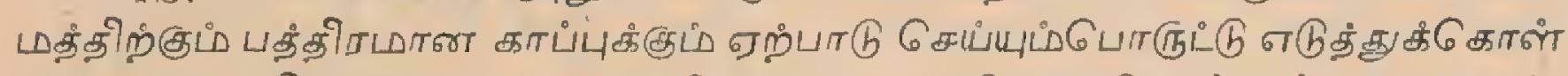

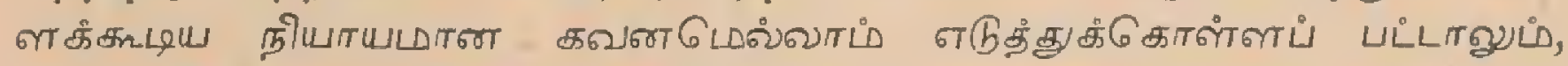

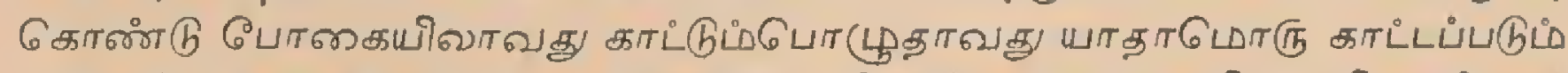

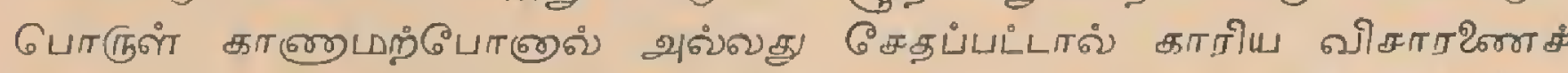

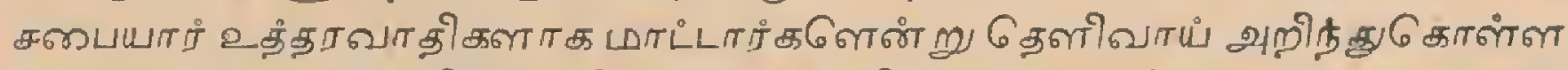

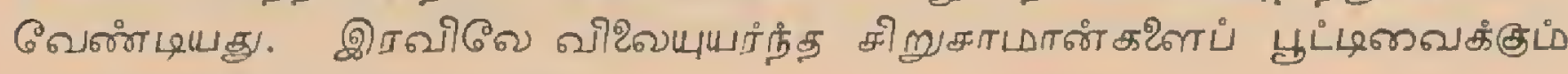
படி யேற்பாடு 6 チயபப்படும்.

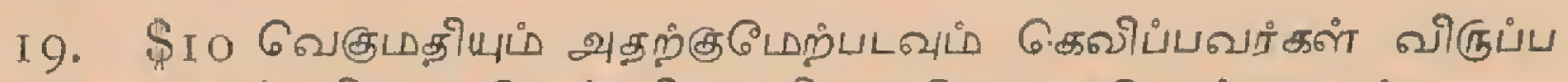

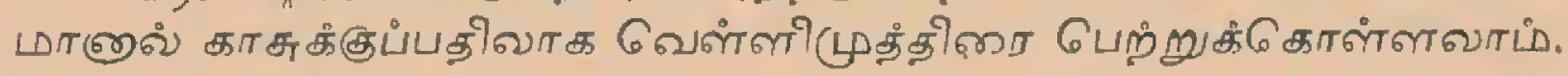


A. பाாकL्ठ.

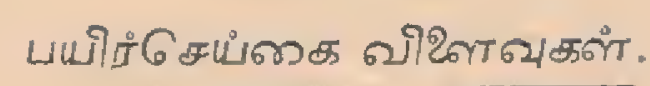

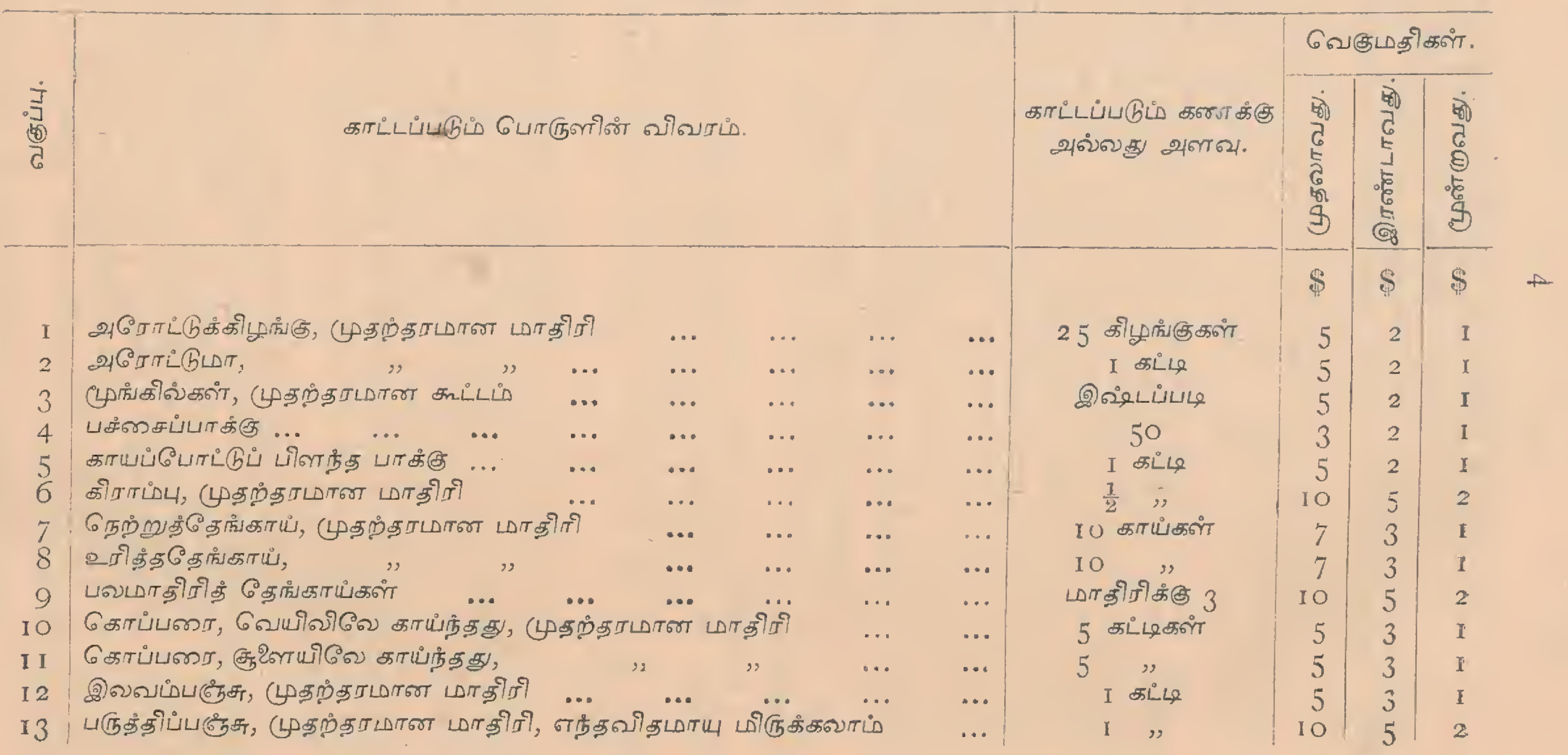


சீரீம கோபீபிக்காய் (

கோப்பி, బவபீரியช์

கோப்பி, வே6றநீதவிதமும்

...

...

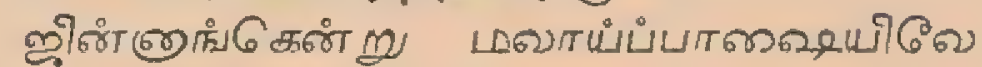
பीภीช

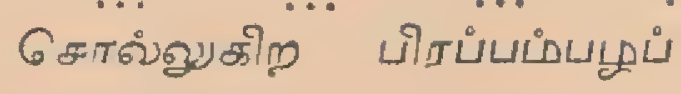
Ђா ஸำำ $\quad \begin{array}{llllll}\ldots & \ldots & \ldots & \ldots & \ldots & \ldots\end{array}$ कमำ कींा

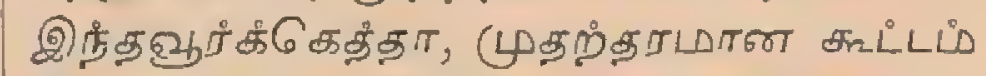

$\cdots \quad \ldots \quad \ldots$

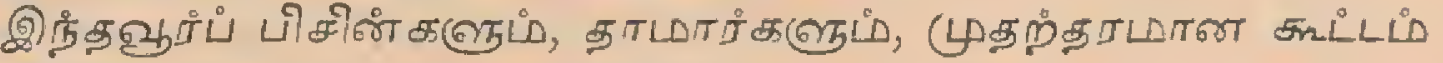

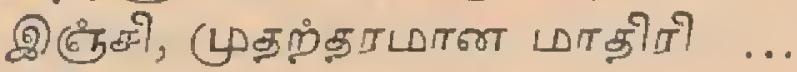
பூரீவเ์,

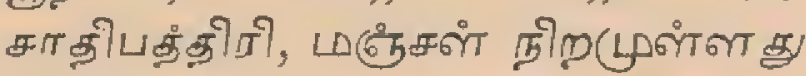

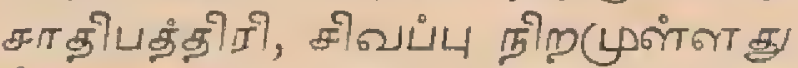

छோளட்

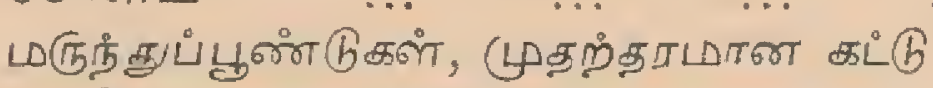

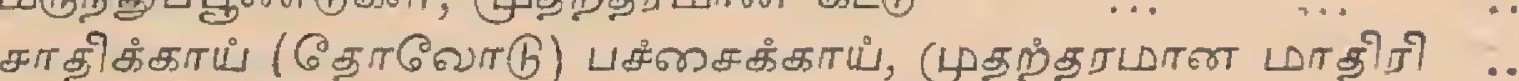

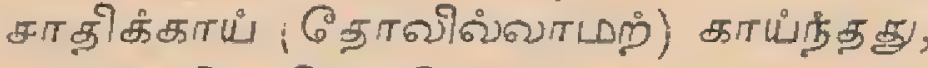

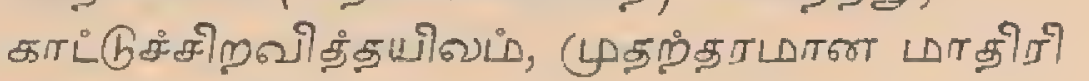
சीрவीத்தயிலเ்,

Gேங்காடயண் Gோ,

Бவீ6ேண் 6ேய,

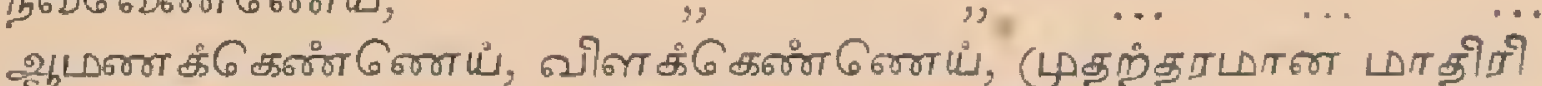

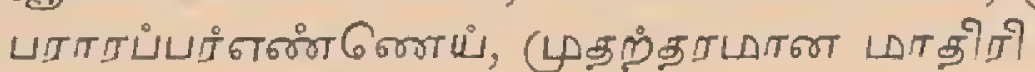
कीராட்புதீதயிலเి,

பிண் ணூ க்கு,

முவிககதீதயிலங்கள், முதற்தாமான कூட்டi

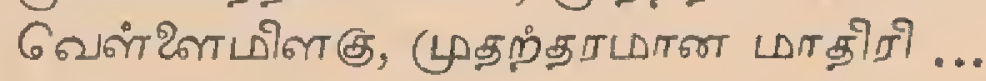

5 காய்கள்

2 கட்டூகள்

I கட்டி

மாதிரிக்கு I கட்டு I कLீடி

மா திரीகீகு $\frac{I}{2}$ கட்டி

20 கிழங்குகள்

I கட்டி

I ",

I "

12 கதிฐீகள்

இぷீடப்படி

50

50

$\frac{1}{2}$ ढோ

நிக்கு $\frac{1}{4}$ போ தீதவ்

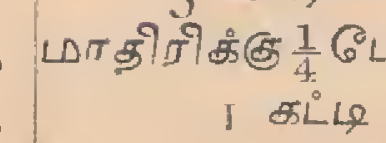

\begin{tabular}{|r|r|r}
5 & 3 & 1 \\
10 & 5 & 2 \\
5 & 3 & 1 \\
& & \\
5 & 3 & 1 \\
15 & 7 & 2 \\
5 & 3 & 1 \\
10 & 5 & 2 \\
7 & 3 & 1 \\
5 & 3 & 1 \\
5 & 3 & 1 \\
5 & 3 & 1 \\
5 & 3 & 1 \\
5 & 3 & 1 \\
10 & 5 & 2 \\
5 & 3 & 1 \\
10 & 5 & 2 \\
10 & 5 & 2 \\
10 & 5 & 2 \\
10 & 5 & 2 \\
5 & 3 & 1 \\
7 & 5 & 1 \\
5 & 3 & 1 \\
5 & 3 & 1 \\
5 & 3 & 1 \\
10 & 5 & 2 \\
10 & 5 & 2 \\
& & \\
\hline & &
\end{tabular}




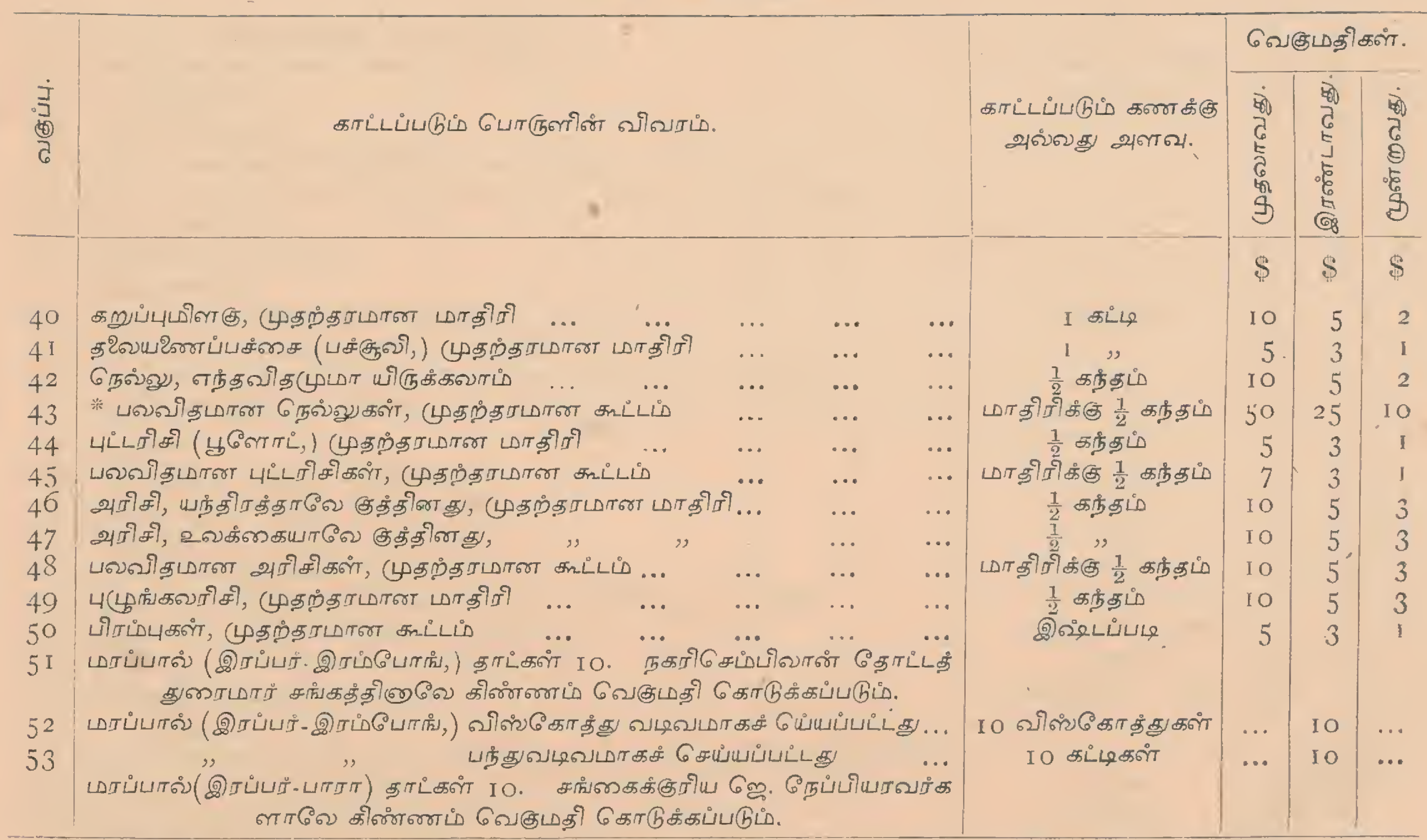

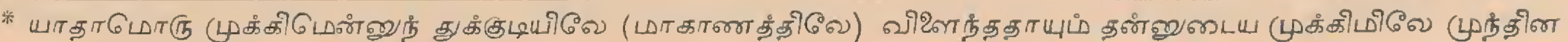

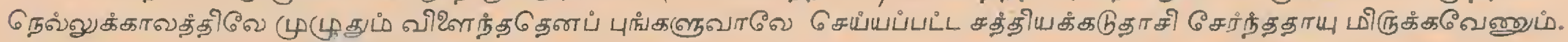




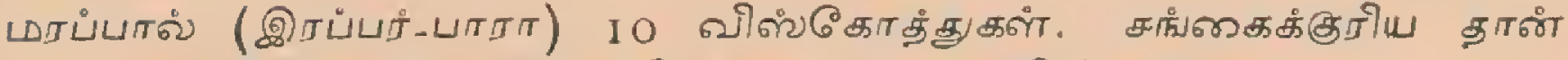

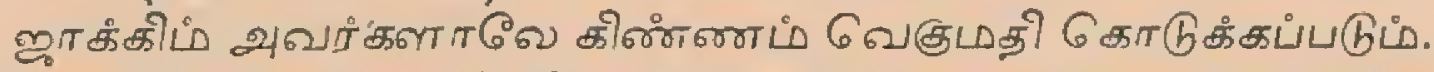

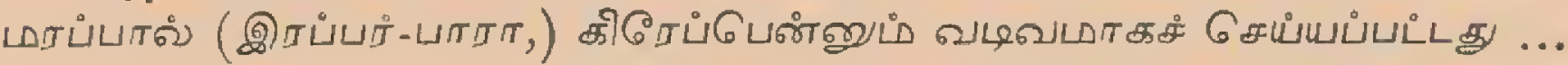

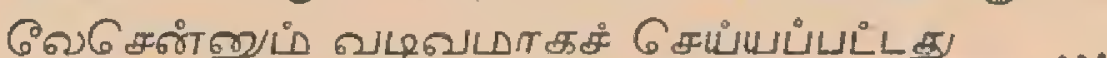

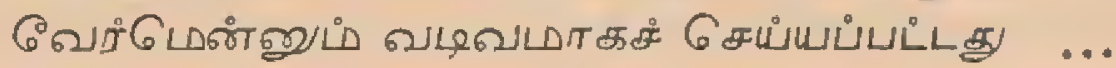

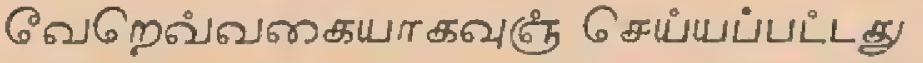
சுரண்டிடயடுதீத தண்டு

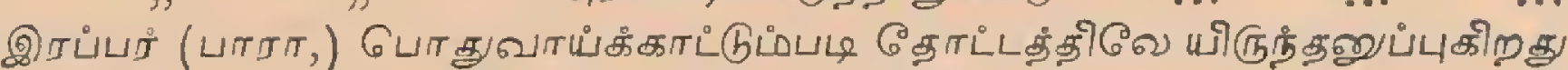

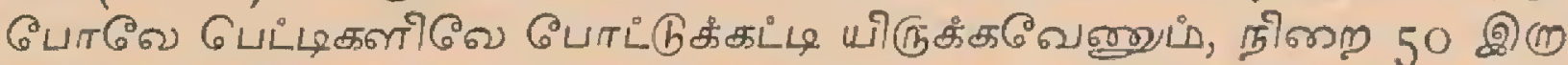

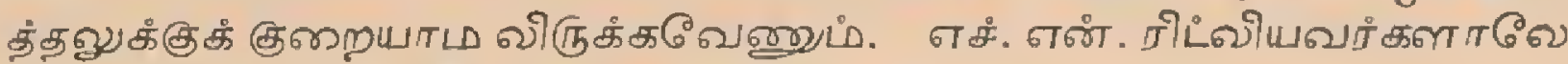
கிண்ணம் 6ேகுபதி 6 कாடுக்கப்படும்

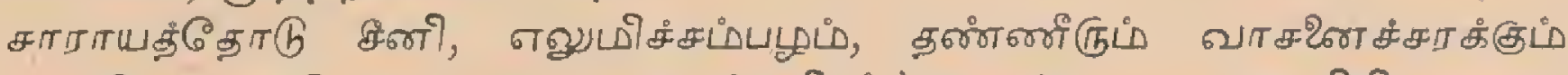

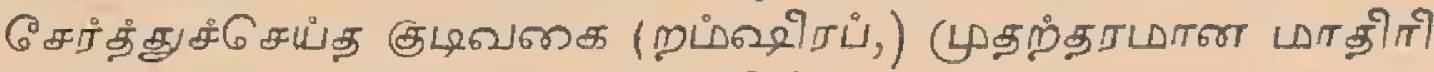

பலவிதமான கரும்புகள், புதற்தாபான கூட்டம்

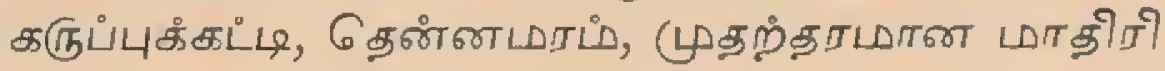
, " நீட்ப கருப்புகீகடிடி, கட்பாங் கறுப்புசீசி (கரூட்பு)

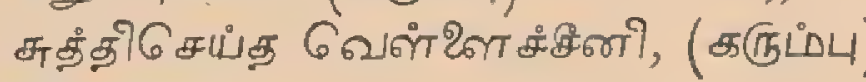
छேனभ மரவள்ளிக் கிழோँ்

\section{,}

,




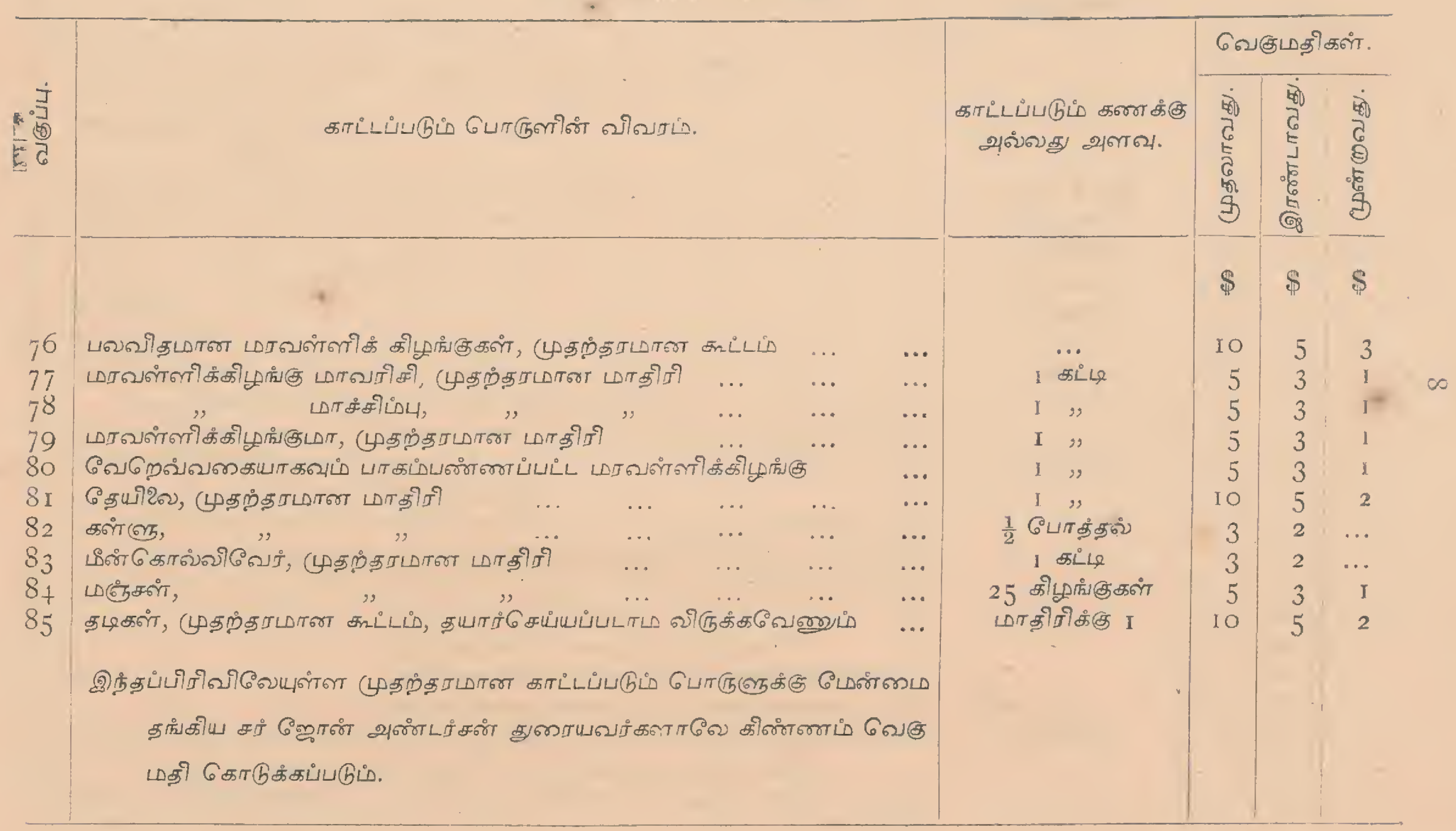




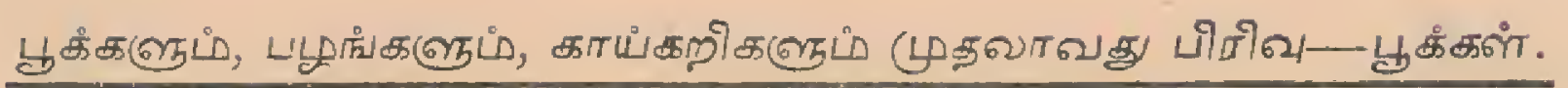

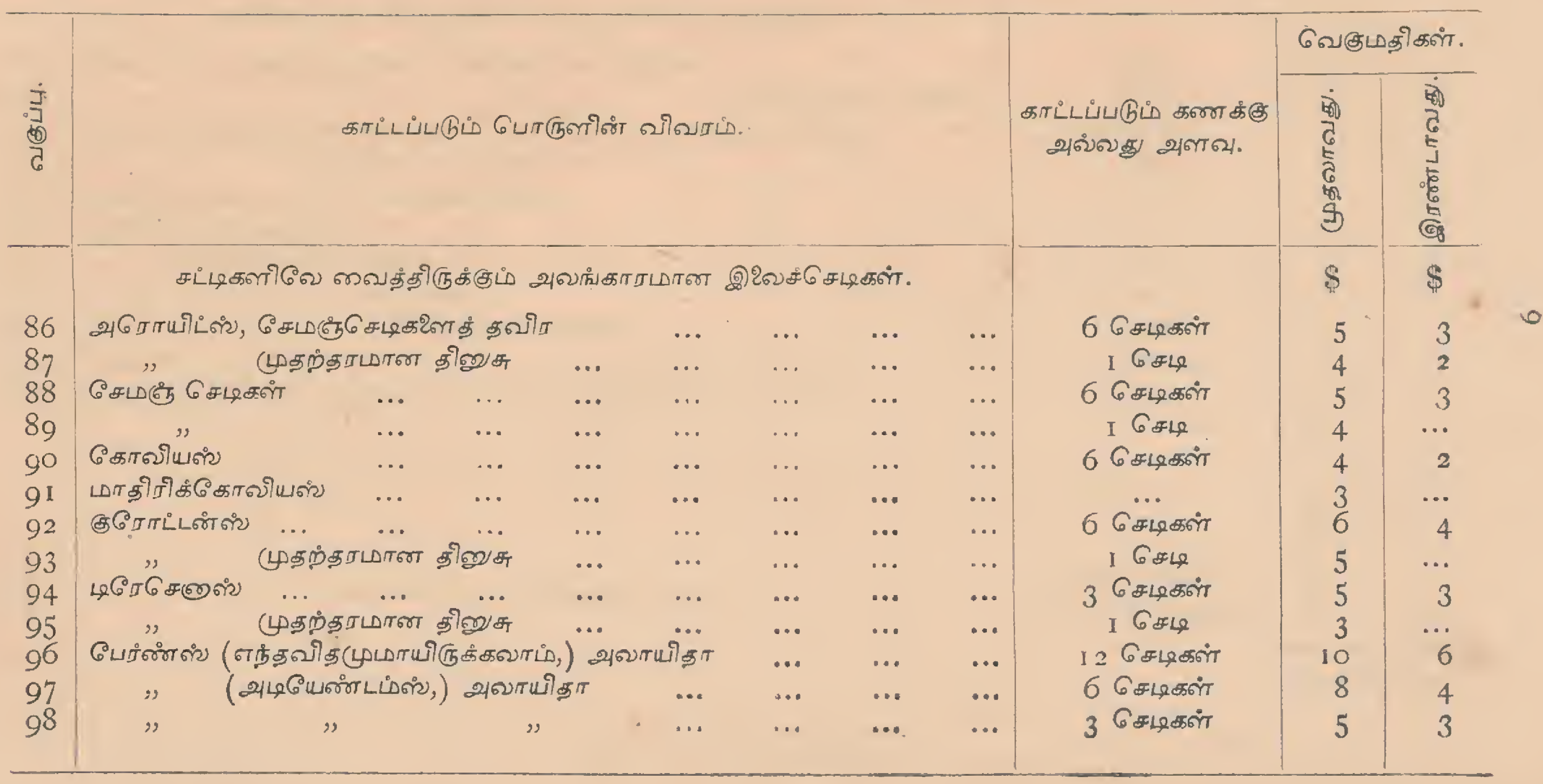




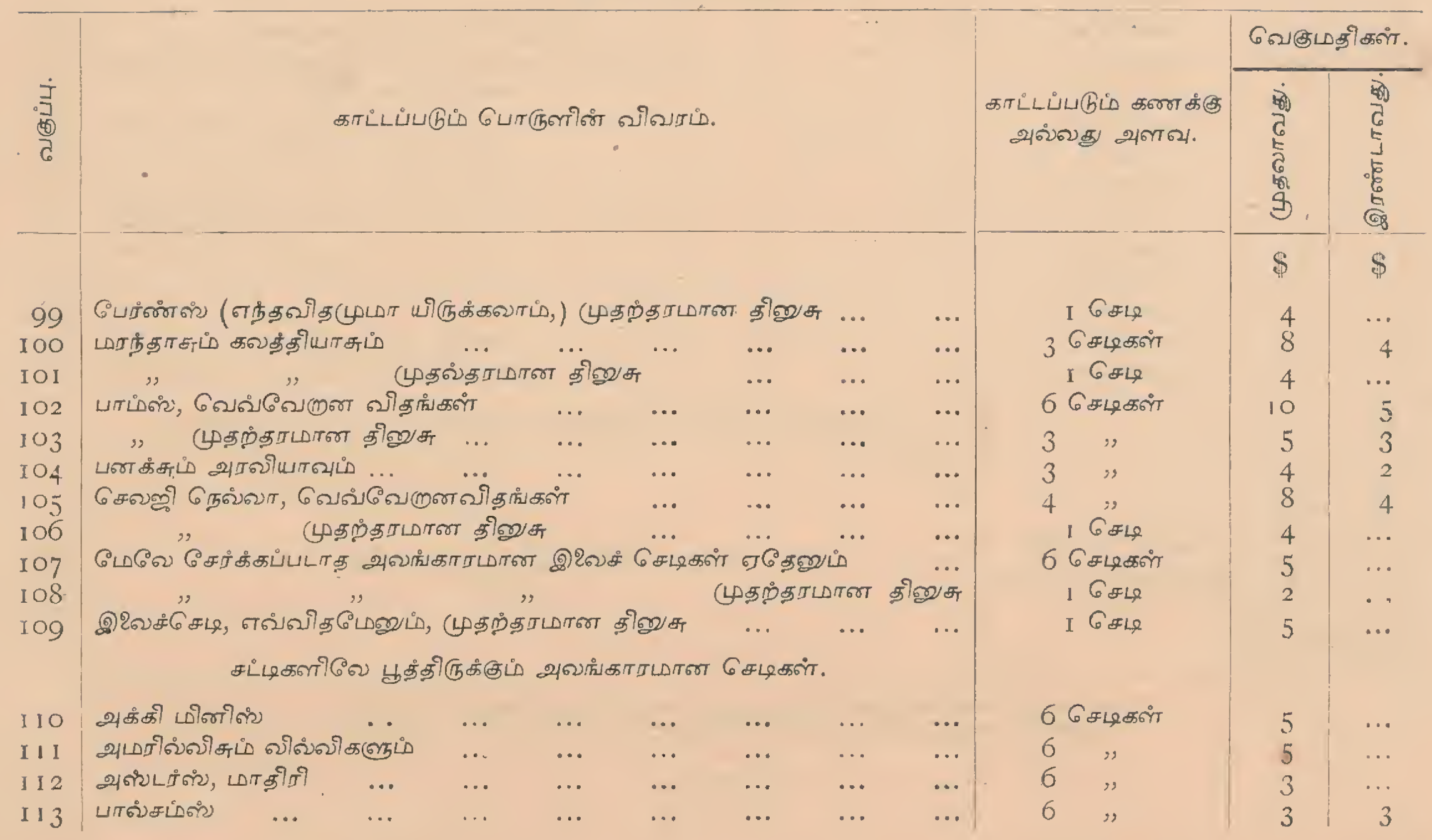




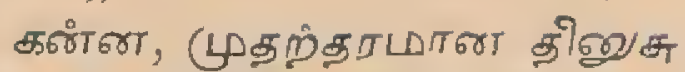

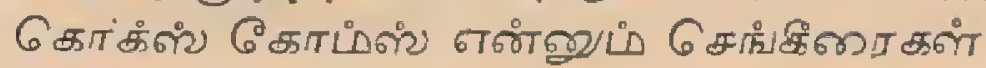

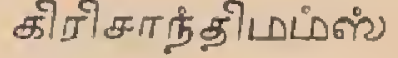

เாவியல்

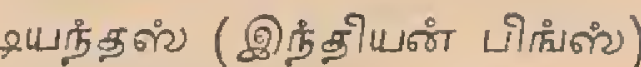

บுக்கநிก่

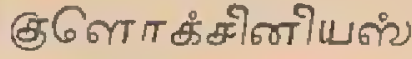

ஓதீகிட்

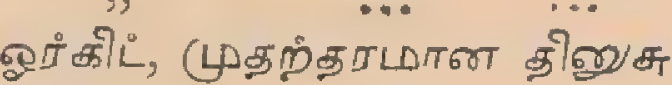
ปी6ோ கீஸे

$\cdots+\ldots$

6ோஜா \&ீ 6 சடிகள்

சின்னிแล่

\section{(ு)தறீதாடாான திख्रசு}

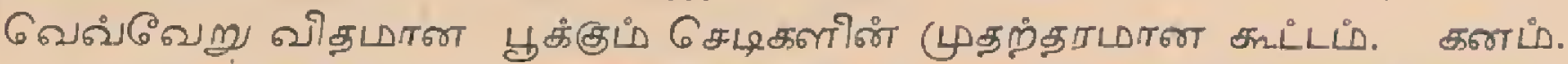

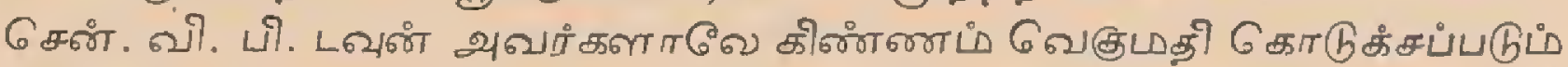

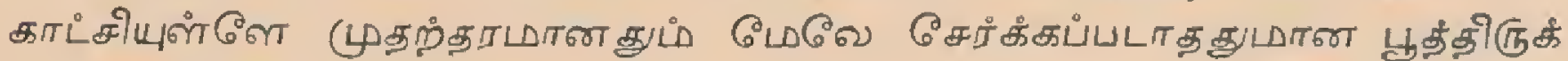
कப் Gசட ..

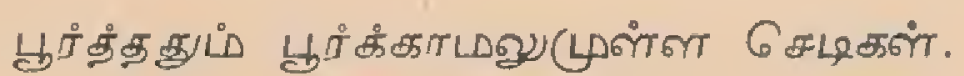

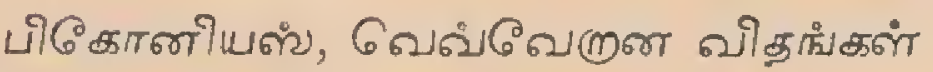

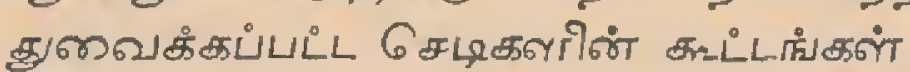

6 சேடிகள்

3 "

3 சேடிகஸ்

3 "

3 ,

3 ,

3 ,

$3 n$

6 ,

3 I 6 푸

6 சேடிகत்

6 ,

3 I 6 சேட

6 சேடிகள்

வாதிரிகீக 6

I 6815

6 சேடிகள்

I Gव्दु

..

...

$\cdots$

...

...

$\ldots$

5

5

...

...

... 


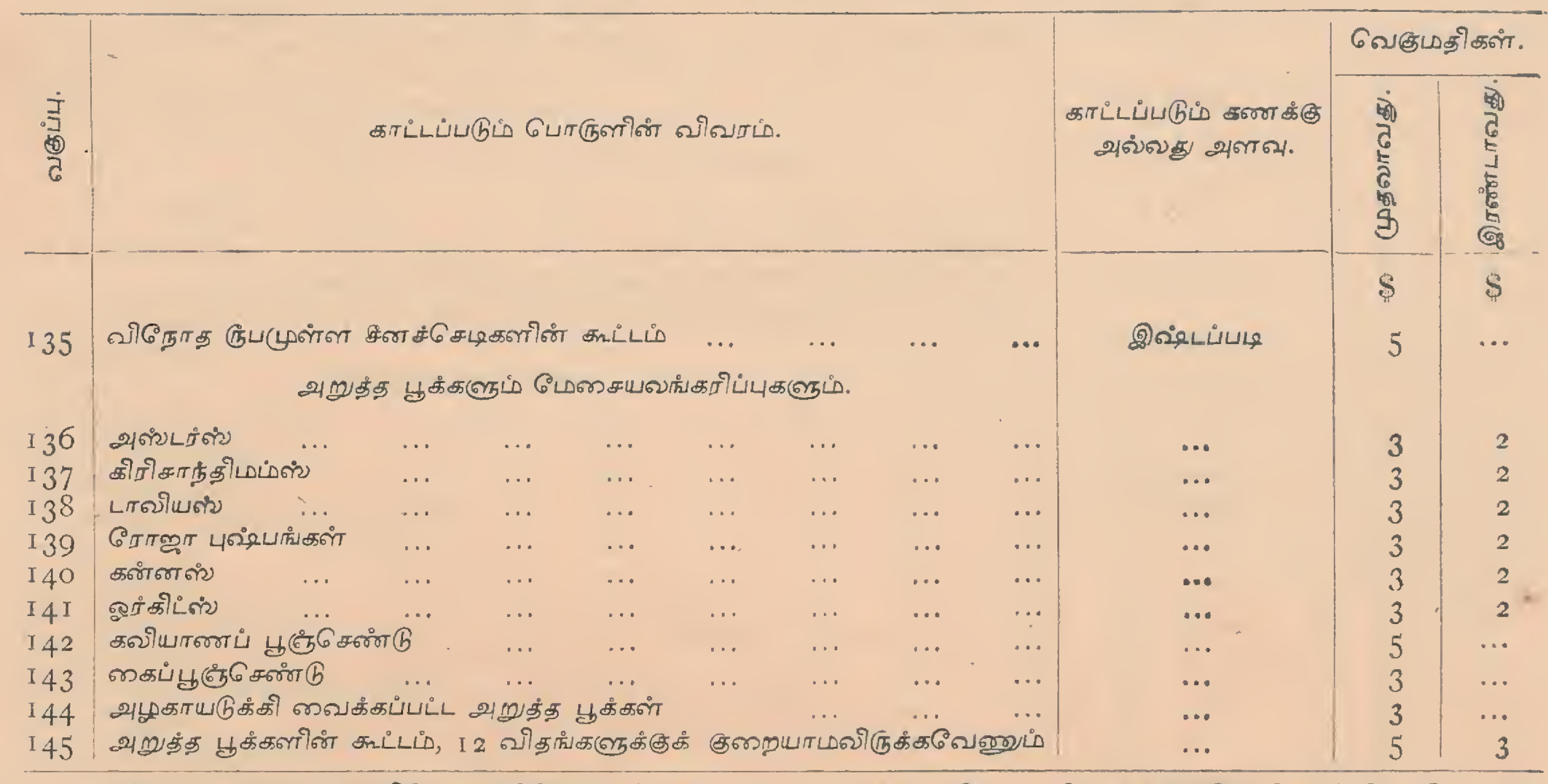

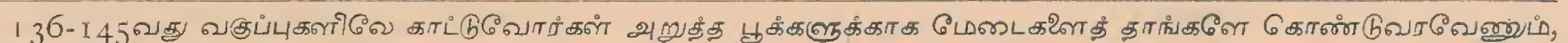

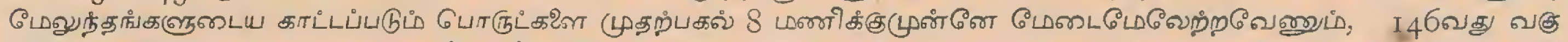

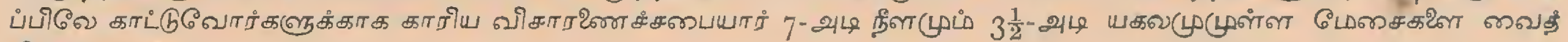
திரिபㄴ 
பேசை யவங்கிப்பு *

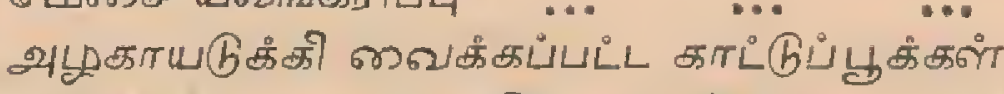

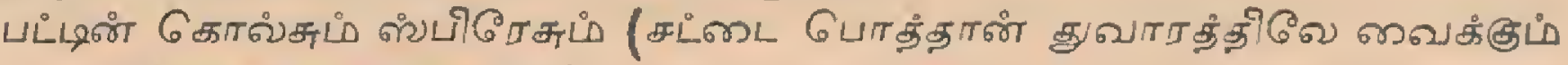
பூகீகளூட் தணைகளூட்)

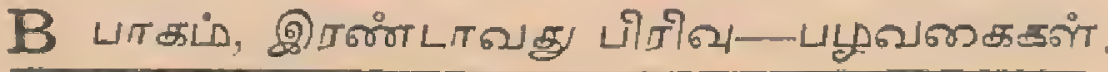

வாலபுப்படுங்கள் ,

,

IT IOULDLO

சிக்க …

2्यदम

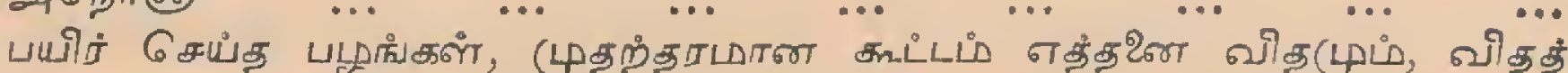

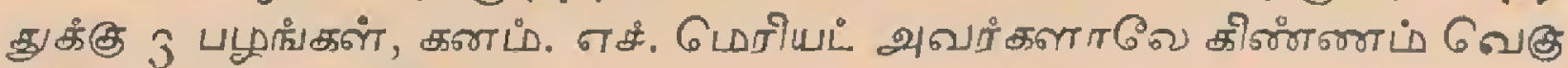
மதி எகாடுகீகப்படுட்.
த/Iியாட்பழு ...

டுக웅

பीำ

பவாப்பழLํ

சட்புநாவற்பழு

வ)ங்

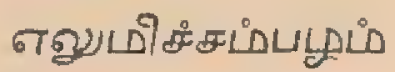

பாட்பதும

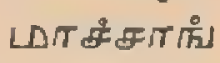

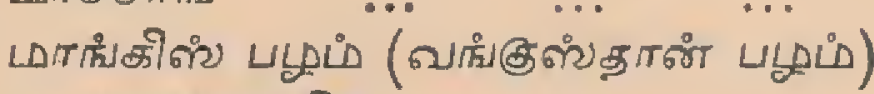

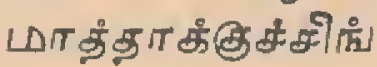

புதறீதாடாான कூட்டம்

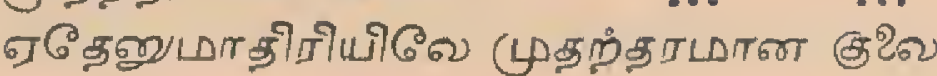

$\cdots$

$\ldots$

$\cdots$

$\cdots$

LัT कीगीकंत 6

I Cक्ष

3 பழூ ஷீ கள்

6 ,

6,

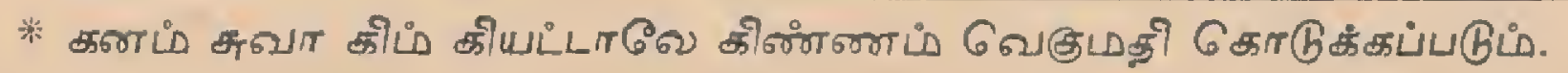




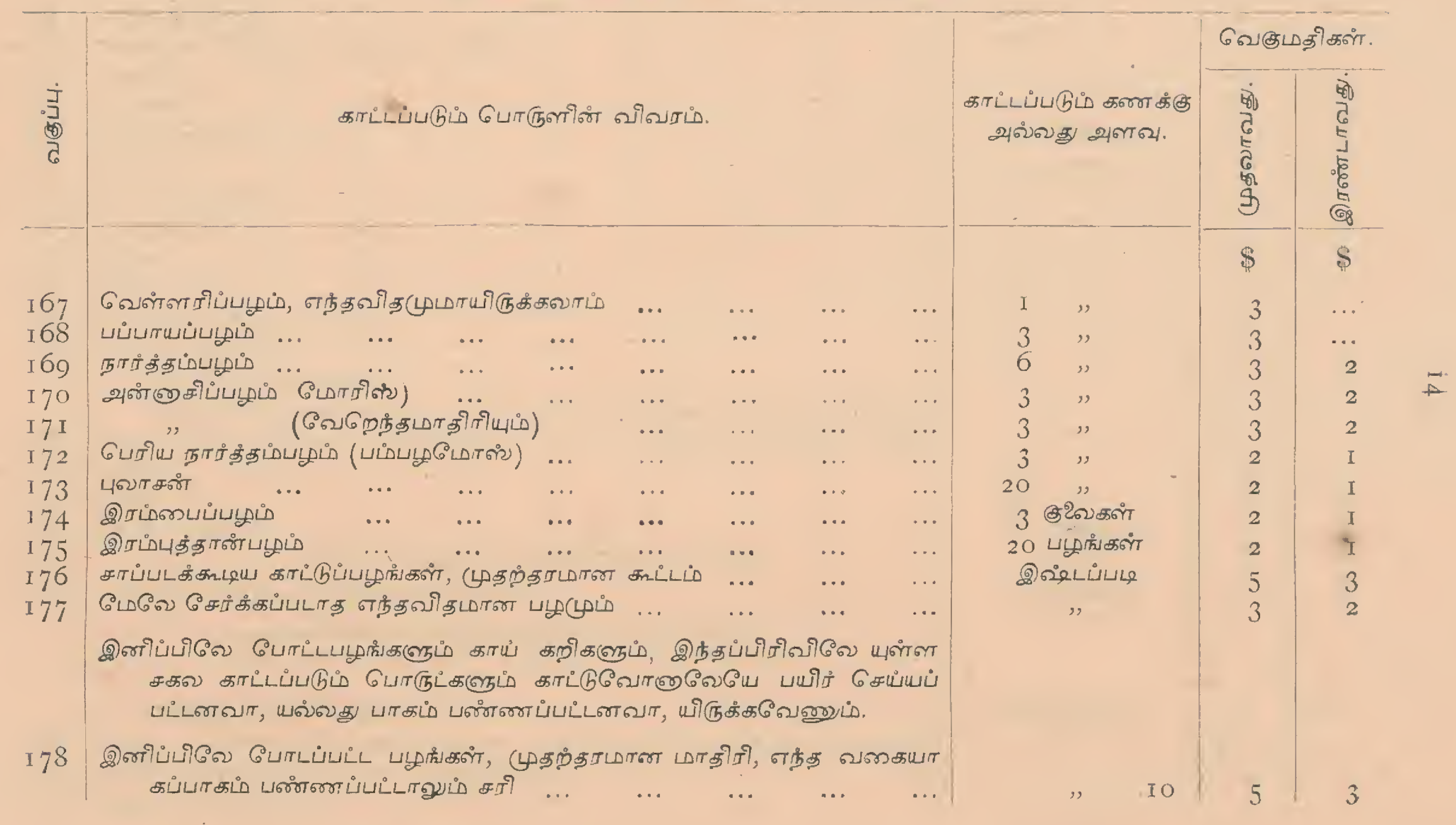




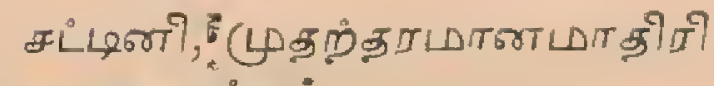

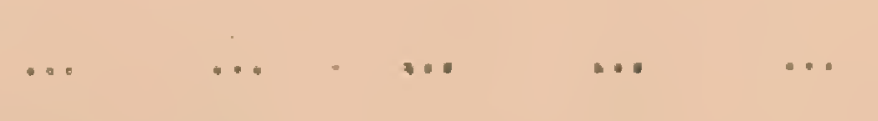

18 I தித்திப்பு कणั

I 82

भூட்டிசீசோக் கிपूங்

$\cdots$

$\begin{array}{lll}\cdots & \cdots & \cdots \\ \cdots & \cdots & \ldots\end{array}$

183

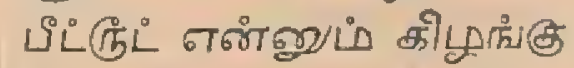

184

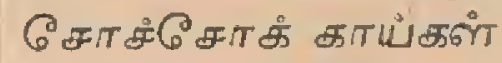

கத்தரிக் காய்கள்

I 86

அவரகீகாய்கள், முதந்தாடான சூட்டப்

187

कேाவीके झँचा

I 88

மிளகாயீகள், (டுதறீதாமாளா கூட்டீ

கக்கரிக்காய்கள்

கறிகளூம் ஈப்பவீகளூப் 6 சய்யுப் கீறைகள்

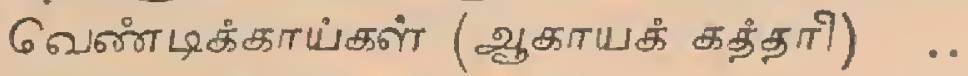

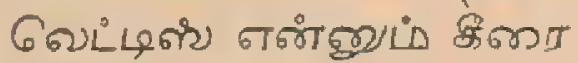

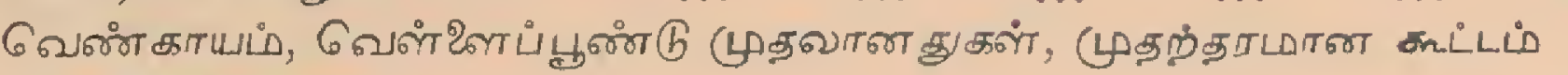
பூசணிகீகாய்கள்

புள்ளங்கிகள்

.. $\quad \cdots \quad \cdots \quad \ldots$ ...

क.

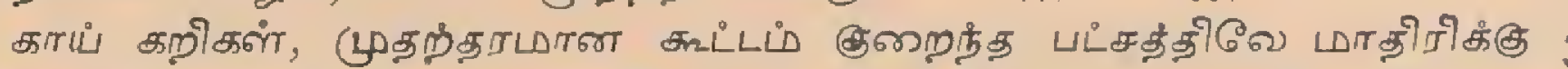

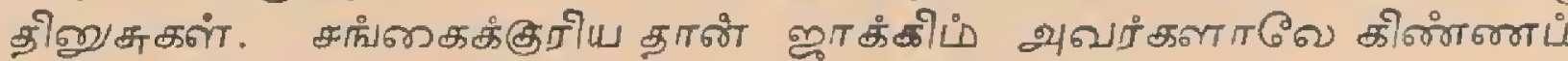
வேகுமதி எோடுக்கப்படும்

வதீதணகக் காய்கள் சுறைக் காய்கள் புதவானதுகள், புதற்தாடான

$$
\text { कூLLLD }
$$

$$
\text { ... }
$$

$$
\text { ... }
$$

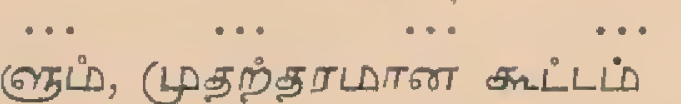

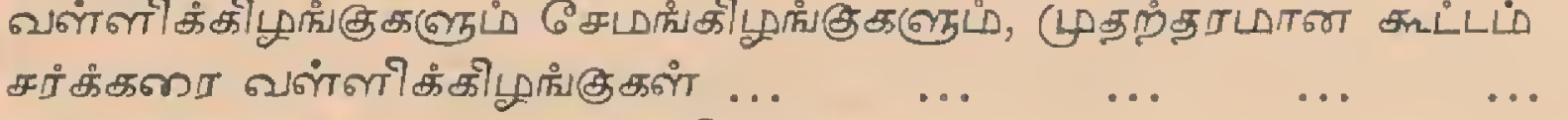

$20 \mathrm{I}$

மேலே சேரீக்கப்படாத காயீகறிகள்

$\cdots$

$\cdots$

$\cdots$

இณீடபบடி

I 2 கிழூங்குகள்

6 தின्ण्मकகते

6 "

6

இパடட்பபடி

மாதிரிகீகு 30

6 कीன् கு கते

1ாதிரிக்கு I கட்டு

6 தीชு சுகள்

4

மாதிரிக்கு I கட்டு

3 कीव्य கள்

IO

6

,

"

6 தினூ சுகள்

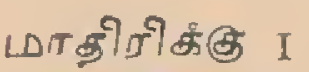

பாதிறிக்கு 6

...

இஷீடியபடி 


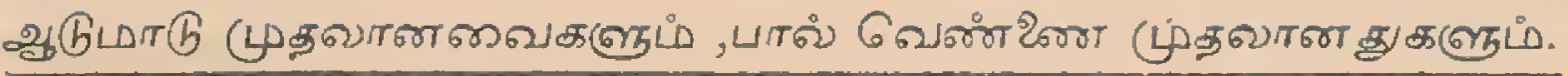

\begin{tabular}{|c|c|c|c|c|c|c|c|}
\hline \multirow[b]{2}{*}{$\frac{J}{3}$} & 1 & & & & \multirow[b]{2}{*}{$\begin{array}{l}\text { காட்Lப்படுப் கணா கீகு } \\
\text { அவ்வது அளவ. }\end{array}$} & \multicolumn{2}{|c|}{ ढேகுமதிकள் . } \\
\hline & \multicolumn{4}{|l|}{ காட்டப்படும் டொருளின் விவரம். } & & $\begin{array}{l}5 \\
\text { C } \\
5 \\
6 \\
\text { की } \\
9\end{array}$ & 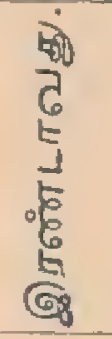 \\
\hline t & & & & & & $\$$ & $\$$ \\
\hline 202 & 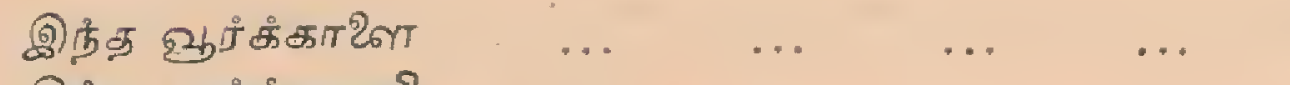 & $\cdots$ & .. & ... & $\cdots$ & 20 & $\cdots$ \\
\hline 203 & இநீத வூர்கீகாரி ... & $\cdots$ & $\cdots$ & $\cdots$ & $\ldots$ & 20 & ... \\
\hline 204 & 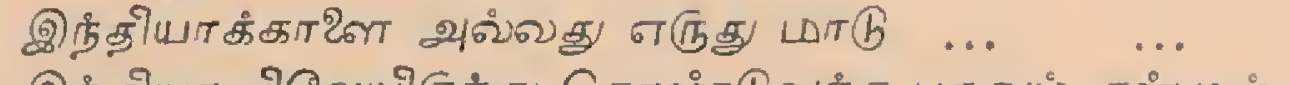 & $\cdots$ & $\cdots$ & $\cdots$ & $\ldots$ & 20 & $\ldots$ \\
\hline 205 & இநீதியாவிடேயிருந் து Cகாண்ரடுவநீத பகுவும் कன் றும் & $\cdots$ & $\cdots$ & $\cdots$ & $\cdots$ & 20 & $\ldots$ \\
\hline $\begin{array}{l}206 \\
207\end{array}$ & 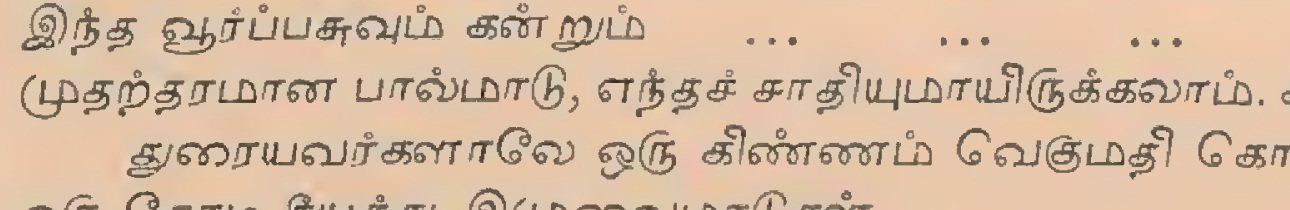 & 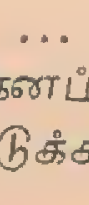 & ㄴ.. & $\cdots$ & ... & 20 & ... \\
\hline 208 & 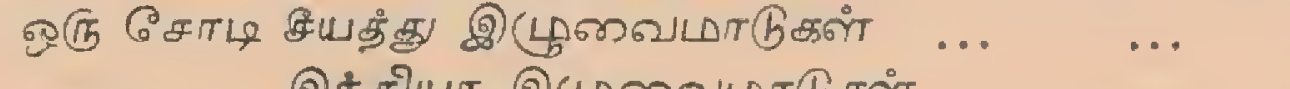 & $\cdots$ & $\cdots$ & $\cdots$ & $\ldots$ & IO & $\cdots$ \\
\hline 209 & 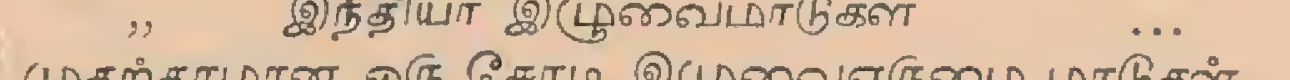 & $\cdots$ & $\cdots$ & $\cdots$ & $\cdots$ & Io & ... \\
\hline $2 \mathrm{IO}$ & 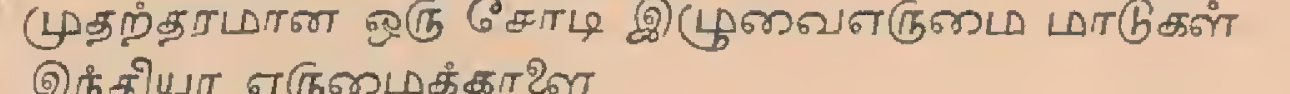 & $\cdots$ & $\cdots$ & $\cdots$ & $\cdots$ & IO & $\cdots$ \\
\hline 211 & 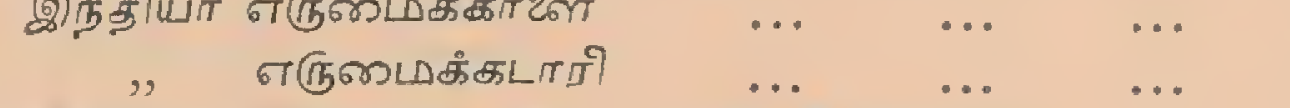 & $\begin{array}{l}\cdots \\
\cdots\end{array}$ & $\cdots$ & $\cdots$ & $\cdots$ & I5 & IO \\
\hline 2 I 3 & 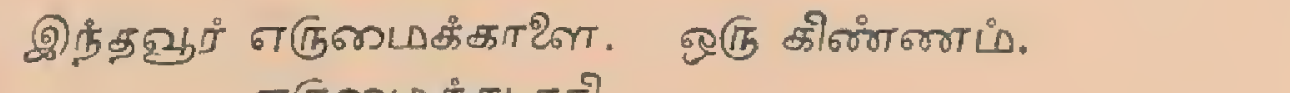 & 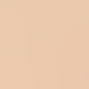 & . & . & $\cdots$ & 15 & $\cdots$ \\
\hline 214 & 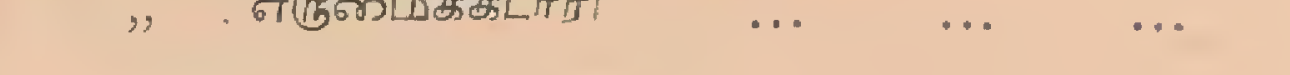 & $\cdots$ & $\cdots$ & $\cdots$ & $\cdots$ & I 5 & IO \\
\hline
\end{tabular}




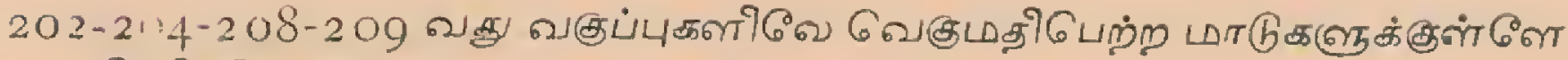

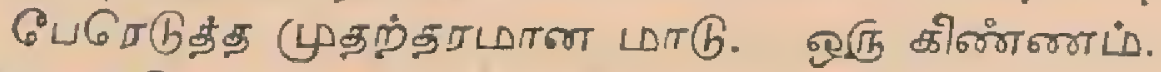

216 भुซ่บช์p? ธபช่นบช์p?

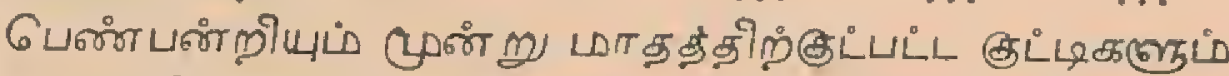

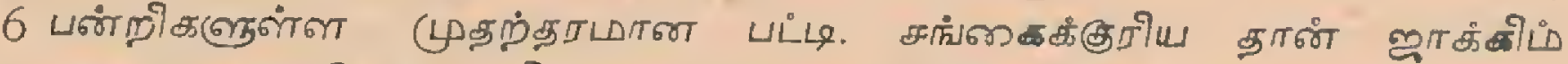

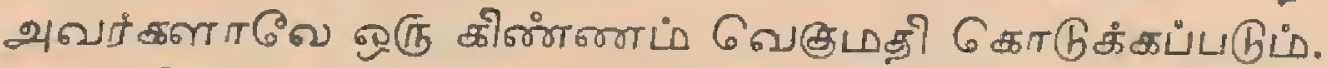

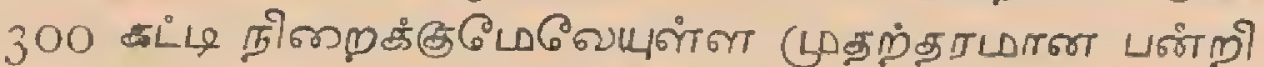

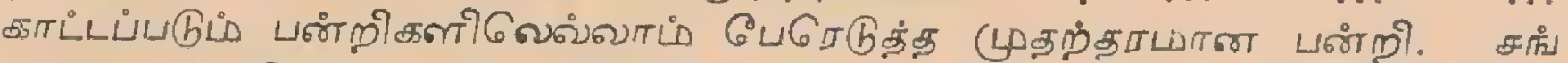

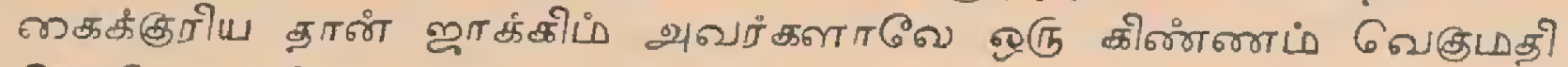
कொடுக்கப்படும்.

பேண் டேศ்ளாடு ம் கட்டிகளுட் முதறீதமாள பாவாடு: 6வள்ளாடு 6 ேட்பறறி

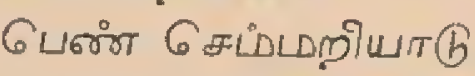

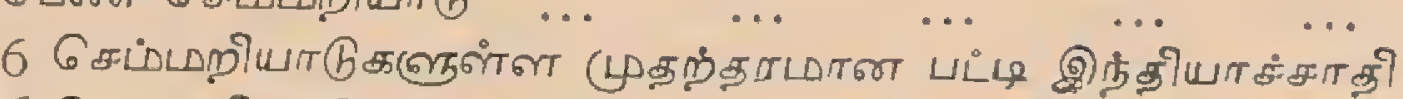

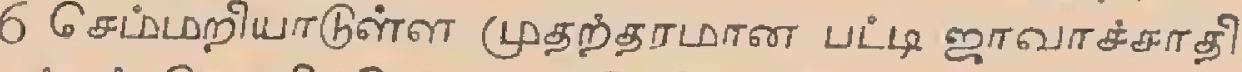

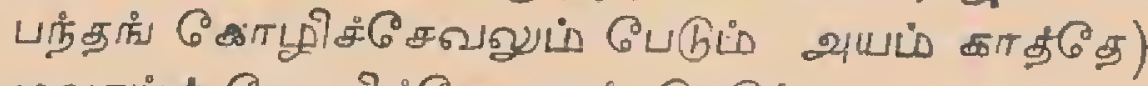

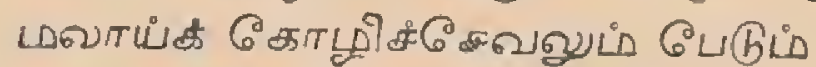

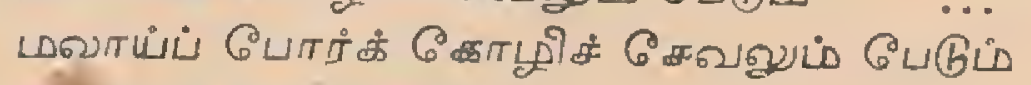

மவाாய கோழிकत்

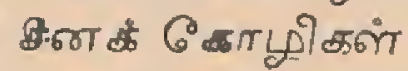

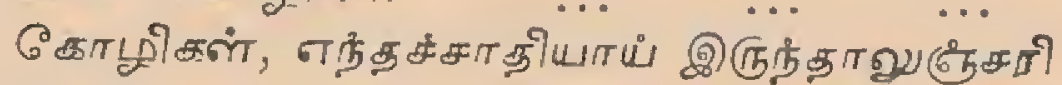

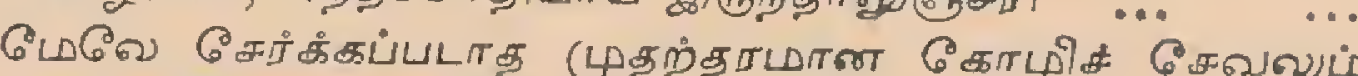

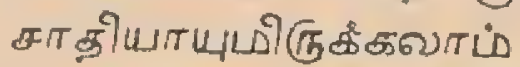

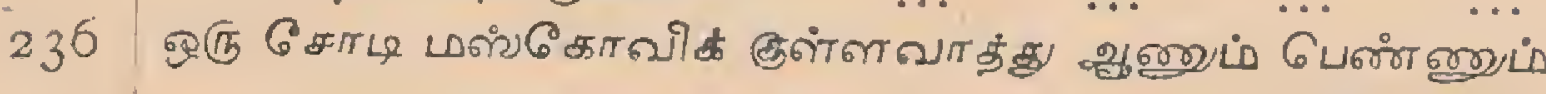




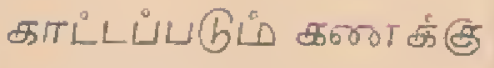

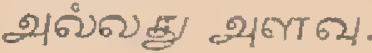

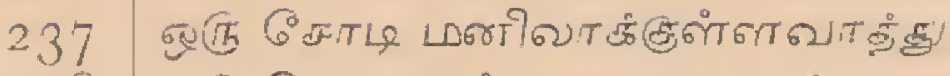

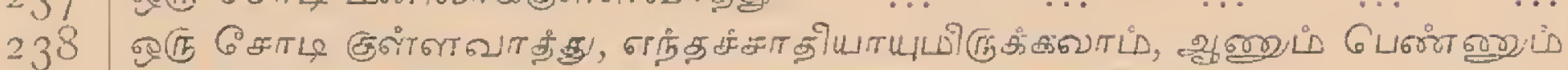

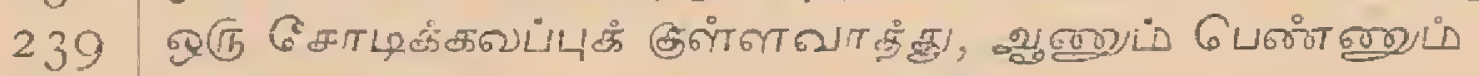

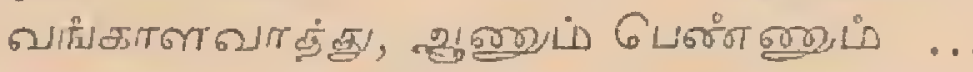

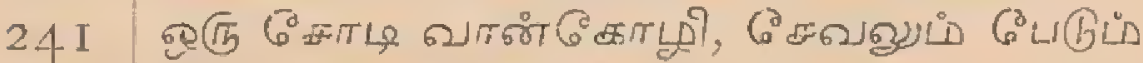

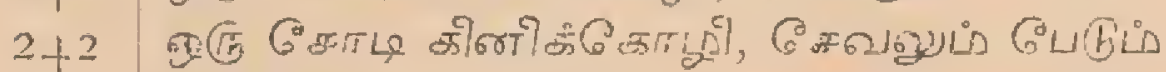

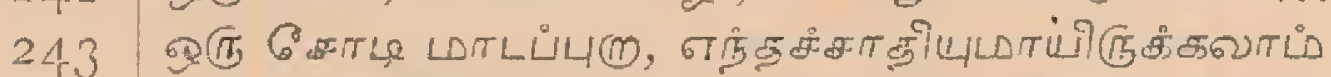

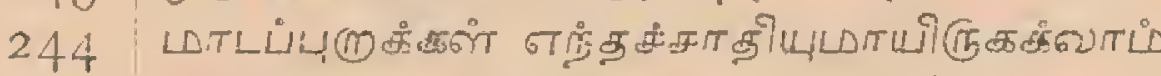

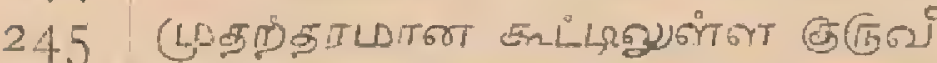

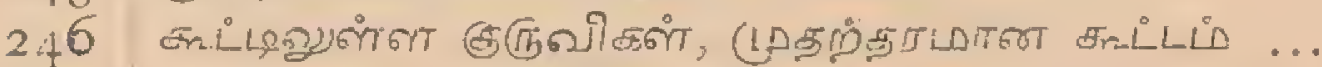

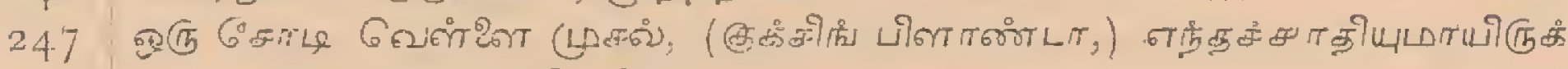

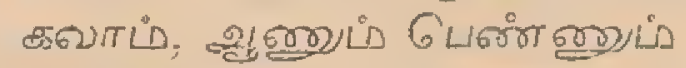

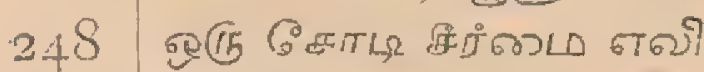

$248 a$ முதற்தரபான பூथन

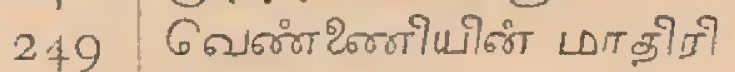

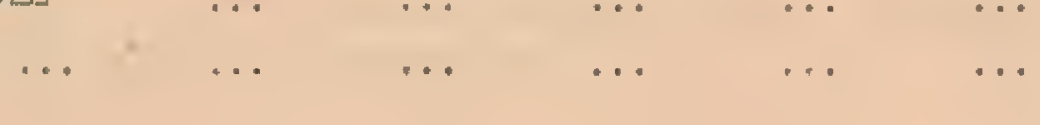

$25^{\circ}$

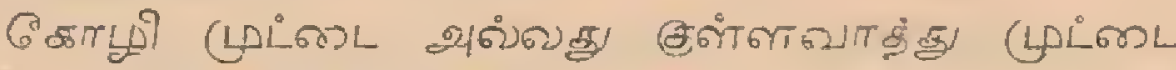
(ு)ட்டீं குவியவீ ..
भல்லத வான்கோழி

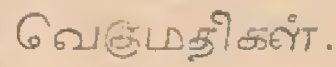

\begin{tabular}{|c|c|}
\hline $\begin{array}{l}\text { की } \\
6 \\
5 \\
6 \\
5 \\
5\end{array}$ & 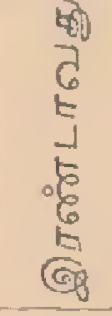 \\
\hline 8 & 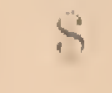 \\
\hline 5 & 3 \\
\hline 5 & 3 \\
\hline 5 & $\begin{array}{l}3 \\
3\end{array}$ \\
\hline 5 & 3 \\
\hline 5 & 3 \\
\hline .5 & 3 \\
\hline 5 & $\cdots$ \\
\hline 5 & $\cdots$ \\
\hline ro & $\cdots$ \\
\hline 5 & $\ldots$ \\
\hline 5 & $\ldots$ \\
\hline I 5 & IO \\
\hline 10 & 5 \\
\hline
\end{tabular}




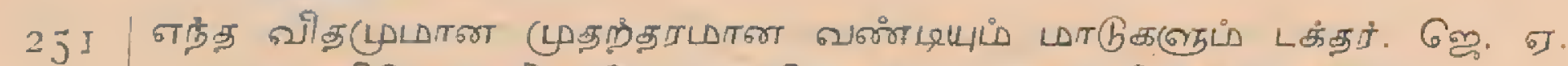

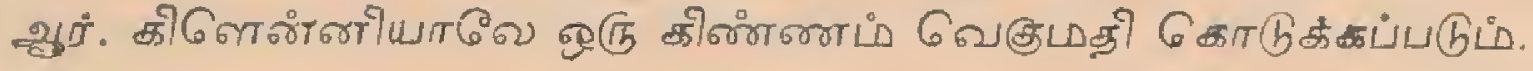

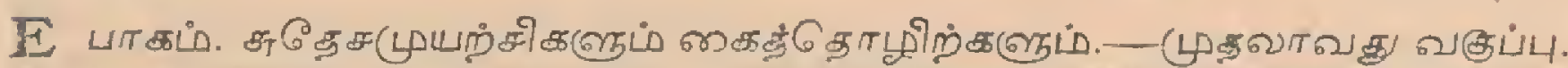

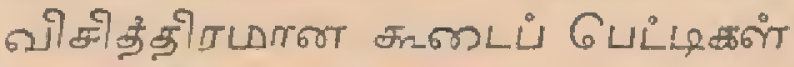

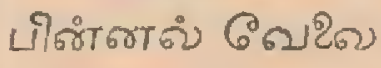

தோவ் ढேथ

278

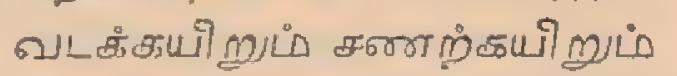

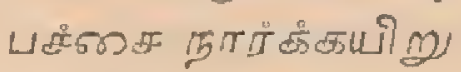

280

281

வ्यத்தாப்புகள்

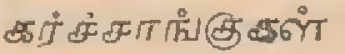

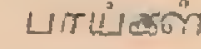

284

285

\section{பிரட்பட்பாய்கள்}

மவாய்பபாய்கள்

Gோபீபியमீं

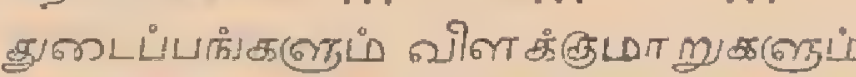

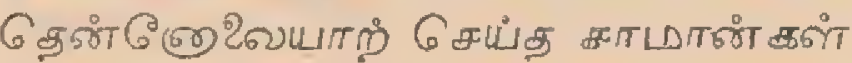

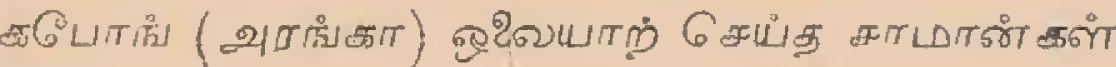

நीढபाई

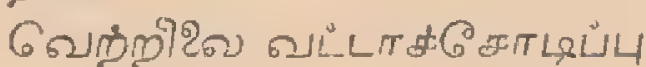

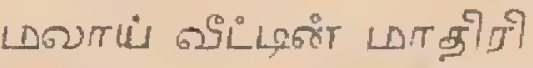

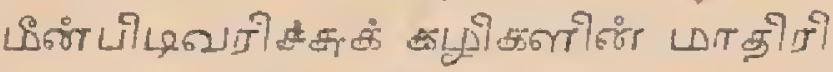

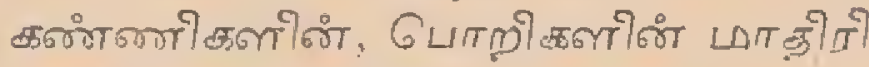

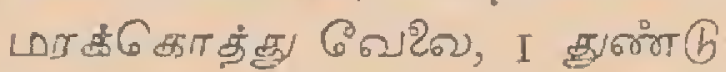

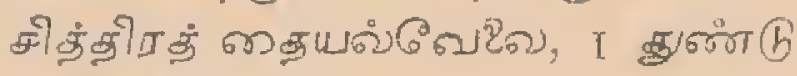

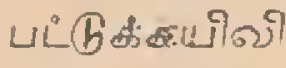

$$
\text { . } \quad \ldots \quad \ldots
$$

$\cdots \quad \cdots$

இவ்டப்படி

$\begin{array}{lllll}\ldots & \ldots & \ldots & \ldots & \ldots\end{array}$

$\cdots \quad \cdots$

$\ldots \quad \ldots$ மாதிगிகீகு 5 ஏவा

\begin{tabular}{ll|l}
$\cdots$ & $\ldots$ & 5
\end{tabular}

\begin{tabular}{ll|l}
$\cdots$ & $\cdots$ & 3
\end{tabular}

\begin{tabular}{ll|l}
$\cdots$ & $\ldots$ & 3
\end{tabular}

‥ $\quad \ldots \quad$ I

… $\quad$... 2

.. $\quad \ldots .3$

\begin{tabular}{ll|l}
$\ldots$ & $\ldots$ & 2
\end{tabular}

$\cdots+\cdots$

$\cdots$

$\ldots$

$\cdots$

..

$\cdots$

... 3

$\cdots$

...

$\cdots$

...

$\begin{array}{llll}\ldots & \ldots & \ldots & \ldots \\ \ldots & \ldots & \ldots & \ldots\end{array}$

$\cdots$

$\cdots$

$\cdots$

$\begin{array}{llll}\cdots & \cdots & \ldots & \ldots\end{array}$

$\begin{array}{llll}\ldots & \ldots & \ldots & \ldots \\ \ldots & \ldots & \ldots & \ldots\end{array}$

$\cdots$

$\cdots$

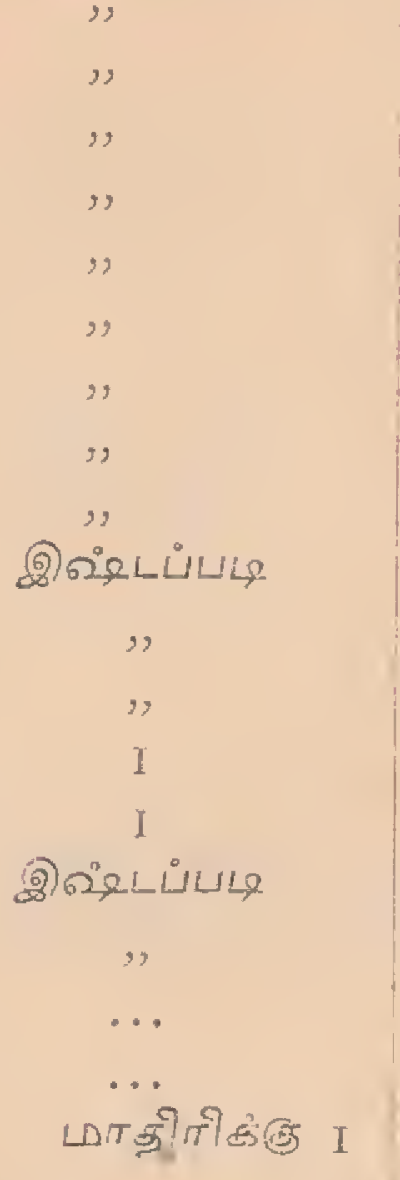




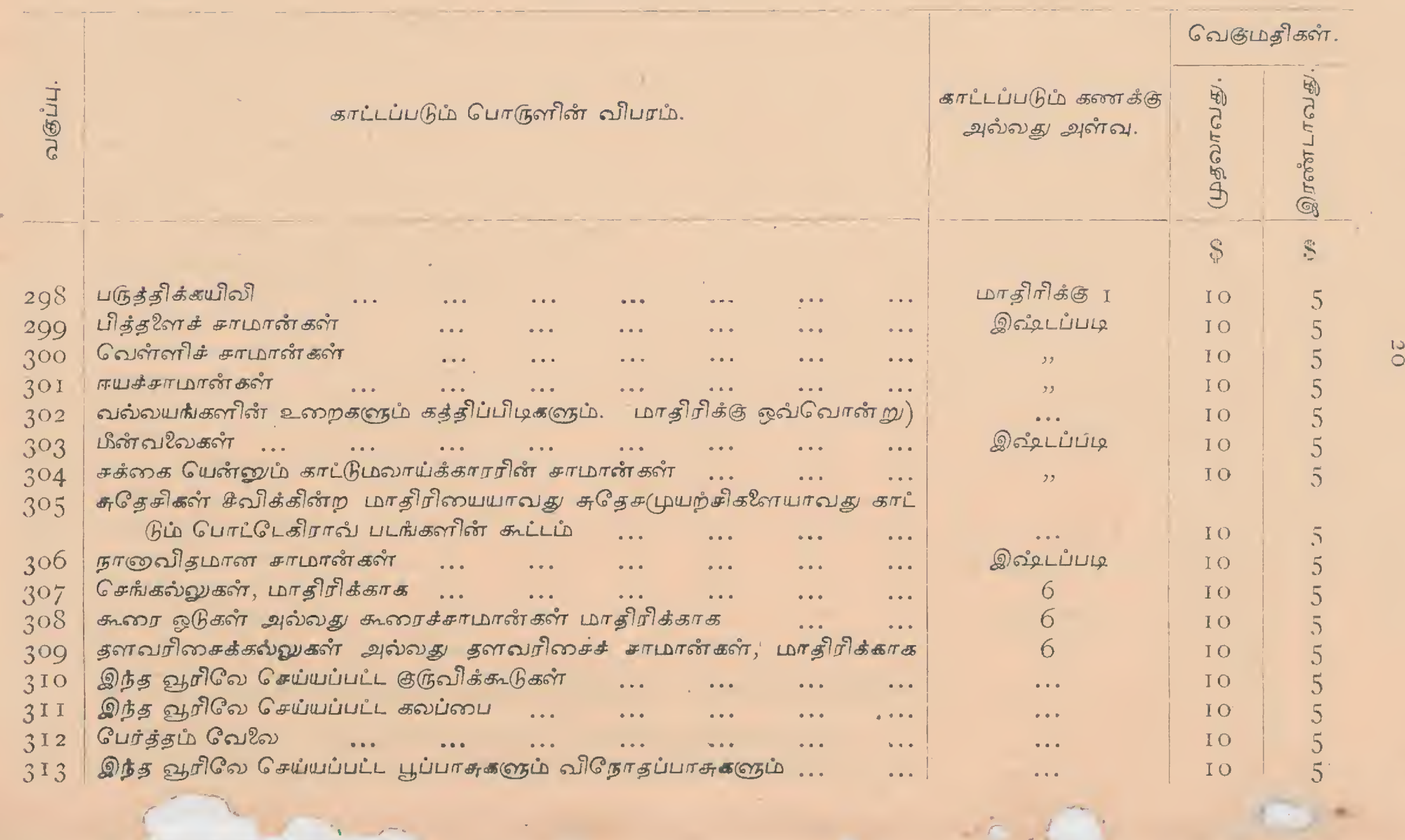




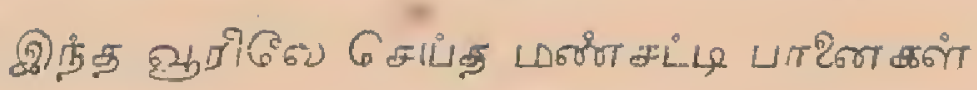

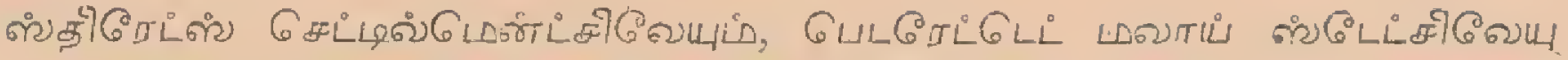
முளำ பสำ

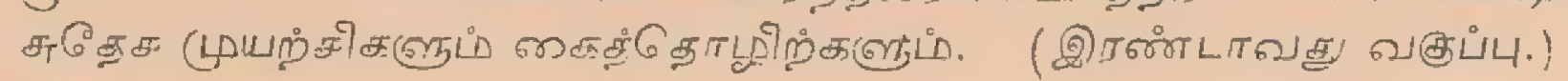

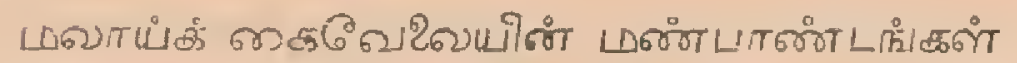

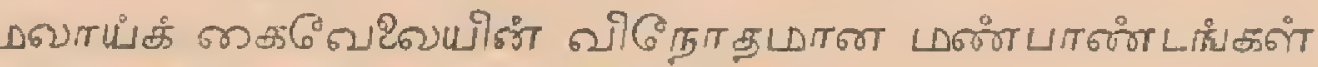

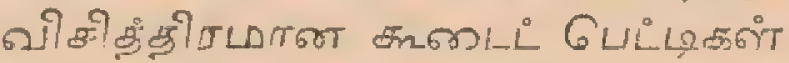

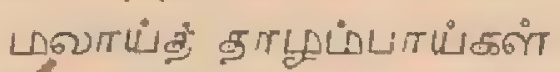

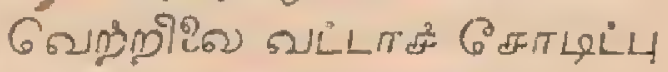

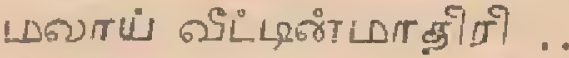

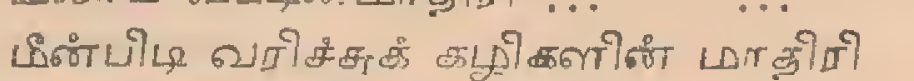

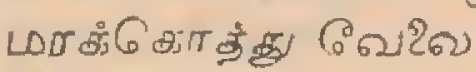

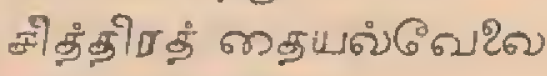

ธயนीखी (

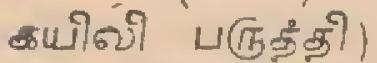

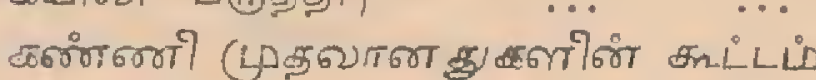

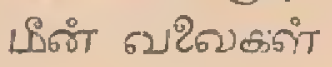

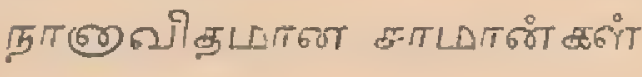

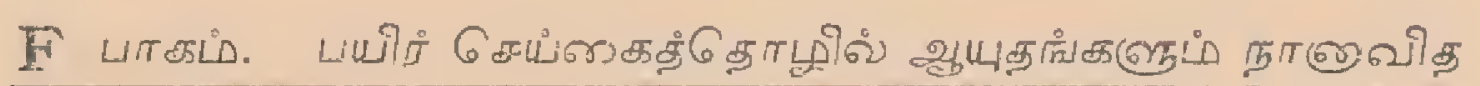

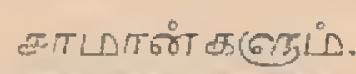

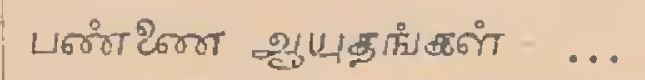

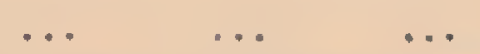

$\ldots$

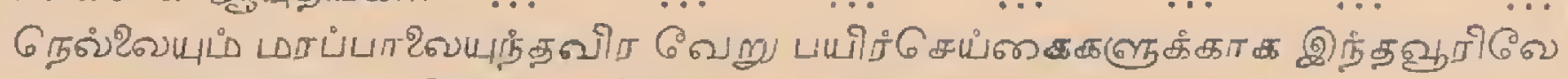

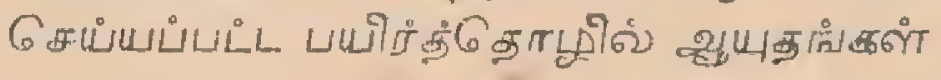

3 தीขு कள்

3

பा लीगीகंब I

3 தोஜु कण

பा தीनीकंक ।

" I

I कु भำ (b

I ,

I ",

I $\because$

இํำบ는

$$
\text { , }
$$$$
\therefore
$$

(

(2)ํํㄴㄴㄴㅇ

\begin{tabular}{l|l}
10 & 5 \\
10 & 5
\end{tabular}




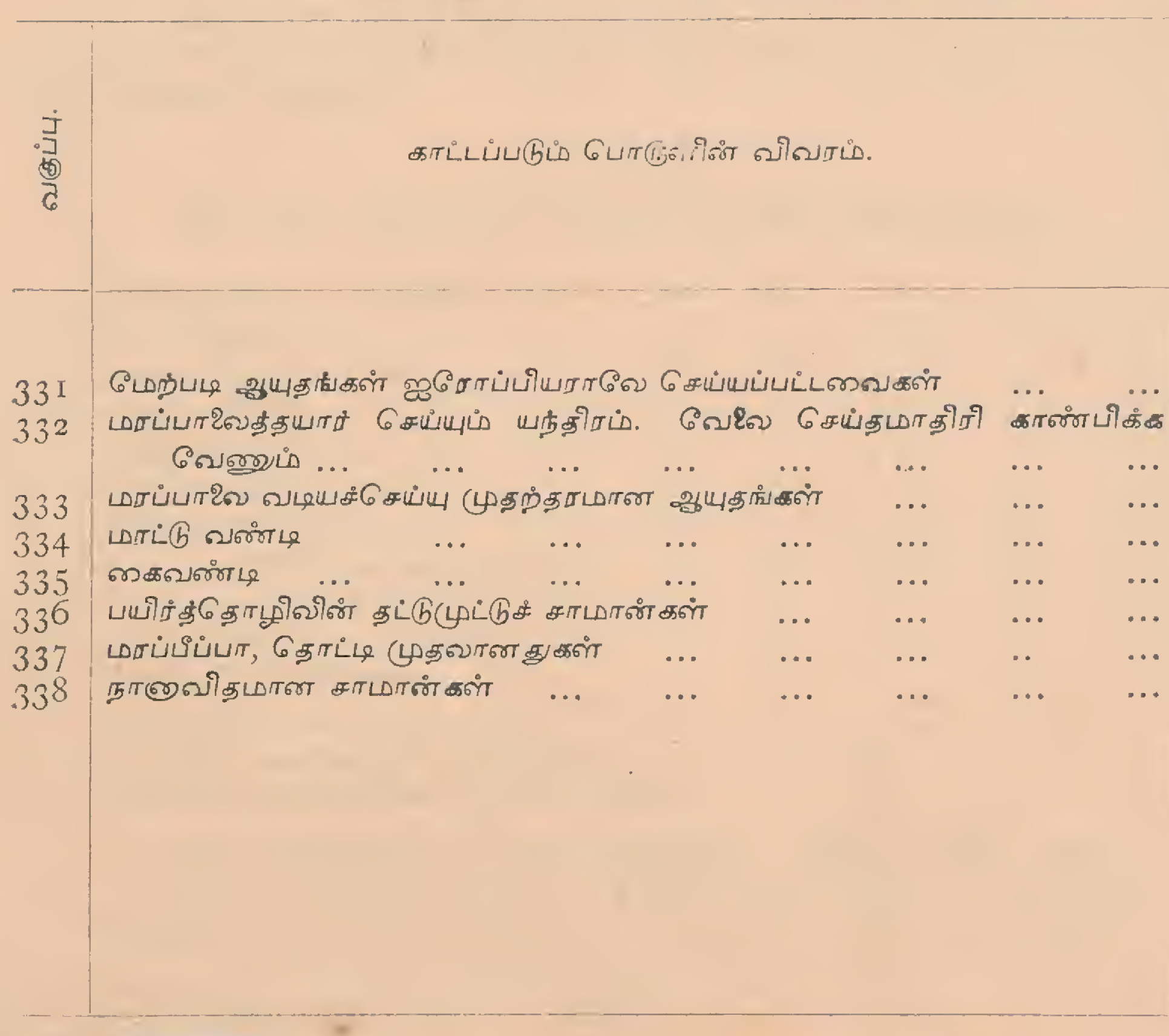

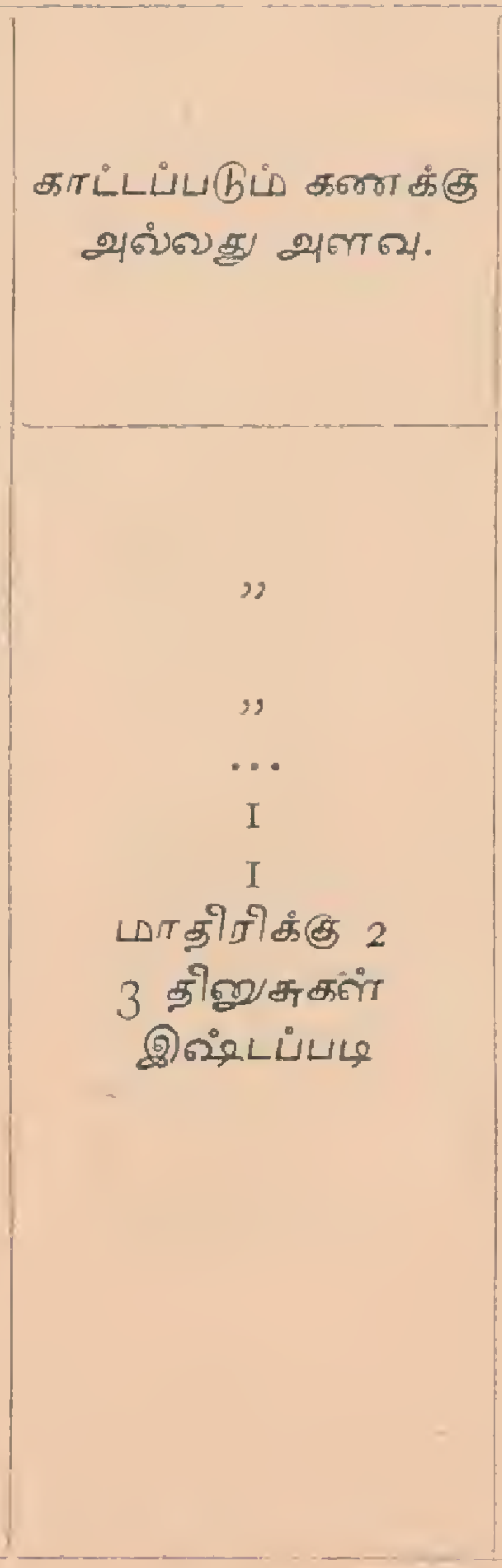

बேகுமதிकள் .

\begin{tabular}{|c|c|}
\hline 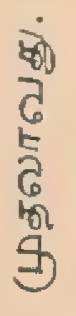 & $\begin{array}{l}80 \\
6 \\
5 \\
5 \\
5 \\
\frac{5}{6} \\
\text { ळ8 }\end{array}$ \\
\hline $\mathbb{N}$ & $\$$ \\
\hline IO & 3 \\
\hline
\end{tabular}

की भं आட

IO 

i

$+4$

$=\frac{-14}{x-1}$

$r$ 

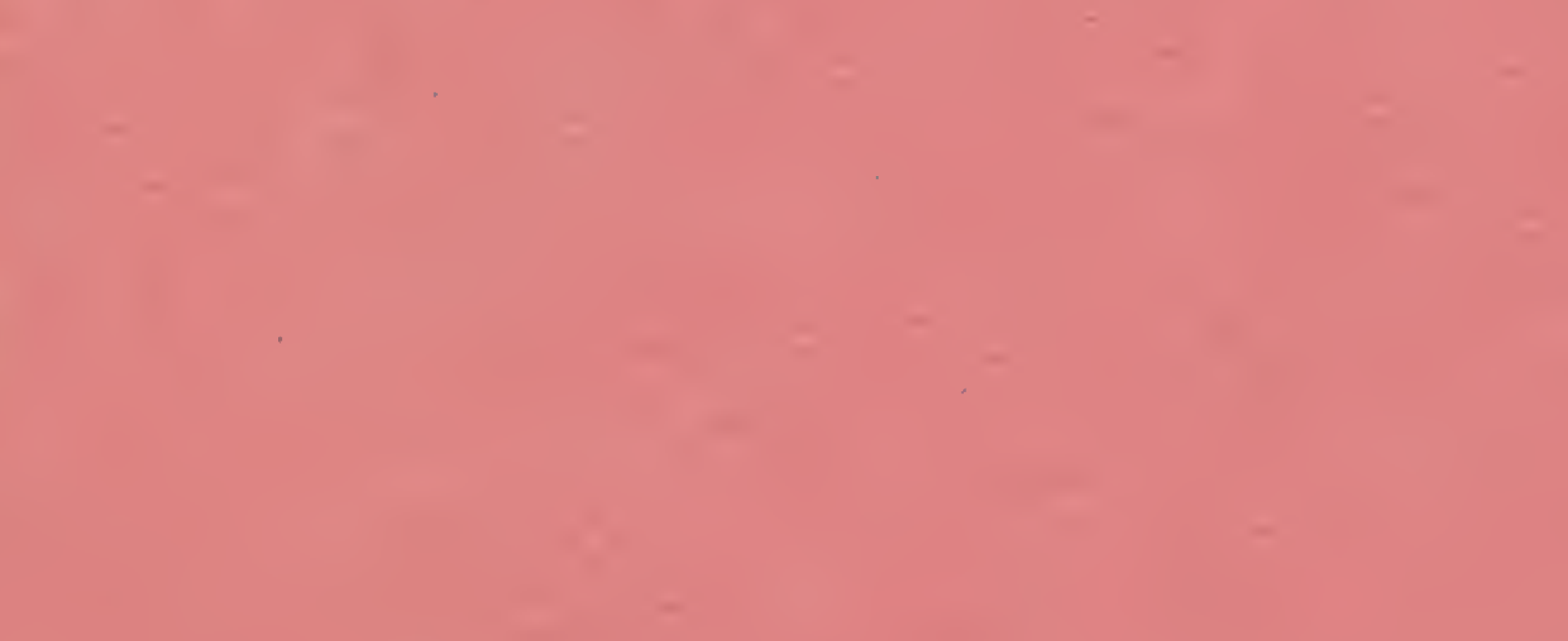

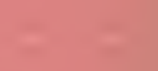

$-1=$
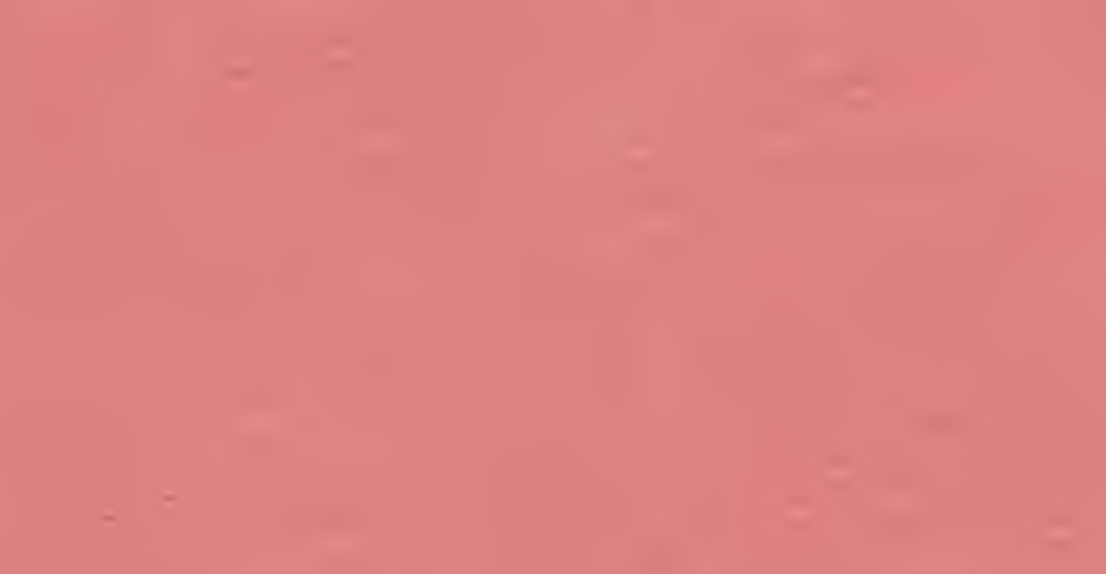

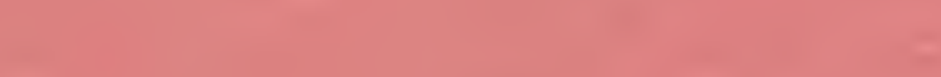

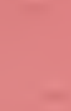

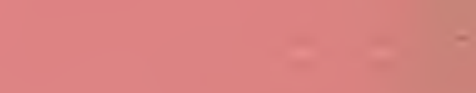

sin " $/ 17 t$ 

18

s

i)

5

is

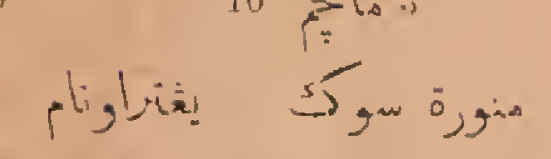

$9+5 \cdot 331$

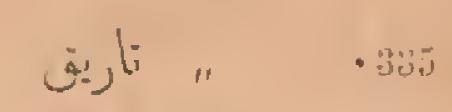

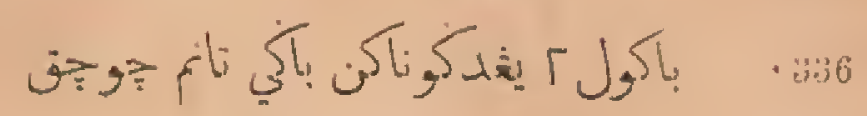

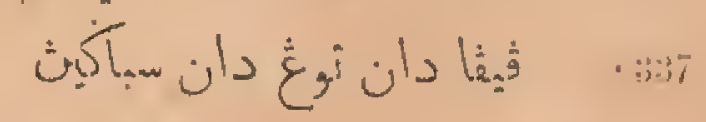

$E_{7}-1 / / 175$ 
17

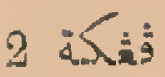

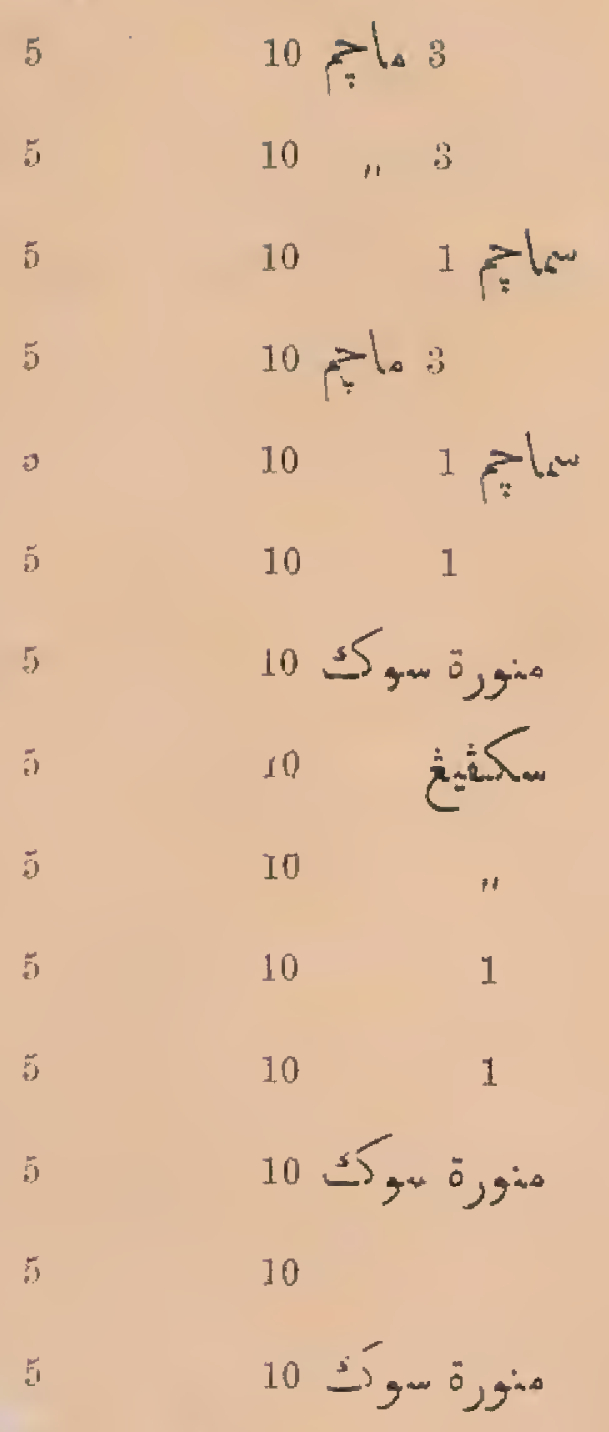

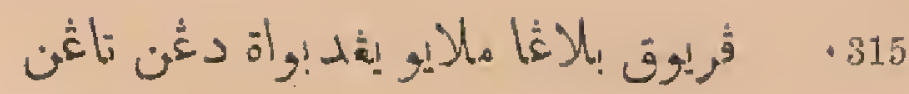

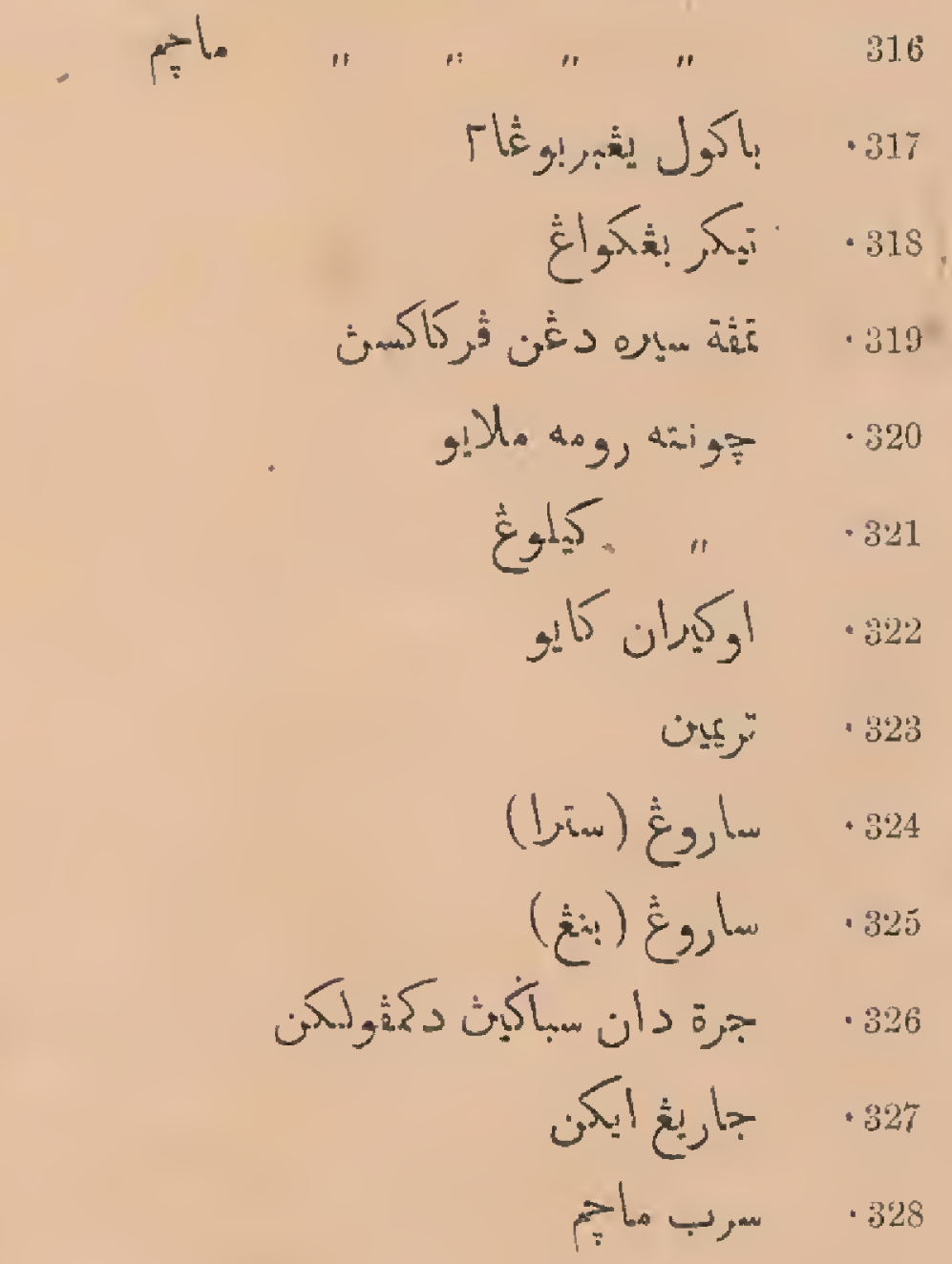

- $: 0:-\cdots$

F ن i is

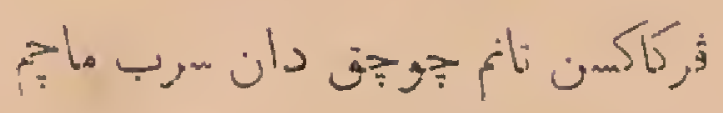

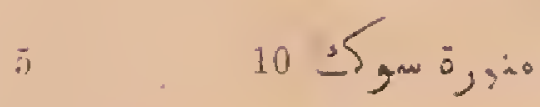

320

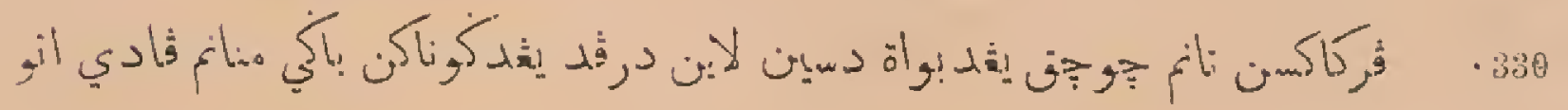

j. 10

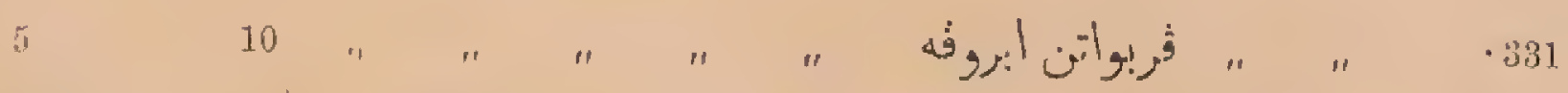

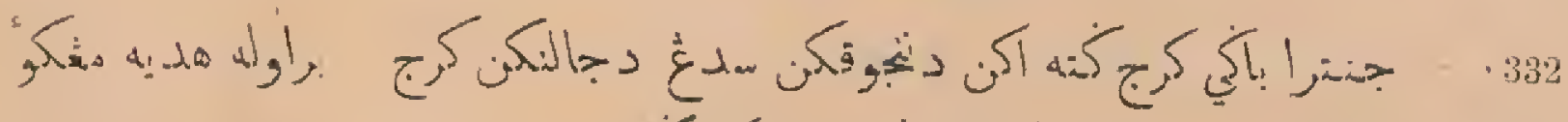

0

10

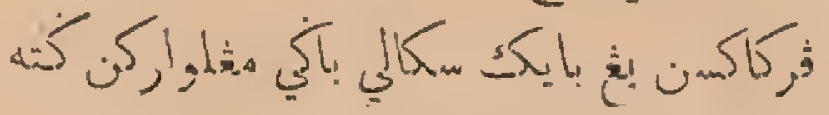

.333 


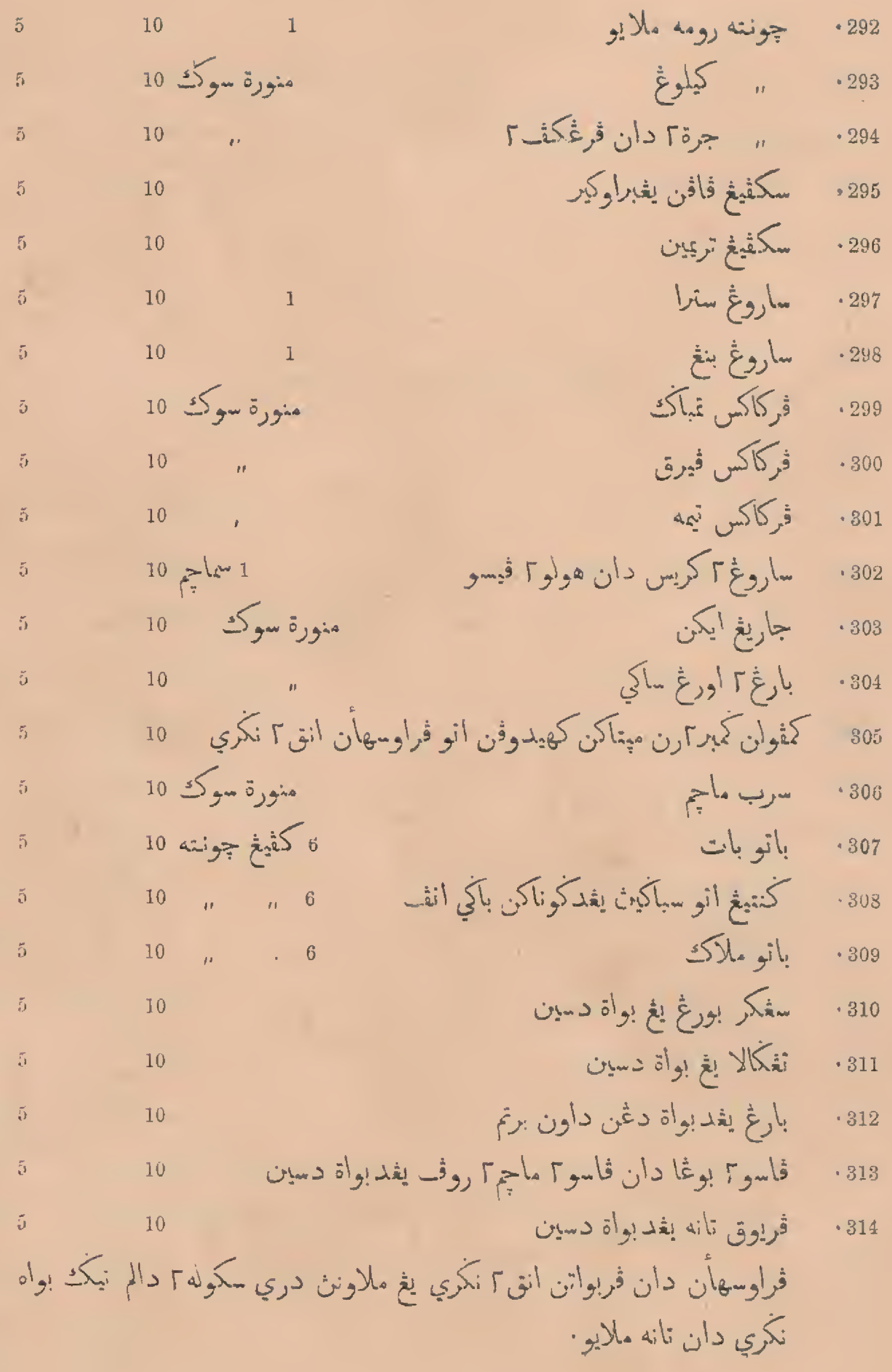


35

250

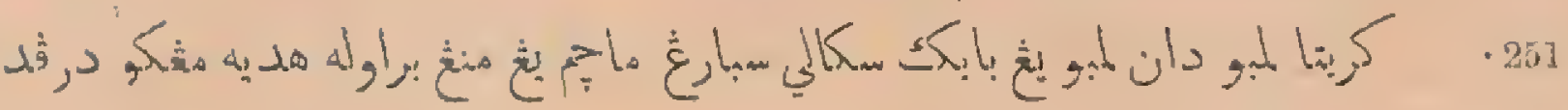

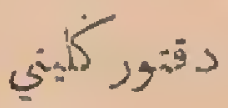

E

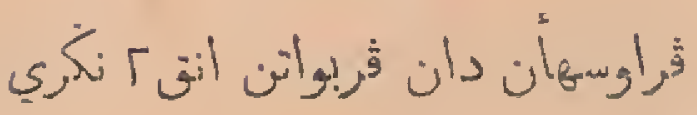

1 的安

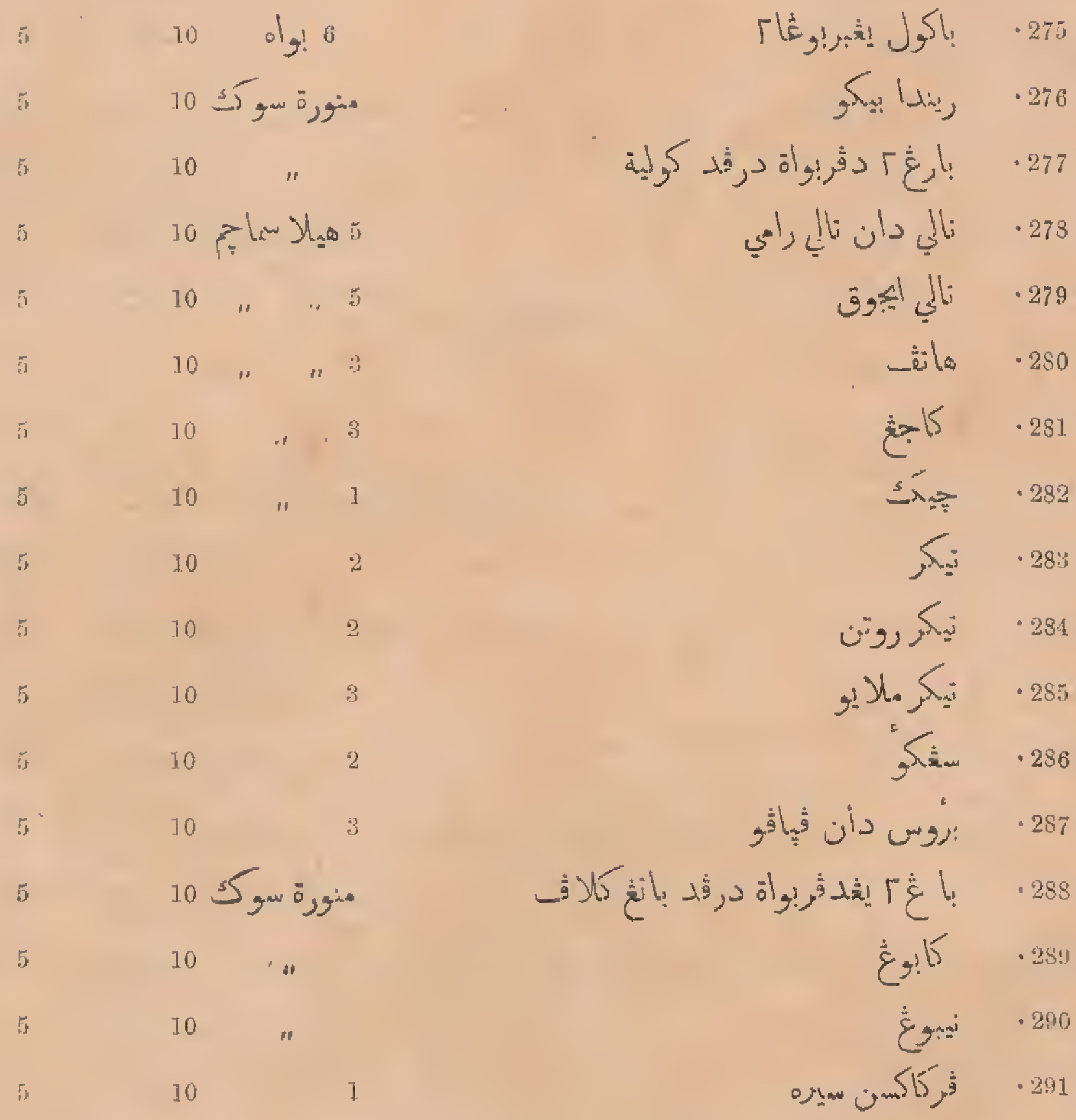


14

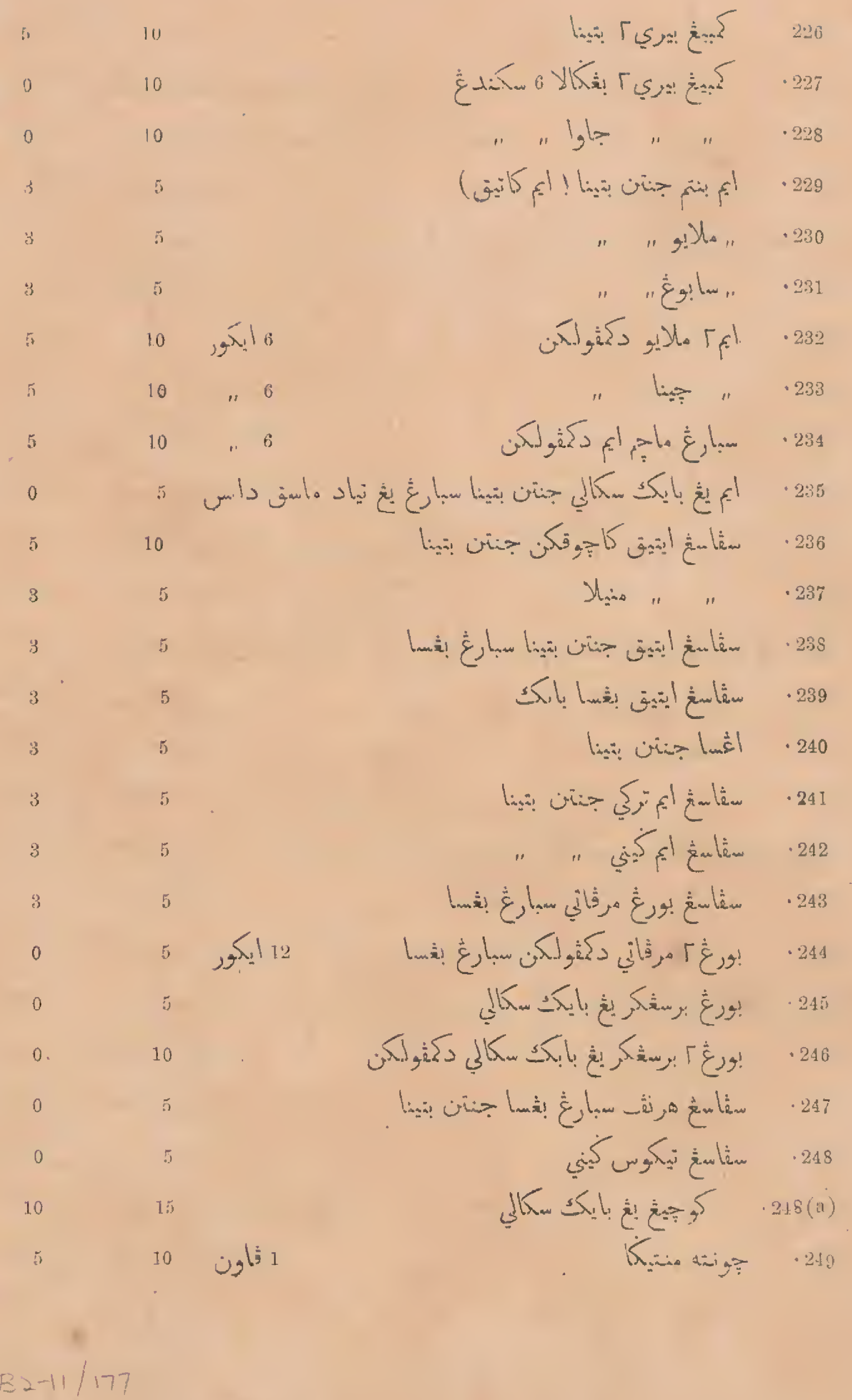




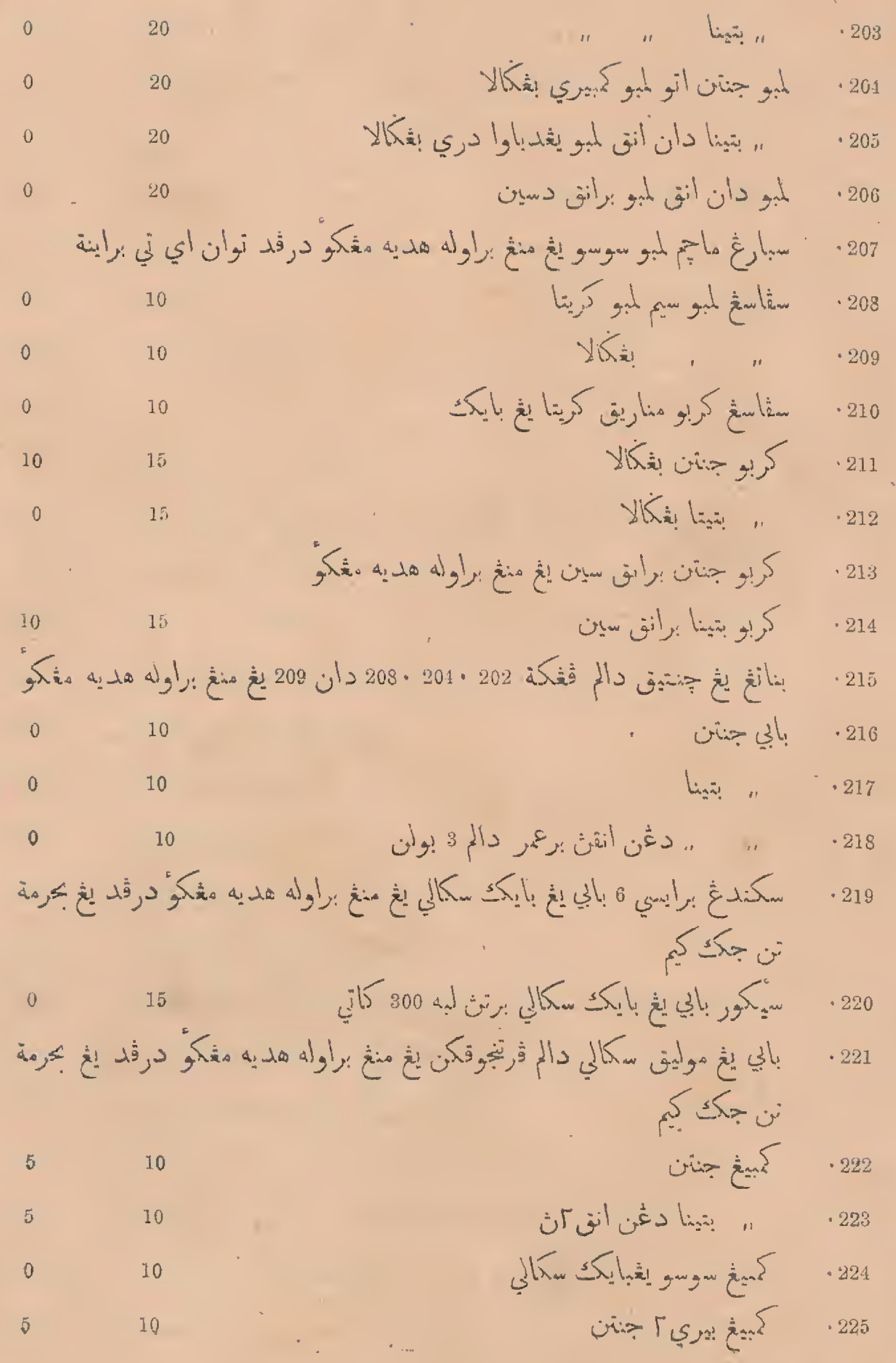


12

0

2

0

$2 \quad 3530 \div 6$

0

$2=6$

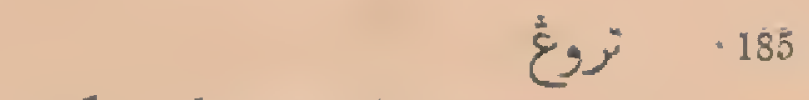

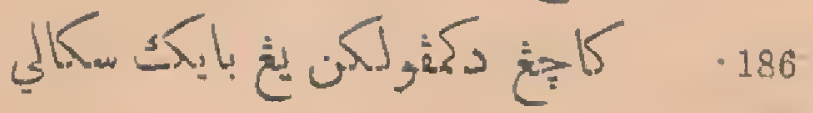

$$
u+5 \cdot 187
$$

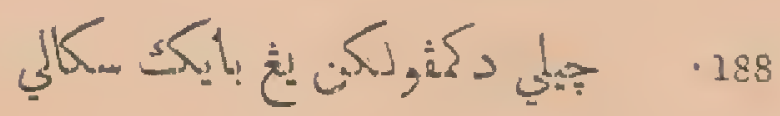

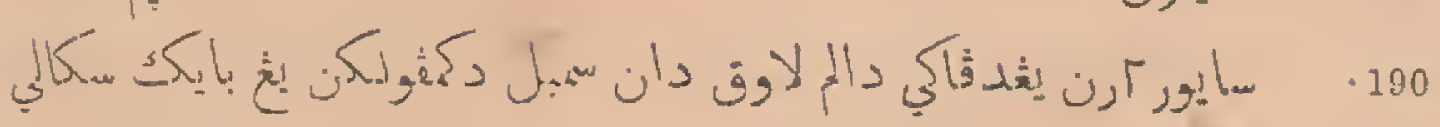

0

$2=$ h. 1

- 51

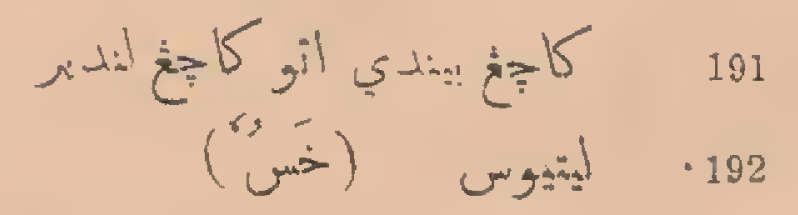

$2 F$

$2 \quad 114$

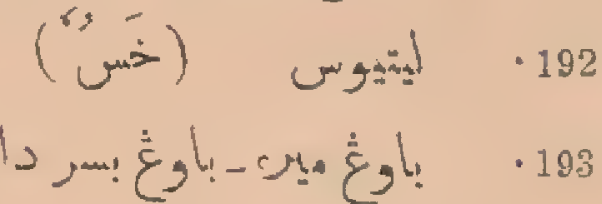

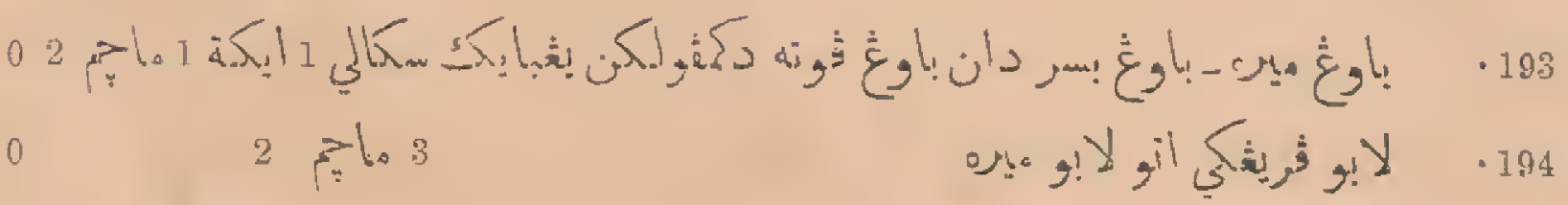

0

$2 \quad \because 10$

i!s $\cdot 195$

3

56

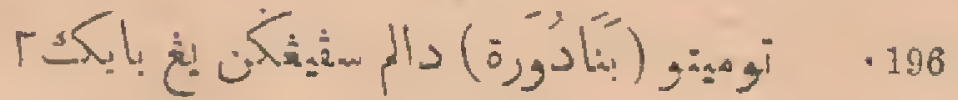

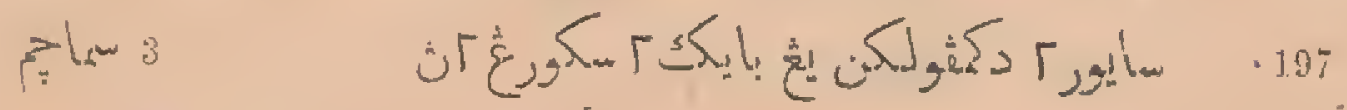

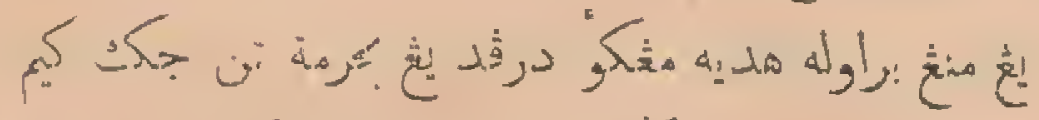

3

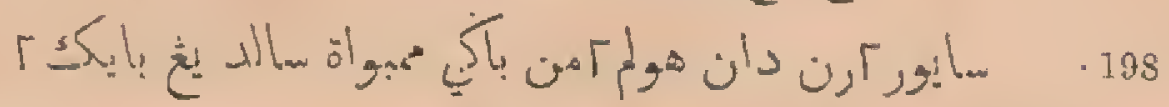

199

3

12

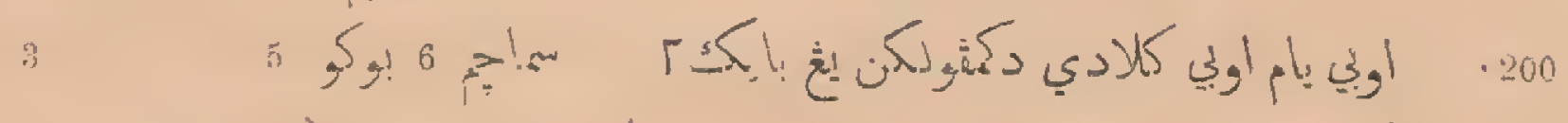

3

(j)

$9: 1.5$

2

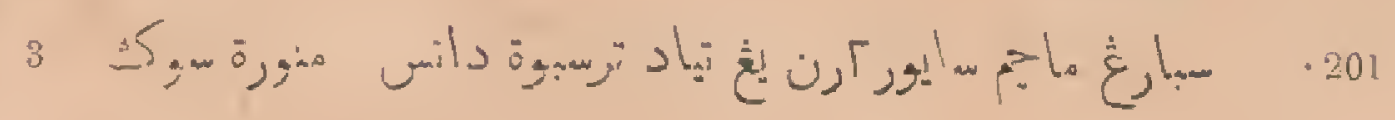

C

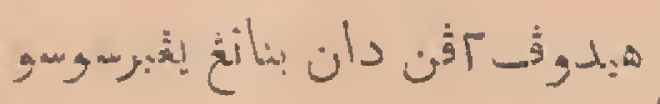

0

20

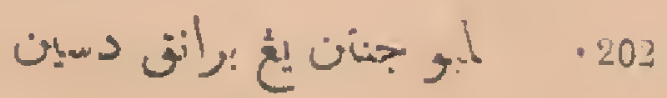

$B 2-11 / 178$ 


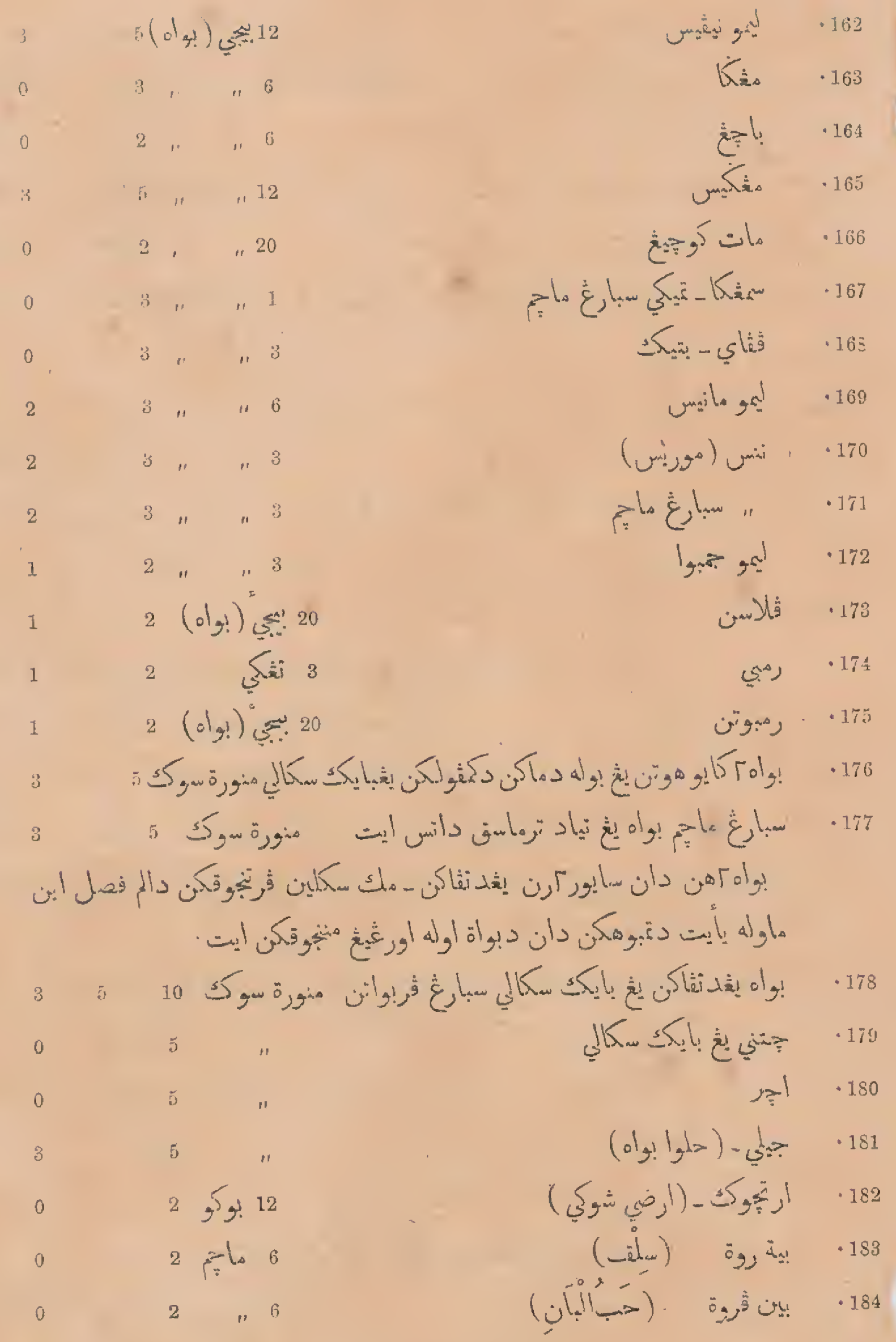




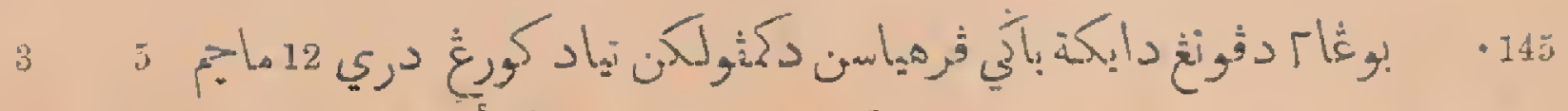

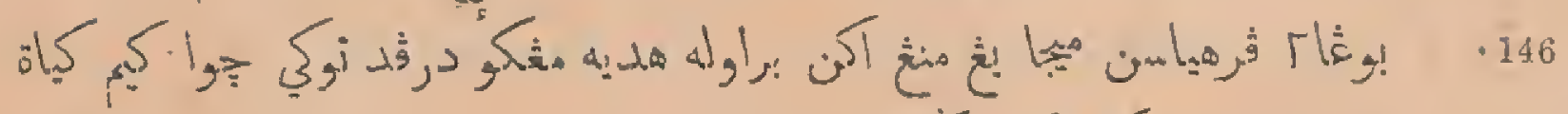
0 3147

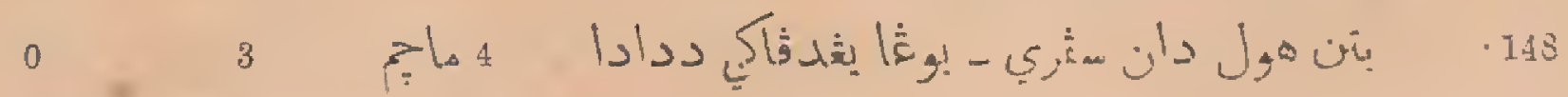

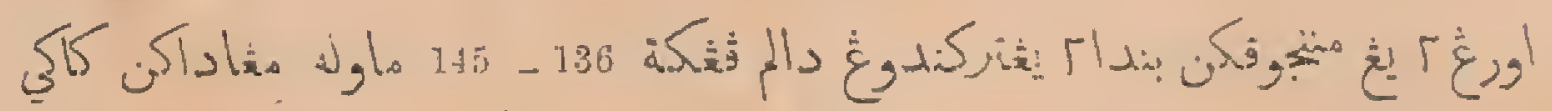

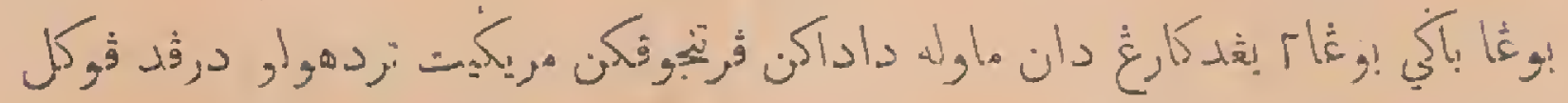

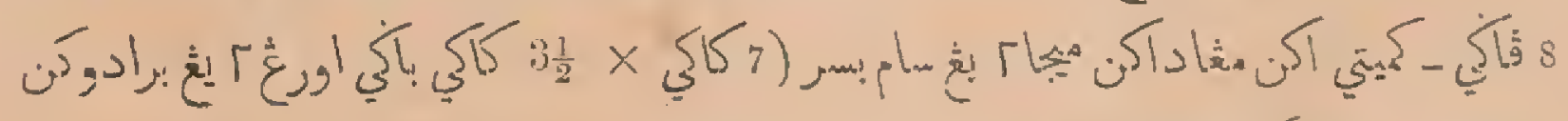

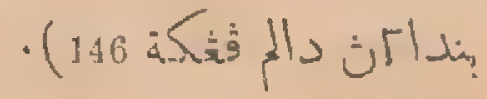

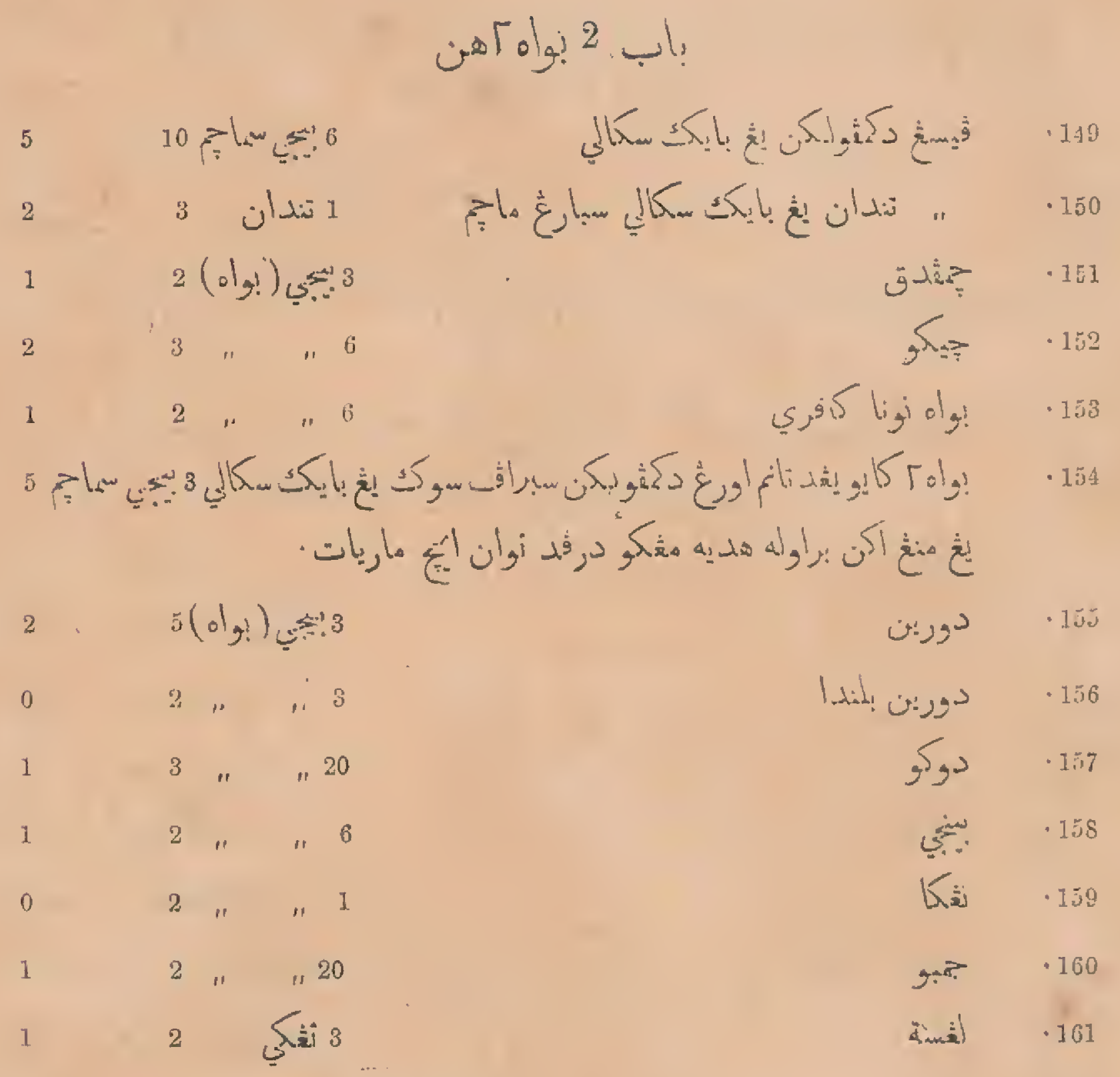


9

$\begin{array}{llll}0 & 5 & " 1 & 1 \\ 0 & 3 & \text { " } & 6 \\ 0 & 3 & n & 6 \\ 3 & 5 & " & 3 \\ 0 & 3 & " 1 & 1 \\ 0 & 3 & n & 6\end{array}$

ل1 124

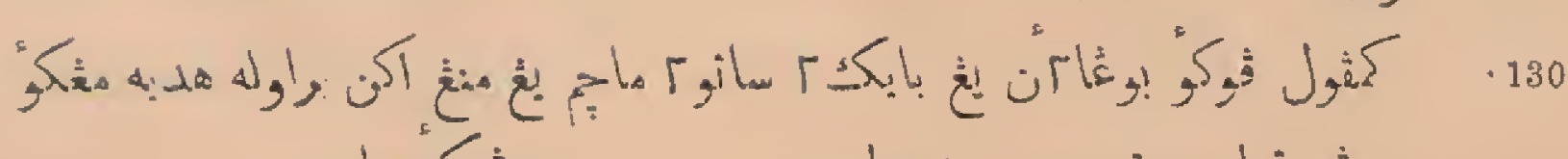

3 plis 6

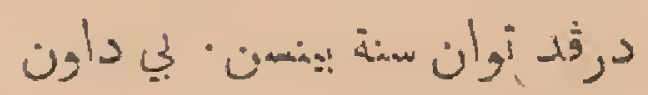

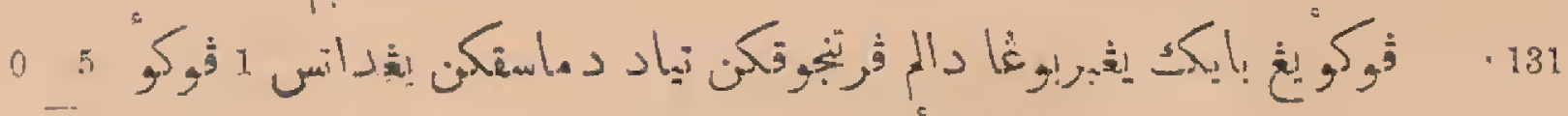
أنو

$3 \quad 5 \quad 5 \%$

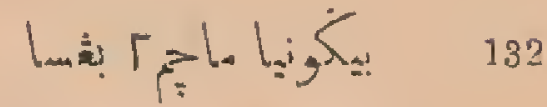

0 31

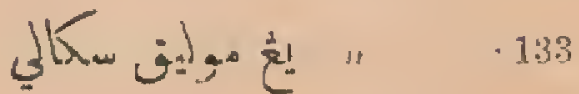

134 5 8

35 , 3 , g.

$0 \quad 5 \quad 35,0$

- 135

客

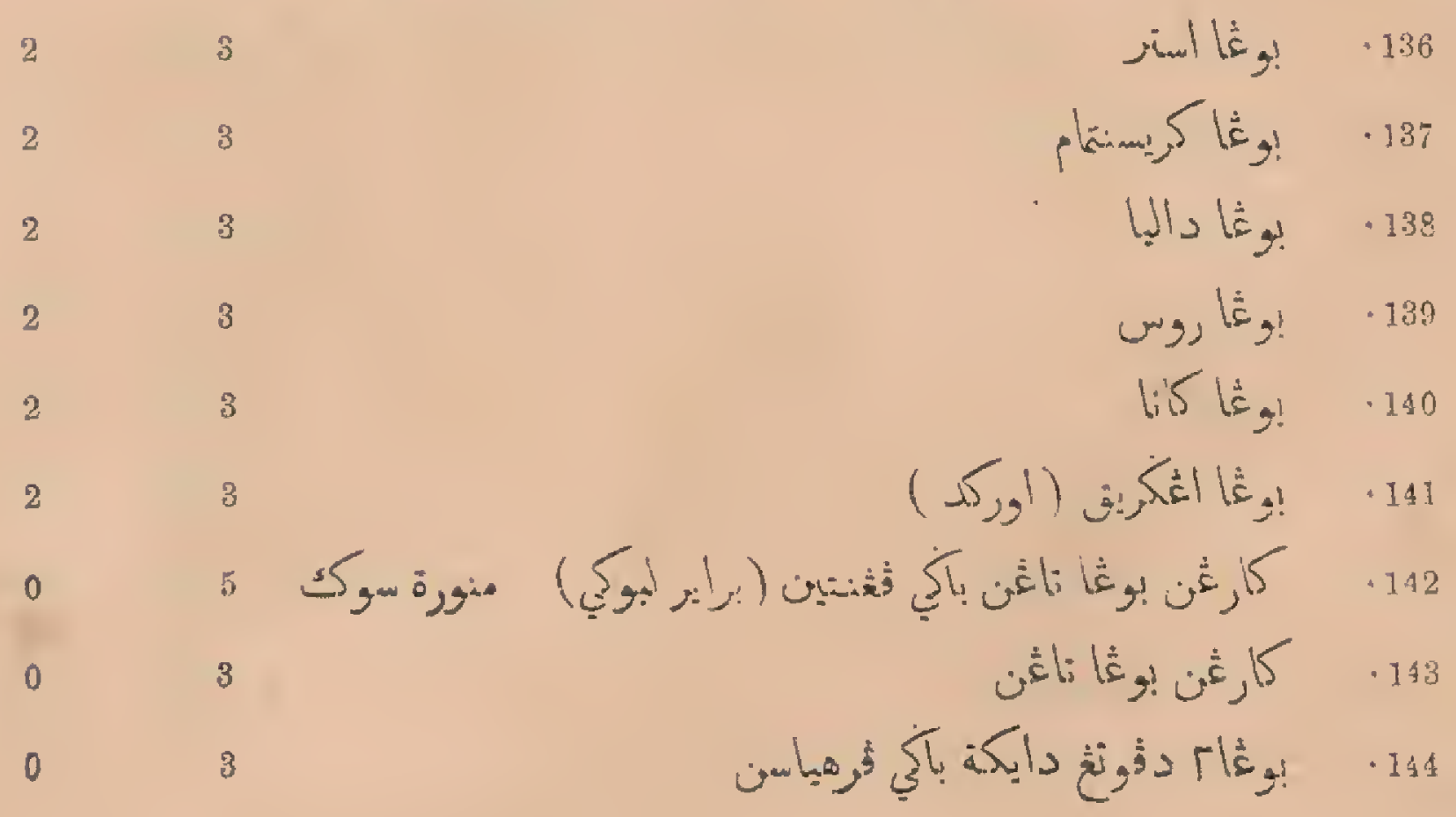


8

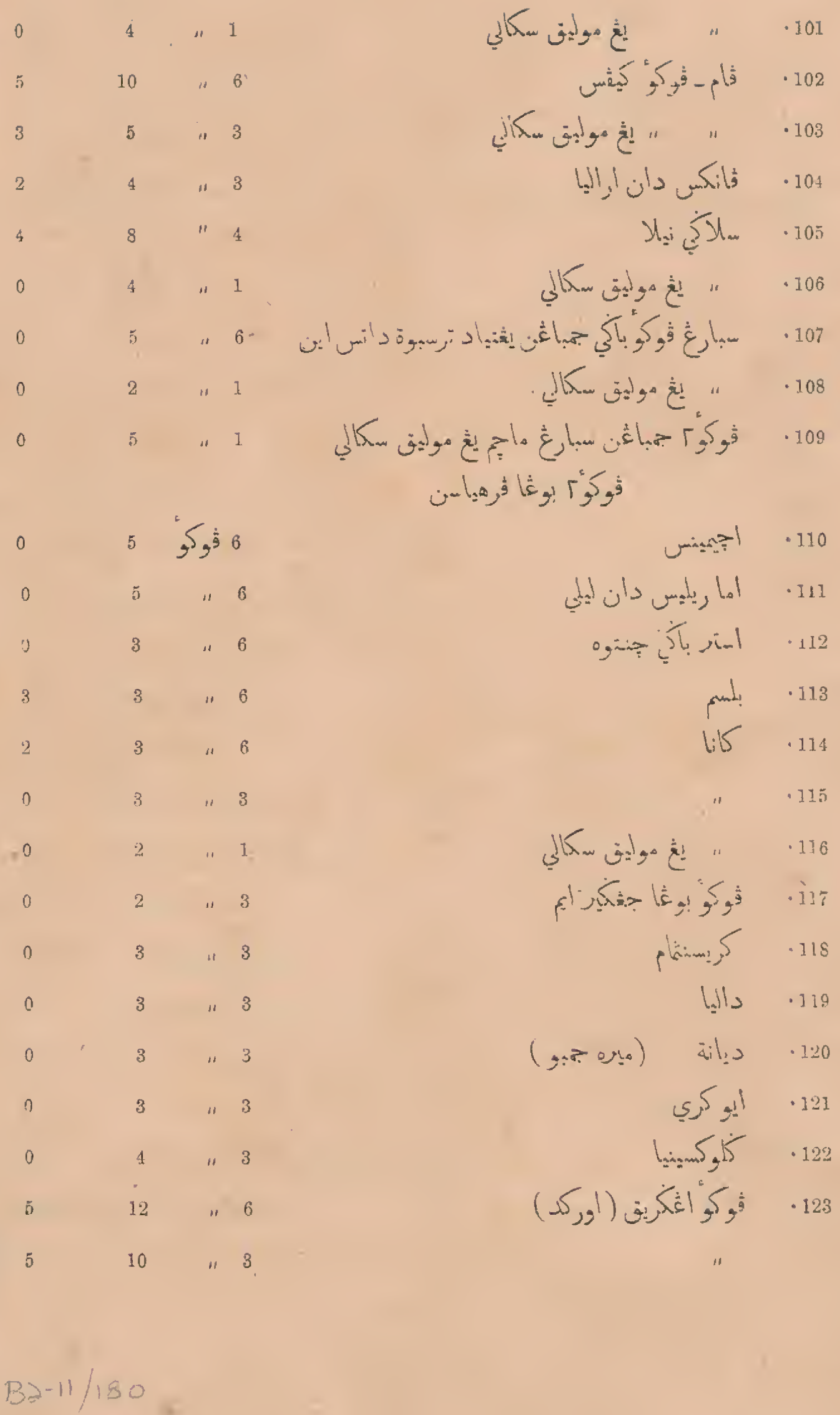


7

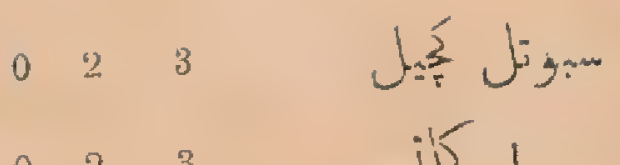

$$
\begin{aligned}
& \text { its } \\
& \text { wig } 25
\end{aligned}
$$

أن

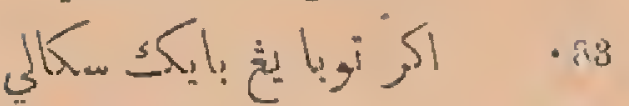

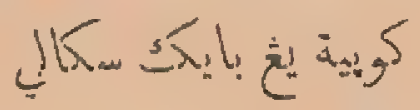

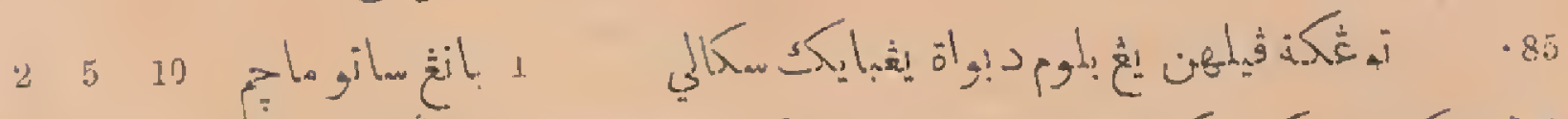

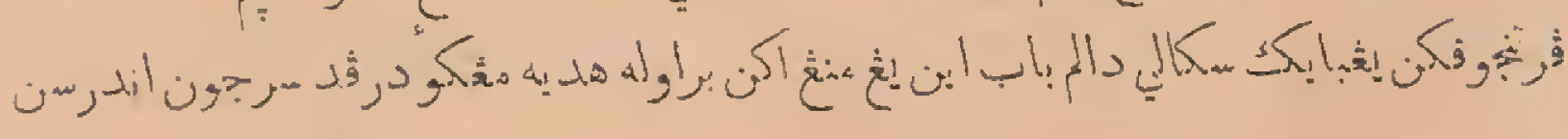

13 ن

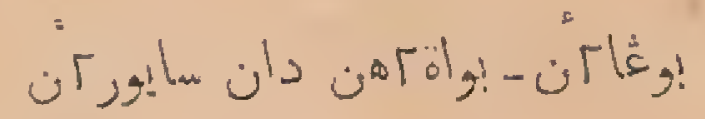

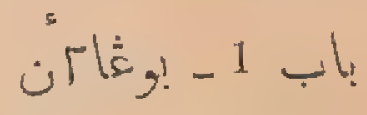

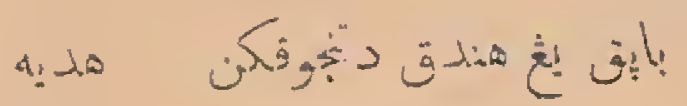

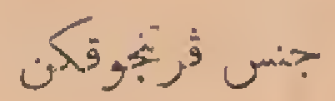

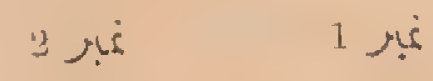

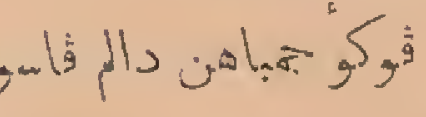

35 क 50

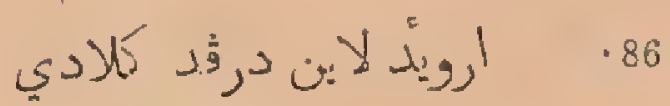

4 "Sو 1

كأل

50

كادي جماثعن

$\cdot 87$

.

65

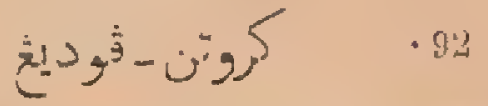

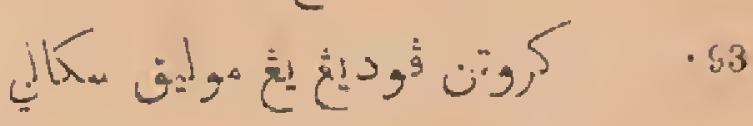

96 94

35113

96

(8)

3

$8 \quad 116$

" " 198

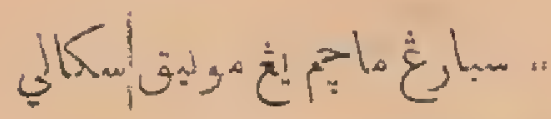

.99

س 100 
6

·

$0 \quad 0 \quad 10 \quad$ J $=5$ Jigm ل61 62

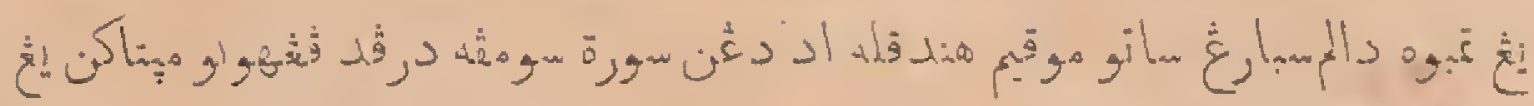

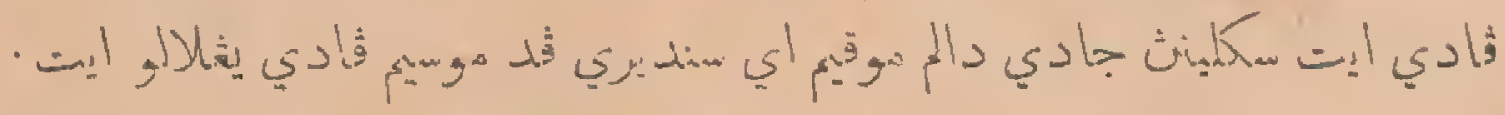

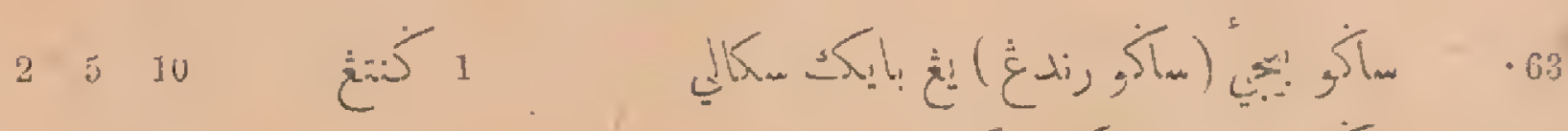

$2 \quad 5 \quad 10$

" 1

管

$13: 500$

65

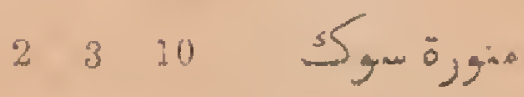

每

$\begin{array}{lll}0 & 0 & 30\end{array}$

îำ 10

(67

1010 pritis

rel.

$\begin{array}{lllll}1 & 3 & 5 & 3 & 3\end{array}$

.69

$\begin{array}{llll}1 & 3 & 5 & 11\end{array}$

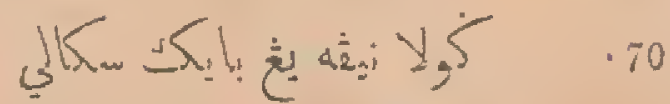

$135 \quad$ " 2

. . . 71

$135 \quad$ " 2

لئس

$2 \begin{array}{llll}2 & 3 & 5 & \end{array}$

淀

025

ث, A 4

135

站

- . 75

$2 \quad 5 \quad 10$

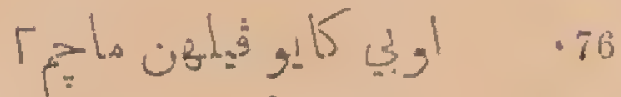

135

ت

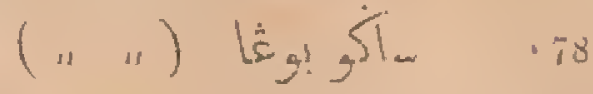

135

135

11 1

1 dै 5

॥ 1

每

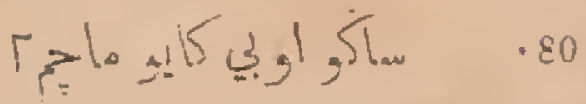

$35 \quad 10$

11 1

"1 1

.81

$B z-11 / 181$ 
5

$\begin{array}{lllll}2 & 5 & 10 & \text { " } 1 \\ 1 & 3 & 5 & \ldots 1 \\ 2 & 5 & 10 & \vdots & \vdots\end{array}$

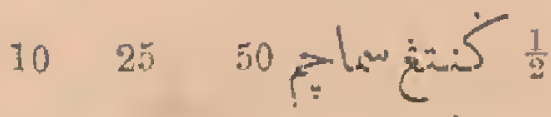

$135 \quad 5 \quad \dot{c} 5 \frac{1}{2}$
لك 150

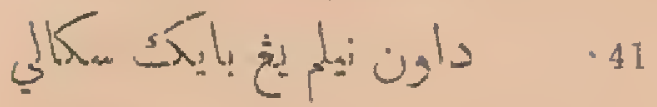

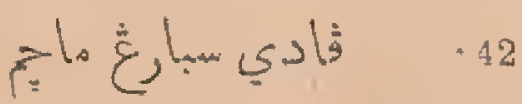

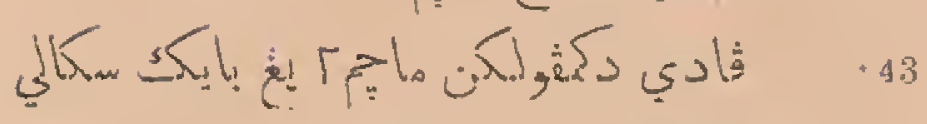

لك.

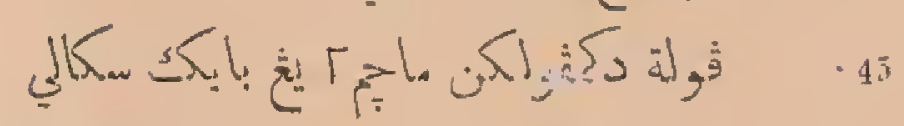

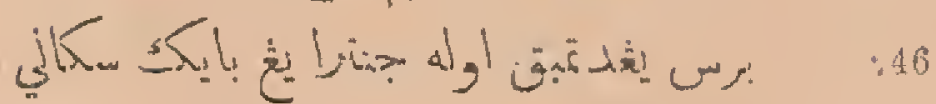

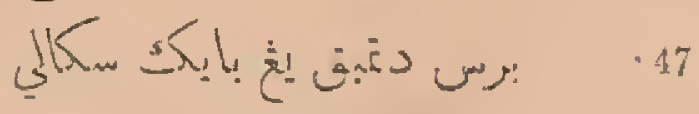

ل

49

لي

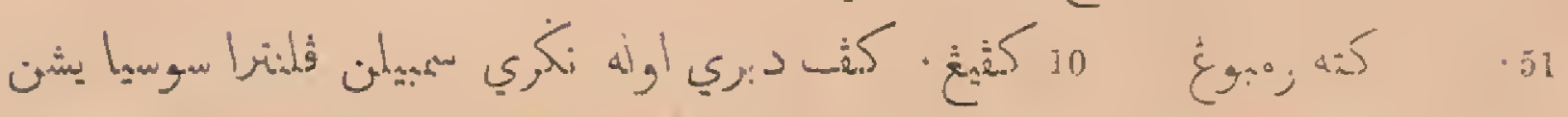

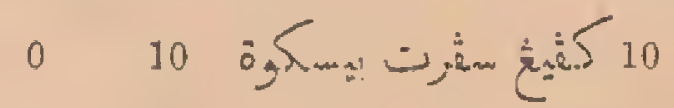

घ90, 4.5

$0 \quad 10 \quad$ لإم 10

$\dot{\xi}, 0,405$

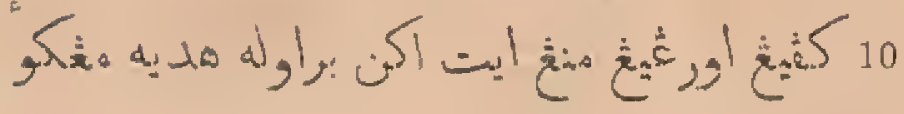

$1,10.5$

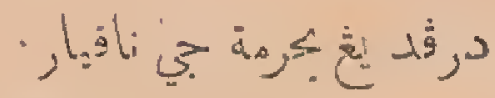

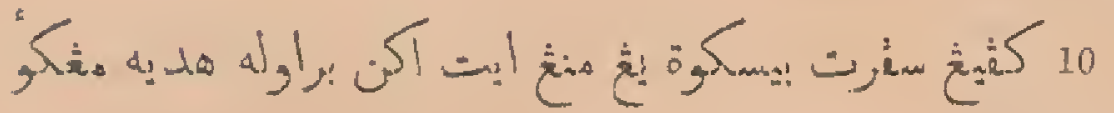

1,1905

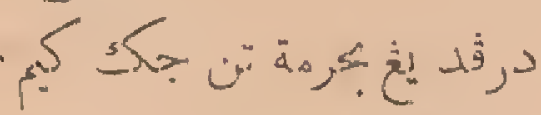

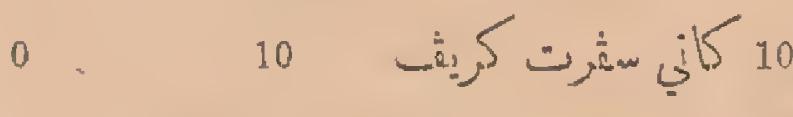

$1,194.5 \quad \cdot 55$

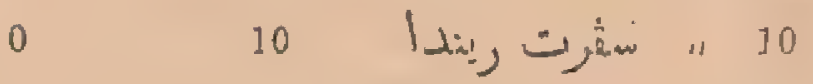

" .56

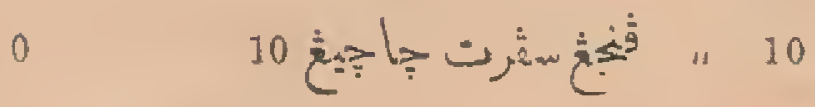

$\begin{array}{ll}\text { " } & .57 \\ \text { " } & .58\end{array}$

0

10

Lls

$0 \quad 10 \Gamma J \pm \leq$

.52

60

دراق 
4

1

$P=\cos \cdot 16$

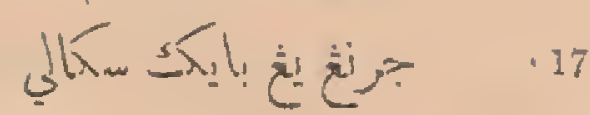

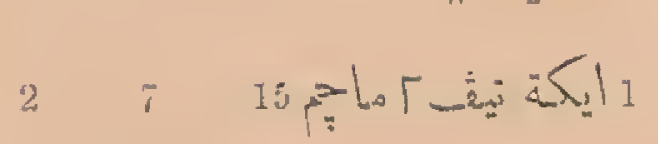

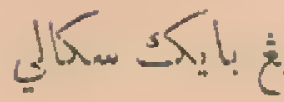

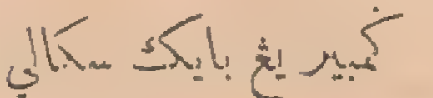

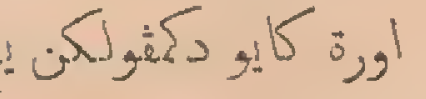

$\cdot 18$

$\begin{array}{lll}1 & 3 & 0 \\ 2 & 5 & 0\end{array}$

$\cdot 19$

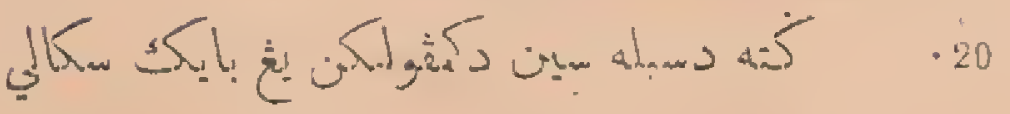

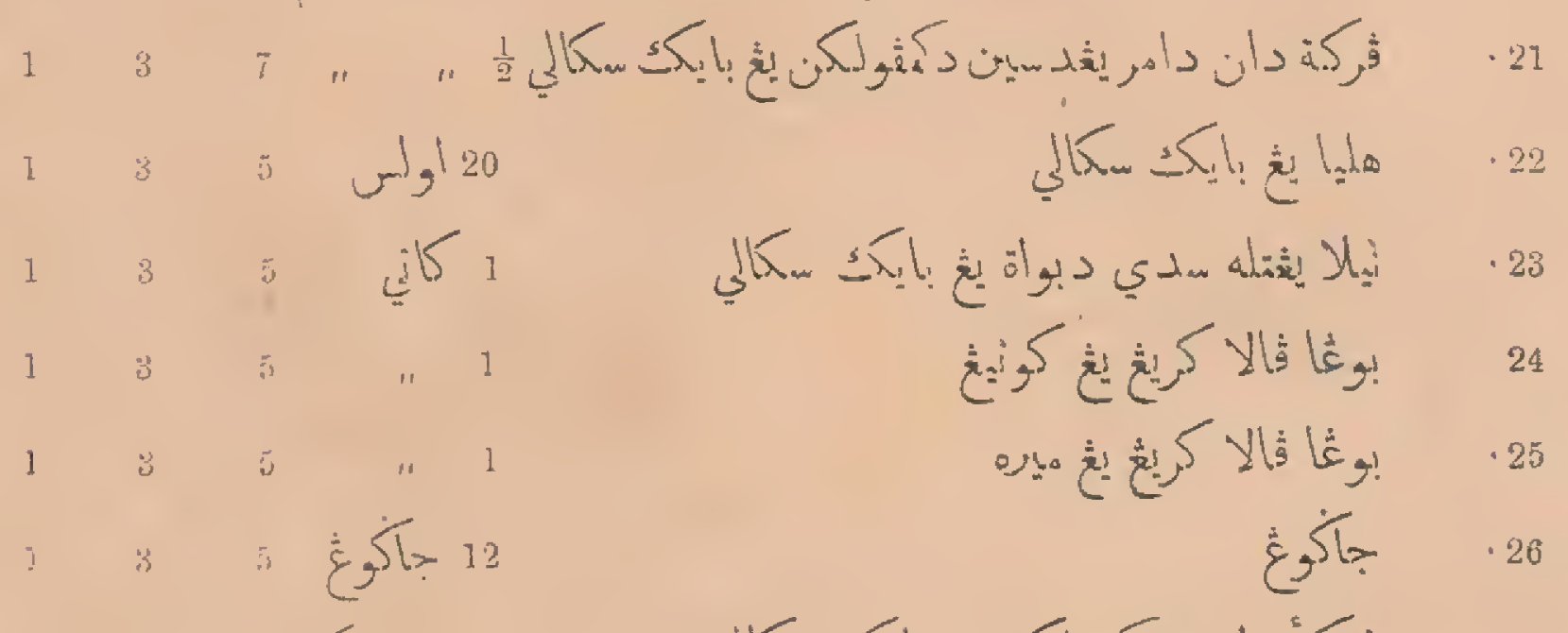

2 i) 10.5 ,

$1350 \lg 50$

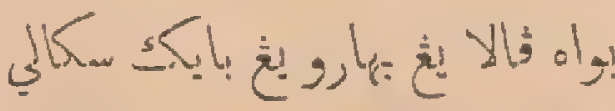

$2510 \quad 50$

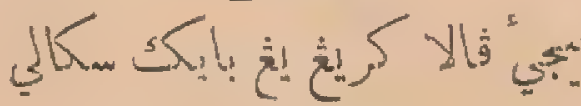

$\cdot 28$

$2010 \mathrm{~J}=5 \mathrm{~S}$

30

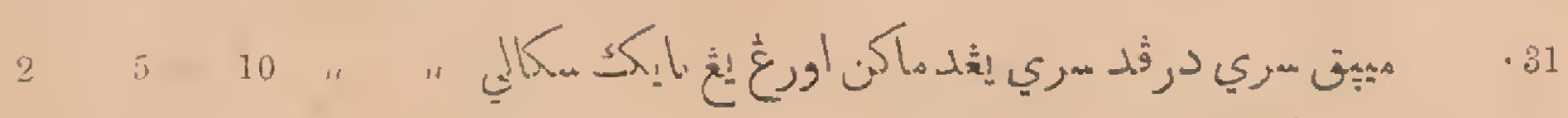

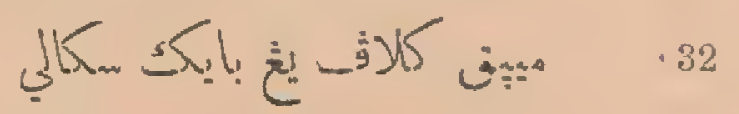

8s

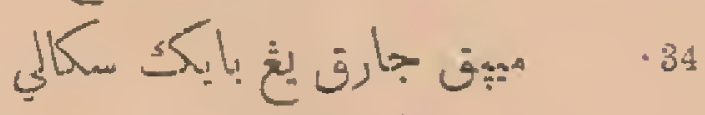

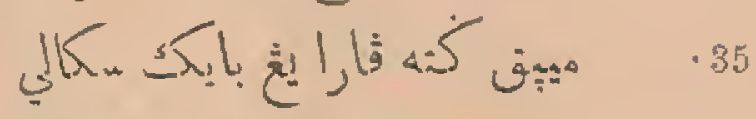

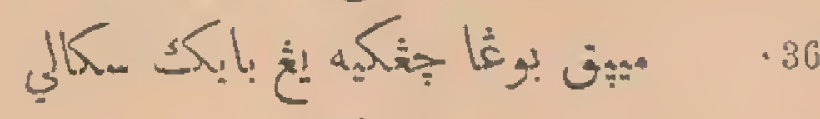

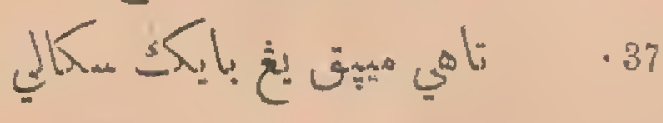

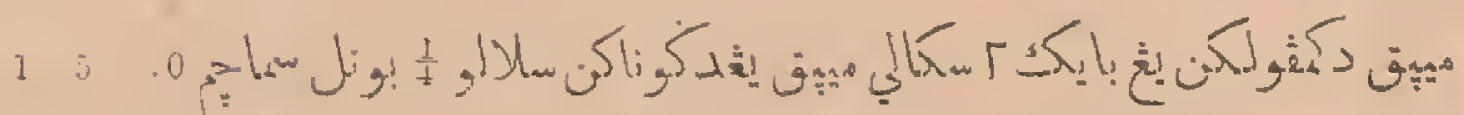

.38

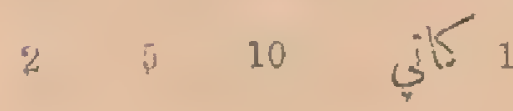

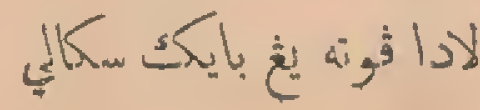

$\cdot 39$

$B 2-11 / 132$ 


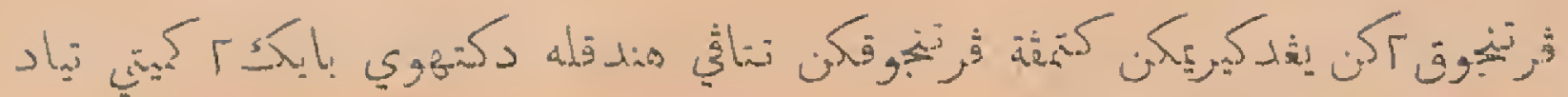

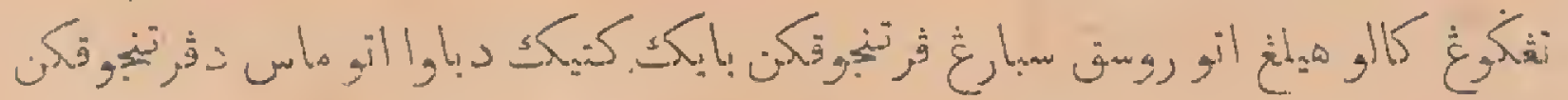

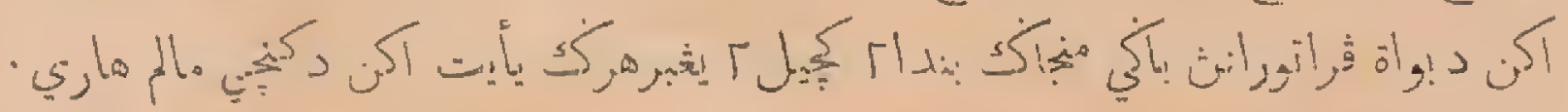

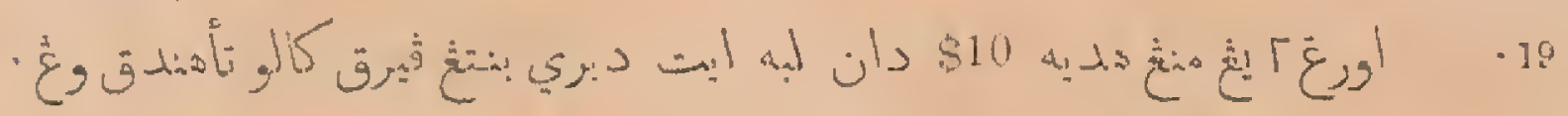

\section{Jy \\ A}

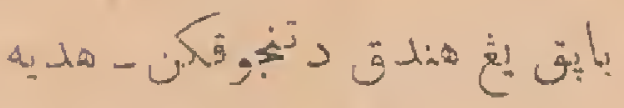

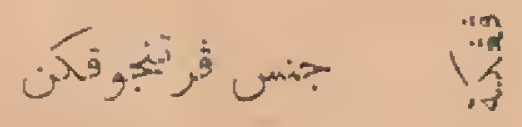

$\begin{array}{ll}32 & 2\end{array}$

$\$ \$ \$$

12525

1

$125 \quad 5 \quad$ is 1 لك

$25 \leq 5$ is 3

$\begin{array}{llll}1 & 2 & 3 & \end{array}$ $($ (r)

255 i 15

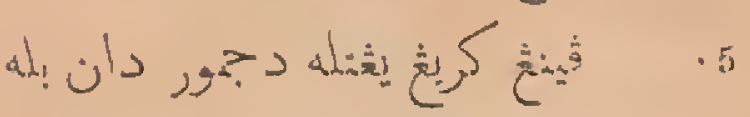

$2510 \quad$ " $\frac{1}{2}$ 6

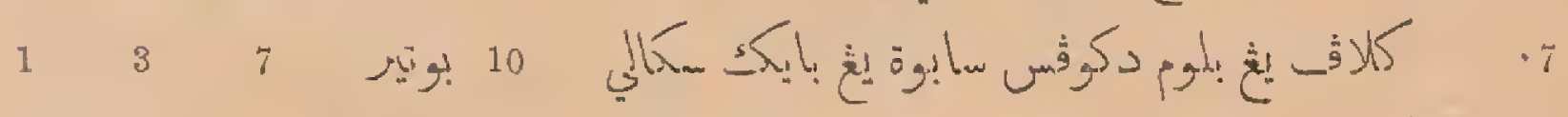

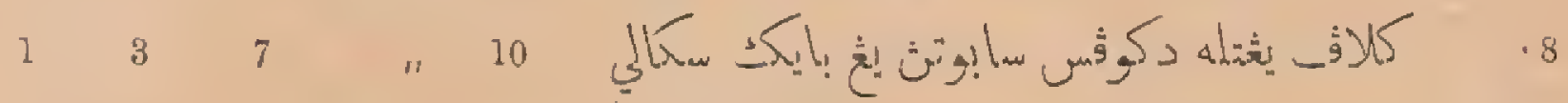

$\begin{array}{llllll}9 & 5 & 0 & 0\end{array}$

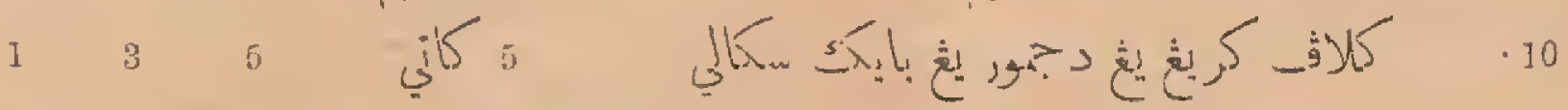

111

$1 \quad 3 \quad$ " 512

$25 \quad 10 \quad 1013$

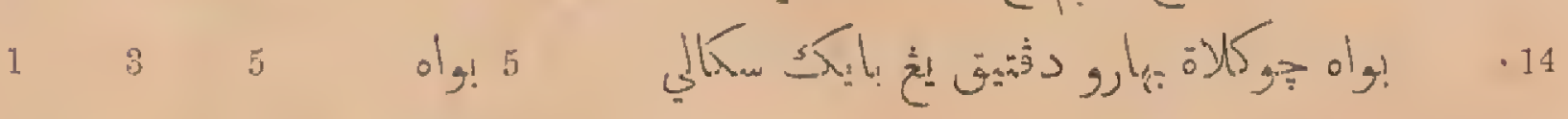

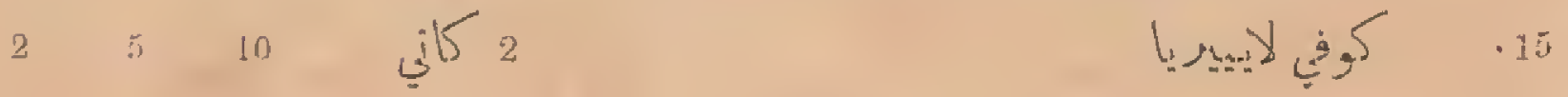


2

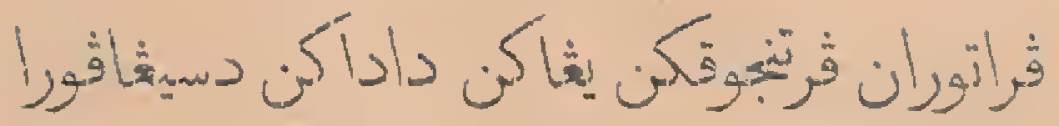

—_-

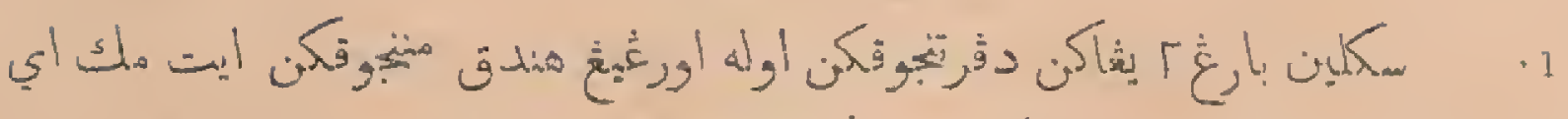

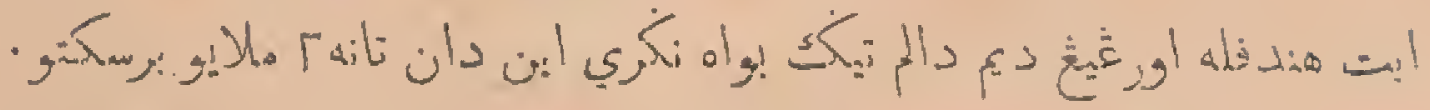

2

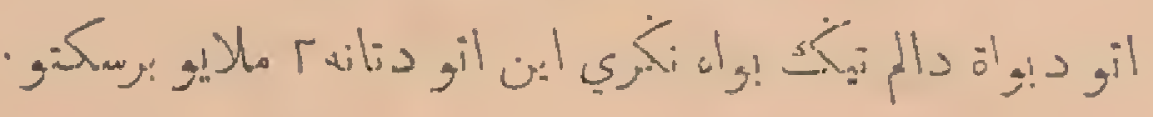

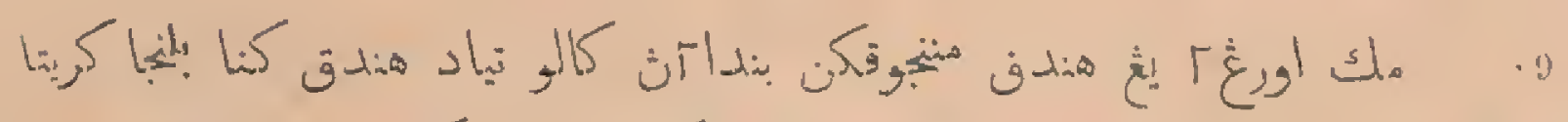

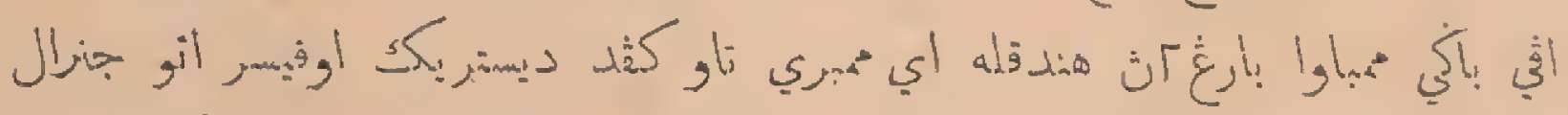

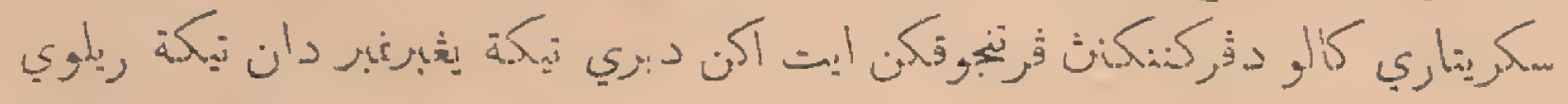

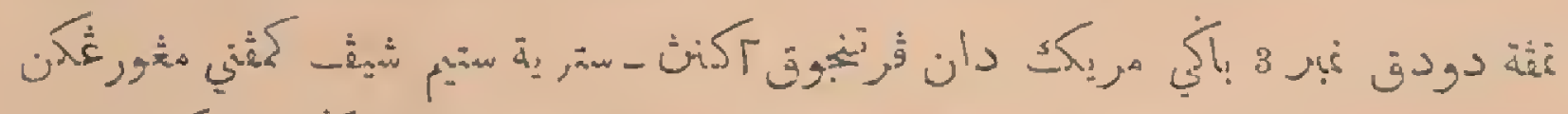

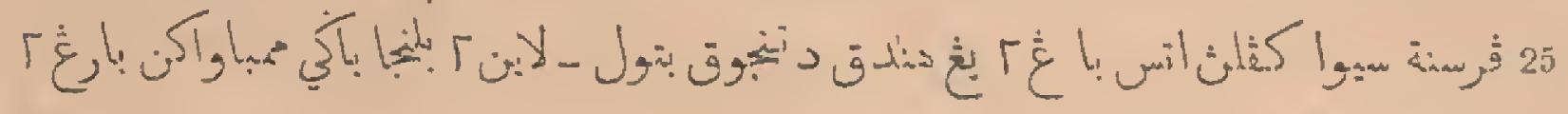
إيت الكن دقيكري أوله كيتئي

10

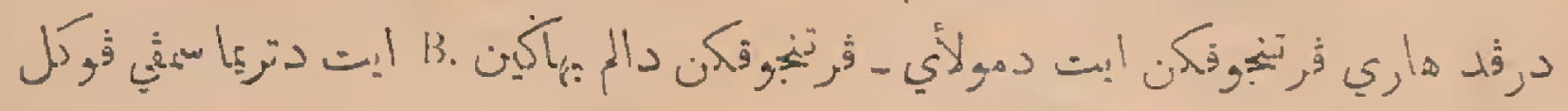
8

12

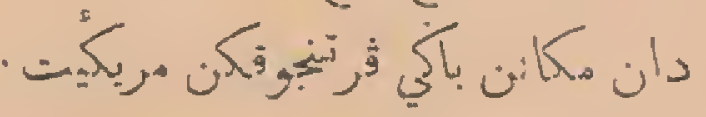

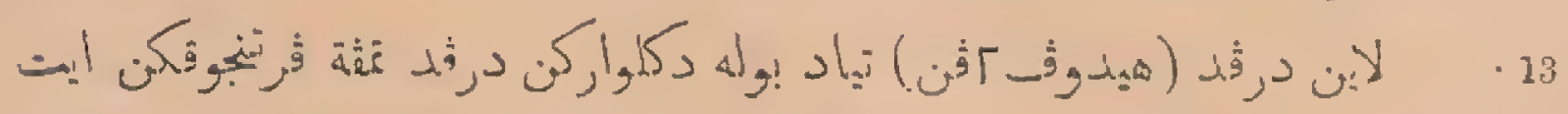

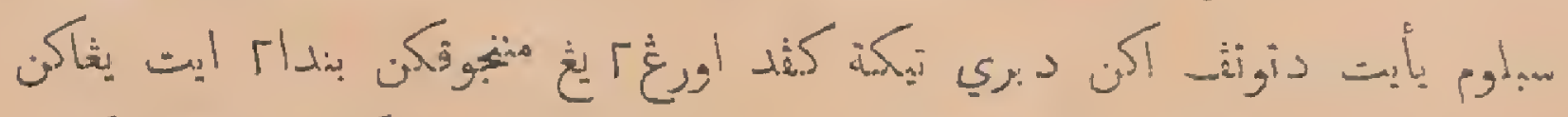

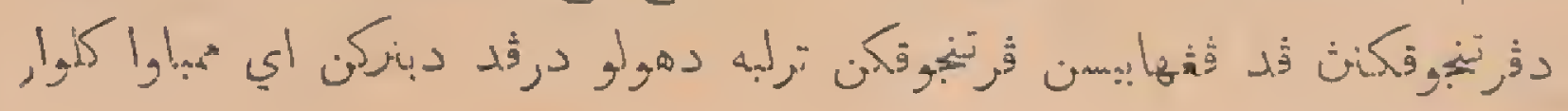

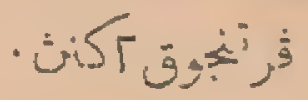

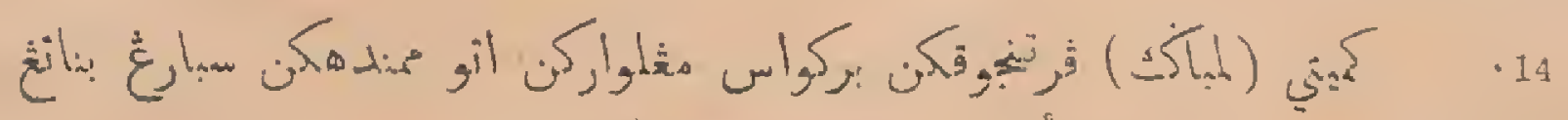

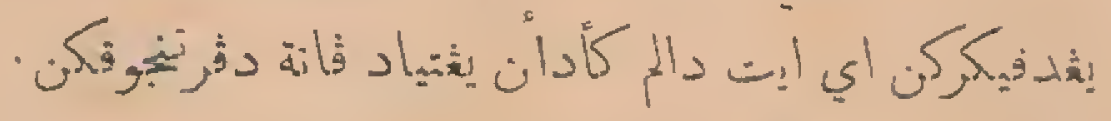

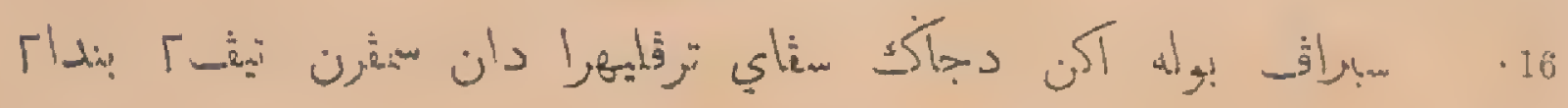
$82-11 / 183$ 
دفر هلدية

-

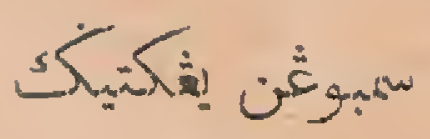

بإ.

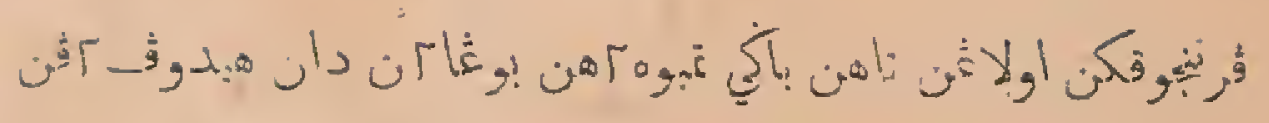

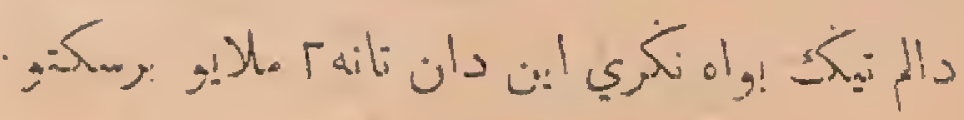

1906

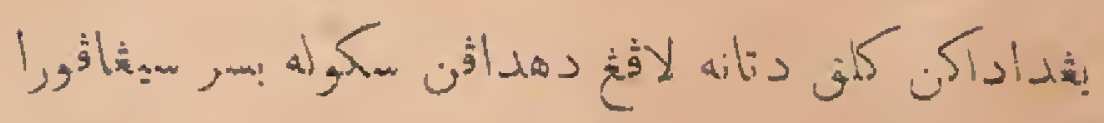

4.

1906 16 19

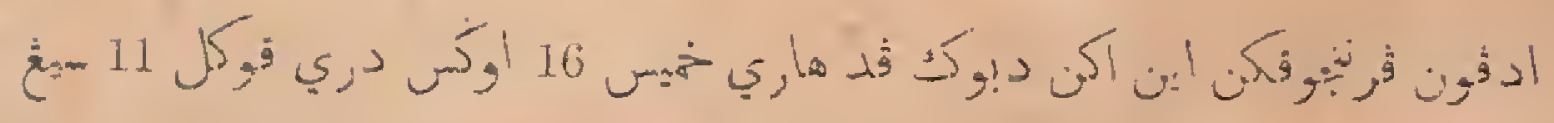

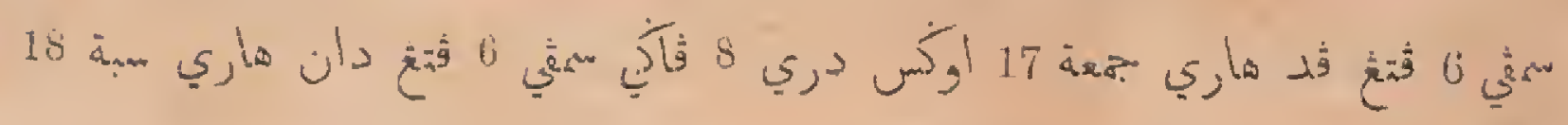

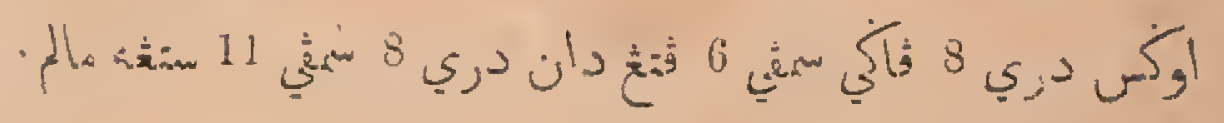

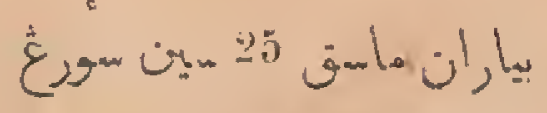

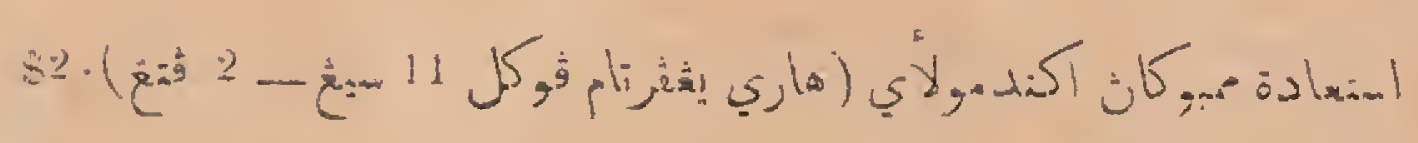

لئل

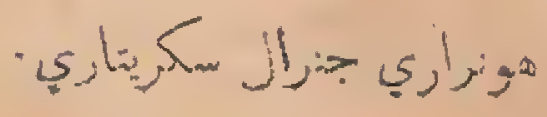



$4-10,03$

$-$

سمبوغن

بن

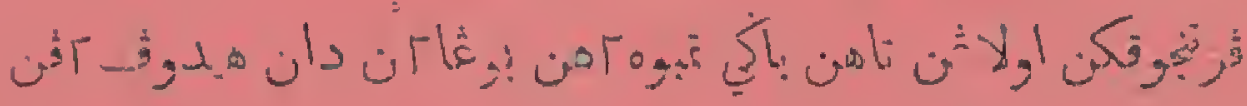

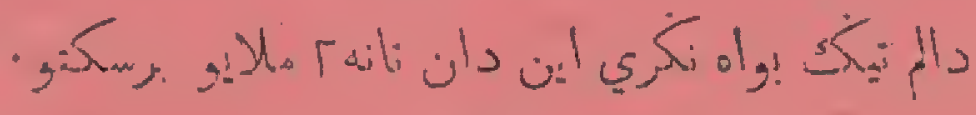

1.906

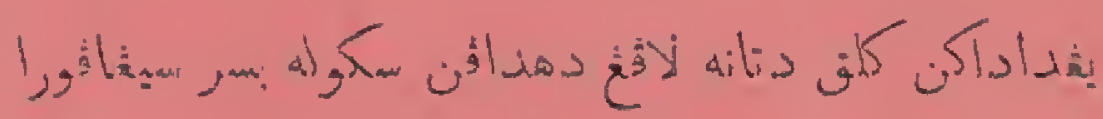

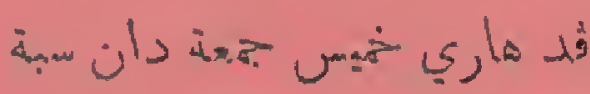

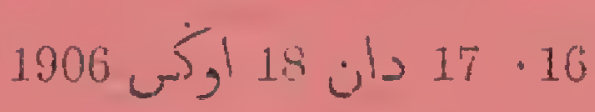

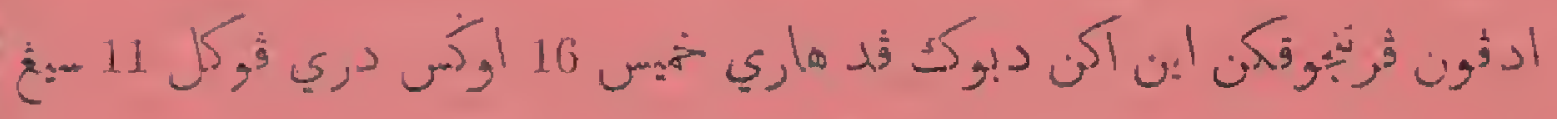

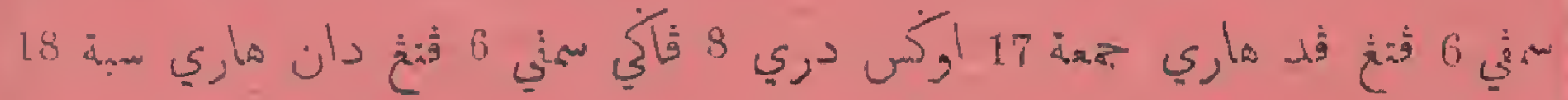

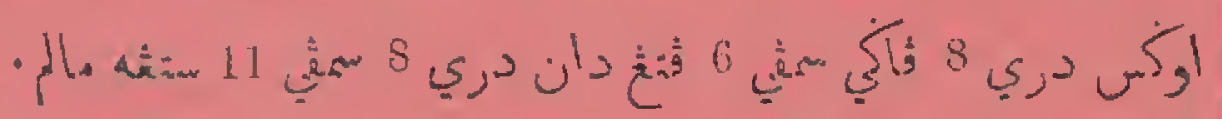

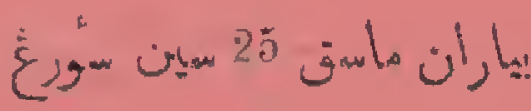

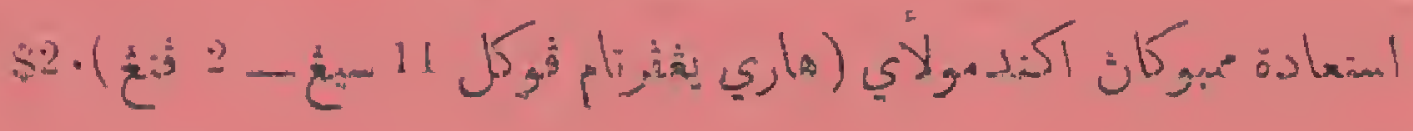

أن

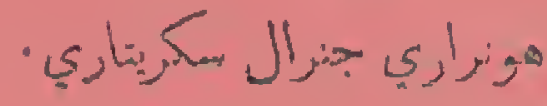

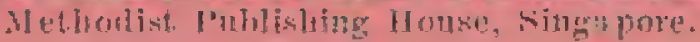


AGRICULTURAL -BULLETIN

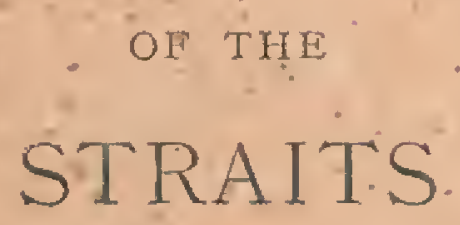

FEDERATED MALAY STATES.

\section{THIRD JOINT ANNUAL AGRI-HORTICUL- TURAL SHOW OF THE STRAITS SETTLE- MENTS AND FEDERATED IIALAY* STATES, 1906.}

Rafrles Reclamation Ground, Singapore, Thursday, Friday and Saturday igth, ifth aNd isth of August.

\section{President.}

The Hon'ble The Colonial Secretary -Capt. Y̌ oung:

$$
\text { Chaimun-Hon. John Anderson }
$$

\section{Committec.}

Col. Alex Murray

Dr. D. J. Galloway

Col. Pennefather

W. C. Michell

H. Payne-Gallwey

Hon. Tan Jiak Kim

Seah Liang Seah

Choa Kim Keat

J. Polglase

Hon. A. T. Bryant
St. V้. B. Down

W. Nañsồn

W. D. Barnes .

W. G. St. Clair

John Somerville

Hans Becker

Rajah Hitam Nongchi

C. B. Buckley

R. W. Egerton Eastwick

Rev. Father Gex

Honorary General Secretary:-H. N. Ridley

Honorary Treasurer:- $\mathrm{Wm}$. Macbean 
Honorary Secretary-Division A-W. Diepenheim

$$
\begin{aligned}
& \text { B-Dr. Ellis } \\
& \text { C-P. S. Falshaw } \\
& \text { D-L.E. Gaunt (Horses) } \\
& \text { D-F. Filton (Dogs) } \\
& \begin{array}{r}
, ", \\
-\quad, "
\end{array} \\
& \text { E-G. A. Hall } \\
& \text { E-E. F. H. Edlin (Schools) } \\
& \text {, F-C. Wreford }
\end{aligned}
$$

Division $A$.

* D. W. Lovell, Robert Little, W. Dumman, L. C. Brown, IV. Diepenlieim, E. Bramall, A. J. C. Hart, F, H. Darke.

\section{Division $B$.}

St. V. B. Down, W. Nanson, Rajah 'Hitam Nongchi, Chua Kim Keat, Dr. Ellis.

\section{Division C.}

Cattle-H. A. Crane, P. S. Falshaw.

- Poultry-C. L. Van Cuylenburg, W. L. Hawtrey.

\section{Division $D$.}

Horses.-Major General Inigo Jones, C.V.O., C.B.

Capt. Leveson Gower (The Sherwood Foresters).

$$
\begin{gathered}
\text { C. Sugden, } \\
\text { Major O. C. Woolley Dod, } \\
\text { L. E. Gaunt, } \\
\text { P. S. Falshaw, } \\
\text { Lt.-Col. Pennefather. }
\end{gathered}
$$$$
\text { J. B. Young. }
$$

Dogs.-J. D. Saunders, Esq., K. A. Stevens, Esq., Capt. The Hon.

C. Baring, F. Hilton, Esq., P. S. Turnbull, Esq.

\section{Division $E$.}

A. Hale, W. S. Coutts, L. Wray,

(Schools) E. F. H. Edlin, Rev. H. C. Izard, C. M. Phillips.

Division $F$.

A. E. G. Keller, K A. Stevens, A. J. Macdonald, F. C. Wreford. 


\section{The Agri-Horticultural Exhibition, igo6.}

The third of the Agri-Horticultural exhibitions of the Colony and Federated Malay States was held at Singapore on August the I6th, I $7^{\text {th }}$ and $I$ Sth, on the reclamation ground in front of the Raffles Hotel. The weather proved fine for the greater part of the time but a heavy shower during the second day caused the ground to become for a time very muddy and wet. This, however, interfered but little with the enjoyment of the general public whose interest in the exhibition was attested by the great crowds which thronged to the entrance on all the three days, giving the working committee no small labour in taking the tickets and giving change. Two ticliet offices were opened but these rvere insufficient for the purpose. A large number of subscribers and exhibitors and their assistants had free tickets but besides these no less than $\$ 5,300$ were talien at the gates, so that upwards of 50,000 people visited the show grounds. Qn the previous occasions two exhibitions at Kuala Lumpur and Penang the gate money amounted respectively to $\$ 300$ and $\$ 622.20$. The price of admission to the opening ceremony was $\frac{\mathbb{T} 2}{2}$ and on all other occasions 25 cents a head. On the second day the school-children were admitted free and added not a little to the crowd of visitors.

Promrammes of events were sold at ten cents each and were reaclily bought. In spite of the vast crowd which attended the exhibition there was no disorder or trouble of any lind; and there were no thefts of any importance. The few losses which did occur of articles in the exhibition were mainly due to the carelessness of exhibitors, who sometimes delayed for days after the exhibition was over to claim their exhibits.

Buildings.-The general arrangement of the buildings tvas designed by a committee consisting of Mr. R. A. J. Bidwell, Mr. Down and Mr. Buckley.

The plan and form of the various buildings was designed and executed by Mr. R. A. J. Bidwell, to whose untiring energy the success of the exhibition was largely due. The plan was very superior and in many ways much more convenient than that of previous shows, and the whole building was on a much more extensive scale. The Agricultural produce and native industries were housed in a long shed of poles and attap roofing, 450 feet long and 50 feet wide, half devoted to each division.

The house for plants and vegetables, 225 feet long and 50 feet wide with two projecting wings of 50 square feet. The house for cut flowers and vegetables was 100 feet long by 50 wide. 50 wide.

The poultry was housed in a separate shed, 75 feet long by

The dogs occupied a space of 125 feet by 50. Besides these a very large area was occupied by the buildings of the different firms, refreshment rooms, lavatories, Secretary's office, a grand stand by the show ring, stalls for horses, and sheds for the cattle. The whole of the ground occupied was $\mathrm{I}, 400$ feet in length and 300 feet broad, or I 4,000 square yards in area. 


\section{Management.}

The Committee for the show was a very large one, but the whole work of the show really devolved on a very small number of worliers. Fortunately they had the assistance of a number of the visitors from the Native States, who materially assisted in the arrangement and judging of the exhibits.

The buildings were planned and executed by Mr. R. A.J. Bidwell and were far the best both in design and plan of any at previons shows, and very much more extensive. The general arrangements for the exhibition were effected by Mr. Bidwell, Mr. Diepenheim, the Hon. Secretary and Mr. J.S. Isaac (Clerk of the Gardens Department) assisted after the opening of the exhibition by Messrs. Marriott, Hall, Edlin, Hilton and Buckley.

The arrangement and judging of exhibits were as follows:Agricultural Section:-Mr. L. Brown, A.J.C. Hart, D. W. Lovell, F. H. Darke, Hon. Sec., Mr. W. Diepenheim, Mr. E. Bramall. Fruits, flowers and vegetables:-Mr. Down, Dr. Ellis, Mr. Pereira, Mr. Campbell, Mr. Main, Mr. Chua Kim Keat. Rubber:Mr. Zacharias.

Native Industries:-Messrs. Collinge, Wray, Simmons, Chevallier, C.M. Phillips, IV.S. Contts, E. F. H. Edlin. Cattle:Mr. Falshaw, Mr. H. A. Crane. Poultry:-Mr. W. L. Hawtrey, Mr. C. L. van Cuylenburg.

Horses:-Mr. Gaunt, Mr. Sugder, Payne-Gallwey, J. B. Young, F. Hilton, J. D. Saunders, Major-General Inigo Jones, Colonel Pennefather.

Dogs:-Messrs ]. D. Saunders, K. A. Sterens, Capt. Hon. C. Baring, F. Hilton and P. S. 'Turnbull.

The thanks of the Committee are due to the Government of the F. M. S. who were good enough to carry exhibits free and exhibitors over theil railways and to the Manager of the Singapore Railway who did the same. The Straits Steamship Company also gave a rebate of 25 per cent. of the freight of exhibits and the native visitors had also the use of the "Sea Belle" to come and return from Malacca and Penang.

The number of native visitors from the Native States was about 750, artel there was some difficulty in housing them at first. The only a vailable building in Singapore large enough for them was the new Tan Tock Seng Hospital ward, but this was so far away from town as to be practically inaccessible to them, and eventually they were housed in various parts of the town, their expenses being paid out of the exhibition funds.

There were also visitors from Ceylon, Burmah, India and two delegates from the Botanic Gardens of Buitenzorg and many others from the Dutch Colonies.

\section{Exhibitors.}

The whole of the Federated Malay States and the different parts of the Colony sent exhibits to the show, viz., Singapore, Malacca, Penang, Province Wellesley, Johore, Muar, Pahang, 
Negri Sembilan, Selangor and Perak. The Siamese States in the north of the Peninsula were however not represented. Of these Perak came first in competition winning 133 prizes of the value of 837 dollars and besides carrying off the Governor's Cup. It is to the credit of this State too that nearly every exhibitor succeeded in securing a prize, showing a careful selection in what was to be sent, by the district officers. Eighty of the prizes were taken by Malays and twenty-one schools also carried off prizes, only seven of the prize winners in this State were Europeans. This in itself shows that the exhilitions are well considered by the natives and that they take a great interest in the competitions. The total number of exhibitors from Perali was three hundred and sixty. four. The exhibits from the Native States would have probably been poor were it not for the energy and perseverance of the various district officers and other officials of the Federated Malay States also. Singapore came next to Perak both in number of exhibitors and in the prizes taken. In this calculation horses and dogs naturally were more easy to show at the seat of the exhibition than from elsewhere, are excluded or Singapore would be first. The small amount of cultivation of agricultural produce in Singapore as might be expected lowered its average, though it had some advantages in the horticultural section. There were 252 exhibitors, the greater part of whom were Europeans, and 124 prizes fell to them valued at 647 dollars.

Penang comes next with 75 prizes but only Io6 exhibitors, of whom the greater number were natives.

Then Malacca with 13 I exhibitors and 49 prizes almost all of whon were Malays.

Selangor sent eighty-nine exhibitors only who obtained 39. prizes of the value of 73 dollars.

Negri Sembilan had a hundred exhibitors but these only obtained nine prizes of the aggregate value of 56 dollars.

Johore sent exhibits from thirty-one exhibitors, all Malays, who carried off twelye prizes valued at 49 dollars.

Pahang which is in the worst position of any State for sending exhibits had sent three exhibitors of whom one scored a first prize for wild guttas.

The total number of exhibitors was 1,006 . This was a smaller number than that of Penang on the previous year, but the fact that while Penang is well supplied with rail to most parts of the Peninsula, exhibits for Singapore had to come by sea, which was inconvenient and no doubt deterred many exhibitors from sending.

\section{Opening Ceremony.}

The Exhibition was visited by His Excellency Sir John Anderson in the early morning when he made a tour of inspection of the various exhibits, and at I a.m. of August $x 6$ th he on being requested by the Hon. Captain Young, the Colonial Secretary, President of the Exhibition, formally declared the Exhibition open in a speech which we herewith give:- 
Speech by H. E. The Governor.

His Excellency the Governor, Sir John Anderson, K.c.M.G., said that this was the third time he had been called upon to receive the great privilege of performing the opening ceremony at functions similar to this one. 'Two years' ago he opened the first of these Shows, and he was suré all those who were at Kuala Lumpur then would remember with satisfaction what an excellent Show it was. Last year he had the privilege of opening the Show at Penang, and every one who saw that one must admit that Penang made an immense advance on the first one. That day they bad the third. This Show had now come to be regarded as an annul exhibition and it had now been fairly established as such. He was sure that those who had been privileged to see the produces of the two previous Shows would admit that in every way this the third one was a distinct advance on the others, not only as regards the extent and variety of the exhibits, but also as to the organisation and accommodation provided for those who came to profit by what they could see and learn there. To those who originated these Shows it must be very gratifying to find they are fulfilling such "useful purposes and, their being organised, managed and run as they were by practical men, was proof enough, if proof were needed, that they were practical in character. To all of them who were so to speak outsiders and belonged to the general public, these Shows had the interesting feature of being so to speak annual stoclitaking times when they saw and heard of the progress which had been made during the year in the agricultural development of the country, and they could also see what kinds of products they had here, thanks to the kindness and enterprise of the merchants of Singapore and elsewhere. If they would make a tour of the ground they would see that there was little that humanity wanted here below which could not be procured in the Malay Peninsula.

The great point about these Shows was the chances they offered to agriculture and to those engaged in work in connection with agriculture. Here they saw the practical results which had been attained by those who were lessons of future success. They add to the development of the Peninsula. They were able to come here to compare notes, to see where each of them had succeeded or failed, and to profit by the success and failure of others. They would see what had been already achieved and learn how it had been achieved. In agriculture and indeed in every branch of life, the battle was not always to the strong, nor the race to the swift, but to him who was ready to learn from success and failure the lesson of future success. They had to remember that it was not only a useful education to those engaged in agriculture, but this Show furnished also a most valuable stimulus to those, who when they saw other people's products better than theirs, were moved to go back and better their own achievements; it was a stimulus to them to resolve to do better next year and so the whole community reaped the great advantage of a continued and growing chain of increased effort and renewed intelligence in agricultural working.

To the Government it was particularly interesting to see these exhibitions because he thought they must all recognize that they 
marked periods of advancement in the development of the community. Hitherto most of the world outside and even those of themselves had been content to regard this as a land where they depended on the rich natural harvest of the earth which they gather without cultivation. He did not think hitherto in the eyes of the world or the merchants of the world that the products of the earth had bulked very largely in an estimate of the wealth of the Malay Peninsula, and whilst the natural harvest of tim, and jungle produce was still very rich and plentiful, it was very gratifying and interesting to find that while that was so, and appeared likely to be so for a long time, our planters were turning themselves with energy and resolution to the permanent cultivation of the soil. So long as we were entirely dependent on the natural harvest of the earth, we could never expect to have any really permanent and abiding community in this part of the world, and he was sure that when they found they were beginning to take themselves seriously as an agricultural community, they should find the world beginning to look to them as a field of some of its most valuable products. They could feel then they were laying the foundations of a legitimate stability of a trade from which Penang and Singapore, the gates of the Peninsula, must reap a great share. When they were privileged to go round this Show they would find much to interest them, and much to profit by. They would see the results of the methods of the European planter, which had been brought to the highest state of perfection possible at present, and they well know the bigh value put upon the products of that work in Europe.

But they would see something if possible more interesting, the results of those native cultivations which had been going on from time immemorial; these inclided not only the exhibits of paddy and other crops, but also models of the implements used in producing those crops. And they would also see numerous specimens of every interesting native art and craft. It had always been a matter of great regret that these native arts and crafts were to such an extent a matter of history, and in spite of efforts made by some energetic officers in different parts of the Peninsula, they were still far from being in a healthy condition. He hoped the result of this and previous exhibitions would be to give a great impetus in that direction. When the native artisan discovered there was a really good and profitable market for what he produces it would do much to encourage the production of those beautiful specimens of Malay silver and weaving, which were now too much things of the past. He thought in that way the people of Singapore could do much to encourage those officers who had been so unselfishly trying to develop these arts. And if it resulted in giving such a stimulus and revival, those officers would feel that they have reaped a very rich reward. The people of Singapore would recognize the debt they owed to the splendid work which has produced the result before them, and the generous co-operation which had been offered to them in the matter not only by the sister Settlements, but by the F. M. S. also. They had here he believed something like 350 Penghulus who had come in charge of various exhibits, some of them from very long distances right away on the northern borders of Perak, and it would be a great stimulus 
and advantage to them to come into contact with European civilisation in one of its busy marts such as Singapore is.

$\mathrm{He}$ thought the visit of these men spoke volumes for the excellent relations between Europeans and the Natives over whom they are placed. And it was very gratifying to Government to see these rulers so sympathetic. Our rule here did not rest upon force, but entirely on the good will of those over whom we were placed, and the fact that he had just alluded to prove that the good will was founded on a rule of sympathy and appreciation on the part of the European officers, who were placed in the various districts of the Peninsula. He was sure that the people of Singapore would recognize what they owed to these people. He was sorry that his friend the Sultan of Johore was not present that day. He had taken very great interest in the Show and had given a large subscription and he was sorry that he was not able to be with them that day.

Having already been round the Show he was amazed at the wonderful organization and extraordinary amount of work, both mental and physical, which it must have involved for the members of the various Committees, who had so generously and disinterestedly taken the matter in hand. When they went round they would see for themselves the extraordinary extent and variety of the exhibits, and the admirable manner in which they were displayed, and they would he thought agree with him that the best thanks of the community were due to those who had worked so wonderfully in the collecting and placing of the exhibits. To the building committee their special thanks were due not only for the admirable arrangements made, but also for the great success achieved in so short a time. And their heartiest congratulations were due to Mr. Bidwell and Mr. Buckley, for their splendid services, and in a hardly less degree to the members of the other Committees. He had much pleasure in declaring the Show open. (Applause).

The exhibition was then opened to the public, closing at $6 \mathrm{p} . \mathrm{m}$. In the afternoon the hack gharries, harness horses over I 4.2 , and hacks under I 4.2 were judged. The Sherwood Foresters and Town bands performed.

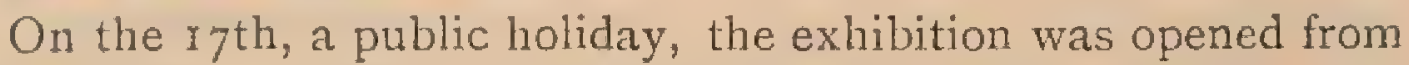
8 a.m. to 6 p.m., and the schools visited the show ground from $2 \mathrm{p} . \mathrm{m}$. to $4 \mathrm{p} . \mathrm{m}$. In the afternoon the polo ponies, hacks over I4.2, harness ponies and lady's hacks were judged. On the I Sth the exhibition was opened as before and in the afternoon there was a dog-show from 2 p.m. to 6 p.m. and driving and jumping competitions in the ring from $3 \mathrm{p} . \mathrm{m}$. to 5 -I $5 \mathrm{p} . \mathrm{m}$. At 5 o'clock Miss Anderson awarded the prize cups to the various winners and the Show closed at 6 p.m.

It was proposed at first to continue to keep the exhibition open for two days more, but as the native visitors had to leave on Sunday afternoon and Monday it was found advisable to close the exhibition as had previously been arranged.

The band of the Sherwood Foresters and the Town band performed during the afternoons, and the former at the opening ceremony. 


\section{Agricultural Section.}

The exhibits of agricultural produce were contained in one half of a long building the other half of which was devoted to native industries. It had been agreed previously to allow competition from any part of the Peninsula from the Isthmus of K'ra to Singapore. Few exhibits howeyer were received from the northern States but all the others including for the first time Johore were well represented. Exhibits of considerable interest not for competition were also shown from Java and Borneo, among them was conspicuous a large series of damars, sugar, tea, oils, sago, nutmeg-butter, spices from Java and the Moluccas exhibited by Mr. Diepenheim.

The whole exhibit of agricultural produce was little if at all inferior to that shown in Penang last year, but it must be remem. bered that the greater distance of Singapore from the main agricultural districts would to a certain extent curtail the amount of produce brought down by rail and boat.

All classes of agricultural produce were shown excepting clove oil and boiled rice.

The judges in this section were Mr. Diepenheim, Mr. L. Brown, Mr. Hart, Mr. Bramall, Mr. Darke, Mr. Zacharias, Mr. Scouler, Mr. Lovell and the Hon. Secretary, while several native penghulus assisted in judging in the classes of articles of native consumption.

Arroreroot. - The prize for the best roots was taken for the third successive show by Mr. H. H. Norris, whose sample would be hard to beat. The whole of the specimens in this class were good.

Prepared arrow-root was also first class. There being several samples of high merit.

Tous-les-mois. - The rhizomes of Canna edulis were shown under arrowroot. One sample was shown last year in Penang and this time two were staged, one of which was exceptionally good and obtained a special prize. This kind of arrowroot is popular with the Javanese and its cultivation might well be developed.

Betel nuts.-Fresh betel nuts were well shown, and there were some fine clusters. A fine bunch of the popular variety known as Pinang Wangi carried off the first prize. Hardly as many were shown this year as last year and the same applies to dry and split betel nuts of which however there were some fairly good and superior samples. Specimens of the large red betel nut known as Pinang Penawar (Actinovlytis Calapparia) were shown. The Malays attach however little value to this fruit, it being chiefly used in medicine and magic.

Sirih leaves.-Were fairly represented with both Chinese and Malay varieties, chiefly the latter which is more popular. The samples were pronounced very good by the Malay connoisseurs who aided in judging them.

Meducinal plants.-The class for the collection of medicinal herbs was very extensive, the largest ever shown. There were forty 
collections some containing over two hundred different herbs and drugs. Mr. Machado carried off the first prize for these with a beautifully prepared series showing the specimens dried as for a herbarium, as well as dried as the drug, and put up in bottles.

Fibres.-This class of produce was better shown and staged than on previous occasions, though there was nothing equal to the collection shown by Mr. Schirmer in the Kuala Lumpur show. There were twenty-two exhibits, Mr. Machado winuing a first prize with a fine set of samples, very well shown. There is doubtless a futtre for fibre in the Malay Peninsula. Many grow extremely readily, and there is a constant demand for high class and even second grade fibres in the European markets.

Cotton.-Some samples of this were decidedly good, but much was very poor. Mr. Machado's Egyptian was very fine. A good deal of that shown by natives was ill-cleaned, and one or two tempting looking baskets were discovered to have a fine sample at the top with very inferior specimens below.

Kapok was largely represented, and most of the samples good, some being very superior.

Cocon pods.-Were much more largely represented this year than on previots occasions, and some samples were very fair and clean, mostly of the red varieties Sangue Toro, and Forastero. The green and yellow varieties do not seem popular. None of the pods were of very large size, and none were fully ripe, still signs of an increase in the cultivation of this product are very welcome.

Dragon's blood was well represented, and the winner of the first prize exhibited a complete series of specimens illustrating the history and manufacture of this product in the form of a living plant of the rattan Damonorops propinqua which produces the drug, a portion of an adult stem with panicles and fruit, the little mat basliet and tripod, with the cockleshells used in separating the resin from the fruit, a bottle of the powdered resin of first class quality and a cake of the pressed dragon's blood as exported. All the samples shown this time seemed to be derived from $D$. propinqua though at previous exhibitions dragon's blood of other species was shown.

Coconuts.-Mr. Lawrence Brown, the Government Inspector of Coconut Trees in the Malay States, acted as judge of coconuts as well as other agricultural produce, and writes: "As at the two previous shows there were a great many fine exhibits of coconuts. and all the classes were not only well filled but well represented. The best collection of varieties of coconuts consisting of no less than thirty-one kinds was that of Hadji Mohamed Yassin of S'tiawan and received the first prize, and Mr. Prior of Golden Hope Estate, Klang, easily obtained the second prize with an excellent exhibit of twenty-two varieties, while another collection of fourteen varieties staged by Latip bin Eusope of Malacca carried the third prize. 
"The husked coconuts were plentiful in numbers and of good selection. The first prize was taken by Penghulu $\mathrm{H}$. Aminudin of Sungei Tinggi, Perak, with a most excellent exhibit, but the judges found it exceedingly difficult to fix upon the second and third in order of merit, there being so many of a very fine standard.

"In unhusked coconuts of which there was an equally large collection the same difficulty arose, and although there was no disputing the superiority of the sample that carried off the first prize the difference between some of the others was very little, though there was no reason to grumble at the final decision."

Mr. Brown brought with him from Pahang, Temai Ulu, a fine young coconut with three strong shoots from one nut and some specimens of the variety known as "Kelapa Dadeh" an uncommon tree. The peculiarity of this nut is that when thoroughly ripe and well shaken up before being opened, it will be found that the whole of the "milk" becomes consolidated and forms as it were a second thick layer of "meat" which if eaten fresh is not only very easily digestible but very pleasant to the palate.

Copra-The samples both of sun-dried and kiln-dried copra were excellent, except that none of them appeared to be sufficiently dry. This horvever might be accidental in samples coming loose from a long distance by sea.

Oils.--These were as usual a large feature in the exhibition, and there was a very extensive series, most prominent and attractive was the collection of Essential Oils from Kamuning Estate, which were not only magnificent samples, but beautifully staged in ornamental bottles on a polished wood and glass frame. Mr. Machado showed samples of oil of calamus, citronella, patchouli, lemon-grass, lemon, and nutmeg, easily winning the first prize.

Katz Brothers staged a nice little lot of citronella and patchouli of high quality from Tampinis Estate.

Coconut oil.-Was as usual represented by a vast array of tottles of very varied values. kind.

Castor oil.-Was somewhat scanty in samples, but good of its

A number of samples of medicinal oils and mixed oils, and other preparations highly esteemed by the Malays as medicaments were shown.

In Mr. Diepenheim's collection from Java and the Moluccas were some excellent samples of pea-nut oil, citronella and kajeput oil, which were very attractive.

Oil-cake.-Was better than usual and some first class samples were shown.

rllipe butter.-Made by the Singapore Oil Mills was shown also, an excellent sample of this valued preparation.

A sample of Nutmeg-butter which has never been seen at these exhibitions previously was shown in his collection of specimens. 
from the Dutch Indies by $\mathrm{Mr}$. Diepenheim. It is used in medicine, and was formerly at least a manufacture of Penang.

Tea. - Was represented by six exhibits, the well-linown product of Durian Tungul Estate carrying off the first prize.

Coffec.-Although the cultivation has of late years almost disappeared from the Peninsula, or more correctly diminished to a very great extent, was better represented than in Penang last year. Golden Hope Estate as on previous occasions holding the front rank with its Liberian. Bailey's Stenoplyylla hybrid was represented by two or more good samples. Should coffee come back again to the Peninsula some time, as we may hope it may, we shall look for a great future for this superb variety.

Sugav-canes.-Were fairly well shown, there being in all fifteen exhibits of specimens and collections, some of these were excellent. With the decadence of the sugar industry nowadays, and the distance which Singapore is from the sugar cane district, it is not to be wondered that eating canes predominated in the collection.

Prepared sugars.-Were abundant especially those of coconut and kabong palms. 'These popular and nutritious sweetmeats were of excellent quality in many samples and gave the judges some trouble to decide on their respective merits. Nipah sugar, never largely represented, was hardly shown at all. Its softness and treacly stickiness make it less popular than the firm dark toffee-like coconut and lxabong sugars. Native cane sugar was also good and well made and brown, white, and refined though not largely shown were of good quality.

Toddy.-Was illustrated by several samples and was as usual judged by Tamils for no European can appreciate the flavour of this native spirit.

Rum and Rum Shrub were shown in a very small series, but the samples were mostly good, and required some care in judging.

Honey.-For the first time at any show in the Peninsula, cultivated honey was shown by Father Gex of the Mission Étrangéres, who may well be called the pioneer of apiculture here. He showed not only a fine lot of combs, run honey and wax, but also had small glass cases of the bees at work which proved a great attraction to the natives, many of whom had the opportunity of watching the bees busily engaged in making up their combs for the first time in their lives. The bees which Father Gex is so skilful in cultivating are not the European hive bee, but the Indian kind, Apis indica, a common bee here. It is a smaller insect than the English bee. It may be hoped that this attractive exhibit may induce some of the natives to cultivate the art of bee keeping, which indeed is not absolutely unknown in the Peninsula, as I have seen in the homes of Chinese near Batu Pahat, hives of cylindric shape made of rattan hung up in the rooms and tenanted by the same kind of bees. Dr. Haviland of Kuching, many years ago devoted some time to apiculture and introduced European bees also from Italy, but they did not thrive as well as the local kind. 
The honey both in comb, and run, shown by Father Gex would be hard to beat and the wax produced was of the highest quality.

Samples of the comb of the large wild bee Apis dorsalis were also exhibited. This is the common large bee which produces only one large comb upon the branch of a tree, and is not suited for agriculture. I s wax collected by natives in the forests is, however, an article of trade and of fairly good quality but usually very yellow.

Spices.- These were hardly as good as we remember to have seen in byegone shows. There were, however, fourteen exhibits of dry nutmegs, some of which were decidedly good, and twelve samples of fresh nutmegs in the husk. The best of the nutmegs competing, however, were not to be compared with the sample of Banda nutmegs shown by Mr. Diepenheim, which of course are a very much larger class of nutmeg than those we usually gret from the Peninsula.

Cloves were fairly good but many better samples have been staged at the earlier shows, and there were only ten exhibits.

Mace both red and yellow were of fairly good quality taken all round, and some of the red mace was quite superior.

Collections of spices for curries were represented by frve exhibits, some of which were very complete in the number of kinds of spices shown. They were in some cases too very nicely put up, but there was a tendency to give very small samples of each kind of spice.

Some excellent samples of Curvy Powder of a very special make were shown by Watt Lee \& Co.

Pepper.-Both black and white was fairly well shown, and some of the samples were first class. But the number of exhibits was not as large as in the former days when pepper was so largely cultivated. There were many fine examples of white pepper which were disqualified owing to their having been grown outside our area.

Ginger and Turmeric.-Were both good and abundantly shown. The turmeric, however, was mostly rather small in the rhizome. The Ginger was good, and some of large size. Both in these classes and in that of arrowroot, some exhibitors have a tendency to send their samples unwashed and coated with mud. Although this does not necessarily count as a point against the exhibit, yet it detracts from the appearance of the sample and is a distinct defect in staging.

Patchouli was well represented. A larger number of exhibits being staged than on previous occasions. Kamuning Estate came well to the front again with some beautifully prepared leaves, and there were other good samples, but some of those shown were too full of stalk.

Tuba root was shown in very extensive series, with roots of all sizes and forms. They were all fair to good and formed a difficult class to judge. Among them several natives showed 
"a new Tuba root" which appeared to be the stems of Tinospora cordifolia.

Dammars.-Were more abundantly shown than on the last occasion and some masses were very large. 'There was a good variety in the collections, but they were chiefly of the Damar daging type, and of other dark resins. A fine series of Moluccan damar's was shown by Mr. Diepenheim. These further eastern damars known mostly as copals in local trade are the most in demand in Europe, being clearer in colour than most of the local ones of the Peninsula.

Rattans.-The collections of rattans were decidedly abore the average of those seen at previous shows and they were also more plentiful. Messrs. Tan Ah Choon exhibited some beautifully prepared Borneo canes, which were awarded a diploma for their careful preparation, though not being produced within the limits of the Peninsula, and being of two varieties only they could not enter for competition with the collections of jungle canes.

Bamboos.-Collections of samples of these were numerous, and there were some good specimens. The curious branched culms, and flexuous culms and other abnormal specimens were as abundant as usual.

Walking-sticls.-Though the prize for these is offered for sticks unfinished and ummounted far the greater part were highly worked up and onnamented. The collection was much larger than on previous occasions and some of the sticks were really very fine, and they were much in demand by purchasers. It would, however, perhaps be preferable in future, considering that most are works of art, to transfer this class to native industries.

rice, paddy, and pulut formed a large feature in the exhibition but it must be admitted that the collection as a whole was not as large as we have seen on other occasions. 'There were several collections for the Mukim prize in which were over fifty varieties, the finest lot containing seventy-two named kinds. Coolied rice was not shown, and as this has commonly happened before, perhaps it would be as well to omit this class in future from the exhibitions. As is usual some of the Malay penghulus were called in to aid in judging the rice and paddy exhibits, but even with their aid the work of examining all the exhibits and judging of their respective merits took the judges a long time.

Tapioca roots were quite up to the average though not as fine as those shown at Penang show, but Tapioca roots are cumbrous things to travel with and the greater distance from the big tapioca culture area is perhaps the reason why fewer large specimens were sent.

Tapioca Flour.--'There were a number of good exhibits.

Five or six samples were very near one another in quality and it was difficult to decide which was the better of the two chosen as leaders.

Flake-There were very few exhibits of really good quality. 
The exhibits from Malacca were rather disappointing, ard were certainly not as good as those shown at the Kuala Lumpur Exhibition.

The sample that was far and away the best in this class was exhibit No. 599, made we believe at the Malakoff Estate, Province Wellesiey.

The same exhibitor shewed a fine specimen of flake siftings the make of which was exceedingly regular.

Pearl. - There were some nice exhibits of Medium Pearl but we only saw one good sample of seed.

Sago Pearl.-There was only one exhibitor in this section, Messrs. Brinkmann \& Co., and the samples shewn were well made and of good colour and quality.

Flour.--The two or three exhibits presented nothing special in the way of quality.

Maize was exceptionally good this year, the cobs shown being mostly large and well-filled, but there was practically only one variety shown.

Biscuits. - A number of specimens of local made biscuits were shown by Messrs. Ho Ho. No prize was offered for competition in products of the bakery.

Soap.-A prize was offered by Dr. Lim Boon Keng for the best locally made soap. Unfortunately the offer came after the printing of the schedule of prizes so that there was no possibility of informing the local manufacturers of this article that there would be a prize offered. A Chinese firm, Messrs. Hock Leong, howerer, brought some excellent samples of this and obtained a medal. The same firm exhibited also a number of sweets, manufactured by themselves.

Dried fish, locally prepared.- - VVas also the subject of a prize offered by Dr. Lim Boon Keng, unfortunately too late to get a competition, and none was shown.

Wet Gambier.--lhere were only three exhibits of this important local produce.

The prize winner was the only fair sample shewn, while that exhibited by the "Gambier Society" was distinctly poor.

In view of the importance of the export trade in this article it would have been more interesting had there been shewn examples of the product in its various stages of manufacture-from int green leaf to the final block.

Cube Gambier.- The exhibits in this section were generally good although some looked as if they had been prepared specially for exhibition purposes.

Gambier Extract.-This was an interesting sample of what looked like Gambier-dust, prepared evidently by some process of crushing the dried gambier extract into very small flakes.

In this state the gambier should be in the most suitable form for export. The only objection we saw to this process was the tendency of the "dust" to become caked by contact with moist atmosphere, but this might be obviated if the stuff were packed in air-tight cases. 
Indigo.-There were a number of bottles of this dye both liquid and in powder, than at recent private exhibitions, and the quality was decidedly better than at the last exhibition. This indeed might have been expected in Singapore where the dyeing business has by no means dind out yet. The samples varied a good deal in quality and one bottle contained liquid of a remarkably brilliant and attractive blue colour which however unfortunately was not indigo-colour.

Cutch.-A sample of the Mangrove cutch made at Santubong in Sarawak was shown. Great improvements in this manufacture have been made in late years, with a corresponding increase in demand.

The Governor's Cup for the best general exhibit in the agricultural section was easily won by Mr. Machado of Kamuning Estate, Perak, who made a good try for it at the last year's show. He took eleven prizes in all, viz., first prize for best collection of essential oils, best sample of patchouli, best collection of medicinal plants, best collection of fibres, best cotton, best kapok, best dried betel-nuts, second prize for best black pepper, best arrowroot, best limes, and third prize for turmeric.

On the whole it may be said that the agricultural produce was very good and representative of the products of the Malay Peninsula, very few of the classes in the schedule were not represented and these consisted only of boiled rice, and clove oil.

At the same time it must be admitted that some classes were not as weIl represented as in previous exhibitions.

Vegetublcs. The exhibition of vegetables was equally poor, probably because the chief vegetable growers, the Chinese, did not understand what the exhibition was about. The idea is utterly foreign to them, and the vegetable-growing class, in spite of explanations, notices in Chinese, etc., failed to send samples of their produce. Mr. Campbell sent from Perak Hills a grand collection of European vegetables as he did last year, with several novelties. Perhaps a time may come when our hills will afford a good supply of European vegetables to the dwellers in the plains. Many of the hills could be opened up for vegetable gardens and the produce brought down and shipped to the larger towns. Mr. Campbeli has shown clcarly that the vegetables can be grown well and can be brought to Singapore in prime condition. That they were highly appreciated was shown by the run on his exhibit at the end of the show. The supply of wholesome and good European vegetables to residents would go a good way in hygienic improvement of the European's life.

Frnit.-Was again not up to standard. Last year the show held in Penang was not as good as it might have been because much of the fruit was not ripe. This year the exhibition was put a little later, and unfortunately the great crop was nearly over. In Singapore at least for several seasons the fruit crops have been very inferior, and the supply las not been what it was in former years. This may be due to pectuliarly unsuitable weather, or it 
may be due to the ageing of the old trees planted many years ago and now dying out not having been replaced by younger plants.

The greater number of the prizes were taken in this section by Malacca, Johore, Perak and Selangor.

No specimens of Binjai, Langsats or mangos were shown.

In the same shed were staged the table decorations and cut Howers, the honey, and the eggs, and also the preserves. In these latter there was a great improvement, chutneys, jellies, preserved fruits, curry powders, pickles and sweetmeats were well staged in abundance and very good were the samples.

\section{Flowers. and Flowering Plants.}

The exhibition of horticulture was distinctly disappointing, what was shown was in many cases good but when one remembers the splendid exhibitions in years gone by, one cannot but feel that a very great deal of the early enthusiasm for gardening in Singapore has disapperted. Some of the old exhibitors whose gardens were a pleasure to see have long gone from us and their plants have been dispersed, others are still with us and showed what they had, but we missed the great masses of Heliconias, Selaginellas, and ferns, Marantas, Crotons and still more perhaps the flowering-plants. The cause of this is perbaps the more frequent home-going of the Europeans. The ordinary resident goes home and auctions all his property including his plants. He does not care to furnish his house well on his return because he may go home agrain in a few years, so he just gets a few common things to fill up the verandah, and leaves their care to the liebun.

We have unfortunately not received any report of the exhibition in this class from the judges. Some good palms were shown, among them a very fine Martinezia erosa, shown by Mr. Down, some fine clumps of Chrysalidocarpus lutcscons by the proprietors of the Raffles Hotel.

A few good orchids were shown, including a fine Cattleya.

Mr. Choa Kim Keat showed a very nice lot of succulents, the dwarf cacti being remarkably good.

Mr. Seah Liang Seah showed a number of quaint contorted trees over eighty years' old.

In many of the classes there was no second prize awarded and there was no award given for coleus, six crotons, panax, selaginellas, best specimen foliage plant, achimenes, amaryllis, asters, balsams, three cannas, dianthus, eucharis, gloxinias, three roses, zinnias, collection of flowering plants, cut asters, chrysanthemums, dahlias, roses, hand bouquet, or wild flowers arranged for effect. Table decorations were entered for by several ladies, and Mrs. Down carried off the first prize with a neat decoration of purple and white cosmos, very light and pretty. Mlle. de Gaspery was sucond with yellow cosmos (Klondyke) and grasses, Mrs. Aptroot showed a very much more elaborate and design of arches in asparagus which, though a little too large for the table, was good and light in style. 
Except some fine palns from Mr. Chesney Duncan in Penang, all the exhibits in this section were from Singapore, the distance from the Native States and the journey by sea preventing its being possible to bring down flowers in grood condition. Mr. Choa Kim Keat, one of our most enthusiastic horticulturists, carried away a large proportion of prizes, and his exhibits formed a large feature of the exhibition.

The thanks of the Committee for the excellent exbibition in these sections was due to the District Officers of the Malay States and colony who did not spare any labour in gretting the natives to send in specimens, and in selecting that was worthy of being sent to the exhibition. Among those who were specially energetic should be mentioned, Mr. Peel, Mr. Firmstone, Mr. Chevallier, Mr. Laidlaw, Mr. Campbell, Mr. Simmonds, Mr. Main and the Residents and Resident Councillors. For the school exhibits the excellent result was due to $\mathrm{Mr}$. Collinge. Rajah Hitam Nongchie did good work both in collecting funds and an excellent series of exhibits from Johore States.

Rubber.-There was really a very good exhibition of rubber of all kinds and forms, mostly as might be expected from the European estates, but there was already some shown by natives. There were 18 entries in Rambong sheets and ball mostly from Perak and Selangor there being only one entry from Singapore and one from Penang. Considering Rambong is a more recent industry than Para the exhibits were fairly representative.

There was a fine display of Para in the various forms of biscuits, sheets, crepe, worm and scrap. Few of the biscuits or sheets however were thoroughly dry, a point all exhibitors should remember, as an exhibit for competition should, whatever class exhibited, be in perfect condition. Undoubtedly deserving of pride of place were the blocks exhibited by Mr. F. Pears of Muar, and the high price recently obtained on the London market for this rubber emphasises the favourable opinion formed at the local show of this form of preparation. In biscuits the first prize fell to Kamuning Estate, Perak, and a diploma to the Highlands and Lowlands, Selangor, there were also some very fine native exhibits in this. class.

The first prize and diploma for crepe went to Lanadron Estate (Mr. F. Pears) while Highland and Lowlands and Kent Estates were highly commended.

Para sheets were well contested, the first prize being secured by the Highlands and Lowlands Estate, Selangor, and the second prize went to Pataling Estate. Mr. Bird of Kuala Kangsar, Perak, obtained a diploma for a sample of excellent sheets which seemed perfect. The Penang Sugar Estates were highly commended. Altogether there were I 5 entries in this class.

In the class "other preparation" which included Mr. Pears blocks there were 19 entries. The diploma for rubber and highest prize going, as said before, to Mr. Pears, but a first prize was awarded to Bertams Estate, a diploma to Highlands and Lowlands, and Yan Seng, Perak, highly commended for long carpet-amber coloured sheets. In this class there were also several other interesting exhibits. 
Class 60, Para-general exhibit, packed in boxes as sent from estate for export included ro entries of 17 boxes of different preparation, the diplom: and cup being carried off by Lanadron (Mr. Pears) the advantage of this preparation as a simple and cheap means of transport being obvious. The second prize going to Vallambrosa Estate, Selangor, who exhibited some splendid rubber but did not meet with usual luck.

Sereral sets of photographs of estates and methods of tapping were shown by Mr. Campbell and Mr. Douglas and others. them.

Para rubber seeds were shown though there was no class for

Among apparatus use for cultivation and preparation of rubber may be mentioned a large series of rubber tools exhibited by McAlister \& Co.

A working plan of a rubber dryer by means of desiccated air was exhibited by Mr. Swan, which may prove an useful adjunct to the planter.

Mr. P. T. Burgess showed an ingenious and very simple girthmeasurer, by means of which any cooly can take the measurements of all the trees in an estate in a very short space of time, so as to give a correct idea of the extent of tapping area in an estate. A description of this simple apparatus is promised for the Bulletin.

\section{Class 51.-Rambong Sheets.}

No.

Exhibitor.

Description of Exlibits. Awards.

2072. Highlands and Lowlands. Latex, Crepe sheetst Ist

Prize.

, Highlands and Lowlands. Dark, Crepe sheetst

"2973. Golden Hope. Crepe, greyt Diplona.

, " Red +

+ " " Block-sheetst

I794. Haj. Md. Ali Yasin Sitiawan. Block-sheets

I 789 .

218.

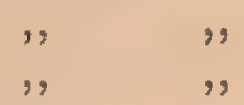

,

Pale sheets

\section{Class 53.- Rambong Ball.}

$\$ 2072$. Highlands and Lowlands. Ball, big $\mid$

I829. Tan Keong Lenggong. Block.

570. Khow Boon Hong, N. Tebal. Lumps.

202. Seet Kee Ann, Singapore. Blocks.

I789. Haj. Mhd. Ali, Sitiawan. Ball.

1500. Kamuning. Lumps.

I503. Ismail, Upper Perak. Block.

\$2599. C. M. Cumming. Crepe, grey and red.

568. Penang Sugar Estates Co. Ball.

\section{Class 53a.-Para Sheets.}

I789. Haj. Mhd. Ali, Sitiawan. Pale thin sheets.

558. Bertam Estate. Brown medium.

tI501. Yam Seng

+18 I4. Plang Estate

do.

do. 


\section{Class 53a.-Para Sheets-(cont.)}

79. H. E. James, Kota Tinggi, Johore. Dark Medium.

\$2502. New Labu Estate. Pale scored.

568. Penang Sugar Estates. Brown Medium Highly Com.

I52I. G. F. Bird, K. Kangsar. Pale Mediumy Diploma.

\section{" ", "Thin'}

+1500. Kamuning. "Pale Mediumt"

†501. Yam Seng. Brown Medium†

12057. Devon. Pale Medium Sheetsł

†2072. Highlands and Lowlands. Brown Scored Sheetsi Ist Prize.

570. Khow Boon Teng N. Tebal. Dark Medium Sheets.

I I. Sandycroft. Thin sheets amber.

+2058. Petaling. Brown Medium Scored.

2nd Prize

\section{Class 54.--Para Biscuits.}

I 50I. Yam Seng. Large brown thin round.

$\$ 2062$. Vallambrosa. Dark Medium Round.

7o. H. E. James, Kota Tinggi, Johore. Dark Medium Round.

203. Haj. Hassain Pengerram. Thick Yellow.

2057. Devon. Pale thin!

12072. Highlands and Lowlands. Diamond dark Squaret

1594. U. C. Thambol K. Kangsar. Pale Medium Oblong.

I500. Kamuning. Pale Medium roundt ist Prize.

250r. Seremban. Black round.

2069. Haron. Pale Medium Round.

\section{Class 55.-Para Crepe.}

12501. Seremban Estate. Pale Crepe.

2501. Seremban Estate. Dark Crepe.

2073. Golden Hope. Medium Crepet

2062. Vallambrosa. Pale Crepe.

2072. H. \& Lowlands. Grey Crepet Highly Commended.

2095. Kent. Palest Crepet Highly Commended.

2095. Kent. Darkish Crepeł

23. Lanadron. Pale Crepel

23. Lanadron. Reddish Crepel

2058. Petaling. Pale Crepet

Diploma and ist Prize.

\section{Class 57.--Para Worm.}

I789. Haj. Md. Ali Sitiawan

Class 58-Para (other preparations).

2 IS Haj.Md. Ali, S'tiawan. Pale Crepe wet.

+2599. C. M. Cumming. Red Medium Sheets smoled.

203. Haj. Hussain Pengaram. Pale Medium Biscuits.

2072. H. \& Lowland. Dark Thin Biscuits sparet

23. Lanadron. Blockt

ti 50I. Yam Seng. Carpet, A mber,

Diploma and Ist Prize. Highly Commended. 


\section{Class 59.--Para Scrap.}

Nu. Exhibitor.

Description of Exhibits.

Awards.

H52I. G. F. Bird, K. Kangsar. Ball

t. Ir. Sandycroft. Lump.

+2095 . Kent. Dark Crepe

2057. Sione. Pressed Scrap

$\$ 2072$. Highlands and Lowlands. Dark Crepet

2072. " ", Block

570. Klyow Boon 'leng, N. Tebal. Loose umpressed.

I789. Haj. Mhd. Ali, Sitiwan. Ball

I789. ", "Sheets, yellow.

202. Seet Kee Ann, Singapore.

2r8. Haj. Mhd. Ali, Peral. Biscuits, yellow.

+2058. Petaling. Grey Crepet

558. Bertam Estate. Loose unpressed ist Prize.

\section{Class 60.-Para General Exhibits.}

+23. Lanadron. Crepe $\dagger$

23. ", Blockt Diploma and ist Prize.

II. Sandycroft. Amber Medium Sheetst.

+ 5 500. Kamuning. Thin Pale Sheetst

558. Bertam Estate. Dark Medium Sheets.

I521. F. G. Bird, K. Kangsar. Pale Medium Sheets.

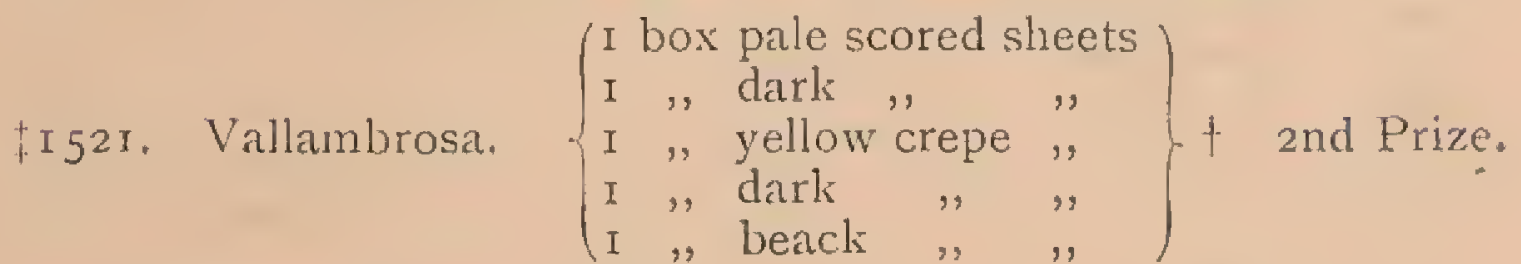

\$2073. Golden Hope. Pale Crepeł

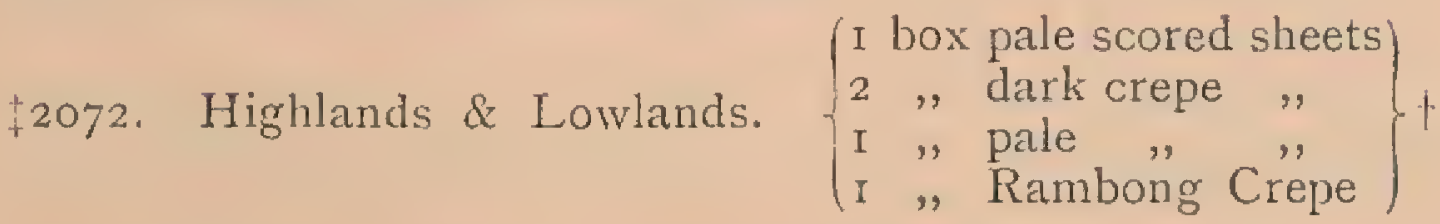

†206g. Haron. I box pale sheets and scrap.

\section{Special Class.}

203. Haj. Hussain Pegaran. Para Seeds

+2057 . Sione.

+2095 . Kent.

Not for Competition.

Singapore Botanical Gardens Pale Sheets.

Pale Biscuits.

I bale 72 lbs.-Scrap.

Photos. From Linsum

Rubber Dryer (with dessicated air) by W. R. Swan working plan.

Girth Measwier by P. J. Burgess.

H. N. R. 


\section{Pourtry.}

As a whole there was a poor show of poultry, only about 50 pens were shown. Two pens of six-Buff, Orpingtons and Minorcas.

The Orpington wimers were a fair average lot, the cock on the small side but the hens were better. The Minorcas, good birds otherwise, failed lamentably in their legs-all were feathered. The Orpingtons were prize winners at Penang also in 1905.

The best pair in the show were a Malay game cock and hen, really good specimens and were easily the hest birds in the show.

Two pairs of Bralumas were shown. If the winning cock and losing hen had been placed together it would have been a rery fine pair. Brahmas do not do well in this damp climate and their plumagre is very much affected. Crossed with or by Malay local game, they should do well, as a pure lird they are disappointing.

Of turkeys only two pair were shown and poor ones at that.

Geese a fair number, but poor and stunted in appearance-only one good and shapely bird amongst the lot.

One pair of indifferent Guinea fowl. Two pair of Victoria pigeon were shown, very handsome birds and easily lept in captivity.

The jungle fow shown was a very scraggy lot and the hens did not strike one as being jungle hens at all, very few were exhibited.

It is an extraordinary thing how many persons think that an agri-horticultural show is an offshoot of Barnum's and any freak or dime-museum monstrosity should find a place in it.

It is a great pity that more interest is not talien in this class of produce-it affects everyone of us and should have better attention paid to it. Poultry repay interest taken in them, but it must be whole-hearted. Natives showed hardly any poultry although they took consiclerable interest in the exhibition.

A few pigeons and canaries were exhibited, none of any very great interest.

Cats.-There were not as many cats shown as might have been expected. There were a few good Persians, notably Mrs. Daly's grey Persian, a beatiful animal which had taken prizes at shows in England. Only three Siamese cats were shown but these were fairly good. English cats were not particularly striking.

Cage birds.-. There was little very striking in this class, a few good parrots, and some canaries, and some birds of paradise. Mr. Lim Lee carried oft a first prize with a very beautiful small bird, black with an orange breast and a long tail. There were aiso a good many Jara sparrows of various colours shown.

Rabbits and guinea pigs were poorly shown, though there are many rabbits of good class kept in Singapore and the exhibition miglit have been better.-C. HATVTREY.

\section{Native Industries.}

'The total number of entries, of which there are records, comes to about 1,000, allowing 50 for the Negri Sembilan and Singapore, the latter contributing very little indeed. Of these I find that 575 came from Perak, 83 from Selangor, I 26 from Penang, and 167 from 
Malacca. These figures show to what extent this Division was indebted to Perak for its exhibits. In embroidery alone Perak exhibited 35 specimens mostly of very fine work; two first prizes being avarded to entries from Kuala Kangsa, and Papan. Two second prizes were awarded to exhibits from Malacca, which sent 20 entries, and was the only country approaching Perak in this class. Other headings under which Perak exhibited largely were ornamental baskets, 22 entries, rope and twine, attaps, mats and serih sets. Models were very numerous. Of the model snares and traps 39 specimens came from Perak, very few indeed coming from elsewhere. Of the old silver-work practically the whole came from Perak (I 8 entries) and Selangor (7 entries) but the first prize was awarded to some new work by a Singapore silversmith. Under brass-ware nearly all the entries were from Perak, although a Penang exhibitor secured the first prize. For ornamental baskets 5 prizes were awarded, all going to the Malacca exhihitors. The tin-ware from Seremban was as usual a very conspicuous exhibit. The Malacca lace workers also attracted much attention, as did the sarong-weavers, and the Chinese drawn thread workers. There were some very handsome old kriss-sheaths shown, but in this, as in other cases, the preference was given to new work, the first prize going to a Butterworth exhibit. The latter part of the prize list, from 307 to $3 \mathrm{I} 4$, calls for no remarks, the prize-list which is in your hands showing the results. Class II (school-exhibits) was separately managed and judged. I enclose a few notes on matters of organisation, arrangement of the prize-list, etc., which may be of use in future shows. Thanks are due to Messrs. Coutts and Marriott for the great assistance rendered by them in the work of this Division.

G. A. HALL,

Honoyary Secretary, Division E.

\section{PRIZES AWARDED AT THE SINGAPORE AGRI-HORTICULTURAL SHOW 1906.}

Division A.-Agriculturat. Produce.

Class No.

Exhibit Amomt.

I.-Arrow-root fresh :-

1 st Prize, H. H. Nonis, Singapore

2nd , A. D. Machado, Kamuning Estate, Perak I 500 S

3rd " Hadji Mohanud Yassim, Perak I 794 I

Special Prize, Din Bin Brahim, Pajak Song, Penang 597 I

2.-Arrow-root, prepared :-

Ist Prize, Penang Sugar Estate, Penang 5685

and "Girls School, S. P. G. Mission, Singapore 92

3rd " Mohamud Kassim, Penang 505 I

3.-Bamboos:-

Ist Prize, Hadji Mohamud Ali, Perak

and "Penang Sugar Estate, Penang

I $790 \quad 5$

3rd "Wan Mohamud Nasic Bukit Gantang, Perak 
Exhibit Amomat.

Ist Prize, Mohamud Noh Lower. Perak

and " Kader Khan, Singapore

3rd "Inche Abdul Rahman, Batu Pahat

5.-Betel Nuts, split and dried:-

Ist Prize, A. D. Machado, Perak

and "Penghulu Abdul Wahab, Perak

3rd " Hadji Mohamud Yassin, Perak

6.-Cloves:-

Ist Prize, S. M. Peralta, Malacca

2nd " Mohamud Zain, Penang

3rd "Hadji Mohamud Yassin, Perak

7.-Coconuts unhusked:-

Ist Prize, Rasit Kuala Kangsar, Perak

and "Ng Ah Soo, Singapore

$3^{\text {rd }}$, Penghulu Hadji A. Majid, Merlimau, Malacca

No, $\$$

$1779 \quad 3$

1072

I33 I

I 5005

15242

1794 I

103310

$548 \quad 5$

17942

I $598 \quad 7$

2 I 3

IIO5 I

8.-Coconuts, husked:-

rst Prize, Penghulu H. Aminudin, Sungei

Tinggi, Perak

15237

and Prize, E. R. Salisbury, Gapis Estate, Perak $\quad$ r520

$3^{\text {rd } ~, ~ H a d j i ~ M a a l i ~ B . ~ H . ~ I s m a i, ~ T a n j o n g ~ K l i n g, ~}$ Malacca

1025 I

9.-Coconuts, best collection:-

rst Prize, Hadji Mohamud Yassin, Perak, Durian

S'tiawan, Perak

and Prize, E. B. Prior, Golden Hope Estate, Klang

3rd Prize, Latip Bin Eusope, Keluban Besar, Malacca

20735

10192

ro.-Copra, sun-dried :-

Ist Prize, E. B. Prior, Klang

and "Penang Sugar, Estate

3rd ", Kanday Penang

$2073 \quad 5$

$568 \quad 3$

532 I

I I,-Copra, kiln dried :-

Ist Prize, E. B. Prior, Klang

$2073 \quad 5$

1073

2nd , Kader Khan, Singapore

1105 I

12.-Cotton "Kapok" :-

Ist Prize, A. D. Machado, Perak

I $500 \quad 5$

I 5333

and ," Said Yusope, Kuala Kangsa, Perak

3rd "Mohamud Zain, Penang

5211

Highly Commended, Inche Din, Penang

13.-Cotton "Kapas": -

Ist Prize, A. D. Machado, Perak

and " D. Logan, Penang

$3^{\text {rd }}$, Mohamud Mansoor, Perak

I 500 IO

$607 \quad 5$

$1792 \quad 2$

I4. - Cocoa :--

Ist Prize, Samiah, Ampangan, Negri Sembilan 25725

and " Hadji Mohamud Akhir, Ampangan, N.S. 25633 
Class No.

I 5.-Coffee Liberian:-

Ist Prize, E. B. Prior, Klang

and " A. D. Machado, Kamuning

3rd " O. Pfenningwerth, Klang

Exhibit Amount.
No.

2073

I $500 \quad 5$

20722

16.-Coffee variety:- -

Ist Prize, O. Pfenningwerth, Klang

and "E. B. Prior, Klang

3rd " Sd. Hussain, Penang

20725

$2073 \quad 3$

600 I

H. C., Nyak Ah Yum, Penang

560

17.-Dragons Blood :-

Ist Prize, Hussain, Perak

and "Penghulu Raja Shabudin, Perak

3rd ," Penghulu Hadji Aminudin, Perak

18315

15253

I 523 I

I8.-Fibres:-

Ist Prize, A. D. Machado, Kamuning Estate

and ", Syed Ahmad, Penang

3rd, K. Hamad, Penang

I 500 I 5

$\begin{array}{lll}5 \times 2 & 7\end{array}$

5182

I9.-Gambier :-

Ist Prize, Inche Hamzah Bin Awang, Muar

and ",

3 rd "Sandycroft Rubber Coy.

Extra Prize.-The Lanadron Estate, Muar

1485

IOI I 3

II I

23 I

20.-Getahs:-

Ist Prize, O. Pfenningwerth, Klang

and ", Datu Luar, Batı, Pahat

3rd ", H. Berkeley, Upper Perak

2072 IO

$136 \quad 5$

15022

21.-Gums and Dammars :-

Ist Prize, District Officer, Kuantan, Pahang

and "Orang Kaya Jaffar, Batu Pahat

$207 \quad 7$

1373

3rd " Hadji Mohamud Ali Perduka Raja, Perak I 790 I

22.-Ginger :-

Ist Prize, Andi, Penang 2nd "Malacca

3rd Prize, R. W. Munro, Kuala Langat, Selangor

5325

IOI 4

219 I

23.-Indigo:-

Ist Prize, Yeop Ibrahim, Perak

and "Abu Bakar, Klang

$\begin{array}{rr}1834 & 5 \\ 2068 & 3 \\ 568 & 1\end{array}$

24.-Mace, yellow:-

Ist Prize, Hadji Hussin, Singapore

25.-Mace, red:-

Is $\downarrow$ Prize, Seet Kee Ann, Singapore

2025

and "Hadji Hussin, Singapore

2033

3rd " O. Pfenningwerth, Perak

2072 I

26. - Maize:-

Ist Prize, Omar, Perak

and "Penghulu Saman, Perak

3rd " Mohamud Mansoor, Perak 
27.-Nedicinal plants:-

ist Prize, $\Lambda$. D. Machado, Perak

and "Hanid Fasir, Perak

3rd , Mohamud Kassim, Penang

Ňo.

I 50010

17935

5052

28. - Nutmegs, fresh :-

Ist Prize, Seet Kee Ann, Singapore

and , Hadji Mohamud Yassin, Perak

3rd , Kulop Mohamud, Perak

2025

I $794 \quad 3$

I 570 I

29.-Nutmegs, dried:-

Ist Prize, Mohamud Kassim, Penang

505 IO

and "Andi, Penang

$3^{\text {rd }}$ " Hadji Hussin, Singapore

5325

2032

30.-Oil, citronella:-

Ist Prize, A. C. Hardouin, Penang

and , Nexander Smith, Perak

3rd "Teban Louise Estate, Tampenis, Singapore

582 Medal

$1805 \quad 5$

42

31.-Oil, lemon grass:-

Ist Prize, A. C. Hardouin, Penang

and "Alexander Smith, Perak

32. - Oil, coconut :-

Ist Prize, Singapore Oil Mills

and " L.S. Aeria, Penang

$3^{\text {rd }}$, ki. Hamad, Penang

H. C., Mohamud Arashad, Penaing

582 Medal

I $805 \quad 5$

I 23 Medal

59 I 5

5 I 2

$5^{\text {I } 3}$

33.- Oil, teel seed:-

Ist Prize, Che Din, Penang

and, Serat B. H. Habil, Penang

$3^{\text {rd }} "$ Odain, Penang

5215

5923

519 I

34. -Oil, castor :-

No award.

35.-Oil, Para Rubber

Ist Prize, H. F. Dupois, Negri Sembilan

and ," Singapore Oil Mills, Singapore

$2502 \quad 5$

1233

36.--Oil, clove:-

No award.

37. -Oil Calie:-

1st Prize, Singapore Oil Mills

H. C., G. Frankland, Singapore

I 235

208

38.-Oil, collection:-

Ist Prize, A. D. Machado, Perak

39.-Pepper white.-Li Ah Pow, Thaiping, Perak

150010

4010

and Prize, E. R. Salisbury, Perak

3rd , Low Kong Yeo, Klang

$1520 \quad 5$

$2082 \quad 2$

40.--Pepper black :-

ist Prize, E. R. Salisbury, Perak

and , A. D. Machado, Perak

152010

$1500 \quad 5$

3rd "Asst. Penghulu Abdul Wahab, Perak

15242 
Class No.

41.-Patchouli:-

Ist Prize, A. D. Machado, Perak

and , Penghulu Abdul Wahab, Perak

3rd ", Lanadron Estate, Muar

Exhibit Amount.

I $500 \quad 5$

$152+3$

231

42.-Padi variety (Ist Prize, presented by Hon. J. K. Bircly):-Ist Prize, Mat Drum, Upper Perak

and "Penghulu Pandak Abdulla, Bagan Serai, Perak

3rd Prize, Kulop Mohamud, Senggang, Kuala Kangsar, Perak

$150+25$

$18+0 \quad 10$

$157^{\circ} \quad 5$

43.-Padi Collection :--

ist Prize, Penghulu Pandak Abdulla, Perak

and ", Penghulu Ijok, Perak

$1840 \quad 50$

I 84225

3rd ", Kulop Mohamud, Perak

I 570 IO

H. C., Penghulu Meor Jaya, Lenggong, Upper Perak I 821

44.-Pulot sample:-

Penghulu Pandak Ibrahim, Kuala Kurau, Perak 18395

and Prize, Kulop Mohamud, Kuala Kangsar, Perak $1570 \quad 3$

3rd " Bahari B. Panchor, Province Wellesley 585 I

45.-Pulot varieties:-

Ist Prize, Penghulu Pandak Ibrahim, Kuala

Kurau, Perak

2nd Prize, Penghulu Puteh Matkief, Briah. Krian, Perak

3rd Prize, Penghulu, Ijok, Perak

I $8+\mathrm{I} \quad 3$

$18+2$ I

46.-Rice prepared by Machinery:-

Ist Prize, Mudin Bin Sulong, Jasin, Malacca

I 102 IO

47.- Rice prepared by pounding:-

Ist Prize, Penghulu Masop Bin Hussin, Bachang,

C. Dist, Malacca

100210

2nd Prize, Kung Theam Seng, Survey effice

Penang

3rd Prize, Penghulu Hadji Abu Bin Ujong, Jasin, Malacca

48.--Rice Collection :--

Ist Prize, H. Berkeley, Upper Perak

150210

and " Hasib, Jaffar, Kampong Bouia, K. Kangsar, Perak

16225

3rd Prize, Ujah thmad, Saiong, Kuala Kangsar, Perali

49.-Rice, boiled :-

No avard.

50.-Rotans:-

Ist Prize, Hadji Mohamud Salley, Johore

and "Syed Bin Kulop, Malacca

2nd "II44 3

3rd " Hadji Mohamud Mlley Peduka Raja, Perak I 790 I

H. C., Tan Ah Choon, Singapore 
Class No.

Exlaibit Amount

51.-Rubber (Rambong) Sheets:-

No.

O. Pfenningwerth, Klang. A Cup presented by United

Planter's Association, N'egri Sembilan 2072

52.--Rubber (Rambong) Biscuits :-

No award.

53.-Rubber (Rambong) Ball :-

O. Pfenningwerth, Klang

207210

53. (a.)-Rubber (Para) Sheets:-

Ist Prize, O.- Pfenningwerth, Klang. A Cup presented by Hon. W. J. Napier

2072

2nd Prize, The Pataling Rubber Estate Coy., Kuala

Lumpur

$205^{8}$ Medal

54.--Rubber (Para) Biscuits :-

rst Prize, Kamuning Estate (A. D. Machado), Perak.

A Cup presented by Hon. Tan Jaik Kim I50o

H. C., O. Pfenningrverth, Klang 2072

55.-Rubber (Para) Crepe :--

Ist Prize, Lanadron Estate, Muar. A Cup presented by United Planter's Association, Selangor 23

H. C., H. M. Darby, Vallambrosa Estate, Klang 2062

56.- Rubber (Para) Lace:-

No award.

57.- Rubber (Para) Worm :-

No award.

58.- Rubber (Para) any other preparation:-

Ist Prize, Lanadron Estate, Muar

23 Medal

59.- Rubber (Para) Scrap :-

Penang Sugar Estate. A Cup presented by United Planter's Association, Selangor

$55^{8}$

6o. - Rubber (Para) Packed :-

Ist Prize, Lanadron Estate, Muar. A Cup presented by H. N. Ridley, Esq.

2nd Prize, H. M. Darby, Klang 2062 ro.

6r.- Rum Sample:ist Prize, Thomas Berja, Perali 1838 ro

62.-Rum shrub :Ist Prize, Penang Sugar Estate, Penang $\quad 56860$

63.-Sago pearl :rst Prize, Messrs. Brinkmann \& Co., Singapore 73 Medal

64.--Sago flour :Ist Prize, Dindang Sauh, Malacca 1052 Io

65.--Sireh :Ist Prize, Jaffar Bin A wang, Malacca III4 5 2nd "Ali Bin Amin, Malacca 10223 3rd ", Baba Bin Rasit, Malacca I030 I

66.-Spices :-

rst Prize, Sd. Hussain, Penang

and , Nayak Ah Yum, Penang

600 10

$560 \quad 3$ 
Class No.

Exhibit Anount.

No. \&

67.- Sugar Cane:-

I Medal

Ist Prize, H. H. Norris, Singapore

and ," Hadji Leman Bin Hadji Hussain, Malacca Io24 5

68.-Sugar Cane varieties:-

Ist Prize, Penang Sugar Estate, Penang

and " Mohamud Kassim, Klang

568 Medal

20705

69.-Sugar (coconut) :-

Ist Prize, Inche Indot Bin Tanjong, Batu Pahat I42 5

and " Hadji Mat Ali Bin Hadji Ismail, Malacca 10253

3rd "Saidin Bin Mahat, Selangor $205^{2}$ I

H. C., Syed Ahmed, Selangor 2042

70.-Sugar (Nipah) :-

Ist Prize, Hadji Ibrahim Selangor $\quad 20845$

and," Koh Boon Cheh Perak $\quad$ I837 3

71.-Sugar (Kabong) :--

ist Prize, Hadji Mohamud Yasin, Perak I794 5

2nd ". Syed Ahmed, Selangor * 20423

3rd "Udah Mat Isah, Peraki I574 I

72. - Sugar (cane) brown:--

Penang Sugar Estate

$568 \quad 5$

73.- Sugar (cane) refined:--

Penang Sugar Estate

5685

74.- Honey in the Comb:-

Ist Prize, Rev Father Gex, Singapore 445

75.-Tapioca Roots:-

Ist Prize, Kee Teck Wee, Penang $\quad 569 \quad 5$

and , Ong Kim Wee, Perak 26022

3rd "Raja Said, Klang I524

H, C., Asst. Penghult Abdul Wahab, Perak I 524

76. - Tapioca varieties:-

Ist Prize, Lee Ah Pow, Perak I858 io

2nd ," J. Slothard, Penang 5995

77.- Tapioca pearl :-

Ist Prize, Messrs. J. Brinkmann \& Co., Singapore 735

2nd " Ong Kim Wee, Malacca 10323

3rd ", Asiatic Produce \& Rubber Coy., Malacca ro89 I

78.- Tapioca flake:-

rst Prize, J. Slothard, Penang 5995

and " Malacca Rubber Plantation Ltd., Malacca i I 383 3rd " Messrs. S. Brinkmann \& Co., Singapore 73 I

79. - Tapioca flour:-

Ist Prize, Lee Keng Hee, Malacca II 22

and "Leng Cheak \& Co., Penang 5093

$\begin{array}{ll}\text { 3rd ." J. Slothard, Penang } & 599 \\ \text { H. C., Penang Sugar Estate, Penang } & 568\end{array}$

8o.--Tapioca preparation:-

Ist Prize, Asiatic Rubber Plantation Ltd., Malacca IoS9 5

and "J. Slothard, Penang 
Class No

81.--Tea:-

Ist Prize, Durian Tongrgol Estate, Malacca

and "The Sione Rubber Estate, Selangor

3rd ", Mohamud Arsat, Penang

Exhibit Amount
No.

I62 Medal

$2057 \quad 5$

5132

82.-Toddy:-

Ist Prize, Sulong Bin Aris, Malacca

and ,"Hussain Bin Abu, Klang

10343

20662

83.-T'uba:-

Ist Prize, Mohamud Kassim, Land Office, Penang 5053

and ," C. Gwah Clu, Bukit Mertajam, Penang 5932

84.-Turmeric:-

Ist Prize, Kong 'Then Seng, Survey Office, Penang 5i I 5

and , Dain, Kelawai, Penang

3rd ", A. D. Machado, Kamuning, Perak $r 500$ I

85.-Walking Sticks:-

Ist Prize, Omar Bin Mohamud Yousop, Singapore I9 Medal

and "Mohamud Abas, Perak

$3^{\text {rd }}$,, Jaffar Tod Kaya, Penang

I $856 \quad 5$

$544 \quad 2$

Division B.

Flowers, Fruits and Vegetables.

86. - Aroids:-

H. M., Mrs. R. A. J. Bidwill, Dalvey Road, Singapore

H. C., O. Mahlenbein, Nassim Road, Singapore 30

87.-Aroids, best specimen :-

Ist Prize, Mrs. R. A. J. Bidwill, Singapore 704

2nd " Mrs. St. V. B. Down, Anderson Road, Singapore

88.---Caladiums (6):-

Ist Prize, Choa Kim Keat, c/o Straits Trading Coy., Singapore

2nd " Mrs. R. A. J. Bidwill, Singapore $\quad 70 \quad 3$

89.-Caladium (I) :-

Ist Prize, Mr. Alex. Gentle's Gardener, Nassim Road 574

90-92.-No award.

93.-Crotons, best specimen :-

Ist Prize, Mrs. L. P. van Cuylenburg, Paterson

Road, Singapore

94.-Dracrenas:-

Ist Prize, Choa Kim Keat, Singapore

95.-Dracaenas, best specimen :-

Ist Prize, Choa Kim Keat, Singapore

I0 5

96.-Ferns, any variety :--

rst Prize, Mrs. St. V. B. Down, Singapore

Io 3

97.--Ferns (Adiantums) :-

Ist Prize, Mrs, van Cuylenburg, Singapore

75 I0

and ," Mrs. St. V. B Down, Singapore 
Class No.

98.-Ferns (Adiantums), distinct:-

Exlutbit Account.
No.

ist Prize, Mrs. R. A.J. Bidwill

99.-Ferns, any variety:-

Ist Prize, Mrs. St. V. B. Down, Singapore

705

754

Ioo.-Marantas:-

Ist Prize, O. Muhlenbein, Singapore

308

IOI.-Marantas, best:-

Ist Prize, Mrs. St. V. B. Down, Singapore

$75 \quad 4$

102.- - Palms, distinct:-

Ist Prize, Choa Kim Keat, Singapore

Io Medal

and ", Joe Constantine, Raffles Hotel, Singapore 1835

ro3.-Palms, best:-

Ist Prize, Chesney Duncan, Penang

$580 \quad 5$

and "Mrs. St. V. B. Down, Singapore

Special Prize, Choa Kim Keat, Singapore

104-ro6.-No award.

ro7.-Foliage plant:- -

Ist Prize, Choa Kim Keat, Singapore

ro8.-No award.

rog.-Foliage plant, best :-

Ist Prize, Choa Kim Keat, Singapore

IO 5

rog (a).-Foliage plant collection :-

ist Prize. A Cup presented by Hon. Tan Jiak Kim,

Choa Kim Keat, Singapore

I I0-I I3.-No award.

II4.-Cannas :-

Ist Prize, Rev. Father Gex, Singapore

I 5.-No award.

II6.-Cannas, best :-

Ist Prize, Rev. Father Gex, Singapore

I17.-Cockscombs:-

Ist Prize,

Singapore

$10,000 \quad 2$

1 8 .-Chrysanthemums :-

Ist Prize, Mrs. D. W. Lovell, Cluny Road, Singapore 563

Ir 9. -Dahlias :-

Ist Prize, Choa Kim Keat, Singapore

IO 3

I 20-I 22.-No award.

123.-Orchids, :-

Ist Prize, Choa Kim Keat, Singapore

IO I2

123 (a).-Orchids, best:-

Ist Prize, Mrs. Sit. V. B. Down,-Singapore

75 IO

124.-Orchids, specimen :-

Ist Prize, Choa Kim Keat, Singapore

ro 5

125.-Phlox:-

Is Prize, Mrs. Pennefather, Scott Rd., Singapore 
Class No.

Exhibit Account.

I 26.-Petunias :-

Ist Prize, P. S. Falshaw, Singapore

I60 3

I 27.- None shown.

128.- Roses, best :-

Ist Prize, Mrs. Pennefather, Singapore

I $6 \quad 3$

129-I 30.-Nंone shown.

131.- Plant in Flower:-

rst Prize, Mrs. Sugden, Tanglin, Singapore

I32.--Begonias:- -

Ist Prize, P. S. Falshaw, Singapore

and "Choa Kim Keat, Singapore

1955

I $60 \quad 5$

ro 3

IO 3

Ist Prize, Choa Kim Keat, Singapore

I34.--Group of plants :--

Ist Prize, Mrs. R. A. J. Bidwill, Singapore

708

I35.-Group of Chinese plants:-

Ist Prize, Seah Liang Seah, Singapore

I 36- I 39.-None shown.

I $40 .-$ Cannas :-

Ist Prize, Father Gex, Singapore

and , Mrs. St. V. B. Down, Singapore

$44 \quad 3$

$75 \quad 2$

I 4 I. - Orchids :-

rst Prize, Mrs. St. V. B. Down, Singapore

753

I 42.- Bridal Bouquet:-

Ist Prize, Miss. J. Gray, Singapore

I IO 5

143.- None shown.

I44.- Cut flowers, arranged:- -

rst Prize, Miss Moss, Singapore

I 23

I 45-Cut flowers, collertion :-

Ist Prize, Choa Kim Keat, Singapore

ro 5

146.--Table Decoration :-

ist Prize, Mrs. St. V. B. Down. A'Cup presented by

Choa Kim Keat Esq.

and Prize, Mlle. de Gaspery, Singapore

I47.- None shown.

I48.-Button-holes:-

Ist Prize, Miss Schomburgk, Singapore

I 7 I 3

Section 2.-Fruits.

I49.--Bananas:-

ist Prize, Mohamud Yunos To-Kaya, Kelawi, Penang

and , Syed Ahmat, Land Office, Penang

I50.--Bananas, best :-

Ist Prize, Penghulu Kakit Goyang, S'Tapa, Kuala

Lumpur

2nd Prize, Yacob, Labu Ulu, Selangor

$2040 \quad 3$

25532 
I 5 I.-Champadak:-

No.

Ist Prize, Penghulu Ahmat Bin Surin, Krubong,

Malacca

152.-Chiku :-

Ist Prize, Rev. Father Gex, Singapore

2nd "Jaludin, Pengkalan Ramah, Malacca

I 53.-Custard Apple:-

Ist Prize, Miss. E. E. Brown, River Valley Rd.,

Singapore

and Prize, H. H. Norris, Singapore

I 54.-Cultivated Fruits (Collection) :Ist Prize, D. Logan c/o Farrer Baynes, Singapore. A

Cup presented by Mr.-H. Marriot and ," H. H. Norris, Singapore

I55.-Durians :-

Ist Prize, Hadji Mat Saman, Batu Kurau, Perak I8or 5 and "Litah Bin Go Mankok, Ulu Langkat, Selangor

156.-Durian Blanda:-

Ist Prize, Sedang Rahim, Pegoh, Malacca

$.106 \mathrm{r} 2$

I 57.-Duku :-

Ist Prize, Penghulu Yusuf Bin Buang, Chobong,

Malacca

2nd Prize, Penghulu Sulong Bin Hussin, Chinchin, Malacca

158.- None shown.

San Bin Hadji Ismail, 40 Pahang St., Singapore

I60.- - Jambu:-

Ist Prize, Mohamud Arsat, Caunter Hall, Penang

I61.-None shown.

I62. - Limes.

Ist Prize, Penghulu Anjang Abdullah, Kampong

Gajah, Perak

and Prize, A. D. Machado, Perak

I $780 \quad 5$

15003

I63.-None shown.

164.-Machang:-

Ist Prize, Chee, Ayer Hitam, Penang

$5 \times 42$

I65.-Mangosteen:-

Ist Prize, Hadji Munahon, Rope Walk, Penang 5475

and ", Hadji Mat Yarsin, Setul, Malacca. 25293

166. - Mata Kuching:-

Ist Prize, Hadji Alam Bin Pongut, Nyalas, Malacca I1o7 2

I67.- Melon :-

Ist Prize, Rajah Hitam Nongchi, Johore

168.-Papaya :-

Ist Prize, Rajah Hitam Nongchi, Johore 
Class No.

Exhibit Amount.

I69.--Oranges :-

Ist Prize, G. F. Bird, Kuala Kangsar, Perak

* and "Along Mohamud Talip, Passir Salak, Perak

I 52 I 3

17782

r 70.- Pine apples (Mauritius) :-

Ist Prize, W. Dumman, Singapore

and "Kauteem Seng, Survey Office, Penang

$124 \quad 3$

5 II 2

I 7I.--Pine apples (any other) :-

Ist Prize, Sri'Ressam, Johore

and " Hukum; Tanjong Malim, Perak

1553

I 8552

I 72.--Pomeloes :-

Ist Prize, G. F. Bird, Kuala Kangsar, Perak I52 I 2

and , R. IV. Munro, Kuala Langat, Selangor 219 I

I 73. - Pulasan :-

Ist Prize, Rajah Hitam Nongchi, Johore

$125 \quad 2$

I74. - Rambai :-

Ist Prize, Sidang Baba Bin Arshad, Duyong,

Malacca

2nd Prize, Penghulu Ahmat Bin Surin, Krubong,

Malacca

175.-Rambutan :-

Ist Prize, Sabut, Bantai, Kuala Lumpur

and " Nohamud Hassim, Kampong Bahru, Penang

IOOO I

$254^{\circ} \quad 2$

$523 \quad I$

I76. - Wild Edible Fruits :-

Ist Prize, Penghulu Rahmat, Batu Pahat

1325

and , Said Ahmat, Penarig

I77.- Any kind of fruits:--

Ist Prize, Sri'Ressam, Johore

and ", R. IV. Munro, Selangor

Special Prize, F. G. Desker, Singapore

I 553

2192

722

I 78.-Preserved Fruits :-

Ist Prize, Rajah Hitam Nongchi, Johore

and "Pengluulu Ahmat Bin Surin, Krubong,

Malacca

125 IO

3rd Prize, Mohamud Kassim, Land Office, Penang 5053

I 79.-Chutney :--

Ist Prize, Francis Alexander, Kuala Lumpur

204 I 5

(Special Prize) Mr. Lesslar, 'Taiping, Perak

17773

I 80.-Pickles:-

Ist Prize, Mrs. A. C. Moses, Singapore

Special Prize, Mr. Lesslar, Perak

$42 \quad 5$

17773

I 8:- - Jellies :-

Ist Prize, Francis Alexander, Kuala Lumpur

$204 \mathrm{I} \quad 5$

I 82-184.-None shown.

185. - Brinjals :-

Ist Prize, Daud Bin Burok, Nyalas, Malacca 
Class No.

I86.-Beans:-

Exhibit Amotnt

ist Prize, Chee, Ayer Hitam Penang

I87.- -None shown.

I88.-Chilis :-

Ist Prize, Kendat Bin Hitan, Nyalas Malacca

and , Francis Alexander, Kuala Lumpur

I I O 3

$204^{1} 2$

1 89.-Cucumber:-

Ist Prize, Mohamud Kassim, Land Office, Penang

$505 \quad 2$

rgo.-None shown.

I9I.-Ladies Fingers:-

rst Prize, Ali Bin Wahid, Penang

5152

r92.-Lettuces:-

Ist Prize, Ali Bin Wahid, Penang

$515 \quad 2$

193.--None shown.

194. - Pumpkins :-

Ist Prize, Penghulu Abdul WTahal, Temerloh Perak I $52+2$ I95.-Radish :-

Mohamud Kassim, Penang

$505 \quad 5$

196.-None shown.

I97-198.-None shown.

т99.-Watermelons, etc. :-

Ist Prize, Ali Bin Wahid, Penang

$515 \quad 5$

200.- - Yams:-

Ist Prize, Penghulu Matsaid Bin Ma'Ali, Tanjong

Minyak, Malacca

and Prize, Rajah Hitam Nongchi, Johore

20r.-Any other Vegetables:-

Ist Prize, Alang, Kuala Kangsar, Perak

and ", Mrs. F.J. Benjafield, Singapore

\section{Division C.}

Stock and Daity Produce.

202.-Bull Locally Bred:-

Ist Prize, Mungol Singh, Serangoon Road, Singapore 8220

and "Es. Galopsah, I7 Burmah Road, Singapore r20 Io

203.-Cow, Locally Bred:-

Ist Prize, Es. Galopsah, Singapore

I 2020

204.-Bull or Bullock, Indian :-

None shown.

205.- Cow and Calf, Imported Indian:-

Ist Prize, Abdul Wahid, Rochore Road, Singapore 8620

206.-Cow and Calf, Locally Bred:-

rst Prize, Es. Galopsah, Singapore 
Class Nio.

207.-Best Milk Cow of any kind:-

Exhibit Amount

Ist Prize, Abdul Wahid, Singapore. A Cup presented by Hon. A. 'T. Bryant

208.-Pair of Draught Oxen, Siamese :- -

None shown.

209.-Pair of Draught Oxen, Indian :-

Ist Prize, Mariappen, Singapore (Silver Medal)

and ", Kader Ali, Marikar, Mohamud, Sultan Road 885

2ro.-Best Pair of Draught Buffalos:-

None shorvn.

2 I I.-Buffalo Bull, Indian :-

rst Prize, Abdul Majid, Macpherson Road, Singapore 97 I5

2 I2.-Buffalo Cow, Indian :-

Ist Prize, Siva Sunga, Serangoon Road, Singapore Io2 I 5

2nd "Sedang, Serangoon Road, Singapore $96 \quad 8$

213-214.-None shown.

215.-Champion Animal :-

rst Prize, Mungal, Sing, Singapore

8220

2i6.-Boar:-

rst Prize, Dr. Lim Boon Keng, Singapore

39 I0

2I7.-None shown.

218-219. -None shown.

220.222.-None shown.

223.--Goat Ewe with Kids:-

Ist Prize, Sheik Mohamud, 4o Buffalo Road,

Singapore

17910

224. - None shown.

225.- Sheep Ram:- -

Ist Prize, Abdul Wahid, Singapore

86 I0

226. - Sheep Ewe:-

Ist Prize, Abdul Wahid, Singapore

86 Iо

227.--Sheep Best Pen of six Indian Breed :-

Ist Prize, Abdul Wahid, Singapore

86 IO

228. - Sheep Best Pen of six Javanese Breed:-Ist Prize, Ali Bin Ahmat, 9I Beach Road, Singapore 62 ro

229. - Bantam Cock and Hen:-

Ist Prize, Mrs. Hawtrey, Singapore

and "Lim Ben Cheng, Singapore

$105 \quad 5$

1963

230.-Malay Cock and Hen:-

Ist Prize, Yu Mat Ali, Kampong Bona, K. Kangsar, Perak

2nd Prize, Hadji Ibrahim, Upper Peral:

16065

I 5073

23I:-Malay Game Cock and Hen:Ist Prize, Mrs. Hawtrey, Singapore

1055

232,-Collection of Malay Fowls:-

and Prize, Hadji Mohamud Yasin, S'Tiwan, Perak 17945 
Class No.

233.- None shown.

234.-Collection of Fowls any Breed:Ist Prize, Mrs. Hawtrey, Singapore and "Mrs. R. A. J. Bidwell, Singapore

235. - Best Cock and Hen any Breed:-

Ist Prize, H. G. Wightwick, 5 I Devonshire Road, Singapore

2nd Prize, Mrs. Hawtrey, Singapore

236.-Muscovy Duck and Drake:Ist Prize, K. A. Stevens, Singapore

237. - Manila Duck and Drake:Ist Prize, Rahlm Bin Hassin, Tabo Naning Malacca 10775

238.-Pair of Duck and Drake any Breed :--

Che Mat Krani, Sungei Ara, Penang

239.-Duck and Drake Hybrid :-

Ist Prize, Hadji Othman, Butterworth, Penang

240.-Gander and Goose :-

Ist Prize, Chuan Keng Peng, 30 South Bridge Road, Singapore

and Prize, K. A. Stevens, Singapore

241.--Pair of Turkey, Cock and Hen :Ist Prize, Mrs. Hawtrey, Singapore

242.-Pair of Guinea Fowl:-

Ist Prize, Mrs. Hawtrey, Singapore

243.-- Pair of Pigeons :-

Ist Prize, Miss. E. Schomburgk, Singapore

and "H.G. James, 28 Syed Ali Road, Sin-

244.- None shown.

245.-Best Cage Bird:--

Ist Prize, Chea Eng Heng, 6 North Boat Quay, Singapore and Prize, Inche Dallah, 75 Anson Road, Singapore

246.-None shown.

247.- Pair of Rabbits:-

Ist Prize, Miss. E. E. Brown, Singapore

and "F. Hilton, Singapore

248.-None shown.

248(a).-Champion Cat in the Show :Ist Prize, Miss Edith Daly. A cup presented by

Hon. A. T. Bryant

and Prize, O. Muhlenbein, Singapore

249.--None shown.

250.-Collection of Eggs:-

Ist Prize, Mrs. Abrams, Singapore

2nd ", M. Stuart, Singapore 
Class No.

250(a).-Best Locally made Soap:-

Ist Prize, C. Hock Leong \& Co., 23 Park Road,

Singapore (Silver Medal)

251.-Best Turnout Bullock cart :--

None shown.

\section{Division D.}

(HORSES AND DOGS.)

\section{Horses.}

252.-Polo Pony :- -

Ist Prize, J. B. Young. A cup presented by H. Payne-Galwey, Esq.

and Prize, Mr. Spooner. A cup presented by the Committee.

253.-Gentleman's Hack (14.2 and under) :-

ist Prize, Mr. J. B. Young. A cup presented by C. Sugden, Esq.

2nd Prize, Mr. J. B. Young. A cup presented by the Committee.

254.-Gentleman's Hack (I4.2 and over) :-

Ist Prize, Mr. C. Sugden. A cup presented by E. F. H. Edlin, Esq.

and Prize, Capt. Mackintyre. A cup presented by the Committee.

255. - Single Harness Pony ( 14.2 and under):-

Ist Prize, Mrs. Diepenheim. A cup presented by Dr. Galloway.

2nd Prize, Miss E. Abrams. A cup presented by the Committee.

256.- Single Harness Horse (14.2 and over):-

Ist Prize, H. Abrams. A cup presented by the Bankers.

and "Mr. Geo. Greaves. A cup presented by the Bankers.

257.-Lady's Hack:-

Ist Prize, Mrs. Stitt. A Silver Bowl presented by J. B. Young.

2nd Prize, Miss Abrams. A cup presented by the Committee.

258.- - Jumping Pony ( 14.2 and under) :-

ist Prize, Capt. Macintyre. A cup presented by W. D. Barnes, Esq.

and Prize, Mr. Pickering. A cup presented by the Committee.

259.- Jumping Horse ( 14.2 and over) :-

Ist Prize, Mr. H. Abrams. A cup presented by the Brokers.

and "Mrs. Stitt. A cup presented by the Committee.

260.-Double Turnout:--

Ist Prize, Messrs. Schmidtmann \& Tan Liang Kee. A cup presented by Noel Trotter, Esq.

and Prize, Mrs. Diepenhein. A cup presented by the Committee. 
Class No.

261.-Gharry 'Turnout:-

Ist Prize, Medde Bin Hadji Hasan

2nd, Barsey

3rd , Seman

262.-Best Horse or Pony:-

C. Sugden, Esq. A cup presented ly D. K. and J. Sommerville, Esq.

Dogs.

263.- Smooth-Haired Fox Terrier Dog.

Ist Prize, H. Kirwan, Esq. A cup presented by L. S. Lewis, Esq.

and Prize, Miss Littlefair. A cup presented by the Committee.

264. - Smooth-Haired Fox Terrier Bitch.

ist Prize, H. C. Sechehay. A cup presented by R. Ker, Esq. and $"$ G. E. Mosley. $\Lambda$ cup presented by the Committee.

265.-Rough-Haired Fox Terrier Dog.

Ist Prize, J. B. Young, Esq. A cup presented by A. R. Linton, Esq.

2nd Prize, T. C. B. Miller. A cup presented by the Committee.

266.-Rough-Haired Fox Terrier Bitch.

Ist Prize, Mrs. Fittock. A cup presented by the Committee.

267.-Chow Dog or Bitch.

Ist Prize, H. P. Kinghorn, Esq. A cup presented by J. Graham, Esq.

268.-Spaniel Dog or Bitch.

Ist Prize, E. M. Janion, Esq. A cup presented by Geo P. Owen, Esq.

and Prize, D. Robertson, Esq. A cup presented by the Committee.

269.-Irish Terrier Dog. Ist Prize, Mrs. Diepenheim. A cup presented by F. Hilton. and "Major Ritchie. A cup presented by the Committee.

270.-Irish Terrier Bitch.

Ist Prize, F. Hilton. A cup presented by A. D. Allan, Esq.

27 I-272.-Kangaroo House Dog or Bitch.

Ist Prize, K. A. Stevens, Esq. A cup presented by Mr. H.C.

Ehrseefells.

273.-Dog or Bitch any Breed.

Ist Prize, Tan Peck Wan. A cup presented bx E. M. Janion, Esq.

2nd Prize, Teo Cheng Hee. A cup presented by the Committee. 3rd , Mrs. St. V. B. Down. A cup presented by the Committee. 
Class No.

274.-Champion Dog or Bitch.

Ist Prize, Tan Peck Wan. A cup presented by the Borneo Coy.

and Prize, E. M. Janion, Esq. A cup presented by the

Committee.

3rd Prize, Mrs. St. V. B. Down. A cup presented by the Committee.

Native Industriles.

275.-Ornamental Baskets:-

5 prizes of 5 each were allowed.

No. 1025 Haji Ma' Ali, 'Tanjong Kling Malacca

, II23 Mahani binte Mamat, Tanjong Kling, Malacca 5

", II24 Nilan binte Lateh, Tanjong Kling, Malacca 5

,, I I 25 Jijah binte Jahudin, Tanjong Kling, Malacca 5

, I 26 Saliah binte Ladi, Tanjong Kling, Malacca 5

276. - Lace work:-

Ist Prize, Io 2 Penghulu Baba, Pringgit, Malacca

and "Senah Binte Salleh, Malacca, Lace work

Io

277. - Leather work no prize :-

278. - Rope and twine-

Ist prize, No. 600 Shuth Hussin, Golf Club, Penang

2nd "No. I804 Wan Mohamed Nasie, Bukit Gantang, Perak

279.-Tali Ijok:-

Ist Prize, No. I048 Nudin bin Kali, Gadok, Malacca and ," , I05x Penghulu H. Saleh, Malaha Pindah

Ist Prize, No, $1786 \mathrm{H}$. Mohamed Tahir, Sungei Durian, Perak

and ," "I 785 Mohamed Nurdin, Kota Stia, Perak

Ist Prize, No. 50 Inche Omar, Kota Tinggi, Johore Medal

and ," I79o H. Mohamed Ali, Peduka Rajah, Pulau Tíga, Perak

282.-Chicks :-

Ist Prize, No. 600 Sheikh Hussin, Golf Club, Penang and Prize, No. 560 Nyak Ah Yum, Klawei, Penang

283.-Mats. None shown.

284. - Rattan Mats:-

ist prize, No. I8 40 Pandak Abdullah, Bagan Serai, Perak Io and Prize, No I 779 Mohamed Noh, Bagan Datch, Perak 5

285 - Malay Mats :-

rst Prize, No. I $8+4$ Penghulu, Ulu, Selama, Peralz Io and ", 575 Mat Bin Mohamed Dain, Rope IValk, Penang 
Class No.

286. - Hats:--

Ist Prize, No. 2620 Senin binte Chee, Port Dickson,

N. Sembilan

and Prize, No. 2625 'Tiah binte Ma' Arip Sungei Rusa,

N. Sembilan

287. - Brushes and Brooms :-

ist Prize, Nil

and ,"No. 600 Sheikh Hussin, Gulf Club, Penangr

288. - Collection of articles made from Coconut palms :-

ist Prize, No. I78I Mohamed Arshad, Clerk, Lower Perak

2nd Prize, No. I 790 H. Mohamed Ali, Pedula Rajah, Perak

289.-Articles made from Kabong:-

Ist Prize, Nil

2nd ", No. I 567 Megat Abdul Magid, Senggang, K.K. 5

290.-Articles made from Nibong:--

Ist Prize I Nil

and

29I.-Set of Sireh apparatus :-

ist Prize, No. 2670 To Mentri Perkasa Sahia, Rambu N. Sembilan

and Prize, No. I552 Mahubuh, Kota. Lama Kiri, Kuala Kangsa

292.-Model Malay houses:--

Ist Prize, I80+ Wan Molamed Nasie, Bukit Gantang, Perak

2nd Prize, No. I538 Pandah Mohamed Asik, Kota Lama, Perak

Special prize $\$ 5$ No. I 787 Haji Mohamed Nasie, Utan Melintang, Perak

293.-Model Fishing Stakes:-

ist Prize, No. I 794 H. Mohamed Yasin, Steawan, Perak 2nd Prize, No. ror 8 Penghulu Usop, Klebang Besar, Malacca

294.--Snares and Traps:-

Ist Prize, No. I794 H. Mohamed Yasin, Stewan, Perak ic and Prize, No. I787 H. Mohamed Nasie, Utan Melintang, Perak

295.-Wood Carving:-

Ist Prize. Nil.

and "No. 529 Yib, Butterworth, Province Wellesley

296.--Embroidery:-

Ist Prize, No. I 577 Datoh Stea, Saing, Kulua Kangsa ro and No. I769 Rajah Bila, Papan, Perak ro

2nd Prize, No. i i 6 Abu bin Dor, Pengkalan Bama, 
Class No.

297.-Silk Sarong:-

Ist Prize, No. I 782 Raja Abdul Malil, Teluk Anson, Perak

2nd Prize, No. I68 Salleh, 7 Java Road, Singapore

298.-Cotton Sarong:-

ist Prize, No. 54 I Sheilk Yusuf, Butterworth, Province

Wellesley

2nd Prize, No. I 502 H. Berheby, Upper Perak

299.-Brass Ware:--

Ist Prize, No. 549 Haji Mohamed Syed, Acheen Street, Penang

and Prize. Nil.

300.-Silver NTare:-

ist Prize, No. I65 Lagid, 135 Arab Street, Singapore Io and "Nil.

301.-Tin Ware:-

Ist Prize, No.25I7 Kwan Lum, Seremban

and ," "2520 Siow Chin, Seremban

302.- Kriss-sheaths and knife handles:-

Ist Prize, No. 536 Haji Othman, Butterworth, P. W.

and ", " I 552 Mehabuh, Kota Lama Kiri, Perals 5

303.-Fishing Nets:-

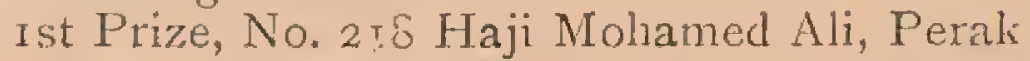

and " ", I788 Abdullah Ali, Teluk Bahru, Perak

30+.-Sakei Articles:-

Ist Prize, No. I 85 I Renghulu, Tapah, Perak

and ", "I034 Sulong bin Aris, Bringin, Malacca

305.-Collection of Photographs:-

Commended No. I 500 A. D. Machado, Kamuning, Perak.

306.--Miscellaneous:-

Ist Prize,

and ', 226 Sit Yow Kun, 27 Duxton Hill, Singapore Io.

and $20 \mathrm{~F}$. A. Langley, Guthrie and Co., Singapore

307.-Building Bricks :-

Ist Prize, No. I856, Mohamed Abas, Tanjong Malim,

Perak

and Prize, No. 45, Alexandra Brick Works, Singapore

I 24 Highly Commended W. Dunman, Singapore I 54 I Honourable mention, Penghulu Indub, Perak

308.-Roofing tiles :-

Ist Prize, No. I 54I, Penghulu Indut, Kota Lama Kanan, Perak

2nd Prize, No. Ioo2, Penghulu Masap, Bachang, Mallacca

309.-Flooring tiles:

Ist Prize, No. 22, Alfred Lea, Hill Street, Singapore and ," , I54I, Penghulu Indut, Kota Lama Kanan, Perak 
Class No.

310.-Bird Cages:--

ist Prize, No. 536, Haji Othman, Butterworth, P. W. Io

and Prize, No. 535, Penghulu Táha Permatang Bertam, P.W.

3 I I, - Plough :-

ist Prize No, roI 8 Penghulu Usop, Klebang Besar

Malacca

2nd Prize. Nil.

312.--Bertam Work:-

ist Prize. Nil.

and "No. 512, Syed Ahmat, Land Office, Penang 5

313.- Flower pots and fancy pots:-

Ist Prize, No. 2094, Li Kong Lam, Selangor

and " " 530, Murugapa, Butterworth, P.W.

IO

3 [4.-Chatties (earthenware):-

rst Prize, No. I79o, H. Mohamed Ali, Peduka Raja, Pulau 'Tiga, Perak

2nd Prize, No, 1792, Mohamed Mansur, Durian

Sebatang, Perak

$314 A .-$

Special Prize, \$1 for drawn-thread work No. I7, Kwong Seng, Singapore.

Special Prize, \$5 for set of carved coconut shells No. 248. Special Prize, \$5 for table and chair, Katingga wood No. I 503, Ismail, Upper Perak.

Class No.

Exhibit Amount No.

Competitors from Schools.

315.-Malay Hand-made Pottery:-

Ist Prize, Kampong Kedah Boys, Perak

I686 Io

316.-Malay Hand-made Fancy Pottery:-

ist Prize, Pulau Tiga, Malay School, L. Perak i67 I ro

317.-Ornamental Baskets:-

Ist Prize, Bagan Serai Girls, Malay School, Krian, Perak

I 696 Io

2nd Prize, Gunong Senanggol, Malay School, Taiping, Perak $\quad 1638 \quad 5$

H. C. Mohamed Bin Mohd. Noor, Kwala Lumpur School

H. M.

do

do

2026

318.-Malay Mats (Pandanus):-

Ist Prize, Lambor Kanan, Malay School, K. Kangsar, Perak

I 756 Io

and Prize, Bagan Serai Girls, Malay School, Krian, Perak

Special Prize, Batu Gajah Girls, Malay School, Kinta, Perak

H. C. Bagan Serai Girls, Perals

H. C. Lombor Kanan, Perak 
Class No.

Exhibit Amount.

3I9.-Sireh Apparatus, set:-

No.

Ist Prize, Lakan Binte Bachee, Kwala Lumpur, School

2nd Prize, Teluk Bahru, Malay School, L. Perak $\quad$ i668 5

320.-Model Malay House:-

Ist Prize, Tanjong Piandang, Malay School, Krian,

Perak

I689 Iо

2nd Prize, Teluk Pial, Malay School, Krian, Perak 16945

H. C. Bulat Teacher, Bernang School, Selangor 2010

32I.-Model Fishing Stakes:-

Ist Prize, Slim, Malay School, Batang, Padang, Perak

I 724 I0

2nd Prize, Temerlok's Boys Malay School, Matang, Perak

16525

322.-Wood Carving:-

Ist Prize, Bulat Teacher, Bernang School, Selangor 2010 Io

2nd ,Sudin Teacher, Bernang School, Selangor 20075

323.-Embroidery:- -

Ist Prize, Bandar Girls, Malay School, L. Perak 1682 ro 2nd " Teluk Anson Girls, Malay School, L. Perak

Special Prize, Minah Binte Bakar, Girls School, Kwala Lumpur

Extra Prize, Teluk Anson Girls, Perak

68 I 5

H. C.

do do

H. C. Bandar Girls

324.- - Sarong (Silk) :-

Ist Prize, Kota, Lama Kiri Girls, Malay School,

K. Kangsar, Perak

and Prize, Kota, Lama Kiri Girls, Malay School,

K. Kangsar, Perak

Special Prize, Taiping Girls, Perak

20 or 5

I68I 3

H. C. Girls, Schools, Malay Settlement, Kwala

Lumpur

I753 IO

$1753 \quad 5$

$1644 \quad 3$

2022

325.--Sarong (Cotton):-

Ist Prize, Matang Girls, Matang, Perak I659 Io

2nd " Lenggong Girls, Upper Perak $\quad$ I765 5

326. - Snares etc, collection:-

Ist Prize, Sungei Saikap, Malay School, Krian, Perak

1690 Io

2nd Prize, Temoh Malay School, Batang Padang, Perak

17215

327.-Fishing Nets:-

Ist Prize, Jeram School Boys, Kwala Lumpur 2000 ro and ", do do 20005 H. C. do do

do

328.--Miscellaneous:-

Ist Prize, Teluk Anson Boys, L. Perak $\quad$ I662 Io

2nd "Sungei Rusa, Boys, L. Perak I678 5

Special Prize, Tanjong Piandang, Krian, Perak $\quad$ r689 3 
The following table shewes the Number of Exhibitors in each Division, places from which they cane, and the prizes gained.

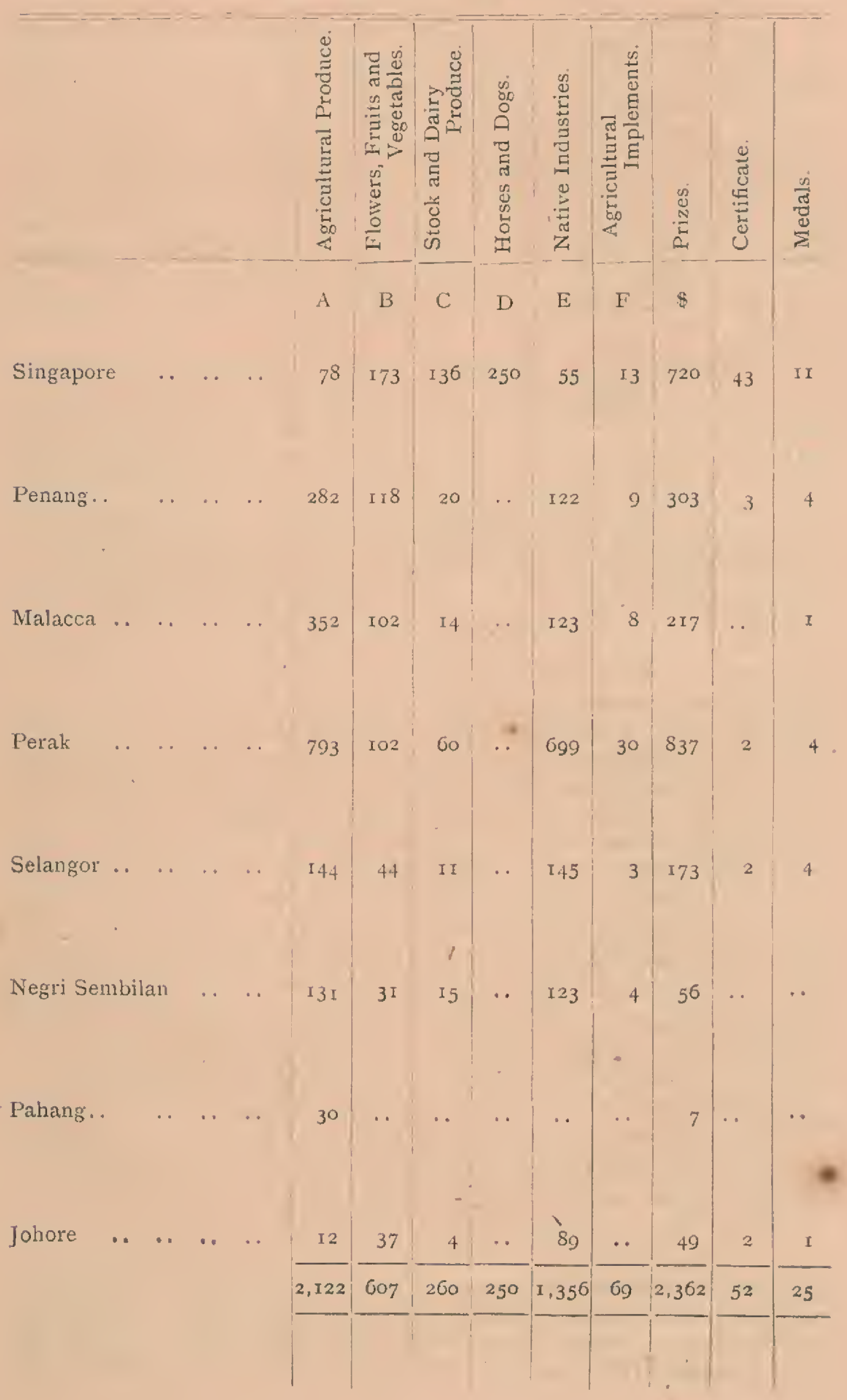


Notes on Management of Exhibitions by

G. A. HALl.

: :

a. As to organisation preceding the opening, it would be an improvement to make a register for each section, as the entries are notified, with a page devoted to each class, so that all the entries for a class, with the exhibitors' names and addresses, would be shown together. The secretary could then accompany the judges with this register, and note down the prizes in it, and the preparation of the prize-list would then be a mere matter of copying. Such an arrangement would save much time and trouble, and would further enable the Secretary to allot his space beforehand, by showing the items under which the largest quantity of exhibits was to be expected. It would also form a record of the show for further reference.

b. The prize-list should not be copied from any former prizelist without careful examination. It should not be adopted by a large and hurried public meeting, but gone through section by section by a few persons who have had actual experience in former shows. Take for instance Division "E." There were complaints that people could not find things because they were not arranged in the order given in the prize-list, but with a prize-list like this it is not possible to keep to the order. First we have ornamental baskets, next lace-work, then leather work, rope, attaps, kajangs, mats, hats, brooms and other collections of articles made from coconut-palms. It is obviously impossible to arrange attaps, kajangs and similar exhibits on shelves alongside lace-work, and curios made of coconut shells. Further down we cane to sireh apparatus then models and then wood-carving and embroidery, fishing nets between krisses and sakei articles and bird-cages between flooring tiles and ploughs. The best way would be to arrange all delicate and valuable articles together so that they could be displayed in the order given in the prize-list. For instance lace, silks, embroidery, silver ware, sireh apparatus, krisses, curios made of coconut shells, brass-watre, and wood-carving might easily be classified in the order in which they could be displayed as could also cumbrous things like attaps, kajangs, chicks, mats, brooms batam-work, and earthenware. Models should have a shed to themselves. They take an immense amount of room and require special accommodation. Perhaps a shed to contain all models, attaps, kajangs, brooms, brushes, fishing-nets, ploughs and agricultural implements generally, would be the best solution of the difficulty. The result of crowding models into the native industries section is that space is filled by them which is badly wanted for displaying embroideries, silver-work, sireh sets, and so on, and many interesting exhibits are so packed that they can hardly be seen, and cannot be arranged at all.

c. The question of exhibiting antique articles requires consideration. Assuming that the object of this section is to stimulate native handicrafts, it is a little difficult to see why ancient jewellery should be allowed any prizes at all. In any case old work should be in a separate class, and should not enter into competition with 
new work at all. For silver-ware it would be as well to provide cases that could be locked so that they need not be removed every evening. The prize-list of Class II of this section for schoolexhibits is far too small, and should have many items added to avoid an unwieldy number of exhibits under "Miscellaneous." It would be better another time to give this class more wall-space, and to separate it entirely from the native industries section.

d. As to the construction of the shed for native industries, rough doors or half-doors should be provided so that all entrances but one could be effectually closed at $60^{\prime}$ clock, and the shed emptied before dark. Plenty of strings should also be fixed between poles and against walls for showing mats. Adequate police arrangements should also be made for clearing the shed, and it should not be left to members of committees to turn people out. Owing to the absence of proper police precautions it was impossible to clear this section before dark, and a certain number of articles are stated to have been stolen. This is a danger to which this section is particularly exposed as many of the exhibits are small and valuable. Arrangements should be made for keeping the ground as dry as possible by digging drains, laying down cinders or sand, and a few coolies should be engaged for each particular section. The want of small supply of labour was much felt, e.g., for clearing up the floor after the exhibits had been arranged, putting up barriers to close entrances, running errands, and stowing away packing cases, and so on. It is no use engaging coolies for the show generally because they are never at hand when wanted. When there are any displays likely to attract a crowd, they should be arranged in a place with one entrance and one exit so that the crowd, can be liept moving in one direction only.

e. Much more care is necessary in the translation of the prize-list into Malay. There were many mistakes which caused annoyance to exhibitors who had been misled by them. For instances under No. 288 the translation implies that the articles must be made out of the trunk of trees. Under 302 the translation means that the exhibits are to be only kriss-sheaths which was perhaps hardly intended. Under 289 and 290 the single word "kabong" or "nibong" is insufficient.

$f$. Large cards showing the numbers and nature of the classes of exhibits should be prepared beforehand. These are of the greatest assistance in arranging exhibits as they enable the space to be allotted beforehand and any members of committee helping in arrangement can direct exhibitors to the proper place, without difficulty or confusion.

g. It seems advisable to make more definite provision beforehand for landing exhibits and housing exhibitors. On the Tuesday before the opening two tongkangs with exhibits were waiting off the reclamation from early morning till $30^{\prime}$ clock in the afternoon before landing anything, and many who were expecting to find house accommodation found nothing ready for them. This however is a simple matter which requires only to be thought of, and entrusted to two or three members of committee in plenty of time. 
h. The question of the size of committees is worth consideration. There is no object in having a huge general committee and in any case meetings of the general committee should be as few as possible. Beyond general questions such as the choice of site and the date of the exhibition there is no use in assembling the general committee at all, and mischief may easily be done by a large meeting which is easily misled through inexperience, e.g., in this case they very nearly shifted the site at the last minute, and, if they had, the financial result would have been disastrous. Also in sub-committees, appoint two or three men who are likely to work, and let them add to their number if they want to. It is useless to nominate ten men if five have not the time or the inclination to assist, and never come near the place except as visitors with free tickets. "Family ticliets" should be abolished altogether.

$i$. The prize-list might be drawn up something in this way. All models, should be cut out of this division altogether. Lace, embroidery and sarongs should come together, krisses and knives, old and new, next. All metal work should form one group, i.e., silver (old), silver (new), sireh sets, brass and tin. The same with all wood-work and carving whether made from coconut trees, liabong, nibong or other wood. Leather-work and rope conld go in anywhere, and baskets, mats, hats, bertam work and bird cages should come together as should bricks, tiles and fancy earthenware. Attaps, chicls, and kajangs could come at the end, but would be better accommodated outside the shed altogether. Further there seems to be no sense in making a cast-iron rule that every first prize is to be $\mathrm{Io}$. An exhibit like kajangs is not worth the same prize as a piece of embroidery, nor is a bird-cage worth the same prize as a silver exhibit or a silk sarong. 'The value of the prizes should certainly bear some relation to the value of the exhibits. 
4th

Ofri: Hoaticuid Shoro

AGRI-HORTICULTURAL SHOW

KUALA KANGSAR. 


\section{PRILE LIST}

OH THY

\section{FOURTH JOINT}

\section{ANNUAL AGR-HORTTCULTURAL SHOW}

$O \mathrm{~F}^{2} \mathrm{THE}$

FEDERATED MALAT-STATES $A N D$

STRAITS SETTLHIENTS,

1907.

\section{KUALA KANGSAR,}

oth, 10th and 11th Angust.

The show will be open on Friday, Sth August, from 11 a.m. until 6 p.m., on Saturday, 10th August, from 9 a.m. until 6 p.m., and on Sunday, 11 th August, from 9 a.m. until 5 p.m.

\section{OPWNING CERENONY,}

FIRST DAY, INAM. 12 NOON, ADINISSION FEEF.

KUALA LUNPUR:

FRINED A'I IHE T.M.S. GOVERNMENT PRINIING OEFICE.

$\overline{1907 .}$ 



\section{PATRONS.}

1. H.E. The Governor and High Commissionmer.

2. H.H. The Sultan of Perak,

3. H.H. The Sultan of Selangor.

4. H.H. The Sutitan of Pahang.

5. H.H. The Sulina or Johore.

6. H.H. The Sultar of Tredah.

7. H.H. The Yang di Pertuan Regar, Neger Semmian.

8. The Resmonemtegeneral, Federated Malay States.

\section{PRESIDENT.}

The British Resident, Perak.

\section{VICE-PRESIDENTS.}

The Hon. The Colonial Secretatix, Sinfapore.

The Hon. The Rusming Coumoinlom, Penana,

The Hon. The Resident Counchlior, Malacca.

The Hon. John Anderson, M.L.C.

The Britrsh Remident, Semangor.

The British Resident', Negri Smmbitan.

The British Resident, Pahang. 


\section{STANDING COMMITTEE.}

\section{STNGAPORE.}

The Drrector of Botanic Garjons, S.S. (Hon. Secretary). Assistant Surerintendent, Botanic Gardens, Singapore. The Colimetor of IiAND Revenue.

PENANG AND PROVINCE WELLESTEY.

The Senior District Offrcer, P.W.

I'he Colifector of tiand Ravenue.

The Supetintendent; Botanic Gardens and Torests (Hon. Serretary).

\section{NALACCA.}

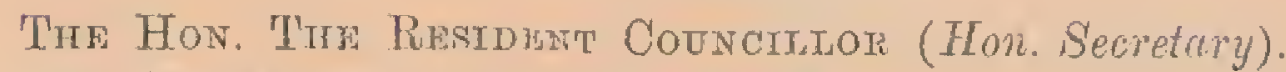

Tha Collector on Land Revenute.

$$
\text { PERAK. }
$$

The Dtrector of Mushums, F.M.S.

'The Drstrict Officher, Kuala Rangsar.

'The Superintrmanent, Government Plantations (Hon. Secretary).

SELANGOR.

The Director of Agriculture, F.M.S.

The Superintendent. Experimental Plantations (Hom. Secretary).

Thr Colmector of tand Revenue, Kuala Lumpur.

\section{NEGRI SEMBLIAN.}

'The District Offiche, Tampin.

l'me Colmector of tand Revende, Shremban.

'The Chatrman, Negri Sembilan Peanters' Assoctamion.

\section{PAHANG.}

'The Senior Magistrate.

'The District Omficer, Raub.

The District Officher, Pekan (Hon. Secretary).

\section{GENERAL PURPOSES COMMITTEE.}
J. B. Carruthers (Chaiman)
R. J. Wilkinson (Vire-Chriman)
E. J. Weld
The Dato Sri Adiki Raja
The Dato Setin
r. Wray
A. Hale
J. W. Camploell
E. R. Salisbury
T. W. Main (Hon. Gen. Secretary)
A. B. Voules (Hon, Treasurer) 


\title{
SUB-COMMITTEES.
}

\author{
Division A. \\ AGRICULTURAL PRODUCE.
}

Shetion I.-PADI.
A. Hale (Convenor)
F. J. Weld

Pandali Abdullah
Sir Adika Raja

SUCTION II.-RUBBLR.

J. B. Carruthers

A. D. Machado

B. C. Knight

T. W. Main (Cunecnur)

SECTHON 111,-MISCELIANEOUS.

J. B. Carruther's

li. J. Clayton (Convenor)

D. McColl

Dito Setia

\section{Division B.}

ERUITS, FLOWERS AND VEGETABLES.

J. IV. Camplyell

T. W. Mitin (Convenor)

H. C, Barnard

A. D. Machido

Diviston C.

CATILE AND POUTHRY.

W. W. Douglias (Convenor)

G. Noil

E. R. Salisbury

Penglima Besal

Division D.

NATIVE INDUSIRTES AND MANURACIUUES.

A. Hale

L. Wray (Convenor)

h. J. Wilkiuson

Sri Adikiu Rajut

Division E.

AGRICULIURAT IMPLEMENTS.

W. Blackslatw (Convenor)

E. R. Salisbury

E. H. I. Day

J. B. Curruthers

A. B. VOULES,

Honorary Treasurer.

T. W. MAIN,

Honorary Generul Secretary. 


\section{RULES AND REGULATIONS RELATING TO EXHIBITS.}

1. All exhibits must be the bona fide property of the exhibitor.

2. Competition for prizes shall be open only to exhibitors resident in the Colony of the Straits Settlements or the States of the Malay Peninsula.

3. Competition (unless otherwise specified) shall be confined to exhibits grown or manufactured in the Colony of the Straits Settlements or the States of the Malay Peninsula.

4. The Conmittee will appoint Judges, from whose decision there shall be no itppeal.

5. The Judges may withhold a prize when they ure of opinion that there is not sufficient merit to justify an award, and may award special prizes for any exhibit not contained in the schedule.

6. No exhibitor shall be awarded two prizes in auy class.

7. The arrangement of the exhibits shall be subject to the directions of the Committee.

8. 'The Committee reserves the right to refuse iny entries that it mily think fit to reject, and to cancel any class at iny time before the 2nd August, 1907.

9. Exhibitors desiring free trensport by Railway, nust give notice to it District ()ficer, or the General Secretiny, who, if the exhibits are arpproved, will provide them with numberer tickets and a free thind class pass on the Railway for themselves and their exhibits. A reduction of 25 per cent. off the usual freight will the made by the Struits stemship Company on all bout fide exhibits. The Committee does not hold itself responsible for exhibitors' expenses.

10. All entries must be in writing and inust reach the Gencral Secretury at least seven days before the first day of the Show.

11. Exhibitors in Division o must provide for the cire and maintenance of their own exhibits.

12. No exhilit (except live animals) may be removed from the ground before the close of the Show.

13. The Committee is empowered to exclude, or remove, any animal which in its opinion is not in a fit state for exhibition. 
14. Exhibitors in Division C will only be permitted to remove their animals from the Show grounds on obtaining an orter of removal from the General Secretary or the Honorary Secretary of the Division.

15. No exhibit in Divisions A, C, D and E will be received later than 6 p.m. on the 8th August, 1907.

16. All reasonable care will be taken to provide for the safety and careful preservation of exhibits of every kind sent to the Show, but it must be clearly understood that the Committee does not hold itself responsible for the loss of, or the damage to, any exhibit, either in transit or during exhilition. Provision will be made for lreeping articles of value moler lock and liey at night. class.

17. Govermment exhibits shall not be eligille for prizes in any

18. Winners of prizes of 810 and upwards may have it silver medal if they prefer it to money. 
DIVISION $\Lambda$.

AGRICULTURAL PRODUCE.

SECTION I.-PADI.

\begin{tabular}{|c|c|c|c|c|c|}
\hline \multirow{2}{*}{ 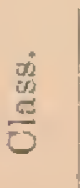 } & \multirow{2}{*}{ Nature of exhibit. } & \multirow{2}{*}{$\begin{array}{l}\text { Number ur } \\
\text { quantity to be } \\
\text { exhibited. }\end{array}$} & \multicolumn{3}{|c|}{ Prize.' } \\
\hline & & & 1st & $2 n d$ & $3 \mathrm{red}$ \\
\hline 1 & Padi, best simple of any named & & & $\$$ & $\$$ \\
\hline 2 & $\begin{array}{c}\text { variety } \ldots \\
\text { Pulut, best sumple of any named }\end{array}$ & & 20 & 7 & 5 \\
\hline 3 & $\begin{array}{l}\text { variety } \ldots \\
\text { Rice, best sample prepared } \ldots\end{array}$ & & 15 & 5 & 3 \\
\hline 4 & $\begin{array}{c}\text { machinery } \ldots \\
\text { Rice, best simple prepared in } \ldots\end{array}$ & 1 gantang & 10 & 5 & :3 \\
\hline 5 & $\begin{array}{l}\text { lesong } \\
\text { Padi, best collection of named }\end{array}$ & & 15 & 7 & 5 \\
\hline 4 & $\begin{array}{cccc}\text { varieties } \ldots & \ldots & \ldots & \ldots\end{array}$ & $\begin{array}{l}\frac{1}{2} \text { chupak of } \\
\text { each variety }\end{array}$ & 30 & 10 & 5 \\
\hline & $\begin{array}{c}\text { Pulut, best collection of named } \\
\text { varicties... } \ldots \\
\text {.... }\end{array}$ & ditto & 10 & 5 & 3 \\
\hline 7 & $\begin{array}{l}\text { Best collection of Padi and Pulnt, } \\
\text { grown in one mulim to be exhi- } \\
\text { bited by the Penghulu of such } \\
\text { mukim and so certified by lim* }\end{array}$ & ditto & $50 \dagger$ & 25 & 10 \\
\hline
\end{tabular}

: Each exhibitor to produce an affiavit swoln before a Magistrate that lis whole exhibit was gromn in his own mulkin last pudi soason.

+ Also a cap presented ty $\mathbb{M}$. A. IIale, District Officer, Larut aud Krias. 


$$
\begin{gathered}
11 \\
\text { ThVtaton A.-(cont.) } \\
\text { SACTTON M.-RUBBER. }
\end{gathered}
$$
Show :

A cup for the best sample of Rubber exhibited in any chass in the Class.

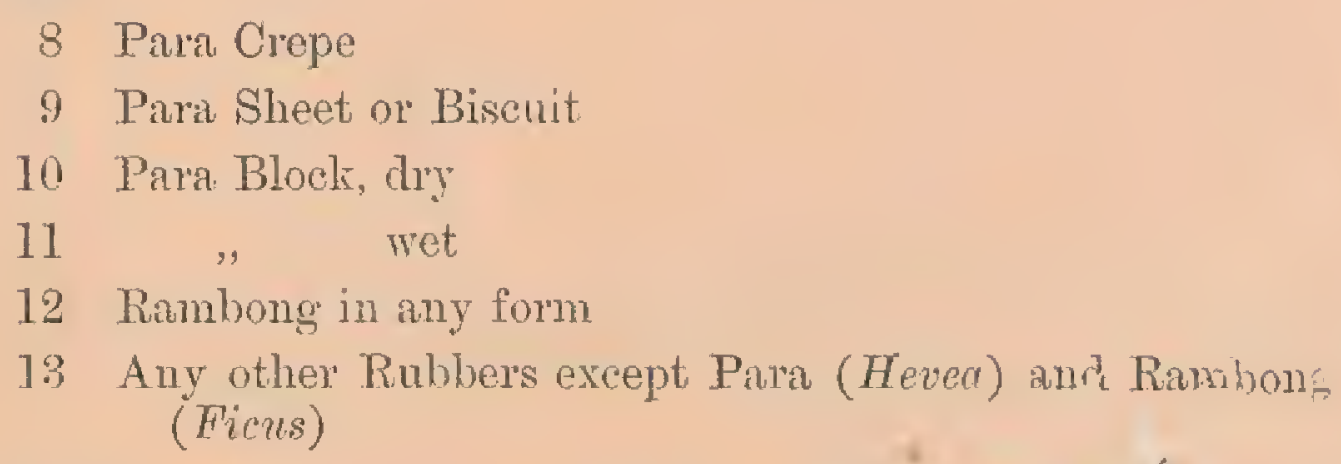

In $8,9,12$ and 13 a minimum of $5 \mathrm{lbs}$, and in $7 / 3 \mathrm{ar}^{2} 11^{\prime}$ a minimum of 10 lbs.

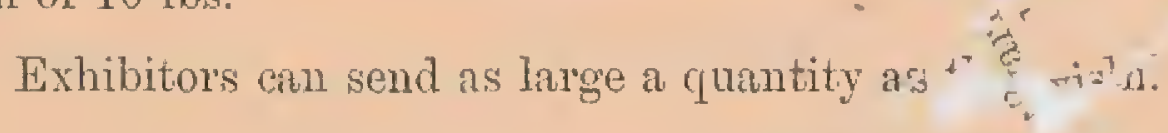


Division A.-(cont.)

SFOTIOX ITI.-MISCELLANEOUS PRODUCE.

\begin{tabular}{|c|c|c|c|c|c|}
\hline \multirow{2}{*}{$\frac{d}{0}$} & \multirow{2}{*}{ Nature of exhibit. } & \multirow{2}{*}{$\begin{array}{l}\text { Number or } \\
\text { quantity to be } \\
\text { exhibited. }\end{array}$} & \multicolumn{3}{|c|}{ Prize. } \\
\hline & & & Ist & $2 \mathrm{ntl}$ & $3 \mathrm{rrl}$ \\
\hline & ta & & $\$$ & $\$$ & $\$$ \\
\hline 14 & Bamboos, best collection ... & optional & 5 & 2 & 0 \\
\hline 15 & Betel-nuts, fresh, best sample ... & 50 & 5 & 2 & 1 \\
\hline 16 & . dried and split, best & & & & \\
\hline & Stimple & 1 catty & 3 & 2 & 1 \\
\hline 17 & C' tves, best sample $\quad \ldots \quad \ldots$ & $\frac{1}{2} \quad$, & 5 & 2 & 1 \\
\hline 18 & "oconts; "ullusked, best sample & $10^{\circ}$ nuts & 5 & 3 & 1 \\
\hline 19 & .. hols $k e d \quad,, \quad \ldots$ & $10 \quad$, & 5 & 8 & 1 \\
\hline 20 & " "est collection of & 3 of each & 10 & 5 & 2 \\
\hline 21 & Coprob, sum rive loat sample ... & 3 catties & 5 & 3 & 1 \\
\hline 22 & $\begin{array}{c}\text { Cotton, "Kat in," any variety, } \\
\text { best simple } \quad . .\end{array}$ & 1 catty & 10 & 5 & 2 \\
\hline 23 & Coffee, any variely & $1 \quad$, & 10 & 5 & 2 \\
\hline 24 & Fibres, best collection $\quad \ldots \quad$... & $\begin{array}{l}1 \text { biundle } \\
\text { of each }\end{array}$ & 10 & 5 & 0 \\
\hline $\begin{array}{l}25 \\
26\end{array}$ & $\begin{array}{l}\text { Gambier, best sample ... } \\
\text { Gums and Dumars, best collection }\end{array}$ & 1 catty & 5 & 3 & 1 \\
\hline & $\begin{array}{llll}\text { of local } & \ldots & \ldots & \ldots\end{array}$ & $\frac{1}{3}$ & 10 & 3 & 1 \\
\hline 27 & Ginger, best sample $\quad \ldots \quad \ldots$ & $20^{-}$roots & 5 & 3 & 1 \\
\hline 28 & Indigo, prepared, best sample ... & 1 atty & 5 & 0 & 0 \\
\hline 29 & Mace, dried & $1 \quad$, & 5 & 3 & 1 \\
\hline 30 & Maize & 10 heads & 10 & 5 & 2 \\
\hline 31 & Medicinal Plants, best collection... & optional & 10 & 5 & 2 \\
\hline 82 & Nutmegs, fresh, best sample $\quad .$. & 25 & 5 & 3 & 1 \\
\hline 38 & ", aried $\quad$, & 25 & 5 & 3 & 1 \\
\hline 34 & Oil, Citronellit & 1 pint & 5 & 0 & 0 \\
\hline 35 & , Lemon grass & $1 \quad$, & 10 & 5 & 2 \\
\hline 36 & "Coconut,$"$, & 1 & 10 & 5 & 2 \\
\hline 37 & " Teel Seed (Minyak Lengah), & & & & \\
\hline & $\begin{array}{ccc}\text { best sample } & \ldots & \ldots \\
\text { Castor best sample } & & \end{array}$ & 1 & 5 & 0 & 0 \\
\hline 38 & $\begin{array}{l}\text { " Castor, best sample ... } \\
\text {, best collection of Essential ... }\end{array}$ & 1 , , of ea. & 10 & $\begin{array}{l}2 \\
0\end{array}$ & $\begin{array}{l}0 \\
0\end{array}$ \\
\hline 40 & Pepper, white, best sample & $\frac{1}{2}$ cattis & 5 & 3 & 0 \\
\hline 41 & Pepper, black & $\frac{1}{2} \quad$, & 5 & 3 & 0 \\
\hline 42 & Patchouli & $\frac{1}{2}, n$ & 5 & 0 & 0 \\
\hline 43 & Rotans, best collection ... & optional & 10 & 5 & 2 \\
\hline 44 & Sago, Pearl, best sample... & 1 gantang & 5 & 3 & 1 \\
\hline 45 & " $\quad \ldots$ & $1, \quad$, & 5 & 3 & 1 \\
\hline
\end{tabular}


Division A.-(cont.)

SECTION TIT-MLSCELLANEOUS PRODUCE-(cont.)

\begin{tabular}{|c|c|c|c|c|c|}
\hline \multirow[b]{2}{*}{$\stackrel{\frac{2}{2}}{\frac{2}{0}}$} & \multirow[b]{2}{*}{ Nature of exlibit. } & \multirow{2}{*}{$\begin{array}{l}\text { Number or } \\
\text { quantity to be } \\
\text { exhibiced. }\end{array}$} & \multicolumn{3}{|c|}{ Prize. } \\
\hline & & & Ist. & $2 n t$ & 3rd \\
\hline $\begin{array}{l}46 \\
47 \\
48 \\
49 \\
50 \\
51 \\
52 \\
53 \\
54 \\
55 \\
56 \\
57 \\
58 \\
59 \\
60 \\
61\end{array}$ & 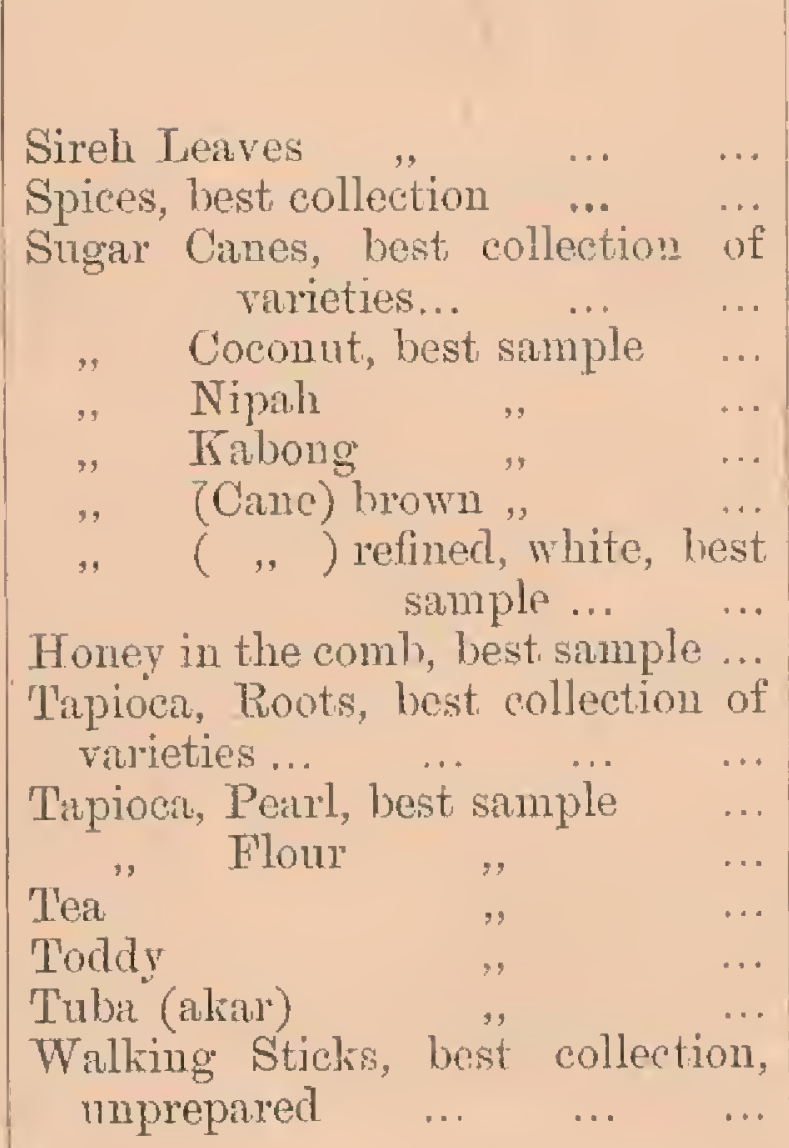 & 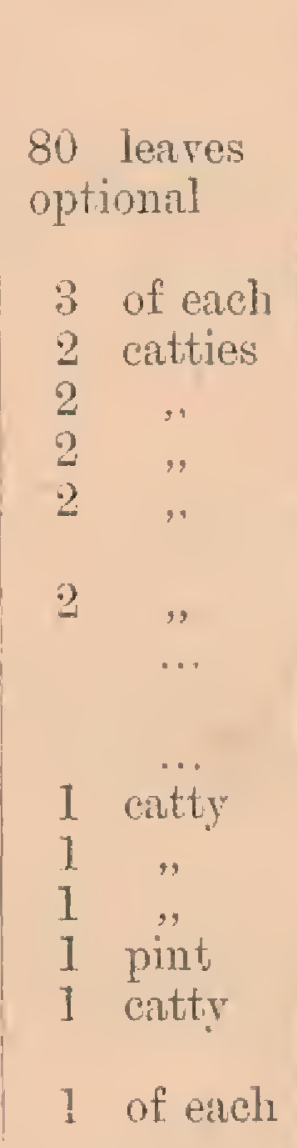 & $\begin{array}{r}5 \\
5 \\
10 \\
5 \\
5 \\
5 \\
3 \\
3\end{array}$ & $\begin{array}{l}3 \\
2 \\
5 \\
5 \\
3 \\
3 \\
3 \\
3\end{array}$ & $\begin{array}{l}3 \\
1 \\
1 \\
1 \\
0 \\
0\end{array}$ \\
\hline
\end{tabular}


DIVISTON $\mathrm{R}$.

FTOWERS, FPUT'S ANT VEGFIABEFS,

SECTION T.- WLOWERS.

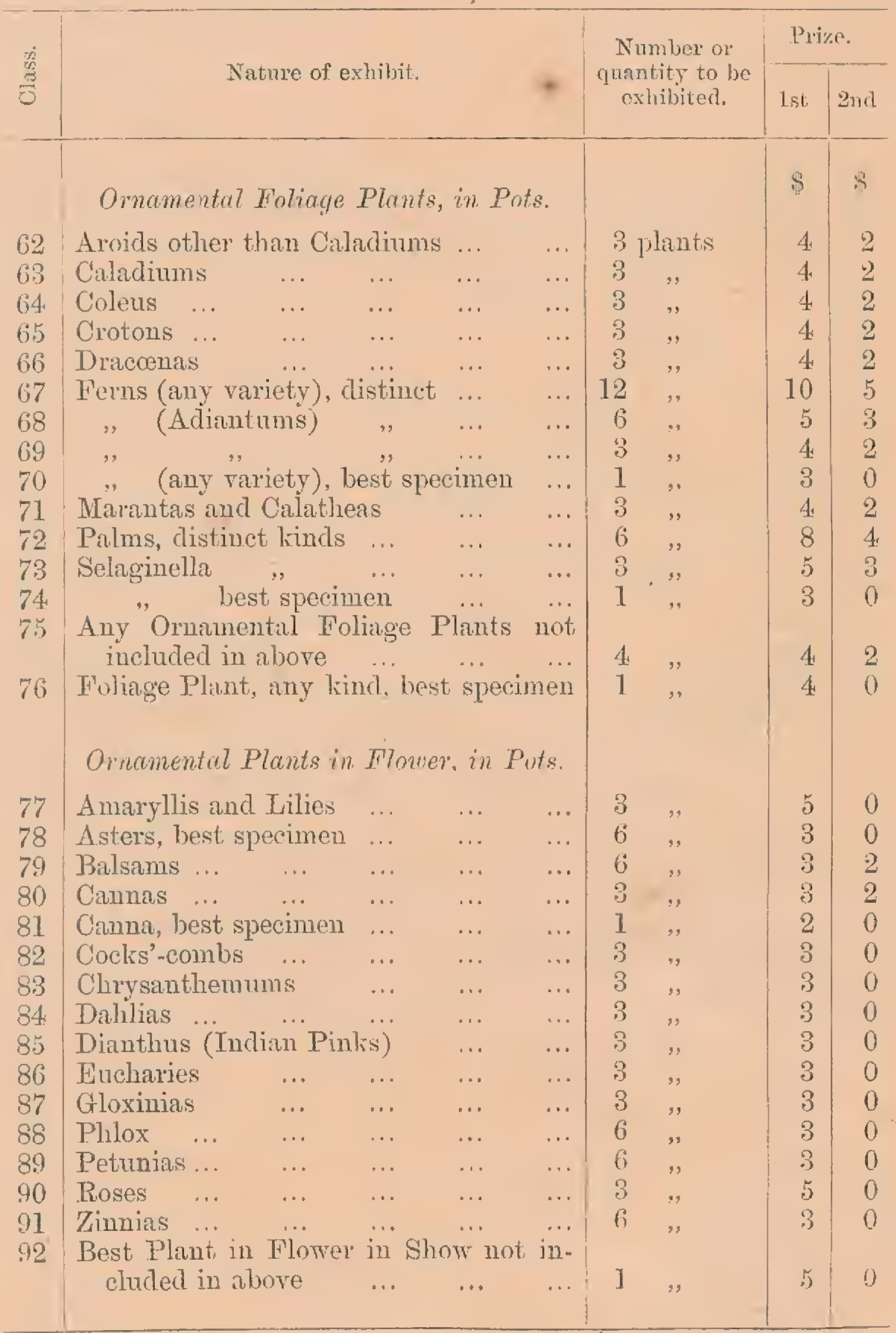


Division B.--(cont.)

Secriox 1.-lidowElis-(cont.)

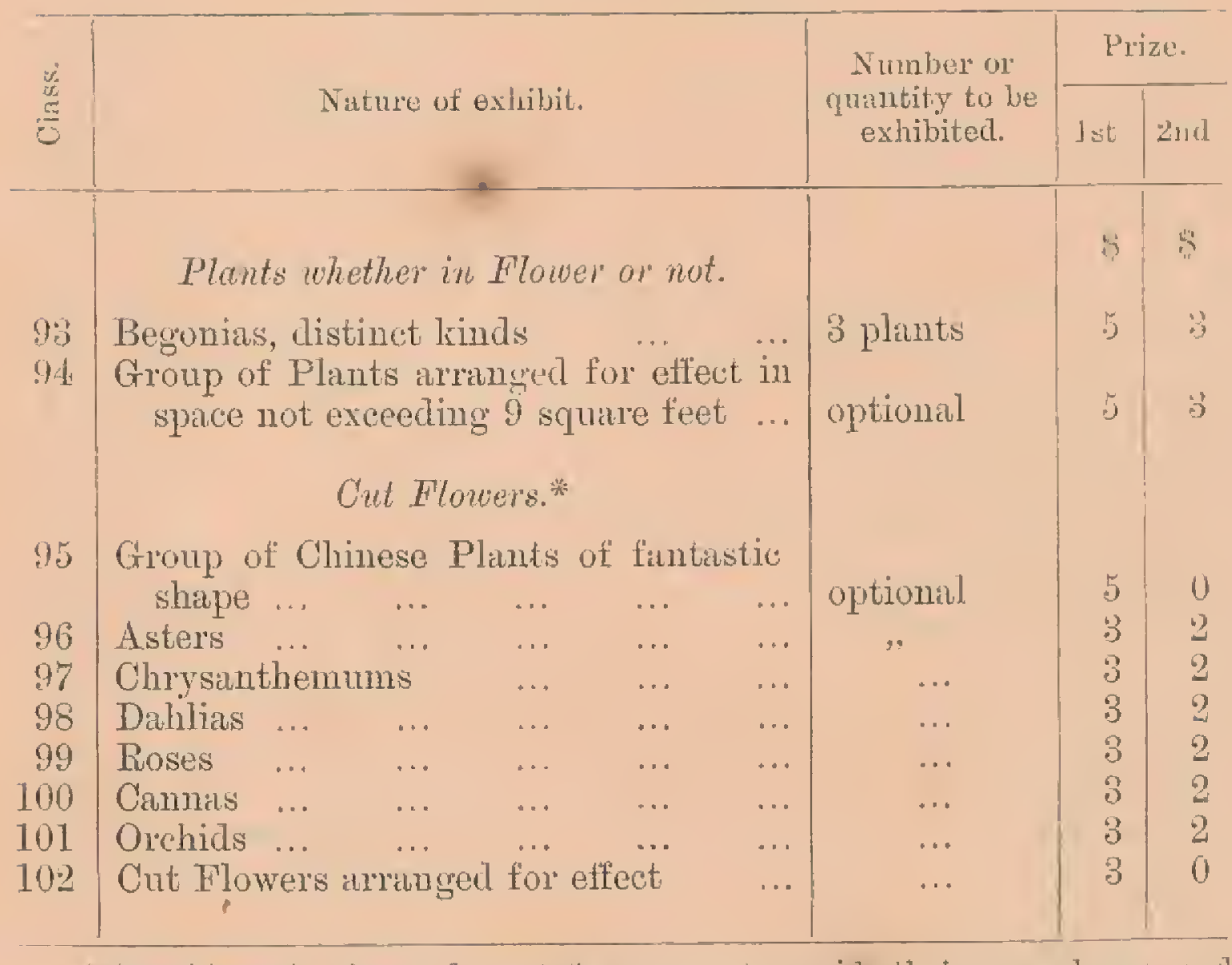

* Exhibitors in classes for cut Howers must provicle their own glasses, and untist stage their exhibits before 8 a.m. 
Division B.-(cont.)

SUCTTON II.-FRUT'S.

\begin{tabular}{|c|c|c|c|c|c|}
\hline \multirow{2}{*}{ 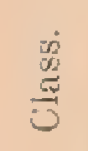 } & \multirow{2}{*}{\multicolumn{2}{|c|}{ Nature of exhibit. }} & \multirow{2}{*}{$\begin{array}{l}\text { Number or } \\
\text { qlantity to bo } \\
\text { exhibited. }\end{array}$} & \multicolumn{2}{|c|}{ Prize. } \\
\hline & & & & 1st & 2nd \\
\hline & & & & $\$$ & 8 \\
\hline 1.03 & Banluas, "Pisangs," best & lection ... & 6 of ed. kind & 10 & 5 \\
\hline 104 & , best bunch, any & d $\quad \ldots$ & 1 bunch & 3 & 2 \\
\hline 10.5 & Champedal $\quad \ldots \quad \ldots$ & $\ldots$ & 3 frouits & 2 & 1 \\
\hline 106 & Chiku... ... & $\ldots$ & ," & 3 & 2 \\
\hline 107 & Custard-ipple ... & $\cdots$ & $6 \quad$, & 2 & 1 \\
\hline 108 & $\begin{array}{c}\text { Cultivated Fruits, best co } \\
\text { number } \quad \ldots\end{array}$ & \begin{tabular}{cc|} 
ction, any \\
$\ldots$
\end{tabular} & 3 of each & $*$ & \\
\hline 109 & Durian $\ldots . \quad \ldots$ & $\ldots \quad \ldots$ & 2 firuits & 5 & 2 \\
\hline 110 & ," Blanda ... & $\ldots$ & , & 2 & () \\
\hline 111 & Duku ... & $\ldots$ & 20 & 3 & 2 \\
\hline 112 & Binjaí $\quad .$. & $\ldots$ & 6 & 2 & 1 \\
\hline 113 & Jach Fruit & $\ldots$ & 1 & 2 & 0 \\
\hline 114 & Jambu $\ldots$ & $\ldots$ & 20 & 2 & 0 \\
\hline 115 & Cungsitu ... & $\ldots$ & $\dddot{y}$ bunclies & 2 & 1 \\
\hline 116 & Limes $\quad \ldots$ & $\cdots$ & 12 finits & 4 & 2 \\
\hline 117 & Mangoes ... & $\ldots$ & $6 \quad$, & 3 & 0 \\
\hline 118 & Machang... & $\ldots$ & 6 & 2 & 0 \\
\hline 119 & Mangosteens & $\ldots$ & 12 & 5 & 3 \\
\hline $1: 20$ & Muta Kuching ... & $\ldots$ & 20 & 2 & 0 \\
\hline 121 & Melon, any kind & ... & 1 & 3 & 0 \\
\hline 1202 & Papaya $\ldots \quad \ldots$ & $\ldots$ & 3 & 3 & 0 \\
\hline 123 & Oranges ... & $\ldots$ & 6 & 3 & 2 \\
\hline 124 & Pine-apples (Mauritius) & $\ldots$ & 3 & 3 & 2 \\
\hline 125 & ," (any other varie & $\ldots$ & 3 & 3 & 2 \\
\hline 126 & Ponneloes & $\ldots$ & 3 & 2 & 1 \\
\hline 127 & Pulasan ... & $\cdots$ & 20 & 2 & 1 \\
\hline 128 & Rumbat ... & $\ldots$ & 3 bumches & 2 & 1 \\
\hline 129 & Rambutan $\quad \ldots \quad \ldots$ & $\ldots$ & 20 fruits & 2 & 1 \\
\hline 130 & Wild Edible Fruits, best c & ection ... & optional & 5 & 8 \\
\hline 131 & Any kind of Fruit not inclu & d in above &, & 3 & 2 \\
\hline
\end{tabular}

* Cup prescoted by A. D. Nachido, Lsq. 
Division B.-(cont.)

SEction III.-VEGETABLES.

\begin{tabular}{|c|c|c|c|c|c|}
\hline \multirow{2}{*}{ 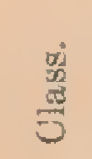 } & \multirow[b]{2}{*}{ Nature of exhibit. } & \multirow{2}{*}{$\begin{array}{l}\text { Number or } \\
\text { quantity to be } \\
\text { exhibited. }\end{array}$} & \multicolumn{3}{|c|}{ Plize. } \\
\hline & & & 1st & 2ud & $3 \times 1$ \\
\hline & $i^{*}$ & & & & \\
\hline & Preserved Fruits and Vegetables.* & & in & 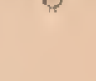 & T \\
\hline 132 & $\begin{array}{c}\text { Preserved Fruits, best sumple, any } \\
\text { method ... } \quad . . \quad \ldots\end{array}$ & optional & 8 & 4 & 2 \\
\hline 133 & Chutney, best sample $\quad \ldots \quad$... & , & 5 & 0 & 0 \\
\hline 134 & Pickles $\quad . . \quad \ldots$ & , & 5 & 0 & 0 \\
\hline 135 & Jellies $\quad \ldots$ & & 4 & 2 & 0 \\
\hline 136 & Articholies... & 12 tubers & 2 & 0 & () \\
\hline 137 & Benny Fruits or Chocho ... & 6 specimens & 2 & 0 & 0 \\
\hline 138 & Brinjals $\quad \ldots \quad \ldots \quad \ldots$ & & 2 & 0 & 0 \\
\hline 139 & Beans, best collection & optional & 3 & 2 & 0 \\
\hline 140 & Cabbages $\ldots$... & & 2 & 0 & 0 \\
\hline 144 & Chillies, best collection ... & 30 of exch & 3 & 2 & 0 \\
\hline 142 & Cucumbers ... $\quad \ldots \quad \ldots$ & 6 specimens & 2 & 0 & 0 \\
\hline 143 & $\begin{array}{c}\text { Herbs used in curries and sambal.s, } \\
\text { best collection ... }\end{array}$ & 1 bundle ea. & 2 & 0 & 0 \\
\hline 144 & Ladies' Fingers $\ldots . \quad \ldots . \quad \ldots$ & 6 specimens & 2 & 0 & 0 \\
\hline 145 & 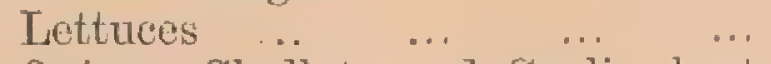 & & 2 & 0 & 0 \\
\hline 146 & $\begin{array}{c}\text { Onious, Shillots and Garlic, best } \\
\text { collection ... }\end{array}$ & 1 bundle ea. & 2 & 0 & 0 \\
\hline 147 & Pumplins ... & 3 specimens & 2 & 0 & 0 \\
\hline 148 & Radishes ... & & 2 & 0 & 0 \\
\hline 149 & Tomatoes, luest dish & & 5 & 3 & 0 \\
\hline 150 & $\begin{array}{l}\text { Vegetables and Herbs for maling } \\
\text { a salad, best collection ... }\end{array}$ & optional & 4 & 2 & C \\
\hline 151 & $\begin{array}{l}\text { Water-melons, Gourds. Squashes, } \\
\text { Luffas, ete, best collection }\end{array}$ & 1 of each & 5 & 3 & 0 \\
\hline 152 & Yams, Kladis, best collection .. & $6 \quad$, & 4 & 2 & 0 \\
\hline 153 & Any Vegetable not in above & optional & 2 & 0 & 0 \\
\hline
\end{tabular}
cxlibitors. 
Division C.

CATTLE AND POULTRY.

\begin{tabular}{|c|c|c|c|c|c|}
\hline 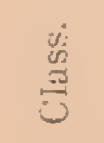 & Nature of exhibit. & & & $\begin{array}{l}\text { Number or } \\
\text { cuantity to be } \\
\text { cxhibited. }\end{array}$ & $\stackrel{\mathscr{c}}{\mathrm{E}}$ \\
\hline & & & & & $\$$ \\
\hline 154 & Bull (locally bred) ... & $\ldots$ & $\ldots$ & 1 & 20 \\
\hline 155 & Cow & $\ldots$ & $\ldots$ & 1 & 20 \\
\hline 156 & Pair of Indian Bulls... & $\ldots$ & $\ldots$ & with cart & 20 \\
\hline 157 &,$\quad$ Bullocks & $\ldots$ & $\ldots$ & , & 20 \\
\hline 158 & Milch Cow, any breed & $\ldots$ & $\ldots$ & 1 & 20 \\
\hline 159 & Cow and Calf, Indian (imported) & $\ldots$ & $\ldots$ & 1 & 15 \\
\hline 160 & , $\quad$ (locally bred) & $\ldots$ & $\ldots$ & 1 & 10 \\
\hline 161 & Buffilo Bull (not Indian) ... & $\ldots$ & $\ldots$ & 1 & 10 \\
\hline 162 & , , Indian (locally bred) & ) ... & $\ldots$ & 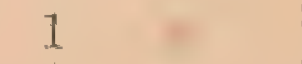 & 10 \\
\hline 163 & , Cow $,, \quad \ldots, \quad \ldots$ & $\ldots$ & $\ldots$ & 1 & 10 \\
\hline 164 & $\begin{array}{l}\text { Champion Animal (bull, cow, } \\
\text { buffalo) }\end{array}$ & bullock & or & 1 & 20 \\
\hline 165 & hain Goat ... ... ... & $\ldots$ & $\ldots$ & 1 & 5 \\
\hline 166 & Goat, with Kid or Kids & $\ldots$ & $\ldots$ & 1 & 5 \\
\hline 167 & Milch Groat $\ldots$... & $\ldots$ & $\ldots$ & 1 & 5 \\
\hline 168 & Sheep, Ram ... ... & $\ldots$ & $\ldots$ & 1 & 5 \\
\hline 169 & " Ewe ... ... & ... & $\ldots$ & 1 & 5 \\
\hline 170 & Bantam Cock and Hen & ... & $\ldots$ & 1 pair & 5 \\
\hline 171 & Malay $\quad,, \quad$, & $\ldots$ & $\ldots$ & & 5 \\
\hline 172 & " Game Couk and Hen & $\ldots$ & & & 5 \\
\hline 173 & Colloction of Malay Fowls ... & $\ldots$ & $\ldots$ & optional & 5 \\
\hline 174 & Chimese , & $\ldots$ & $\ldots$ & , & $\dot{v}$ \\
\hline 175 & Fowls, any breed & ... & $\ldots$ & , & 5 \\
\hline 176 & Vranila Drake ind 2 Ducks... & $\cdots$ & $\ldots$ & $\ldots$ & 5 \\
\hline 177 & Javanese $\quad, \quad,, \quad \ldots$ & ... & $\ldots$ & & 5 \\
\hline 178 & Gandex and Goose. & ... & $\ldots$ & 1 pair & 5 \\
\hline 179 & T'urkeys, Cock and Hen & ... & $\ldots$ & 1, & 5 \\
\hline 180 & Guinea Fowls, Cock and Hen & $\ldots$ & $\ldots$ & 1, & $\tilde{5}$ \\
\hline 181 & Pigeoms, auy breed ., , , & ... & $\ldots$ & & 5 \\
\hline 182 & Best Cage Bird & $\ldots$ & $\ldots$ & 1 bind & 5 \\
\hline 183 & Collection of Cage Birds & ... & $\ldots$ & optional & 5 \\
\hline 184 & Rabbits, Buck and Doe & $\cdots$ & $\ldots$ & 1 paix & 5 \\
\hline 185 & Champion Cat in Show & $\ldots$ & $\ldots$ & 1 & 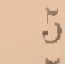 \\
\hline $186^{\circ}$ & Butter, best sample ... & $\ldots$ & $\ldots$ & not less $\frac{1}{2} 1 b$. & 5 \\
\hline 187 & Collection of Egggs, Fuwls ... & ... & $\ldots$ & $12-2$ & 5 \\
\hline 188 & , Ducks ... & $\ldots$ & $\ldots$ & 12 & j \\
\hline 189 & "Turlieys & & $\ldots$ & 6 & 5 \\
\hline
\end{tabular}


Diviston $\mathrm{C}$ - (ent.)

DOGS.*

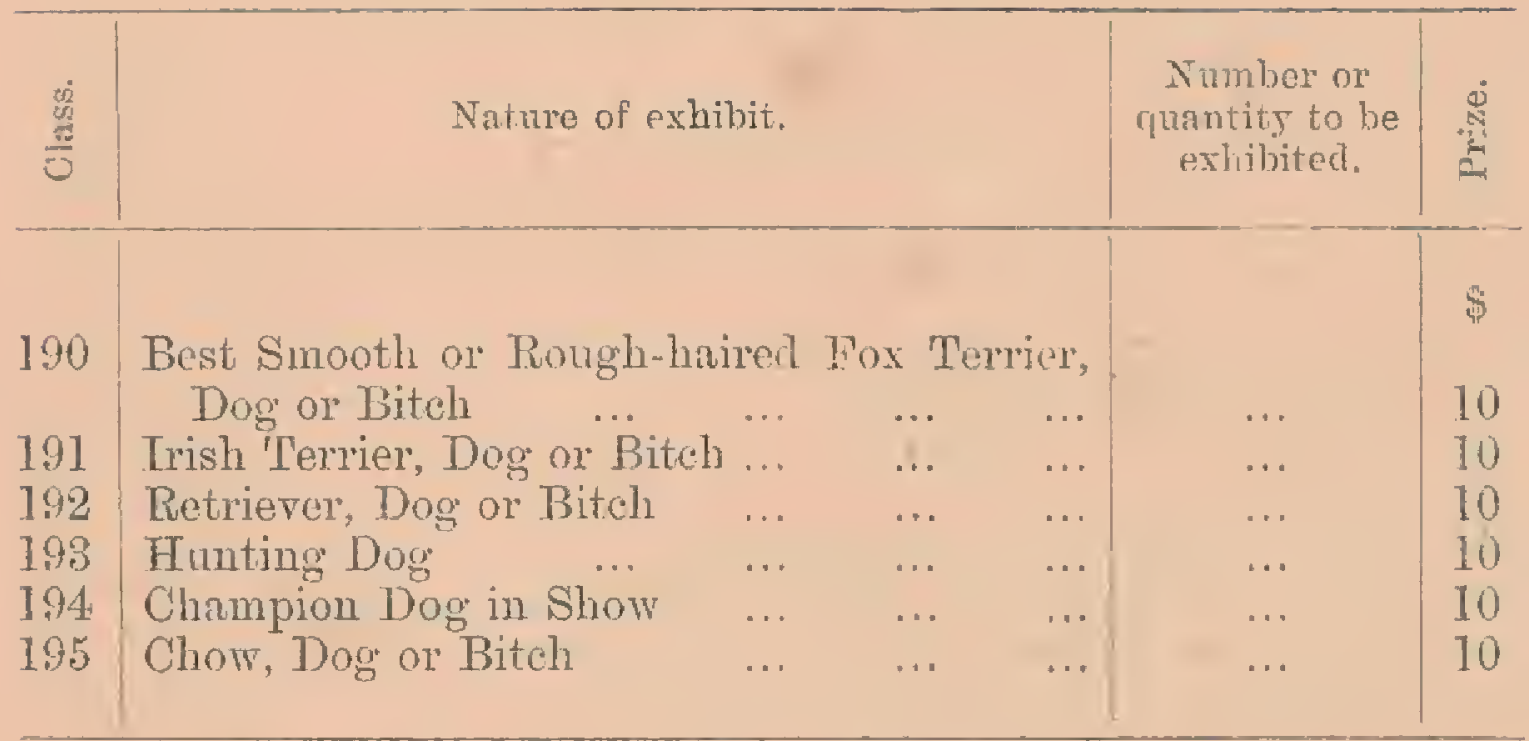

* All Dogs for competition must bo locally bred. 
Diviston $D$.

MALAY ARTS AND INDUSTRIES.

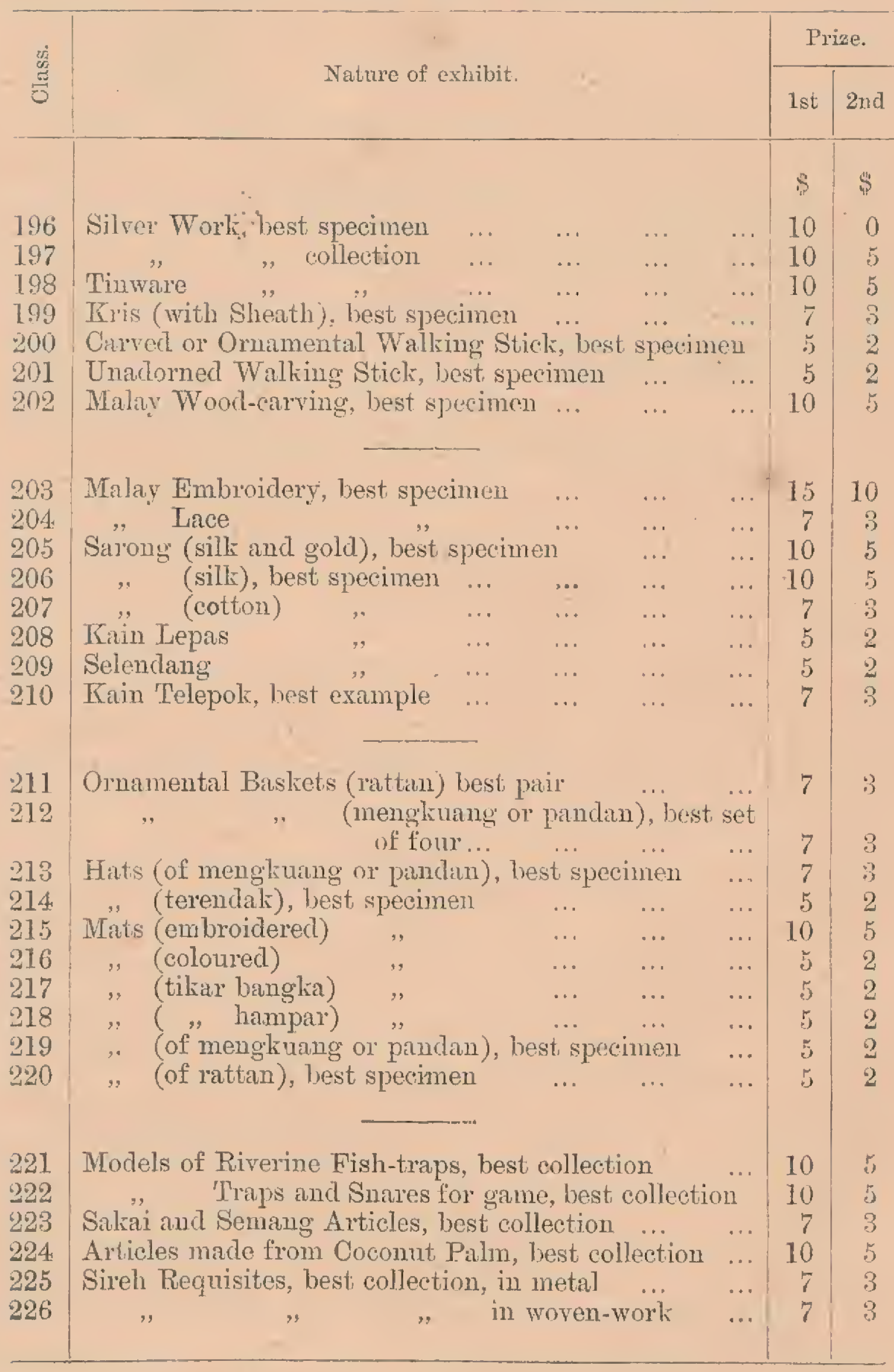




cultivated soil was added, the plants recovered, formed nodules on the roots, and also became capable of absorbing nitrogen. These nodules, upon examination, were found to be full of organisms which, since the sand in which the plants were growing had been sterilized, could only have been derived from the water extract of the cultivated soil that had been added. It was concluded from these observations that the assinilation of free nitrogen by leguminous plants takes place after the formation of root nodules, which are caused by some organism present in cultivated soil.

These organisms have been isolated, and further observation has shewn that the different forms associated with different leguminous plants are all modifications of one species, to which the name, Pseudomonas radicicola, Beyerinck, has been assigned.

As regards the actual way in which the bacterial organism enables the plant to assimilate nitrogen from the atmosphere, since it has been proved that the organism itself, even when isolated from the plant nodule, can in certain forms, take up nitrogen, and store it up in itself as nitrogenous matter, there seems little doubt that it also absorbs nitrogen in this way when in the nodule.

The present view of the case, briefly stated, is that, firstly, the bacterium enters the root of the plant, where its originally minute form changes into a rod-like shape, multiplies, assimilates nitrogen, and stores up nitrogenous compounds, and then finally, in the nodule, the rod-like form changes to the branched form, which is ultimately destroyed by an enzyme, or ferment, produced within the plant. The nitrogenous matter is dissolved and absorbed by the plant, and the nodules gradually diminish in size.

In consequence of this power of leguminous plants to obtain supplies of nitrogen from the air, it is obvious that they are of much greater value for green manuring purposes than non-leguminous crops, as apart from adding organic matter to the soil, their growth and subsequent ploughing-in are equivalent to the application of an expensive nitrogenous manure, such as sodium nitrate or sulphate of ammonia.

It has been shewn that some soils, though capable of growing: leguminous crops, are deficient in the specific organisms which enable these crops to assimilate nitrogen. As the result of a complete scientific investigation of the nature and mode of action of the organism, however, the U.S. Department of Agriculture now supply pure cultures of the bacteria in question, by which the soil of any given field, or the seed about to be sown, may be inoculated with the nitrogen-fixing organism. It is stated in the Bulletin dealing with the above investigation that the following conclusions have been drawn, as the result of the observations made by the Department: Inoculation is not likely to produce any beneficial effect upon soils which already contain the necessary bacteria, or upon soils rich in nitrogen, or again upon soils which, on account of their acidity, are unsuitable for the growth of leguminous plants. Inoculation is undoubtedly of value where the bacteria do not already exist 
in the soil, or lave lost their activity, as indicated by failure in the growth of leguminous crops and absence cf root nodules.

$$
\text { H. N. R. }
$$

\section{The Outlook for Camphor.}

The following extract from the Pharmaceutical Fournal for June 22 last, refers to the possibility of a substitute being found for camphor in the manufacture of celluloid, and to the production of camphor by synthetic processes:-

There have been rumours of a substitute ror camphor in the celluloid industry, ard although there may be little foundation for such rumours, there is no doubt that research in this direction has been encouraged by high prices. Then there is the problem of synthetic camphor; several processes are being worked in England, in Switzerland, and in the United States, and there is a probability that before long (provided the price of turpentine, on which these prccesses depend, remains reasonably normal) the synthetic prouluct will be offered at a price lower than the present price of the natural article.

H. N. R.

\section{THE 4TH JOINT ANNUAL AGRI-HORTICUL- TURAI SHOW OF STRAITS SETTLEMENTS AND FEDERATED MALAY STATES.}

The th annual Agri-Horticultural Show was held this year at Kuala Kang-ar on the 9 th, roth and I th August. So far as beautiful surroundings are concerned no finer spot could have been selected, but th $=$ want of accommodation for lixhibitors and visitors attending the Show was severely lelt, a fact which must be recogrized in selecting the venues for future Shows.

Beautiful weather prevailed for the three days. The form of the buildings was to some extent similar to the Penang Show i.e, three sides of a square, with a band stand and Kiosk in the centre. The shed for agricultural implements and the rubber coagulating machine were in an annexe at right angles to the building containing native industries. The Poultry were housed a little distance from the lastnamed shed whilst the cattle-sheds were some distance away near the river.

As was to be expected Perak shewed up magnificently and may be said to have monopolised the Show, fully four-fifths of the exhibits belonging to Perak. His Excellency the Governor accompanied by Miss. Andirson, Hi; Highness the Sultan of Johore etc. amid a brilliant throng of Europeans and natives opened the Show at 1 i a.m. His Highness the Sultan of Perak who spoke in Malay which was interpreted by the Resident (Mr. E. W. BrRCH) welcomed His Excellency the High Commissioner and thanked him for his 
presence, he contrasted the country before the British occupation and now and said how delighted he was when he heard that the Show was to be in Yerak, and that he ras glad to point out the interest taken in the Shows by his people of their free will. His Excellency who spoke for about a quarter of an hour, after thanking His Highness for his kind words referred to agriculture gentrally and Coconuts and Rubber in particular. He also dwelt upon the labour question and the methods Govermment was adopting for putting it on a satisfactory basis, after a tribute of praise to the hard working Committee he declared the Show open.

Exhibits:--Speaking generally the Exhibits were of a high standard and as might have been expected the Rubber exhibiled was an advance on previous Shows both as regards quantity and improved appearance and gave the Judges a difficult task in deciding the relative merits. Padi and Pulut was in strong force and shewn in a variety of bags, bas, ets, etc. Betel-nut: were good, as likewise were the Coconuts which were an even lot. Copra was very fair, some thirty odd lots competing, the various other classes call for no special remark except perhaps the Exhibits of Medicinal and dried plants shewn by Mr. MACHADO of Kamuning Estate, of the former some 222 varieties were shewn, the labels in most cases giving the uses to which they were put. The dried plants were also neatly arranged as were the samples of fibres put up by the same gentleman.

The Horticulture Division so far as plants and fiowers were concerned was a failure, the few poor specimens that were shewn only served to accentuate the fact On the other hand the fruit was distinctly good and more particularly the Durians sime of which were of enormous size, all the classes in the Fruit Section filled well.

The usual excellent collection of vegetables from Taiping Hills was again in evidence, very nicely arranged by the Honorary General Secretary Mr. T. W. MAIN.

There were a few interesting Exhibits of preserved fruits, Pickles, Chutnies and Eggs which were really very goor..

Unquestionably the strength of the Show lay in the Division devoted to native Industries. In the three former Shows Perak has ahways been the backbone of this Division, and it was expected that on the present occasion that Perak would improve on previous efforts. An enormous number of the most beautiful Exhibits were staged, many of which found purchasers. In this Department some excellently bound books were shewn that had been done in Kuala Kangsar Gaol, as well as a miscellaneous lot of furniture, chairs, etc. made at the same place, they all shewed very careful workmanship, and large urders were booked for duplicates.

In the Division for Agricultural Implements the chief attention was drawn to the Federated Malay States Engineering Company's Exhibit, of their washing and coagulating machine, from some latex supplied by Kamuning Estate some excellent crepe rubber was 
turned out in less than half an hour, and, could have been ready dried and packed for shipment home in a few hours if need be.

Mention should be made of an exhibit in this Section by Mr. A. B. STEPHENS of Taiping of a corrugated iron sheet, perforated with slits on the arched or convex surface, it is designed for a covering for plant houses where light is essential without heavy rain, by having the slits on the convex portion only, and the concave left intact the latter forming water channels. Mr. STEPHEN was awarded a Diploma for his invention.

The Divisions for Cattle and Dogs were of local interest only and call for no special remark. The grand collection of Perak Elephants gathered together for the occasions however was a source of never ending admiration.

Mention should be made of a collection of Miscellaneous Exhibits from Labuan the most interesting of which were the Gongs and Metal works of very unique designs and apparently old. Many of the best specimens were secured for Raffles Museum.

IV. FOX.

\section{Rubber at the Agri-Horticultural Show.}

The Rubber shewn at the Peninsular Show this year exceeded all previou: Malayan Exhibitions in quantity and in quality. The Para sheet and biscuit class contained the greatest number of Exhibits, though crepe ran it close In both so many exhibits approached a high standard that the Judges found it difficult to separate the prize winners. Very little of the material, particularly in sheet and biscuit, was of that pale colour, which, according to advires from home, seems to be favoured by buyers just now. Possibly the spell of dry hot weather immediately before the Show had something to do with the prevalence of dark colour. Many Planters consider that lightcoloured crepe or sheet is more difficult to produce in dry weather than at other times. Dry block was more poorly represented than the first two classes, and wet block even more so. The standard was high and the quality very uniform in dry block. The wet block exhibits were indifferent.

There was a fair number of exhibits, in the Rambong class, and, excepting the first few which were well ahead of the others, the quality was more uniform than in any other group. The Special Cup for scrap drew few competitors, probably due to the fact that notification of it was given very late. Rubbers other than Para or Rambong were very poorly represented, though the winning exhibit-crepe from Willughbeia sp.-was very good. A good collection of various wild rubbers and seeds came from the Government Gardens, Kuala Kangsar; their Ceara sheet was good in 
appearance and of fair quality. There are not enough people willing to devote the amount of attention needed for collecting and experimenting on rubbers of comparatively little financial value. The Guttas were few ; the winning exhibit was a Gutta Taban.

It is extremely difficult owing to the lack of knowledge of definite criteria in rubber to separate lots differing slightly in quality.

The following table gives the points on which the Judges marked the exhibits; the maximum of marks under each heading and the number actually awarded, which is the average of the different judges, judging by points is the fairest method but is of necessity a longer process than the rough and ready process of judging by selection, so much so that the judges were unable to complete their work on the first day. Fifty marks being the total by doubling their total competitors arrive at the percentage of the total maximum awarded to them.

It appears that most exhibits arrived late on the day belore the Show opened; this caused considerable confusion, and accounts for some exhibits appearing without numbers. It is hoped that in future there will be no grounds to make this complaint. Further it is desirable for Exhibitors sending in two or more exhibits of different merit, to label them $\mathrm{A}, \mathrm{B}, \mathrm{C}$, etc.

The judges, the Acting Director of Gardens, Singapore, Mr. W. Fox, the Chairman of the United Planters Association, Mr. R. W. HARRISON, and the Director of Agriculture, Federated Malay States, Mr.J.B. CARruTHERS, were assisted in their task by Mr. W. G. Gallagher, Government Mycologist and Assistant to the Director of Agriculture whose help hastened considerably an interesting but lengthy task.

J. B. CARRUTHERS. 
LIST OF PRIZE IVINNERS, RUBBER EXHIBITS,

KUALA KangSAR, I gO7.

\section{SPECIAL CLASS.}

FOR THE BEST SAMPLE OF RUBBER IN ANY CLASS IN THE SHOW.

PRIZE A SILIER CUP.

Presented by the United Planters Association. - Won by Messrs. Pears, Lanadron Estate, Muar--Sample of dry block.

\section{Class 8-Para Crepe.}

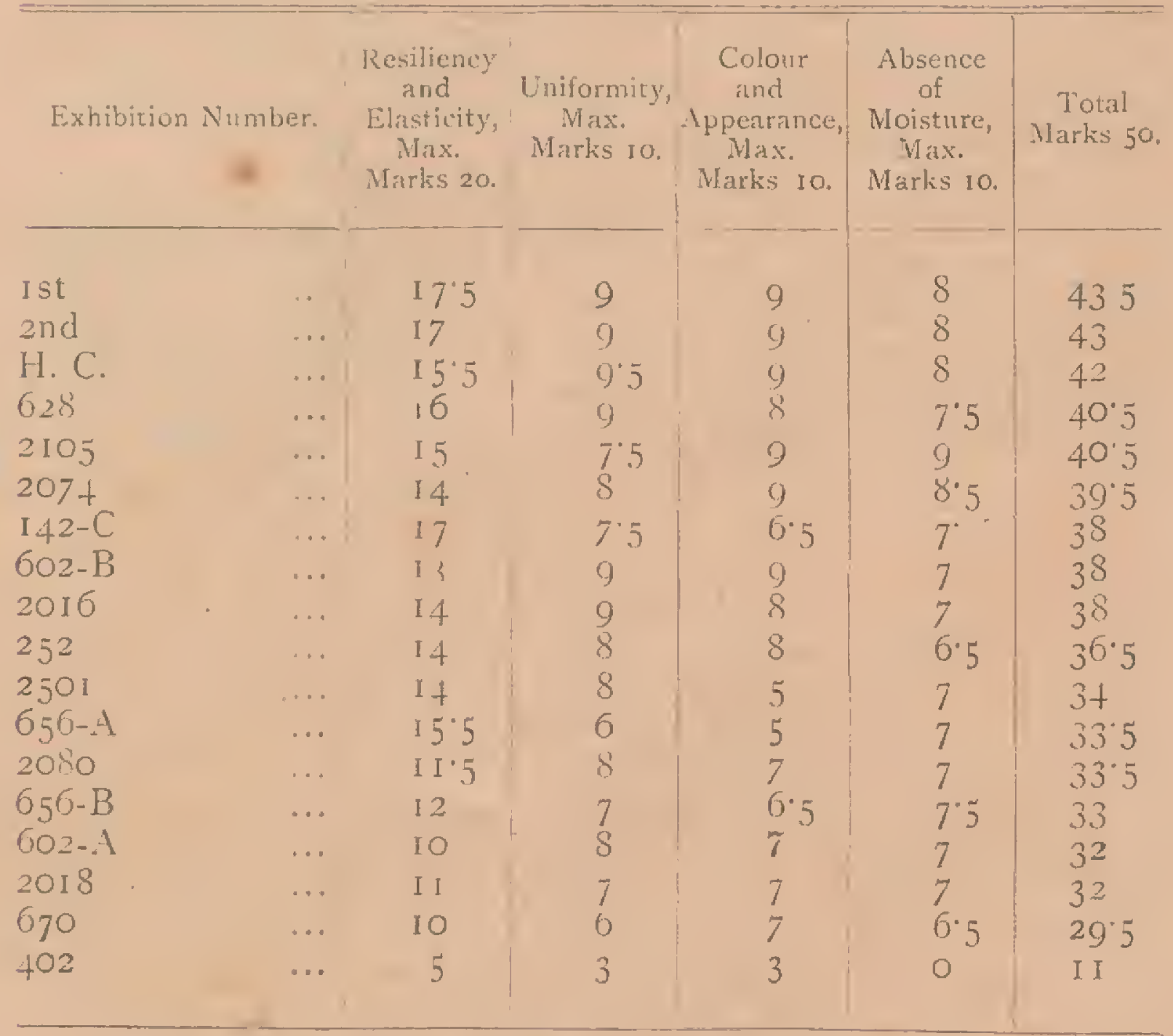

First Prize Silver Cup.-Presented by Lanadron Estate.

E. B. PrIOR, Golden Hope Estate, Klang.

Sccond Prize Silver Cup.-

B. C. N. KNIGHT, Jebong Estate, Taiping.

Highly, Commended.-

B. C. N. KNight, Jebong Estate, Taiping. 
Class 9.-Para Sheet on Biscuit.

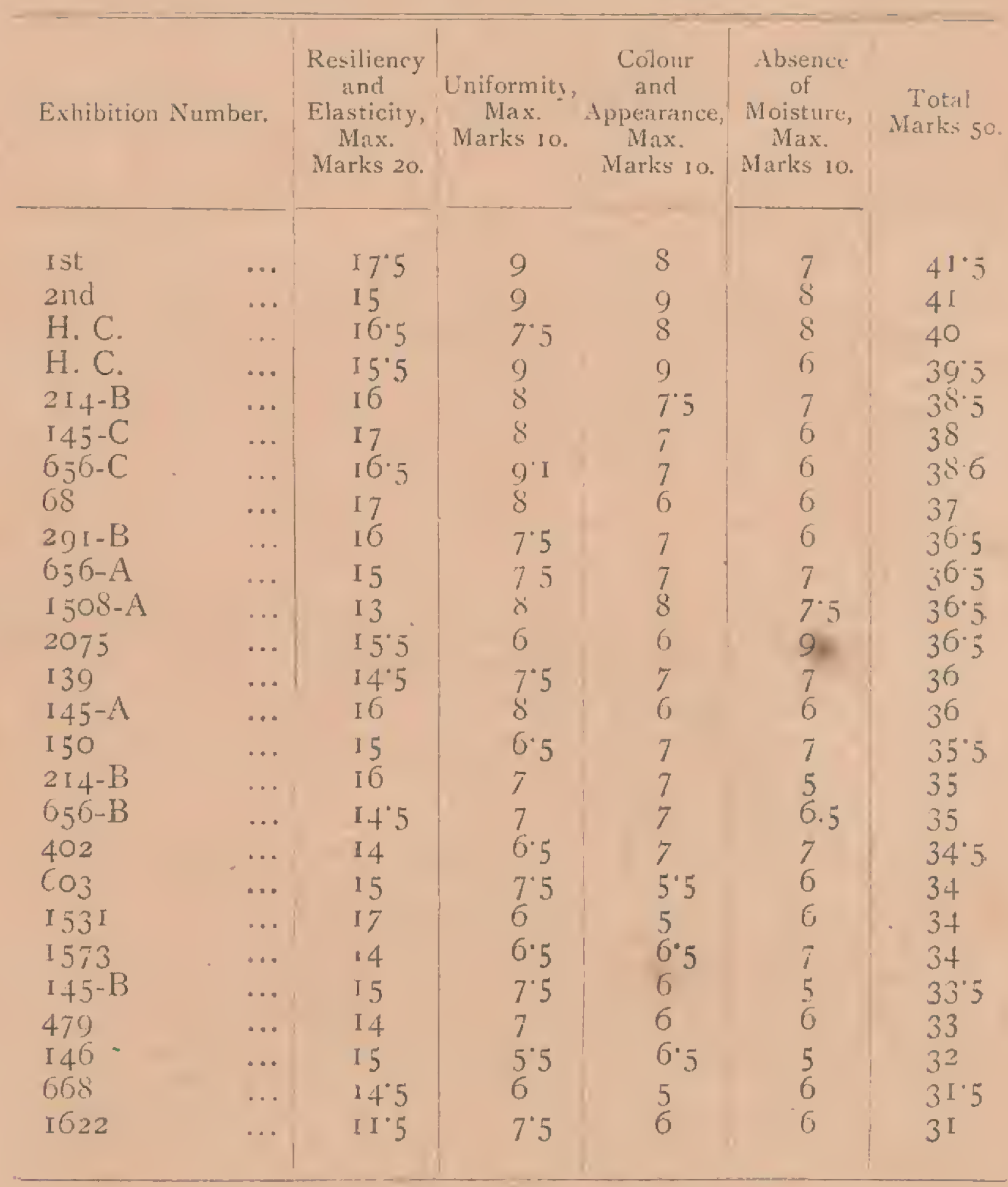

First Prize Silver Cup.-Presented by Selangor Rubber Co. B. C. N. KNIGHT, Jebong Estate, Taiping.

Second Prize Silver Cup.-

Jofin Lami, Bertam Estate, Province Wellesley: Highly Commended.-

A. D. MaChado, Kamuning Estate, Sungei Siput.

Highly Commended.-

M. MaUne, Cicely Estate, Telok Anson. 
Class 10.-Dry Para Block.

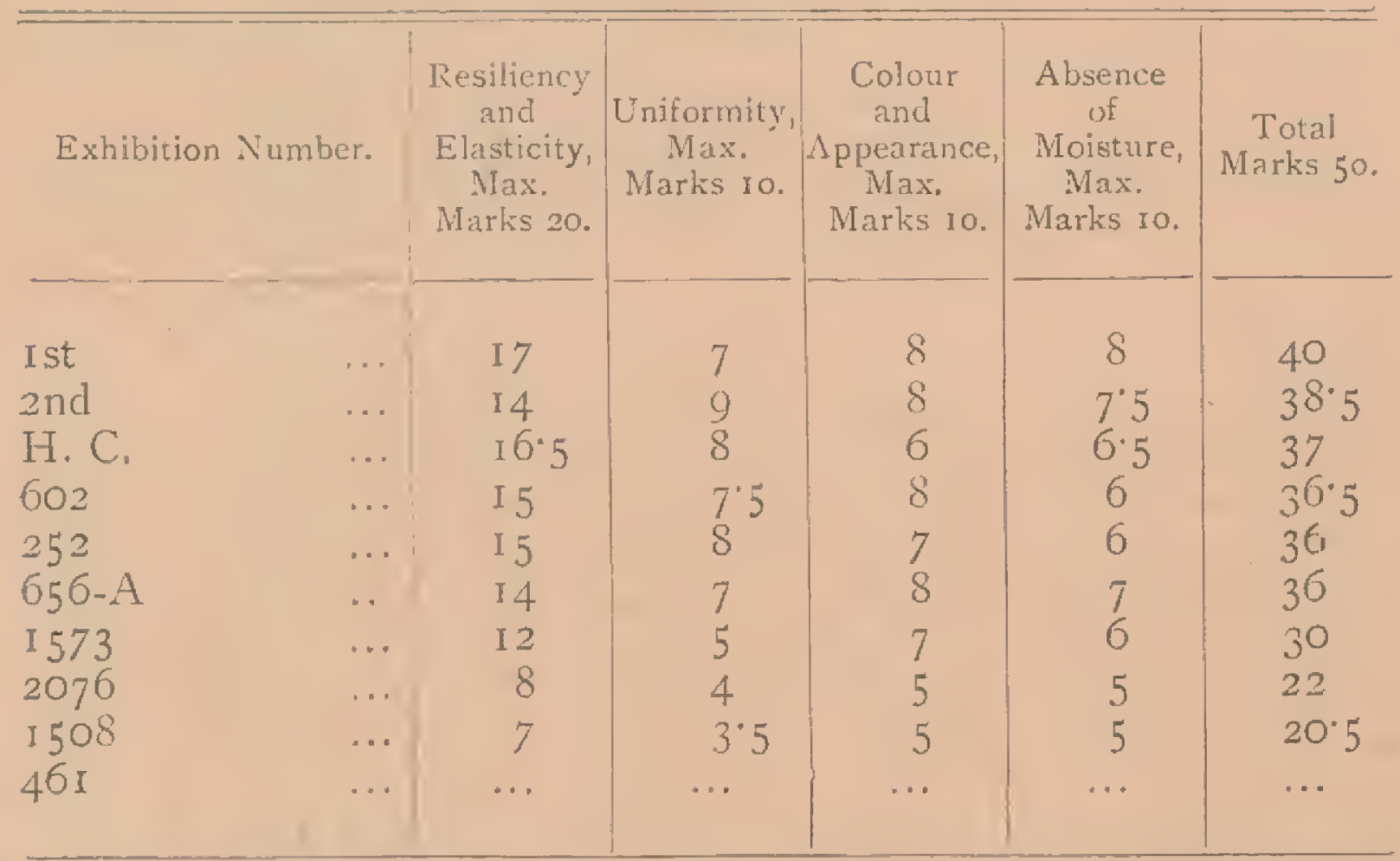

First Prize Silocr Cup.-Presented by Highlands and Lowlands Estate. F. PEAlRS, Lanadron Estate, Johore.

Second Prize Silver Cup.-

O. Pfeñigwerth, Highlands and Lowlands Estate, Klang.

Highly Commended.-

C. T. Hamerton, Bukit Rajah Estate, Klang.

Class 11.-Wet Para Block.

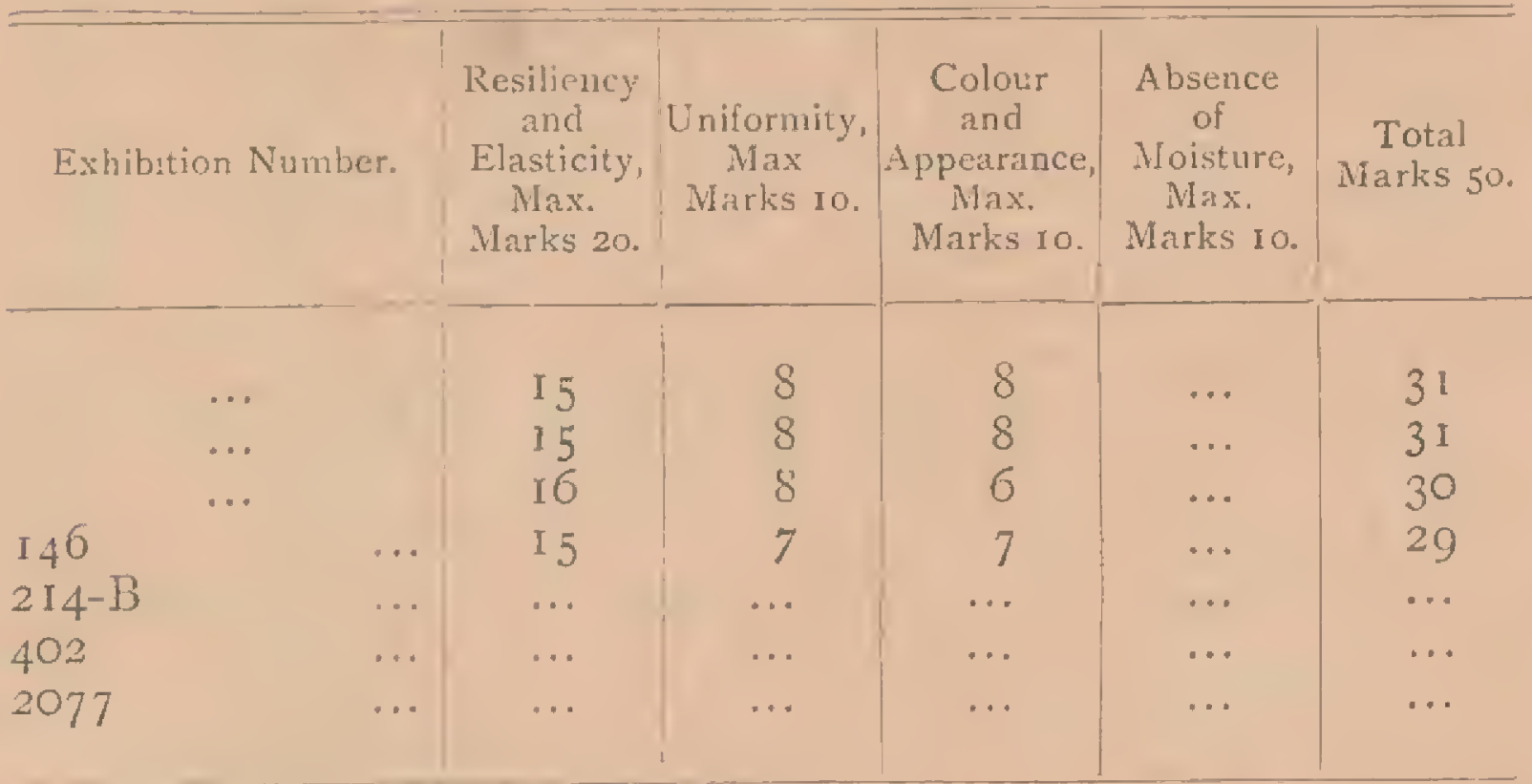

First Prize. - The Lauderdale Cup.

H. M. DARBY, Vallambrosa Estate, Klang.

Second Prise.-

O. PFEnNigwerth, Klang.

Highly Commended.-

A. D. Machado, Kamuning Estate, Sungai Siput. 
Class 12.-Rambong in any Form.

\begin{tabular}{|c|c|c|c|c|c|c|}
\hline Exhibition & Number. & $\begin{array}{c}\text { Resíljency } \\
\text { and } \\
\text { Elasticity, } \\
\text { Max. } \\
\text { Marks } 20 .\end{array}$ & $\begin{array}{c}\text { Uniformity, } \\
\text { Max. } \\
\text { Marks Io. }\end{array}$ & $\begin{array}{c}\text { Colour } \\
\text { and } \\
\text { Appearance, } \\
\text { Max. } \\
\text { Marks Io. }\end{array}$ & $\begin{array}{c}\text { Absence } \\
\text { of } \\
\text { Moisture, } \\
\text { Max. } \\
\text { Marks Io. }\end{array}$ & $\begin{array}{c}\text { Total } \\
\text { Marks } 50 .\end{array}$ \\
\hline I st & $\ldots$ & I 8 & 9 & $\ldots$ & 8 & 35 \\
\hline 2nd & $\ldots$ & I 6.5 & 8 & $\ldots$ & 8 & $32 \cdot 5$ \\
\hline 2078 & $\ldots$ & 15 & 9 & $\ldots$ & 8 & 32 \\
\hline 479 & $\ldots$ & 14 & 8 & $\ldots$ & $\delta$ & 30 \\
\hline $609-B$ & $\ldots$ & I6 & 7 & $\ldots$ & 7 & 30 \\
\hline 67 & $\ldots$ & I 5 & 6 & $\ldots$ & 8 & 29 \\
\hline 69 & $\ldots$ & 16 & 5 & ... & 8 & 29 \\
\hline $46 x$ & $\ldots$ & I 6 & 6 & $\ldots$ & 7 & 29 \\
\hline 252 & $\ldots$ & $16 \cdot 5$ & 5 & .. & 6 & $27 \cdot 5$ \\
\hline I 573 & ... & 15 & 6 & $\ldots$ & 6 & 27 \\
\hline 2208 & $\ldots$ & I 5 & 6 & $\ldots$ & 6 & 27 \\
\hline $2 \mathrm{I} 4-\mathrm{B}$ & $\ldots$ & I 4 & 6 & ... & 6 & 26 \\
\hline $67 \mathrm{I}$ & $\ldots$ & I 2 & 5 & ... & 8 & 25 \\
\hline
\end{tabular}

First Prize Silver Cup.-Presented by Vallambrosa Estate.

O. PFennigiverth, Highlands and Lowlands Estate, Klang.

Second Prize Silver Cup.-

E. B. PrIOR, Golden Hope Estate, Klang

Highly Commended.-Exhibition Number 2078.

Class 13 A.-Rubbers other than Para or Rambong.

\begin{tabular}{|c|c|c|c|c|c|}
\hline Exhibition Number. & $\begin{array}{l}\text { Resiliency } \\
\text { and } \\
\text { Elasticity, } \\
\text { Max. } \\
\text { Marks } 20 .\end{array}$ & $\begin{array}{l}\text { Uniformity, } \\
\text { Max. } \\
\text { Marks Io. }\end{array}$ & $\begin{array}{c}\text { Colour } \\
\text { and } \\
\text { Appearance, } \\
\text { Max. } \\
\text { Marks Io. }\end{array}$ & $\begin{array}{l}\text { Absence } \\
\text { of } \\
\text { Moisture, } \\
\text { Max. } \\
\text { Marks Io. }\end{array}$ & $\begin{array}{c}\text { Total } \\
\text { Marks } 50 .\end{array}$ \\
\hline $25^{2}$ & $\begin{array}{r}20 \\
7\end{array}$ & $\begin{array}{l}10 \\
15\end{array}$ & $\begin{array}{l}\text { IO } \\
9 .\end{array}$ & $\begin{array}{r}10 \\
7\end{array}$ & $\begin{array}{l}50 \\
38\end{array}$ \\
\hline
\end{tabular}

First Prize Silver Cup.--Presented by Bukit Rajah Estate. A. D. Machado, Kamuning Estate, Sungai Siput. 
Class 13 B.-Guttas.

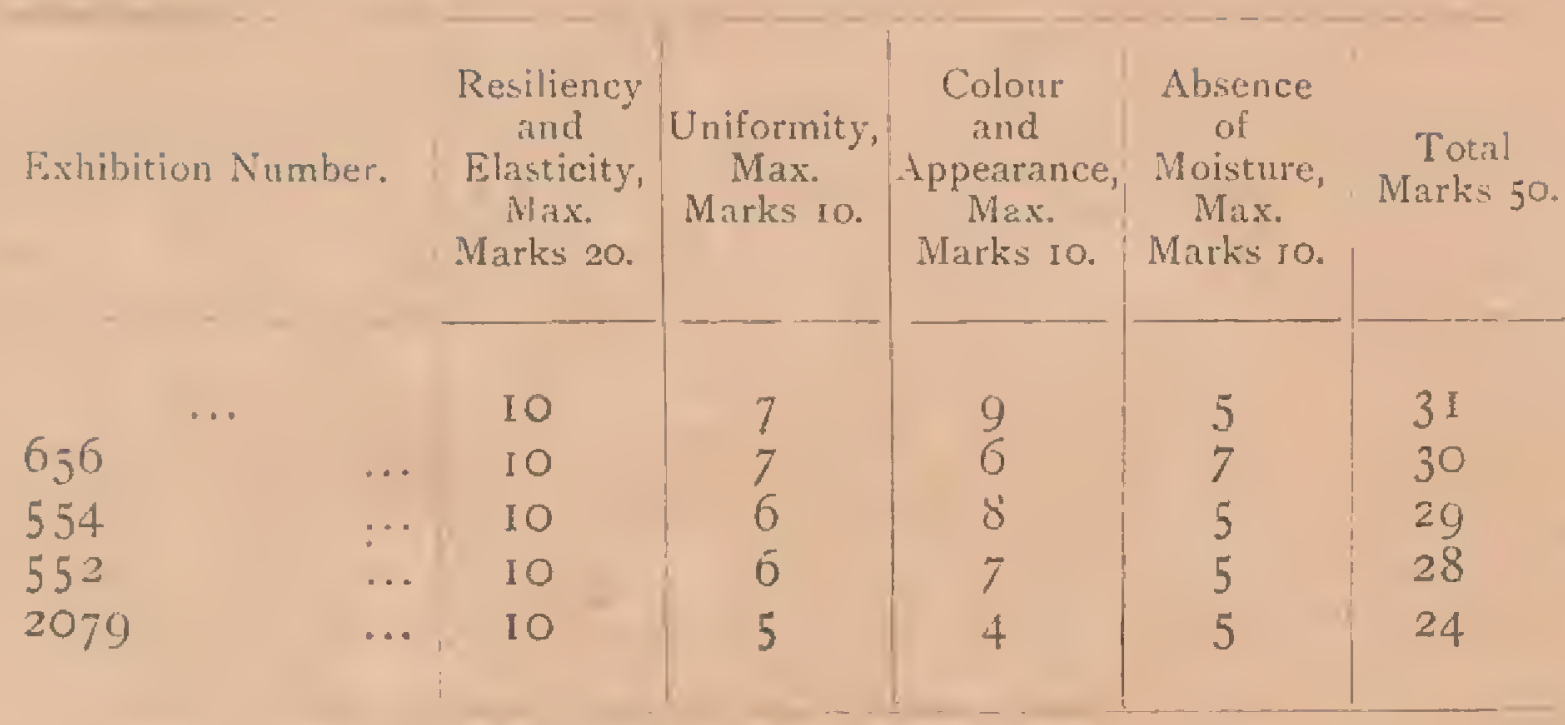

First Prise.-Ismail, Perak.

Class 13 C.-Scrap.

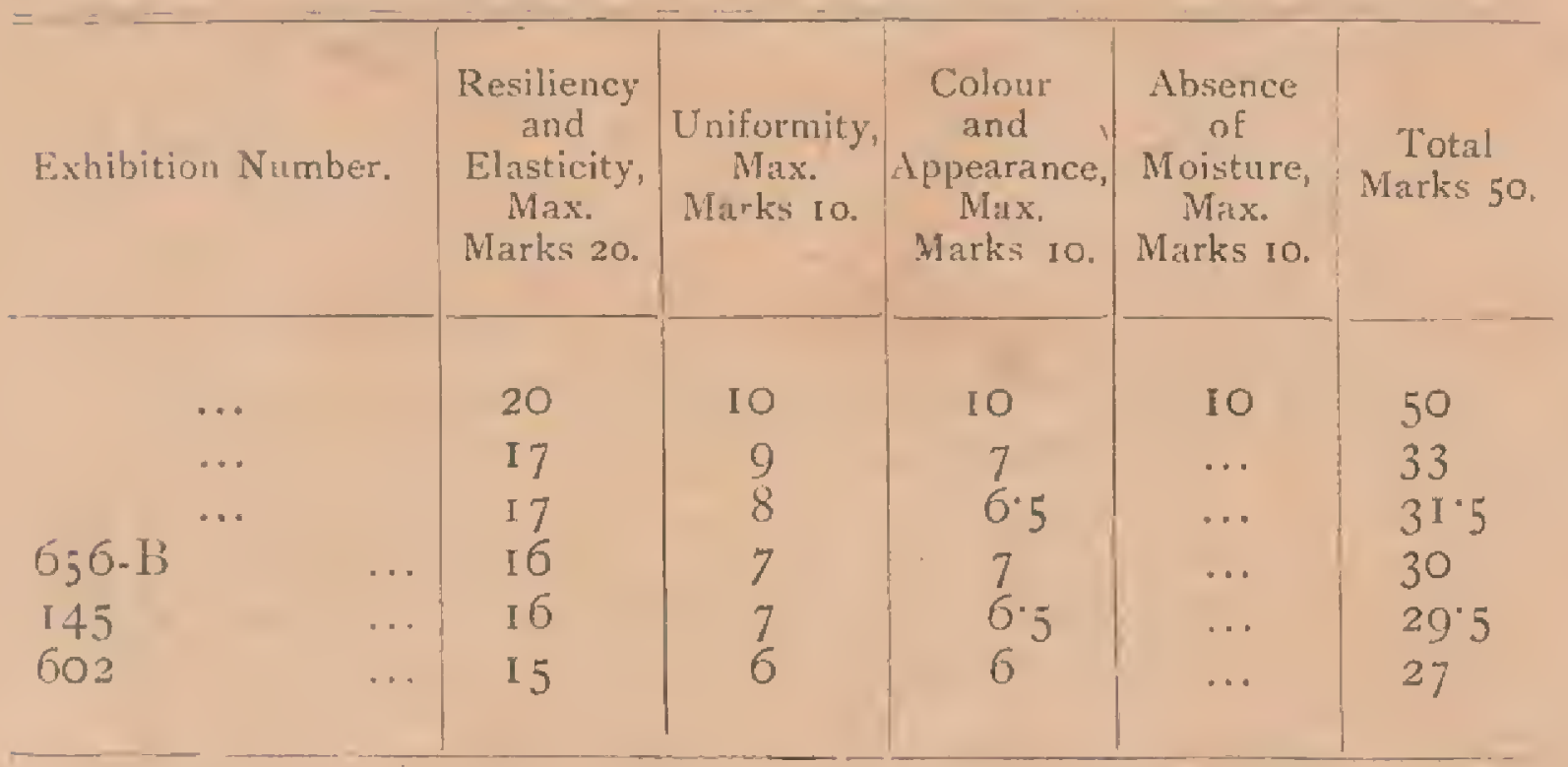

First Prize.-Presented by E. H. Bratt, Esq.

O. Pennigwerth, Highlands and Lowlands Estate, Klang.

Sicond Prize-_JoIn LAMb, Bertam Estate, Province Wellesley. 


\section{PRIZES AWARDED AT THE FOURTH ANNUAL EXHIBITION.}

Drvision $A$.

\section{Agricultural Produce.}

Cu.iss No. I. - Padi, best sample of any named zuriety :Ist Prize to Mohamud bin Tukang, Krian and " Hea Wood Estate, Sungei Siput 3rd " Hamad bin Awang, Krian

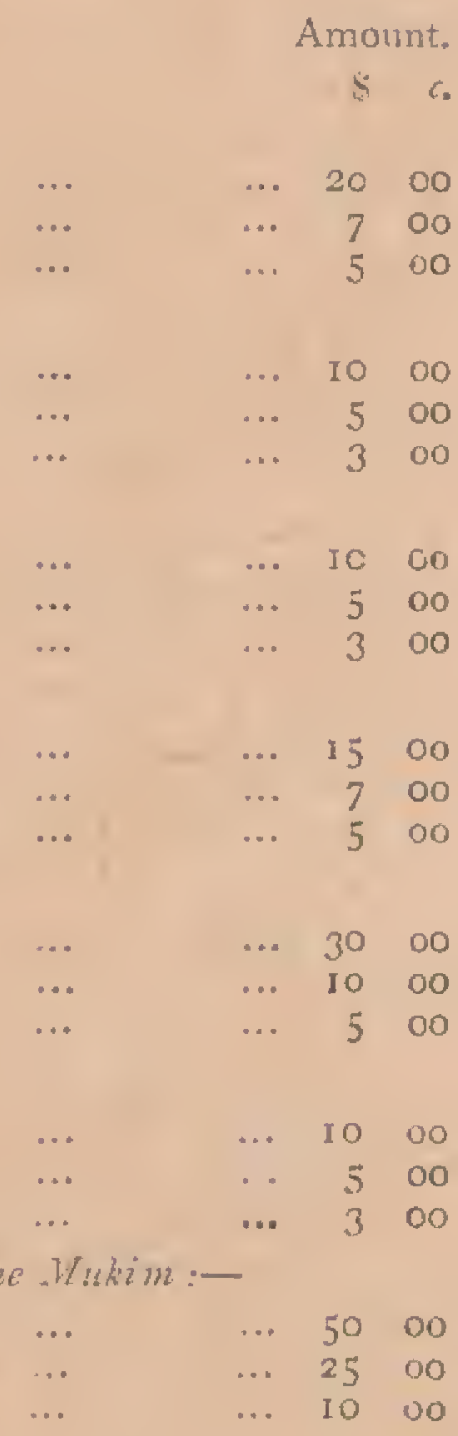

Ci.4ss No. 2.-Pulut, best sample of any named arriety :Ist Prize to Hadji Osman, Province Wellesley and " to Penghulu Pandak Abdullah, Bagan Serai 3:d " to Penghulu Pandak Ibrahim, Kuala Kuran

Cisss No. 3.-Pulut, best sample preparcd by machinery:Ist Prize to Whee Eng Bec, Penang and " to Mohamud, Dito Kramat, Penang ... $3^{\text {rd } " ~ t o ~ P e n g h u l u ~ H a m i d ~ b i n ~ A w a n g, ~ K r i a n ~}$

CuAss No. 4.-Rice, best sample prepared in a lesong:Ist Prize to Ngah Bogok, Kuala Kangsar $\quad \ldots$
and to Mohamud Kassim, Penang 3rd " to Penghulu Yesop Ibrahim, Parit Buntar

Class No. 5.-Padi, best collection of named arriety:Ist Prize to ljok Mukim, Selami and " to Kulop Abdul Kahim, Kota Lama K̈̈ri $3^{\text {rd }}$ " to Sidang Hamid Pongout, Malacca ...

CLiss No. 6.-Pulut, best collection of named sariety :1st Prize to Penghulu Hamid bin Awang, Krian 2nd " to Grila Selama, Selama ... ... $3^{\text {rd }}$ " to Mat Esah, Kuala Kangsar $\cdots$

Cuass No. 7.-Best collection of Padi and Pulut grown in one Mukim:1st Prize to Kulop. Mohamud, Senggong, K. K.
and " to Said Yusop, Chigar Galah, K. K. 3rd , to Penghulu Pandak Abdullah, Bagan Serai

\section{SECTION III.}

\section{Miscethaneols Produce.}

Cuass No. 14.-Bamboos, best collertion:-

Ist Prize to Mohamud Salleh, Perali

and " to Datoh Paduka Rajah, Perak

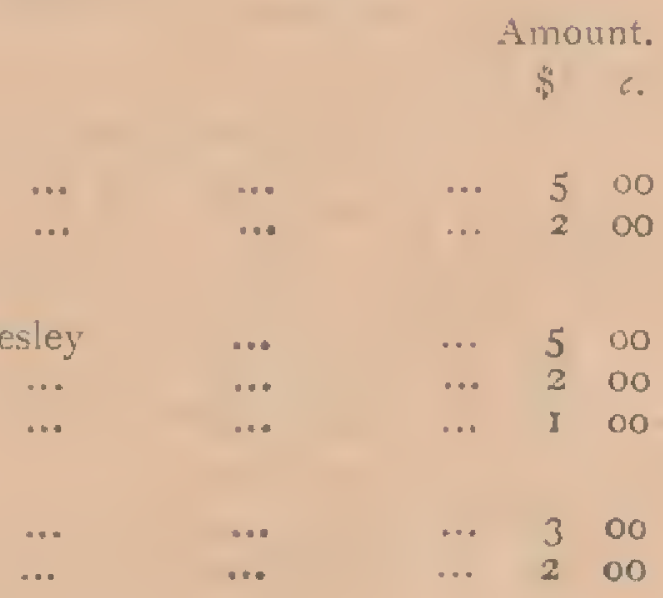

Cisss No. 15.-Betel unts, fresh:-

ist Prize to Penghulu IIassan, Province Wellesley

2nd " to Mat, Penang

3rd " to Normen, Perak

Ciass No. 16.-Betel nuts, dried:Ist Hize to kiung Thean Seng, Penang and " to Rajah Basah, Perak

Cuss No. 17.-Cloves, best sample:-

Ist Prize to Mohamud bin Mohamud Salleh, Penang and " to Mohamud Noor, Ferak 3rd "to Mohamud bin Noor Said, Penang

\begin{tabular}{|c|c|c|}
\hline . & $\ldots$ & \\
\hline ." & $\ldots$ & \\
\hline .. & $\ldots$ & \\
\hline
\end{tabular}


CLASs No. 18,-Coconuts unhusked:-

Amount.

ust Prize to Sidang Idis bin Hasin, Malacca

Ctass No. 19.-Coconuts husked : - No award.

Cuss No. 20.-Coconuts, best collection:-No award.

Cuass No. 21.-Copra, sun dried:-

Ist Prize to Tan Lo Hcong, Perak ...

and " to Federal Oil Mills

3rd "to Sidang Matgin bin Ali, Malacea ...

Class No. 22.-Catton "Kakabu":--

wst Prize to Law Chit Mun, Penang

2nd " to Mohamud Kassim, Penang

3rd ", to Kamuning Estate, Perak

$4^{\text {th }}$, Indut, Perak ... ..

Class No. 23.-Coffee:- -

Ist Prize to Goldenhope Estate, Klang

and " to Jugra Estate

Cunss No. 24. Fibres:-

Ist Prize to Syed $\Lambda$ hmad, Penang ... and " to Shaik Ismail, Penang ... Special to Kamuning Estate, Perak

Cr.Ass No. 25.-Gambir:Ist Prize to Mohamud bin Hadji Said, Penang .

Class No. 26.-Gums and Damars:-

Ist Prize to Datoh Pandak Raja, Perak and ", to Kulop Abdul Rahim

.. 500

... 300

... I 00

... $10 \quad 00$

... 500

... 300

. I 00

... 1000

.. 500

... $10=00$

... 500

... I0 00

... 500

... 1000

... 3 vo

Class No 27.-Ginger:-

Ist Prize to Andi, Penang

and " to Raja Ali, Selangor ...

.. 500

.. 300

Class No. 28. -Indigo, locally prepared:-

Ist Prize to Tan Lo Heong, Perak

Class No. 29.-Mace, dried:-

Ist Prize to Nyak Ahgum, Penang ...

... 500

.. $5 \quad 00$

Ciass No. 30.-Maize:-

Ist Prize to Unda Mat Isah, Perak ...

and " to Datoh Pandak Raja, Perak

... $10 \quad 00$

... 500

Ceass No. 31.-Medicinal Plants:-

1st Prize to Kamuning Estate, Perak

and " to Haji Hassan Ketiwa, Perak

3rd " to Wango, Penang

.. 10 00

.. 500

... 200

Cluss No. 32.-Nutmers, fresh :-

Ist Prize to Megat Hassan, Perak ...

and " to Ngah Mohamud Lateh, Perah

3rd " to Said Musah, Perak

.. 500

.. 300

Cuass No. 33.-Nutmegs, dried:-

Ist Prize to Mat, Penang

and " to Naron bin AbduI Rahim, Penang . 3rd " to Mohamud Kassim, Penang

Class No. 34-Oil. Citronella:Ist Prize to Heawood Estate, Perak 
SEC'TION III,-Continued.

\section{Miscellaneous Produce.}

Class No, 35-Oil, Lemon Griss:-

Amount

$\$ c$

Ist Prize to A. C. Hardoun, Penang

and " to Kamuning Estate, Perak

$\ldots \quad 10 \quad 00$

.. 500

Class No. 36.-OH, Coconut:-

Ist Prize to Singapore Oil Mills, Singapore ...

and " to Caledonia Estate, Province Wellesley, Penang $\quad \ldots \quad 500$

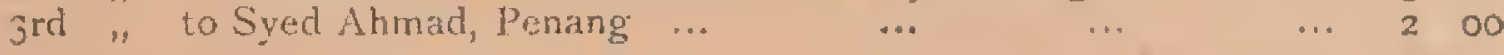

Ciass No, 37. - Oil, Teel seed:-..

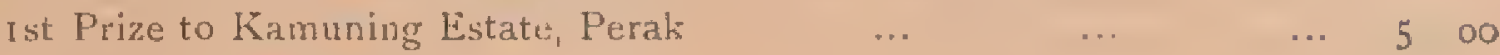

Chass No. 38 -Oil, Castor:-

Ist Prize to Mohamud Kassim, Penang

and "to G.S. Hussain, Penang

Highly Commended, J. R Bruce, Penang

CLAsS No. 39.-Oil, best collection Essential :-

Ist Prize to Kamuning Estate:

Highly Commended, A. C. Hardoun, I'rovince Wellesley

Cunss No. 40.-Pepper, white:

Ist 1 rize to Tong Kat Pow, Penang

and " to Penghulu Shin, Penang

Cuass No. 41.-Pepper, black:-

Ist Prize to E. R. Salisbury, Perak ...

and "Tong Kat Pow, Penang ...

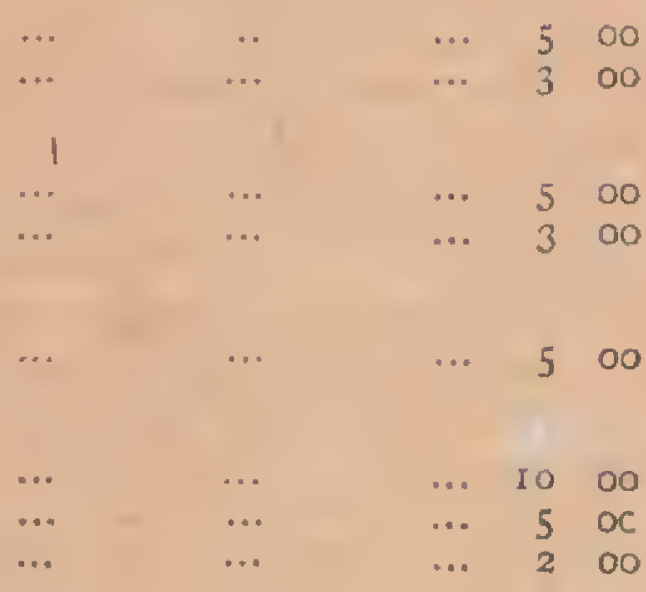

Cists No. 42.-Patchouli:-

ist Prize to Hadji Osman, Perak ...

‥ 500

Class No. 43.-Rotans:-

rst Prize to Raja Ngah Abubakar, Perak

and " to Mohamud Salleh, Perak

3rd "s to Ljok, Peral...

Class No. 44.-Sago, pearl. No award.

Class No. 45.-Sago, flowr.

rst Prize to $(\mathrm{No}, 1614 \%):-$

Class No. 45 A.-Arrowroot, flour :ist Prize to Mohamud Emjara, Penang

Class No. 46.-Sireh leaves:-

Ist Prize to Ibrahin, Perak

and " to Krani Kassim, Perak ...

3rd " to John Lamb, Province Wellesley

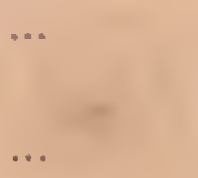

... 500

... 300

.. 700

… 3 OC

Class No. 47.-Spices:-

Ist Prize to G S. Hitssain, Penang

and " to Mohamud Ibrahim, Perak

Class No. 48. - Sugar Care:-

Ist Prize to Caledonia Estate, Province Wellesley

and " to H. S. bin H. Salleh, Malacca

3rd " to Nyak Abas, Perak

$\cdots$

... 1 1 00

CuAss No. 49.-Sugar coconut:-

Ist Prize to $\left(\mathrm{No}, 473^{\circ}\right)$...

$\cdots 5 \quad 500$

$\cdots 200$

ss No. 50--Sugar Nipah:-

1st L'rize to Caledonia Estate, Province Welleslcy

$\begin{array}{rrr}\ldots & 10 & 00 \\ \cdots & 5 & 00\end{array}$

... 200

*. 500

$\cdots 5 \quad 50$ 


\section{Miscellaneous Produce.}

Cuass No. 51--Sugar Kabong:-

ist Prize to Ahmad bin Penglima, Selangor

and "to Ngah Ahmad Garang, Perak

3rd " to Nalum bin Nanin, Malacca

\section{(n)}

Amount.

Class No. 52.-Sugar (cane), bronen:-

Ist Prize to Caledonia Estate, Province Wellesley

2nd " to Towkay Ong Meah Hah, Perak

Class No. 53.-Sugar (cane), white:-

Ist Prize to Caledonia Estate, Province Wellesley

Cuass No. 54.-Honey in the Comb:-

Ist Prize to Penghulu Hassan bin Senan, Perak

Class No. 55.-Tapioca roots:-

Ist Prize to Bahir, Penangr

2nd " to Caledonia Estate, Province Wellesley

$3^{\text {rd }}$ "to Ali bin Penghulu Garang, Selangor

Cinass No. 56.-Tapioca pearl:-

1st Prize to Sandilands Battery \& $\mathrm{Co}_{\text {., }}$ Penang

Class No. 57.-Tapioca fiour:-No award.

Class No. 58.-Tea:-

Ist Prize to Nyak Salleh, Penang

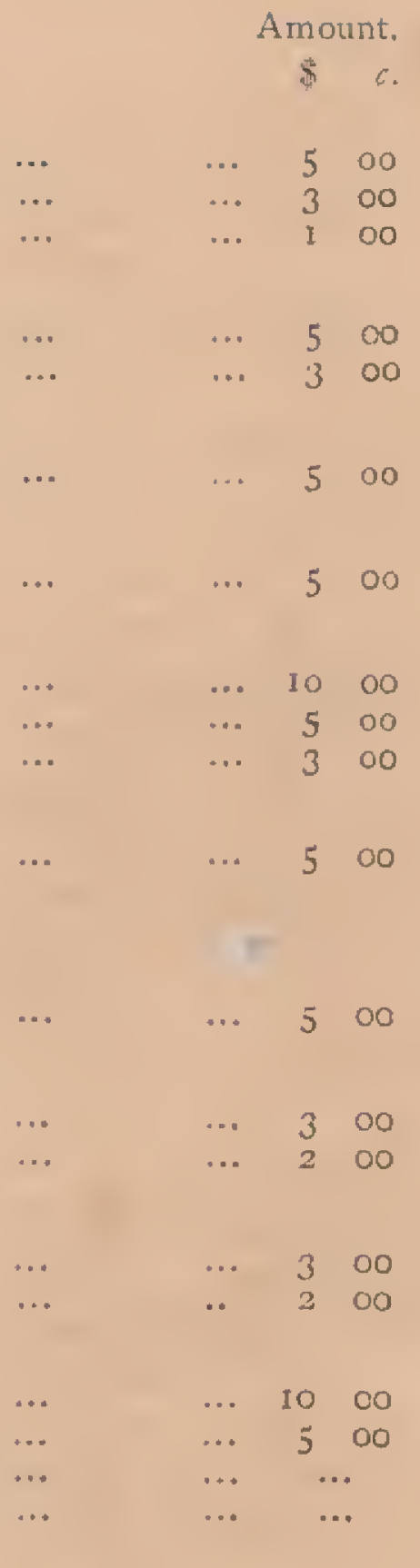

Class No. 59--Toddy:-

1st Prize to Caledonia Estate, Province Wellesley and " to K. Arrumugum, Perak ...

Division $B$.

Flowers, Fruits and Vegetables.

\section{SECTION I.}

Amount.

\&.

Cuss No. 62.-Aroids, other than Caladizms:-No award.

Class No. 63.-Caladiums:--

Ist Prize to Mr. Hughes, Perak

Class No. 64.-Coleus:--No award.

Cuass No. 65-Crotons:--No award.

Class No. 66.-Dracoenas:-No award.

Class No. 67.-Ferns, any variety:-

Ist Prize to H. C. Barnard, Perak

Cuass No. 68.-Ferns, Adiantums:--

ist I'rize to H. C. Barnard, l'erak 
Class No. 69.-Ferns, Adiantums distinct:--No award.

Class No. 70.-Ferns, best specimen :-

Ist Prize to H. Velge, Perak

...

Cuass No. 7I.-Marentas and Calatheas :-No award.

Class No. 72. - Palms:-

rst Prize to Kamuning Estate, Perak

$\cdots$

Cuass No. 73.-Selaginellas:-No award.

Class No. 74.-Selaginella, best specimen:-No award.

Class No. 75.-Foliage Plants:-No award.

CLass No. 76.-Foliage plants, best specimen:-No award.

Class No. 77.-Amaryllis and Lillirs:-No award.

Class No. 78.-Asters :- No award.

Class No. 79,-Balsams:-No award.

Class No. 80.-Cannas:-No award.

Ctass No. 8I.-Cannas, best specimen:-No award.

Class No. 82.-Cock's-comb:-No award.

Class Nö. 83.-Chrysanthemums:-No award.

Class No. 84.-Dahlins:-No award.

Class No. 85.-Dianthus:-No award.

Class No. 86.-Eucharies :-No award.

Class No. 87.-Gloxinias:-No award.

Crass No. 88.-Phlox:-No award.

Class No. Bg,-Petunias:-No award.

Class No. go.-Roses:-

Ist Prize to Mr. Hughes, Perak

Class No. 91.-Zinnias:-No award.

Class No. 92.-Best plant in flower:-No award.

Ciass No. 93.-Begonias:-No award.

Class No. 94.-Group of plants:-No award.

CLass No. 95.-Group of Chinese plants:-

ist Prize to Chung Yee Sing, Perak

Crass No. 96.-Aster's (cut flowers) :-No award.

Cr.Ass No. 97.-Chrysanthemums (cut flowers):-No award.

Class No. 98.-Dahlias (cut flowers) :-No award.

Crass No. 99.-Roses (cut flowers) :-No award.

Class No. roo.-Camas (cut flowers) :-No award.

Class No. Ior.-Orchids (cut flowers) :-No award.

Class No. IC2.-Cut flowers, arranged:Ist Prize to Mat Tahir, Perak

\section{SECTION 1 I.}

Fruits.

Amount.

Ciass No. Io3.-Bananas, best collection:- 
Cl.ass No. I04.-Bananas, best bunch :-

Ist J'rize to Sidang Mat Jin bin Ali, Malacca 2nd , to Soh Lebai Hamat. Penang

Class No. I05.-Champedak:ist Prize to Sedang Leman, Malacca and " to Menti Tengat bin Sedih, Malacca ...

Class No. I06.-Chiku:Ist P'rize to Hadji Salleh, Penang ... and " to Mat Hasim, l'enang

Class Nn. Io7.-Custard Apple:Ist Prize to Edwin I'hilips, Perak

CLASS No. I08.-Cultivated Fruits:Ist I'rize to Tan Lo Jeong, l'erak and " to Kamuning Estate, l'erak

Class No. 109.-Durian:-

Ist Prize to Sidarg Ali bin Tahir, Malacca and "to Mohamud bin liassim, Perak Highly Commended, Penghulu Indut, Perak

CuAss No. IIO.-Durian blanda:Ist Prize to Mat bin Hadji Mohamud Saman, Penang

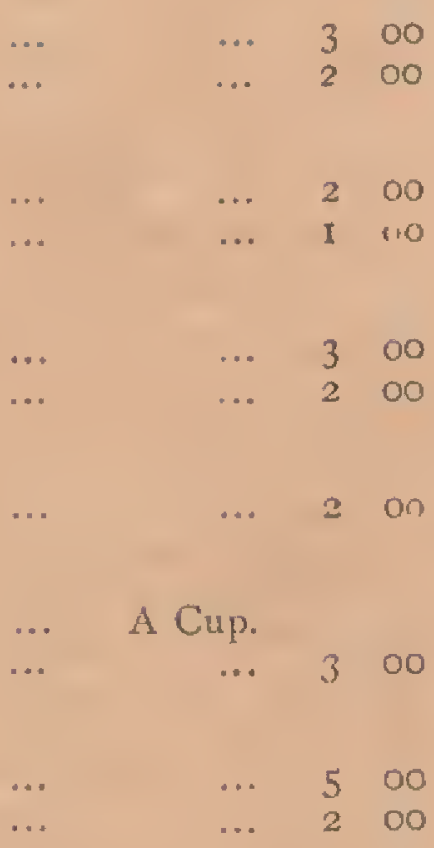

Class No. II I.-Duku:-No award.

Class No. I12,-Binjai:-

Ist Jrize to Sidang Hadji bin Mohanud Musa. Malacca and "to Hadji Mohamud Saman, l'onang

$\cdots 200$

Ciass No. II 3. - Fack fruit:-

Aenghulu Hassan Machang Bali, J'rovince Wellestey

oo

Class No. I14.-Fambu:-

ist Prize to Kung Thean sing, Penang

Ciass No. I1 5. - Langsat:-

Ist Prize to Mohamud Ali, l'erak

and , to Kulop Ibrahim, Perak ...

-. 2 OC

Highly Commended, Kulop Abdul Rahman, l'erak

Class No. II6.-Limes:-

Ist J'rize to Ibrahim, Penang

and ", to Kamuning Estate, Perak

Highly Commended, İenghulu Wahab, Perak

CJASS No. I17.-Mangors:-No award

Ciass No. I 8.- Machang:-No award.

Class No. I 19.-Mangosteens:-

Ist Prize to Hadji Osman bin Hadji Jalil, Jenang and to Hadji Mohamud Saman, J'enang ...

Extra K. Hamad, Penang

.. 5 oo

… 300

... 200

Class No. I20.- Mata Kuchéng:-

Ist Prize to Mata-mata Sahat, Malacca

Class No. I21.-Nelon, any kind:-

Ist Prize to Mohamud Daud, Perak

Cl.Ass No. 122.-Papaya:-

Ist Prize to Abu Omar, l’erak 
Amount, \$.

Ci.ass No. 123.-Oranges: :-

Ist l'rize to Kamuning Estate, Perak and " to Luje

Crass No, 124-Pine-apple:-Mauritius. Ist I'rize to Penghulu Hassan, Province Wellesley

CL.ıSS No. 125.-Pine-apple, any other taricty:Ist l'rize to Kamuning Estate, Jerak and " to Raja Hadji Jahaya, Penang ...

Class No. I26.-Pomeloes:Ist l'rize to (No. $423 \%$ ) ... and "to Kashi Ahmat Kellmah, Perak ..

Cluss No. 127.-Pulasen:Ist T'rize to Hadji Omar, Negri Sembilan ......... and " Ibrahim, J'enang

CLAss No. I 28. - Nambai :-

Ist Jrize to Bahab bin Syed, Selangor and " to Mohamud Daud, T'erak

Ci.lss No. 129.-Rambutan:Ist Prize to Bahari, Province Wellesley and " to Mat bin Hadji Mohamud Siman, Penang

... 200

CLASs No. 130.-Wild edible fruits:Ist l'rize to (No. $554 \mathrm{~A}$ )

CLASS No. 131.-Any kind of fruits not included rabove :Ist l'rize to Law Chit Mun, l'enang and " to Mohamud Sah I'enghulu, Selangor Highly Commended, Kamuning Estate, l'erak

\section{SECTION III.}

VEGETABLES.

CLASS No, I32.-P'resered fruits:-

Ist Prize to E. E. Lessler, l'erak and "to Francis Alexander, Selangor

Cr.Ass No. 133.-Chutuey:1st Prize to Francis Alexander, Selangor

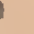




\section{SECTION IIL-Continucd.}

Amount. $\$ c$.

Cisss No. I39.-Beans:-

Ist Prize to Belal Mohamud Daud, Perak 2nd " to Ali, Penang

...

$\cdots$

Crass No. 140.-Cabbage:-No award.

Cinss No. 141.-Chillies:-

Ist Prize to Ngah Alimat, Perak 2nd ", to Pandak Jenal, Perak

... $\quad$..

Cuass No. 142.-Cucumbers:-

Ist Prize to Penghulu Hassan, Province Wellesley

Class No. 143.-Herbs used in curry:-No award.

Class No. 144.-Ladies fingers:-

Ist Prize to Nyah Amin, Perak

Cuass No. 145.-Lethuces:-

Ist P'rize to Ahmad, Penang

CiAss No. 146, - Onions:-

1st Prize to Kung Sin Woe, l'enang

Class No. 147.-Pumpkins:-

Ist Prize to Kamuning Estate, Perak

Class No. 148.-Radishes:-

Ist Prize to Kung Sin Woe, Penang

CLASS No. I49.-Tomatoes:-No award.

Class No. $150 .-V e g e t a b l e s$ and Herbs for making salad:Ist Prize to G. S. Hussain, Penang

Class No. I 5 I.-Water Mclons:-

Pengbulu Hadji Osman, Perak

$\cdots$

CLASS No. I 52, - Yams, etco:-

Ist Prize to Alang Abdul Rahman, Perak and ", to Abdul Aziz, Perak

Class No. I 53. - Any vegetable not in above:Ist Prize to Kulop Mat Ali, Perak ...

\section{Division $C$.}

\section{Cattle and Poultry.}

Amount.

$\$ c$.

Cuiss No. I54.-Bull, locally bred _.. Bruseh Hyd. M. Co. 1st l'rize 20 oo

Cr.Ass No. 155.-Coni, do. d.. Ramasamy, K. K. ... " $\quad 2000$

Cr.Ass No. 157.-Pair Indian Bullocks ... M. Vallipuram, K. K. " 2000

Crass No. 160.-Corw \& Calf, locally bred Ramasamy, K. K. ... " I0

Cuass No. 161.-Buffaloe bull

Crass No. 163.-Buffaloe Con", locully bred Abdul Raman ... " " Io 00

Class No. 164.-Champion animal _.. Bruseh Hyd. M. Co. , , 20 o0

Ciass No. 165.-Kam goat _... Hadji Ali Saiong ... " 500

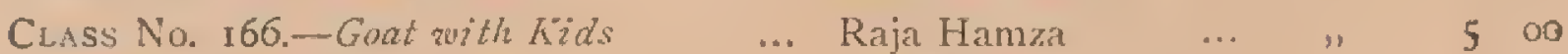

Class No. i68.-Sheep ram $\quad$... Uda Mohamud Ali ... " " 500

Class No. 169.-Do. Eque _.. Edwin Phillips ... " ", 500

Class No. r7o.-Bantan Cock and Hon .. Sidang Hadji Madali " 500 


\begin{tabular}{|c|c|c|c|c|}
\hline Cuass No. 171.-Mralay Cock and Hon ... & $w$ & & & \\
\hline Cuass No. 173-Collection, Nalay fouls... & Anjang Aris & & ,. & \\
\hline Ass No. 174.- Do. Chinese foruls... & Pandak Kemal, K. K & & & \\
\hline Liss No. 175.- Do. any breed & A. D. Machado & $\cdots$ & " & \\
\hline Ass No. 176-Manila Drakes \& 2 Duks & B. O. Stoney & .. & & \\
\hline Luss No.:77.-Favanese & & ... & & \\
\hline cuss No. I78.-Gander and Goose & Hadji Jabar & ... & & \\
\hline LASS No, i8o.--Guinea forvls, Cock \& Hen & Ngah Abass & $\ldots$ & & \\
\hline Ass No. 181.-Pigeons, any breed & C. G. Simons & $\ldots$ & & \\
\hline Lass No. I82.-Best cage bird & Tunku Sleiman & ... & & \\
\hline Lass No. 183. -Collectron, cage birds ... & Vangoo (Balik Pular & & & \\
\hline LASs No. 184.-Rabibits, Buck \& Doe $\ldots$ & A. D. Machado & ... & & \\
\hline iss No. 185.-Champion Cat in Show... & Pandak Lamal & $\ldots$ & & \\
\hline Ass No. I 86.-Best Butter & J.S & $\ldots$ & & \\
\hline LAss No. 187 -Collection of Eggs & A. D. Machado & $\ldots$ & & \\
\hline Lass No. 188.- Do. do. Duiks... & lỉang Trean Sung & ‥ & & \\
\hline Latss No. 190--Best Fox Terrier & W. W. Douglas & ... & & o \\
\hline Lass No. 192.- Do. Retriover & $\because$ & $\ldots$ & & Io \\
\hline Ass No. I93-Do. Hunting dog & " & .. & & 10 \\
\hline ASS No. I94.-Champion dog & , & $\cdots$ & ,. & 10 \\
\hline
\end{tabular}

Class No. $154 .-$ Bull

Cuass No. I68.--Sheep ram

EXTRA PRIZES.

... Mr. Machado ... 2nd Prize ro 00
... Sagomader (Taiping) " 300

\section{Division $D$.}

\section{Malay Arts and Industries.}

Class No. 196.-Silver Works, best specimen:--No award.

CLass No. 197.-Silver Works, best collection :2t.d Prize to District Officer, Kinta, Perak

Class No. 198.-Tintuare:Ist Prize to Ngu Seng, Seremban ... and " to "Tai Shin, Seremban ...

Class No. I99.-Kris:Ist Prize to Penghulı Indut, Perak ... and " to Mohamud Ali, Perak ...

CLASS No. 200.-Carved or Ornamental Walking Stich :Ist Prize to Hamad, Perak

..

Class No. 201.-Unadorned Walking Stick:and Prize to Ngah Ahmat, Perak ...

Class No. 202. - Malay Wond-carving:Ist Prize to Raja Ali, Selangor ... ... and " to Hadji Abdullah bin Mohamud Ahmad, Penang

Class No. 203. Malay Embroidery :Penghulu, Batu Gajah, Perak and "to Sidang Arslad bin Osman, Malacca

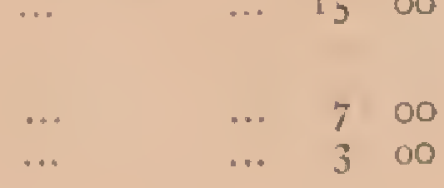




\section{DIVISION D.-Continued}

Cl.ass No. 205-Sarong, silk and yold:Ist l'rime to Penghulu, Papan, P'erak and "to Penghulu, Ipoh, Perak ...$$
1000
$$

Class No. 206 -Sarong, silk:Ist Prize to Tunku P'uan Besar, Pahang and " to Inche Ngah bin Hadji Abdul Rahman, Pahang

Amount. $\$ \quad \epsilon$.

Cuass No. 207.-Saro gr, cotton:Ist Prize to Slaiman, l'erak 2nd " to Mohamud bin Hadji Jumol, Penang

Class No. 208. -Kuin Lepas:Ist Prize to Hadji Mohamud $A l i$, Penghulu, Perak

Class No. 209--Selendang:and Prize to Sulaiman, l'erak

Class No. 2ro.-Krin Telepok:Ist I'rize to Penghulu Jugra and Banda, Stangor

Class No. 2rr.-Ornamental baskets, rattan:Ist l'rize to E. E. Lessler, l'erak

$\cdots$

Class No. 212.-Ornamental baskets, pandan:Ist l'rize to Sidang Hadji Mat $A l i$, Malacea and "to Tijah, Malacca

Class No. 213.-Hats, pandan:Ist Prize to Collector of Land Revenne, Seremban? and " to Collector of Land Revenue, Seremban:

CLAss No. 214.-Hats, terendak:Ist l'rize to Saib bin .lin, Penang ... and ," to Nohanud bin Kassim, lerak ..

CLASS No. 215.-Mats, embroidered:Ist l'rize to Hadji What swin, l'erak and "to kajit Abdul Mali, Perak

Cuass No, 2I6.-Mats, coloured:Ist Prize to Che Pandak Abdullah, Perak and "to H. Berkley, Perak ...

CLass No. 217.-Mats (tikar bangka):Ist Prize to Mohamud Arsad, Perak and "to V.V. l'eters, l'erak

CI.ASS No. 2I8. - Mats (tikar hampar):Ist Prize to Mohinud Mansoor, Perak and ," to Datoh l'aduka Rajah, J'erak

C.LASS No. 2ig. -Mats, pand n:Ist Prize to Mohamud Osman bin Mohimud Taib, Penang and to Nyah Abas, Perak

Cisss No. 220.-Muis, rattan:Ist J'rize to Che l'andak Abdullah, Perak $\quad \ldots \quad$... $\quad$... 5 oo

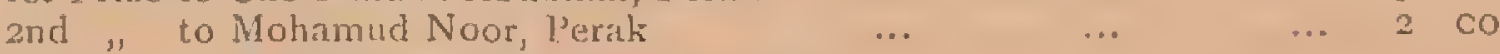

Cuss No. 221.-Models of Riverine Fish-traps:Ist Prize to Mohamud Mansoor

Class No. 222.-Molels, Trops and Snares for Game:Ist Irize to Mohamud Mansoor, l'erak and "to Ismail, Perak

Ci.ASS No. 223. - Sakai and Semang Articles:Ist l'rize to Penghulu Batang l'adang, Perak ... and " P'anjang Abu Baker, P'eralk
... $10 \quad 00$

... 500

.. $10 \quad 00$

... 500

... 700

.. 500

.. 2 00

‥ 700

‥ 700

‥ 700

... 300

... 700

... 300

... 500

.. 200

... 1000

... 500

... 500 oO

.. 500

... 200

.. $5 \quad 00$

... 200

... 500

... 200

... $10 \quad 00$

... 500

.. 700

... 2 OD
... 300 
Chass No. 224. - Articles male fiom Coconat Paluin:Ist Prize to Che Onar, Selangor
and," to Hadji Sirit K. S. Hadji Mohamud, Perak

CuAss No. 225. - Sireh Requisites, Metal:1st l'rize to Hadji Osman, I'enang and " to Hadji Mohamud Sahis, Penang ...

Class No. 226. - Sireh Requisites, in Worcn.riork :- Ist Prize to IKulop Abdul Rahman, Perak

Amount.

Class No. 227.-Chicks:-

Ist Prize to H. Berkley, I'erak 2nd " to Anjany Idris, l'erak

Class No. 228. - Kajang, pandan (Hengkuangr):Ist l'ize to Datoh Paduka Rajah and , to Penglulu Mohamud Noordin, l'erak

Class No. 229. -Kajangs, nipat:Ist Prize to Abdul Mli, lerak and ," to Alang Mohamud Talip. Perak

Class No. 230 - Ataps:Ist Prize to Abdul Ali, Jerak

Ci.Ass No. 23 1.-Rope and Trvine:Ist Prize to Sidang Hadji Mat Ali, Perak and , to Syed Ahmad, Penang ...

Ci,Ass No. 232.-bird's Conge:Ist I'rize to Ngah thmat, Perak and ", to FIadji Salib, Penang

$\cdots$

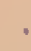

L.Ass No. 233.-Strainer (tapisan) :Ist P'rime to $\mathrm{S}$, Ahmad, Selangor and " to Omar, J'erak

CLASS No. 234.-Niru:Ist Prize to Haron bin Abdul Rahaman, l'enang 2nd " to IIadji Mohamud Jasin, l'erak

CLASs No. 235.-Parans (with handle):-No award.

Ciass No. 236 .- Golok (with sheath):and Prize to Penghulu Ali, Malacca

Cidss No. 237. - Flonery Pots:Ist Prize to Meena Mariuppen, Province Wellesley and ", to Wan Kichil, L'erak

Crass No. 238-Cooking Pots:Ist 'Prize to Wan Kichil, l'erak and " to Do.

Do.

Class No. 239.-Water-vessels:Ist ]rize to District Officer, Kuala Kangsar, Perak 2nd " to Datoh Paduka Rajah, Perak

\section{...}

Class No. 240.-Roofing Tiles: Ist Prize to Tan Ong Peng, l'erik ... and " to P'enghulu Indut, Perak ..

Class No. 241.-Flooring Tiles:Ist Prize to Mohamud, Penarg 2nd " to l'enghulu Indut, Perak

Class No. 242.-Bricks:Ist Prize to Tan Ong l'eng, Perak ... and in to Seng Hin, Perak 


\section{Division $E$.}

\section{Agricultural Implements.}

Class No. 253.-Padi Implements:-

Ist Prize to Anjang Mohamud Daud, Kuala Kangsar.

2nd "to Haji Mat Jassin bin Haji Ali, Bagan Serei.

3rd ", Not awarded.

CLass No. 254.-Agricultural Implements other than for Padi or Rubber locally. made:-

Ist Prize to Penghulu Usof bin Haji Kechil, Malacca.

and Mohamed Kassim, Pealang.

Class No. 255.-Agricultura, Implements, European made:Ist Prize to C. R. Salisbury, Gapis Estate, Perak.

Class No. 256.-Rubber Muchinery in motion:Ist Prize to Federated Engineering Company, Kurala Lumpur.-(Cup).

Ciass No. 257.-No entries.

C.ass No. 258 . - Bullock Cart:1st Prize to M. Vallipuram, Kuzla Kangsar. and " to T. Hisman, Kuala Kangsar.

Cuass No, 260,-Agricultural Bajkets:ist Prize to Syed Ahmat, P'ening. 2nd ", to Jaudin bin Abu, Pengkalan Rama, Malaccia.

Class No. 262.-Miscellaneons :Special Prize to Penghulu Che Puteh, Krian. 
LIST OF MEDALS.

\begin{tabular}{|c|c|c|c|c|}
\hline No. & Names. & Place. & Exhibits. & $\begin{array}{c}\text { Number } \\
\text { of } \\
\text { Medals. }\end{array}$ \\
\hline I & Mr. A. C. Hardowin & Bukit Tamboon & $\begin{array}{cc}\text { Lemon } & \text { Grass } \\
\text { Oil } & \ldots\end{array}$ & I Medal. \\
\hline 2 & $\begin{array}{l}\text { Bruseh Hydraulic } \\
\text { Tin Mining Co. }\end{array}$ & Bidor & Cattle $\quad \ldots$ & 2 Medals. \\
\hline 3 & Mr. E. B. Prior ... & $\ldots$ & Coffee $\quad \ldots$ & I Meda. \\
\hline 4 & Mr. A. D. Machado & Sungai Siput. & $\begin{array}{l}\text { Medicinal } \\
\text { Plants, Fibre } \\
\text { and Essential } \\
\text { Oils }\end{array}$ & 3 Medals. \\
\hline 5 & Singapore Oil Mills & Singapore $\quad .$. & Cocoanut Oil & I Medal. \\
\hline 6 & Caledonia Estate & P. Wellesley... & Sugar Canes & I $\quad$, \\
\hline 7 & Mr. Tan Oon Peng & K. Kangsa & Roofing Tiles & I \\
\hline 8 & Mr. Tan Oon Peng & K. Kangsa & Bricks & I \\
\hline
\end{tabular}

LIST OF DIPLOMAS.

\begin{tabular}{|c|c|c|c|c|}
\hline No. & Names. & Place. & Exhibits. & $\begin{array}{c}\text { Number } \\
\text { of } \\
\text { Diplomas. }\end{array}$ \\
\hline I & Mr. H. B. Collinge & $\begin{array}{l}538,252 \text { and } \\
\text { Teluk Anson } \\
\text { Girls' School }\end{array}$ & Embroidery & 2 Diplomas. \\
\hline 2 & Mr. A. D. Machado & Sungai Siput & Eibre & I Diploma. \\
\hline 3 & & Kuala Kangsar & Padi \& Pulut & I \\
\hline 4 & Fed. Eng. Coy. Ltd. & Kuala Lumpur & $\begin{array}{l}\text { Rubber } \\
\text { Machinery }\end{array}$ & I \\
\hline 5 & $\begin{array}{cc}\text { Highlands \& } & \text { Low- } \\
\text { lands } & \ldots\end{array}$ & Selangor & Rubber & I \\
\hline 6 & Mr. A. B. Stephens & Taiping & Tin Roofing & I \\
\hline 7 & Tungku Tuan Besar & Pahang & Silk Sarongs & I \\
\hline 8 & Brunei Exhibit & .. & $\ldots$ & $\ldots$ \\
\hline
\end{tabular}




\section{AGRICULTURAL SHOW 1907.}

BALANCE SHEET.

RECEIPTS.

By Government Contributions, S. S.

\$ $c$.

By Government Contributions, F. M. S. ...

... $2,000 \quad 00$

By Public Subscriptions

By sale of sheds, materials etc.

... $2,000 \quad 00$

... 2,695 00

… 7 I 875

Total

$\ldots \$_{7,4} I_{3}, 75$

\section{PAYMENTS.}

Cost of Buildings

Cost of Prizes

$\begin{array}{llll}\ldots & \cdots & 3,300 & 00 \\ \ldots & \ldots & 2,010 & 00\end{array}$

Printing, \$196; Compensation, \$427.20; Wages, \$75:

Accommodation, \$225.66; Rent, \$1 30: Advertisements, \$76.67; Transport, \$123.68: Sundries, \$I I.58; Telegrams and Postage \$25.38; $\quad \ldots \quad$ r,39 I 17 Balance handed to Secretary to Resident, Perak $\quad \ldots \quad 712 \quad 58$ Total $\ldots \$ 7,413 \quad 75$

\section{GOW, WILSON \& STANTON, LIMITED- India Rubber Market Report.}

I3, ROOD LANE, LONDON, E.C. August 9 th, 1907 .

At to-day's auction, about 843 packages of Ceylon and Malaya Plantation grown rubber were under offer, of which, about 427 were sold.

The auction having been postponed until after the holidays, offerings were rather larger than usual, the total weight amounting to 45 tons, Ceylon contributing over 10, and Malaya about 35 tons.

Competition was in most instances somewhat irregular, and a decline of about $\mathrm{I} d$. to $2 d$. per $\mathrm{lb}$. was recorded for the finer grades, except in the case of a few lots shewing particularly attractive quality which were well competed for, and sold up to 5/Io per lb.this price being realized for some Ceara biscuits from Rangbodde Estate. 
The chillies me well nigh exhauster about four months from the time of planting. They are generally succeded by a crop of sweet potatoes, after which the land is allowed to fio fallow for a comple of months. Then the weeds we ploughed up and used as green manure mixed with farm-yard compost.

Briefly the orter may be-stated categorically.

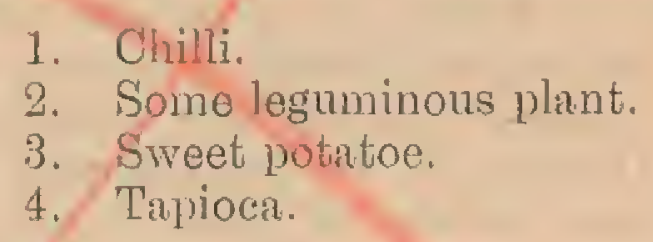

Indigo is a farourite leguminous plant to be cultivated. It requires very careful manuring, and generally yields three crops of cuttings - after which the land is allowed fo rest for a few montils.

- By careful tillage and judicious manuring, these Chinese regetable gardenersare able to make use of the poolest land available and to obtain good retuyns for their toil and investment. When lalang land has been thus rechimed, it is not unprofitable to plant it up with rubber between the vegetables grown.-Dr. Lim Boon Heng.

\section{FIFTH JOINT ANNUAL AGRI-HORTICULTURAL SHOW OF THE MALAY PENINSULA, HELD AT KUALA LUMPOR 10th, 11th, and 12th, AUGUST, 1908.}

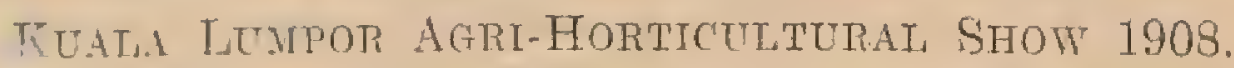

The fifth Agri-Horticultural Show of the Malay Peninsula was beld in Kuala Lumpol on the 10th, 11th, and 12th August. The Show which was opened by His Excellency Sir John Anderson K. C. M. G. was farouled by excellent weather and was by general consent consitereil very successful.

Working arrangements.-The system of working this yenr's Show was somewhat different to that of previous years in that the whole management of the Show was undertaken by one small Committee of nine mombers. No Suh-Committees were appointed with the exception of a committee of three who drew up the mize list in the native art section, and the Horse Committee which had entire charge of that portion of the Show.

In place of the usual sub-committees in each division Stewards were appointel for exch section, whose duty it was to attend to the arrangements of the exhibits on arrival, and facilitate the work of the Judges in their section.

This change in working arrangements is I think one to be recommender, the small committee is more workable, and the selection of Stewarls ench having a definite section to look after is preferable to the old system of having a sub-committee of say five persons, who ench left the work to the other with the result on many occasions when most needed, they were not to be found. 
Show and Buildings. The site of this year's Show was the Malay Settlement Padang which is most conveniently situated and made an admirable ground for the purpose. The buildings were arranged all round the grounds with the Horse-Ring in the centre. The main buildings three in number $150^{\prime} \times 50^{\prime}$ were used for Division $A$. Agricultural Produce Division B. Flowers, Fruits \&c., and Division D. Native Arts. 'The Agricultural Tmplements \&.c., had a shed $100^{\prime} \times 50$ ' allotted to them. A similar shed $\left(100^{\prime} \times 50^{\prime}\right)$ was put up for trade exhibitors. Special sheds were put up for Horses, Fowls and Cattle. The total cost of buildings and staging was $\$ 3,120.85$.

Exhitits. The Forest Depastment put up a most interesting and instructive exhibit of forest produce including fine collection of Rotans, Gums, Diumars and Getahs \&.e., \&c. The exhibit ins a whole was most creditable to the Department and was awarded a Diploma. good.

Agricultural Produce. In this section entries were in most cases

Padi. The entries in padi were very large and the samples good. The principal prizes went to the Krian District.

Rubber. It is to be regretted that entries in the rubber section were not so numerous as in previous years. This may perhaps be accomted for by the fact that the quantities required were increased to a minimum of $25 \mathrm{lbs}$. There appears to be a disinclination on the part of some owner's and Directors to enter into competitions of this sort. In one or two cases the reason given for not competing, where Estate Minagers were seen was that "their Directors had forbidden them to enter." The quality of the rubber shown was on the whole excellent. The prize for the best rubler in the Show was won by Mr. F. G. Harrey, Petaling Estate, with very fine sample of crepe.

An excellent exhibit of rubber packed for shipment shown by Mr. B. C. N. Knight, Jebong Estate, was awarded the Goremor's Cip for: the best Agricultural Exhibit in the Show.

The Sultan's Cup awarded "by points" for the most successful exhibitor in the Rubber section was won by Mr. A. D. Macharo, Kamming Estate.

Coconuts and Copra. Entries in Coconuts and Copra were numerous. The samples submitted were reported by the Judges to have heen excellent and caused a good deal of trouble in placing the awards. The Cup for Copra was won by Mr. Molesworth of Jugra Estate.

Gencral Prodnce. The principal items that call for attention under this heading are the very fine cloves and nutmegs from Penang. The exhibits of Tapioca in all forms were good. The entries in Cotton were numerous and the samples in many instances very good.

The Oil exhibits as a whole where not up to the standard of previous years. Coconut oil being a remarkable exception. Penang cloves oil and tapioca flour from Malacca were awarded Diplomas in this section.

Fruits and Flowers. The principal fenture in this shed was the fine group of plants exhibited by Mr. Tong 'Tak Io of Iraala Limpor, which. 
was awarded a cup as a special prize. The Exhibition of plants and flowers wias as a whole very disappointing.

The group put up by Mr. Coomaraswamy Pillai was perhaps the best exhibit in the section.

Fruits were not vely numerons this year. Small fruits in particular being very poor. Pine-ajples, Limes, Oranges, Pisangs and Durians were fair. The vegetable section does not yet receive the support it merits and it is probable that better prizes will have to be offered to induce entries in this section. An excellent stand of regetables from the Tripeng Fills was jut up by Mr. Long, Superintendent Government Plantations, Perak, and was much admired.

A very clroice group of plants was shown by Mr. Fox, Superintendent of Gardens and Forests, Penang, amongst which were some fine plants of Gesuer as and the pretty Habenaria carnea from Lankawei.

In the section for Preserved Fruits, Jellies and Pickles, Chntneys filled well. The prineipal prizes went to Mr. F. Alexander, Kuala Lumpor, who was awarded a Diploma for his exhibit collectively.

Divsion D. Native Arts and Industries. This section was as usual the largest in the Show and the exhibits in most cases quite up to the standard of previous years. The Perak schools put up a very large exhibit which taken as a whole was good but not quite up to the standard set up at former' shows.

The Selangor schools put up some rotan work and wood carving. The embroidery and wearing section was well filled. The specimen of embroidered mat from Tower Perak was very fine. The specimen of Lian Telepoly shewn by Penghulu Morrib, Kruala Langat, was very fine and was awarded the Resident's cup as the best exhibit in native orts and industrics in the Show.

Pahang sent a linge number of Sarongs and took a number of prizes for them.

Malacca Lace Makers. A most interesting feature in this section was the exhibit of lacemaling from Malacea by five small children in charge of a school mistress. They showed themselves to be expert at their work and were a great centre of attraction at all times. The metal work section did not fill so well as it ought to have done. The principal feature of the section being the Brunei exhibits which took a large number prizes. Seremban Tin-ware was exhibited in large quantities. The new class opened for minature Malay models was a huge success as far as entries was concerned but much of the interest in this class was lost throngh over crowding and for want of descriptive tickets. Both prizes in this class went to Lower Peral.

It might be well another year to give all models, Basket work and Rotan work a shed to themselves. The Malacea Baskets were exhibited in quantity and attracted a good deal of attraction.

Brunei Government put up a stand of silver and bronze work also sarongs \&c., in addition to what they entered in open classes. The exhibit which was one that attracted considerable attention, was awarded a diploma.

The Malay Art School, Kuala Kangsar, put up a stand of silver work and pottery. 
Division $C$. It is to be regretted that the entries in the Cattle section were not more numerous. Practically all that did come were from Perak and Malacca. The prize for the champion animal went to Mr. Brett Brussi, Pelak.

There were no entries in Buffaloes and Pigs.

Poultry. The entries in the poultry section were much larger than in previous Shows with the result that there was a shortage of pens for housing. As a result arrangements in this section were bad. At the time the entries closed oflicially, the number of pens was full, and in addition some fifty entries were accepted on the Show ground, people bringing their birds along, some from it distance, it was not thought advisuble to discourage them by not allowing them to compiete. This fact accounts for the confusion in this section.

Ayricultural Implements. Entries in this division were poor. The Federated Fngineering Company, Kuala Lumpor, took first prize for rubber tapping tools, also for Agricultural Implements European macle.

Two exhilbits were shewn of coagulating machinery the one being a circular iron tank with a hand propelled paldle and the other a similar mechanism arranged so that it could be attached to the ordinary form of Shanghai bath which has a smooth glazed interior. Both these machines did their work elfectively and were awarded a silver and bronze medal respectively.

Horse Section. In all 78 horses and 16 gharry ponies were entered. The champion prizes were won by Messrs. Yap Brothers for black mare over 15 hands, and for horse under 15 hands hy Mr. C. E. Spooner, C. M. G. with Catucha.

Prize Vinners. Amongst prize winners Penang and Province Vellesley were the most successful. The percentage of prizes to entries being very high indeed.

Next on the list como Malacca, Lower Pelak, Fuala Langsar and Krian. The later districts prizes chiefly for padi. The State of Negri Sembilan sent a large number of entries. Selangor with the exception of Kuala Langat District was poorly represented considering the fact that the Show was held in the State.

A great deal of the success of the Shows as far as entrios go depends on the interest the District Officers take in the matter and without their co-operation results would be poor indeed.

Crencral. Athough entries officially closed on 3rd Ausust "the majority were received on the last two days (2nd and $31 . d$ )" and a large number came in late.

This caused a tremendous rush of work on the week before the Show. The entries up to Wednesday 5th August numbered 5994; in addition to this some 2000 entries were accepted after this date most of them being taken on the ground.

Articles for sale were not so roadily disposed of as in previous years. This is probably due to the scarcity of money. The sellers can scarcely agree with press comments as to Museum people snapping up 
the best articles before the public liave the opnortunity of buying, a crititicism that cannot be said to apply to this yoar's Show.

The Show being favoured by such fine weather, realized a good sum of money from the gate which at once placed it in a sound position financially. Fach subscriber of $\$ 10 /-$, and all judges, Stewards and Lxhibitors were admitted free. Orer 1,000 exhibitor's tickets were given out. About 1,500 school children were admitted free.

The F. M. S. Railways Department again undertook the conveyance of exhibitor's 3 rd class and exhibits free of charge. The British India Steam Narigation Company offered 50 free tickets from Penang to Port Swettenham. The Straits Stemmship Company allowed a $25 \%$ reduction on all bonatide exhibitors and exhibits concessions that were much appreciated.

Accommodalion of visitors. Special arrangements were made for the accommodation of visitor's to the Show. The Victoria Institution was turned into id Temporary Hotel and under the stewardship of $\mathrm{Mr}$. T. H. B. Phillips. The Bulls Heal Mless House was put at the disposal of the committee by the Government and the management of it undertaken by Mr. Van Donop.

\section{J. T. Campbell, Suporintendent Exporimentul Plantations,}

Finala Lumpor.

\section{GOVERAOR'S SYEECH.}

No prettier of more convenient site could have been found tiran that selected on the Padang of the Malay Settlement for the tifth annual Agri-Horticultural Slsow of the Matay Peninsula, now being held at Fuala Lampor. The that plain in a small valley, surrounded by the bills that beautify the catpital of the Federated Malay States. was an ideal site, while Krualir Lumpor itself is a convenient centre, not only for exhibitors but for visitors from all parts of the Peninsula. The officials lean something from each successive Show, and it was the opinion of those who have visited previous Shows that the arrangements for the displaty of the exhibits in classes and sections and for expeditious judging of the exhibits wele distinctly in advance of anything hitherto experienced.

There were over 6,000 individual exhibits this year, and in the class for Pati no loss than 608 entries were made, for Cotton 78, and for models illustrative of native life and occupations no less than 246 in the two classes. The Committee were wisely advised when they introduced the award of diplomas in aldition to prizes, which will have a mole pemanent value to successful competitors than the money awards.

\section{NATIVE INTLRLST.}

That the Show is upreciated and valued by the natives was apparent to anyone who visited the showgurd on Saturday and Sunday. From all purts of the Peninsula, from Pekan ibnd Malacca, from Perak and Negri Sembilan, as well as from Selangol and Pahang, and even from Brunei, collections of native produce manufucture and native were arriving and being placed in position by busy officials, under the superintendence of $\mathrm{Mr}, \mathrm{J}, \mathrm{B}$. Carruthers, Chairman of the Committee, and 
Mr. J. IV. Camploll, the hari-worked but courteous Honorary Secretary, whose readiness to answer the numerous questions nddressed to him be token a knowledge of detail acguired by weeks and months of caleful organisation. It is fitting at this point to set down the names of the Committee by whom the final arrangements were carried out:-Mr. J. B. Caruthers, Diroctor of Agriculture (Chairman); Mr. E. Burnside, Collector of Sand Revenue, Selangor (Vice-Chaiman); the Raja Muda, M. C. Towkay Chan Sow Lim, Mr. C.; Mr. R. C. Grey, Mr. D. A. Dalziel (Hon. Treasurer); Mr. W. J. P. Hume, Chinese Secretariat; Mr. H. R. Moullin, Public Works Depantment; and Mr. J. W. Catnpbell, Superintendent, Experimental Plantations (Hon. Secretary).

\section{ODENING CERTMONY.}

By ten o'elock on Monday, most of the exhibits had been judged, and everything was in readiness for the formal opening of the Show by His Excellency the High Commissioner, Sir John Anderson, K.C.M.G. His Excellency was escorted from the Residency by the Malay States Mounted Infantry under the command of Cuptain A.J. Fox, and was received at the showyard by a guand of honour of the Maliay States Volunteer Rifles under the command of Captain Shaw. The guard of honour presented ams upon His Excellency's arival, and were inspected by him before His Excellency took un his position on the steps of the Pavalion for the opening celemony.

Here a large number of ladies and gentlomen were assenbled, and the scene presented by the pretty costumes of the ladies, the uniforms of the Sultun of Selangor and his suite and the civil ofticials and rolunteers, and the gaily-colonred dressed of the natives, was most picturesque.

\section{Mli. BLtefledu's SPEECH.}

Mr. Belfield, Resident of Selangor, sati--Your Excellency; on behalf of the committee, subseribers and oxhibitors at this our fith annual Agri-Horticultural Exhibition, I have the bonour to express our thanks to you for again alranging to be present to open our proceedings, and for thus evincing your continued interest in a movement which we all hope is conducing to the furtherance of agricultural develojment in the Colony and F.M.S. I ma be permitted to remind Your Excellency that the periol during which these anmual Shows have been held is synchronous with that of your own residence in the Colony." The first Show was held in Kuala Lumpor in 1904, a fow months only after your arrivai at Singupore, and 'on that occusion and at every succeeding fixture you have been foremost in aiding local endeavours by sympathetic interest and practical assistance. His Highness the Sultan desires me to express his gratification that Iíuala Lumpor should for the second time have been selected as the venue of the Exhibition. His Highness expresses the belief that those of our visitor's who have not been here since the first Show was held will have observel some material improvements in the town and neighbourhood. He also voices the hope that Your Excellency and the visitors here to-day will find that the standard of the present Show is not in any. way inferior to those which have preceded it, notwithstanding the recent period of commercial depression which has reacted prejudicially 
upon all classes of the community and upon all forms of business interests. I have the honour to ask Your Excellency to declare the Show open.

\section{SPEECH BY HIGH COMMISSIONER.}

In declating the Show open, His Excelleney the High Commissioner said that Mrr. Belfield had been unkind enough to remind him that he hivd been more than four year's in this part of the world, and that, in the ordinary course of cireunstances his time as Figh Commissioner was more than half finished. Mr. Belfield had also reminded them that this was the second time the Show had been held in Kuala Lumpor, and he was sure that those who saw it would agree that the present one showed an enormous advance, not only in the number, variety and quality of the exhibits, but also a great aivance in the manner and method of arrangements which the Committee had shown. It was a great advantage to those who had come to the Show that it had been so well laid out, and in consequence they had not to double on their tracks and waste time going from one end to the other in search of particular exhibits. At each succeoding stage they would find something as interesting as where they had gone before. The exhilits of native industries were most interesting. He was sure, that those who looked as the basket work, and not only the basket work but the silver work, and the other varieties of native craft, would see what great strides had been made in the last few years. Not only wis Malacca to the front again, but Porak had begun to progress in this direction. Ladies, he was sure would find the Perak, work far more useful than the Malacca basket work, which was, after all, rather a curiosity, whereas the Perak was very useful,

\section{BAD TIMES.}

As the Resident had said, they had been passing through a time of commercial depression. Some prophets said they had got to the bottom and were now on the up-grade. He was sure everyone hoped it was so. But as far as the planting industry was concerned he was not sure they had not been it little in need of a bad time. Everything wis so prosperous and prices were so high that there was a disposition on the part of planter's to think they had fallen into a fortune and had only to sit still and reap it. It was one of the advantages of bad times that it made people study economy as use their brans to devise improved methods of produtetion and of handling the product. If the bad times through which they had been passing had that result with the planting industry of the Federated Malay States, he felt sure, instead of coonsidering it a bad time, they would all look upon it as a blessing in disgrise. Whether or not, all cordially wished the bad times at an end. Towards the end of last year, the planting industry was not only feeling the stress of bad times, but also there was not quite so much harmony among themselves as they ought to expect in such a happy family. "Usually, the only enemy of the planting community was the Government; perhaps he should say the industry had now two enemies, the Government and the white ant. (Laughter). He scarcely knew which was the worst. Perhaps on the whole the white ant left a more permanent mark on the industry than the Government. 


\section{ADVICE FROM PLANTERS.}

Last year the planters were doing a little quarrelling among themselves-only a family disagreement, - but now it seemed to have died out. Anyhow the newspaper's had dropled it, and so he supposed it had ceased to the intoresting. But he taught it was partly due to that incident that they had the formation of that useful body - the Planters Association of Malaya. The formation of the Association was not only of importance to the planting industry but also to the Government, and was, His Excellency thought, a step in the right direction. The Association not only served to focus the opinion of the planting community as to their. wants, which everyone knew were numerous, but it also holpod the Government by giving them it representative body to which they might look for advice and from which they might always expect criticism which was helpful. Since his arrival in Kuala Lumpor, he had heard from his colleague, the Resident-General, that, at a recent meeting, they had again been going for theil old onemy, the Government, thinking, apparently, that has planting had been having a bad time, Gorernment ought to have a bad time as well, and that the planters ought to have a little more of that attractive thingthe surplus. Well, when the Government embarked, a few years ago, on what many considered the hazardous policy of helping the resident ylanter, because they did not want to lose him, they said they would advance up to half a million dollars for the purvose of helping to clear off mortgages and to help them to weather bad times, which were certainly then much worse than now, and worse than at any time since he had been here. When that policy was started thoy thought there would be a rush for the half million dollars. Curiously onough, on the contrary it was a long time before people came forward. Perhaps they did not think the Government was in earnest when they said the money was there and they could have it on application, provided they gave good security.

\section{GOVERNMENT LIABILITLES.}

About the same time it was found that the British public was very eager to take a hand in planting, and very little call was made upon Government funds. When the British public had enough shares in the planting industry, the planters turned again to Government, and the half million was soon gone. Then the planters came again to the Resident General, and he (the High Commissioner) agree to go another half million. That went very quickly, and then they offered a third. With that third half million the question arose whether Government was to go on and where it was to stop. The matter was considered anxiously and carefully. Government had many duties, responsibilities and liabilities, of which the outside public knew little or nothing. Of these liabilities, some were prospective, some actually incurred; some were liable to be increased and some were not yet settled. The Government had to bear in mind all these, for the surplus was not so very large. He assured them that the Government could dispose of every dollar of it. However, that did not weigh so much with the Government as the fact that the loans were to a large extent going to those who could not be described as resident planter's, the class they 
wished to attract and keep in the country, but to the man who took up planting as a speculation, and wanted a loan to save the speculation until he was able to place it on the market at a profit.

\section{SPliculdtion.}

It was no function of the Government to assist speculation of that sort, and they came to the conclusion that the time had come to restrict loans exclusively to those for whon they were originally intended, the resident plinters, the men they wished to come into the country to form a strong planting community with their interests bere. There were also instances of estates which in the hope of the Government purse proving bottomless had been starter with insulticient capital, and there was some likelihood, if they stopper, that the estates would be closed down. There could be no hard and fast rule, but they decided after consideration to make further advances strictly for maintenance. They dicl not want land under cultivation to fall back or to be abandoned, and thus be a roproach to the F.M.S. and perhips become an injury to the industry. Therefore, for the present, no more loans woukl be granted, except for the purpose of maintaining existing plantutions. While that might disappoint some, he was satisfied it was best in the interest of the couni:y, and those who came out on speculation, with no intention of becoming resident planters had better clear out of their speculations as soon as possible. The man who went into a concern merely with the intention of making a little money out of it as an incidental spoculation was not as likely to contribute materially to the advincement of the industry as he who went into planting and lived and worked himself on his land. He was the man they wanted to encourage, and to him they har to look for the real development of the industries of the Malay Peninsula.

\section{IALBOLT:}

One other matter of interest to the planting community-the labour question. It was gratifying to know that labour had been coming in freely. In the last year just orer 60,000 labourers had come, and in the first half of this year 30,000 , very nearly. With these numbers he thought there should be no more complaints of the shortage of labour. The means taken to increatse the stipply bad been subjected to a good deal of criticism. He could not say that he thought the Tamil tax an unfarir one. At any rate, they must continue it for some few years. Possibly, by and by, when the country was wellknown and the demands for fresh labour diminished, it might be possible to remove it, but he was afraid that, so far as they could seo at present, it had come to stay. When the proposal for levying a tax was under discussion in Singapore, he had stated on behalf of Government that there were certain considerations which Governmest would have to look to later, when it was seen how the money went. The other day, an amending Bill had been introduced to deal with some of the considelations that had heen pressed on Government, mainly by the older-cstablishorl estintes. The Govermment, naturally, did not wish to press unfairly upon them, and the amendments they had introduced -and which would be submitted to the Planter's' Associations so that Government might have the benefit of their criticism-were two. 
First of all, they wanted, out of the profits of the tax, to give some acknowledgment to those who had already establisher a considerable labour force when the tax came into existence. The equity of this. he was sure, they would agree with. Whether the moner, when it was distributed, would come up to thoir expectation, was another malter. He was afraid not.

\section{SELF-HeTP REWARDHD.}

Another point was to give relief to those planters who were in the habit of sending their own kungunis to Indiat to recruit labour. One important object the Committee and the Government hat in view was to get rid of the great evil of crimping, and by making the tax universal they thought they had destroyed whatever incentive there was to crimp. They thought there was now sufficient labour and no inducennent to at man to steal another's coolies. But so long as some sent their own kanganis to Indit to recruit obviously these planters were doing more than men who sat down and waited for the labour to cone to him, and only took it of the hands of the Government. Therefore, the Committee had recommended-und the Government was prepared to agree--that some allowance should bo made to those who sent theil own kanganis to India. It was only fair, His Excellency thought they would agree, to take away this grievance and to do something to relieve them from the acute evil of crimping.

His lixceliency concluded by apologising for trospassing upon their patience, but he did not, often have the opportunity of speaking to them. He then declared the Show open.

Reprint from Straits Times August 12.

LIST OF IVINNLRS.

(As Reported by The Maluy Mail)

DITISION A. I.

1. Padi, best sample of any named variety.

ist prize. Peng. Haji Ahmat, Malacca.

2nd prize. Che Wang, I. O., Penang.

3rd prize. syed Usup, Chigar Galah, K. Kangsar.

llighly Commerded Penghulu Long, Malacca.

2. Pulut, best sample ol any named variety.

lst prize. Mohamed Bin Yupe, L. O., Penang.

2nd prize. Raji Bosah Penghulu, Krian.

òrd prize. Peng. Mat l'edong, Malacca.

3. Rice, best sample, prepared by machinery.

1st prize. Wan Yeop Ibrahim, Parit Buntal.

2nd prize. Khy Heng Bee, Sungei Penang.

3rd prize. Mohamed Datoh, Karamat Rd, Penang.

4 Rice, best sample, prepared in a lesong.

ist prize. Peng. II ussain, G. Semmengol, Perak.

2nd prize. Penghulu, Jebong Matang, Perak.

srd prize. Kamaludinbin Haji Baludin, Penang. 
5. Best collection of different Padi, in the ear, 10 heads in each sampile.

1st prize. Penghulu Ijok, Perak.

2nd prize. Puceh Penghulu, Alor Pongou. Perak.

3rd prize. Nat Noh. Penang.

6. Best Padi and Pulut, grown in one mukim, to be exhibited by the

Penghulu of mukim, and so certified by him.

Each exhibitor to produce an affidavit, sworn before a Magistrate, that his whole exhibit was grown in his own mukim last padi season.

1st prize Hussin Penghulu, Griong Semmenggol, Krian.

2nd prize Hussin bin Katib Hassan, K. Kangsar.

3rd prize Kamaludin bin Haji Baludin, Penang.

$$
\text { DIVISION A. II. }
$$

Govemor's cup. A warded to Mr. B. C. N. Knight of Jebong Estate, Simpang, Perak, for his best Sample of Rubber in the Show.

Sultan's Cup. A warded to A. D. Machado, Kamuning Estate.

7. Para Crepe, best sample.

1st prize. F. J. Harvey, Petaling, Fstate.

2nd prize. J. B. Douglas, Terentang Estate, Negri Sembilan. 3rd prize. A. D. Machado, Kamuning Estate, Perak.

H. C. B. C. N. Knight, Jebong Estate.

8. Para sheet or Biscuit, Packed in boxes as shipped from the estate.

1st prize. O. Pfenningworth, Hirhlands and Lowlands Estate. 2nd prize. Crawford, Ualedonia Estate, P. IW.

9. Para Block, dry.

1st prize. A. D. Machado, Kamuning Lstate, Perak.

10. Para Block, wet.

1st. prize. A. D. Machado, Kamuning Estate, Perak.

11. Rambong in any form.

1st prize. Bukit Rajah Estate.

2nd prize. Atherton Estate, Port Dickson.

Brd prize. J. Molesworth, Jugra Estate.

12. liubber, (Para) packed in boxes as shipped from the Estate.

ist prize. 13. (1. N. Knight, Jebong Estate.

?nd prize. F. J. Ilarvey, Petaling Estate.

3rd prize. H. M. Darby, Tallambrosa Fstate.

13. Any other Rubber. (Plant from which obtained must be stated with each).

1 st prize. A. D. Machado, Kamuning Estate.

2nd prize. O. Pfenningworth, IIighlands and Lowlands Estate.

13A. Penghulu Md. Nomot, Malacca.

13A. Sinnaiah, Kuala Kangsar.

13B. Penghulu Atdul Gani, Malacca.

Best Rubber in the Show, Petaling Estate, 2 sorts crepe.

$$
\text { DIVISION A, III. }
$$

14. Coconuts, unhusked, best simple.

1st prize. Penghulu Hassan, Penang.

2nd prize. Penghulu, Sungei Pringgi, Matang.

3rd prize. Haji Latib, Setapak: Kuala Lumpor. 
15. Coconuts, husked, best sample.

1st prize. Penghulu Sungei Pringgi, Matang, Perak.

2ud prize. Penghulu Mat Suh, Kuala Selangor.

3rd prize. Haji Noordin, Setapak, Kuala Lumpor,

Highly Commended. Mat Mahin Rambau, Negri Sembilan.

16. Coconuts, best bunch.

1st prize. Penghulu, Jugra and Bandar, Kuala Langat.

2nd prize. H. M. Darby Vallambrosa Lstate.

Special prize. Penghulu Semmiah Ulu, Langat.

17. Coconuts, husked, best sample.

lst prize. R. M. Connolly, Ipoh, Perak.

2nd prize. Penghulu Ilji Nasan, Malacca.

3rd prize. Penghulu Ijok, Perak.

Highly Commended, H. M. Darby, Vallambrosa, Klang.

18. Copra, best sample, 5 catties.

1st prize. Mr. Valpy, Klanang Estate, Kuala Langat.

2nd prize. A bu Bakkar bin Ishak, Penang:

3rd prize. Penghulu Mat Sub, Kuala Selangor.

Ilighly Commended. Penghulu, Sungei Pring gi, Matang, Perak.

18a. Copra, 50 catties.

1st prize. (Cup). N. Molesworth, Jugra Estate, Jugra.

2nd prize. (Silver Medal). K. T. Koomarasawuy Pillai, Kuala Lumpor.

Highly Commended. Mr. Talpy, Klanang, Kuala Langat.

19. Tapioca, roots, best sample, 3 roots.

1st prize. Brahin, Penang.

2nd prize. Kulop Mat Jiri, Kuala Kangsar.

20. Tapioca, pearl, best sample. 2 catties.

lst prize. S. Moorehouse, Malacea.

2nd prize. Osmainbin Isak, Penang.

21. 'T'apioca, flake, best sample. 2 catties.

1st prize. A. Crawford, Caledonia Futate, Province Wellesley".

2nd prize. S. Moorehouse, Malacca.

22. 'lapioca, flour, best sample. 2 catties.

1st prize. S. Moorehouse Malicea.

and prize. A. Crawford, Caledonia Kitate, Province Wellesley.

23. Arrowroot, fresh, 25 roots.

1st prize. H. H. Norris, Singapore.

2nd prize. Bahu Lin, 'lapah.

24. Arrowroot, prepared, best sample. 1 catty.

1st prize. Kamala Din, Penarg.

2nd prize. A. Crawford, Caledonin Estate, Province Wellesley.

25. Sago, pearl, best sample. 2 catties.

1st prize. A. Crawford, Caledonia tistate, Province Wellesley.

26. Sago, flour, best sample, 1 catty.

1st paize. A. Crawford Caledonia, Estate, ProvinceWellesley.

2nd prize. Hussin Penghulu, Perak.

27. Maize, best sample. 12 heads.

1st prize. Bidul, Perak.

2nd prize. Mahomed Esah, Kuala Kangsar.

3rd prize. Nooh, Batu, Kuala Lumpor. 
28. Ginger, best sample, 20 roots.

1st prize. Penghulu, Samman, Malacea.

2nd prize. Sidek, Penang.

3rd prize. Daluman bin Awang, Penang.

29. 'l'urmerie, best sample, 25 roots.

lst prize. Цaji Mohamed Samman, Penang.

2nd prize. Penghulu Abu, Malacca.

srd prize. Kamaludin, Penang.

80. 'l'uba (akar), best sample, 1 catty.

Ist prize. P.J. Cornelius. Penang.

2nd prize. T'oow Beng Huat, Penang

3rd prize. Hussain bin Haji Isak, Penang.

31. Sugar, cane, best sample, 10 canes.

lst prize. Bulat, Serendah.

2nd prize. Sidang Arshad, Malacca.

3rd prize. Datoh Mohamed Casim, Klang.

32. Sugar, coconut, best sample, 2 catties.

1st prize. Penghulu Yusup, Malacca.

2nd prize. Penghulu Abr, Malacca.

Brd prize. Abdul Majid, Klang.

Highly Commended. Mohamed Sahid, Penang.

33. Sugar, nipah, best sample. 2 catties.

1st prize. Mohamed Arsat, Penang

2nd prize. Penghulu Abdul Raman, Penang.

3rd prize. Raja Tahir, Kuala, Langkat.

34. Surar, kabong, best sample. 2 catties.

lst prize. Mohamed Mansur, Lower Perak.

2nd prize. Siakap, Negri Sembilan.

ird prize. Kulop Mohamed, Kuala Kangsar.

35. Sugar cane, brown, best sample.

1st prize. Nohamed Casim, Penang. (Silver Medal).

znd prize. A. Mohamed Sherilf, Penang. (Bronze Medal).

Highly Commended. A. Crawford, Caledonia listate, Province IVellestey.

36. Sugar cane, white, best sample. 2 cattiss.

1st prize. A. Crawford, Ualedonia Estate, Province IVellesley (Silver Medal).

2nd prize. Mr. Boyò, Kalampang Estate, Bagan Serai, (Bronze Medal).

37. Colfee, Arabian, best sample. 2 catties.

lst prize. Haji Mohamed Sahid, Penang.

2nd prize. No award.

38. Coffee, Tiberian, best sample.

lst prize. O. Pfenningworth, Klang. (Silver Medal).

2nd prize. R. A. Gibson, Golden Hope Estate, Klang. (Bronze Medal).

Highly Commended. J. Molesworth, Jugra Listate, Jugra.

39. Uoffee, any another variety. 2 catties.

lst prize. R. A. Gibson, Golden Hope Estate, Klang (Silver Medal).

2nd prize. J. Molesworth, Jugra Fitate, Jugra (Bronze Medal). 
40. Cocoa, fresh pods, best sample. 10 pods.

1st prize. Haji Mohamed Nasin Lower Perak.

2nd prize. Kulop Mohamed, Kuala Kangsar

41. Toddy, best sample. 1 pint.

1st prize. Penghulu Anjang, Malacca.

2nd prize. Mohamed Sultan, Kuala Lumpor.

42. Rum, best sample. 1 pint.

1st prize. Mr. Boyd, Kalumpang Estate, Bagan Serai.

2nd prize. A. Crawford, Caledonia listate, Province Wellesley.

43. Rum shrub, best sample. 1 pint.

Ist prize. A. Crawford, Caledonia Estate, Province Mellesley.

2nd prize. Mr. Boyd, Kalumpang Lstute, Bagan Serai.

\section{DIVISION A. IY.}

44. Betel-nuts, fresh, best sample. 50.

Ist prize. Harm, Kuala Kangsar.

2nd prize. Mohamed Bedin, lower Perak.

3rd prize. Alang 'Tahib, Kuala Kangsar.

45. Betel-nuts, dried and split, best sample. 1 catty.

1st prize. Brahim, Penang.

2nd prize. Kung 'Thean Sung. Penang.

3rd prize. Mohamed Bin Salleh, Penang.

46. Sireh leaves, hest sample. 100 leaves.

1st prize. Sidang Haji Mohamed, Malacca.

2nd prize. Llasanudin Bin Long, Malacea.

3rd. prize. Penghulu Baba, Malacca,

47. Gloves, best sample, 1 catty.

1st prize. Low Chit Neun, Penang.

2nd prize. Lean Shak Foong, Penang.

3rd prize. Cheah Chee Eong, Penang.

48. Nutmegs, fresh best sample. 50. .

lst prize. C. 'T'uah Choo, Penang.

2nd prize. Kulop Mohamed, Kuala Kangsar.

ird prize. Kamuludin Bin Maji, Bahndin, Penang.

49. Netmegs dried, best sample. 50.

lst prize. Low Chit Nem, Penang.

2nd prize. Mat (Paya 'l'rubong), Perrang.

Brd prize. Osman Bin Ishark, Penang.

50. Mace, dried, yellow. 1 catty.

1st prize. No award.

2nd prize. Osman Bin Ishark, Penancr.

3rd prize. No a ward.

ol. Mace, dried, red. 1 catty.

1st prize. Mchamed Bin Molramed, Salleh Penang.

2nd prize, Haji Mahomed Saman, Penang.

3rd prize. Osman Bin Ishark, Penang.

52. Pepper, white, best sample. 1 catty

lst prize. Mohamed Abu, 'Tapah, Perak.

2nd prize. Joh Kong Yew, Sipang, Kuäla Langat.

3rd prize. Ol.u Woh Estate, Negri Sembilan. 
53. Pepper, black, best sample. 1 catty.

1st prize. Khoo Choo Boo, Penang.

2nd prize. Cheah Chee Eong, Penang.

3rd prize. Assistant Penghulu T'emerloh, Perak.

54. Spices, best collection. optional.

1st prize. Sh ik Hussin, Penang.

2nd prize. Ilaji Mohamed Saman, Penang.

DIVISION A. V.

55. Putchuli, best sample. 1 catty.

1st prize. PenghuIu Sungei T'rap, Batu Gajah.

2nd prize. P. J. Cornelius, Penang.

3rd prize. Low Chit Neun, Penang.

56. Oil, Citronella, best sample. 1 pint.

No award.

57. Oil, lemon grass, best sample. 1 pint.

No award.

58. Oil, coconut, best sample. 1 pint.

lst prize Moliamed Serajudin, Sungei, Perak.

2ıd prize. J. Molesworth, Jugra Estate, Juora.

3rd prize. Pengulu, Sungei Trap, Perak.

Highly Commended. Federal Oil Mills, Kuala Selangor.

59. Oil, gingelly, teal seed (minyak lengah), best sample. I pint.

ist prize. Kung Thean Sung, Penang.

2nd prize. M. Nanih Municipality, Penang.

3rd prize. 'T'uah Cheow Kay, Penang.

60. Oil, castor, best sample. I pint.

1st prize. Mohamed, Penang.

2nd prize. Mohamed, Kassim, Penang.

sod prize. Shaik Hussin, Penang.

61. Oil clove, best sample 1 pint.

No a ward.

62. Oil, Para Rubber seed, best sample, 1 pint.

1st. prize. A. Crawford, Caledonia Estate, Province IV ellesley. 2nd prize. No award.

63. Oil, Kabu Kabu seed, best sample, 1 pint.

No award.

64. Oil, any other kind, best sample, I pint.

1st prize. Indat Penghulu sulim, P. Molim.

2nd prize. Ahamat Penghulu, Lower Peral.

65. Oil, cake, coconut, best sample. is catties.

1st rize. Federal Oil Mills, Knala Selangor.

2nd prize. Kasi Dollah Lengerong, Upper Perak.

nighly Commended. Sidang LIaji Mohamed, Malacca.

66. Oil, cake, Para seed, best sample, 5 catties.

1st prize. A. Crawford Caledonia Estate, Province Wellesley.

2nd prize. No a ward

67. Oil, cake, Kabu kabu seed, best sample, 5 catties,

No award.

68. Seed, Kabu kabu : best sample, 2 chuppas.

1st prize. Mohamed Bin Ismail, Penang.

2nd prize. Mohamed Omor, Lower Perak.

Uighly Commended Abdullah Serat, Sungi Siput, Kuala Kangasr. 
69. Oils, best collection $\frac{1}{4}$ pint of each.

1st prize. No award.

2nd prize. 'T'uah Cheow Kay (Shooft), Penang.

sid prize. No award.

Division A. VI.

70. Getah, best collection of local. $\frac{1}{2}$ catty of each. 1st prize. No award.

2nd prize. Penghulu Ijok Slmah Perak.

3rd prize. Hiji Mat Yasin Situl, Negri Sembilan.

71. Getah taban, best sample, 1 catty of each.

1st nrize. Haji Mohamed, Negri Sembilan.

2nd prize. Mohamed Serajudin. Sungkai, Perak.

3rd prize. Penghulu Ijok, Perak.

72. Gambier, best sample. 1 catty of each.

1st prize. Ilussain Bin Haji Isak, L. O. Penang.

2nd prize. Penghulu Beranang, Kuala Langat.

3rd prize. Penghulu Haji Lintang Malacca.

Highly Commanded. Long Kong Yew, Kuala Langat.

73. Dragon's blood, best sample $\frac{1}{2}$ catty of each.

1st prize. Hussain, Penghuli K rian, Perak.

2nd prize. Aujong Said, Kuala Kangsar.

3rd prize. Penghulu Ijok Selama, Perak.

74. Gums and damars, best collecilion of local $\frac{1}{2}$ catty of each.

1st prize. Daloh Paduka Raja, Lower Perak.

and prize. Penghulu Ijok, Selama, Perak.

3rd prize. Said Omar, Kuala Kangsar. Commended. Megat Abu Sammah.

75. Cotton (Kakabu) best sample, 1 catty.

1st prize. A. Crawford, Caledonia, Estate, Province Well. eseley.

2nd prize. J. Molesworth, Jugra Estate, Kuala Tangat.

3rd prize. Haji Mohaned Ali, Lower Perak, Special. Mat Bin Hashim, Penang.

76. Cotton any other variety, best samples 1 catty.

1st prize. Mohamed Ali, Kuala Kangsar.

2nd prize. Anjang Abdultah-Pasir Panjang, Lower Perak.

3rd prize. Lean ah Keon, Penang. Special. MLhamed Man-

sur, Lower Perak, Highly Commended. Jusoh, Butterworth.

77. Fibres, best collection, 1 bundle of each.

1 st prize. Khoo Soo Ee, Penang.

2nd prize. G. Baptist, Penang.

3rd prize. G. F. Lachlan, Telok Anson. Commended.

Federated Oil Mill, Kuala Selangor.

78. Rotans, best collection, optional.

1st prize. Haji Mohamed Sahid, Penang.

2nd prize. Mat Saleh, Kuala Kangsar.

3rd prize. Kulop Samat, Kuala Kangsar.

Commended. Jatoh Paduka Raja, Lower Perak.

79. Bamboos, best collection, optional.

1st prize. Dadoh Puduka Raja, Lower Perak.

2nd prize. Siakap Panti, Negri Sembilan.

3rd prize. Penghulu Pupai, Larut, Perak. 
80. Walking sticks, best collection (unprepared). 1 of each. 1st. prize. Shuckor Konn Buaia, Kuala Kangsar.

2nd prize. Mrohamed Yasin bin Itaji Mohamed, Penang.

srd prize. Along T'usup, T'apah.

81. Medicinal plants, best collection (openj optional.

1st prize. No awards.

2nd prize. No awards.

1 82. Medicinal plants (Natives only) optional.

1st prize. Penghulu Jurian Sebatang, Lower Perak.

2nd prize. Mr. Kassim c/o Land oftice Penang.

srd prize. Said Usop, Ghigagalah' Kuala Kangsar.

Highly Commended. Penghulu Ijak Selama, Perak.

\section{DIVISION. B. I.}

83. Avoids other than Caladiums. 3 plants.

\section{No entry.}

84. Caladiums. 3 plants.

1st prize. Tong Tak In, Kuala Lumpor.

2nd prize. Mrs, Carruthers, Kuala Lumpor.

8.5. Coleus. 3 plants.

86. Crotons. 3 plants.

No entry.

1 st prize. Lee Kong Lam, Kuala Lumpor.

2nd prize. No award.

87. Dracenas. 3 plants.

$$
\text { No entry. }
$$

88. Ferns, any distinct variety. 6 plants.

1st prize. No award.

2nd prize. 'Tong Tak In, Kuala Lumpor.

3rd prize. No a ward.

89. Ferns, Adiantum, distinct.

1st prize. No award.

2nd prize. Mrs. Caruthers, Kuala Lumpor.

3rd prize. To award.

90. Ferns, any variety, hest specimen. 1 plant.

1st prize, Mrs. W. E. Smith, 'lelok Anson.

2nd prize. No award.

91. Marantas and Calatheas. 3 plants

No entry.

92. Palms, distinct kinds. 3 plants.

1st prize. Kong 'Tak In, Kuala Lumpor.

2nd prize. Lee Kong Lam, Kuala Lumpor.

3rd prize. Lee Kong Lam, Kuala Lumpor.

93. Palms best specimen. 1 plant.

1 st prize. No award.

2nd prize. Lee Kong Lam, Kuala Lumpor.

Ilighly Commended. Tong 'tak In, Kuala Lumpor.

94. Selaginella, best specimen. 1 plant.

No entry.

95. Any ornamental foliage plant not included in above, 3 plants.

ist prize. 'long 'l'ak In, Kuala Lumpor.

and prize. N. Siew Hin, Kuala Lumpor. 
96. Foliage plant, any kind; best specimen. 1 plant.

1st prize. Choo Kia Peng, Kuala Lumpor.

and prize. No award.

$$
\text { DIVISION B. II. }
$$

97. Amaryllis and Lilies. 3 plants.

Mrs. Spooner, Kuala lumpor.

08. Asters, best specimens. 3 plants.

No entry.

99. Balsams, best specimens. 3 plants.

No entry.

100. Cannas, best specimens. 3 plants.

No entry.

101. Cocks' combs best specimen. 3 plants.

No entry.

102. Chrysanthemums, best specimens. 3 plants.

1st prize. Nouward.

2nd prize. Mrs. Burn Murdoch, Kuala Lumpor.

103. Dahlias, best specimens. 3 plants.

No entry.

104. Dianthus (Indian Pinks) 3 plants.

No entry.

105. Eucharis, best specimens. 3 plants.

106. Gloxinias, 3 plants.

$$
\text { No entry. }
$$

107. Orchid, best specimens. 1 plant.

1st prize. No award.

2nd prize. Choo Kia Peng, Kuala Lnmpor

108. Phlox best specimens. 3 plants.

$$
\text { No entry. }
$$

109. Petunias, best specimens, 3 plants

No entry,

110. Zinnias best specimens. 3 plants.

No entry.

111. Roses, best specimens. 3 plants.

No entry.

DIVISION B. III.

112. Begonias, distinct kinds. 3 plants.

1st prize. Lee Kong Lam, Kuala Lumpor.

2nd prize. Mrs. Carruthers, Kuala Lumpor.

Commended. Mrs. W. F. Smith, Telok Anson.

113. Begonia, best specimen. 1 plant.

ist prize. Lee Kong Lam, Kuala Lumpur.

2nd prize. Mrs. Smith, T'elok Anson.

114, Group of plants arranged for effect in space not exceeding 9 sq. $\mathrm{t}$ 1st prize. K. T. Coomarasawny Pillai, Kuala Cumpor. 2nd prize. No award.

11\%. Group of Chinese plants, fantastic shape.

ist prize. 'T'. Seow 'l'eng, K uala Lumpor.

2nd prize. No award.

Special prize. 'l'ong 'l'ak In, Kuala Lumpur.

Special prize. Uhian Ah Peng, Kuala Lumpor, 
DTYTSTON B. IV.

116. Asters, optional.

No entry.

117. Chrysanthemums, optional.

]st prize. Noaward.

2nd prize. Mrs. Burn Murdoch, Kuala Lumpor.

118. Dalilias, optional.

119. Roses, optional.

No entry.

120. Cannas, optional.

No entry.

121. Orchids. optional.

No entry.

No entry.

122. Cut flowers arranged for effect.

No award.

123. Collection of cut flowers, not less than 12 kinds.

1st prize. Mrs. 'Trump, Kuala Lumpor.

2nd prize. Mrs. Carruthers, Kuala Lumpor.

3rd prize. No award.

124. Table decoration.

1st prize. Miss V. Grey, Kuala Iumpor.

2nd prize. Mrs. Nutt, Kuala Lumpor.

3rd prize. Hes. Walsh, Kuala Lumpor.

\section{DIVISION B. T.}

125. Pisangs (Bananas) best collection. 6 of each kind.

Itt prize. Kulop Mat Jiu, Kuala Kangsar.

2nd prize. Haji Mohamed Nasir, Lower Perak.

3rd prize. Mat Bin ILasin, Penang.

Commended, Usop, Batu, Kuala Lumpor.

126. Pisangs (Bananas) best buach, any kind. 1 bunch.

1st prize. Saleh, Kurla Lumpor.

and prize. 'l'alib, Kuala Lmmpor.

Highly Commended, Haji Mohamed, Kuala Kangsar.

commended, Reja 'Tahir, Kuala Langat.

127. Champedak, 3 fruits.

1st prize. H. H. Norris, Singapore.

2nd prize Penghulu Salim Alei, Malacca.

Highly Commended, Hasamudin Bin Long, Malacca.

Commended, Penghulu Mat Din, Malicca.

128. Jack fruit. 1 fruit.

Ist prize. Penghulu Ilasan, Penang.

Highly Commended. Sidang Mamat, Malacca.

Commended. Ugal Alimat, Kuala Kangsar.

129. Durian. 2 fruits.

1st prize. Raja Tahir, Kuala Langat.

2nd prize. Marip bin Mat Said, Malacca.

3rd prize. Sohariah, Kuala Langat.

Mighly Commended, Penghulu II. Amat, Malacea.

Comnended, Penghulu Jugra and Bandar, Kuala Langat. 


\section{DIVTSTON B. IV.}

116. Asters, optional.

$$
\text { No entry. }
$$

117. Chrysanthemums, optional.

jst prize. No award.

2nd prize. Mrs. Burn Murdoch, Kuala Lumpor.

118. Dahlias, optional.

119. Roses, optional.

$$
\text { No entry. }
$$

120. Cannas, optional.

No entry.

121. Orchids. optional.

$$
\text { No entry. }
$$

122. Cut flowers arranged for effect.

No award.

123. Collection of cut flowers, not less than 12 kinds.

1 st prize. Mrs. Trump, Kuala Lumpor.

2nd prize. Mrs. Carruthers, Kuala Lumpor.

3rd plize. No award.

124. Table decoration.

1st prize. Miss V. Grey, Kuala Lumpor.

2nd prize. Mrs. Nutt, Kuala Lumpor.

3rd prize. Mrs. Walsh, Kuala Lumpor.

\section{DIVISION B. Y.}

125. Pisangs (Bananas) best collection, 6 of each kind.

1 tt prize. Kulop Mat Jin, Kuala Kangsar.

2nd prize, Ilaji Mohamed Nasir, Lower Perak.

3rd prize. Mat Bin Ilasin, Penang.

Commended, Usop, Batu, Kuala Lumpor.

126. Pisangs (Bananas) best buach, any kind. 1 bunch.

1st prize. Saleh, Kunla Lumpor.

2nd prize. 'Talib, Kuala Lumpor.

Highly commended, ILaji Mohamed, Kuala Kangsar.

Commended, Raja 'l'ahir, Kuala Langat.

127. Champedak, 3 fruits.

1st prize. H. H. Norris, Singapore.

2nd prize Penghulu salim $\Lambda$ lei, Malacca.

Highly Commended, Hasamudin Bin Long, Malacca.

Commended, Penghulu Mat Din, Malacca.

128. Jack fruit. 1 fruit.

Ist prize. Penghulu Llasan, Penang.

Highly Commended. Sidang Mamat, Malacca.

Commended. Ugal Alimat, Kuala Kangsar.

129. Durian. 2 fruits.

lst prize. Raja I'ahir, Kuala Langat.

2nd prize. Marip bin Mat Said, Malacca.

srd prize. Sohariah, Kuala Langat.

Mighly Commended, Penghulu II. Amat, Malacea.

Commended, Penghulu Jugra and Bandar, Kuala Langat. 
130. Melon. 1 fruit.

1st prize. No award.

2nd prize. Penghulu Beranang, Llu Langat.

131. Pineapple (Mauritius). is fruits.

1 st prize. Syed Hamid, Penang.

2nd prize. L. Hamad, Penang.

3rd prize. Che Wang. Penang.

132. Pineapple (any other variety). 3 fruits.

1 st prize. Usop, Batu Kuala Lumpor.

and prize. IIaji saret, Kuala Lumpor.

3rd prize. Sidang Bakar, Malacca.

Commended. Jamin, Kuala Lumpor.

133. Pomeloes. 3 fruits.

1st prize. Pun Sun, Kuala Iumpor.

2nd prize. R. II. Conolly, Ipoh, Perak.

131. Papaya. 3 f'uits.

ist prize. Siakap, Negri Sembilan.

2nd prize. 'Tuah Choo, Penang.

srd pri\%e. Haji Akub, Serendab.

Commonded. Nahulah, Batu, Kuala Lumpor.

135. Oranges. 6 fruts.

1st prize. Penghulu sam Ratu Berendan, Malacca.

and prize. R. M. Conolly, Ipoh, Perak.

3rd prize. R. W. Munro, Permating Estate. Jugra.

\section{DIVISION B. $Y$.}

136. Limes. 12 fruits.

1st prize. Abdul Rais Senggang. Kuala Kaugsar.

2nd prize. D. Paduka Paja, Lower Perak.

3rd prize. R. W. Munro, Permatang Estate, Jugra.

137. Lemons. 12 fruits.

1st prize. Abdul Rais Senggang. Kuala Kangsar.

2nd prize. Abulaker Bin Issahak, Penang.

3 rd prize. Kamaludin, Penang.

138. Chiku. 6 fruits.

1st prize. Mohamed Hussain Bin Maji Mli. Penang.

2nd prize. Mohamed Hussain, Kampong Bharu, Penang.

139. Custard Apple. 6 fruits.

1st prize. Mohamed Jafer, Kuala Langat

2nd prize. Mat bin Din, Penang.

1.0. Duku. 20 fruits.

1st prize. Jamidin bin Abu, Malacca.

znd prize. Haji Darus bin H. Nahomed, Saman, l'enang.

141. Binjai, 6 fruits.

1st prize. Sodong Laman, Malacca.

2nd prize. Haji Hassan, Malacca.

112. Jambu. 20 fruits.

1st prize. Umpu, Kuala Lumpor.

Commended. Kung 'T'hean Sung, Penang.

143. Langsat. 3 bunches.

1st prize, No award.

2nd prize. Jamudin bin Abu, Malacca, 
111. Mangoes. 6 fruits.

1st prize. Penghulu llassan, Penang.

145. 6 fruits. Bachang.

1 st prize. Penghulu Jugraand Bandar, Kuala Langat.

1.6. Wangosteens. 12 fruits.

lst prize. Sidany H. Mohamed, Malacca.

ond prize. Penghulu Salim, Malacca.

3rd prize. Sidang Haji Ma'ali, Malacca.

Highly Commended. L. ILanid, Penalig.

147. P'ulasan. 20 fruits.

1st prize. Mat, Penang.

2nd prize. Penghuia Jugra and Bandar, Kuala langat.

148. Rambai, 3 bunches.

1st prize. Hussain, Penang.

2nd prize. Said thmad, Penang.

149 Rambutan. 20 fruits.

1st prize. T'eoh Beng Пuat, Penang.

2nd prize. Mat, Penang.

àld prize. H. Mohamed Saman, Penang.

150. Mata kuching. 20 fruits.

1st prize. Syed Ahmad, Penang.

2nd prize. IIussain biin Haji Issark, Penang.

srd prize. Hussain Datols Kramat, Penang.

151. Durian blanda. (Sour-Sop) \& fruits. 1st prize. Ilaji Osman, Lower Perak.

152. Any fruit not included in above. Optional. Ist prize. Mohamed bin Mohamed Saleh, L'enaurs.

and prize. Peng Mat, Malacca.

153. Cuitivated fruits, best collection. Any number of kinds. 1st Prize (Cup). Low Chit Mun, Penang.

and prize. Tuah Choo Kay, Penang.

3rd prize. Latip bin Usop, Malacca.

1j1. Wild edible fruits, best collection.

1 st prize. IH. G. Bahiri, Penang.

2nd prize. Abdullah dli, Lower Perak.

$$
\text { DIVISION B. VI. }
$$

155. Preserved fruits, best sample any method. Optional.

1st prize. Girls' School K. L. Kiri, Kuala Kangrar,

2nd prize. Datoh Mohamed Kassim, Klang.

156. Chutney, best sample, any method: Optional.

1st prize. (Bronze Medal) $\mathrm{F}$. Alexander $2 \frac{1}{2}$ mile, Palang lioad, Kuala Lumpor.

2nd prize. Girls' School, 'laipeng, Perak.

157. Pickles, best sample, any Method. Optional.

1st prize. F. Alexander, $2 \frac{1}{2}$ nile, Pahang Road. Kuala Jumpor.

2nd prize D. Podusingho 'Trolak, 'Tapah.

1วS. Jellies, best sample, any Ilethod. Optional.

1st prize. (Bronze Medal) E. Alexander, $2 \frac{1}{2}$ mile, Pahang

Road, Kuala Lumpor.

2nd prize. (Bronze Medal.) Girls' School, Jernam Perak. 
159. Honey, in comb. $1 \mathrm{lb}$.

1st prize. Abdullah dli Lower Perak.

2nd prize. Penghtulu H. Alimat, Malacca.

160. Butter, $1 \mathrm{lb}$.

1st prize. Shaik IIussain, Penang.

2nd prize. Rahamathullah, Penang.

161. Eggs, best collection, fowls, Aucks, or turkeys. Optional.

1st prize. Kung 'Thean Sung. Penang.

2nd prize. Ahmat, Kuala Lumpor.

162. Artichokes, best sample. 12 tubers.

Ist prize. No entry.

163. Beetroot, best sample. 6 specimens. 1st prize. Ahmat Ulu Yam.

161. Benny fruits or chocho. 6 specimens. No entry.

165. Brinjals. 6 specimens.

1st prize, Penghulu. Abdulgany, Malacca.

and prize. Straits Settlements Bertam Rubber Company, Province Wellesley.

166. Radishes. 10 specimens.

1st prize. Cheng Hun, Penang.

and paize. No award.

167. Ladies' finger's. 6 specimens

1st prize Mohamed Arshat, Penang.

Highly Commended. Brahim, Penang.

168. Uucumbers, 6 specimens

lst prize. Straits Settlements Bertam Rubber Company, Province Wellesley.

2nd prize. Nohamed Tahib, bin Mohamed Nor, Pennng.

Highly Commended. Mohamed Arshat, Penang.

Commended. Ahmat, Lower Perak.

169. 'Tomatoes, best dish. 6 specimens.

1st prize. I. M. Uonolly, Ipoh. Perak.

2ud prize. No award.

\section{Divisiun B. VII,}

170. Beans, best collection. Optional.

1st prize. Jayah, Penang.

2nd prize. Ahmin, Kuala Kangsar.

171. Uabbages, 3. Uptional.

1st prize. P. Y. Seow 'Teng, Kuala Lumpor.

Wighly Commended. R. M. Conolly, Ipoh.

172. Lettuces, 6. Optional.

1st prize. Cheng Hun, Penang.

2nd prize. Said Abmad, Penang.

173. Onions, shallots and garlic, best collection. One bundle of eact. 1 st prize. Kung Thean Sang, Penang.

Ilighly Commended. Musim, Rawang.

17. Llerbs used in curries and sambals, best collection. One bundle of each.

1st prize. Ah Seng, Penang.

and prize. Molianed Arshad, Penang. 
17. Chillies, best collection, 30 of each.

1st prize. Kutop Mohamed bin Wahab, Tanjong Malim, Perak.

2ud prize. Lamah, Kuala Langat.

High!y Commended. Nohamed Bidin, Lower Perak.

176. Chillies, best sample any kind. 30 specimens.

lst prize. Penghulu Sau, Malacca.

znd prize Unji Mastafa, Penang.

Highly Commended. Cheng II un, Penang.

177. Verretables and herbs for making a salad, best callection. 3 of each.

J st prize. Phaik Hussain, Penang.

2nd prize. Mohamed Arsat. Penang.

178. Tegetables, best collection. 3 of each.

1st prize. Al Seng, Penang.

znd prize. Lai Lang, Rawang.

Highly Commended. Low Chit Mun, Penang.

179. Pumpkins. 3̈ specimens.

1 st prize. Datoh Mohamed Kassim, Klang.

Ilighly Commended. Ang Yew, Ulu Yam.

180. Wrater-melons, gourds, sifuashes, luffas, etc., best collection. of each.

1 st prize. Mohamed Omar, Lower Perak.

2nd prize. saleh, Kuala Lumpor

:ird prize. No a ward.

181. Yams, kladies, best collection. 6 of each.

1st prize. Penghulu Sam, Nalacca.

2nd prize. Penghulu Abuat, Malacca.

3rd prize. Abmat Penghulu, Lower Perak.

182. Any regetables not included in above. Optional.

1st prize. R. M. Conolly. I poh, Perak.

2nd prize, Peng Mat, Malacca.

lligbly Commended, Sidang Dris Malacca.

$$
\text { Division C. I. }
$$

183. Bull (locally bred).

1 st prize. Ilasan, Kampong Baru, Kuala Tumpor.

znd prize. K. T. Koomarasawmy Pillai, Kuala Lumpox.

184. Cow and calf.

1st prize. No award.

and prize. K. 'I' Koomarasawmy Pillai, Kuala Lumpor.

185. Bull (imported).

1st prize. No award.

2nd prize. "

186. Cow and calf (imported).

1st prize. R. M. Gonnolly, Ipoh.

and prize. K. 'T' Koomarasawmy Pillak, Kuala Lmmpor.

187. Pair of draught bullocks with cart, (Indian).

1st prize. K. 'T. Koomarasawmy Pillai, Kuala Lumpor.

2nd prize. 'Taku, 10th Mile, Sungei Besi.

188. Pair of draught bullocks with cart, (Siamese).

1st prize. No award.

2nd prize. Penghulu H. Ahmat, Malacca.

189. Champion animal in section. Horace Brett, Perak. 


\title{
DIVISION O. II
}

No Entries.

\author{
DHISION C. ILI.
}

$19+$ to 196, No Entries.

197. Ram goat.

1st prize. Sidang 11. Mohamed, Biu L. Musah, Malacca, 2nd prize. Mat Talir Bin Abdul Rani, Malacca.

198. Ewe goat with kids

1st prize. Sidang Marshat, Malıcca.

2nd prize. No award.

\section{I)IVISION C, 1Y,}

199. Bantam cock and lien.

1st. prize. syed Harrou Alynite, Penang.

zud prize. Sidang IIaji Mohamed B. H. Husa, Malaced.

200. Malay cock and hen.

Ist prize. Mohamed Esah, Kuala Kangsar.

2nd prize. Mohamed Kasim, Penang.

201. Malay game cock and hen.

1 st prize. Hohamed Bedin, Lower Perak.

2nd prize. Ah Bum, Kuala Langat.

202. Collection of Malay fowls. 6.

1st prize. IV. IV. Douglas, Kuafa Lumpor.

2nd prize. Mohamed Omar, Lower Perak.

203. Collection of Chinese fowls. 6.

1st prize. No award.

2nd.prize. Penghulu Sungei Tiergrgi, Matang, Perak.

201. Cullection of fowls, any breed.

$$
\text { No award. }
$$

205. Pair of Muscovy ducks.

1st prize. Jamir, Kuala Lumpor

2nd prize. J. G. Dragon Kualí Lumpor.

206. Pair of ducks, any bieed.

1st prize. Mohamed Kassim, Penang.

2nd prize. Penghulu Ijok, Perak.

207. Pair of ducks, hybrid.

1st. prize. No award.

2ud prize. Kulop Samat, Bruas, Kuala Kangsar.

208. Gander and goose.

1st prize. Abmat, Kuala Lumpor.

2nd prize. Laiman, Kampong Buaia, Kuala Kangsar.

209. Pair of pigeons.

ist prize. W. H'. Douglas, Kuala Lumpor.

2nd prize. J. Carrol, Recreation Club, Kualı Lumpor

210. Best domesticated wild bird.

1st prize. Mohamed Bin Mohamed Saleh, Penang.

znd prize. Raja Nlang, hawang.

211. Best domesticated wild animal.

1 st prize. 'I. H. Sarnelis, Pudoh, K uala Lumpor.

2nd prize. Allang Mat Daud, Lungki, Perak 


\section{DIVISION D. I.}

212. Collection of Malay jewelry: not more than 8 pieces. No alward.

213. Pinding, any metal. 1. 1st prize. Mohamed Alsop, Brunei. znd prize. No award.

214. Sireh requisites, any metal. 1 set. 1st prize. Penghulu Jugra and Bandar, Kuala Langat. 2nd prize. Omar, Penang.

Highly Commended. IIaji Mohmed Shaid, Penang.

215. Muka bantal, rold suasa or silver. 1 pair. No award.

216. Oruamental boxes, any metal except tin. 1 pair,

1st prize. Mat Hasan, Brunei. 2nd prize. Mohamed Usop, Brunei.

Gommended. Waji Mobamed Shaid, Penang.

217. 'lalam or semerit, any metal. 1 pair. 1st prize. Jalaludin, Brunei. 2nd prize. Ilaji Mohamed Shaid, Penang.

218. Piring or semerit, any metal. 1'paic.

$$
\text { No award. }
$$

219. Batil, any metal. 1 pair.

1st prize Penghulu Jurra and Bandar, Kuala Langat. 2nd prize. Pengliulu Ijok, Kinta, Perak.

220. Dulung, any metal. 1 pair.

1st prize. Bin Mohamed Usop, Brunei.

2nd prize. Pandak Lazin, Kuala Langat.

221. Krusang, gold suasa or silver, 1 set.

\section{No award.}

222. Any kind of metal work not included in above. Optional. 1st prize. Haji Mat Daud, Brunei. 2nd prize. Mohamed Usop, Brunei.

223. Kris with sheath. 1. 1st prize. Penghulu Sungei Raia, Perak. 2nd prize. Penghulu Ipoh, Perak.

Commended. Mohamed bedin, Sungei Durian, Lower Perak.

224. Tumbok lada with sheath. 1 1st prize. Prae Bin Husin, Remban, Negri Sembilan. 2nd prize. Pengian Yakop, Brumei.

225. Parang with sheath. 1 .

1st prize. Pengian Yakop, Brunei. 2nd prize. Aluamod 'l'asir, Lower Perak.

226. Golok with sheath. 1. 1st prize. Penjirian Yacob, Brunei. 2nd prize. Sultan of Pahang.

227. Ormamental tin box. 1. 1 st prize. Ng Seng, Serendah. and prize. Swe Heng, Serendah.

228. Collection of tinware. Not more than 8 . 1st prize. Foh Seng, Seremban. 2nd prize, Tye Shin, Seremban. 
223. Malay gong. 1

1 st prize. Assistant Penglulu Tanjong Poh Alang: Perak

2nd prize. Penghula Irian Abdulnhaman. Brunei.

\section{DIVISION D. II.}

230. Carved wooden box. 1.

1st prize. Bulat School Master, Bermang.

2nd prize. Kidan Bin Dolah, Negri Sembilan.

231. Wood carring, any other form. 1.

Ist prize, Zabadi, Brunei.

2nd prize. II. B. Ellerton, 11, O. Negri Sembilan.

Special prize. Hazi Dollah Bin Nohodu Mat, Penang.

23:. Model of a Mlalay house.

1st prize. Indot K. L. Kanan Kuala Kangsar.

2nd paize. Mohamed Mansur, Lower Perak.

Special prize. Abdul Hamid Penghulu, Ulu Selangor.

233. Nalay models : best set of models illustrative of any one of the following subjects :- Hish-trapping, game suaring, weaving, navigation, rice cultivation, Malay ceremonies, Malay magic.

Surface measument of each exhibit not to exceed $4 \mathrm{sq}$. ft. lst prize. Mohaned Manswer, Lower Perak.

2nd prize. Mohamed Bidin, Lower. Perak.

Special prize. Penghulu, l'apah, Batang Padang.

234. Malay children's playthings, best collection, Optional.

1 st prize. Molumed Ali-Senggang, Kuala Kangsar.

2nd prize. Mohamed Mansur, Lower Perak.

235. Articles made from the coconut palm, best collection, Optional. 1 st prize. No award.

2nd prize. Haji Serat Rungkup, Lower Perak.

236. Sumpitan with quiver. Optional.

1st prize. Mohamed Serajudin Sungkai. Perak.

2nd prize. Said Yusuf, Chigar Gatah, Kuala Kangsar.

237. Set of Malay musical instruments. Not more than 8 . No award.

Special prize. Mohamed Casim, Bukit Raja, Klang.

$$
\text { DIVISION D. III. }
$$

238. Malay embroidery, best specimen. 1.

lst prize. Mohamed Omar, Lower Perak.

2nd prize. No award.

special price. Penghulu Bernang, Llu Langkat.

239. Limbroidered slippers. 1 pair

1st prize. Roja Kudin, Klang

2nd prize. Penghulu Ipoh, Perak

240. Lmbroidered muka bantal. I pair.

1st prize. Haji Mohamed Yasin, Lower Perak.

2nd prize. Abdullah Ali, Lower Perak

211. Embroidered mats. 1 pair.

1st prize. Mohamed Omar, Lower Perak.

2nd prize. Kajah Zyobidah, Kuala Lumpor

ord prize. No award.

212. Embroidered tudong saji. 1.

1st prize. Penghulu, Durian Sibatang, Lower Perak.

2ud prize. No award. 
243. Malay lace, 1 piece.

1 st prize, Sena Gurau, Malacca,

2nd prize. No award.

244. Sarong (silk and gold). 1.

1st prize. Wan Usop, Kuala Lumpor.

znd prize. Dayang Siti Eajijah, Brunei

245. Sarong (massuli), 1

1st prize, No a ward.

and prize Penghulu, Pulan Rusa, Pahang.

2ud prize. 'l'unku 'luan Besar, Pahang.

246. Sarong (silk). 1.

1st prize. Penghulu, Pulan Rusa, Puluang.

2nd prize. 'T'unku Tuan Besar, l'ahang.

2.17. Sarong (colton) 1.

Ist prize. Siti Esah, Brunei

2nd prize. Indot, Kuala Kangsar.

218. Silk for Malay baju, best pieco, 1 piece.

1st prize. 'T'unkn 'l'uan Besar, Pahangr.

249. Best collection of varieties of Malay weaving. Optional

ist prize. Mohamed Amin, Kurla Kangsar,

2nd prize. Bedul, Kuala Kangsar.

250. Kain lepas. 1.

1st prize. Penghulu, Ipoh, Perak.

2ud prize. No award.

231. Kain telepok. 1.

Ist prize. Penghulu Morilen, Kuala Langkat.

and prize. Haji Hamad Kuala Langkat.

252. Kain pelangi. 1.

list prize. No award.

zud prize. Mohaned Amimu, Kuala Kangsar.

253. Kain tudong kepala. 1.

lst prize. Ilaji Darus, Penang.

2ud prize. Punghulu Morib, Kuala Langkat.

25\%. Seleudan.

1st prize. Wan Usop, Kuala Lumpur.

2nd prize. Girls' School, Telok Anson.

\section{DIVISION D, IV.}

255. Onmmental basket (rattan) 1 pair.

1 st prize. Pengnulu Ijok, Selama Perak.

2ud prize. Mobamed Martin, Bedor, perak.

256. Ornamental baskets (mengkuang or pandan) Set of 4.

1 st prize. Penghulu Yusup. Nalacca,

2nd prize. Chi Itam bti : Jasir, Negri Sembilan.

257. Llats (mengkuang or pandan). 1.

1st prize. Sinen Binti Chu, Port Dickson.

2ud prize. Ilaji Abdul Carim, Negri Sembilan.

258. Mat (coloured).

1st prize. Abdul Raman, Palani, Negri Sembilan.

2nd prize. Penghulu Ujong, Permatang, Kuala Selangor

259. Hat (tikar bangka).

No award, 
260. Mat (tikar hampar).

1st prize. Datoh Paduka Raja, Lower Perak.

2nd prize. Sow 'Thok, Ampang, Kuala Lumpor.

261. Nat (mongkuang or pandan).

1st prize. Haji Mohamed Yasin, Lower Perak.

2nu prize. Mohamed Arshat, Lower Perak.

Special prize. Pandan Mat.

\section{DIFISION $\mathrm{V}$.}

262. Kajang (mengkuang), 1 piece.

ist prize. Datoh paduka Raja, Lower Perak.

2nd prize. Mohamed T'oh Maharaja, Derva Lam+Kanan Kuala Kangsar.

263. Attap. 10 pieces.

1st prize. Haji Mohamed Nasir, Lower Perak.

2nd prize. Mohamed Bedin, Lower Perak.

264. Set of cooking utensils, Not more than 8.

265. Labu. 1. No award.

1st prize. Ngah Ahmat, Kuala Kangsar.

2nd prize. Datoh Paduka Raja, Lower Perak.

266. Gelok. 1.

1st prize. Muda Jamsah, Negri Sembilan.

2nd prize. No award.

267. Buyong. 1.

1st prize. D. Dinas de Silva, Ruala Lumpor.

2nd prize. No award.

268. Ornamental pottery, best collection. Not more than 6 pieces 1 st prize. Murugapah, Province Wellesley. 2nd prize. No award.

269. Industrial earthenware, best collection Not more than 6 pieces No award.

270. Rope and twine. Optional.

1st prize. Penghulu Abdul Gani, Malacca.

2nd prize. Bala-Rassah, Negri Sembilan.

271. Malay Pottery, hand-made. 3 pieces.

1 st prize. Boys' School, Pulan Packat, Perak.

2nd prize. Boys' School, Pulan 'liga, Perak.

272. Ornamental baskets. 1.

1st prize. Girls' School, Telok Anson.

2nd prize. Girls' School, Kam: Buaia Perak.

273. Malay Mats. 3.

1st prize. Boys' School, Klang.

2nd prize. Girls' School, 'Trong, Perak.

274. Sireh requisites. 1 set.

1st prize. Boys' School, Pasir Panjanghlis. Perak.

2nd prize. Pasangan Boys' School.

27i. Model Malay house. 1.

1st prize. Boys' School, Kuala Kangsar

2ud prize. Boys' School, Kajang.

276. Wood-carving. 1.

1st prize. Malay School, Bernang Ialish.

2nd prize. Boys' School, Bagan Dalish. 
277. Embroidery. 1. piece.

1st prize. Girls' School, Tenish.

2nd prize. Girls' School, 'leluk Anson.

278. Sarong. 1.

1st prize. Giris' School, Kila Lama Kiri, Kuala Tumpor.

2nd prize. Girls' School, Lenggong.

279. Fishing nets. Optional.

1st prize. Boys' School, Jeram.

2nd prize. Boys' School, Pasir Panjang, Iarut, Perak.

280. Collection of miniature Malay models. Optional.

1st prize. Boys' School, Kampong, Padang Gajah.

2nd prize. No award.

281. Best article of rattan furniture.

1st prize. Boys' School, 'Taipeng.

2nd prize. Malay School, Setapak.

282. Collection of models of Malay boats. Optional.

1st prize. Boys' School, 'lelok Anson.

2nd prize. No award.

283. Finbroidered mat. 1.

1st prize. Girls' School, Bandar.

2nd prize. Girls' School, Kata Lama, Kanan.

Highly Uommended. Girls' School, T'elok Anson.

Higbly Commended. Girls' School, 'l'rong.

DIVISION E.

284. Agricultural implements, locally made. Optional.

1st prize. Abdul Shuckor, Kuala Kangsar.

2nd prize Sidang Drisbin Hasan, Malacea.

Special prize. Ahmet, Kuala lumpor.

Commended. Datoh Puduka Raja, Lower Perak.

Highly Commanded." F. E. Co., Kuala Lumpor.

28\%. Agricultural implements, European made.

1st prize. liederated Engineering Company, Kuala Iumpor.

2nd prize. Noaward.

286. Machinery in connection with preparing rubber, to be exhibited at work. Diploma \&c.

1st prize. F. J Harvey, S. M.

2nd prize. J. B. Douglas, T'erantang Estate.

Commended. Federated Eugineering Company, Knala Lumpor

287. Spraying apparatus. Optional.

lst prize. No award.

2nd prize. No award.

Commended. Federated Engineering Company, K uala Isumpor.

28. Best tools for tapping rubber. Optional,

1st pri\%e. Federated lingineering Company, Kuala Iumpor

2nd prize. Messrs. Brown and Davidson, Ceylon.

289 and 290. Bullock carts and hand carts. No entries.

291. Agricultural baskets. 2 of each kind.

1st prize. Penghulu, Ulu Langkat, Kajang.

2nd prize. Said Ahmad, Penang.

2.2. Casks, tubs, etc, 3 speciuens. No entry. 
293. M[iscellaneous. Optional.

1st prize. Iussain Bin Haji Ishak, Penang.

(For a model of cotton spinning apparatus.)

Commended. Nain Lenggong; Negri Sembilan.

Special prize. 'Tan Ong Pin, Kurla Lumpor. (For making tricks.)

Special prize. H. B. Ellerton D. O. (for implements collecting wood oil).

\section{PERSONAL.}

Mr. H. C. Pratt, the Government Entomologist, has been transferred from the Institute for Medical Research to the staff of the Director of Agriculture and will be engaged in the first instance in completing his investigations published last May in this Bulletin into the life history of Temes Cestroi, the white ant attacking living rubber trees. He will also carry on experiments in the direction of prevention and cure of this ulbiquitons pest.

\section{GROWTH OF GUTTA PERCHA.}

The Ejtor Agriculturat Bulletis,

\section{Singapore.}

DEAR SIR,

In reference to Mr. Bum Murdoch's interesting article on the rate of growth of forest trees in the F. M. S. the following mensurements of two Getah Tahan trees growing amongst my young Para trees may be of interest. The trees I may mention wre situater on a gentle slope and have been practically clean weeded the whole time. Measurements taken 3 ft. from the ground.

TREE No. 1.

$\begin{array}{rlr}\text { 18th January } & 1906 \\ \text { 25th } & \text { April } & 1906 \\ \text { 18th } & \text { October } & 1906 \\ \text { 18th } & \text { October } & 1907 \\ 5 \text { th } & \text { September } & 1908\end{array}$

$14 \frac{11}{8}$
$14 \frac{1}{43}$
$15 \frac{1 "}{2}$
$17 "$
$18 "$

TrRE No. 2.

$$
\begin{aligned}
& 11 \frac{11}{4} \\
& 11{ }^{\prime \prime} \\
& 12 \frac{1}{4} \\
& 14{ }^{1 \prime \prime} \\
& 155^{\frac{1}{2 \prime \prime}}
\end{aligned}
$$

Therefore during 31 months the two trees have increased in girth $4 \frac{1}{4}$ and $4 \frac{11}{4}$ respectively, or an average increase of about $1.65^{\prime \prime}$ per annum, against $1.69^{\prime \prime}$ of the 100 Taban trees at Taiping, vefered to by Mr. Murdoch.

In a year or two these two Taban trees will be entirely shut in by. Para trees, when there will be probably a falling off in the rate of growth, so far as girth is concerned. I shall continue my measurements.

Yours Faithfully, G. A, L. 


\section{EMERGENCY MEETING OF THE PLANTERS' ASSOCIATION OF MALAYA.}

Meeting held at 2.30 p.m., on August 27th, 1908, at the Selangor Club, Kuala Lumpor. Present:--For the Perak Planter's' Association : Mr. C. L. Gibson, Mr. H. S. Whiteside; Negri Sombilan Planters، Association: Mr. J. le P. Power"; Johore Planters' Association: Mr. A. H. Malet, Mr. A. L. Buyers: Kuala Selangor D. P. A: Mr. A. Irving; Ǩlang D. P. A: Mr. Jno. Gibson, Mr. A. B. Tuzke, Mr. R. W. Harrison; Kapal: D. P. A: Mr. H. M. Darby, Mr. C. T. Hamerton; Kuala Langat D. P. A: Mr. E. Macfadyen; Kuala Lumpor D. P. A: Mr. E. B. Skinner, Mr. F. G. Harrey, Mr. C. Burn Murdoch, Mr. A. J. Fox, Mr. H. C. L. Zacharias. Batu Tiga D. P. A: Mr. H. F. Browell, Mr. P. W. Parkinson, Mr. H. L. Jarvis. Mr. R. W. Harrison Chairman. and Mr.H.C.E. ZaCharias, Secretary. Mr. L. H.ChAYTon, (Visitor).

1. The Secretary, having read the notice convening the Meeting, places before the Meeting the following letter's :-

No. $3278 / 1908$.

KuAla Tumpor, 13h August, 1908 . SIR,

I am directed to forward herewith copies of the Bill to repeal and re-enact with the amendments the Tamil Immigration Fund Ordinance (Straits Settlements) 1907, the second reading of which is moposed to be taken in the Legislative Council of the Colony on the 21st Angust.

2. As it is considered desimble that the Bill should be proceeded with without unnecessary delay, I $\mathrm{mm}$ to request that any observations which tho Planters' Association may wish to make may be submitted as soon as possible.

I have, etc.,

(Sgd.) S. W. MACARTHUR,

Federal Secrelary.

KILANG, 19th Angust, 190 S.

The Secretary to H. E. The Governor, SINGAPORT:

SIR,

Enclosed I have the honour to hand you copies of my telegrams of to-day's date with reference to prostponing the final reading of the revised Immigration Ordinnce.

An emergency meeting of the Planters' Association of Malaya cannot be called with less than 7 days' notice, and as the first intimation we received of the Second Reading of the Bill being taken on the 21 st inst., was in a letter from the Federal Secretary dated 14 th inst., it was impossible for us to call a meeting in time.

I have, etc.,

(Sgd.) R. W. HARrison,

Chaiman, P.A. M. 


\section{STRAITS SETTLEMENTS}

AND

FEDERATED MALAY STATES.

THE SIXTH JOINT ANNUAL

\section{Agri=Horticultural Show, 1909.}

WILL BE HELD ON THE PARADE GROUND

AT

PENANG

ON

Monday, Tuesday and Wednesday, 9th, Ioth and IIth August, 1909.

\section{PRIZE LIST.}

The Show will be open on Monday, 9th August. from 11 a.m. until 6 p.m.; and on Tuesday and Wednesday, 10th and $1 \mathrm{lth}$ August, from 8 a.m. until 6 p.m.

ADMISSION: TWENTY-FIVE CENTS. Opening Ceremony (1st Day, 11 a.m.-2 p.m.) \$2. 

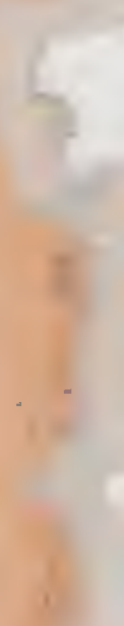

I

,

1 


\section{PATRONS.}

H. E. The Governor and High Commissioner, (Sir John Anderson, B.C.M.G.)

H, H. The Sultan of Perak.

H. H. The Sultan of Selangor

H. H. The Sultan of Pahang

H. H. The Sultan of Kedah

1.H. The Yang Di Pertuan Besar, Negri Sembilan

( Resident-General, Federated Malay States, (Sir W. Taylor, K.C.M.G.)

\section{PRESIDENT.}

Hon Rewident Councillor, Penang, (Hon'ble R. N. Bland)

\section{VICE.PRESIDENTS.}

H E The General-Oficer Commanding the Troops, (Majol-General T. Perrott, C B.)

The Hon'ble The Chief Justice, (Sir H. H. Hyndman Jones)

The Hon'ble Colonial Secretary, (Captrin Sir Arthur Youug, K.C.M.G.)

The Hon'ble The Resident Councillor, Malacca, (W. Evans, Esq)

The British Resident, Perak

The British Resident, Selangor

The British Resident, Negri Sembilan.

The British Resident, Pahang

The Rajah Mudah, Selangor

Tho Prosident, Municipal Commissionors, Penang.

The President, Chamber of Commerce and Agriculture, Perang. 


\section{STANDING. COMIMITTEE.}

\section{SINGAPORE.}

The Director of Botanic Gardens, S. S. (Hon Secretary.) The Curator, Botanic Gardens, Singapore

\section{PENANG and PROVINCE WELLESLEY.}

The Senior District Officer, P W

The Collector of Land Revenue

The Superintendent, Botanical Gardens aud Forests, (Hon Secretary

\section{MALACCA.}

The Collector and Land Revenue

'l'he Hon'ble The Resident Councillor, (Hon Secretary)

\section{PERAK.}

The Director of Museums, $\mathrm{F}$ M S

The Superiuteudent, Goverument Plantations

The District Officer, Kuala Kangsar

The District Officer, Larut and Krian (Hon. Secretary)

\section{SELANGOR.}

The Collector of Land Revenue, Kuala Lumpur

"The Chairman, United Planter's' Association

The Superinteudeut, Experimental Plantations, (Hon Secretary)

\section{NEGRI SEMBILAN.}

The District Officer, Tampin

The Collector of Land Revenue, Seremban

'The Chairman, Negri Sembilan Planter's' Asssociation.

\section{PAHANG.}

The Senior Magistrate

The District Officer, Raub

'l'se District Officer Pekan 


\section{GENERAL PURPOSES COMMITTEE.}

Cavendish, A

Gilmour; D W

Guinness, C

(xan Gnoh Bee

John 'I urner, Hon'ble

Jamieson, Dr

Fangham Carter, W

\author{
Pigott, F G \\ Linton, S E A \\ Yearrood, P A \\ Elton Bell \\ Hamilton, A W B \\ Nunn, B \\ Muir, $\mathrm{H}$
}

Chairman, A. Cavendish.

Hon. Secy, W. Fox.

\section{SUB-COMMITTEE.}

Division A. - Aqricultural Produce.

Baruett, C W

Begg, Hood A

Chasseriau, E

Lamb, J

Stothard George

Turner, Fon'ble $\mathrm{J}$.

Hon. Secy., P. A. Yearmood.

Division B.-Flowera, Fruits and Vhatadieș.

Adams, Hon'ble, A R

Avetoom, $\mathrm{Dr}$

Buckland, R G S

Deunys, A
Gan Gnoh Bee

Loong Lok Heng

Lees, $\mathrm{G}$ H

Whitehead, C B

Hon. Secy., S. E. A. Linton.

Division C.-Stock and Dairy Produce.

Hogan, E

Locke, $\mathrm{Dr}$

MacFarlane, J R
Merican, A O

Stark, J

Kee Tek Kwee

Hon. Secy, Elton Bell.

\section{Division D.-Horses and DoGs.}

Faber, A G

Guinness, C

Macintyre, Oapt
McArthur, W H MoCallum, Capt Wreford, J F

Hon, Secy,, A, W, B, Hamilton, 


\title{
Divianon F,-Native Imdostries.
}

Allan, P ' Ambrose, $\mathrm{S} \mathrm{C}$ De Hamel, Majo:
Hallifax, $J$ W Thomes, O V

\author{
Hon, Secy., B. Nunn. \\ Divigion E.-Natipe Industrifes.
}

For Schools.

Hon. Secy., Brother James.

Divigion F,-Manufaoture and IMphements.

Allan, J G

Bell, L

Buckell, C P.

Goh Taik Chee

Robertson, J H

Wemyss, J Is

Young, $\mathrm{R}$

Hon. Secy,, H. Muir.

Hon. Veterinary Suryeons, W. H. MacArthur and Elton Bell.

Honorary Treasurer, D. W. Gilmour, The Chartered Bank.

\author{
W. FOX, \\ Hon. General Secretary.
}




\section{Rules and Regulations Relating to Exhibits.}

1. All exhibits must be bona fide the property of the Exhibitor.

2. Competition for prizes shall be open only to Exhibitors resident in the Colony of the Straits Settlements or the States of the Malay Peniusula.

3. Competition in Divisions A. B. and E. shall be confined to exhibits grown or manufactured in the Colony of the Straits Settlements, the the States of the Malay Peninsula.

4. The Committee will appoint Judges, from whose decision there all be no appeal.

5. The Judges will have authority to withhold a prize when they are of opinion that there is not sufficient merit to justify an award, and also to award special prizes for anything not contained in the schedule.

6. No Exhibitor shall be awarded two prizes in any one class, or more than five prizes in the same division.

7. The arrangement of the exhibits shall be subject to the directions of the Committee.

8. The Committee reserves to itself the right to refuse any entries that they may think fit to roject, and to cancel any class at any time up to seven days previous to the first day of the Show.

9. Exhibitors desiring free transport by Railway, must give notice to a District Officer, or the General Secretary, who will if they approve of their exhibits, provide them with numbered tickets and a free third class pass on the Railway for themselves and theil exhibits. A reduction of 25 per cent. off the usual freight will be made by the Straits Steam Ship Co. on all bona fide exhibits. Other reasonable expenses in connection with transport of exhibits will be considered by the Committee.

10. Entries in all Divisions must be in writing, and reach the General Secretary at least seven days before the opening day. In the cake of Horses and Dogs three weeks.

No exhibit in Divisions A. C. E. and F. will be receired later than $6 \mathrm{p}, \mathrm{m}$ on the same day previous to the opening day.

Exhibits in Divisions B, and D. will be recoived up to 8 a.m, on the morning of the Show.

11. Entries for Horses undor Classes 223, 224, 225, 226, 227, 229, $230,232,233,236$ and 237 , must be accompanied by a certificate of height from any Sporting, Turf or Polo Club in the Colony or F.M.S, or from a qualified Veterinary Surgeon.

12. Exhibitors in Divisions C. and D, must provide for the care and maintenance of their own exhibits. 
13. No exhibit (except live animals) may be removed from the ground before the close of the Show. Exhibitors will be provided with a card which must be produced at the close of the Show before they can bo allowed to remove their exhibits.

14. The Committeo is empowered to exclude, or remore, any animal which in their opinion is not in a fit state for exhibition.

15. Exhibitors in Divisions C. and D. will only be permitted to remeve their animals from the Show Ground on obtaining an order of removal from the General Secretary or the Hon. Secretaries of these Divisions.

16. All reasonable care will be taken to provide for the safety i careful preservation of exhibits of every kind sent to the Show but it $\mathrm{m}$. be clearly understood that the Committees does not hold itself responsibre for the loss of, or the damage to, any exhibit either in transit or during exhibition. Provision will be made for keeping small articles of value under lock and key at night.

17. Space will be provided for tradesmen and others wishing to exhibit articles for sale, but not for competition. The sale price must be clearly specified.

18. Government exhibits shall not be eligible for prizes in any class. Winners of prizes of $\$ 10$ and upwards, may have a silver medal if they prefer it to money. 


\section{DIVISION A.}

\section{AGRICULIURAL PRODUCE.}

Nature of Exhibit.

Nuinber or quantity to

Prizes. be exhibited.

Ist 2 nd $3 \mathrm{rd}$

\section{SECTION I.PADI.}

Padi, best sample of any named variety

2 Pulut, best sample of any named variety $\quad \ldots \quad \ldots$

3 Rice, best sample, prepared by machinel'y

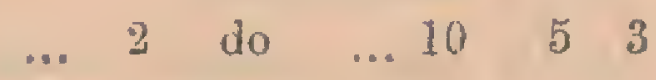

4 Rice, best samplo prepared in a lesong

... 1 gautang... $10 \quad 5 \quad 3$

5 Best collection of different Padi in the ear, 10 heads in each sample

1 do $\quad \ldots 10 \quad 5 \quad 5$

6 Best Padi and Pulut, grown in one mukim to be exhibited by the Penghulu of such mukim, and so certified by

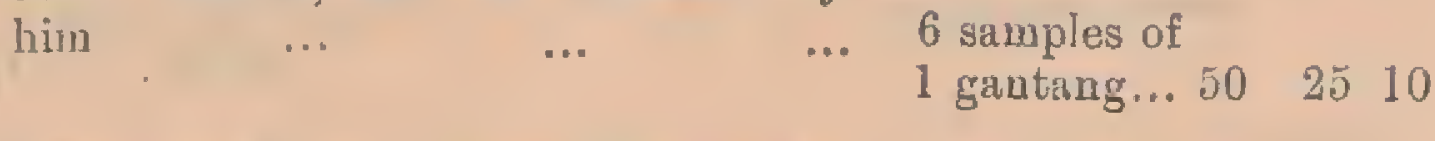

Exhibitols from the Krian Districts are not eligible to compete in Classes 1 to 6, Classes 7, 8, and 9, are open to them ouly.

Each exhibitor to produce au affidavit, sworn before a Magistrate that his whole oxhibit was grown in his own mukim last Padi season.

7 Padi, best sample of any named variety ... $\quad$... 2 chupals... $15 \quad 10 \quad 5$

8 Best collection of different Padi in the ear, 10 heads in each 'sample...

9 Best Padi and Pulut, grown in one mukim to be exhibited by the Penghulu of such mukim, and so certified by him

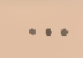

6 simples of

1 gantang.. *25 1510

"Prize Presented by ERIC MAXWELL, Esq. 


\section{SECTION II,-RUBBER,}

10 Para Crepe, best sample

11 Do Sheet do

12 Do Biscuit do

13 Do Block dry do

14 Do best sample ready for shipment. The Exhibit to be one case with not less than 50 lbs. of crepe, sheet, or, block, and one case of $\begin{array}{llll}\text { serap } \quad \ldots & \ldots & \ldots\end{array}$
... not less than

25 lbs.

..(a) Cup

do

(b) Cur

do S.M.

do

(c) Cup

15 Do best sample of Commercial Rubber $\ldots$... 100 lbs, weight (e) Cup

16 Do best sample of sheet, crepe, biscuit, block, and scrap

not less than 100 lbs. ... (f) Cup

17 Rambong, any form ... $\quad \ldots$ not less than 25 lbs. ... S.M. B.M:

18 Any other Rubber, plant from which obtained must be stated with each (... S.M. B.M,

(a) Cup presented by Hon'ble John 'Turuer.

(b) Do by . do

(c)

Do by E. Lauder Watson, Esq.

(d) Caledonia Cup presented by Caledonia Estate.

(e) Cup presented by Francis Pears, Esq.

(f) Do by Malay Peninsula Agricultural Association. N.B.-S.M. meaus silver medal.

B. M. " bronze unodal. 
Nature of Exhibit.

SECTEON IIT - FOOD PRODUCTS.
Number or quantity to

be exhibited. 1st 2nd 3rd

19 Cocoanuts, unhusked, best sample.

20 Do liusked do

21 Do best, bunch

22 Do Collection of varieties

(n)

23 Copra best simple

24 Do do

T Tapioca roots, best samplo

6 Do pearl do

27 Do flake do

28 Do flour do

29 Arrowroot, fresh

30 Do propared, best sample

31 Sago pearl, best sample

32 Do flour

33 Maize, best sample ...

34. Ginger do ...

35 Turmeric

36 Tuba (akar), best sample

37 Sugar cane do

38 Do cocoanut do

39 Do nipah do

40 Do kabong do

41 Do (cane), brown, best sample

\section{$\cdots$}

$\cdots$

$\cdots$

$\cdots$

$\cdots$

...

42 Coffee, arabian, do ...

43 Do liberian do

44 Coffee, any other variety

45. Cocoa, fresh pods best sample

46 Toddy, best sample

47 Rum, best simple

48 Rum shrub, best sample

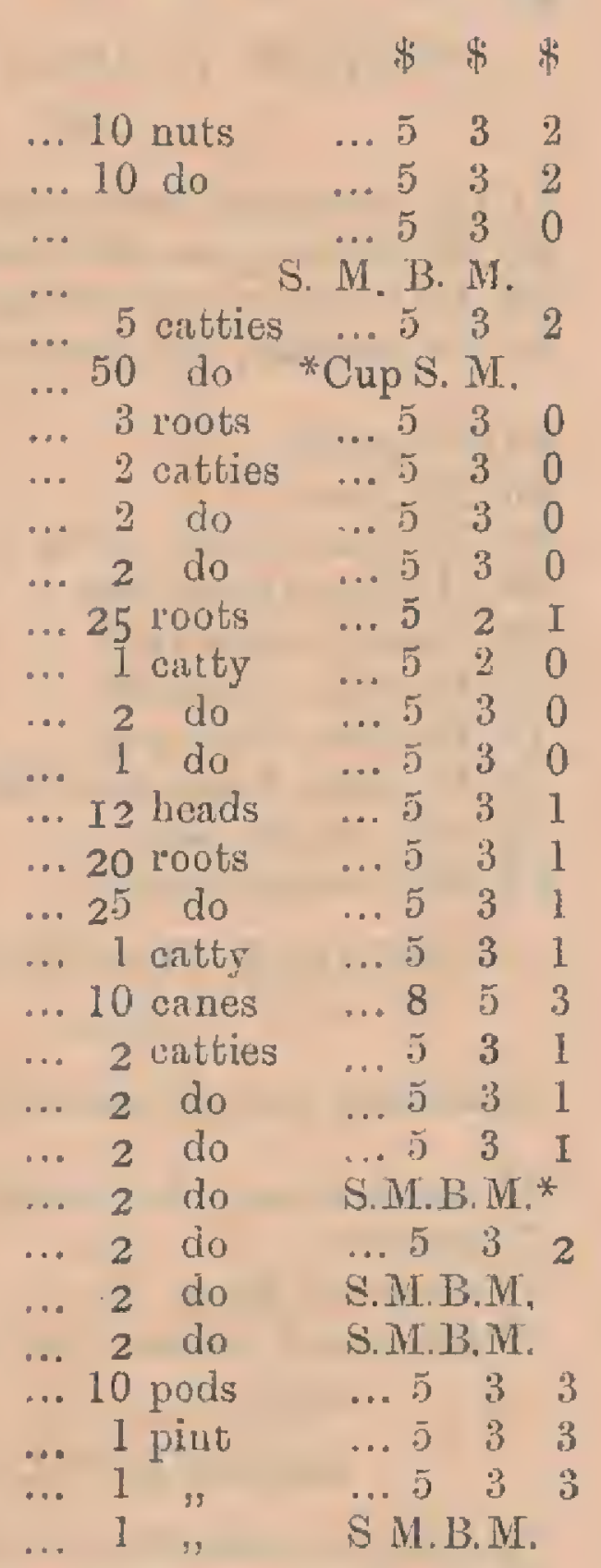

* Preserted by C. E. S. Baxendale, Esq.

SECTION IV.-SPICES, ETC.

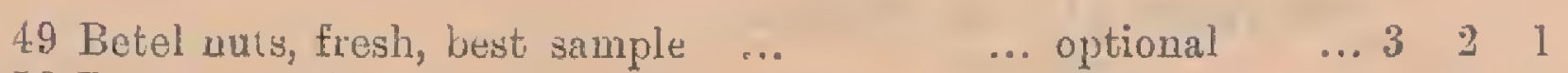

50 Betel nuts, dried and split, best sample $\quad \ldots \quad 1$ catty $\quad \ldots 4$ i $\quad$ I

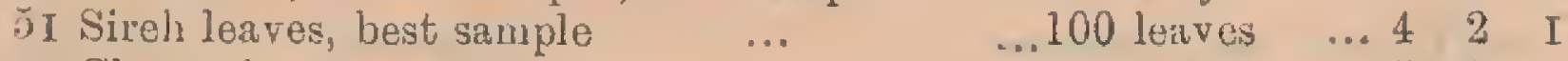

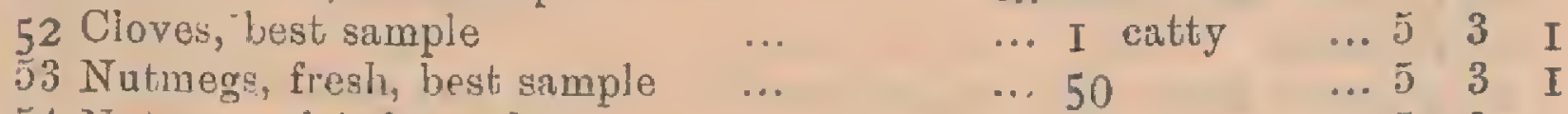

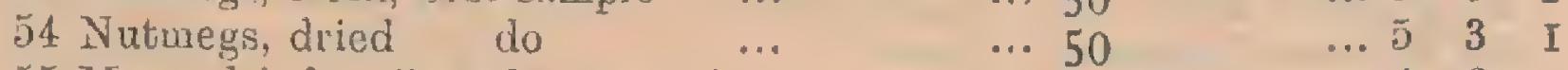

55 Mace, dricd, yellow, best simple $\quad \ldots$ I catty $\quad \ldots 4433$ I

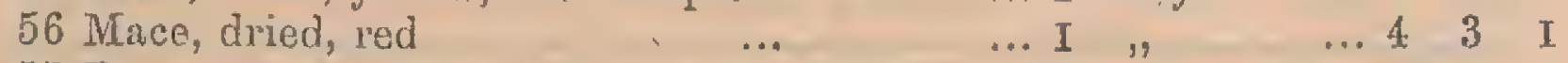

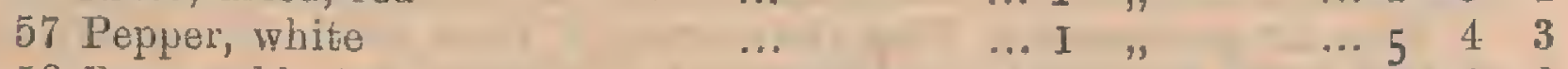

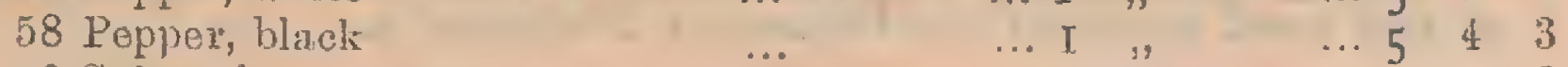

$\begin{array}{lllrrr}59 \text { Spices, best collection } & \ldots & \ldots \text { optional } & \text { I0 } & 5 & 0 \\ 60 \text { fatchouli, best sample } & \ldots & \ldots \text { I catty } & \text {. } & 5 & 3\end{array}$ 
- Nature of Exhibit.

SECIION V.-OILS, OIL CAKE, ETC.

6 I Oil, citronella, best sample

62 Do lemon griss, best sample ...

63 Do coconut, best sample

64 Do gingelly, teal seed (minyak length)

65 Do castor hest sumple

66 Do clove

67 Oil, Para Rubber seed, best sumple

68 Do Kabu kabu seed do

69 Do any other kiud do

70 Do cake, cocounut do

7 I Do cake, Par'u seed do

72 Do cake, Kabu kabu seed, best sample

73 Seed, Kabu kabu

74 Oils, best collection

do

do
Number or quantity to be exhibited. 1st 2 nd $3 \mathrm{rd}$

\$娄

... I pint

$\ldots 840$

... I :

.. 8 4 4.0

... I ,

I0 53

...

‥ I ,

... I ,

... I

... I ,

... 1 ,

... 5 catties

... 5

... 5 ,"

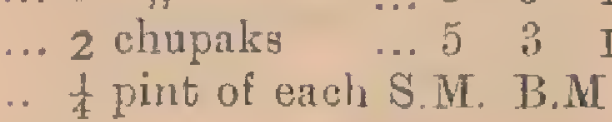

$\begin{array}{lll}\ldots & 5 & 3\end{array}$

$\ldots 53$

... 53

S.M. B.M.

... $53 \quad 3 \quad$ I

.. 53 I

... 53 I

... 533

... $53 \quad 3 \quad I$

$\ldots 53$ I

SEOIION VI.-GLIAHS, GUMS,

ETC.

75 Getahs, best collection of local

... $\frac{1}{2}$ catty of

76 Getah taban, best sample

77 Gumbier do

78 Dragon's blood do

79 Gums and damars, best collection of local

‥

each $10 \quad 5 \quad 2$

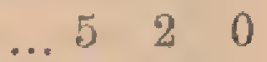

$\ldots 5 \quad 2 \quad I$

.. 521

$\ldots 7 \quad 3 \quad$ I

SECTION VII, - FIBRES.

80 Cotton (Kakabul), best sample

8I Do any other variety, best simple

82 Fibres, best collection
... I catty $\quad \ldots 66 \quad 3 \quad 2$

... I , $\quad \ldots 6630$

... I bundle of S.M. B.AI. each

\section{SECTION VII.-MISCELLA- \\ NEOUS.}

83 Rotans, best collection

84 Bamboos do

85 Walking sticks do (unprefared)

86 Medicinal plants do (open

87 Do du (Natives only)
. optional $\ldots 53 \quad 3 \quad 1$

$\begin{array}{llllll}\ldots & \text {.. } & \ldots & 5 & 2 & 1\end{array}$

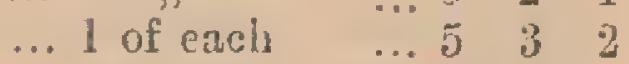

... optional S.M. B.M.

His Excellency's The Governor's Cup will be awarded to the best general exhibited in Division $A$.

Entries close in Division A., August, 2nd. 


\section{DIVISION B.}

FLOWERS, FRUTTS AND VEGETABLES.

$\begin{array}{ccc}\text { Number or } & \text { Prings. } \\ \text { Nature of Exhibit. } & \text { quatity to } & \text { 1st 2nd 3rd } \\ \text { be exhibited } & \$ \$ \$ \$ \$\end{array}$

SECTION I.-ORNAMENTAL FOLTAGE PLANT'S IN POTS.

88 Aroids other than Caladiums

89 Caludiums

90 Coleus

91 Crotons

92 Dracenas

93 Ferns, (any variety), distinct ...

94 Do Adiantum

95 Do any variety, best specimen

96 Marantas and Calatheas

97 Pulms, distinct kinds

98 Do besti specimen

99 Selaginella, best specimen

... 3 plants

$\begin{array}{llll}\ldots & 3 & 0 & 0\end{array}$

3 ,

$\begin{array}{lll}. .3 & 0 & 0\end{array}$

... 3 "

…3,

$\ldots 3$

6 "

... 3 ,

.. 1 ,

... 3

... 3

100 Any ornamental foliage plant not included in above

101 Foliage plant any kind, best specinen

... 3 "

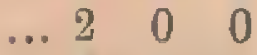

$\therefore \begin{array}{lll}3 & 0 & 0\end{array}$

... 3 r 00

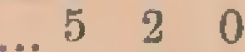

$\ldots 3 \quad 0 \quad 0$

a

... $3 \quad 0 \quad 0$

$\begin{array}{lll}7 & 5 & 0\end{array}$

$\begin{array}{llll}\ldots & 5 & 2 & 0\end{array}$

... 1

.. 3300

SECTION II-ORNAMENTAL P.LANTS

IN FLOWER IN POTS.

102 Achimenes

$103 \mathrm{~A}$ maryllis and Lilies

$104 \mathrm{~A}^{\mathrm{s}}$ ters, best specimen

105 Balsams Do

106 Camnas Do

107 Cocks-combs Do

108 Chrysanthemums, best specimen

109 Dahlias, best specimen

110 Dianthus (Tudian Pinks)

11 Eucharis, best specinen

112 Gloxinias

113 Orchid

114 Orchid

115 Phlox

116 Petunias

117 Zinnias

118 Roses
Do

Do

Do

Do

Do

Do

Do

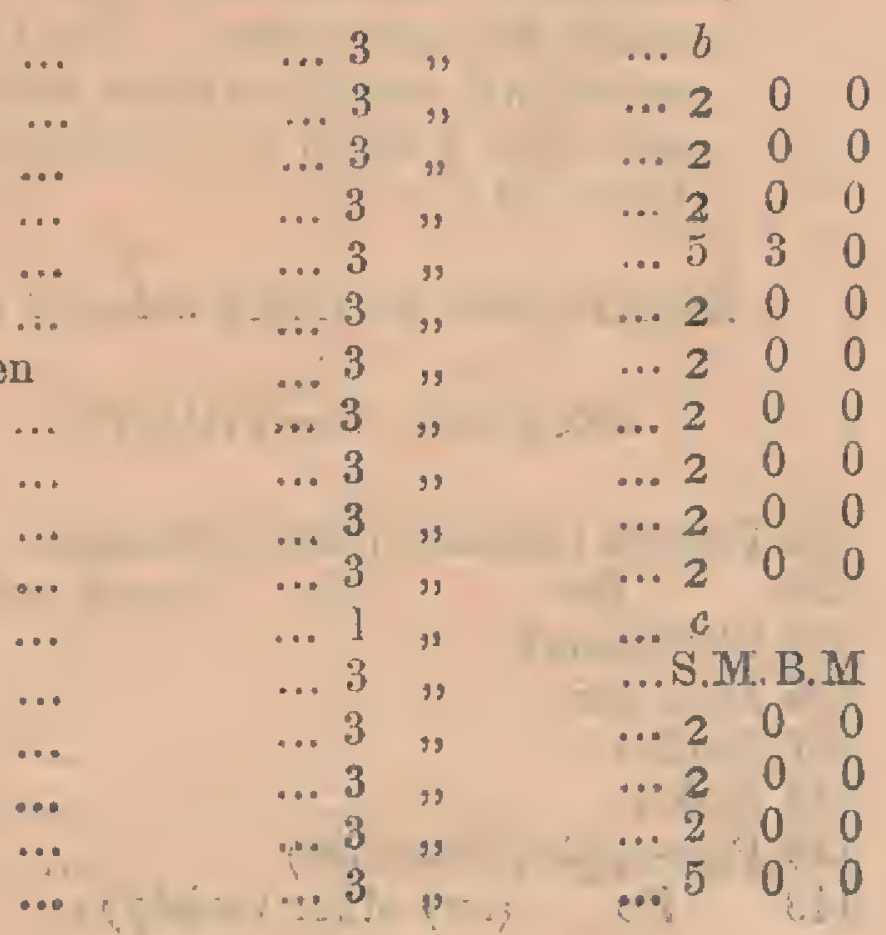


Number or quantity to be exhibited
Prizes. I st 2 nd $3 r d$ $\$ \$ \$$

\section{SECTION III,-PLANTS WHETHER}

IN FI,OWER OR NOT.

119 Begonias, distinct kinds

120 Begonias, best specimen

... $\quad$...3 plants ... $3 \quad 0 \quad 0$

121 Group of plants arranged for effect in space not exceeding 9 sq. ft.

... optional $\ldots 2 \quad 0 \quad 0$

122 Group of Chinese plants, fantastic shape

$1_{2} 3$ Group of flowering Annuals (Subscribers) $\ldots$

SECTION IV.-CUT FLOWERS AND

TABLE DECORATION.

\section{Asters}

125 Chrysanthemums ...

126 Dahlias

127 Roses

128 Cannas

129 Orchids

130 Cut flowers arranged for effect...

13] Collection of cut Hower's not less than 12 kinds

132 Table decoration ...

\begin{tabular}{|c|c|c|c|c|}
\hline$\ldots$ & $\ldots$ & : & $\ldots 5$ & 0 \\
\hline ". & $\ldots$ & 1, & & 0 \\
\hline . & $\ldots$ & , & $\ldots 2$ & 0 \\
\hline . & ... & " & $\ldots P$ & \\
\hline -• & $\ldots$ & ", & $\ldots 3$ & 0 \\
\hline & $\because \cdot$ & " & $\ldots 4$ & 0 \\
\hline des & $\cdots$ & ", & $\ldots 5$ & 3 \\
\hline & $\ldots$ & " & $\ldots 5$ & 3 \\
\hline & $\cdots$ & " & $\ldots f$ & \\
\hline
\end{tabular}

Exhibitors must provide their orm stands for cut flowers. The Committee will provide table of uniform size ( $7 \mathrm{ft} . \times 3 \frac{\mathrm{ft}}{2}$ ) fol exhibitors in Class 132.

Exhibits must be staged before 8 a.m.

\section{SECIION V.-FRUITS.}

133 Pisangs (Banunas) best collection

134 Do

135 Champedak

136 Jack fruit

137 Durian

138 Melon

139 Pine-apple (Mauritius)

140 Do (any other vurioty)...
... 6 of each kind $10 \quad 5 \quad 0$

... 1 bunch $\ldots 3 \quad 20$

... 3 fruits $\quad \ldots 2210$

$\begin{array}{llllll}\ldots 1 & 1, & \ldots & 2 & 1 & 0\end{array}$

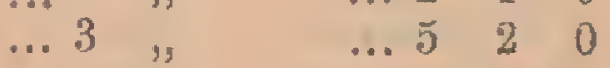

$\begin{array}{llllll}\ldots 1 & \ldots & \ldots & 1 & & \end{array}$

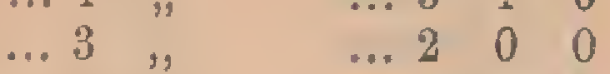

$\begin{array}{lllll}\ldots 3 & \ldots 2 & \ldots & 0 & 0\end{array}$ 


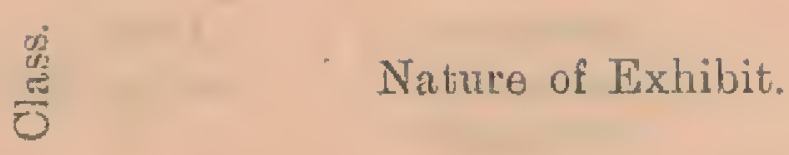

SECTION V.-FRUTTS-cte.
Pomeloes

142 Papaya

143 Oranges

144. Linnes

145 I.cmons

146 Chiku

..7 Custard Apple ...

148 Duku

149 Binjai

150 Jambu

151 Langsat,

152 Mangoes

153 Machang

154 Mangosteens

155 Pulasan

156 Rambai

157 Rambutan

158 Mata kuching

159 Durian blanda

160 Any fruit not included in above

161 Cultivated fruits, best collection, any number of kinds

162 Wild edible fruits, best collection

\section{SECTION VI.-PRESERVED} FRUTLS, ETC.
Number or

quantity to

be exlibited.
PIRIZES.

1st 2nd 3rd

$\$ \$ \$$

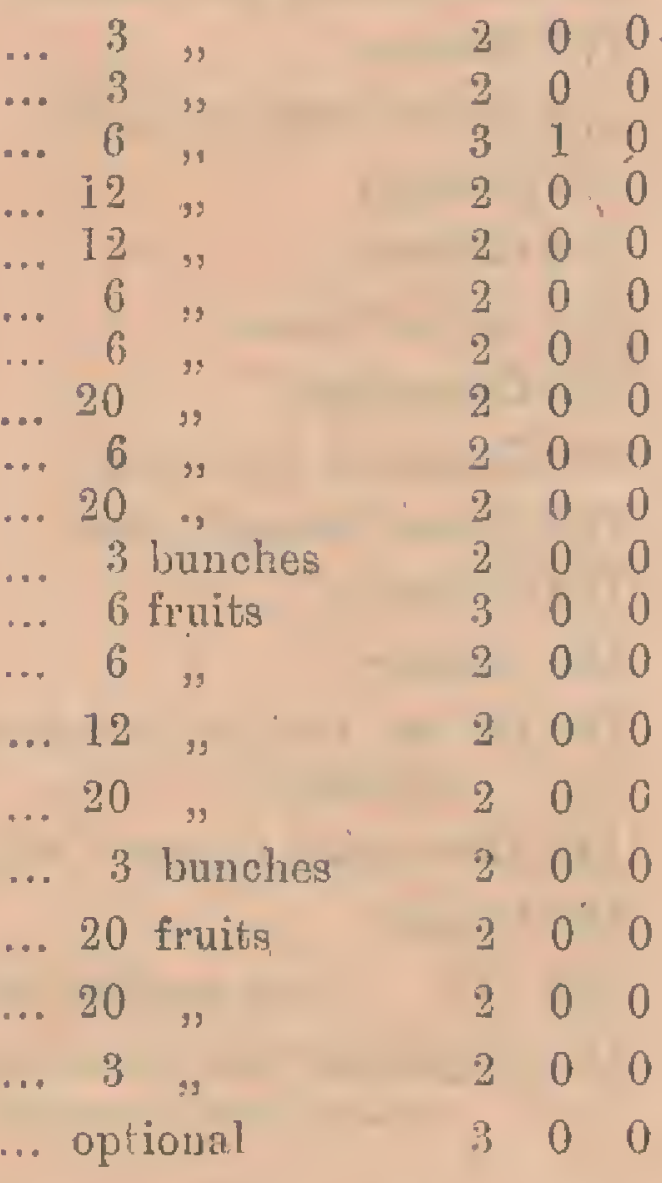

… , ,

$g$

5.30

163 Preserved fruits, hest sample, any method

... optional

164. Chutney, best sample, any method

165 Pickles

Do

166 Jellies

Do

do

do

,...

‥
... $1 \mathrm{lb}$.

$\times \quad$,

168 Butter method

$169 \mathrm{Eggs}$ best collection, fowls, ducks or tarkeys

Exhibits in Classes 163-166 and Class 168 must have been prepared by exlibitors. 


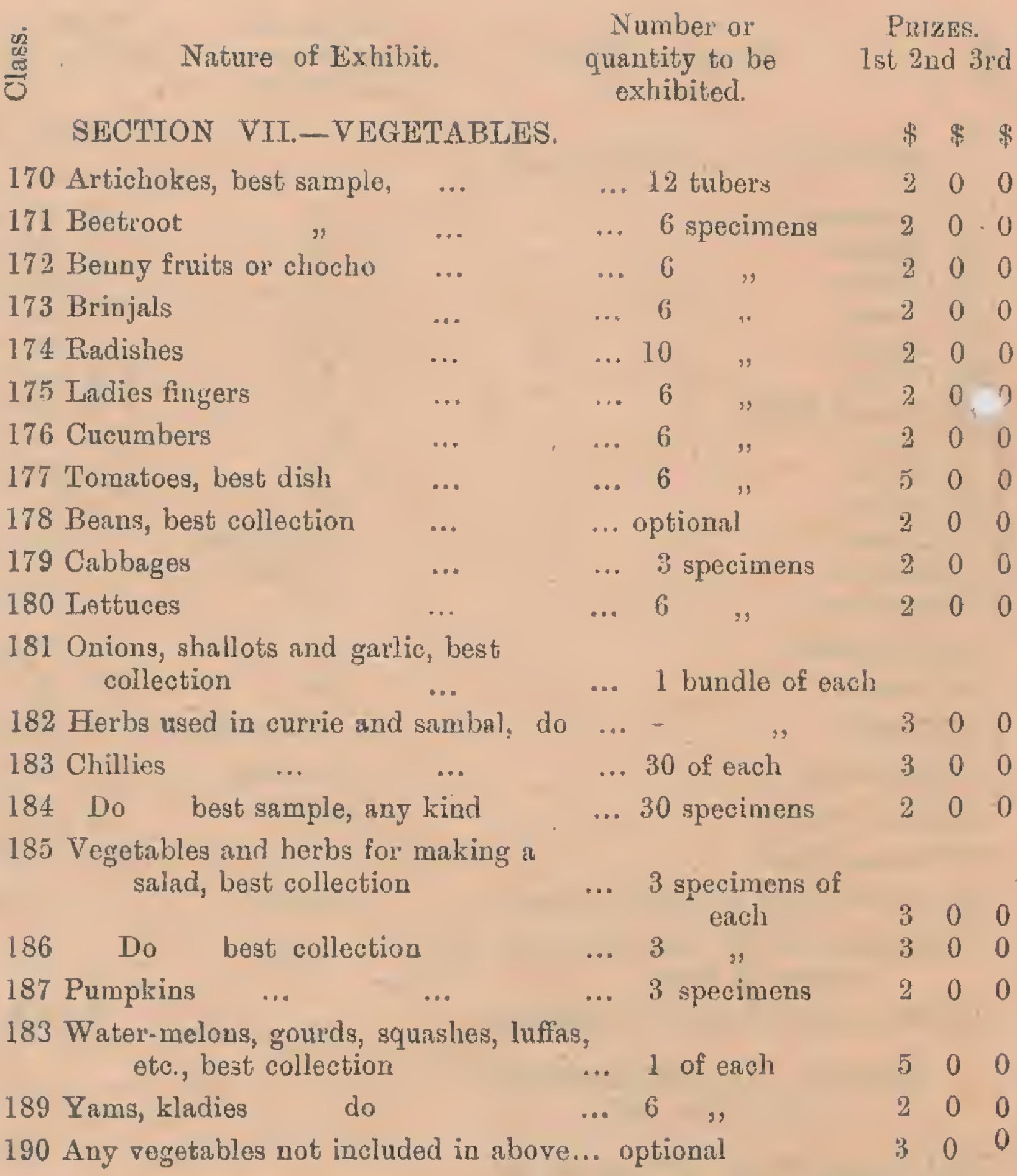

All exhibits in this section must have been grown by Exhibitor.

(a) Prize presented by J. W. Stokes.
(b)
Do.
Gan Gnoh Bee, Esq.
(c)
Do.
F. Duxbury, Esq.
(d)
Do.
Cheah Choo Yew, Esq.
(e)
Do.
W. Hutson, Esq.
$(f)$
Do.
'I. Gawthome, Esq.
(g)
Do.
Gan Gnoh Bee, Esq. 


\section{DIVISION C.}

\section{CATTLE AND POULTRY.}

PRIZES. quantity to be exhibited 1st 2 nd $3 \mathrm{rd}$ $\$ \$ \$$

SECTION I-CAIILE.

191 Bull (locally bred)

192 Cow and calf (locally bred)

103 Bull (imported)

194 Cow and calf (imrotted)

195 Pair of draught Bullocks with cart, Indian

196

Do

do

$\begin{array}{ll} & \ldots \\ \ldots & \ldots \\ \ldots & \ldots \\ \text { caurt, Indian } & \\ \text { Siamese } & \ldots\end{array}$

197 Champion animal in section

\author{
...
}

198 Best animal for Butchers' purpose

SECTION II.-BUEFALOES.

199 Malay buffalo, bull

200 Do do cow SECIION TII - PIGS

201 Boar

$202 \mathrm{Sow}$

203 Best pen of 6 pigs

204 Ram goat

205 Ewe do with kids

206 Sheep Raw

207 Do Nwe

208 Best pen of 6 sheep

SECTION IV.-POULTRY, ETC.

209 Bantam cock and hen

210 Malay

do

2II Do game cock and hen

2 I2 Collection of Malay fowls

2 I3 Collection of Chinese fowls

2 I4 Do fowls, anv hreed

215 Pair of Muscovy ducks

2 I6 Do ducks, any breed

217 Do do liybrid

2 I8 Gander and goose

219 Pair of pigeons

220 Best domesticated wild bird

22 I Rabbits Buck and Doe

222

(a) Prize presented by A. O. Merican, Esq.
(b)
Do
(c)
Do
The Penang Butchers.
(d)
Do
(e)
Do
(f)
Do
(g)
Do
Yerm Kee, Esq.
Ah Khoon, Esq.
The Penang Butchers. Do
The Iudian Mutton Trading Co.

$\begin{array}{llll}\ldots & 10 & 5 & 0\end{array}$

$a$ cup $0 \quad 0$

.. $10 \quad 5 \quad 0$

.. $10 \quad 5 \quad 0$

$\begin{array}{llll}\cdots & 10 & 5 & 0\end{array}$

$\begin{array}{llll}\ldots & 10 & 5 & 0\end{array}$

b 25

c 25

$\begin{array}{llll}\ldots & 10 & 5 & 0\end{array}$

... $10 \quad 5 \quad 0$

i 25

e 25

f 25

... $10 \quad 5 \quad 0$

.. $10 \quad 50$

.. $10 \quad 50$

... $10 \quad 5 \quad 0$

g 25

$\begin{array}{llll}\ldots & 5 & 3 & 0\end{array}$

... 5530

.. 530

530

$\begin{array}{lll}5 & 3 & 0\end{array}$

530

$\begin{array}{llll}\ldots & 5 & 3 & 0\end{array}$

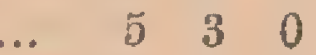

$\begin{array}{llll}\ldots & 5 & 3 & 0\end{array}$

$\begin{array}{llll}\cdots & 5 & 3 & 0\end{array}$

‥ $\begin{array}{llll}\cdots & 5 & 3 & 0\end{array}$

$\begin{array}{llll}\ldots & 5 & 3 & 0\end{array}$

... $53 \quad 30$

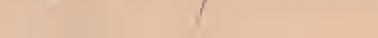




\section{DIVISION D.}

\section{HORSES AND DOGS.}

Class.

223 Polo Pony, 14-2 or under, tho bond ficle property of a nuember of any recognised Polo Club in the Colony or the Federated Malay States. To be showu under saddle and tested will stick and ball if required.

1st Prize Cup presented by Stephen Anthony, Esq

2nd Prize Silver medal

224 Gentleman's Hack, \$4-2 and under, to be shown muder sadule

1st Prize Cup presented by Cecil Guimess, Esq

2ud Prize Silver medal

225 Gentleman's Hack, over I 1-2, to be shown under saddle

1st Prize Cup presented by Hon. A. R. Adams

226 Lady's Hack over I4-2, to bo shown under saddle and ridder by a Lady

1st Prize Cup presented by G. H. Lees, Esq

2nd Prize Silver medal

227 Lady's Hack 14-2 and under, to be shown under saddlo and ridden b甲 a Lady

Ist Prize Cup presented by D. Gilchrist, IEsq

2nd Prize śliver medal

298 Best Gharry 'furnount and Pony, 14-2 and under, to bo shown in harness

1st Prize $\$ 20$

2ud Prixe $\$ 10$

229 Single harness Pony 14-2 and under, to be shown in harness, any description of velicle

Ist Prize Cup preseuted by Lawrence Aroozoo, Esq

zud Prize Silver medal

230 Single harness Horse over 14-2, to be shown in harness, any description of vehicle

1st Cup presentad by Luwrusce Aroozoo, Esq

2ud Silver medal 
Class.

231 Registered native bred Gharry Pony of any description : used for hire and the bona ficle property of a licensed Gharry owner.

1st. Prize. $\$ 20$.

2nd. Prize. \$10.

232 Jumping Pony 14-2 and under, to be riddeu over at least four jumps. Judging on performance only.

lst Prize. Cup presented by J. G. Berkhuijsen, Esq.

2nd Prize. Silver medal.

¿33 Jumping Horse over 14-2, to be ridden over at least four jumps Judging on performance only.

lst Prize. Cup prosented by Mr. Jamieson.

and Prize. Silver medal.

234. Double 'Turnout, of any description, pairs, tandems, etc.

lst Prize. Silver medal.

2ud Prize. Bronze medal.

235 Shandrydan 'l'ournout, the bond fide property of a licensed Shaudrydan owner.

Ist Prize. \$25 presented by Lawrence Aroozoo, Esq.

236 The Horse o1 Mare over 14-2, wimning the highest number of marks in competitions at the Bhow, to be shown on the halter.

Cup presented by J. F. Wreford, Esq.

237 'The Horse of Mare 14-2 and under', winning the highest number of marks in competitions at the Show, to be shown on the halter.

lst Prize. Cup presented by the Turf Club.

238 The best native bred Horse or Mare any age, over 12 hands, to be shown on the Lalter.

1st Prize. Silver modal.

2nd Prize. Bronze medal.

\section{DOGS.}

239 Smooth-hared Fux Terrier Dog 240 Smooth-haired Fox Texrier Bitch 241 Rough-haired Fox Terrier Dog 242 Rough-haired Fox 'L'errier Bitch 243 Chow Dog 244 Chow Bitch lst Prize Silver medal Bronze medal Do Do Do Do Do 2nd Prizo

do

do

do

do

do 
DOGS. $\rightarrow$ contrl.

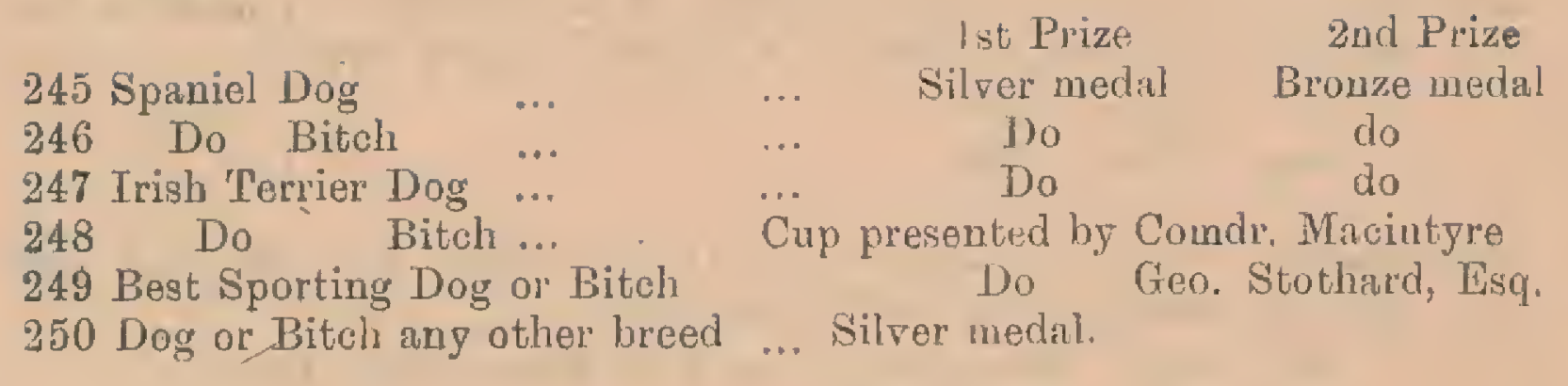

Entries close July, I9th.

DIVISION E.

\section{NATIVE ARTS AND INDUSTRIES.}

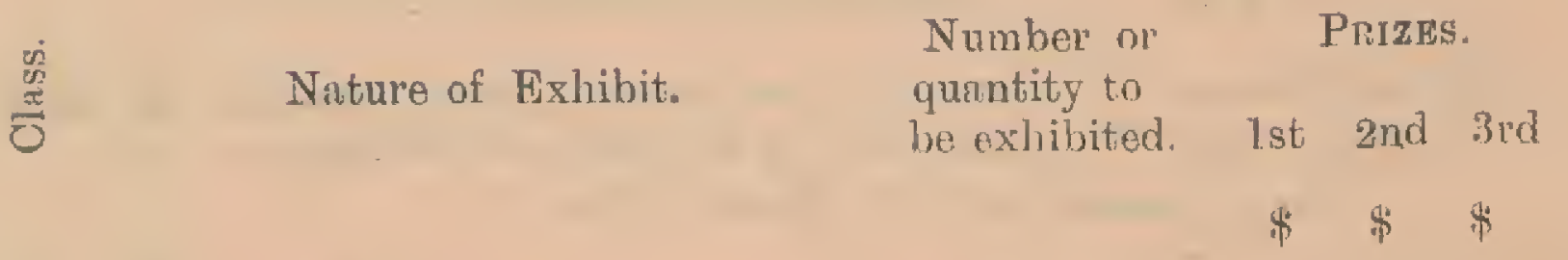

SECTION 1.-METAL WORK.

251 Collection of Malay

not more than

$$
\text { jewellery }
$$

252. Pinding, any metal

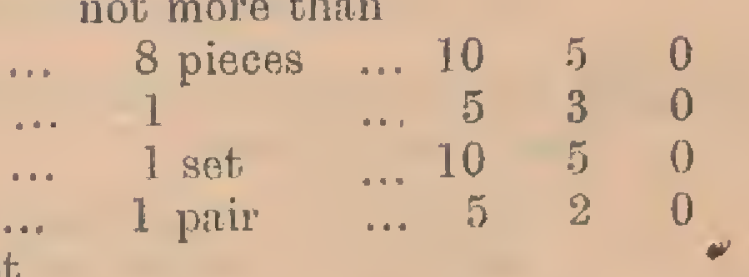

253 Sireh requisites, any metal

254 Muka bantal, gold suasa or silver ...

255 Ornamental boxes, any metal except

$\begin{array}{lllllllrrr}\text { tin } \ldots & \ldots & \ldots & 1 & & \ldots & 7 & 3 & 0 \\ 256 & \text { Trays and bowls } & \ldots & \ldots & 4 & \text { pieces } & \ldots & 10 & 5 & 0 \\ 257 & \text { Krusang, gold suasa or silver } & \ldots & \text { l set } & \ldots & 5 & 3 & 0 \\ 258 & \text { Any kind of metal work not included } & & & & & \end{array}$ in above

259 - Kris with sheath.

$\begin{array}{llllll}\ldots & \text { optional } & \ldots & 5 & 3 & 0 \\ \ldots & 1 & \ldots & 5 & 3 & 0\end{array}$

260 Tumbok lada with sheath

261 Parang Do do

262 Golok Do do

263 Collection of Tin-ware

264 Malay gong

$\begin{array}{lllllll}\ldots & 1 & \cdots & \ldots & 5 & 3 & 0\end{array}$

$\begin{array}{llllll}\ldots & 1 & \ldots & 5 & 3 & 0\end{array}$

$\begin{array}{llllll}\cdots & 1 & \cdots & 5 & 3 & 0 \\ \cdots & 1 & \ldots & 5 & 3 & 0\end{array}$

... not more than $810 \quad 5 \quad 0$

... $1 \ldots+\ldots=5$ 
Number or quantity to be exhibited.
PRIZES,

1.st 2nd $3 r$ d

SECIION TI.-WOOD CARVING, ETC.

265 Wood carving

266 Model of a Malay house

267 Sot of models of snares and traps (land)

268 Set of models of fish traps and stakes (sea)

269 Set of models of fish traps and stakes (river')

270 Malay children's playthings, best collectiou

271 Best collection of Sakei articles

272 Best collection of Semang articles

273 Set of Malay nusical

\section{SECTION IIT.-EMBROIDERY}

instruments

AND WIA AVING.

274. Malay ombroidery, best

\section{specinen}

275 Chinese embroidery, best specimen

276 Embroidered slippers

277 Embroidesed muka bantal

278 Embroidered mat

279 Embroidered tudong saji

280 Malay lace

281 Surong (silk and gold) Tolepok

282 Sarong (mastuil)

283 Sarong (silk)

284 Silk for Malay baju, best piece

285 Best collection of varieties

286 Kain lepas of Malay weaving

287 Do telepok

288 Do pelangi

289 Do tudong kepala

290 Do seleudang

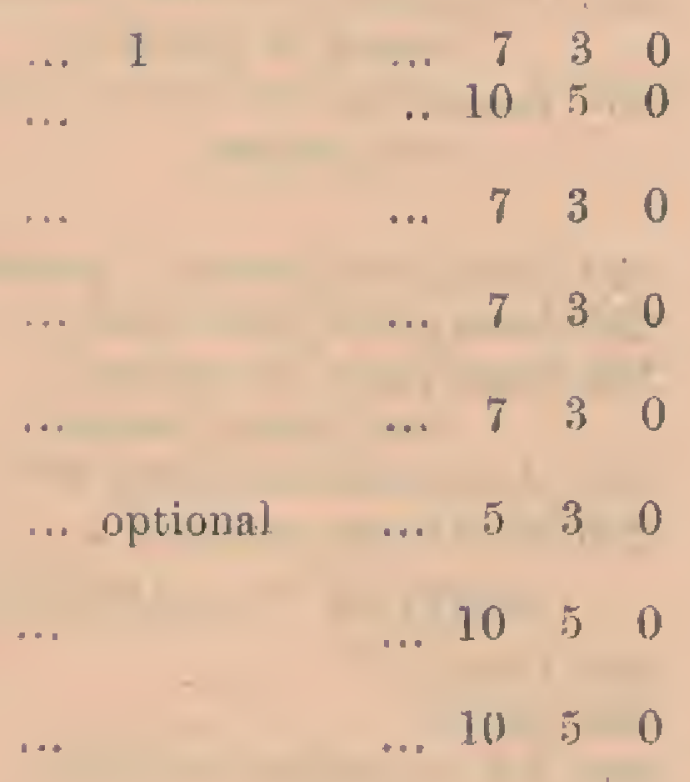

.. not more than 8530 


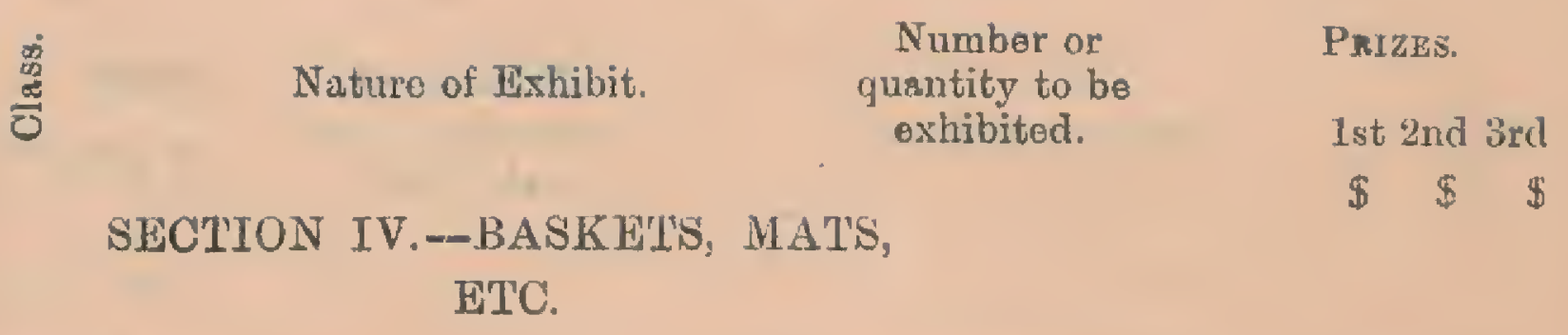

291 Ornamental baskets (rattan)

292 Ornamental baskets (mengkuang or pandau)

293 Best collection of articles raade from mengkuang

294 Huts (mengkuang or paudau) 295 Mats plain, house wear 296 Mlats plain, for drying

$$
\text { padi (tikal hampar) ... }
$$

297 Mats coloured, house wear

298 Mats fancy varieties not more than 4

$\begin{array}{lllll}\text {... articles } & \ldots & 5 & 3 & 0\end{array}$

530

... prize presented

by Hon'ble R. N. Bland J5 100

... $1 \quad 530$

... not more than $4 \quad 5 \quad 30$

$\begin{array}{cccc}\ldots 1 & 5 & 3 & 0 \\ \ldots \text { not more than } 4 & 5 & 3 & 0 \\ \ldots \text { not more than } 4 & 5 & 3 & 0\end{array}$

SECTION V.-MISCHLLANEOUS.

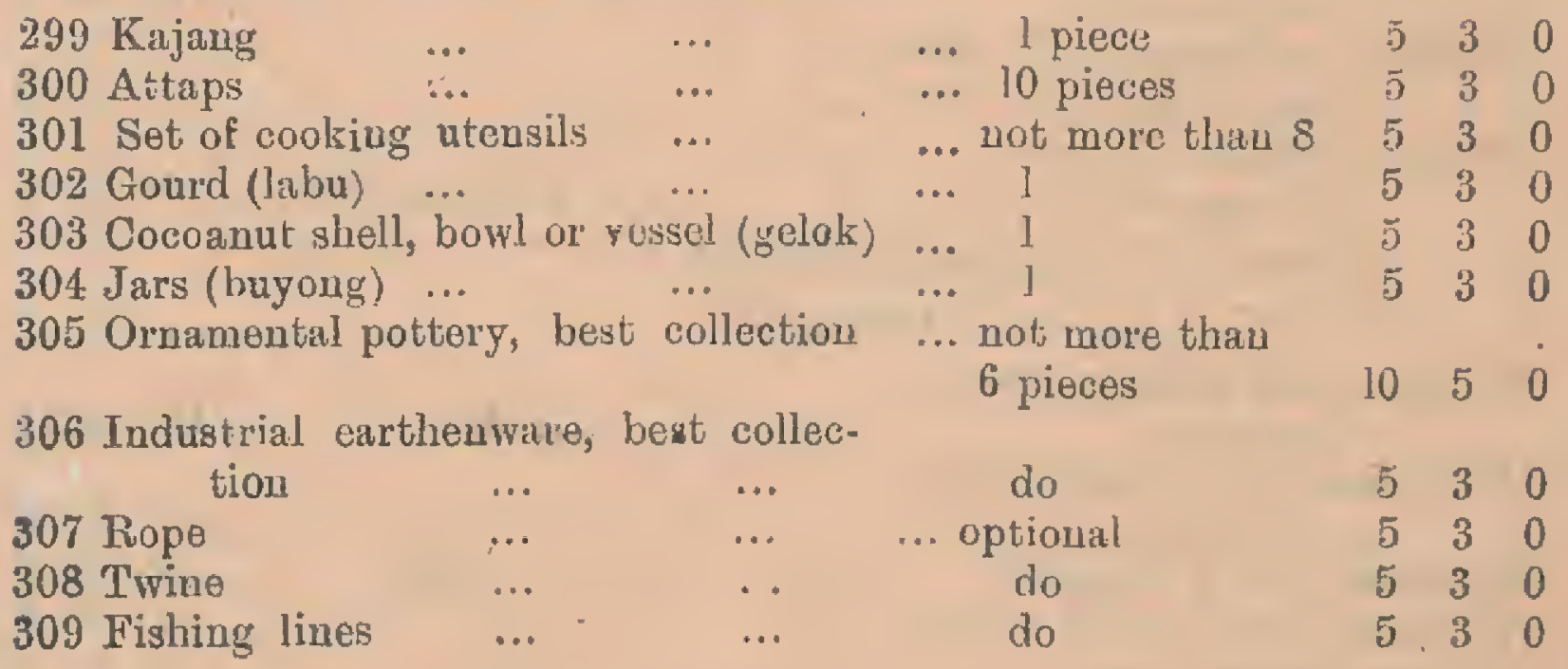

$N, B$-Articles for competition in all the above classes, except Class 285, must have boen mad within the last five years.

SECIION VI._FOR MALAY

SCHOOLS ONLY.

310 Malay pottery, haud-mado

311 Ornamental baskets

312 Malay mats

313 Sireh requisites

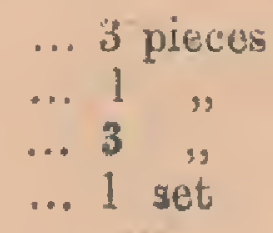

530

530

530

530 


\section{1}

意

$$
\text { Nature of Exhibit. } \quad \begin{gathered}
\text { Number or } \\
\text { quantity to be } \\
\text { exhibited. }
\end{gathered}
$$

Prizes.

314 Model Malay house

315 Wood-carving

316 Emoroidery

317 Sarong

318 Fishing nets

319 Collection of miniature Malay mindels

320 Best article of rattan furniture

... 1 ",

... 11

... 1 ",

... 1 p ece

... optional

1 st 2 nd $3 r$ d

$\$ \$$

$10 \quad 5 \quad 0$

$\begin{array}{lll}10 & 5 & 0\end{array}$

$\begin{array}{lll}7 & 4 & 0\end{array}$

$\begin{array}{lll}5 & 3 & 0\end{array}$

$\begin{array}{lll}5 & 3 & 0\end{array}$

321 Collection of models of Malay boats 1 "

322 Embroidered mat

... 1 ,

$1, \quad 1 \quad \ldots \quad 10 \quad 5 \quad 0$

All articles for competition in this section must be bond fide the work of pupils in Malay Schools.

Entries close August, 2nd. 


\section{DIVISION F.}

\section{Agricultural Implements and Miscellaneous.}

Nature of Exhibit.

323 Padi Implements (collection)

324 Agricultural Implements, other than for Padi or Rubber

325

Do Eurnpean made

326 Plongh suitable for Estate work ...

327 Reaping Machiue

328 Spraying Machine

329 Machine in convection with the preparation of Rubber to be exlibited at Work

330 Rnives, best collection for tapping Rubber 'l'rees

331 Bullock Cart (inproved)

332 Do. do. do.

333 Roofing tiles, locally made

334 Do. do. imported

335 Flooring tiles, locally made

336 Do. do. imported

337 Pottery Malayan, hand-made

338 Do. fancy

3:99 Baskets Agricultural ...

340 Casks, Tubs, ete.

341 Carriage four wheeled, locally made

342 Do, two do. do do.

343 Do. suitable for Estate work, locally made

344 Flower Pots

345 Garden Furniture,

346 Do. do.

347 Hack Gharry (improved construction), locally inade

348 Shandridan do. do.

349 Mining Implements ...

350 Miscellaneous
Number or quantity to be exhibited
$\$ \$ \$$

$\begin{array}{lll}10 & 5 & 0\end{array}$

(a) Cup S.MT.

(b) Cup S.M. S.M. B.M. $\begin{array}{lll}10 & 5 & 0\end{array}$ $\begin{array}{lll}10 & 5 & 0\end{array}$

(c) Cup S.M.

(d) Cup B.M. $\begin{array}{lll}10 & 5 & 0\end{array}$

1050

(e) Cup B.M . $\begin{array}{lll}10 & 5 & 0\end{array}$

11) 50

$10 \quad 50$

$10 \quad 5 \quad 0$

$\begin{array}{lll}10 & 5 & 0\end{array}$

$\begin{array}{lll}10 & 5 & 0\end{array}$

$\begin{array}{lll}10 & 5 & 0\end{array}$

S.M. B.M.

S.M. B.M.

S.M. B.M. 1050

(f) Cup S.M.

$10 \quad 5 \quad 0$

S.M. B.M, $10 \quad 50$

(a) Presented by Messrs. Sundilands, Buttery \& Co.

(b) Do. do. Riley Hargreaves de Co.

(c) Do. do. Huttenbach Bros \& Co.

(d) Do. C, E. Paterson, lisq.

(e) Do. J. R. Nicholson, Ksq.

(f) Do. Whiteaway, Laidaw \& Co.

Entries close August, 2nd. 




\section{REPORT OF THE PENANG AGRI-HORTICULTURAL SHOW.}

\section{Held on the 9th, 10th and IIth August, 1909.}

President-HON. R. N. BLAND.

General Purposes Committec-C. GUINnEss, GAN QUOH BEE, HON JOHN Turner, Dr. Jamieson, W. Langham CARThr, F. G. PigotT, S. E. A. Linton, P. A. YearwOOd, Elton Bell, W. H. MACARTHUR, B. NUNN, H. MUIR.

Chairman-A. CAVENDISH.

Hony. Treasurer-D. W. GILMOUR.

Hony. General Secretary-WALTER FOX.

The Sixth Annual Agri-Horticultural Show was held in Penang, on the gth, Ioth and IIth of August. The last Show here was held exactly four years ago and by a coincidence on the same dates.

The occurrence of two public holidays in the week fixed for the Show was the primary reason for the selection of these dates, a factor of considerable importance to the officials connected with the management of the Show and also to the public in general.

H. E. The Governor, Sir John Anderson. G.C.M.G., opened the Show in brilliant weather, and spoke at length on Agricultural topics and commented in detail on the advance, made since the previous Show.

The statistics given below set forth the advance that has been made, and justify the freely expressed opinion that it was the most successful Show of the series hitherto held.

2. The Prize Schedule was divided as before into the six following Divisions :--

Division (A) Agricultural Produce

87 Classes

(B) Flowers, Plants, Fruits and Vegetables.. I03

(C) Stock and Dairy Produce .. $\quad$. 32

(D) Horses and Dogs _. . 28

(E) Native Arts and Industries of which

13 Classes were confined to Schools .. 59

(F) Agricultural Implements \& Miscellaneous 30 "

3. The Show attracted I,935 Exhibitors as against I,54I in I905 who came from the following places:-

\begin{tabular}{|c|c|c|c|c|c|}
\hline Penang and Pro & nce & esley & . & .. & $\mathrm{I}, 00 \mathrm{I}$ \\
\hline Perak & .. & .. & . & .. & 322 \\
\hline Malacca & .. & .. & .. & .. & I 80 \\
\hline Negri Sembilan & . & $\cdots$ & . & .. & 267 \\
\hline Selangor & $\cdots$ & . & . & .. & $\mathrm{I} 34$ \\
\hline Brunei & . & . & . & . & 4 \\
\hline Singapore & $\cdots$ & . & * & . & 9 \\
\hline Kedah & . & .. & $\cdots$ & $\cdots$ & 6 \\
\hline Tohore & .. & . & $\cdots$ & - & 2 \\
\hline
\end{tabular}


4. The following table shows the number of Exhibitors in each Division, the place from which they came and the prizes gained:-

\begin{tabular}{|c|c|c|c|c|c|c|c|c|c|c|}
\hline & 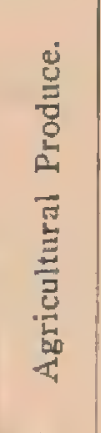 & 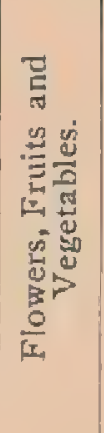 & 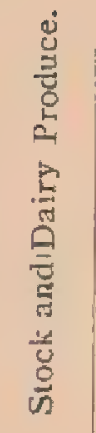 & 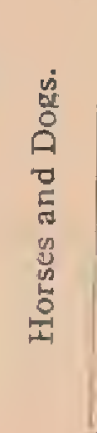 & 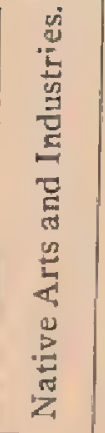 & 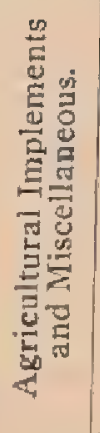 & & & & \\
\hline & A & B & C & D & E & F & Total. & $\begin{array}{c}\text { Prizes. } \\
\$\end{array}$ & Cups. & Medal: \\
\hline $\begin{array}{l}\text { Penang and Pro- } \\
\text { vince Wellesley }\end{array}$ & 329 & 236 & 147 & 82 & 183 & 24 & $I, 00 I$ & 1,206 & 17 & $4 I$ \\
\hline Perak .. & 130 & 67 & 27 & 2 & 88 & 8 & 322 & 701 & . & 4 \\
\hline Malacca & 79 & 47 & 9 & $\cdots$ & 43 & 2 & I 80 & IO4 & . & . \\
\hline Negri Sembilan & 83 & 32 & I3 & I & I 37 & I & 267 & $6 I$ & .. & . \\
\hline Selangor & 63 & 33 & 3 & $\cdots$ & 35 & .. & I 34 & 89 & 5 & 4 \\
\hline Singapore & 2 & . & .. & 7 & $\cdots$ & .. & 9 & ro & 4 & 3 \\
\hline Brunei .. & 2 & . & $\cdots$ & $\cdots$ & 12 & .. & 14 & 42 & . & - \\
\hline Johore... & 2 & . & .. & . & . & .. & 2 & .. & I & . \\
\hline Kedah .. & . & . & . & 5 & I & . & 6 & . & I & 2 \\
\hline Total .. & 690 & 415 & 199 & 97 & 499 & 35 & $\mathbf{I}, 935$ & $\$ 2,204$ & 28 & 54 \\
\hline
\end{tabular}

5. The figures work out to about the same proportion, as regards the percentage of prizes gained to the number of competitors as was the case at the previous Show, Penang and Province Wellesley, heading the list with $54 \%$ of the prizes and $51 \%$ of competitors.

6. The Show was patronised to an unprecedented extent as shown by the gate money which amounted to $\$ I, 030.69$ on the first day, $\$ 808.96$ on the second day, and $\$ 460.65$ on the third day. The prices of admission were $\$ 2$ for the opening ceremony on the first day up till 2 p.m., afterwards 25 cents. The charge of admission on the second day was 25 cents and on the third day ro cents. The second day presented an unusually animated appearance when school children of all denominations were admitted free.

7. Housing of Native Exhibitors. - The Committee were fortunate in being allowed to use the barracks till lately occupied by the F.M.S. Guides overlooking the Show grounds to accommodate Native Exhibitors and their families and thus what at one time seemed to be a difficult problem to solve was rendered comparatively easy. The 
thanks of the Committee are due to Messrs. Thomas and Hamilton for the excellent arrangements made for transporting native visitors with their Exhibits to and from the Show grounds.

Mr. Thomas organised and supervised a special tram service from the Jetty to th: main entrance to the Residency whilst Mr. Hamilton spent a good deal of time and trouble in meeting and seeing off relays of native visitors.

8. The thanks of the Committee are again due to the Government of the F.M.S. and the Railway authorities for granting free transport to and from Penang over their lines to the numerous Exhibitors and Exhibits. But for this assistance it is doubtful whether half the number of natives who came would otherwise have attended.

The Straits Steam Ship Company were good enough to grant a rebate of $25 \%$ off the freight of all bona fide Exhibits whilst Messrs. Huttenbach, Liebert and Company kindly offered 50 free deck passages to and from Port Swettenham by the s.s. "Kistna."

9. Finances.-The usual contribution of $\$ 2,000$ each from the Governments of the Colony and F.M.S. were given and a sum of $\$ 1,000$ was voted from the Penang Municipal funds which together with a balance from the last Penang Show of $\$ 748.65$ formed a nucleus of $\$ 5,748.65$. The subscriptions amounted to $\$ 5,337.7 \mathrm{I}$ and Silver Cups to the value of $\$ 600$ were generously presented by certain Associations, Firms and private individuals.

At first the subscription came in very slowly but later the total receipts came to $\$ 1 \mathrm{I}, 086$ which was considered sufficient to cover all expenses. The Committee wish to thank the Penang and Province Wellesley public for their generosity and support. It should be added that had the Committee known earlier that such a magnificent sum would have been at their disposal, much improvement in the way of arranging the Exhibits could have been effected, thus the somewhat ugly rectangular sheds for housing Native Industries would have been discarded for a series of sheds for the different states so as to have presented a more realistic view of the life and industries of the natives; perhaps the best shape of sheds for this Division would have been circular or octagonal with radiating avenues. The central building could be reserved for articles of common manufacture in the several states, whilst the avenues could be devoted to each of the different states, or might have been set apart for industries peculiar to any particular State or District, such an arrangement would not only be picturesque but would foster emulation and competition in rival industries.

I0. Buildings.-The buildings were on a much more extensive scale than before; whereas at the former Show the floor area was 45,427 square feet, the area on this occasion was no less than $7 \mathrm{I}, 999$ square feet. The arrangement of the buildings were also a great improvement; they were erected around the sides of the Parade Ground in a continuous scries, whilst the upen space in the centre 
served as the ring for competition in the Horse Classes. For intormation the following table shows the sizes of the sheds for each Division, and the cost for the whole :-

\begin{tabular}{|c|c|c|c|c|}
\hline \multirow{2}{*}{\multicolumn{2}{|c|}{ Secretary's Office }} & & & Square \\
\hline & & .. & .. & 450 \\
\hline \multicolumn{2}{|c|}{ Ticket Boxes at Entrance } & . & .. & 72 \\
\hline \multicolumn{2}{|c|}{ Flowers, Fruits and Vegetables } & . & . & 10,665 \\
\hline Trade Section & .. & .. & .. & 21,000 \\
\hline Poultry and Birds & $\cdots$ & . & .. & $I, 080$ \\
\hline Dogs & .. & $\cdots$ & .. & $\mathrm{I}, 080$ \\
\hline Buffaloes and Cattle & .. & .. & .. & 3,072 \\
\hline Sheep & . & .. & .. & 720 \\
\hline Pigs & . & . & .. & 2,860 \\
\hline Native Industries & . & . & .. & 15,000 \\
\hline Agricultural Produce & . & . & .. & 16,000 \\
\hline & & & & $7 \mathrm{I}, 999$ \\
\hline Including cost & of above & .. & . & $4,634.90$ \\
\hline
\end{tabular}

The course for exhibiting horses, ponies and carriages measured 145 feet along its parallel sides and the ends were connected by semicircles of 80 feet radius, the total distance round being nearly 550 feet.

Much credit is due to Mr. S. E. A. Linton, of the P. W. D., for the care and trouble he took in drawing the plans and supervising the erection of the buildings.

Nature and Quality of the Exhibits.-Speaking generally the standard of excellence was equal to that of former Shows, and in some cases far exceeded it. Taking the Divisions in their order it will be useful to note the points of interest and improvement shown.

\section{DIVISION A.}

12. Agricultural Produce--This Division was in charge of $\mathrm{Mr}$. P. A. Yearwood as Honorary Secretary, and whether looked at from the number of exhibits, or their importance, easily stands out as the back bone of the Show. In the 87 classes no less than 690 competitors took part. Foriconvenience of classification it was divided into the following Sections: I. Paddy, II. Rubber, III. Other Food Products, IV. Spices, V. Oil Cakes, etc., VI. Getah Gums and Resins, VII. Fibres and Miscellaneous. In almost every class the keenest competition took place, and especially in the staple products of rubber, rice, cocoanuts, copra and tapioca. Undoubtedly the most important was the Rubber Section in which magnificent samples were on view.

It is unfortunate, however, that in several instances competitors did not comply with the conditions laid down, and thus two magnificent-cups offered for competition were not awarded by the Judges. 
It would perhaps be better in arranging the Prize Schedule for future Shows, to offer at least three prizes in each class, and to ask the Judges to specify the number of marks gained by the Ist, 2nd and 3rd prize Exhibits respectively, to indicate the Exhibits in their order of merit. It would certainly be more satisfactory to competitors, and also more interesting for the public, to learn the relative value of the different Exhibits, for instance when only one prize was awarded there was no means of knowing the comparative excellence of the next Exhibit in merit, which might possibly have been only slightly inferior to that gaining the first prize. The method of judging by points would enable one to see how the winning Exhibits in rubber for instance, obtained their marks, whether for strength and resiliency, colour, uniformity, and so on. A most instructive rubber Exhibit was sent by Messrs. Behn, Meyer and Company, as Agents, consisting of a series of long sheets which had been coagulated by the new coagulant called "Purub." The merits claimed for this method of preparation are its rapidity of action (the specimens shown had been rolled up six hours after coagulation) and the fact that it is at better preservative, and does not in any way injure the quality of the rubber. The F.M.S. Agricultural Department are to be congratulated on the excellent and instructive series of Exhibits staged by them, consisting of an Entomological Section, showing the noxious termites in their various forms; specimens of their nests, and sections of trees riddled by them. Adjoining was the Mycological Section showing various plant diseases, especially those which attack rubber. Examples of actual trees showing bad, and good, methods of tapping and the results of each were on view. Close by were collections of fibres, oils, and a small camphor still at work. The next Exhibits in value to the rubber were the cocoanuts. These as usual were sent in vast numbers, 2I3 Exhibitors showing in one or other of the four classes; Perak carried off most of the Prizes. Copra, which has been in such great demand lately, with steadily rising prices, and for which an improved method of preparation has been suggested, was very well represented, and srme very good samples were shown. The common fault, however, was noticed that some of the samples had been made from unripe, and others from badly dried, nuts. A small sample sent from Ceylon for comparison was of good quality, but received no award from the Judges. Tapioca was well shown, and contested, the first prize being easily won by the Manager of Malakoff Estate. Cloves and Nutmegs were sent in by the Penang growers in excellent samples, and had no difficulty in excelling those sent from other places. Penang Exhibitors were also successful in another important Section, Paddy, in which they more than held their own. It was disappointing to see the few poor specimens sent from Krian District, where the finest rice in the Peninsula is grown, under an irrigation scheme carried out by Government at great expense. The other Exhibits such as Oils, Pepper, Arrowroot, Dragons-blood, Gambier, Gums, Dammar, Tree Cotton, and Coffee were all more or less good in quality and quantity but call for no special remarks. 
DIVISION B.

13. Flowers, Fruits and Vegetables. - This Division which held at most prominent position just at the Entrance Gate was in charge of Mr. S. E. A. Linton who must be congratulated on its very neat and effective arrangement. It may be said that so far as the plants and flower sections are concerned it was the best display that has as yet been seen. At the entrance to the buildings (which was in the form of a cross) and on both sides some fine groups of plants sent by the Botanic Gardens, not for competition, were most artistically arranged, whilst central tables filled with flowering plants, such as Dahlias, Cockscombs, Chrysanthenums, Asters, Orchids, etc., made a picturesque vestibule to the competitive groups. The collection of vegetables from the Government Gardens on the Perak Hills was again an attractive feature, occupying the central portion of the building, the various vegetables being all excellently grown. The plants in the competitive classes were numerous and on the whole fairly well-grown-flowers, including table decorations, were good Considering that so far as Penang was concerned the fruit season was practically over, the Fruit Section generally was well filled, the Dukus, Chikus, and Mangoes being the best. Enormous numbers of limes were staged, and Pisangs were also largely shown, one Exhibitor showing no less than 52 varieties. The Section for Preserved Fruits was also good: Mr. Alexander's fine collection of Chutneys, Pickles, and Jellies being far the most prominent Exhibit of this kind.

\section{Division C.}

14. Cattle and Ponltry.-This Division was in charge of $\mathrm{Mr}$. Elton Bell; speaking generally, the cattle were more numerously shown than at the previous Shows. Pigs, Sheep and Goats were about the same in number as on previous occasions. The Section for poultry, however, was the outstanding feature of this Division; no less than 290 Exhibitors entered. The standard of excellence on the whole was not very high, there were few excellent birds, and in many cases, ordinary fowls were sent in that stood no possible chance of gaining a prize.

\section{DIVISION D.}

15. Horses and Dogs.-This popular Division was in charge of Mr.W.H. MacArthur, and was very well patronised. Mr. Macmillan's fine English horses sent up from Singapore carried away first honour's with the utmost ease, but notwithstanding this, competition in several classes was keen. Dogs were well shown and some splendid animals were sent in.

\section{DIVISION E.}

I6. This is the second largest Division and probably the most interesting, was in charge of Mr. B. Nunn ably assisted by Mr. G. A. Hall; they had to deal with 499 Exhibitors whose Exhibits were more than usually representative. The Exhibits coming from all places, from Brunei on the one hand to Kedah on the other, in most 
cases filled the various classes; and the curious and ornate workmanship of some of the Exhibits was fully equalled by their artistic designs. The Silver and Bronze Metal work from Brunei, the tin ware from Seremban, the Rambong baskets, and the delicate lace work from Malacca, and the rattan fumiture, and carving from the Schools, went to make up a Show of surpassing interest. The display of weapons, mats, and embroidery generally was very good indeed, and although there were few or no Exhibits of an entirely novel character, still the Division was more than equal either in quantity orquality to anything previously seen.

\section{DIVISION F.}

I7. Agricultural Implements and Miscellaneous.-This Division was under the care of Mr. H. Muir, and covered a wide field of Exhibits from tiny models, to steam ploughs and motors. Some classes had no entries but on the whole the Division was far better represented than formerly. Amongst the most notable Exhibits were the carriages from Messrs. Chin Seng and Company, Penang. The garden furniture consisting of trellis work, chairs, tables, rustic arches and a band stand, manufactured by the Eastern Shipping Company, and the coloured concrete tiles made at Batu Feringgi by Messrs. Huttenbach, Leibert and Company.

18. The Trade Section.-This covered a floor area of 21,000 square feet, and extended the whole lengtl of Residency Road. It is difficult to say what articles could not be found among the various firms who exhibited, perhaps the most interesting were the Electrical and Machinery appliances.

19. Judging. -27 Judges were appointed by the Committee, they were assisted by Native experts in the difficult matter of judging the Paddy classes; and by Rajah Puteh who kindly gave her valuable advice in the Malay Embroidery Section. The Judges are to be congratulated on their decisions, which appear to have given general satisfaction, and the Committee tender their thanks to them for the great labour involved in carrying out their arduous duties.

20. Standing Committee.-The Standing Committee met on the second day of the Show, they decided that the Show be held in Singapore next year. Their attention was also directed to various suggestions for improving the usefulness of future Shows, notably in the matter of having Conferences on Agricultural subjects. It was suggested that Papers should be read on interesting topics, and experts appointed to personally conduct Exhibitors and others round the Show and explain the merits and other characteristics of the winning Exhibits.

21. Protection.-Unfortunately the Committee were without the valuable assistance of the F.M.S. Guides, who on a former occasion did such excellent service in guarding the Show by night. The police, however, carried out this difficult task satisfactorily, notwithstanding the few thefts that were reported. 
22. Presentalion of Cups and Medals.-Miss Anderson kindly presented the valuable cups offered for competition; and the 36 Silver and 24 Bronze Medals, at the close on the third day, thus bringing to an end certainly one of the most successful Shows of the series.

In conclusion the Committee take the opportunity of thanking all those ladies and gentlemen, who in their various capacities worked with such energy and good-will, and without whose hearty co-operation the Show would not have been such a great success as it was by general consent pronounced to be.

W. Fox,

Hon. General Secretary.

\section{Statement of Receipts and Expenditure.}

\section{RECEIPTS.}

I. BY S. S. Government Grant

2. „ F. M. S. Government Grant ..

3. " Municipal Grant

4. "Balance from last Show, Penang

5. "Subscriptions

6. "Gate Money

7. " Charges for Space (Trade Section)

8, " Refund per Bank Account .. .

9. , Interest to 30th June, Ig09 ..

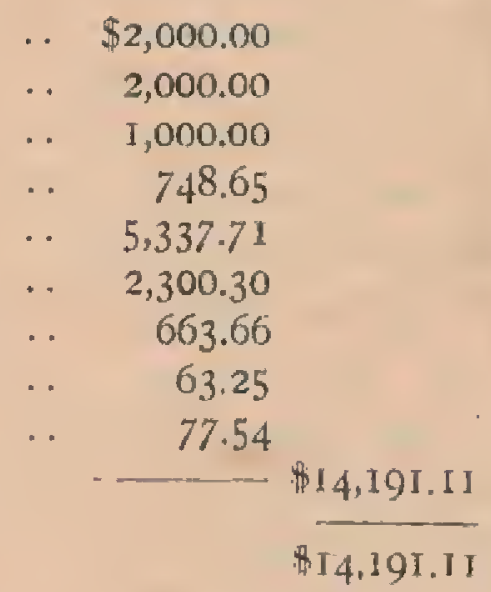

EXPENDITURE.
1. To Erection of Show Buildings ..
. $\$ 4,634.90$
2. " Amount of Prizes .. ..
. $\quad 2,204.00$
$\begin{array}{cccccc}\text { 3. }, \text { Cost of Medals } & \ldots & \ldots & \ldots & 379.05 \\ 4 . & , & \text {, Silver Cups } & \ldots & \ldots & \text { 105.78 }\end{array}$
5. "Printing, Advertisements \& Stationery 1,070.68
6. ", Transport and Subsistence Money .. I,457.63

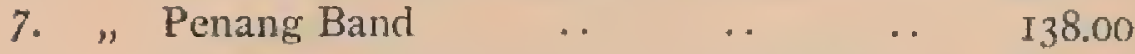
8. "Exhibitors' Expense and Compensation $\quad 583.39$

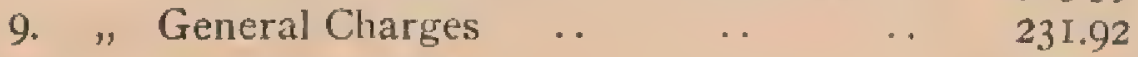

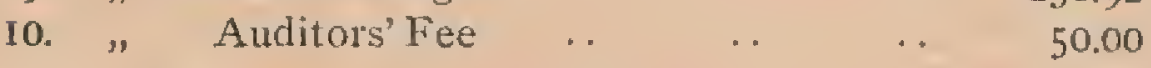
II. " Balance in Cliartered Bank

We have examined the Vouchers and Bocks connected with the Agricultural Horticultural Show, I909, and the ahove is, to the best of on belief, a true and correct account of the same.

DAVID BROWN \& CO., Auditors.

D. W. GILMOUR, Hon. Treasurer. 


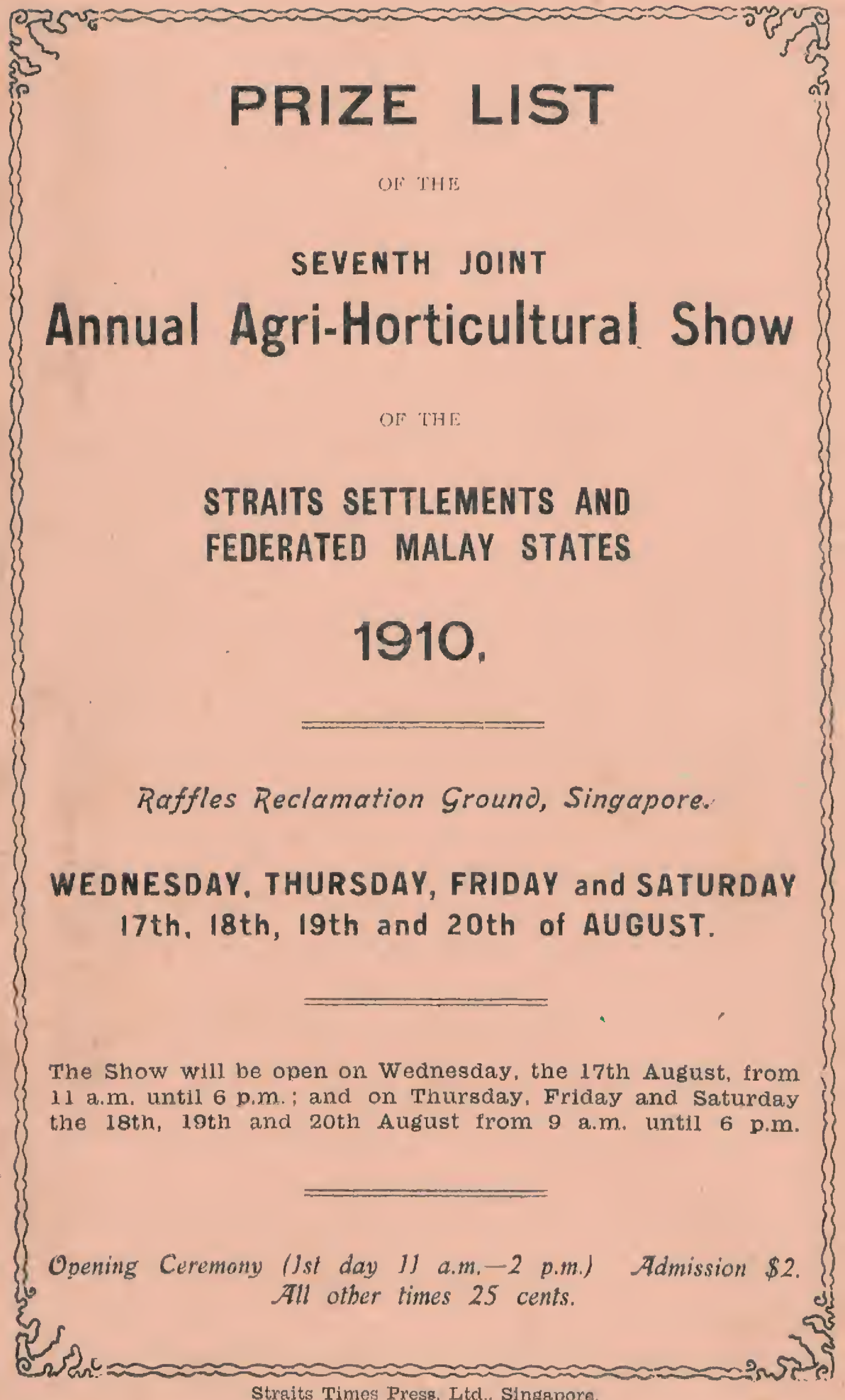





\section{Datrons.}

His bxcellency 'the Governor and High CoumissionerThe Resident-General. Federated Malay States

H. H. The Sultan of Perak.

H. H. The Sultan of Selangor.

H. H. The Sultan of Pahang.

H. H. The Sultan of Tohore.

H. H. The Yang Di Pertuan Besar, N. Sembilau.

H. H. The Sultan of 'Trengganu.

H. H. 'The Sultan of Brunei.

H. H. The Rajah of lielantan.

H. H. The Sultan of Kedah.

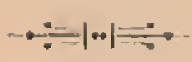

\section{Dresident.}

The Hou: The Colonial Secretary, S.S.<smiles>[CH]=[N+]=[N-]</smiles>

\section{Vice= Presidents.}

H. E. The General-OHicer Commanding the T'roops. The Hon: The Chief Justice.

The Hon : The Resident Councillor, Penang.

The Hon: 'The Resident Councillor, Malacea.

The British Resident, Perak.

The British Resident, Selangor.

The British Resident, Negri Sembilan.

The British Resident, Pabang.

'The President, Municipal Commissioners, Singapore-

The President, Chamber of Commerce. Singapore. 


\section{standing Committec.}

\section{জับinapote.}

The Birector of Botanic Gardens, S.S. (Hon : Secretary).

'The Curatci, Botanic Gardens, Singapore.

The Collector of Land Revenue, singapore.

$$
\because 1 \cdots
$$

\section{frenang and porotince Zdellesley.}

"We Senior District Officer. P. W.

The Collector of Land Revenue (Hon : Sceretary).

(i. A. Hall, Esq.

$$
\therefore=101 \div
$$

\section{Minlacen.}

The Hon : The liesident Comncillor (Hon : Secretary).

The Collector' of Land Revenuc.

$$
\therefore|\cdot|=
$$

\section{Derak.}

The Director of Museums, F. M. s.

The sunerintendent, Government Plantations.

T'he District Officer, Kuala Kangsar.

The District Officer, Larut and Krian, (Hon : Secretary).

$$
\div-\cdots \mid=
$$

\section{जelanger.}

The Collector of Land Revenue. Kuala Lumpur.

The Chairman United Planters Association.

The Assistant to the birector of Agriculture (Hon : Secretary).

$$
\therefore 1 \cdot 1=
$$

\section{Hacari gembilan.}

The District ()ticer, 'L'ampin.

"The Collector of Land Revenne. Seremban, (Hon : Secretary). "I'he Chairman, Negri Senbilan J'lanters" issociation.

$$
\therefore|\cdot| \div
$$

\section{Pabang.}

The senior Magistrate.

The District ()flicer. Raub.

The District ()fficer, Pekan, (Hon : Secretary.) 


\section{ffimance and General Purposes Committee.}

E. G. BHoAmRILK.

'The Hon: The Colonial Secretary, S.S. Chairman.

The Hon. 'lax. Juk lian.

F. E. . TAGO.

W. PATChI'T.

D. T. Boyı,

W. MaKHPHCH.

Syli Onar Alsagohl.

H, SPAKLER.

A. D. Asmus.

E. II. TลNTUx.
R. Scouldi.

O. A. Kinguta

ST, V. B. DOWR.

A. G. Hatidgaton.

F. HILTON.

H. ELPHTEt,

M. RUDESSF.

A. D. ALIAN.

A. W. BHAs:

IV. IV. Cook.

A. Wr. S'TITL.

\section{Jouildoing Committee.}

The Hon: F, J. Pitotri (Chaiman)

R. Scoular (Hon: Sec:).

M. W. PE'T',

F. HiLton.
R. Princr.

I. H. Garrite

IV. B. DRден.

R. A. T. BIDWhLt.

\section{ฐub=conmmittees.}

$\therefore \mid-1 \equiv$

Division $\mathfrak{z}-\mathcal{E}$ gricultural Proonce.
H. N. RidLMY,
E. Bramall.
C. E. Craiti.
R. T. Andorti.

O. A. Jinnum (Hon: Sec.)

W. N. GaWLLR.

A. D. Machados.

P. Cuntifle.

Choa Giangr Thyt.

\section{$\because \cdots \cdots$ \\ Division is-fints, jflowers allo vegetables. \\ ST. V. B. Down (Hon: Sec.) THE REv. H. C. IFARD. The Rev. Father Gex. W. MaCbrax.}

The Hon: J. O. Anthosisz.

Dr, Gilmoru Exis.

F. E. J $J_{(\div 0}$,

Seat Lranti Seah.

P. S. FaLshaW.

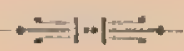

Division C-Gtock ano झुate Droonce.

P. S. FALSHAW。

Dr. W. R. C. MTHDLETUN.

W. E. HoOprit.
A. T. BRYANT.

Dr. P. Fowhik.

A. (H. Harringtor (Hon: Sec.) 


\section{4 \\ Division $\$$ - Borses ano Dogs.}
A. D. Allan.
F. Hilton (Hon: Sec., Dogs.)
D. K. Somerville.
H. FupHIck (Hon : sec. Horses.)
M. Surt.
G. E. T. Thomsin.
MAMOR H.YYS.
The Bon LALT.

H. H. H. EDLIN.

\section{$\because-1 \cdot 1 \div$}

Division E-Hative fnoustries and namufactures.

A. Gentle.

J. B. ELCUM.

Ong Teck Lim.

Low LoN' Teng.
W. C. Michell.

Rev. W. T. Cherry.

M. Rodesse (Hon : Sec.)

Tax Kexa Hoon.

\section{$\therefore=1 \cdot 1=\div$}

Division ff-Agricultual Fmplements ano Jiscellancous.

k. A. Siprests.

TAx hiM Hock.

R. Pejece.

'TH: Hon. DI. I. .T. G.LLoway.
A. D. Michado.

A. D. Allax (Hon: Sec.)

A. E. Bhoneley.

R. M. Goldis.

R. I. FARRER,

Howorary 'Treasurer.

II. W, MAIN,

Hou: General Secretary. 


\section{Rules and Regulations Relating to Exhibits.}

1. All exhibits must be bonk fide the property of the exhibitor.

2. Competition for prizes shall be open only to exhibitors resident in the Colony of the Straits Settlements or the States of the Malay Peninsula.

3. Competition in Divisions $A . B$, and E. shall be confined to exhibits grown or manufactured in the Colony of the Straits Settlements, or the States of the Malay Peninsula.

4. The Committee will appoint Judges, from whose decision there shall be no appeal.

5. The Judges will have anthority to withhold a prize when they are of opinion that there is not sufficient merit to justify an award, and also to award special prizes for anything not contained in the schetule.

6. No Exhibitor shall be awarded two prizes in any one class, or more than five prizes in the same division.

7. The arrangement of the exhibits shall be subject to the directions of the Committee.

8. The Committee reserve to itself the right to refuse any entries that they may think fit to reject, and to cancel any class at any time up to ten days previons to the first day of the Show.

9. Exhibitors desiring free transport by Railway must give notice to a I istrict Officer, or the General Secretary, who will, if he approves of their exhibits, provide them with numbered tickets and a free third class pass on the Railway for themselves and their exhibits. A reduction of 25 per cent. oft the usual freight will be made by the Straits Steamship Co. on all bona fulle exhibits. Other reasonable expenses in comnection with transport of exhibits will be considered by the Committee.

10. Entries in all Divisions must be in writing, and reach the General Secretary at least ten days before the opening day. In the case of Horses and Dogs three weels.

No exhibit in Divisions A. C. E. and F. will be received later than 6 p.x. on the day previous to the opening day.

Exbibits in Divisions $B$, and $D$. will be received up to 8 a.n. on the morning of the Show.

11. Entries for Horses under Classes 247, 248, 249, 250, 251, 252, 253, $254,25,256,257,259,260,261$ must be accompanied by a certificate of height from any Sporting, Turf or Polo Club in the Colony or F.M.S. or from a qualited Veterinary Surgeon. 
12. Exhlibitors in Divisions (4. and D. must proride for the care and maintenance of their own exhibits.

13. No exhibit (except live animals) may be removed from the ground before the close of the Show. Exhibitors will be provided with a card which must be produccd at the close of the Show belore they can be allowed to remove their exhibits.

14. The Committee is empowered to exclude, or remove, any animal which in its opinion is not in a fit state for exhibition.

15. Exhibitors in Divisions C. and D. will only be permitted to remore their auimals from the Show Ground on obtaining an order of removal from the General Secretary or the Fon. Secretaries of these Divisions.

16. All reasonable care will be taken to provide for the salcty and careful preservation of exhibits of every kind sent to the Slow but it must be clearly understood that the Committee does not hold itself responsible for the loss of, or the damage to, any exhibit either in transit or during exhibition. Provision will be made for leeping small articles of value nuder lock and key at night.

17. Space will be provided for tradesmen and otheis wishing to exhibit articles for sale, butnot for competition. The sale price must be clearly specified.

18. Government exhibits shall not be eligible for prizes in any class. Winners of prizes of 10 and upward. may have a silver medal if they prefer it to money.

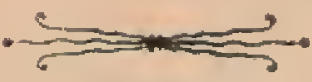




\section{DIVISION A.}

\section{AGRICULTURAL PRODUCE.}

Nature of Exhibit.

\section{SECTION I. -PADI.}

1 Padi, best sample of any named $\begin{array}{llll}\text { variety } . . . & \ldots & \end{array}$

2 Pulut, best sample of any named $\begin{array}{llll}\text { variety } \quad . . & \ldots & \end{array}$

3 Rice, best sample, prepared by machi. nery Rice, best sample prepared in a lesong

4 Best collection of different Padi in the ear, 10 heads in each sample

6 Best Padi and Pulut, grown in one mukim to be exhibited by the Penghulu of such mukim, and so certified by him

Each exhibitor to produce an aftidavit, sworn before a Magistrate that bis whole exhibit was grown in his own mukim last Padi season.

\section{SECTION II,-RUBBER.}

7 Para-Crepe, best sample

8 "Sheet

9 "slieet, smoked,

10 "Block (dry)

11 "Best exhibit ready for shipment. The exhibit to be one case of Crepe, one case of Sheet and one case of Scrap

iail

12 Para, Best sample of Commercial Rubber

13 Rambong, any form

14 Any other Rubber, plant from which obtained must be stated with each
(a) Cup presented by
(b)
(c)
(d)
(e)
(f) of Malaya.
NOTE. G.M.= Gold Medal.
S.M. = Silver Medal.
B.M. = Bronze Medal.

Championship" Cup presented by 'The United Planter's Issociation
Number or
quantity to
be exhibited. 1st. 2nd. 3rd.

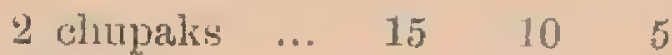

$\begin{array}{llllll}2 & \text { do. } & \ldots & 10 & 5 & 3\end{array}$

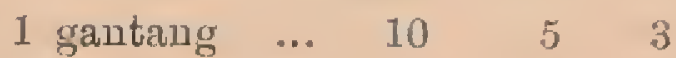

1 do. $\quad$... $10 \quad 10 \quad 5 \quad 3$

$\begin{array}{lllll}\ldots \ldots & \ldots & 20 & 10 & 5\end{array}$

6 samples of

$\begin{array}{lllll}1 \text { gantang } & \ldots & 50 & 25 & 10\end{array}$

not less than 50 lbs. each. Cup (c)

not less than $100 \mathrm{lbs}$.

in weight. $\quad \operatorname{Cup}(f)$

not less than 25 lbs. S.M.B.M.

SM.B.M. 
Nutuber ux quantity to be exhibited.
Plizizhas

1st. 2nd. : sid.

\section{SECTION III.-FOOD PRODUCTS.}

\section{SECTION IV,-SPICES, ETC.}

46 betel nuts, fresh, best sample

47 Retel nuts, dried and split, best sample

48 Sireh leaves, best sample

49 Clores, best sample
... 50 nuts

1 catty

100 leaves

... 1 catty $\begin{array}{lllll} & 4 & \$ & \\ \ldots & 5 & 3 & 2 \\ \ldots & 5 & 3 & 2 \\ \ldots & 5 & 3 & 0\end{array}$

... Cup S.MI.

... Cup S.M.

... Cuy S.M.

.. 530

.. $5 \quad \$ \quad 0$

.. $5 \quad 3 \quad 0$

... $5 \quad \ddot{3} 0$

.. 530

...5 21

... $5 \quad 2 \quad$ (1)

.. 5 : 0

.. 5 ; 0

... 5 31

.. $5 \quad 3 \quad 1$

.. 5 $\$ 31$

... 5 51

... 85

... 531

‥ $5 \quad 3 \quad 1$

‥ $5 \quad 91$

... S.M. B.M.

... S.M. B.M.

... S.M. B.M.

.. 53

.. 533

‥ 538

... S.M. T.M.

To be shown in sacks ready for shipment.

+ Cup Presented by Brinkman \& Cu.

Cup Presented by R. J. Addie. Esu.

1 Cup Presented by C. E. S. Baxendale. Esy. 
SECTION IV.-SPICES, ERC.-comt.

50 Nutmegs, fresh, best sanple

51 Nutmogs, dried,

Mace, dried, yellow, ",

Mace, dried, red.

Pepper, white,

Pepper, black.

Spices,

best collection

Patchouli, best sample

Camphor (Chmmommm Camphom)

best simple

$$
\begin{array}{lrl}
\ldots & 50 & \text { nuts } \\
\ldots & 50 & , \\
\ldots & 1 & \text { catty } \\
\ldots & 1 & , \\
\ldots & 1 & , \\
\ldots & 1 & \\
\ldots & \text { optioual } \\
\ldots & 1 \text { catty }
\end{array}
$$

$\begin{array}{rrrr} & \$ & \$ & \$ \\ \ldots & 5 & 3 & 1 \\ \ldots & 5 & 3 & 1 \\ \ldots & 4 & 3 & 1 \\ \ldots & 4 & 3 & 1 \\ \ldots & 5 & 4 & 3 \\ \ldots & 5 & 4 & 3 \\ \ldots & 10 & 5 & 0 \\ \ldots & 5 & 3 & 1\end{array}$

... optional

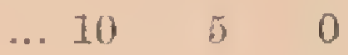

\section{SECTION V.-OILS, OIL CAKE, ETC.}

59

60

61.

62

63

64

65

66

67

68

69
Oil, citronella, best sample

. lemon grass, best sample .

, coconut,

. cingelly, teal seed, best sample

". castor, best sample ...

". clove,

"Para Kubber seed, best sanuple

" Kábu kabn seed,

\begin{tabular}{|c|c|c|c|c|}
\hline & $\operatorname{int}$ & $\ldots$ & $s$ & \pm \\
\hline 1 &, & $\ldots$ & 8 & 4 \\
\hline 1 & 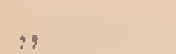 & $\ldots$ & 10 & 5 \\
\hline 1 &. & $\ldots$ & 5 & 3 \\
\hline 1 & $"$ & $\ldots$ & 5 & 3 \\
\hline 1 & ", & $\ldots$ & 5 & 3 \\
\hline 1 & ," & $\ldots$ & S.M. & B.M. \\
\hline 1 & $"$ & $\ldots$ & 5 & 3 \\
\hline 1 & ", & $\ldots$ & $\overline{5}$ & 3 \\
\hline & atties & $\ldots$ & 5 & 3 \\
\hline 5 & , & $\ldots$ & 5 & $\ddot{3}$ \\
\hline 5 &. & $\ldots$ & 5 & 3 \\
\hline & hupals & $\ldots$ & 5 & $\ddot{3}$ \\
\hline & jint of ea & & S.M. & B., 11 \\
\hline
\end{tabular}

" any other kind,

"calie, coconut,

" cake, Para seed,

" cake, Kabu liabu seed, .

Seed, Kabu kabu,

Oils, best collection

SECTION VI.GETAHS, GUMS, ETC.

$7:$ Getah Jelutong best sample

74 Getahs, best collection of local

75 Getah taban, best sample

76 Gambier, cubes ", ...

77 Gambier, block

78 Dragon's blood

79 Gums and Damars, best collection of local ...
... 10 catties $10 \quad 50$

... $\quad \frac{1}{2}$ catty of cacil $10 \quad 5 \quad 2$

... $\quad \frac{1}{3}$ catty $\quad \ldots \quad 5 \quad 2 \quad 0$

... 5 catties $\ldots 10 \quad 5 \quad 2$

$\ldots 5 \quad 5 \quad, \quad \ldots 10 \quad 5 \quad 2$

\section{SECTION VII.-FIBRES.}

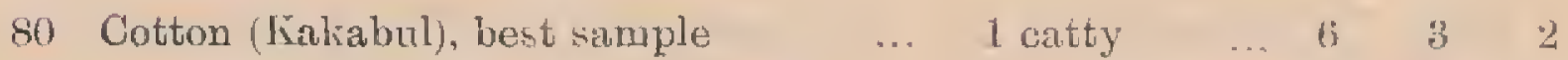

81 " any other variety, best sample $\quad 1$ catty $\quad \ldots \quad 6 \quad 3 \quad 2$

82 Fibres, best collection ... 1 bundle of S.M. B.M. each 


\section{SECTION VIII.-MISCRLLANEOUS.}

84 Bamboos,

85 Walking sticks

86 Medicinal plants, do.

$8 \%$

Do. do

\section{(umprepared)}

(open)

(Natives only) ..

$\begin{array}{ccccc}\text { optional } & \ldots & 10 & 5 & 3 \\ \text { " } & \ldots & 5 & 2 & 1 \\ 1 \text { of each } & \ldots & 5 & 3 & 2 \\ \text { optional } & \ldots & \text { S.M. } & \text { B3.M. } & \\ ,, & \ldots & 8 & 4 & 2\end{array}$

\section{DIVISION B.}

FLOWERS, FRUITS AND VEGETABLES.

$\frac{\dot{0}}{0}$

\section{SECTION I.-ORNAIMENTAL} FOLIAGE PLANTS IN POTS.

Aroids other than Caladiums

Do. bert spccimen

Caladiums

Do. best specimen

Coleus

Do. best specimen

Crotons

Do. best specimen

Dracaenas

1). bust specimen

Ferus. (any variety), distinct

Do. (adiantums). do.

Do.

do.

do.

Do. (any variety), best specimen

Marantas and Calatheas

Do. best specimen

Palms, distinct kiuds

Do. best specinen

Panax and Aralia
Number or: quantity to be exllibited.

Prizks.

1st. 2 nd.

His Excellency The Governor's Cup will be awarded to the best general exhibit in Division $A$. 


\section{Nature of Exinibit, \\ SECTION I.-ORNAMENTAL \\ FOLIAGE PLANTS IN POTS.-Cont.}

Number or quantity to be exhibited.

Prizess.

1st. 2nd.

107 Selaginella, distinct kinds

108 Selaginella, best specimen...

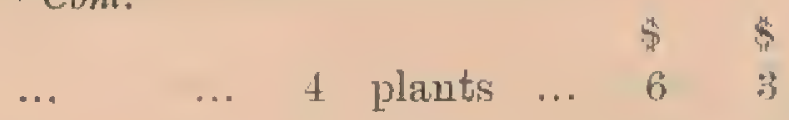

109 Any ornamental Foliage Plant not inchied in the above $\quad . . \quad$... ..

Do. best specimen

111 Foliage Plant of any limd, best specimen

112 Bost collection of Foliage Plants, distinct kinds

$$
\begin{array}{cccccc}
\ldots & 6 & , & \ldots & 5 & 0 \\
\ldots & 1 & , & \ldots & 3 & 0 \\
\ldots & 1 & , & \ldots & 5 & 0 \\
& & & & & \\
\ldots & & & \ldots & \text { (u) } \text { Cup }
\end{array}
$$

\section{SECTION II.- PLANTS IN FLOWER, IN POTS.}

11: Amaranthus

114 Amaryllis and Lilies

115

116

Asters

Balsams...

$\cdots$

Cammas, Orchidaceons Flowered

\section{Cannas}

Canna, best specimen

Cocks combs

Chrysanthemums

124

$1 \cdot 5$

126

127

128

129

130

131

132

Dahlias

Dianthus, (Indian Pinks)

Eucharis

Gloxinias

Orchids

Do.

Orchid, best specimen

Carnations

Phlox

Petunias

Roses

133

134 Kinnias

135 Best collection of flowering plauts dis tinct lrinds, not less thin

136 Rest plant in Flower in Show not in. cluded in above ...

$\begin{array}{lllllll}\ldots & \ldots & b & , & \ldots & 4 & 2\end{array}$

$\begin{array}{llllll}\ldots & 6 & \prime & \ldots & 4 & 2\end{array}$

$\begin{array}{llllll}\ldots & 6 & & & & \\ \ldots & 4 & 4\end{array}$

$\begin{array}{llllll}\ldots & 6 & , & \ldots & 4 & 2\end{array}$

$\begin{array}{llllll}\ldots & 6 & 4 & \ldots & 4 & 2\end{array}$

$\begin{array}{llllll}\ldots & 6 & , & \ldots & 4 & 2\end{array}$

$\begin{array}{llllll}\ldots & 1 & , & \ldots & 3 & 0\end{array}$

$\begin{array}{llllll}\ldots & 6 & \cdots & \ldots & 4 & 2\end{array}$

$\begin{array}{llllll}\ldots & 6 & 1 & \ldots & 4 & 2\end{array}$

$\begin{array}{llllll}\ldots & 6 & 1 & \ldots & 4 & 2\end{array}$

$\begin{array}{llllll}\ldots & 6 & 1 & \ldots & 4 & 2\end{array}$

$\begin{array}{llllll}\ldots & 6 & , & \ldots & 4 & 2\end{array}$

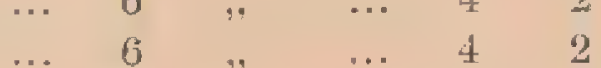

$\begin{array}{llllll}\cdots & 6 & 1 & \ldots & 4 & \\ \cdots & 6 & \cdots & \ldots & 10 & 5\end{array}$

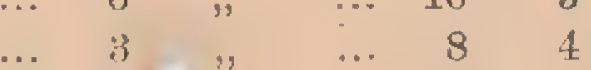

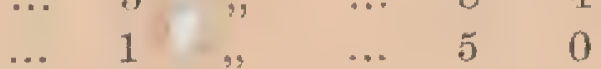

$\begin{array}{llllll}\ldots & 6 & , & \ldots & 4 & 2\end{array}$

$\begin{array}{llllll}\ldots & 6 & 1 & \ldots & 4 & 2\end{array}$

$\begin{array}{llllll}\ldots & 6 & , & \ldots & 4 & 2\end{array}$

$\begin{array}{llllll}\ldots & 6 & , & \ldots & 5 & 3\end{array}$

... 1 1

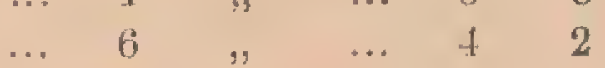

SECTION III.-PLANTS WHETHER

$$
\begin{array}{lll}
6 & \text { linds ... } & \text { (b) cup } \\
1 & \text { plant ... } & \text { (c) cup }
\end{array}
$$

IN FLOWER OR NOT, IN POTS.

137 Begonias, distinct kinds

138 Begontia, best specimen

139 (iroup of plants arranged for effect in space not excerding $9 \mathrm{ft}$. square.

140 (troup of Chinese or Tapanese plants, of fantastic shape, including dwarfed plants.

$\begin{array}{cccc}6 \text { plants } & \ldots & 5 & 3 \\ 11 & \ldots & : 3 & 0 \\ \text { optional } & \ldots & 8 & 5 \\ & & & \\ , & \ldots & \text { (d) } & \text { cup }\end{array}$




\title{
ADDENDA TO PRIZE LIST.
}

\author{
Division A.
}

\section{SECTION 1.-PADI.}

CLASS.

I A. Padi, light - to $4 \frac{L}{2}$ months old $\quad \ldots \quad \ldots \quad \$ S, 4,2$.

I B. Padi, medium-to 5 months old _. $\quad \ldots \quad 8,4,2$.

I C. Padi, heavy-6 months old $\quad \ldots \quad \ldots \quad 3,4,2$, 


\section{SECTION IV. CUT FLOWERS AND} TABLE DECORATIONS.

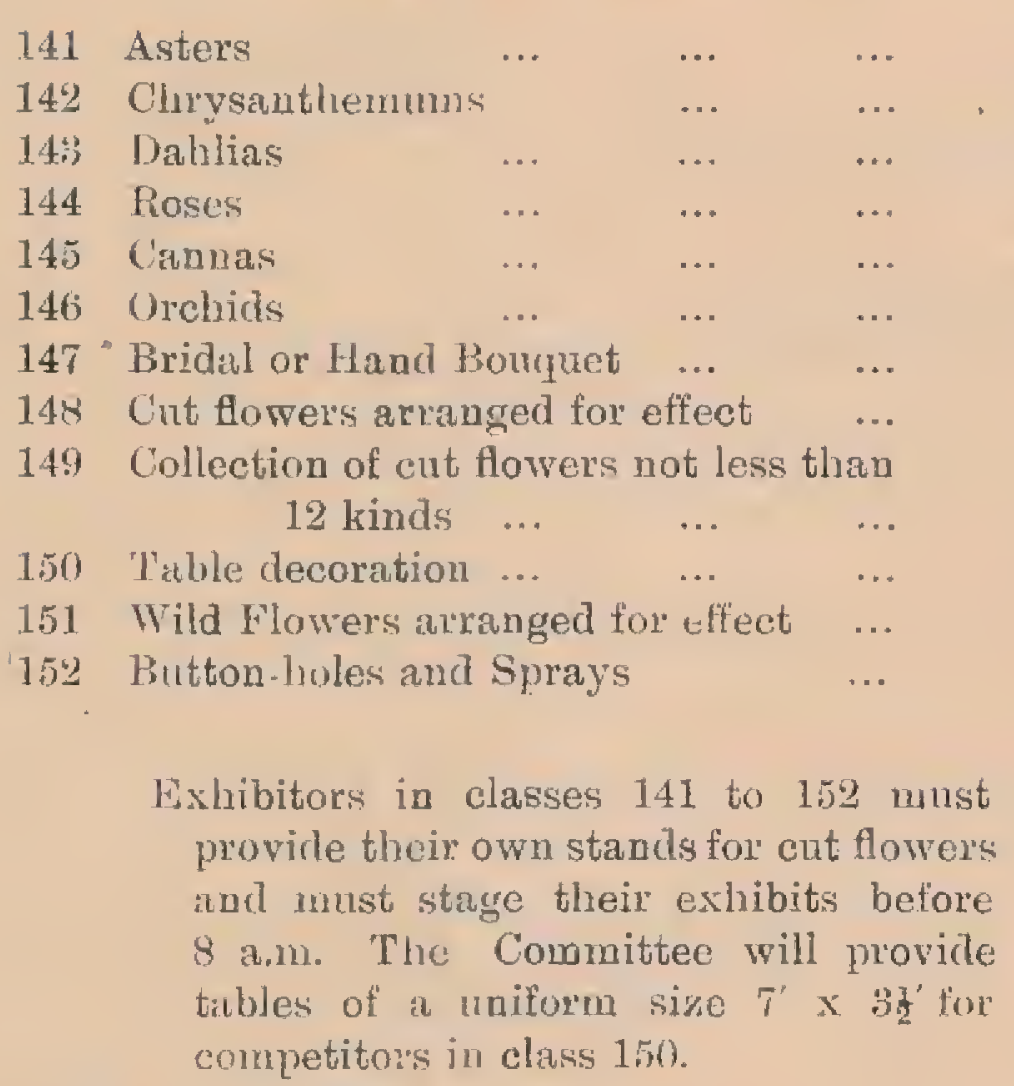

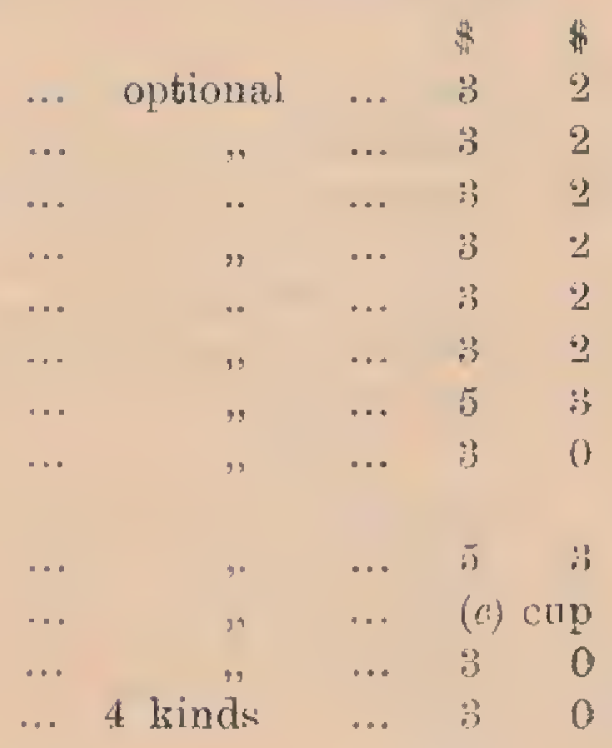

\section{SECTION V.-FRUITS.}

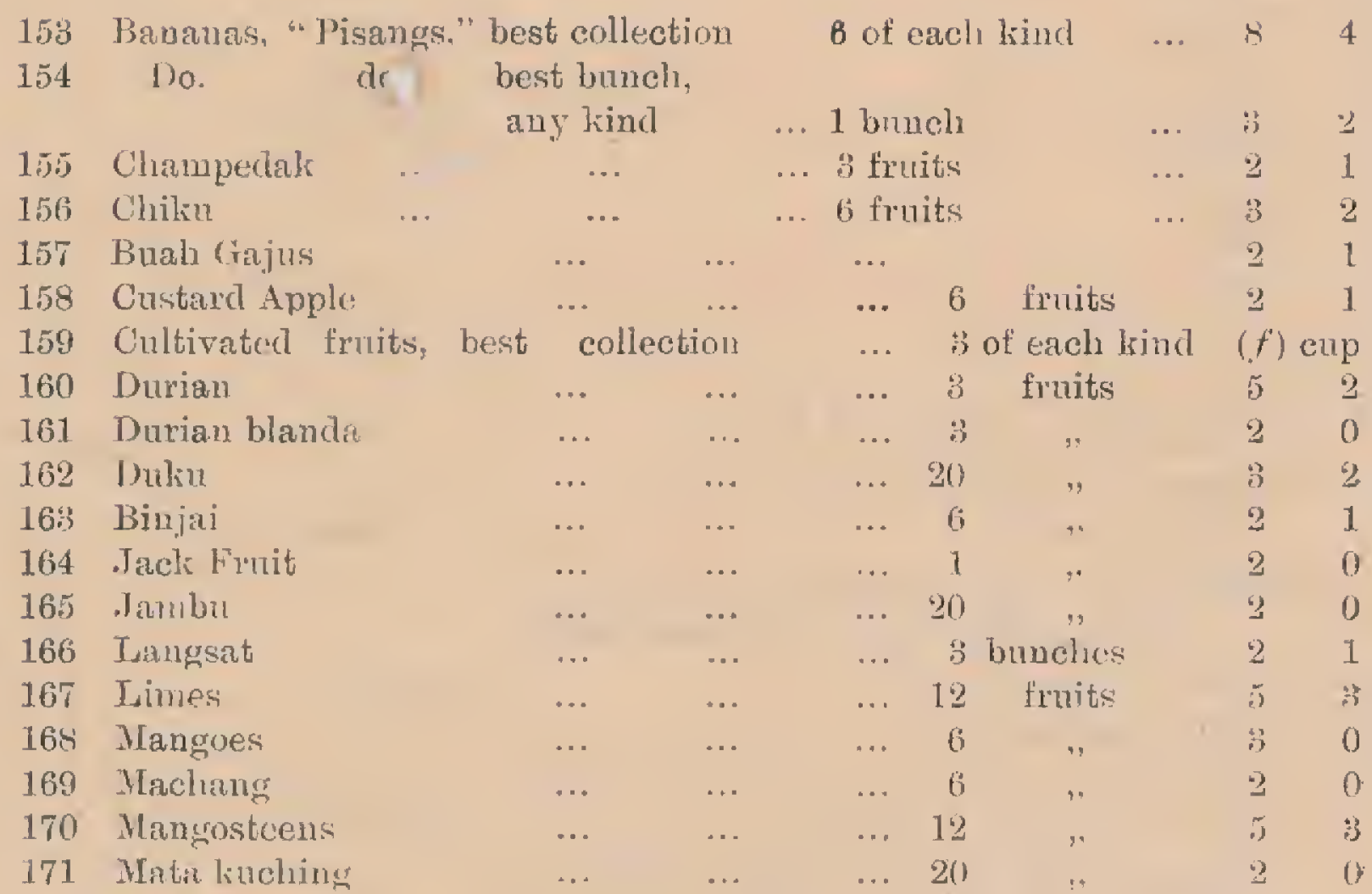


SECTION V.-FRUITS.-Cmt.

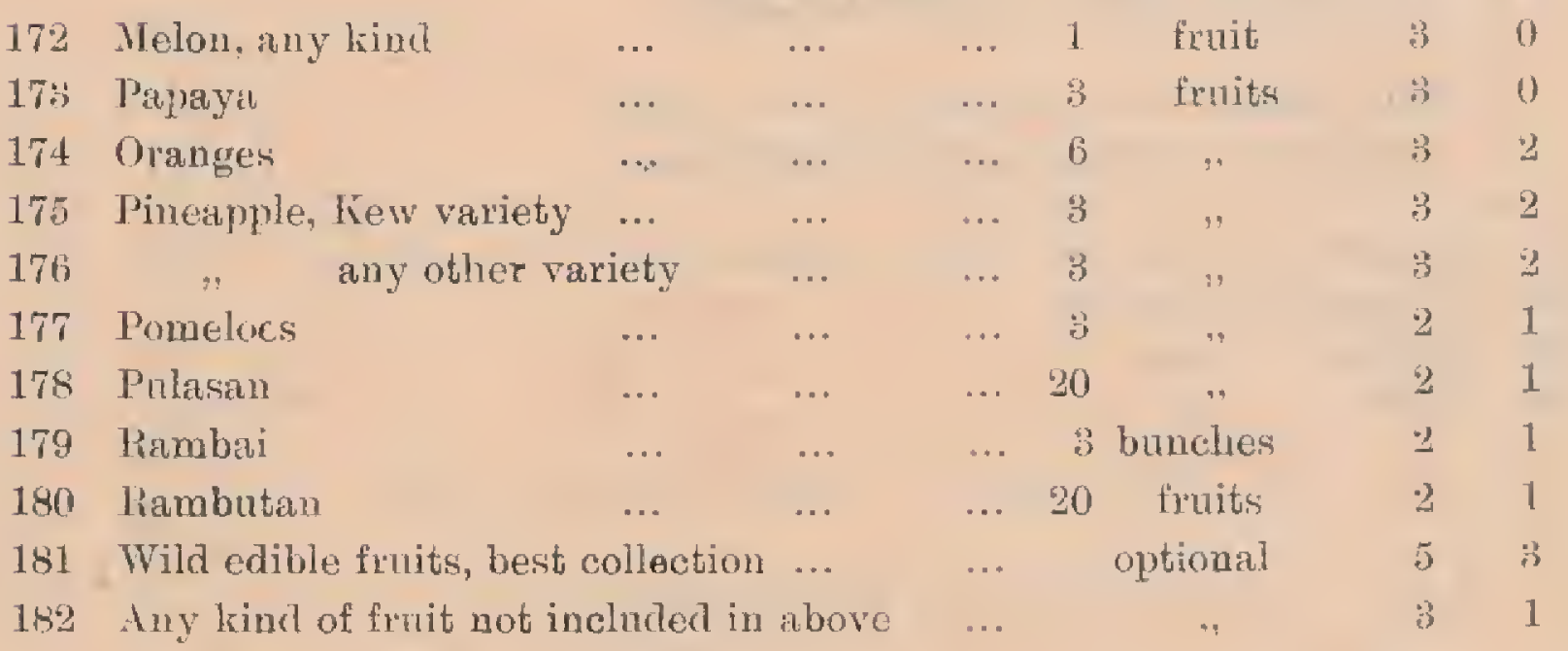

\section{SECTION VI.-VEGETABLES.}

\begin{tabular}{|c|c|c|c|c|c|}
\hline 3 & Articholies & $\ldots$ & $\ldots$ & tubers & 2 \\
\hline & Bectroot & $\ldots$ & $\ldots$ & 6 specimens & 3 \\
\hline & Benny funits or chochu & $\ldots$ & $\ldots$ & " & 2 \\
\hline & Brinjals & $\ldots$ & $\cdots$ & 6 & 2 \\
\hline & Beans, best collection & $\ldots$ & $\ldots$ & optional & : \\
\hline & Cabbages & $\ldots$ & $\ldots$ & $\dddot{g}$ speciuneus & $\ddot{2}$ \\
\hline & Chillies, best collection & .. & $\cdots$ & 30 of each & $: ;$ \\
\hline & Lincumbers & $\ldots$ & $\ldots$ & 6 specimens & ; \\
\hline & \multicolumn{3}{|c|}{ Herbs usd in curres it sambals, best collection } & 1 bundle of each : & $::$ \\
\hline & Ladies' fiugers & $\ldots$ & $\cdots$ & 6 specimens & 2 \\
\hline & Lettuces & $\ldots$ & $\ldots$ & 4 & 2 \\
\hline & Marrows & $\ldots$ & $\cdots$ & 6 & $\because$ \\
\hline & \multicolumn{3}{|c|}{ Onions, shallots and garlic. best collection } & \multicolumn{2}{|c|}{1 bundle of each: :3 } \\
\hline & Pumplins & $\ldots$ & $\ldots$ & $\because$ specimens & 2 \\
\hline & Radishes & $\ldots$ & $\ldots$ & - 10 & 2 \\
\hline & Spinach & $\ldots$ & $\cdots$ & optional & 2 \\
\hline & T'omatoes, best dish & $\ldots$ & $\ldots$ & 6 & $\therefore$ \\
\hline & \multicolumn{3}{|c|}{ Vegetables, best collection, European, at least } & \multicolumn{2}{|c|}{$\begin{array}{l}: 3 \text { specimens of } \\
\text { each (u) Cu }\end{array}$} \\
\hline & \multicolumn{3}{|c|}{$\begin{array}{l}\text { Vegetables and herbs for making a Salad, } \\
\text { best collection } \quad \ldots\end{array}$} & & Cup. \\
\hline & \multicolumn{3}{|c|}{$\begin{array}{l}\text { Water-melons, Gourds, Squasiles, Luffas, } \\
\text { etc, best collection }\end{array}$} & 1 of vach. & 5 \\
\hline & \multicolumn{2}{|c|}{ Yams, Kladis, best collection } & ... & 6 & 5 \\
\hline & \multicolumn{3}{|c|}{ Any Tegetnble not in the above } & optiounl & 3 \\
\hline
\end{tabular}




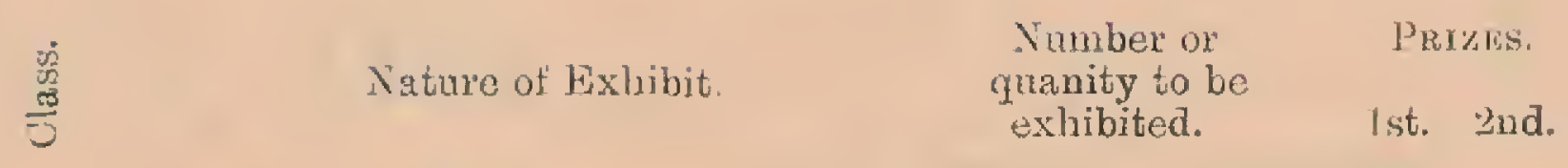

\section{SECTION VII.-PRESERVED FRUITS AND VEGETABLES.}

All exhibits in the lollowing classes nust be grown or prepared by the exhibitors.

205 Preserved Fruits, best sample, any method

206 Chutney, best sample

207 Pickles do.

208 . Tellies do.

Entrie's close in Division B, Angust 8th.

(a) Cup presenter by F. E. Jago, Esq.

(b) $"$ St. T. B. Jown, Esy.

(c)

(d) $\quad$.. $\quad$ F. E. Jago, Esq.

(c) " $\quad$ R. Scoular, Esq.

(j)

$(g)$

(h)

\section{DIVISION C.}

\section{STOCK AND DAIRY PRODUCE.}
Class.
Nature of Exhibit.
PRIYK.
1st. 2nd. 3rd.

\section{SECTION I.-CATTLE.}

209 Bull

210 Cow and Calf (Australian exchded)

212 Pair of draught bullocks, Siamese

$21:$ Do. do. Indian $\quad \ldots$. ...

214 Champion animal in classes 210 \& 211

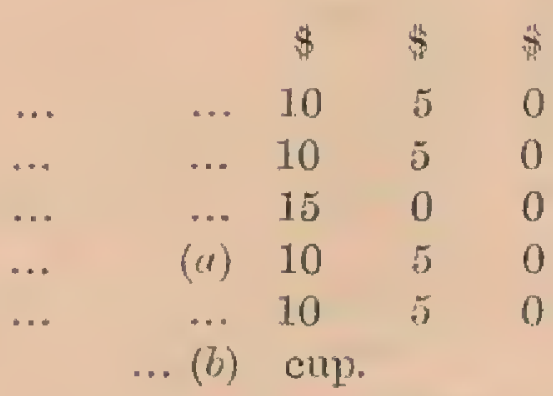

SECTION II.-BUFFALOES. 
Class.

Nature of Exhibit.

Plíis:.

1st. 2nd. sid.

\section{SECTION III. GOATS AND SHEEP.}

217

218

219

220

221

222

228

224

He Goat

She Goat with kits

Ram Sheep

Hwe Slieep

Best pen of forl Sheep Malay breed

Do. do any other breed

Champion Goat in classes 217 \& 218

Do. sheep do. 219, 220, 221,222 .

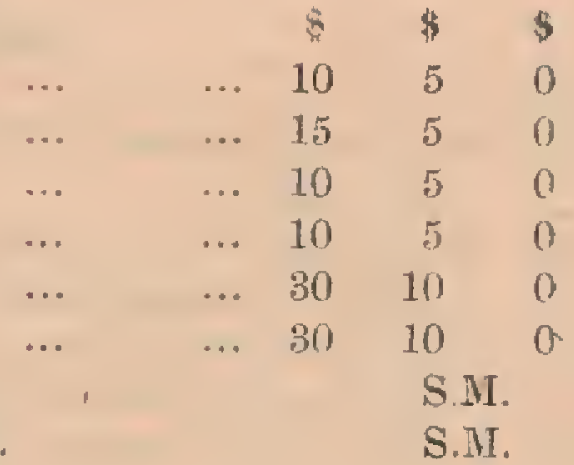

\section{SECTION IV.-PIGS.}

225 Boar

226 Sow

227 Best pen of four pigs

228 Champion pig in classes 225, 226, 227

$\begin{array}{ccccc}\ldots & \ldots & 15 & 10 & 5 \\ \ldots & \ldots & 15 & 10 & 5 \\ \ldots & \ldots & 20 & 10 & 0 \\ & \ldots & & (c) & \text { cup. }\end{array}$

\section{SECTION V,-POULTRY Etc.,}

229 Bantam Cock \& Hen

230 Malay Cock \& Hen

231 Malay Game Cock \& Hen

232 Chinese Cock \& Hen

$23:$ Cock and Hen any other breed

234 Best pen of six fowls for table use

$\begin{array}{rrrrrr}\ldots & \ldots & \ldots & 5 & 3 & 0 \\ \ldots & \ldots & \ldots & 5 & 3 & 0 \\ \ldots & \ldots & \ldots & 5 & 3 & 0 \\ \ldots & \ldots & \ldots & 5 & 3 & 0 \\ \ldots & \ldots & \ldots & 5 & 3 & 0 \\ \ldots & \ldots & \ldots & 10 & 5 & 0 \\ 2 & & & & & \end{array}$

285

Best fowl in classes $229,230,231,2 \hat{3} 2$. 238,234

236 Muscovy duck and drake

237 Duck and Drake, any other breed

238 Gander and Goose

239 Turkey cock and hen

240 Pair of pigeons

241 Best cage bird

242 Rest collection of cage birds

243 Rabbits, buck and doe

\section{SECTION VI.-DAIRY PRODUCE.}

244 Best sample of butter locally proctuced,

Medal and ... 10

(d) c..p.

245

Do. milk in bottle,

Medal and ... 10

..

Best collection of 12 eggs, fowls, ducks or turkeys optional

246A Honey in Comb, Best Sample

2461: Honey, clear,

(a) Presented by E. G. Broadrick, Hisq.

(b) Presented by W. Patchitt, Esq.

(c) P'resented by Seah Fing Kun, Esq.

(d) Prescnted by A. 'I. Bryant, Ėsণ1.

Entries close in Division C, August 8th. 


\section{DIVISION D.}

\section{SECTION I.-HORSES.}

Class.

247 Polo Pony registered 14.2 or under. To be shown in saddle and tested by judges.

248 Gentleman's Hack over 14.2. To be shown in saddle at wall, trot and canter, mounting and dismounting.

249 Gentleman's Hack 14.2 and under. To be shown in saddle at walk, trot and canter, mounting and dismounting.

250) Single Harness Horse over 14.2 To be shown in harness in any description of vehicle. Open only to Australian and Native Bred Horses.

"251 Single Harness Pony 14.2 and under. To be shown in barness in any description of vehicle. Open only to Australian and Native Bred Ponies.

25.2 Single harness horse, any height. 'T'o be shown in any description of velicle. Open only to horses inported from the United Kingdom.

253 Lady's Hack, any height. 'To be shown in saddle at walk, trot and canter and ridden by a lady. This event will be divided into two classes if there are sufficient entries. One for horses over 14.2 and one for ponies 14.2 and under.

254 . Irmping conpetition for horses over 14.2. To be ridden over 4 or 5 jumps, judging on performance only.

255 Inmping competition for ponies 14.2 and under. 'l'o be ridden over 4 or 5 jumps, judging on performance only.

256 High jumping contest for horses of any height.

257 Pouble turnont of any description, pairs, tandems, etc.

258 Gharry turnout: bona tide property of a licensed Gharry owner. (Australian horses barred.)

259 Gharry turnout (Native bred ponies bar'ed.) Ponies that compete in this event will not be eligible for other harness events.

260 Champion Horse in the Slow over 14.2 to be shown on a halter.

261 Champion Pony in the Show 14.2 and under to be shown on a lialter.

'lwo prizes will be presented for each event provided there are five or more horses or ponies competing.

No horse or pony may take more than two first prizes, or one first and two sccond, exclusive of a championship prize.

Winners of all cvents with the exception of classes 258,259 must compete for the championship prizes.

"A Native Bred Horse or Pony" shall mean any horse or pony bred in India, the Malay Peninsnla, Burma or Dutch fndies.

EXTRIES must reach the Hon. General Secretary, on or before Saturday Botl. July, 1910.

Wintrance foes $\$ 2$ for each class with the exception of class $25 \%$ which is free and elass $25 \%$ for which the entrance fee will be $\$ 2$ per pair.

ENIRY FORMS may be obtained from the Hon. General Secretary or from H. Elphick, Esq., the Hon. Secretary, Division "D" (Horses), and from the Secretaries of the varions 'Iurf Clubs throughont the Straits Settlements and the Feterated Malay States. 


\section{SECTION II-DOGS.}

Glass.

262 Smooth Haired liox Ierrier Dog.

263 Smooth Haired Fox Tervier Bitch.

264 Rough Haired Fox Terrier Dog or Bitch.

265 Chow Dog.

266 Chow Bitch.

267 Spaniel, Dog or Bitch.

268 Irish 'Terrier, Dog or Bitch.

269 Best Puppy under 6 months old. Any Breed.

270 Dog or Bitch any other breed.

271 Ghampion Dog in the Show.

272 Champion Bitoh in the Show.

'I'wo prizes will be presented for each event provided there are tive ol' more dogs competing.

No dog may take more than two first prizes, or one first and two second exchusive of a championship prize.

Winuers of all events wust compete for the championship prizes.

ENIRIES must reach the Hon: Gen. Secretaly, on or before Saturday 30th July, 1910.

Entrance feo 2 for each event.

ENTRY FORMS may be obtained from the Hon: Gen. Secretary or from F. Hilton, Esq., the Hon. Secretary, Division "D" (Dogs), and from the Secre. turies of the various Turf Clubs throughout the Straits Settlements and the Eederated Malay States.

Entries close in Division D, July 30th.

\section{DIVISION E.}

\section{NATIVE INDUSTRIES AND MANUFACTURES.}

\section{Nature of Exlibit. \\ SECTION I.-METAL WORK.}

273

274

275

276

277

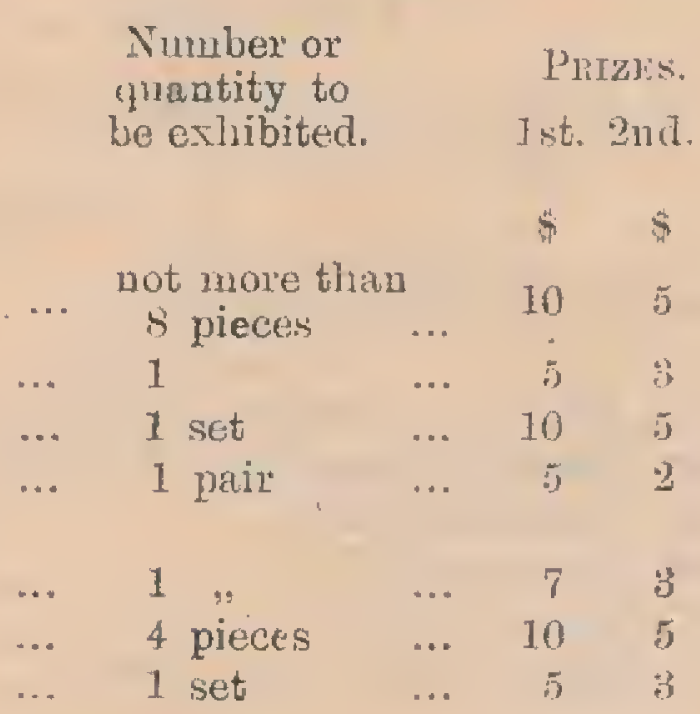




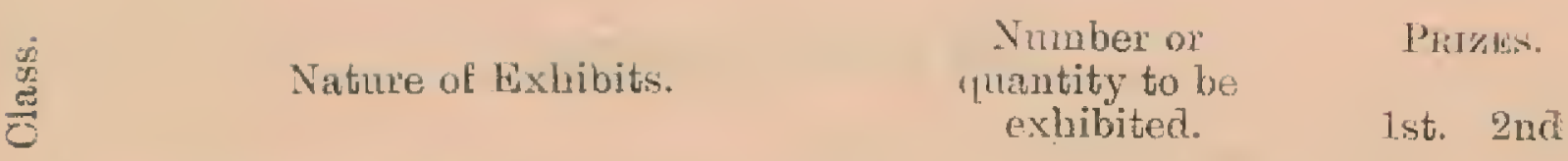

SECTION I.-METAL WORK.-Cont.

280 Any kind of wetal trork not included in above

281 Kris with sheath

282 Tumbol lada with sheath

288 Parang Do. do.

284 Golok Do. do.

285 Collection of tin-ware

286 Malay gong

287 Malay spears

288 Collection of Chinese jewellery

289 Do. of Indian "'
290 Do. of Chinese joss table

290 Do. of Chinese joss table

291 Native made lamps and candlesticks

$\begin{array}{lccrc} & & & \$ & \$ \\ \ldots & \text { optional } & \ldots & 5 & 3 \\ \ldots & 1 & \ldots & 5 & 3 \\ \ldots & 1 & \ldots & 5 & 3 \\ \ldots & 1 & \ldots & 5 & 5 \\ \ldots & 1 & \ldots & 5 & 3 \\ \ldots & \text { not more than } 8 & 10 & 5 \\ \ldots & 1 & \ldots & 5 & 2 \\ \ldots & \text { not more than } & 6 & 5 & 3 \\ \ldots & 8 \text { pieces } & \ldots & 10 & 5 \\ \ldots & 8 \text {.. } & \ldots & 10 & 5 \\ & & & & \\ \ldots & \text { optional } & \ldots & 10 & 5 \\ \ldots & 2 \text { pieces } & \ldots & 5 & 3\end{array}$

\section{SECTION II.-WOOD CARVING.} ETC.

292 Wood carving

293 Model of a Malay house

294 Set of models of snares and traps (land)

295 Set of motels of fish traps and stakes (sea)

296

Set of models of fish traps and stalies (river)

297

298

\section{SECTION III.-EMBROIDERY AND WEAVING}

299 Malay embroidery ( 1 ) large pieces (mats curtains (ve.,)
(b) small pieces

(slippers, caps \&c.,)

300 Malay lace (a) pillow lace

(b) crochet lace

301 Clinese embroidery, best specimen

302 Sarong (silk and gold Telepoli)

303 Sarong (mastuli)

304 Sarong (silk)

305 Sarong (thread)

306
Malay scarves \& head wear

$\begin{array}{lllrl}\ldots & 1 \text { piece } & \ldots & 10 & 5 \\ \ldots & 1 \text { model } & \ldots & 10 & 5 \\ \ldots & 1 \text { set } & \ldots & 7 & 3 \\ \ldots & 1 \text { set } & \ldots & 7 & \xi \\ & & & & \\ \ldots & 1 \text { set } & \ldots & 7 & 3 \\ \ldots \text { optional } & \ldots & 10 & 5 \\ \text { not more than } 6 & \ldots & 5 & 3\end{array}$

$\begin{array}{lllll}\ldots & 2 \text { pieces } & \ldots & 15 & 10\end{array}$

$\begin{array}{lllll}\ldots & 2 \text { pieces } \quad \ldots & 10 & 5\end{array}$

$\begin{array}{llll}\ldots & 1 \text { piece } \quad \ldots & 10 & 5\end{array}$

$\begin{array}{lllll}\ldots & 1 & & & \end{array}$

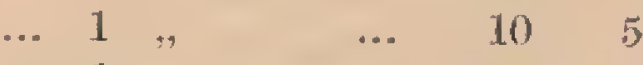

$\begin{array}{llllll}\ldots & 1 & & & & \end{array}$

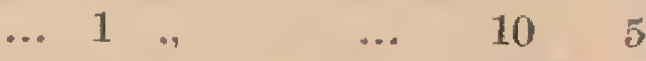

$\begin{array}{lllll}\ldots & 1 & 1 & \ldots & 10\end{array}$

$\begin{array}{lllll}\ldots & 1 & & & \\ & \ldots & 5 & 8\end{array}$

$\begin{array}{llll}\ldots & 3 \text { pieces } \quad . . & 15 & 10\end{array}$ 


\section{SECTION IV.-BASKETS, MATS,} ETC.

307

305
Onamental baskets (rattan)

Unanemial baskets (mengkuang)

Best collection of articles made trom nengkuang

Hats (mengkuang)

Ilats plain, hotnse wear

Wats plain, for drying padi (tikar hampar:

Mats coloured, house wear

Mats, lancy varietics

Bamboo or rattan furniture A cluicks not more than 4

articles $\ldots \quad 5 \quad$;

$\begin{array}{llll}\ldots & \text { optional } & \ldots & 10\end{array}$

... 1 piecs 7 j

... not mole tlian + articles

.. 1 piece ... 5 5

... not more than $4 \ldots$ j 3

... not wore than $4 \ldots$..

not more than 4

$$
\text { pieces }
$$

\section{SECTION V.-MISCELLANEOUS.}

:36 Coconut shell bowl or vessel (gelok)

317 Jay's (bityong)

3180 uremental pottery best collection

... 1. piece

... 1 ,

ojs Orumental portery, best collection

not mole tlian

\pm pieces

$$
\text { di. }
$$

... optional

320 Fishing lines

321 Fisling nets

322 Collection of nutive made toys of any

$$
\text { sort }
$$

523 Paper lantems or ormanents

... optional not more thian.

... 10

10

\section{SECTION VI.-FOR MALAY SCHOOLS ONLY.-}

321. Wialay mats

$\$ 25$ Woor-carving ...

326 Einbroidar

i27 Sarong

328 Collection of miniature Malay nodels

329 Best article of ratian fumiturc

$\$ 30$

$3: 1$
.. ij pieces

... 1 piece

... 1 ,

... 1,
..

.

.. 10 5

.. 53

‥

. $5-5$




\section{DIVISION F.}

AGRICULTURAL IMPLEMENTS AND MISCELLANEOUS.

\section{Nature of Exhibit.}

Padi Implements (collection) Agricultural Implements. than for Padi ox Rubber Do. European made Plough suitable for Estate work Reaping Machine Tree stump extractor ... ... Spraying Machine ... ... Hachinery in connection with the nreparation of linbber to be exhibited at Work ... ... 'The best collection of appliances for tapping and collecting Rubber Bullock Cart

Rooting tiles, locally made Do. imported

Flooring tiles. locally mate 1). jomported

Íutrely, MLalayau. Jand-mado

Do. funcy

Baskets, Agricultural

Casks, 'T'ubs, etc.

Carriage, fon wheeled, locally made Do. two Do. suitable for Estate work. locally made

Flover Pots. Iu sizes 6 of ea size

Hack Ghary'y, locally made Mining Tools \& Implements Miscellaneous Garden Furniture Number or
quantity to
be crabibed.

optional
(11) Cup S...I.

(b) Cin S H.

$S$ II. B.MI.

$10 \quad 5$

105

$10 \quad 5$

optional (v) (t.MI. (1) Cill

S.MI. B MI. 105

(v) Cup B.M. 10) 5

105

105

$10 \quad 5$

$10 \cdot 5$

$10 \quad 5$

105

S M. B.M.

S.M. B.M.

S.X. B.MI. 105

optioual (f) Cup 13.M. 105

S.MI. F.M. $10 \quad 5$

(a) Cup presented by Messis. Guthie \& Un. Lta.

(b) Do. do. Messrs, Piley Hargieaves it Co., Ltd.

(c) Gold Mcdal presented by Messrs. McAlister id ('G., Ltd.

(d) Cup presented by A. I). Machado, Esq.

(e) Do. do. Dr. D. J. Galloway.

(f) Do, do. K. A. Stevens, Esq.

Entries clost, Disision F., August Sth. 


\section{List of Prizes Presented.}

All the prizes for Padi are kindly presented by the Chinese Chamber of Commerce.

Additional prizes will be given by the Committee to the value of $\$ 200$.

\section{RUBBER.}

Ci.Ass,

IST PRIZE, CLI.

2ND PRIZE, CUP.

PRESENTED BY

PRESENTED BY

7. Para crence Messrs. Barlow \& Co. Messrs. Hogg \& Co.

8. Para sheet? Malay Peninsula

Agricultural Asso. Ruffles Hotel

9. Parasheet smoked H. N. Ridley, Esq. E. M. Janion. Esq.

ro. Para block Dry The Straits Times. Messre, Hoge \& Co.

Ir. Best exhihit ready Messsrs. Tan Jiak for shipment Kim, Tan Jiak

Clwo and Tan Jiak Hoe

"J. I. inle \& Ca.

12. Best sample of

H. H. the Sultan

Commercial Rubber of Johore and Johore Planters. Tan Chey Yan, Esq.

I3. Rambong Chan Kang Swi

14. Any other rubleer Kelantan Planters Association

144 Inmanufactured? F. Valpy, Essc!. Pala Scrap ;

Championship Cup for best single exhibit in the above presented by The Planter's' Asmociation of Mulsya. 


\section{NON-MALAYAN RUBBER OF ANY KIND.}

Cups by Gino Pertile, Esq., and Dr. Lim Boon Keng.

\section{FOOD PRODUCTS.}

19. Copra, 1st I’rize Cup, presented by Messrs. Brinkman \& Co.

20. Do. Ist

R. J. Addie, Esq.

21. Do. Ist

C. E. S. Baxendale, Esq.

Best general exhtbit in Division $A$. Prize presented by H. E. The Governor. S.S.

\section{FLOWERS, FRUITS \& VEGETABLES.}

CUPS PRESLNTED BY

112. Best collection of Foliage plants F. E. Jago, Esq.

135.

Do.

flowering plants

St. V. B. Down

140. Groups of Chinese or Japanese
plants of fantastic shape
F. E. Jago, Esq.

160. Table Decoration
Jst Prize
R. Scoular, Esq.
2nd Prize
Messrs Robinson \& $\mathrm{Co}$.

159. Cultivated fruits

Anonymous

200. Europen Verotables

Messrs, Robinson \& Co.

\section{LIVE STOCK.}

209 Bull
2 I0 Cow and Calf ' Ist prize presented by J. R. Belilios \& Co. 


\section{POULTRY.}

212 Pair of draught bullocks, Siamese 1st prize presented by

E. G. Broadrick, Esq.

214 Champion cow, - Cup presented by W. Patchitt, Esq.

228 " pig - " , " Seah Eing Kun, Esq.

229 Bantam Cock and Hen. Ist prize presented by

Dr. Croucher, Esy.

231. Malay Game Cock and Hen. Ist prize presented by

Di. Croucher, Esq.

233A Houdans $\$ 10$

B Plymouth rocks 10

C Polisin 10

D Brahmas 10

E Orpingtons $\quad$ 10

Prizes presented by the Singapore Poultry Farm.

I. Leghorns I0

G Wyandotes 10 !

234 Best pen of 6 fowls for table use 1st prize presented by

T. R. Nicholson, Esq.

235 Best fowl Cup presented by A. T. Bryant, Esq.

245. Best Milk, in bottle. - Ist prize presented by Dr. Fowlie

\section{MACHINERY, Etc.}

333 Agricultural Implements, - Cup presented by Messis. Guthric \& Co., Ltd.

334 Agriculumal Implements(European macle). Cup presented by Messrs. Riley Hurgreaves \& Co., Ltd.

339 Rubber Michinery at work. (Gold medal presented by Messrs. McAlister \& Co., Ltd. Cup presented by

(Tin Kheam Hock, Esq.

342 Ronfing tiles (local). - Cup meented by Dr. Galloway.

35. Garden Fumitude. Cup presented by K. A. Stevens, Esq. 



